\title{
Protein NMR studies of two systems involved in bacterial pathogenicity
}

\author{
Dissertation \\ zur Erlangung des Doktorgrades \\ der Mathematisch-Naturwissenschaftlichen Fakultäten \\ der Georg-August-Universität zu Göttingen
}

vorgelegt von

Sigrun Rumpel

aus Göttingen

Göttingen 2006 
D7

Referent: Prof. Dr. Christian Griesinger

Korreferent: Prof. Dr. Axel Zeeck

Tag der mündlichen Prüfung: 01.11.2006 


\section{Zusammenfassung}

Die vorliegende Arbeit beinhaltet neue Strukturdaten über bakterielle Proteine, die an der Transkriptionsregulation und Proteinsekretion beteiligt sind, sowie einen Beitrag zur Verbesserung der Strukturaufklärung von homodimeren Proteinen mittels NMRSpektroskopie. Ein Hauptaugenmerk liegt auf der Untersuchung von zwei homodimeren bakteriellen Proteinen: Dem Transkriptionsfaktor CylR2 aus Enterococcus faecalis und dem Chaperon CesT aus enteropathogenem Escherichia coli.

Enterococcus faecalis hat sich zu einer Hauptursache von Infektionen in Krankenhäusern aufgrund von Antibiotikaresistenzen entwickelt, wobei die schwere der Infektion mit einem von Enterococcus faecalis sekretierten Protein, dem Cytolysin, zusammenhängt. Die Produktion dieses Cytolysins wird von einem zwei-Komponenten CylR1/CylR2 System über einen autoinduzierten Quorum-Sensing Mechanismus reguliert. In dieser Arbeit wird gezeigt, dass das regulatorische Protein CylR2 ein rigides Dimer formt. Die Kristall- und NMR-Struktur sind im wesentlichen identisch. Jedes Monomer besteht aus einem fünf Helix-Bündel, das ein Helix-Turn-Helix DNA-Bindingsmotiv enthält und durch ein zweisträngiges antiparalleles $\beta$-Faltblatt verlängert ist. Die Lösung der NMR-Struktur erfolgt mit Hilfe einer neuartigen Strukturbestimmungsmethode für homodimere Proteine. Um die beiden Untereinheiten als rigide Körper aneinander zu docken, werden experimentelle residuale dipolaren Kopplungen der Rückgrat-Amide und intermolekulare, langreichweitige Distanzen, die über paramagnetische Relaxationsverstärkung ermittelt werden, verwendet. Eine Modellstruktur für CylR2 im Komplex mit seiner spezifischen palindromischen DNA innerhalb des Cytolysinpromotors wird basierend auf der Veränderung der chemischen Verschiebungen von CylR2 bei DNA-Bindung erstellt. Diese Ergebnisse deuten auf eine Rolle von CylR2 als Repressor der Cytolysintranskription hin.

Enteropathogenes Escherichia coli ist eine Hauptursache von Durchfallerkrankungen, die durch Sekretion bakterieller Proteine über ein Type III Sekretionssystem in die menschliche Wirtszelle ausgelöst werden. CesT ist an dem Sekretionsmechanismus beteiligt indem es Effektorproteine vor der Sekretion spezifisch im bakteriellen Cytoplasma bindet und dadurch den Effektor in einem sekretionsbereiten Zustand hält. Die genaue Funktion des Chaperons und damit der Mechanismus der bakteriellen Type III Proteinsekretion ist noch ungeklärt. Mittels dipolarer Kopplungen wird gezeigt, dass CesT in Lösung, anders als im Kristall, eine den homologen Proteinen ähnliche Struktur ausbildet. Viele hydrophobe Interaktionen werden für die Erkennung von Effektoren durch CesT als wichtig identifiziert und abschliessend wird ein Modell für die Erkennung des Chaperon/Effektor Komplexes durch das Type III Sekretionssystem vorgeschlagen. 


\section{Abstract}

The present work provides new structural information about bacterial proteins involved in transcription regulation and protein secretion, as well as a contribution to the improvement of structure determination of homodimeric proteins by NMR spectroscopy. A main focus is placed on two homodimeric bacterial proteins: the transcription factor CylR2 from Enterococcus faecalis and the chaperone CesT from enteropathogenic Escherichia coli.

Enterococcus faecalis has emerged as a leading agent of hospital-acquired antibioticresistant infections and the severity of its infections has been linked to a secreted protein called cytolysin. Production of this enterococcal cytolysin is regulated by the two-component CylR1/CylR2 system through an autoinduction quorum-sensing mechanism. Here, the regulatory protein CylR2 is found to form a rigid dimer with an essentially identical crystal and solution NMR structure. Each monomer contains a helix-turn-helix DNA-binding motif as part of a five helix-bundle, which is extended by an antiparallel $\beta$-sheet. The determination of the solution NMR structure involves the development of a novel method for homodimeric proteins. This method applies rigid-body docking driven by backbone amide residual dipolar couplings and intermolecular long-range distances from paramagnetic relaxation enhancement. A model structure for CylR2 in complex with its specific palindromic DNA within the cytolysin promotor region is derived based on NMR chemical shift perturbation experiments. These results suggest that CylR2 acts as a repressor of cytolysin transcription.

The other studied homodimer, the chaperone CesT, originates from enteropathogenic Escherichia coli which is a chief cause of diarrhea and involves secretion of bacterial proteins via a type three secretion system into the human host cell. CesT participates in the secretion mechanism by specifically binding effector proteins in the bacterial cytoplasm prior to their secretion; the effector is thereby kept in a secretion-competent form. However, the exact function of the chaperone is still open and remains a key question for understanding the bacterial type three protein secretion mechanism. A novel application of NMR dipolar couplings is used to elucidate the structure of CesT in solution. In this way CesT is shown to form a structure similar to its homologues. Many hydrophobic interactions are identified to be important for the complex formation with at least two of its effectors. Finally, a model is suggested for the recognition

of the chaperone/effector complex by the type three secretion system. 


\section{Acknowledgements}

During the years of this thesis many people have contributed in many different ways to the work. I have been very fortunate to work under a good atmosphere in a department with great scientists and with the most modern equipment. So I am deeply grateful and would like to express thanks.

Most importantly, my direct supervisor Dr. Markus Zweckstetter, for the interesting projects, his good ideas, patience and advice.

To Prof. Christian Griesinger for giving me the opportunity to do my PhD. in his department with the very stimulating scientific environment.

Dr. Stefan Becker for helpful guidance concerning protein expression, purification and crystallization.

To Prof. Axel Zeeck for accepting me as an external PhD. student in the Chemistry Faculty of the Georg-August University, Göttingen.

Vinesh Vijayan for the company during the time of this thesis and for a lot of help with the measurement of NMR spectra.

All technical assistants and especially Karin Giller for her many technical tricks. Kerstin Overkamp for the breaths of fresh air, the organization of volleyball games and other events and of course all the HPLC runs and mass spectra.

All members of the department "NMR based structural biology": in particular, Dirk Lennartz, Monika Bayrhuber and Daniela Fischer, my officemates during different years of this work, for their cordiality, helpfulness and a lot of useful discussions. Pierre Montaville and Fernando Rodriguez-Castañeda for company and advice in the lab. Young-Sang Jung, Jochen Junker, Karel Kubíček, Marcel Reese and Jegannath Korukottu for help with different computational aspects. My trainees, Ekaterina Torbashevich and Hai-Young Kim, who have contributed to the work about the type three secretion system.

All my friends, from tennis, school and studies, who have made these years special and well-balanced between a lot of scientific work and "real" life.

Last but not least I would like to thank my parents for their endless support, my brother for all the joint adventures, and Christophe for all the special moments that gave me the strength to finish this thesis. 


\section{Publication list}

This thesis is based on the following papers:

Chapter 4

[1] S. Rumpel, A. Razeto, C. M. Pillar, V. Vijayan, A. Taylor, K. Giller, M. S. Gilmore, S. Becker and M. Zweckstetter. Structure and DNA-binding properties of the cytolysin regulator CylR2 from Enterococcus faecalis. EMBO Journal, 23(18):3632$3642,2004$.

Chapter 6

[2] S. Rumpel, H. Y. Kim, V. Vijayan, S. Becker and M. Zweckstetter. Backbone resonance assignment of the homodimeric, $35 \mathrm{kDa}$ chaperone CesT from enteropathogenic Escherichia coli. Journal of Biomolecular NMR, 31(4):377-378, 2005.

Chapter 5

[3] S. Rumpel, S. Becker and M. Zeckstetter. High-resolution structure determination of the CylR2 homodimer using intermonomer distances from paramagnetic relaxation enhancement and NMR dipolar couplings. submitted. 


\section{Table of contents}

Abbreviations xvii

\begin{tabular}{|ll}
\hline 1 Introduction & 1
\end{tabular}

1.1 Bacterial pathogenesis $\ldots \ldots \ldots \ldots \ldots \ldots$

$1.2 \quad$ NMR spectroscopy in structural biology $\ldots \ldots \ldots \ldots \ldots \ldots$

1.3 Macromolecular complexes . . . . . . . . . . . . . . . . . . 3

1.4 Protein symmetry and function $\ldots \ldots \ldots \ldots$

1.5 Rationale and Outline . . . . . . . . . . . . . . . . 4

$\begin{array}{lll}2 & \text { Materials } & 7\end{array}$

$2.1 \quad$ Bacterial strains and plasmids $\ldots \ldots \ldots \ldots \ldots \ldots$

2.2 Oligodesoxyribonucleotides and peptides $\ldots \ldots \ldots \ldots$

2.3 Culture media and antibiotics $\ldots \ldots \ldots \ldots$

2.4 Buffers and solutions . . . . . . . . . . . . . . . . . . . . . 11

$2.5 \quad$ Enzymes and Chemicals $\ldots \ldots \ldots \ldots \ldots$

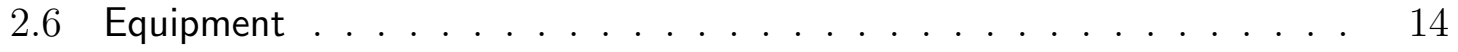

2.7 Software $\ldots \ldots \ldots \ldots \ldots \ldots$

\begin{tabular}{lll}
\hline 3 Methods & 17
\end{tabular}

$3.1 \quad$ Molecular biology methods $\ldots \ldots \ldots \ldots \ldots$

$3.1 .1 \quad$ Agarose gel electrophoresis $\ldots \ldots \ldots \ldots$

3.1 .2 Isolation and purification of DNA $\ldots \ldots \ldots \ldots 17$ 
$3.1 .3 \quad$ Enzymatic modifications of DNA $\ldots \ldots \ldots \ldots$

3.1 .4 Polymerase chain reaction . . . . . . . . . . . . . . . . 18

3.1 .5 Site-directed mutagenesis . . . . . . . . . . . . . . . . . . . 19

$3.1 .6 \quad$ DNA sequencing . . . . . . . . . . . . . . . . . . . . . . . . . . 19

3.1 .7 Concentration and purity of DNA $\ldots \ldots \ldots \ldots$

3.1 .8 Transformation of E. coli . . . . . . . . . . . . . . 20

3.2 Protein expression $\ldots \ldots \ldots \ldots \ldots$

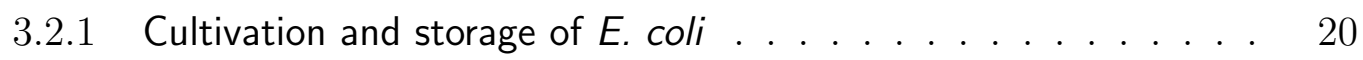

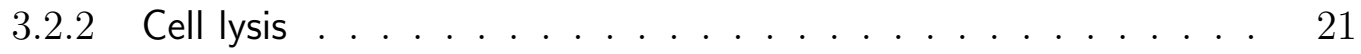

3.3 Protein methods . . . . . . . . . . . . . . . . . . . . . . . . . . 21

$3.3 .1 \quad$ Nickel-nitrilotriacetic acid agarose affinity chromatography . . . . 21

3.3 .2 lon exchange chromatography . . . . . . . . . . . . . . . 22

3.3 .3 Gel filtration . . . . . . . . . . . . . . . . . . . . . . . 22

3.3 .4 Reversed phase-high performance liquid chromatography . . . . . 22

3.3 .5 Cleavage with TEV-protease . . . . . . . . . . . . . . . . . 22

3.3 .6 Concentrating of proteins . . . . . . . . . . . . . . . 23

3.3 .7 Protein storage $\ldots \ldots \ldots \ldots$

3.3 .8 Sodium dodecyl sulphate-polyacrylamide gel electrophoresis . . . 23

3.3 .9 Edman degradation . . . . . . . . . . . . . . . . . . 24

3.3 .10 Limited proteolysis . . . . . . . . . . . . . . . . . . . . . . 24

3.3 .11 Peptide synthesis . . . . . . . . . . . . . . . . . . . . . . 24

3.3 .12 Introduction of a paramagnetic center. . . . . . . . . . 25

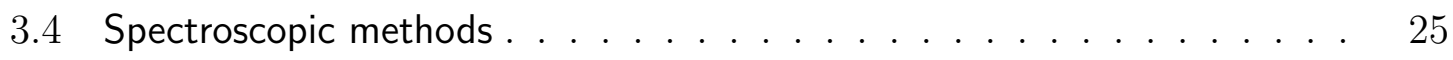

3.4 .1 Determination of protein concentration . . . . . . . . . 25

3.4 .2 Circular dichroism spectroscopy $\ldots \ldots \ldots \ldots$

3.4 .3 Electrospray ionization mass spectrometry . . . . . . . . 26

$3.5 \quad$ NMR spectroscopy $\ldots \ldots \ldots \ldots \ldots$

3.5 .1 Chemical shift assignment . . . . . . . . . . . . . . . 27

3.5 .2 Secondary structure determination $\ldots \ldots \ldots \ldots . \ldots 27$

3.5 .3 Residual dipolar couplings . . . . . . . . . . . . . . . . 28 
$3.5 .4 \quad$ NMR relaxation data. . . . . . . . . . . . . . . . . . . . . . . . . 29

3.5 .5 Mapping binding surfaces $\ldots \ldots \ldots \ldots$. . . . . . . . 31

3.5.6 Determination of protein-ligand dissociation constants . . . . . . 31

$\begin{array}{lll}3.5 .7 & \text { Long-range distances from paramagnetic relaxation enhancement } 32\end{array}$

3.6 Structure calculation . . . . . . . . . . . . . . . . . . . . . . . . . . . 34

3.6 .1 Monomer structure calculation . . . . . . . . . . . 34

3.6 .2 Dimer structure calculation . . . . . . . . . . . . . . . 34

$3.6 .3 \quad$ Ab initio docking $\ldots \ldots \ldots \ldots \ldots$

3.6 .4 Protein/DNA docking . . . . . . . . . . . . . . 35

3.7 Structure analysis $\ldots \ldots \ldots \ldots \ldots \ldots \ldots$

4 Structure and DNA-binding properties of CyIR2 37

4.1 Introduction . . . . . . . . . . . . . . . . . . . 37

4.2 Materials and methods . . . . . . . . . . . . . . . . . . . 40

$4.2 .1 \quad$ Expression and purification $\ldots \ldots \ldots \ldots$

$4.2 .2 \quad$ Preparation of ds DNA $\ldots \ldots \ldots \ldots \ldots$

$4.2 .3 \quad$ NMR experiments $\ldots \ldots \ldots \ldots \ldots$

4.2 .4 DNA titration . . . . . . . . . . . . . . . . . . 41

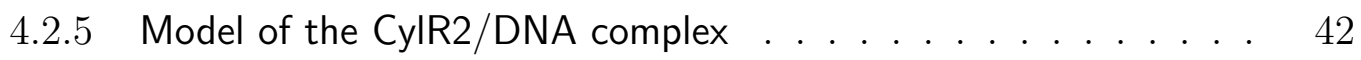

4.3 Results and discussion . . . . . . . . . . . . . . . . . . . . . . 43

$4.3 .1 \quad$ Three dimensional structure of CylR2 $\ldots \ldots \ldots \ldots$

4.3 .2 Dimerisation . . . . . . . . . . . . . . . . . . . . . 45

4.3 .3 Solution NMR studies of CylR2 and CylR2/DNA complex . . . 47

4.3 .4 Mapping of the DNA-binding site . . . . . . . . . 50

4.3 .5 Model for the CylR2/DNA complex . . . . . . . . . . . 53

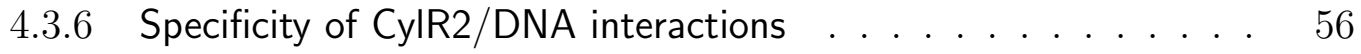

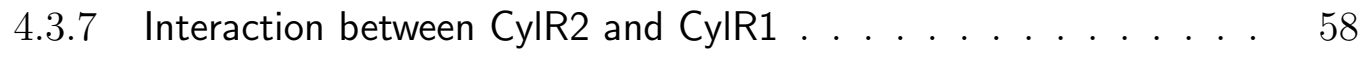

4.4 Conclusions. . . . . . . . . . . . . . . . . . . . . . . . . . . . . 59

5 Structure of a homodimer using RDC and PRE data 61

5.1 Introduction . . . . . . . . . . . . . . . . . . 61 
5.2 Materials and methods . . . . . . . . . . . . . . . . . . . 63

5.2 .1 Construction of CylR2-mutants $\ldots \ldots \ldots \ldots$

5.2 .2 NMR sample preparation $\ldots \ldots \ldots \ldots$

5.2 .3 NMR spectroscopy $\ldots \ldots \ldots \ldots$

5.2 .4 Monomer structure calculation . . . . . . . . . . . . . . . 64

5.2 .5 Intermonomer distances . . . . . . . . . . . . . . . . 65

5.2 .6 Homodimer structure calculation $\ldots \ldots \ldots \ldots$

5.2.7 Ranking of homodimer models from ab initio docking . . . . . 66

5.3 Results . . . . . . . . . . . . . . . . . . . . . . . . . 66

5.3 .1 Monomer solution structure $\ldots \ldots \ldots 6$

$5.3 .2 \quad$ Mutagenesis and spin-labeling . . . . . . . . . . . . . . 68

5.3 .3 Long-range distances from PRE . . . . . . . . . . . . . . . 72

5.3 .4 Homodimeric solution structure $\ldots \ldots \ldots \ldots$. . . . . . . 74

5.3 .5 The ATCUN-motif as a paramagnetic position . . . . . . . 78

$5.3 .6 \quad$ Ranking of homodimer structures from ab initio docking . . . . . 79

5.4 Discussion . . . . . . . . . . . . . . . . . . . 80

5.5 Conclusions $\ldots \ldots \ldots \ldots \ldots \ldots$

6 Function of the chaperone CesT in protein secretion 85

6.1 Introduction . . . . . . . . . . . . . . . . . . 85

6.2 Materials and methods . . . . . . . . . . . . . . . . . . . 93

$6.2 .1 \quad$ Cloning . . . . . . . . . . . . . . . . . . . . . . . . 93

6.2 .2 Protein expression . . . . . . . . . . . . . . . . . . . 93

6.2 .3 Purification . . . . . . . . . . . . . . . . . . 94

$6.2 .4 \quad$ NMR experiments $\ldots \ldots \ldots \ldots$

6.3 Results and discussion $\ldots \ldots \ldots \ldots$

$6.3 .1 \quad$ Experimental difficulties $\ldots \ldots \ldots \ldots$

6.3 .2 Extent of the assignment of CesT $\ldots \ldots \ldots \ldots$

6.3 .3 Secondary structure and dynamics of CesT in solution . . . . . 99

6.3 .4 Solution structure of CesT . . . . . . . . . . . . . . . 100 
6.3 .5 CesT $_{V 116 R}$ aggregates $\ldots \ldots \ldots \ldots \ldots \ldots$

$6.3 .6 \quad$ Structural characterisation of Tir and Map . . . . . . . . . 103

6.3 .7 Crystallisation of the CesT/Map complex . . . . . . . . . . 105

6.3 .8 Backbone assignment and secondary structure of Tir108 . . . . 105

6.3 .9 Effects of CesT binding on Tir and Map . . . . . . . . . 107

6.3 .10 Effect of CesT on effector regions outside the CBD . . . . . . 110

6.3 .11 Effects of Tir and Map binding on CesT . . . . . . . . . 110

6.3 .12 Properties of the CesT/Map84 complex in solution . . . . . . . 117

6.3 .13 Conclusions . . . . . . . . . . . . . . . . . . 120

7 Summary and future considerations 123

\begin{tabular}{ll}
\hline References & 128
\end{tabular}

A Appendix: Sample preparation $\quad 149$

A.1 CylR2 . . . . . . . . . . . . . . . . . . . . . . . . . . . . . . . . . . . . 149

A.2 CesT . . . . . . . . . . . . . . . . . . . . . . . . . . . . . 150

A.3 Map . . . . . . . . . . . . . . . . . . . . . . . . 151

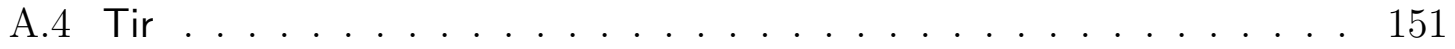

A.5 CesT/effector-complexes . . . . . . . . . . . . . . . . . 153

B Appendix: NMR experiments and chemical shift assignments $\quad 155$

B.1 NMR experiments . . . . . . . . . . . . . . . . . . . . 155

B.2 Chemical shift assignments $\ldots \ldots \ldots \ldots \ldots \ldots$

\begin{tabular}{ll}
\hline C Appendix: NMR restraints & 165
\end{tabular}

C.1 Experimental residual dipolar couplings . . . . . . . . . . . . . . 165

C.2 Distance restraints for CylR2 _ . . . . . . . . . . . . . . . . 170

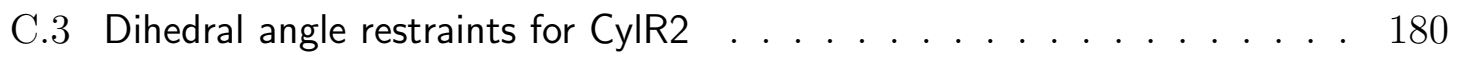

$\begin{array}{ll}\text { D Appendix: Xplor input files } & 181\end{array}$

D.1 Water refinement . . . . . . . . . . . . . . . . . . . . . . . 181

D.2 Rigid body docking $\ldots \ldots \ldots \ldots \ldots \ldots \ldots$ 
D.3 Simulated annealing . . . . . . . . . . . . . . . . . . . . 195

\begin{tabular}{ll}
\hline Curriculum Vitae & 203
\end{tabular}

\begin{tabular}{ll}
\hline Lebenslauf & 204
\end{tabular} 


\section{List of figures}

$2.1 \quad$ Plasmid map of pET16bTEV and pETDuet1 . . . . . . . . . . 8

4.1 Model of cytolysin maturation and regulation of cytolysin production 38

4.2 Cytolysin promotor region $\ldots \ldots \ldots \ldots$

4.3 The 22-mer DNA sequence of IR1 used for the NMR experiments. . . 41

4.4 Sequence of CylR2 and stereo view of the crystal structure of CylR2 . 44

4.5 Superposition of the CylR2 monomer with SinR and 434 repressor . . 44

$4.6 \quad$ Dimer interface of CylR2 $\ldots \ldots \ldots \ldots \ldots$

4.7 Dimer interfaces of CylR2 and GerE superposed . . . . . . . . . 46

4.8 Superposition of one subunit of CylR2 to 434 repressor $\ldots \ldots \ldots$. . . 47

4.9 Correlation between experimental and back-calculated HN-RDC for free CylR2 and DNA-bound CylR2 . . . . . . . . . . . . . . . . 48

$4.10^{15} \mathrm{~N}-{ }^{1} \mathrm{H}-\mathrm{NOE}$ of CylR2 and CylR2 in complex with its DNA . . . . 50

4.11 Overlay of ${ }^{15} \mathrm{~N}-\mathrm{HSQC}$ spectra of free and DNA-bound CylR2 . . . . . 51

4.12 Chemical shift changes upon CylR2/DNA complex formation. . . . . 52

4.13 Surface representation of CylR2 $\ldots \ldots \ldots \ldots \ldots$

4.14 Model of the CylR2/DNA complex structure . . . . . . . . . 55

4.15 Overlay of NMR titration ${ }^{15} \mathrm{~N}-\mathrm{HSQC}$ experiments of CylR2 with IR2 57

4.16 Chemical shift changes upon binding of CylR2 to unspecific DNA . . 58

4.17 Prediction of transmembrane helices for CylR1 . . . . . . . . . . 59

$5.1 \quad$ Monomer solution structure of CylR2 $\ldots \ldots \ldots \ldots$ 
5.2 Reaction of the MTSL spin label with the -SH group of a protein . . 69

5.3 Chemical shift changes due to single cysteine mutations . . . . . . . . 69

5.4 Overall strategy to derive intermonomer distances from PRE in homodimers. . . . . . . . . . . . . . . . . . 70

$5.5{ }^{15} \mathrm{~N}-\mathrm{HSQC}$ spectra of paramagnetic and diamagnetic CylR2 $2_{T 55 \mathrm{C}}$. . . 71

5.6 Accuracy of the intermolecular distances determined from PRE . . . 73

5.7 Homodimeric solution structure of CylR2 . . . . . . . . . . . . 75

5.8 Comparison of the solution and crystal structure of CylR2 . . . . . . 77

5.9 Overlay of ${ }^{15} \mathrm{~N}-\mathrm{HSQC}$ spectra of CylR2 and ATCUN-CylR2 . . . . . 79

5.10 NMR-based ranking of structural models from $a b$ initio docking . . . 80

6.1 Schematic representation of a type three secretion system . . . . . . . 86

6.2 Structures of TTSS chaperones . . . . . . . . . . . . . . . . 89

6.3 Structure of CesT . . . . . . . . . . . . . . . . . . . . . . . 90

$6.4 \quad$ Structures of chaperone/effector complexes . . . . . . . . . . . . 91

$6.5{ }^{15} \mathrm{~N}-\mathrm{HSQC}$ spectrum of $\mathrm{His}_{6}$-CesT . . . . . . . . . . . . . . . . . . . . 97

$6.6{ }^{15} \mathrm{~N}$-TROSY-HSQC spectrum of CesT . . . . . . . . . . . . . . . . 98

6.7 Secondary structure of CesT . . . . . . . . . . . . . . . . . . . . . 99

6.8 Surface representation of the model and X-ray structure of CesT . . . 100

6.9 Correlation between experimental and predicted or back-calculated RDCs for CesT . . . . . . . . . . . . . . . . . . . 101

6.10 ESI-MS spectrum of coexpressed and copurified CesT/Map101 . . . . 104

6.11 Far-UV-CD spectra of His7-Map84 and Tir108 . . . . . . . . . . . . . 104

6.12 Secondary chemical shifts of Tir108 . . . . . . . . . . . . . 106

6.13 Overlay of ${ }^{15} \mathrm{~N}-\mathrm{HSQC}$ spectra of free and bound Tir108 . . . . . . . . 107

6.14 Chemical shift changes of Tir108 upon CesT/Tir108 complex formation 108

6.15 Overlay of ${ }^{15} \mathrm{~N}-\mathrm{HSQC}$ spectra of free His; - Map84 and bound Map84 . 109

6.16 Overlay of ${ }^{15} \mathrm{~N}-\mathrm{HSQC}$ spectra of TirN and TirN bound to CesT . . . 111

6.17 Overlay of ${ }^{15} \mathrm{~N}-\mathrm{TROSY}-\mathrm{HSQC}$ spectra of free and effector-bound CesT 112

6.18 Secondary structure of CesT bound to Map84 . . . . . . . . . . . . . 113 
6.19 Chemical shift changes of CesT upon formation of the CesT/Map84

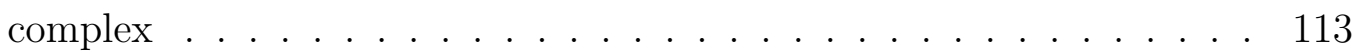

6.20 Structure-based sequence alignment of TTSS chaperones . . . . . . . 114

6.21 Mapping of shift changes upon Map84 binding to the surface of CesT 115

6.22 Chemical shift changes of CesT upon formation of the CesT/Tir108 complex . . . . . . . . . . . . . . . . . . 116

$6.23{ }^{15} \mathrm{~N}^{-1} \mathrm{H}-\mathrm{NOE}$ of CesT and Map84 in complex with each other . . . . . 117

$6.24{ }^{15} \mathrm{~N}$-TROSY-HSQC spectrum of ${ }^{15} \mathrm{~N}$-labeled CesT/Map84 complex. . 119

6.25 Model of effector targeting to the TTSS through CesT . . . . . . . . 121

A.1 $\quad$ Expression and purification of CylR2 . . . . . . . . . . . . . . . . . . 149

A.2 ESI-MS spectrum of CylR2 $2_{T 55 C}$. . . . . . . . . . . . . . . . 150

A.3 $\quad$ ESI-MS spectrum of perdeuterated ${ }^{2} \mathrm{H}-\mathrm{His}_{7}$-CesT $\ldots . . . . .150$

A.4 Expression and purification of His Hap84 $_{7}$.M. . . . . . . . . . . . . 151

A.5 Expression and purification of Tir108 . . . . . . . . . . . . . . . . . . 151

A.6 $\quad$ ESI-MS spectrum of Tir108 . . . . . . . . . . . . . . . . . . . . . . . 152

A.7 $\quad$ ESI-MS spectrum of TirN . . . . . . . . . . . . . . . . . . . . . . . . 152

A.8 Coexpression and copurification of CesT/Map84 . . . . . . . . . . . 153

A.9 ESI-MS spectrum of coexpressed and copurified CesT/Map84 . . . . 153

A.10 ESI-MS spectrum of coexpressed and copurified CesT/Tir108. . . . . 154

B.1 Dimerization of CylR2 . . . . . . . . . . . . . . . . . . . 155 


\section{List of tables}

$2.1 \quad$ Name, genotype and reference for the used E. coli strains. . . . . . . 7

2.2 Plasmids used in this work. . . . . . . . . . . . . . . . . . . . . . 8

2.3 Name, sequence and application of the applied oligonucleotides. . . . 9

2.4 Name, sequence and residues of CylR1 of the applied peptides. . . . . 10

2.5 Culture media and antibiotics . . . . . . . . . . . . . . . . 10

2.6 Buffers and solutions . . . . . . . . . . . . . . . . . . . . . . . . . 11

2.7 Enzymes and chemicals . . . . . . . . . . . . . . . . . . . 13

2.8 Equipment and supplier . . . . . . . . . . . . . . . . . . . . 14

2.9 Software . . . . . . . . . . . . . . . . . . . . 15

3.1 Composition of the $17.5 \%$ SDS-gels . . . . . . . . . . . . . . . . 24

4.1 Best-fit of HN-RDCs for DNA-bound CylR2 to the X-ray structure of free CylR2. . . . . . . . . . . . . . . . . . . . . . . 49

$5.1 \quad$ Statistics for CylR2 monomer solution structure determination. . . . 67

5.2 Statistics for CylR2 dimer solution structure determination. . . . . . 75

B.1 NMR spectra for assignment and structure determination of CylR2 . 156

B.2 NMR spectra for backbone assignment of CesT . . . . . . . . . . . 156

B.3 NMR spectra for backbone assignment of Tir108 . . . . . . . . . . . . 156

B.4 NMR spectra for backbone assignment of CesT in complex with Map84 157

B.5 NMR spectra for backbone assignment of CesT in complex with Tir108 157

B.6 $\quad$ NMR spectra for backbone assignment of Map84 in complex with CesT 157 
B.7 Assigned chemical shifts of CylR2 in complex with its DNA $\ldots . .158$

B.8 Assigned chemical shifts of CesT bound to Map84 . . . . . . . . . . . 159

B.9 Assigned chemical shifts of CesT bound to Tir108 _ . . . . . . . . 160

B.10 Assigned chemical shifts of Tir108 . . . . . . . . . . . . . . . . 161

B.11 Assigned chemical shifts of Map84 in complex with CesT . . . . . . . 162

C.1 HN-RDCs of CylR2 . . . . . . . . . . . . . . . . . . . . . . . . 165

C.2 HN-RDCs of DNA-bound CylR2 $\ldots \ldots \ldots \ldots \ldots$

C.3 HN-RDCs of CesT . . . . . . . . . . . . . . . . . . 167

C.4 NC'-RDCs of CesT . . . . . . . . . . . . . . . . . . . . 167

C.5 C $\alpha \mathrm{C}^{\prime}-\mathrm{RDCs}$ of $\mathrm{CesT} \ldots \ldots \ldots \ldots \ldots$

C.6 HN-RDCs of Tir108. . . . . . . . . . . . . . . . . . . . . . . 169

C.7 Intramolecular NOE distances of CylR2 . . . . . . . . . . . . . 170

C.8 Intermolecular distances of CylR2 from PRE . . . . . . . . . . . 178

C.9 Intermolecular NOE distances of CylR2 $\ldots \ldots \ldots \ldots \ldots$

C.10 Dihedral angle restraints for CylR2 $\ldots \ldots \ldots \ldots \ldots \ldots$ 


\section{Abbreviations}

A

\begin{tabular}{|c|c|}
\hline A & adenine \\
\hline $\mathbf{A}_{\lambda}$ & absorption at wavelength $\lambda$ \\
\hline Ala & $\underline{\text { alanine }(\mathrm{A})}$ \\
\hline Ap & $\underline{\text { ampicillin }}$ \\
\hline Arg & $\underline{\operatorname{argininine}(\mathrm{R})}$ \\
\hline Asn & asparagine $(\mathrm{N})$ \\
\hline Asp & 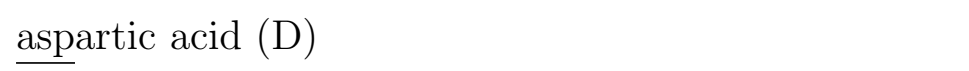 \\
\hline ATCUN & amino terminal $\underline{\mathrm{Cu}}(\mathrm{II})-$ and $\underline{\mathrm{Ni}}(\mathrm{II})$-binding \\
\hline $\mathrm{ATP} / \mathrm{ADP}$ & adenosine $5^{\prime}$-triphosphate / adenosine $5^{\prime}$-diphosphate \\
\hline \multicolumn{2}{|l|}{ B } \\
\hline bp & base pair \\
\hline BMRB & $\underline{B}$ iological Magnetic $\underline{\text { Resonance } \underline{B} a n k}$ \\
\hline BSA & 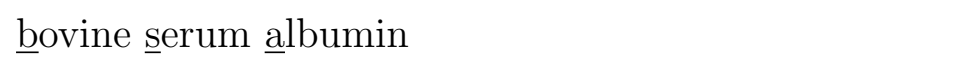 \\
\hline \multicolumn{2}{|l|}{$\mathrm{C}$} \\
\hline CBD & chaperone binding domain \\
\hline $\mathrm{CD}$ & circular dichroism \\
\hline CesT & 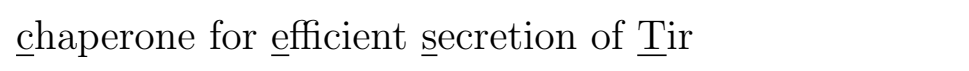 \\
\hline CIAP & calf intestine alkaline phosphatase \\
\hline CylR1 & $\underline{\text { cytolysin }} \underline{\text { regulator }} \underline{1}$ \\
\hline CylR2 & cytolysin regulator $\underline{2}$ \\
\hline
\end{tabular}




\begin{tabular}{|c|c|}
\hline Cys & cysteine $(\mathrm{C})$ \\
\hline \multicolumn{2}{|l|}{ D } \\
\hline $\mathbf{D}_{a}$ & magnitude of the alignment tensor \\
\hline DNA & $\underline{\text { desoxyribonucleic acid }}$ \\
\hline DNase & desoxyribonuclease \\
\hline dNTP & desoxyribonucleotide triphosphate \\
\hline ds & double-s tranded \\
\hline DTT & dithiothreitol \\
\hline$\delta$ & chemical shift \\
\hline \multicolumn{2}{|l|}{$\mathbf{E}$} \\
\hline E. coli & Escherichia coli \\
\hline E. faecalis & Enterococcus faecalis \\
\hline EHEC & enterohemorrhagic Escherichia coli \\
\hline ELISA & enzyme-linked immunosorbent assay \\
\hline EPEC & enteropathogenic Escherichia $\underline{\text { coli }}$ \\
\hline EDTA & ethylene diamine tetraacetic acid \\
\hline ESI & electrospray ionization \\
\hline
\end{tabular}

$\mathbf{F}$

FPLC fast protein liquid chromatography

G

G g guanine

Gln glutamine (Q)

Glu glutamic acid (E)

Gly glycine $(\mathrm{G})$

$\gamma \quad$ nuclear gyromagnetic ratio

H

h Planck's constant $\left(=6.6260693 \times 10^{-34} \mathrm{Js}\right)$

$\hbar \quad$ reduced Planck's constant $\left(=1.05457168 \times 10^{-34} \mathrm{Js}\right)$

HEPES 2-[4-(2-ḩydroxyethyl)1-1 piperazinyl] ethansulfonic acid

His $\quad$ histidine $(\mathrm{H})$ 


\begin{tabular}{|c|c|}
\hline HPLC & high performance liquid chromatography \\
\hline HSQC & heteronuclear single-quantum coherence \\
\hline НТН & $\underline{h e l i x-t u r n-\underline{h e l i x}}$ \\
\hline
\end{tabular}

I

Ile

INEPT

isoleucine (I)

IPAP

insensitive nuclei enhancement by polarization transfer

IPTG

in-phase / anti-phase

IR

isopropyl- $\beta$-D-thiogalactopyranoside

inverted repeat

K

$\mathbf{K}_{d}$

dissociation constant

$\mathrm{kDa}$

kilo-Dalton $\left(=10^{3} \mathrm{~g} / \mathrm{mol}\right)$

L

LB

Luria Bertani

Leu

leucine $(\mathrm{L})$

Lys

lysine $(\mathrm{K})$

M

M

$\mathrm{mol} / \mathrm{l}$

Map

mitochondrial associated protein

MES

2-(N-morpholino)ethane-șulfonic acid

MCS

multiple loning site

$\mathrm{MeOH}$

methanol

Met

methionine $(\mathrm{M})$

MOPS

3-(N-morpholino)propane-șulfonic acid

MS

mass spectrometry

MTSL

(1-oxy-2,2,5,5-tetramethyl-3-pyrroline-3-methyl)-

methanethiosulfonate spin-label

MW

molecular weight

MWCO

molecular weight cutofff

$\mu_{0}$

permeability of vacuum $\left(=1.25663706144 \times 10^{-6} \mathrm{NA}^{-2}\right)$ 


\begin{tabular}{|c|c|}
\hline Ni-NTA & $\underline{\text { nickel-nitrilotriacetic a acid }}$ \\
\hline NMR & nuclear magnetic resonance \\
\hline NOE & $\underline{\text { nuclear }}$ Overhauser effect \\
\hline NOESY & 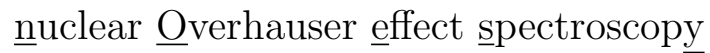 \\
\hline
\end{tabular}

\section{$\mathrm{O}$}

OD

$\mathbf{P}$

PAGE

PCR

PCS

PDB

Pf1

Phe

PMSF

ppm

PRE

Pro

R

$\mathbf{R}$

$\mathbf{R}_{1}$

$\mathbf{R}_{2}$

RDC

rmsd

RP-HPLC

optical density

polya acrylamide gel electrophoresis

polymerase chain reaction

pseudocontact shift

Protein Data Bank

filamentous phage

phenylalanine $(\mathrm{F})$

phenylmethylsulphonyl fluoride

parts per million $\left(=10^{-6}\right)$

paramagnetic relaxation enhancement

proline $(\mathrm{P})$

rhombicity of the alignment tensor

longitudinal or spin-lattice relaxation rate

transversal or spin-spin relaxation rate

residual dipolar coupling

root mean square deviation

$\mathrm{S}$

$\begin{array}{ll}\text { SDS } & \text { sodium dodecylsulfate } \\ \text { SDSL } & \text { site- } \underline{\text { directed }} \text { spin-labeling } \\ \text { Ser } & \underline{\text { serine }(\mathrm{S})} \\ \text { ss } & \underline{\text { single-stranded }}\end{array}$


SVD $\quad$ singular value decomposition

$\mathrm{T}$

$\mathrm{T}$

$\mathbf{T}_{1}$

$\mathbf{T}_{2}$

TEMED

TEV

Tir

TFA

Thr

TOCSY

Tris

TROSY

$\operatorname{Trp}$

TTSS

Tyr

$\tau$

$\tau_{c}$

V

$\begin{array}{ll}\text { Val } & \text { valine }(\mathrm{V}) \\ \mathbf{Y} & \\ \mathbf{Y T} & \text { yeast/tryptone }\end{array}$

thymine

threonine $(\mathrm{T})$

tyrosine $(\mathrm{Y})$

yeast/tryptone longitudinal or spin-lattice relaxation time

transversal or spin-spin relaxation time

N,N,N',N'-tetramethylethylenediamine

tobacco etch virus

translocated intimin receptor

trifluoroacetic acid

total correlation spectroscopy

tris(hydroxymethyl)-amino-methan

transverse relaxation optimised spectroscopy

tryptophan (W)

type three secretion system

correlation time for the electron-nuclear interaction

global or rotational correlation time 


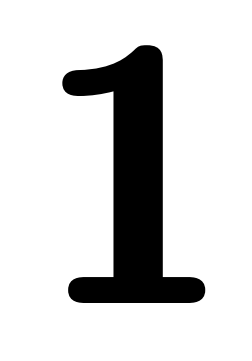

\section{Introduction}

Proteins (greek: protas $=$ of primary importance) are essential to all life processes. Association of proteins with each other or with DNA leads to the formation of enzymatic and regulatory complexes that are integral to cellular control processes such as transcription, signal transduction and protein translocation. Numerous of these processes are involved in bacterial pathogenicity.

\subsection{Bacterial pathogenesis}

Bacterial pathogenicity is defined as the sum of biochemical mechanisms whereby micro-organisms cause disease in a host organism [1]. These infectious diseases are a main cause of death worldwide. Emerging new infectious diseases, re-emerging deadly infectious diseases and increasing prevalence of antimicrobial resistant strains mark a formidable threat to public health and welfare. Pathogenic bacteria utilize a versatile and flexible repertoire of mechanisms by which they exert influence over their hosts. Yet, as these mechanisms become better understood, there is significant new evidence that very different microbial pathogens use common strategies. Two general abilities underlie bacterial pathogenicity. Firstly, the capacity of tissue invasion: this encompasses mechanisms for adherence and initial multiplication, the ability to bypass or overcome host defence mechanisms and the production of extracellular substances which facilitate invasion. Secondly, the competence to produce toxins. There are two types of toxins. The first type, the exotoxins, are released from bacterial cells and may act at tissue sites remote from the site of bacterial growth while the second type, 
the endotoxins, are cell-associated substances that are structural components of the cell walls of Gram-negative bacteria [2, 3].

Our understanding of biological systems and their interaction networks in general and of bacterial pathogenesis in particular, relies largely on the three-dimensional (3D) structures of proteins as well as protein/DNA- and protein/protein-complexes. Unraveling their mechanisms and functions may render them feasible for control and correction by drugs. Moreover, 3D structures play an important role in rational drug design by enabling the examination of interactions between proteins and potential drug compounds at atomic detail.

\subsection{NMR spectroscopy in structural biology}

Currently, NMR spectroscopy and X-ray crystallography are the most prominent techniques capable of determining 3D structures of macromolecules at atomic level. The most important technique used in the present study is NMR spectroscopy. For a detailed treatment of NMR spectroscopy, the reader is invited to consult the wellestablished books [4, 5, 6, 7].

The basic phenomenon of NMR, that energy levels of atomic nuclear spins are split by a magnetic field and that transitions between these energy levels can be induced by irradiating with a frequency equal to the energy difference between the two levels, was discovered in 1946 [8, 9]. In the last two decades, NMR spectroscopy has evolved enormously and become a powerful alternative structural elucidation tool to X-ray crystallography. In addition, NMR spectroscopy provides at least two important advantages compared to X-ray crystallography. First, the 3D structures are solved in aqueous solution and thus under physiologically-relevant conditions. Second, NMR spectroscopy offers a unique perspective on dynamics. Advances in NMR spectroscopy were acknowledged by Nobel prizes in 1991 [10] and in 2002 [11] and were accompanied by an ever-growing number of structures solved by NMR and deposited in the protein data bank (PDB, http://www.rcsb.org/pdb) [12]. In spite of the success of NMR spectroscopy, limits on the size of the studied systems remain. Yet, the symbiotic development of deuteration strategies [13], transverse relaxation 
optimized spectroscopy (TROSY) [14, 15] and new computational methods [16, 17] have expanded the maximum molecular weight amenable to high-resolution structure determination by NMR spectroscopy from 10-15 kDa up to 30-40 kDa. However, the development of such novel methods remains a main research focus in NMR spectroscopy.

\subsection{Macromolecular complexes}

The vast majority of the reported structures in the PDB were solved by X-ray crystallography and often represent only monomeric proteins. This is due to the difficulty in production and crystallization of macromolecular assemblies compared to individual proteins. It is foreseeable that this gap between solved monomeric and complex structures will even widen as structural genomics efforts increase and mainly contribute monomeric structures. Hence, complex structures will continue to represent an important bottleneck in our understanding of cellular processes [18]. Improvement of existing methods as well as development of new tools for macromolecular structure determination are thus required. Most promising is the application of hybrid methods to improve efficiency, accuracy and completeness [19]. A prime example of combined approaches to complex structure determination is the combination of NMR, crystallography and computation. First, the structures of the free components of a macromolecular assembly can be solved by NMR or crystallography. Then, in the context of complex assemblies, efficient NMR tools can be combined with computational methods. Contact surfaces between the individual monomers can be identified by chemical shift perturbation or slight conformational changes upon complex formation can be identified by residual dipolar couplings (RDCs). Reviews covering the manifold theoretical, experimental and computational aspects of RDCs are available [20, 21, 22, 23]. 


\subsection{Protein symmetry and function}

A particular case of macromolecular assemblies are homooligomers which are presumed to form symmetrical complexes. They have evolved due to functional genetic and physicochemical needs and are favored because of stability [24]. In fact, most of the soluble and membrane-bound proteins form homooligomers. However, structural characterization of oligomeric states of proteins still remains a challenge. This is illustrated by the fact, that although $66 \%$ of the structures in the PDB in 1993 were monomeric, only about one fifth of the proteins annotated in the SWISS-PROT for $E$. coli is monomeric. Out of the remaining four fifth of oligomeric proteins, $79 \%$ form homooligomers [24]. In general, oligomerization plays a key role in protein function. These functions include the possibility of allosteric control, higher local concentration of active sites, larger binding surfaces and new active sites at subunit interfaces [25]. The over-representation of monomers and lack of homooligomers in the PDB is one reason for the lack of knowledge about the assembly of oligomers. One mechanism involved to form a dimer or higher oligomer is 3D domain swapping [25]. This 3D domain swapping is defined as the replacement of a portion of the tertiary structure of a protein with an identical structural element from a second protein molecule [26]. 3D domain swapping has gained recent interest because it can serve as a mechanism for reversible oligomerization and is implicated in amyloidosis [27].

\subsection{Rationale and Outline}

The work presented in this thesis brings new insights into two mechanisms involved in bacterial pathogenicity with a focus on advances in solution NMR structure determination of homodimeric proteins. Overall, an emphasis is placed on the interpretation of the complementary data from X-ray crystallography and NMR spectroscopy combined with essential in vivo data. Thus, the work attempts to achieve an in-depth understanding of molecular functions and mechanisms through structure determination or identification of interaction surfaces between biomolecules with very recent tools for liquid state NMR. 
The work at hand is organized as follows: Chapter 2 specifies commercially available and self-made materials followed by the methods applied to gain experimental results given in Chapter 3 . The subsequent three Chapters 446 provide experimental details, results and conclusions. Specifically, two different mechanisms of bacterial pathogenicity which are involved in the virulence of Enterococcus faecalis (E. faecalis) and enteropathogenic Escherichia coli (EPEC) are studied in the Chapters 6 and 4 . respectively. Chapter 6 presents aspects of chaperone/substrate interactions in the bacterial cytoplasm preceding protein translocation via a type three secretion system. In the first part of Chapter 6, structural details of the homodimeric chaperone are studied using experimental measurement of residual dipolar couplings and a novel application for their prediction to unravel the potential functional relevance of a 3D domain swap. The second part of this Chapter 6 deals with the characterization of the interactions of the chaperone with its substrate. In Chapter 4 a structural model for a protein/DNA complex is presented based on X-ray crystallographic and NMR spectroscopic data. This model is interpreted together with in vivo data to propose a role for the protein in the transcriptional regulation of the exotoxin cytolysin which is of major importance for the pathogenicity of E. faecalis. Using the same protein as in Chapter 4, a novel method for homooligomeric protein structure determination by NMR is demonstrated in Chapter 5. Finally the results are summarized and future experiments are suggested in Chapter 7 . 
Protein NMR studies of two systems involved in bacterial pathogenicity 


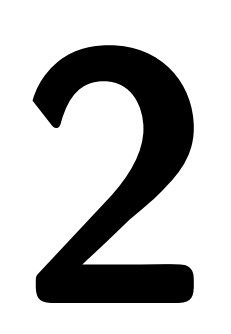

\section{Materials}

In this chapter, a general description is provided for the most important materials used in this work.

\subsection{Bacterial strains and plasmids}

Bacterial strains used in this work are summarized in Table 2.1.

Table 2.1: Name, genotype and reference for the used E. coli strains.

\begin{tabular}{llrr}
\hline \hline STRAIN & GENOTYPE & REFERENCES \\
\hline BL21 & $\mathrm{F}^{-}$, omp $T, h s d \mathrm{~S}_{B},\left(\mathrm{r}_{B-}, \mathrm{m}_{B-}\right), d c m$, gal & {$[28]$} \\
BL21(DE3) & $\mathrm{F}^{-}$, omp $T, h s d \mathrm{~S}_{B},\left(\mathrm{r}_{B-}, \mathrm{m}_{B-}\right), d c m$, gal, $\lambda(\mathrm{DE} 3)$ & {$[28]$} \\
XL2-Blue & recA1 endA1 gyrA96 thi-1 hsdR17 supE44 relA1 lac [F'proAB & Stratagene \\
& lacI ${ }^{q} \mathrm{Z} \Delta$ M15Tn10 $\left(\mathrm{Tet}^{r}\right)$ Amy Cam $\left.{ }^{r}\right]$ & & \\
\hline \hline
\end{tabular}

Plasmids containing the desired gene were transformed into the Escherichia coli (E. coli) expression strains BL21 or BL21(DE3). For expression of CylR2, the pET32a vector was used and a modified pET16b vector, referred to as pET16bTEV (Figure 2.1A), was used for cloning the coding sequences of the TTSS chaperone CesT and its effectors Tir and Map. For coexpression of protein-protein complexes, the genes were cloned into the pETDuetI vector (Figure 2.1B). All plasmids used in this work are listed in Table 2.2 . 

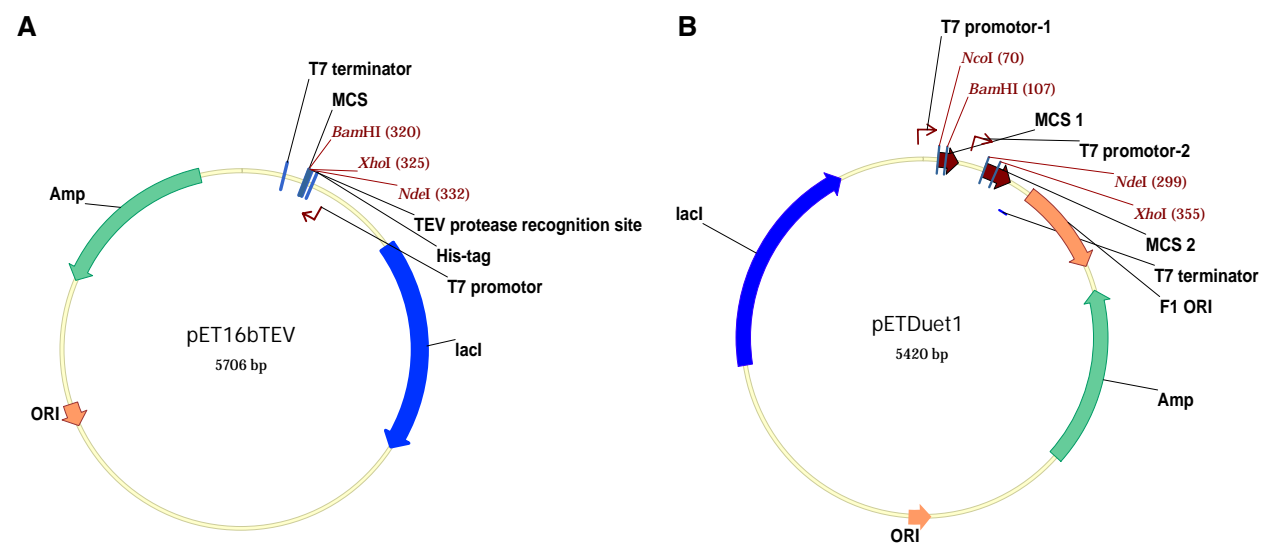

Figure 2.1: Plasmid map of pET16bTEV (A) and pETDuet1 (B)

Table 2.2: Plasmids used in this work.

\begin{tabular}{|c|c|c|c|}
\hline PLASMID & RESISTENCE & PROPERTIES & REFERENCE/ORIGIN \\
\hline pET32a & Ap & expression vector & Novagen, Madison, USA \\
\hline pET32a-CylR2 & Ap & pET32a with inserted CylR2-gene & $\begin{array}{l}\text { K. Giller, Max Planck Institute } \\
\text { Göttingen. Chapters } 4 \text { and } 5\end{array}$ \\
\hline pET32a-MGSHG-CylR2 & Ap & $\begin{array}{l}\text { pET32a with inserted MGSHG- } \\
\text { CylR2-gene }\end{array}$ & this work, Chapter 5 \\
\hline pET32a-MGHG-CylR2 & Ap & $\begin{array}{l}\text { pET32a with inserted MGHG-CylR2- } \\
\text { gene }\end{array}$ & this work, Chapter 5 \\
\hline pQE30-His 6 CesT & Ap & pQE30 with inserted $\mathrm{His}_{6}$-CesT-gene & 29 \\
\hline pET28a-Map & $\mathrm{Km}$ & pET28a with inserted Map-gene & 30 \\
\hline pET28a-Tir & $\mathrm{Km}$ & pET28a with inserted Tir-gene & 31 \\
\hline pET28a-TirN & $\mathrm{Km}$ & pET28a with inserted Tir-gene & 32 \\
\hline pET16bTEV & Ap & $\begin{array}{l}\text { modified pET16b with an additional } \\
\text { TEV-protease recognition site for re- } \\
\text { moval of the N-terminal His-tag }\end{array}$ & $\begin{array}{l}\text { K. Giller, Max Planck Institute } \\
\text { Göttingen, Chapter } 6\end{array}$ \\
\hline pET16bTEV-CesT & Ap & pET16bTEV with inserted CesT-gene & this work, Chapter 6 \\
\hline pET16bTEV-Map & Ap & pET16bTEV with inserted Map-gene & this work, Chapter 6 \\
\hline pET16bTEV-Map101 & Ap & $\begin{array}{l}\text { pET16bTEV with inserted Map101- } \\
\text { gene }\end{array}$ & this work, Chapter 6 \\
\hline pET16bTEV-Map84 & Ap & $\begin{array}{l}\text { pET16bTEV with inserted Map84- } \\
\text { gene }\end{array}$ & this work, Chapter 6 \\
\hline pET16bTEV-Tir & Ap & pET16bTEV with inserted Tir-gene & this work, Chapter 6 \\
\hline pET16bTEV-TirN & Ap & pET16bTEV with inserted TirN-gene & this work, Chapter 6 \\
\hline pET16bTEV-Tir110 & Ap & $\begin{array}{l}\text { pET16bTEV with inserted Tir110- } \\
\text { gene }\end{array}$ & this work, Chapter 6 \\
\hline pETDuet1 & Ap & expression vector & Novagen, Madison, USA \\
\hline pETDuet1-CesT-Map101 & Ap & $\begin{array}{l}\text { pETDuet1 with inserted CesT- and } \\
\text { Map101-gene }\end{array}$ & this work, Chapter 6 \\
\hline pETDuet1-CesT-Map84 & Ap & $\begin{array}{l}\text { pETDuet } 1 \text { with inserted CesT- and } \\
\text { Map84-gene }\end{array}$ & this work, Chapter 6 \\
\hline $\begin{array}{l}\text { pETDuet1-Ces } T_{1-135^{-}} \\
\text {Map84 }\end{array}$ & Ap & $\begin{array}{l}\text { pETDuet } 1 \text { with inserted CesT } \mathrm{T}_{1-135^{-}} \\
\text {and Map84-gene }\end{array}$ & this work, Chapter 6 \\
\hline pETDuet1-CesT-Tir108 & Ap & $\begin{array}{l}\text { pETDuet1 with inserted CesT- and } \\
\text { Tir108-gene }\end{array}$ & this work, Chapter 6 \\
\hline
\end{tabular}




\subsection{Oligodesoxyribonucleotides and peptides}

All oligonucleotides were ordered from Invitrogen (Karlsruhe, Germany), IBA (Göttingen, Germany) or Eurogentec (Liège, Belgium) and are presented in Table 2.3.

Table 2.3: Name, sequence and application of the applied oligonucleotides.

\begin{tabular}{|c|c|c|}
\hline NAME & SEQUENCE 5' to 3' & APPLICATION \\
\hline ir1_5' & ttgtcaagtgacacttgacaaa & oligonucleotides to construct IR1 \\
\hline ir1_3' & ttgtcaagtgtcacttgacaaa & for NMR studies \\
\hline ir1short_5' & gtcaagtgacacttgaca & oligonucleotides to construct the \\
\hline ir1short_3' & gtcaagtgtcacttgact & central 18-bp region of IR1 \\
\hline ir2_5' & ttgtaacatattatgttatgca & oligonucleotides to construct IR2 \\
\hline ir2_3' & tgcataacataatatgttacaa & for NMR studies \\
\hline ACTG_5' & actgactgactgactgactgac & oligonucleotides to construct a \\
\hline ACTG_3' & gtcagtcagtcagtcagtcagt & random dsDNA for NMR studies \\
\hline CylR2_T55C5' & gcttactacctgaattgtccactagaagatatttttcaatggc & mutagenesis of the gene for CylR2 \\
\hline CylR2_T55C3' & gccattgaaaaatatcttctagtggacaattcaggtagtaagc & to $\mathrm{CylR} 2_{T 55 C}$ \\
\hline CylR2_N40C5' & ggtatagaaaaaaataaatattgccettctttacagttagcattaaaaattgc & mutagenesis of the gene for CylR2 \\
\hline CylR2_N40C3' & gcaatttttaatgctaactgtaaagaagggcaatatttatttttttctatacc & to $\mathrm{CylR} 2_{N 40 C}$ \\
\hline MGSHG-CylR2_5' & ggaggacatatgggcagccatggcatgataatcaataacttaaaattaattag & attaching the ATCUN-motif to \\
\hline MGSHG-CylR2_3' & ggaggactcgagtttattcaggttg & CylR2 \\
\hline ATCUN-CylR2_5' & gaaggagatatacatatgggccatggcatgataatcaataac & mutagenesis of the gene for \\
\hline ATCUN-CylR2_3' & gttattgattatcatgccatggcccatatgtatatctccttc & MGSHG-CylR2 to MGHG-CylR2 \\
\hline CesT5' & ggaggacatatgtcgtcgcgatctgaacttttattagataggtttgcgg & amplification of the \\
\hline CesT3' & ggaggaggatcctttatcttccggcg & gene for CesT \\
\hline $\operatorname{CesT}_{V 116 R^{5}}$ & gagaatgaaatagaagtccgcgttaagtcgatgg & mutagenesis of the gene for CesT \\
\hline $\operatorname{CesT}_{V 116 R^{3}}$ & ccatcgacttaacgcggacttctatttcattctc & to $\operatorname{Ces}_{V 116 R}$ \\
\hline $\operatorname{Ces}_{E 135}{ }^{3}$ & ggaggaggatccttattcgttttctaatgttattccctgattatg & $\begin{array}{l}\text { amplification of the gene for } \\
\text { CesT }_{1-135}\end{array}$ \\
\hline Map5' & ggaggacatatgtttagtccaacggcaatggtag & amplification of the gene for Map \\
\hline Map3' & ggaggactcgagttacagccgagtatcctgcacattg & \\
\hline Map101_3' & ggaggaggatccttatgccgaattattactcatgcaatagtc & $\begin{array}{l}\begin{array}{l}\text { amplification of the gene for } \\
\text { Map101 }\end{array} \\
\end{array}$ \\
\hline Map84_3' & ggaggactcgagttaggatagaaaagtaatctgctcttgcttg & $\begin{array}{llll}\text { amplification of the gene for } \\
\text { Map84 }\end{array}$ \\
\hline Tir5' & ggaggacatatgcctattggtaaccttggtaataatg & amplification of the gene for Tir \\
\hline Tir108_3' & ggaggactcgagttaagagggtccaatttgcgtattgag & $\begin{array}{l}\text { amplification of the gene for } \\
\text { Tir108 }\end{array}$ \\
\hline
\end{tabular}

The peptides comprising predicted intracellular loops of CylR1 were synthesized by Kerstin Overkamp. The names, sequences and residues of the CylR1 peptides are summarized in Table 2.4. 
Table 2.4: Name, sequence and residues of CylR1 of the applied peptides.

\begin{tabular}{lll}
\hline \hline NAME & SEQUENCE & RESIDUES of CylR1 \\
\hline CylR1A & KKDERILYTISL & residue 24 to 35 \\
CylR1B & DSKKDERILYTISL & residue 22 to 35 \\
CylR1C & KKDERILYTISLTS & residue 24 to 37 \\
CylR1D & FFNKRTS & residue 88 to 94 \\
CylR1E & LLFFNKRTS & residue 86 to 94 \\
\hline \hline
\end{tabular}

\subsection{Culture media and antibiotics}

All culture media were prepared according to Table 2.5 followed by autoclave sterilisation. Before use, $100 \mu \mathrm{g} / 1$ of ampicillin or $70 \mu \mathrm{g} / 1$ of kanamycin was added to every medium. For agar plates $15 \mathrm{~g}$ agar per $1 \mathrm{l}$ medium were added before autoclaving.

Table 2.5: Culture media and antibiotics

\begin{tabular}{|c|c|c|c|}
\hline NAME & AMOUNT & CHEMICAL & REMARK \\
\hline \multirow{4}{*}{$2 \times$ YT-medium } & $16 \mathrm{~g}$ & tryptone & \\
\hline & $10 \mathrm{~g}$ & yeast extract & \\
\hline & $5 \mathrm{~g}$ & $\mathrm{NaCl}$ & \\
\hline & up to 11 & $\mathrm{H}_{2} \mathrm{O}$ & \\
\hline \multirow{4}{*}{ LB-medium } & $10 \mathrm{~g}$ & tryptone & \\
\hline & $5 \mathrm{~g}$ & yeast extract & \\
\hline & $10 \mathrm{~g}$ & $\mathrm{NaCl}$ & \\
\hline & up to 11 & $\mathrm{H}_{2} \mathrm{O}$ & \\
\hline \multirow{10}{*}{ M9-minimalmedium } & $6.8 \mathrm{~g}$ & $\mathrm{Na}_{2} \mathrm{HPO}_{4}$ & \\
\hline & $3 \mathrm{~g}$ & $\mathrm{KH}_{2} \mathrm{PO}_{4}$ & \\
\hline & $0.5 \mathrm{~g}$ & $\mathrm{NaCl}$ & \\
\hline & $1 \mathrm{~g}$ & $\mathrm{NH}_{4} \mathrm{Cl}$ or ${ }^{15} \mathrm{NH}_{4} \mathrm{Cl}$ & \\
\hline & $4 \mathrm{~g}$ & glucose or ${ }^{13} \mathrm{C}_{6}$-glucose or $\mathrm{D}_{8}$-glycerol & \\
\hline & $2 \mathrm{ml}, 1 \mathrm{M}$ & $\mathrm{MgSO}_{4}$ & \\
\hline & $50 \mu \mathrm{l}, 2 \mathrm{M}$ & $\mathrm{CaCl}_{2}$ & \\
\hline & $0.03 \mathrm{~g}$ & thiaminechloride hydrochloride & \\
\hline & $10 \mathrm{ml}$ & trace elements & \\
\hline & up to 11 & $\mathrm{H}_{2} \mathrm{O}$ or $99.9 \% \mathrm{D}_{2} \mathrm{O}$ & \\
\hline \multirow{9}{*}{ trace elements } & $0.6 \mathrm{~g}$ & $\mathrm{FeSO}_{4} \cdot 7 \mathrm{H}_{2} \mathrm{O}$ & \\
\hline & $0.094 \mathrm{~g}$ & $\mathrm{MnCl}_{2} \cdot 2 \mathrm{H}_{2} \mathrm{O}$ & \\
\hline & $0.08 \mathrm{~g}$ & $\mathrm{CoCl}_{2} \cdot 6 \mathrm{H}_{2} \mathrm{O}$ & \\
\hline & $0.07 \mathrm{~g}$ & $\mathrm{ZnSO}_{4} \cdot 7 \mathrm{H}_{2} \mathrm{O}$ & \\
\hline & $0.03 \mathrm{~g}$ & $\mathrm{CuCl}_{2} \cdot 2 \mathrm{H}_{2} \mathrm{O}$ & \\
\hline & $0.002 \mathrm{~g}$ & $\mathrm{H}_{3} \mathrm{BO}_{3}$ & \\
\hline & $0.025 \mathrm{~g}$ & $\left(\mathrm{NH}_{4}\right)_{6} \mathrm{Mo}_{7} \mathrm{O}_{24} \cdot 4 \mathrm{H}_{2} \mathrm{O}$ & \\
\hline & up to $100 \mathrm{ml}$ & $\mathrm{H}_{2} \mathrm{O}$ & stir $10 \mathrm{~min}$ \\
\hline & $0.5 \mathrm{~g}$ & EDTA & stir over night \\
\hline ampicillin stock solution & $100 \mathrm{mg} / \mathrm{ml}$ & ampicillin sodium salt & steril filtrated, stored at $-20^{\circ} \mathrm{C}$ \\
\hline kanamycin stock solution & $70 \mathrm{mg} / \mathrm{ml}$ & kanamycin & steril filtrated, stored at $-20^{\circ} \mathrm{C}$ \\
\hline IPTG stock solution & $1 \mathrm{M}$ & IPTG & steril filtrated, stored at $-20^{\circ} \mathrm{C}$ \\
\hline
\end{tabular}




\subsection{Buffers and solutions}

The buffers and solutions used for the methods and protein preparations described in this work are subsumed in Table 2.6.

Table 2.6: Buffers and solutions

\begin{tabular}{|c|c|c|c|}
\hline METHOD & NAME & AMOUNT & CHEMICAL \\
\hline \multirow{6}{*}{ Affinity chromatography } & wash buffer 1 & $\begin{array}{l}20 \mathrm{mM} \\
500 \mathrm{mM} \\
10 \mathrm{mM}\end{array}$ & $\begin{array}{l}\text { Tris/HCl pH } 7.9 \\
\mathrm{NaCl} \\
\text { imidazole }\end{array}$ \\
\hline & wash buffer 2 & $\begin{array}{l}20 \mathrm{mM} \\
500 \mathrm{mM} \\
40 \mathrm{mM}\end{array}$ & $\begin{array}{l}\text { Tris/HCl pH } 7.9 \\
\mathrm{NaCl} \\
\text { imidazole }\end{array}$ \\
\hline & & $20 \mathrm{mM}$ & Tris $/ \mathrm{HCl} \mathrm{pH} 7.9$ \\
\hline & elution buffer 1 & $500 \mathrm{mM}$ & $\mathrm{NaCl}$ \\
\hline & & $100 \mathrm{mM}$ & imidazole \\
\hline & elution buffer 2 & $\begin{array}{l}20 \mathrm{mM} \\
500 \mathrm{mM} \\
500 \mathrm{mM}\end{array}$ & $\begin{array}{l}\text { Tris/HCl pH } 7.9 \\
\mathrm{NaCl} \\
\text { imidazole }\end{array}$ \\
\hline \multirow[t]{2}{*}{ Agarose gel } & $5 \times$ DNA loading buffer & $\begin{array}{l}12.5 \mathrm{~g} \\
2.5 \mathrm{ml}, 0.5 \mathrm{M} \\
0.5 \mathrm{~g} \\
25 \mathrm{mg} \\
25 \mathrm{mg} \\
\text { up to } 50 \mathrm{ml}\end{array}$ & $\begin{array}{l}\text { Ficoll } 400 \\
\text { EDTA pH } 8.0 \\
\text { SDS } \\
\text { bromphenol blue } \\
\text { xylene cyanol FF } \\
\mathrm{H}_{2} \mathrm{O}\end{array}$ \\
\hline & $10 \times$ TBE buffer & $\begin{array}{l}108 \mathrm{~g} \\
55 \mathrm{~g} \\
40 \mathrm{ml}, 0.5 \mathrm{M} \\
\text { up to } 1 \mathrm{l}\end{array}$ & $\begin{array}{l}\text { Tris } \\
\text { boric acid } \\
\text { EDTA pH } 8.0 \\
\mathrm{H}_{2} \mathrm{O}\end{array}$ \\
\hline \multirow{3}{*}{ Cell lysis } & denaturing lysis buffer & $\begin{array}{l}8 \mathrm{M} \\
20 \mathrm{mM} \\
500 \mathrm{mM} \\
10 \mathrm{mM}\end{array}$ & $\begin{array}{l}\text { urea } \\
\text { Tris/HCl pH } 7.9 \\
\mathrm{NaCl} \\
\text { imidazole }\end{array}$ \\
\hline & native lysis buffer & $\begin{array}{l}20 \mathrm{mM} \\
500 \mathrm{mM} \\
10 \mathrm{mM}\end{array}$ & $\begin{array}{l}\text { Tris/HCl } \mathrm{pH} 7.9 \\
\text { NaCl } \\
\text { imidazole }\end{array}$ \\
\hline & lysis buffer for CylR2 & $\begin{array}{l}50 \mathrm{mM} \\
100 \mathrm{mM} \\
1 \mathrm{mM} \\
1 \mathrm{mM} \\
5 \mathrm{mM} \\
0.1 \mathrm{mg} / \mathrm{l}\end{array}$ & $\begin{array}{l}\text { HEPES pH } 6.0 \\
\mathrm{NaCl} \\
\text { EDTA } \\
\mathrm{MgCl}_{2} \\
\text { DTT } \\
\text { DNase I }\end{array}$ \\
\hline Gel filtration & gel filtration buffer & $\begin{array}{l}20 \mathrm{mM} \\
150 \mathrm{mM}\end{array}$ & $\begin{array}{l}\text { HEPES pH } 7.0 \\
\mathrm{NaCl}\end{array}$ \\
\hline \multirow{4}{*}{ Ion exchange } & anion exchange buffer A for CesT & $\begin{array}{l}20 \mathrm{mM} \\
0.1 \mathrm{M} \\
0.5 \mathrm{mM} \\
1 \mathrm{mM}\end{array}$ & $\begin{array}{l}\text { Tris/HCl pH } 8.2 \\
\text { NaCl } \\
\text { EDTA } \\
\text { DTT }\end{array}$ \\
\hline & anion exchange buffer $\mathrm{B}$ for CesT & $\begin{array}{l}20 \mathrm{mM} \\
1 \mathrm{M} \\
0.5 \mathrm{mM} \\
1 \mathrm{mM} \\
\end{array}$ & $\begin{array}{l}\text { Tris/HCl pH } 8.2 \\
\mathrm{NaCl} \\
\text { EDTA } \\
\text { DTT }\end{array}$ \\
\hline & ion exchange buffer A for CylR2 & $\begin{array}{l}50 \mathrm{mM} \\
0.1 \mathrm{M} \\
1 \mathrm{mM} \\
1 \mathrm{mM} \\
5 \mathrm{mM}\end{array}$ & $\begin{array}{l}\text { HEPES pH } 6.0 \\
\mathrm{NaCl} \\
\text { EDTA } \\
\mathrm{MgCl}_{2} \\
\text { DTT }\end{array}$ \\
\hline & ion exchange buffer $\mathrm{B}$ for CylR2 & $\begin{array}{l}50 \mathrm{mM} \\
1 \mathrm{M} \\
1 \mathrm{mM} \\
1 \mathrm{mM} \\
5 \mathrm{mM}\end{array}$ & $\begin{array}{l}\text { HEPES pH } 6.0 \\
\mathrm{NaCl} \\
\text { EDTA } \\
\mathrm{MgCl}_{2} \\
\text { DTT }\end{array}$ \\
\hline
\end{tabular}




\begin{tabular}{|c|c|c|c|}
\hline METHOD & NAME & AMOUNT & CHEMICAL \\
\hline \multirow{4}{*}{ Ligation } & \multirow{4}{*}{ T4-DNA ligase buffer } & $40 \mathrm{mM}$ & Tris/HCl pH 7.8 \\
\hline & & $10 \mathrm{mM}$ & $\mathrm{MgCl}_{2}$ \\
\hline & & $1 \mathrm{mM}$ & DTT \\
\hline & & $0.5 \mathrm{mM}$ & ATP \\
\hline \multirow{12}{*}{ NMR } & \multirow{3}{*}{ NMR buffer for CylR2 } & $50 \mathrm{mM}$ & HEPES pH7.0 \\
\hline & & $250-600 \mathrm{mM}$ & $\mathrm{NaCl}$ \\
\hline & & $5-10 \%$ & $\mathrm{D}_{2} \mathrm{O}(\mathrm{v} / \mathrm{v})$ \\
\hline & \multirow{5}{*}{ NMR buffer for CesT } & $50 \mathrm{mM}$ & potassium phosphate $\mathrm{pH} 6.8$ \\
\hline & & $100 \mathrm{mM}$ & $\mathrm{NaCl}$ \\
\hline & & $1 \mathrm{mM}$ & DTT \\
\hline & & $0.5 \mathrm{mM}$ & EDTA \\
\hline & & $5-10 \%$ & $\mathrm{D}_{2} \mathrm{O}(\mathrm{v} / \mathrm{v})$ \\
\hline & \multirow{4}{*}{ NMR buffer for Tir108 } & $50 \mathrm{mM}$ & potassium phosphate $\mathrm{pH} 6.8$ \\
\hline & & $100 \mathrm{mM}$ & $\mathrm{NaCl}$ \\
\hline & & $5 \mathrm{mM}$ & DTT \\
\hline & & $5 \%$ & $\mathrm{D}_{2} \mathrm{O}(\mathrm{v} / \mathrm{v})$ \\
\hline \multirow{6}{*}{ PCR } & \multirow{6}{*}{ Cloned Pfu buffer } & $20 \mathrm{mM}$ & Tris/HCl $\mathrm{pH} 8.8$ \\
\hline & & $2 \mathrm{mM}$ & $\mathrm{MgSO}_{4}$ \\
\hline & & $10 \mathrm{mM}$ & $\mathrm{KCl}$ \\
\hline & & $10 \mathrm{mM}$ & $\left(\mathrm{NH}_{4}\right)_{2} \mathrm{SO}_{4}$ \\
\hline & & $0.1 \%$ & Triton X-100 \\
\hline & & $0.1 \mathrm{mg} / \mathrm{ml}$ & BSA \\
\hline \multirow{4}{*}{ RP-HPLC } & \multirow{2}{*}{ RP-HPLC buffer A } & $99.9 \%$ & $\mathrm{H}_{2} \mathrm{O}$ \\
\hline & & $0.1 \%$ & TFA \\
\hline & \multirow{2}{*}{ RP-HPLC buffer B } & $99.9 \%$ & acetonitrile \\
\hline & & $0.1 \%$ & TFA \\
\hline \multirow{16}{*}{ SDS-PAGE } & APS & $10 \%$ & $(\mathrm{w} / \mathrm{v})$ in $\mathrm{H}_{2} \mathrm{O}$ \\
\hline & \multirow{2}{*}{ destaining solution } & $100 \mathrm{ml}$ & acetic acid \\
\hline & & $900 \mathrm{ml}$ & $\mathrm{H}_{2} \mathrm{O}$ \\
\hline & \multirow{5}{*}{$4 \times$ protein loading buffer } & $1.7 \mathrm{~g}$ & SDS \\
\hline & & $7.5 \mathrm{ml}, 1 \mathrm{M}$ & Tris/HCl pH 6.8 \\
\hline & & $23 \mathrm{ml}$ & glycerol \\
\hline & & $\begin{array}{l}50 \mathrm{mg} \\
0.5 \mathrm{ml}\end{array}$ & $\begin{array}{l}\text { bromphenol blue } \\
\beta \text {-mercaptoethanol }\end{array}$ \\
\hline & & up to $50 \mathrm{ml}$ & $\mathrm{H}_{2} \mathrm{O}$ \\
\hline & \multirow{4}{*}{ running buffer } & $1 \mathrm{~g}$ & SDS \\
\hline & & $3.03 \mathrm{~g}$ & Tris \\
\hline & & $14.4 \mathrm{~g}$ & glycine \\
\hline & & up to 11 & $\mathrm{H}_{2} \mathrm{O}$ \\
\hline & \multirow{4}{*}{ staining solution } & $2.2 \mathrm{~g}$ & Coomassie Brilliant blue G250 \\
\hline & & $100 \mathrm{ml}$ & acetic acid \\
\hline & & $250 \mathrm{ml}$ & isopropanol \\
\hline & & $650 \mathrm{ml}$ & $\mathrm{H}_{2} \mathrm{O}$ \\
\hline \multirow{3}{*}{ TEV-cleavage } & \multirow{3}{*}{ TEV-buffer } & $50 \mathrm{mM}$ & Tris/HCl $\mathrm{pH} 8.0$ \\
\hline & & $1 \mathrm{mM}$ & DTT \\
\hline & & $0.5 \mathrm{mM}$ & EDTA \\
\hline
\end{tabular}




\subsection{Enzymes and Chemicals}

Enzymes and chemicals used in this thesis can be found in Table 2.7.

Table 2.7: Enzymes and chemicals

\begin{tabular}{|c|c|}
\hline CHEMICALS/ENZYMES & COMPANY \\
\hline Agar, DNase I, IPTG & AppliChem, Darmstadt, Germany \\
\hline Pf1 NMR co-solvent & ASLA $^{\text {biotech }}$, Riga, Latvia \\
\hline ammonium chloride $\left(>98 \%{ }^{15} \mathrm{~N}\right)$ & Cambridge Isotope Laboratories, Andover, USA \\
\hline${ }^{13} \mathrm{C}_{6}$-D-glucose $\left(>98 \%{ }^{13} \mathrm{C}\right)$ & Spectra Stable Isotopes, Columbia, USA \\
\hline Wizard Screen ${ }^{T M}$ I/II & Emerald BioSystems, Bainbridge Island, USA \\
\hline $99.9 \% \mathrm{D}_{2} \mathrm{O}, 98 \% \mathrm{D}_{8}$-glycerol & Eurisotop, Gif-sur-Yvette, Frankreich \\
\hline $\begin{array}{l}\text { BamHI, CIAP, } \lambda \text { HindIII, NcoI, NdeI, } \phi \mathrm{X} 174 \mathrm{DNA} / B s u \mathrm{RI}(H a e \mathrm{III}), \\
\text { T4-DNA ligase }\end{array}$ & Fermentas, St. Leon-Rot, Germany \\
\hline $\begin{array}{l}\text { ammonium molybdate tetrahydrate, ascorbic acid, Coomassie Bril- } \\
\text { lant Blue R-250, copper chloride dihydrate, iron (II) sulfate hep- } \\
\text { tahydrate }\end{array}$ & Fluka, Neu-Ulm, Germany \\
\hline DTT & Gerbu, Gaiberg, Germany \\
\hline agarose, kanamycin sulphate & GibcoBRL, Karlsruhe, Germany \\
\hline Crystal Screen $^{T M}$ I/II, Index Screen, PEG/Ion Screen & Hampton Research, Aliso Viejo, USA \\
\hline Ficoll 400 & ICN Biomedicals Inc, Costa Mesa, USA \\
\hline Bench Mark protein ladder & Invitrogen, Karlsruhe, Germany \\
\hline $\begin{array}{l}\text { acetic acid, } \alpha \text {-D }(+) \text {-glucose monohydrate, ammonium acetate, am- } \\
\text { monium chloride, ammonium hydrocarbonate, boric acid, disodium } \\
\text { hydrogen phosphate, ethanol, glycerol, glycine, hydrochloric acid, } \\
\text { manganese chloride dihydrate, magnesium sulfate heptahydrate, } \\
\text { sodium chloride, sodium dihydrogen phosphate, sodium hydroxide, } \\
\text { TFA, thiaminechloride hydrochloride, Tris, urea }\end{array}$ & Merck, Darmstadt, Germany \\
\hline BSA & New England Biolabs, Ipswich, USA \\
\hline $\begin{array}{l}\text { Hot Star Taq, Ni-NTA Agarose, QIAGEN Plasmid Midi Kit, QI- } \\
\text { Aprep Spin Miniprep Kit, QIAquick Gel Extraction Kit, QIAquick } \\
\text { PCR Purification Kit }\end{array}$ & Qiagen, Hilden, Germany \\
\hline cobalt chloride hexahydrate & Riedel-de Haën, Seelze, Germany \\
\hline $\begin{array}{l}\text { Complete }{ }^{T M} \text { protease inhibitors, Complete }{ }^{T M} \text { protease inhibitors } \\
\text { EDTA-free, elastase, protease K, trypsin }\end{array}$ & Roche Diagnostics, Mannheim, Germany \\
\hline $\begin{array}{l}\text { acetonitrile, ampicillin sodium salt, APS, dipotassium hydrogen } \\
\text { phosphate, ethidium bromide, EDTA, HEPES, imidazole, magne- } \\
\text { sium chloride hexahydrate, MES, MOPS, potassium dihydrogen } \\
\text { phosphate, Rotiphorese Gel 30, sodium acetate, TEMED, tryptone, } \\
\text { yeast extract }\end{array}$ & Roth, Karlsruhe, Germany \\
\hline SDS & Serva, Heidelberg, Germany \\
\hline ATP, chymotrypsin & Sigma, Deisenhofen, Germany \\
\hline Turbo Pfu DNA polymerase, dNTP & Stratagene, La Jolla, USA \\
\hline MTSL & Toronto Research Chemicals, Toronto, Canada \\
\hline
\end{tabular}




\subsection{Equipment}

In the following, laboratory instruments and consumables are tabulated.

Table 2.8: Equipment and supplier

\begin{tabular}{|c|c|}
\hline COMMON NAME & IDENTIFIER/COMPANY \\
\hline Balances & $\begin{array}{l}\text { Sartorius B } 3100 \text { S, Sartorius, Göttingen, Germany } \\
\text { Sartorius AC } 210 \text { S, Sartorius, Göttingen, Germany }\end{array}$ \\
\hline Centrifuges & $\begin{array}{l}\text { Beckmann-Coulter Avanti J-20 and J-301, rotors: JLA 8.100, JLA 9.100, JLA } 16.250 \text {, } \\
\text { JA } 25.50 \mathrm{Ti} \text {, JA } 30.50 \mathrm{Ti} \text {, Krefeld, Germany } \\
\text { Eppendorf Centrifuge 5415D, Wesseling-Berzdorf, Germany } \\
\text { Eppendorf Centrifuge 5804, Wesseling-Berzdorf, Germany } \\
\text { Heraeus Biofuge primo, Kendro, Hanau, Germany }\end{array}$ \\
\hline Concentrators & $\begin{array}{l}\text { Microcon, YM-3 and YM-10, Amicon, Bedford, USA } \\
\text { Centricon, YM-3 and YM-10, Amicon, Bedford, USA } \\
\text { Centriplus, YM-3 and YM-10, Amicon, Bedford, USA } \\
\text { Vivaspin } 2 \mathrm{ml} \text { MWCO } 10.000 \text { PES, Vivascience, Hannover, Germany }\end{array}$ \\
\hline Crystallization & $\begin{array}{l}\text { TECAN Genesis RSP 150, Crailsheim, Germany } \\
\text { Mosquito Crystal, TTP LabTech Ltd., Melbourn, UK }\end{array}$ \\
\hline Desalting & $\begin{array}{l}\text { NAP }^{T M}-10, \text { Amersham Pharmacia Biotech, Freiburg, Germany } \\
\text { PD }^{T M}-10 \text {, Amersham Pharmacia Biotech, Freiburg, Germany }\end{array}$ \\
\hline Dialysis & $\begin{array}{l}\text { Slide-A-Lyzer Dialysis Cassettes, MWCO 3500, 0.1-0.5 ml Capacity, Pierce Biotechnol- } \\
\text { ogy, Inc., Rockford, IL, USA } \\
\text { Slide-A-Lyzer Dialysis Cassettes, MWCO 10000, 0.1-0.5 ml Capacity, Pierce Biotechnol- } \\
\text { ogy, Inc., Rockford, IL, USA } \\
\text { Spectra Por membranes, MWCO 10000, Roth, Karlsruhe, Germany } \\
\text { Spectra Por membranes, MWCO 3500, Roth, Karlsruhe, Germany }\end{array}$ \\
\hline Electrophoresis & $\begin{array}{l}\text { Kodak Electrophoresis documentation and analysis system 120, Eastman Kodak Co., } \\
\text { New York, NY, USA } \\
\text { Power Pac 300, BioRad, München, Germany } \\
\text { Polyacrylamide gel electrophoresis: Mini-PROTEAN } 3 \text { Cell, BioRad, München, Germany } \\
\text { Agarose gel electrophoresis: Mini-Sub Cell GT, BioRad, München, Germany }\end{array}$ \\
\hline$-80{ }^{\circ} \mathrm{C}$ freezer & MDF-U71V Ultra-low temperature freezer, SANYO Electric Co., Ltd, Osaka, Japan \\
\hline Filtering & sterile filter $0,20 \mu \mathrm{m}$, Sartorius, Göttingen, Germany \\
\hline FPLC & $\begin{array}{l}\text { Äkta prime, Amersham Pharmacia Biotech, Freiburg, Germany } \\
\text { Äkta basic, Amersham Pharmacia Biotech, Freiburg, Germany } \\
\text { Disposable } 10 \text { ml polypropylene columns, Pierce Biotechnology, Inc., Rockford, IL, USA } \\
\text { Frac-100, Amersham Pharmacia Biotech, Freiburg, Germany } \\
\text { HiLoad Superdex } 75 \text { prep grade } 16 / 60 \text {, Amersham Pharmacia Biotech, Freiburg, Ger- } \\
\text { many } \\
\text { HiTrap Q-Sepharose, Amersham Pharmacia Biotech, Freiburg, Germany } \\
\text { HiTrap S-Sepharose, Amersham Pharmacia Biotech, Freiburg, Germany }\end{array}$ \\
\hline HPLC & $\begin{array}{l}\text { system 1: UV-975, PU-980, LG-980-02, DG-980-50, AS-2055Plus, CO-200, JASCO In- } \\
\text { ternational, Groß-Umstadt, Germany } \\
\text { system 2: MD-910, PU-980, LG-1580-04, DG-1580-54, AS-950-10, CO-200, JASCO In- } \\
\text { ternational, Groß-Umstadt, Germany } \\
\text { system 3: MD-2010Plus, PU-2080Plus, LG-2080-04, DG-2080-54, AS-2055Plus, CO-200, } \\
\text { JASCO International, Groß-Umstadt, Germany } \\
\text { Vydac C4 8x250 mm, Hesperia, CA, USA } \\
\text { Vydac C18 8x250 mm, Hesperia, CA, USA }\end{array}$ \\
\hline Incubator & $\begin{array}{l}\text { Infors Multitron HT, Einsbach, Germany } \\
\text { Certomat R, B. Braun Biotech International, Melsungen, Germany }\end{array}$ \\
\hline Lyophylisation & Christ Alpha 2-4, B. Braun Biotech International, Melsungen, Germany \\
\hline NMR & $\begin{array}{l}\text { AVANCE 400, Bruker, Karlsruhe, Germany } \\
\text { AVANCE 600, Bruker, Karlsruhe, Germany } \\
\text { DRX 600, Bruker, Karlsruhe, Germany } \\
\text { AVANCE 700, Bruker, Karlsruhe, Germany } \\
\text { DRX 800, Bruker, Karlsruhe, Germany } \\
\text { AVANCE 900, Bruker, Karlsruhe, Germany } \\
\text { Quality NMR Sample Tubes } 5 \mathrm{~mm} \text {, Norell, Inc., Landisville, NJ, USA } \\
\text { Shigemi NMR tube } 5 \mathrm{~mm} \text {, Shigemi Corp., Tokyo, Japan }\end{array}$ \\
\hline
\end{tabular}




\begin{tabular}{ll}
\hline \hline COMMON NAME & IDENTIFIER/COMPANY \\
\hline Peptide synthesis & 433A Peptide Synthesizer, Applied Biosystems, Darmstadt, Germany \\
\hline pH-Meter & PB11 PY-P10, Sartorius, Göttingen, Germany \\
\hline \multirow{2}{*}{ Spectroscopy } & $\begin{array}{l}\text { UV/VIS-Spectrophotometer, Hewlett-Packard 8453, Böblingen, Germany } \\
\text { Mass spectropolarimeter J-720, JASCO International, Groß-Umstadt, Germany } \\
\text { France }\end{array}$ \\
\hline Thermocycler & HYBAID PCR sprint, Perkin Elmer, Wellesley, MA, USA \\
\hline Ultrasound & SONOPLUS HD 2200, Bandelin, Berlin, Germany \\
\hline \hline
\end{tabular}

\subsection{Software}

The software used for processing and analysing NMR spectra, for calculating and analysing structures and for preparing figures is summarized in Table 2.9.

Table 2.9: Software

\begin{tabular}{|c|c|}
\hline PROGRAM & REFERENCE/ORIGIN \\
\hline AQUA 2.0 & 33 \\
\hline BOBSCRIPT & 34 \\
\hline $\mathrm{CCP} 4$ & 35,36 \\
\hline ClusPro & http://nrc.bu.edu/cluster, 37, 38, 39 \\
\hline CYANA 2.0 & 40 \\
\hline DINO & 41 \\
\hline InsightII & MSI 2000 release, San Diego, CA, USA \\
\hline MARS & 42 \\
\hline MOLMOL & 43 \\
\hline MOLSCRIPT & 44 \\
\hline MSMS & 45 \\
\hline NMRPipe/NMRDraw & 46 \\
\hline NMRView 5.0.4 & 47 \\
\hline PALES & 48 \\
\hline PROCHECK & 49 \\
\hline PROCHECK-NMR & 33 \\
\hline PyMOL & http://www.pymol.org, 50 \\
\hline RASTER3D & 51 \\
\hline Sparky 3 & T. D. Goddard and D. G. Kneller, University of California, San Francisco \\
\hline TALOS & 52 \\
\hline XPLOR-NIH 2.9.7 & 53 \\
\hline X-WINNMR 3.5 & Bruker, Karlsruhe, Germany \\
\hline
\end{tabular}




\section{3}

Methods

Here, all general methods applied in this thesis are presented. The detailed purification strategies for the studied proteins are given in Chapters 4 and 6 .

\subsection{Molecular biology methods}

\subsubsection{Agarose gel electrophoresis}

DNA fragments were separated on horizontal agarose gels. The gels were prepared by melting $1 \%(\mathrm{w} / \mathrm{v})$ agarose in 1 x TBE buffer and adding $25 \mu \mathrm{l} / \mathrm{l}$ ethidium bromide. Each DNA sample was mixed with $1 / 5$ volumes of 5 x DNA-load. Gels were run at a constant voltage of $100 \mathrm{~V}$, imaged under UV-light and digitized for documentation.

\subsubsection{Isolation and purification of DNA}

$10 \mathrm{ml}$ E. coli XL2-blue overnight cultures were used for purification of up to $20 \mu \mathrm{g}$ plasmid DNA with the help of the QIAprep Spin Miniprep Kit and $200 \mathrm{ml}$ E. coli XL2-blue overnight cultures were used for purification of up to $100 \mu \mathrm{g}$ plasmid DNA with the help of the QIAGEN Plasmid Midi Kit. Plasmid DNA was isolated from the cell pellets according to the producer's instructions.

For removal of enzymes, salts and buffers, columns with a silica gel membrane (QIAquick spin columns) were used according to the user manual.

DNA fragments were purified from $1 \%$ agarose gels with the QIAquick Gel Extraction Kit. 


\subsubsection{Enzymatic modifications of DNA}

\subsubsection{Fragmentation with restriction enzymes}

DNA was digested with 2-10 units restriction enzyme per $\mu \mathrm{g}$ DNA under buffer conditions recommended by the manufacturer. The reaction was performed at $37^{\circ} \mathrm{C}$ for 1-2 h with BamHI or overnight for all other restriction enzymes.

\subsubsection{Dephosphorylation}

To avoid self-ligation of restricted vectors, 5'-overhanging phosphate groups were cleaved by adding 1 unit CIAP (calf intestine alkaline phosphatase) per $1 \mu \mathrm{g}$ DNA and incubating for $1 \mathrm{~h}$ at $37^{\circ} \mathrm{C}$.

\subsubsection{Ligation}

To ligate DNA-fragments with overhanging ends, 50-100 ng vector DNA were mixed with insert-DNA at a ratio of $1: 3$ to $1: 25$ in T4-DNA ligase buffer and incubated overnight at $16{ }^{\circ} \mathrm{C}$ with 4 units T4-DNA ligase.

\subsubsection{Polymerase chain reaction (PCR)}

For amplification of DNA fragments by PCR [54], $25 \mu \mathrm{l}$ reaction mixture containing 20-30 ng plasmid DNA template, 10 pmol of each primer, $100 \mathrm{nmol}$ dNTPs and 2.5 units Pfu Turbo DNA polymerase in Cloned Pfu buffer were prepared on ice. The PCR was carried out after an initial denaturing step of 2 min at $95^{\circ} \mathrm{C}$ with 30 cycles comprising the following steps: 1.) denaturing for $30 \mathrm{~s}$ at $95^{\circ} \mathrm{C}, 2$.) annealing for $30 \mathrm{~s}$ at $55^{\circ} \mathrm{C}$ and 3.) elongation for $1 \mathrm{~min}$ per $\mathrm{kb}$ of fragment length at $72{ }^{\circ} \mathrm{C}$. The final elongation step was extended by $10 \mathrm{~min}$ at $72{ }^{\circ} \mathrm{C}$.

Colony PCR was used to verify a successful cloning directly from single colonies without prior preparation of plasmid DNA. Each $25 \mu \mathrm{l}$ PCR reaction contained 10 pmol of each primer, $50 \mathrm{nmol}$ dNTPs and 0.3 units HotStar Taq polymerase. Bacterial cells from single colonies were transferred into PCR reaction mixtures with the help of sterile pipette tips, which were afterwards used to inoculate $10 \mathrm{ml}$ LB medium containing ampicillin. The LB cultures were incubated overnight at $37^{\circ} \mathrm{C}$. The PCR 
reaction mixtures were initially heated to $95^{\circ} \mathrm{C}$ for 15 min to activate the HotStar Taq polymerase. Afterwards, the PCR was performed with 25 cycles comprised of: 1.) denaturing for $30 \mathrm{~s}$ at $95^{\circ} \mathrm{C}, 2$.) annealing for 1 min at $55^{\circ} \mathrm{C}$ and 3.) elongation for 2 min per $\mathrm{kb}$ of fragment length at $72^{\circ} \mathrm{C}$. The final elongation step was extended by $10 \mathrm{~min}$ at $72{ }^{\circ} \mathrm{C}$. The PCR products were analysed by agarose gel electrophoresis (see 3.1.1). Plasmid DNA isolated from LB cultures (see 3.1.2) with a positive PCR result was confirmed by DNA sequencing (see 3.1.6).

\subsubsection{Site-directed mutagenesis}

Single amino acid changes were generated using the QuikChange site-directed mutagenesis kit. Primers used for mutagenesis were designed according to the instruction manual and are included in Table 2.3. The $50 \mu \mathrm{l}$ PCR reaction mixtures contained $125 \mathrm{ng}$ of each primer, $10 \mathrm{ng}$ plasmid DNA template, $6.25 \mu \mathrm{mol}$ dNTPs and 2.5 units Turbo Pfu DNA polymerase in Cloned Pfu buffer. The cycling after an initial step at $95^{\circ} \mathrm{C}$ for $30 \mathrm{~s}$ was performed as follows: 1.) denaturing for $30 \mathrm{~s}$ at $95^{\circ} \mathrm{C}, 2$.) annealing for $1 \mathrm{~min}$ at $55^{\circ} \mathrm{C}$ and 3.) elongation for $2 \mathrm{~min}$ per $\mathrm{kb}$ of plasmid length at $68^{\circ} \mathrm{C}$. For creating single nucleotide changes and for creating double or triple nucleotide changes the number of cycles was 12 and 16, respectively. Following temperature cycling, 10 units of Dpn I restriction enzyme were directly added to each PCR reaction mixture and incubated for $1 \mathrm{~h}$ at $37^{\circ} \mathrm{C} .1 \mu \mathrm{l}$ of the reaction mix was transformed into E. coli XL2-blue (see 3.1.8), plasmid DNA was purified (see 3.1.2) and correct introduction of the desired mutation was confirmed by DNA sequencing of the insert region of the plasmid DNA (see 3.1.6).

\subsubsection{DNA sequencing}

Sequencing of purified plasmid DNA was performed via the extended Hot Shot DNA sequencing service of Seqlab (Göttingen, Germany). $200 \mu \mathrm{l}$ PCR tubes with a flat lid were loaded with a total volume of $7 \mu \mathrm{l}$ containing $20 \mathrm{pmol}$ of primer and $0.6-0.7 \mu \mathrm{g}$ plasmid DNA in $\mathrm{H}_{2} \mathrm{O}$. 


\subsubsection{Concentration and purity of DNA}

The absorption at $260 \mathrm{~nm}\left(\mathrm{~A}_{260}\right)$ was measured to determine the DNA concentration. An $\mathrm{A}_{260}$ of 1 equals $50 \mu \mathrm{g} / \mathrm{ml}$ dsDNA [55]. The ratio of $\mathrm{A}_{260} / \mathrm{A}_{280}$ was calculated. A ratio between 1.8 and 2 indicates reasonably pure DNA, suitable for DNA sequencing.

\subsubsection{Transformation of $E$. coli}

$1 \mu \mathrm{l}$ plasmid DNA or $5 \mu \mathrm{l}$ ligation assay were added to $40 \mu \mathrm{l}$ competent cells and incubated for $30 \mathrm{~min}$ on ice. Cells were subjected to a heat shock of $42{ }^{\circ} \mathrm{C}$ for $60 \mathrm{~s}$ and supplemented with $1 \mathrm{ml}$ of $2 \mathrm{x}$ YT medium. After incubation for $1 \mathrm{~h}$ at $37{ }^{\circ} \mathrm{C}$ and centrifugation for $1 \mathrm{~min}$ at $7500 \mathrm{x}$ g the cell pellet was resuspended in $\sim 50 \mu \mathrm{l}$ medium and plated onto LB-agar plates containing ampicillin.

\subsection{Protein expression}

\subsubsection{Cultivation and storage of E. coli}

All proteins were expressed in the E. coli strain BL21(DE3). Agar plates and all liquid nutrient media contained the appropriate antibiotic. Cells were grown at $37^{\circ} \mathrm{C}$ if not denoted differently. A single colony of E. coli BL21(DE3) harbouring the appropriate plasmid was grown on an agar plate and used to inoculate a $1 \mathrm{ml}$ LB overday preculture. $20 \mu \mathrm{l}$ of this preculture were transferred to a $20 \mathrm{ml}$ overnight preculture. The $20 \mathrm{ml}$ overnight preculture was then used to inoculate a $1 \mathrm{l} \mathrm{LB}$ culture. For producing labeled protein, the $20 \mathrm{ml} \mathrm{LB}$ and the $1 \mathrm{l} \mathrm{LB}$ culture were replaced by a $50 \mathrm{ml}$ and a $1 \mathrm{l}$ M9-minimal medium culture containing the required nitrogen and carbon source. In the case of deuterium labeling, M9-minimal medium culture was prepared with $99.9 \% \mathrm{D}_{2} \mathrm{O}$. For perdeuteration, $2 \mathrm{ml}$ M9-minimal medium precultures with the deuterium concentration increasing from $33 \%, 50 \%, 75 \%$ to $99.9 \%$ and $4 \mathrm{~g} / \mathrm{l} \mathrm{D}_{8}$-glycerol as the sole carbon source were grown for 10-22 $\mathrm{h}$ each. Each preculture was centrifuged at $7500 \mathrm{x}$ g and the pellet was resuspended in the medium with the next deuterium concentration. Cell growth was monitored 
by measuring the $\mathrm{OD}_{600}$. At an $\mathrm{OD}_{600}$ of 0.6-0.8 the protein expression was induced by addition of $1 \mathrm{M}$ IPTG to a final concentration of $1 \mathrm{mM}$. The cells were harvested at an $\mathrm{OD}_{600}$ of $1.2-1.6$ by centrifugation at $7500 \mathrm{x} \mathrm{g}$ and $4{ }^{\circ} \mathrm{C}$ for $15 \mathrm{~min}$. The cell pellets were stored at $-80^{\circ} \mathrm{C}$.

For long term storage of the E. coli strains, $350 \mu \mathrm{l}$ stationary LB culture were mixed with $150 \mu$ l sterile $50 \%$ glycerol and stored at $-80{ }^{\circ} \mathrm{C}$.

\subsubsection{Cell lysis}

For cell lysis under native conditions, cell pellets from 11 cultures were thawed on ice and suspended in 40-50 ml lysis buffer containing $0.5 \mathrm{mM} \mathrm{PMSF}$ and half a tablet of Complete $^{T M}$ protease inhibitors. The suspension was sonicated $9 \times 20 \mathrm{~s}$ on ice. The lysate was centrifuged for $30-45 \mathrm{~min}$ at $48000 \mathrm{xg}$ and $4{ }^{\circ} \mathrm{C}$.

Cell lysis under denaturing conditions was performed in 40-50 ml lysis buffer containing $8 \mathrm{M}$ urea. Pellets were suspended and stirred for $1 \mathrm{~h}$ or overnight at room temperature. The lysate was centrifuged for $1 \mathrm{~h}$ at $14{ }^{\circ} \mathrm{C}$ and $48000 \mathrm{x}$ g.

\subsection{Protein methods}

\subsubsection{Nickel-nitrilotriacetic acid (Ni-NTA) agarose affinity chro- matography}

Components of the required buffers can be found in Table 2.6. For purification of sevenfold histidine $\left(\mathrm{His}_{7}\right)$-tagged proteins under denaturing conditions, the wash and elution buffers (see 2.6) contained $8 \mathrm{M}$ urea in addition.

Proteins with a N-terminal His - -tag were purified via a Ni-NTA agarose column. $3 \mathrm{ml}$ of resin were used per protein solution from $1 \mathrm{l}$ of expression culture. The resin, equilibrated with wash buffer 1, was incubated with the supernatant from the cell lysis (see 3.2.2) for $1 \mathrm{~h}$ at $4{ }^{\circ} \mathrm{C}$ under native conditions or at room temperature under denaturing conditions. After washing the column with 10 column volumes of wash buffer 1 , bound proteins were eluted by increasing the imidazole concentration in three steps: 1.) 5 column volumes of wash buffer 2,2.) 5 column volumes of elution buffer 1 and 3.) 5 column volumes of elution buffer 2 . 


\subsubsection{Ion exchange chromatography}

Ion exchange chromatography was performed on an ÄKTA prime low pressure liquid chromatography system with HiTrap columns. Before each run, the HiTrap column was prepared by washing at first with 5 bed volumes of buffer A and then with 5 bed volumes of buffer B. Finally, the column was equilibrated with 5-10 bed volumes of buffer A. Samples were loaded at a flow rate of $1 \mathrm{ml} / \mathrm{min}$ onto the equilibrated column. Unbound protein was washed with 5-10 column volumes of buffer A. The elution was performed applying a linear gradient of 0-100 \% buffer B at a flow rate of $1-3 \mathrm{ml} / \mathrm{min}$ and collecting 2 or $3 \mathrm{ml}$ fractions. Protein elution was detected by measuring the absorption at $280 \mathrm{~nm}\left(\mathrm{~A}_{280}\right)$.

\subsubsection{Gel filtration}

An ÄKTA basic medium pressure liquid chromatography system was used for gel filtration with a HiLoad Superdex 75 prep grade 16/60 column. 2-5 ml of the concentrated protein solution containing 10-20 mg protein were loaded onto the column, equilibrated with gel filtration buffer. The gel filtration was performed at a constant flow rate of $1 \mathrm{ml} / \mathrm{min} .2 \mathrm{ml}$ fractions were collected.

\subsubsection{Reversed phase-high performance liquid chromatography (RP-HPLC)}

RP-HPLC systems were operated by Kerstin Overkamp or Gerhard Wolf.

A semi-preparative $\mathrm{C}_{18}$-HPLC column was used for final purification of all CylR2 samples and a semi-preparative $\mathrm{C}_{4}$-HPLC column was used for final purification of all Tir samples. The column was pre-equilibrated with RP-HPLC buffer A (Table 2.6). The elution was performed at a flow rate of $3 \mathrm{ml} / \mathrm{min}$ with a linear gradient of $0-100 \%$ RP-HPLC buffer B in 50 min for Tir and in 30 min for CylR2.

\subsubsection{Cleavage with TEV-protease}

N-terminal His-tags were cleaved with the TEV-protease. At first, His-tagged proteins after affinity chromatography were dialysed at $4{ }^{\circ} \mathrm{C}$ against two times 51 TEV-buffer 
(see 3.3.1). Then the protein concentration was determined (see 3.4.1) and 0.5-2 $\mu \mathrm{g}$ of TEV-protease per $100 \mathrm{mg}$ of protein were added. The cleavage reaction was performed for 16-48 $\mathrm{h}$ at room temperature. As the TEV-protease contains a His-tag it was removed via a second affinity chromatography step. After completed cleavage, the protein solution was dialysed against $5 \mathrm{l}$ of wash buffer 1 and applied to a 1-ml NiNTA Agarose column. The flow through and the first $5 \mathrm{ml}$ from wash step 1 were collected for further purification.

\subsubsection{Concentrating of proteins}

Protein solutions were concentrated by ultrafiltration using $0.5,2$ or $20 \mathrm{ml}$ concentrators with the appropriate molecular weight cutoff (MWCO). The centrifugation was performed at $4{ }^{\circ} \mathrm{C}$ as recommended by the supplier (Table 2.8). The progress of the ultrafiltration was monitored by measuring the $A_{280}$.

\subsubsection{Protein storage}

Proteins in $\mathrm{H}_{2} \mathrm{O}$ were shock-frozen in liquid nitrogen and dried by lyophilisation. Lyophilised proteins were stored at $-20{ }^{\circ} \mathrm{C}$. Proteins in buffer solutions were shockfrozen in liquid nitrogen and stored at $-80^{\circ} \mathrm{C}$.

\subsubsection{Sodium dodecyl sulphate-polyacrylamide gel electrophore- sis (SDS-PAGE)}

The discontinuous Tris-glycine buffer system as described by Laemmli [56] was used to separate proteins by molecular weight. The components of the stacking and separating gel are given in Table 3.1. All gels had a separating gel with an acrylamide concentration of $17.5 \%$ and a $3 \%$ acrylamide stacking gel. For detection of proteins with a molecular weight smaller than $8-10 \mathrm{kDa}, 2 \mathrm{M}$ Tris/HCl $\mathrm{pH} 8.8$ was used as the separation gel buffer instead of the $1 \mathrm{M}$ buffer [57]. Protein samples were mixed with $4 \mathrm{x}$ protein loading buffer and denatured at $100{ }^{\circ} \mathrm{C}$ for $5 \mathrm{~min}$. Gels were run at $25 \mathrm{~mA}$ and stained with Coomassie blue R-250 by heating for $90 \mathrm{~s}$ in the microwave and 10-30 min incubation. For destaining, several 90 s heating steps in the microwave 
were performed and gels were completely destained by shaking overnight. Finally, the SDS-gels were imaged and digitized for documentation.

Table 3.1: Composition of the $17.5 \%$ SDS-gels

\begin{tabular}{lll}
\hline \hline & Seperating gel & Stacking gel \\
\hline & & \\
Rotiphorese Gel 30 & $3 \mathrm{ml}$ & $250 \mu \mathrm{l}$ \\
$1 \mathrm{M}$ or $2 \mathrm{M} \mathrm{Tris} / \mathrm{HCl} \mathrm{pH} 8.8$ & $1.88 \mathrm{ml}$ & - \\
$1 \mathrm{M}$ Tris/HCl pH 6.8 & - & $313 \mu \mathrm{l}$ \\
$\mathrm{H}_{2} \mathrm{O}$ & $30 \mu \mathrm{l}$ & $1.88 \mathrm{ml}$ \\
& & \\
$10 \%$ SDS & $50 \mu \mathrm{l}$ & $25 \mu \mathrm{l}$ \\
TEMED & $2.5 \mu \mathrm{l}$ & $2 \mu \mathrm{l}$ \\
$10 \%$ APS & $50 \mu \mathrm{l}$ & $25 \mu \mathrm{l}$ \\
\hline
\end{tabular}

\subsubsection{Edman degradation}

N-terminal protein sequencing was performed via Edman degradation [58] by U. Pleßmann in the group of Prof. Dr. Klaus Weber.

\subsubsection{Limited proteolysis}

Limited proteolysis was used to identify the rigid core of Map in complex with CesT [59]. Proteolysis was performed with the proteases trypsin, proteaseK, elastase and chymotrypsin. A $\sim 30 \mu \mathrm{M}$ protein solution in $20 \mathrm{mM}$ HEPES pH 7.0 and $150 \mathrm{mM}$ $\mathrm{NaCl}$ was digested at protein:protease ratios of 50:1, 100:1, 1000:1 and 10000:1 at room temperature. The reaction was stopped after 5, 10, 20, 30, 60 and 120 min by addition of SDS-PAGE loading buffer and boiling for $\sim 5 \mathrm{~min}$ at $100{ }^{\circ} \mathrm{C}$. All samples were analysed by SDS-PAGE.

\subsubsection{Peptide synthesis}

Solid-phase peptide synthesis (SPPS) was performed by Kerstin Overkamp. Raw peptides were purified via RP-HPLC. 


\subsubsection{Introduction of a paramagnetic center \\ 3.3.12.1 Site-directed spin-labeling (SDSL)}

SDSL is a technique to introduce a spin label into a protein based on a specific reaction between the spin label and an amino acid. In the most commonly used procedure, a unique sulfhydryl group is selectively modified with a nitroxide reagent [60]. Single cysteine mutants for the attachment of a spin label were generated by sitedirected mutagenesis (see 3.1.5) and modified with the thiol-specific nitroxide spin label reagent (1-oxy-2,2,5,5-tetramethyl-3-pyrroline-3-methyl)-methanethiosulfonate (MTSL). $100 \mu \mathrm{g} / \mu \mathrm{l}$ MTSL in cold acetone was added with a 3 to 5 -fold molar excess and incubated overnight at room temperature. Unreacted spin label was removed by size exclusion chromatography via a PD-10 column. Complete labeling with MTSL was verified by ESI-MS (see 3.4.3).

\subsubsection{Copper-binding}

Introduction of paramagnetic $\mathrm{Cu}^{2+}$ was achieved by prepending the amino terminal $\mathrm{Cu}$ (II)- and $\mathrm{Ni}(\mathrm{II})$-binding (ATCUN) motif to the N-terminus of CylR2 via recombinant cloning. $\mathrm{Cu}^{2+}$ was bound by adding a 3 to 5 -fold excess of $\mathrm{CuCl}_{2}$ dissolved in $\mathrm{H}_{2} \mathrm{O}$. Excessive $\mathrm{Cu}^{2+}$ was removed by dialysis against MOPS buffer. MOPS buffer was used to minimize the interaction between buffer molecules and $\mathrm{Cu}^{2+}[61$.

\subsection{Spectroscopic methods}

\subsubsection{Determination of protein concentration}

The concentration of proteins in solution was determined by measuring the absorption at $280 \mathrm{~nm}\left(\mathrm{~A}_{280}\right)$.

$$
A_{\lambda}=\epsilon_{\lambda} \cdot c \cdot d
$$

$\begin{array}{ll}\mathrm{A}_{\lambda}: & \text { absorption at wavelength } \lambda \\ \epsilon_{\lambda}: & \text { molar extinktion coefficient at wavelength } \lambda \text { in } \mathrm{M}^{-1} \mathrm{~cm}^{-1} \\ \mathrm{c}: & \text { protein concentration }(\mathrm{M}) \\ \mathrm{d}: & \text { thickness of the cuvette }(\mathrm{cm})\end{array}$




\subsubsection{Circular dichroism (CD) spectroscopy}

CD spectra were recorded from 200 to $260 \mathrm{~nm}$ in a $0.1 \mathrm{~cm}$ cuvette at a scan-rate of $20 \mathrm{~nm} / \mathrm{min}$ and with $0.5 \mathrm{~nm}$ resolution for $200 \mu \mathrm{l}$ of a 20-30 $\mu \mathrm{M}$ sample in the NMR buffer. The temperature was kept constant at $25^{\circ} \mathrm{C}$. The mean molar ellipticity per amino acid was calculated from the measured ellipticity according to the formular 62]:

$$
[\Theta]_{M R W}=\frac{10^{5} \cdot \Theta}{c \cdot d \cdot N}
$$

$[\Theta]_{M R W}$ : mean molar ellipticity per amino acid $\left(\mathrm{deg} \cdot \mathrm{cm}^{2} \cdot \mathrm{dmol}^{-1}\right)$

$\Theta: \quad$ measured ellipticitity

c: $\quad$ protein concentration $(\mu \mathrm{M})$

$\mathrm{d}$ : thickness of the cuvette $(\mathrm{cm})$

$\mathrm{N}$ : number of amino acids

\subsubsection{Electrospray ionization mass spectrometry (ESI-MS)}

ESI-MS was performed after the final purification of the first preparation of each protein sample to confirm the identity by the molecular weight and verify the purity. Fractions from RP-HPLC (see 3.3.4) were analysed directly by ESI-MS. All other samples were transferred into $20 \mathrm{mM} \mathrm{NH}_{4} \mathrm{OAc}$ by dialysis or via a $\mathrm{NAP}^{T M}-10$ column.

\subsection{NMR spectroscopy}

The preparation of the NMR samples is described under materials and methods of Chapters 4,5 and 6.

All NMR experiments were acquired at 600,700, 800 or $900 \mathrm{MHz}$ on Bruker AVANCE 600, 700, and 900 or DRX 600 and 800 spectrometers running under X-WINNMR 3.5. All NMR spectra were processed using X-WINNMR 3.5 or NMRPipe/NMRDraw [46] and analysed using NMRView 5.0.4 [47] or Sparky 3 (T. D. Goddard and D. G. Kneller, University of California, San Francisco). 


\subsubsection{Chemical shift assignment}

All NMR experiments performed for chemical shift assignments of the different samples are summarized in the Appendix B.1.

\subsubsection{Backbone chemical shift assignment}

Backbone $\mathrm{C}_{\alpha}, \mathrm{C}$ ', $\mathrm{N}$ and $\mathrm{H}_{N}$ assignments were obtained using standard triple resonance experiments for proteins smaller than $30 \mathrm{kDa}$ [63]. The assignments were derived from 3D HNCA, $\mathrm{HN}(\mathrm{CO}) \mathrm{CA}, \mathrm{CBCA}(\mathrm{CO}) \mathrm{NH}$ or $\mathrm{HN}(\mathrm{CO}) \mathrm{CACB}$, HNCACB, $\mathrm{HNCO}$ and $\mathrm{HN}(\mathrm{CA}) \mathrm{CO}$ experiments. For proteins and protein complexes bigger than $30 \mathrm{kDa}$, TROSY versions of the experiments were measured with deuterium decoupling [64, 65, 66]. In addition, the magnetization was completely transferred from $\mathrm{C}_{\alpha}$ to $\mathrm{C}_{\beta}$ in $\mathrm{HN}(\mathrm{COCA}) \mathrm{CB}$ and $\mathrm{HN}(\mathrm{CA}) \mathrm{CB}$ experiments to detect $\mathrm{C}_{\beta}$ carbons for molecules bigger than $30 \mathrm{kDa}$ [67]. The manual assignment of backbone resonances was assisted by using the automatic assignment program MARS [42].

\subsubsection{Side chain chemical shift assignment}

Aliphatic side chain resonances were derived from 3D HCCH-TOCSY, CCONHTOCSY and HCCONH-TOCSY spectra. Refocusing and mixing of ${ }^{13} \mathrm{C}$ magnetization was performed using adiabatic pulses [68, 69]. Additional data provided by 3D $\left[{ }^{15} \mathrm{~N},{ }^{1} \mathrm{H}\right]$-NOESY-HSQC and $\left[{ }^{13} \mathrm{C},{ }^{1} \mathrm{H}\right]$-NOESY-HSQC experiments were used for further assignment as well as confirmation of the through-bond data. Aromatic ring proton and carbon resonances were assigned using $2 \mathrm{D}\left[{ }^{13} \mathrm{C},{ }^{1} \mathrm{H}\right]-\mathrm{HSQC},(\mathrm{H} \beta) \mathrm{C} \beta(\mathrm{C} \gamma \mathrm{C} \delta) \mathrm{H} \delta$ and $(\mathrm{H} \beta) \mathrm{C} \beta(\mathrm{C} \gamma \mathrm{C} \delta \mathrm{C} \epsilon) \mathrm{H} \epsilon$ experiments [70].

\subsubsection{Secondary structure determination}

Regions of secondary structure can be identified by calculating the difference between the experimental chemical shift $\left(\mathrm{C}^{\exp }\right)$ and the random coil chemical shift $\left(\mathrm{C}^{\text {coil }}\right)$. Tabulated random coil values were used for folded proteins. For unfolded proteins, sequence corrected random coil values as empirically determined at $\mathrm{pH} 3.0$ by Schwarzinger et al. were used for all residues with the exception of His, Glu and 
Asp [71]. Since His, Glu and Asp are particularly sensitive to $\mathrm{pH}$, the experimental random coil chemical shifts at pH 5.0 from Wishart et al. were applied for these residues [72]. Secondary chemical shifts $(\Delta \delta \mathrm{C})$ for $\mathrm{C}_{\alpha}, \mathrm{C}_{\beta}$ and $\mathrm{C}^{\prime}$ were calculated as the difference between measured ${ }^{13} \mathrm{C}$ chemical shift $\left(\delta \mathrm{C}^{\exp }\right)$ and random coil ${ }^{13} \mathrm{C}$ chemical shift $\left(\delta \mathrm{C}^{\text {coil }}\right)[73]$ :

$$
\Delta \delta C=\delta C^{e x p}-\delta C^{c o i l}
$$

To derive secondary structure information, the secondary chemical shifts were combined using the following formular:

$$
\Delta \delta\left(C_{\alpha} C_{\beta} C^{\prime}\right)=\Delta \delta C_{\alpha}-\Delta \delta C_{\beta}+\Delta \delta C^{\prime}
$$

or by adding $\Delta \delta \mathrm{C}_{\alpha}$ and $\Delta \delta \mathrm{C}^{\prime}$ if $\mathrm{C}_{\beta}$ chemical shifts were not available. $\mathrm{C}_{\beta}$ chemical shifts were not used for unfolded proteins.

\subsubsection{Residual dipolar couplings (RDCs)}

Anisotropic media for measurement of RDCs were prepared by addition of filamentous phages (Pf1) to a concentration of 7-12 $\mathrm{mg} \mathrm{m}^{-1}$ [74, 75]. RDCs were back-calculated from X-ray or NMR structures using singular value decomposition (SVD) as implemented in PALES [48]. The correlation coefficient and the quality or Q-factor were used to evaluate the agreement between a structure and the observed RDCs. The Q-factor was determined as $\operatorname{rms}\left({ }^{1} \mathrm{D}_{H N}^{\text {measured }}-{ }^{1} \mathrm{D}_{H N}^{\text {calculated }}\right) / \operatorname{rms}\left({ }^{1} \mathrm{D}_{H N}^{\text {measured }}\right)[76]$.

\subsubsection{HN-RDCs}

${ }^{15} \mathrm{~N}-{ }^{1} \mathrm{H}$ splittings were measured under isotropic and anisotropic conditions using $2 \mathrm{D}$ IPAP- ${ }^{15} \mathrm{~N}-{ }^{1} \mathrm{H}$ HSQC experiments [77]. HN-RDCs were extracted by substraction of the ${ }^{1} \mathrm{~J}_{N H}$ scalar coupling measured for the isotropic sample [78]. Alternatively, HNRDCs were determined from the ${ }^{15} \mathrm{~N}-{ }^{1} \mathrm{H}$ splittings of a modified 3D TROSY-HNCO experiment for strongly overlapped peaks in large molecules [79]. 


\subsubsection{C'N- and $C^{\prime} \mathrm{C}_{\alpha}-\mathrm{RDCs}$}

The quantitative J correlation method was used to determine one-bond dipolar ${ }^{13} \mathrm{C}$ '${ }^{15} \mathrm{~N}$ couplings and one-bond dipolar ${ }^{13} \mathrm{C}^{\prime}-{ }^{13} \mathrm{C}_{\alpha}$ couplings. ${ }^{1} \mathrm{~J}_{C^{\prime} N}$ or ${ }^{1} \mathrm{~J}_{C^{\prime} C_{\alpha}}$ values were measured under isotropic and anisotropic conditions for ${ }^{2} \mathrm{H},{ }^{13} \mathrm{C},{ }^{15} \mathrm{~N}$-labeled CesT using a 3D TROSY-HNCO [80] or a 3D TROSY-HN(CO)CA-QJ experiment [81], respectively.

\subsubsection{Prediction of RDCs}

The electrostatic module of the software PALES was used to predict RDCs from the surface charge distribution of proteins in filamentous phage medium [48, 82] in order to validate structures or to rank results from ab initio docking (see 3.6.3). Predictions were performed for the experimental Pf1 concentration, for different $\mathrm{NaCl}$ concentrations $(0.005,0.01,0.015,0.02,0.03,0.04,0.05,0.06,0.07,0.08,0.09,0.10,0.15,0.20$, $0.25,0.30,0.35,0.40,0.45$ and $0.50 \mathrm{M})$ and with the default charge attached to all ionizable residues. The correlation between predicted and experimental couplings was finally multiplied with the correlation obtained from a best-fit of experimental values to the structure using singular value decomposition.

\subsubsection{NMR relaxation data}

\subsubsection{Approximation of the molecular weight}

The rotational correlation time $\left(\tau_{c}\right)$ is proportional to the molecular weight (MW) and thus the aggregation or oligomerization state of a macromolecule:

$$
\tau_{c}[n s] \sim \frac{1}{2} M W[k D a]
$$

An approximate value of $\tau_{c}$ can be derived from the $\mathrm{T}_{2}$ times of the backbone amide protons

$$
\tau_{c}[n s] \approx \frac{1}{5 T_{2}[s]}
$$

or from the $\mathrm{T}_{1 \rho}$ times of the backbone amide nitrogens:

$$
\tau_{c}[s]=\frac{5 r_{H N}^{6} 16 \pi^{2}}{\gamma_{H}^{2} \gamma_{N}^{2} \hbar^{2} \mu_{0}^{2} T_{1 \rho}},
$$


where $\mathrm{r}_{H N}$ is the distance between amide proton and nitrogen, $\gamma_{i}$ is the gyromagnetic ratio of nucleus $i, \hbar$ is the reduced Planck's constant and $\mu_{0}$ is the permeability of vacuum. For unlabeled samples transverse relaxation times $\left(\mathrm{T}_{2}\right)$ of backbone protons were estimated from two 1D one-one echo experiments [83] measured with a relaxation delay of $100 \mu \mathrm{s}\left(\Delta_{A}\right)$ and a relaxation delay of $3.1 \mathrm{~ms}\left(\Delta_{B}\right)$ using the following formular:

$$
T_{2}=\frac{2\left(\Delta_{A}-\Delta_{B}\right)}{\ln \left(I_{B} / I_{A}\right)}
$$

For ${ }^{15} \mathrm{~N}$-labeled samples $1 \mathrm{D} \mathrm{T}_{1 \rho}$ experiments with a spin-lock power of $2.5 \mathrm{kHz}$. Two 1D $\mathrm{T}_{1 \rho}$ experiments were acquired. One with a spin-lock pulse duration of $2 \mathrm{~ms}\left(\Delta_{A}\right)$ and a second with a spin-lock pulse duration $\Delta_{B}$ which corresponds to $\sim 1.3$ times the relaxation time calculated from the theoretical MW. The average relaxation time of all backbone nitrogens was calculated using the following formular:

$$
T_{1} \rho=\frac{\Delta_{A}-\Delta_{B}}{\ln \left(I_{B} / I_{A}\right)}
$$

\subsubsection{2 ${ }^{15} \mathrm{~N}$ transverse relaxation times $\left(\mathrm{T}_{2}\right)$}

${ }^{15} \mathrm{~N}$ relaxation data were acquired with modified versions of pulse sequences described earlier [84, incorporating pulsed field gradients and a WATERGATE for solvent suppression. $\mathrm{T}_{2}$ times were sampled using seven different ${ }^{15} \mathrm{~N}$ relaxation delays $\left(\Delta_{\text {relax }}\right)$ : $7.6,50,90,130,160,190$ and $220 \mathrm{~ms}$. To extract ${ }^{15} \mathrm{~N} \mathrm{~T}_{2}$ relaxation times by measuring the peak volumes (I) for all spectra recorded with different relaxation delays and fitting the volumes to a single-exponential function:

$$
y=I(O) \exp \left(-\frac{\Delta_{\text {relax }}}{R_{2}}\right)
$$

where $R_{2}$ is the transverse relaxation rate $\left(T_{2}=1 / R_{2}\right)$.

\subsubsection{3 ${ }^{15} \mathrm{~N}-{ }^{1} \mathbf{H}$ steady state NOE}

The heteronuclear nuclear Overhauser effects (NOEs) of ${ }^{15} \mathrm{~N}$ nuclei in $\mathrm{NH}$ groups were measured using the Bruker standard pulse program invinoef3gpsi. ${ }^{15} \mathrm{~N}-{ }^{1} \mathrm{H}$ NOE 
values were calculated as the intensity ratios of the ${ }^{15} \mathrm{~N}-{ }^{1} \mathrm{H}$ correlation peaks from pairs of interleaved spectra acquired with and without ${ }^{1} \mathrm{H}$ presaturation during the recycle time of $5 \mathrm{~s}$.

\subsubsection{Mapping binding surfaces}

An easy and rapid way to gain qualitative information about the interaction between two molecules is to study the chemical shift values. Chemical shifts depend on the local environment. The chemical shifts of ${ }^{1} \mathrm{H}$ and ${ }^{15} \mathrm{~N}$ are particularly sensitive to changes of the environment. Therefore ${ }^{15} \mathrm{~N}$ and ${ }^{1} \mathrm{H}$ chemical shift perturbations recorded via ${ }^{15} \mathrm{~N}-{ }^{1} \mathrm{H}-\mathrm{HSQC}$ spectra throughout an NMR titration are commonly combined in order to map a binding site on a protein according to the following equation [85]:

$$
\Delta \delta_{H N}=\sqrt{\frac{\left(\Delta \delta_{N} / 5\right)^{2}+\left(\Delta \delta_{H}\right)^{2}}{2}}
$$

$\Delta \delta_{H N}: \quad$ average amide chemical shift perturbation

$\Delta \delta_{N}: \quad$ amide nitrogen chemical shift perturbation

$\Delta \delta_{H}: \quad$ amide proton chemical shift perturbation

The binding interface is defined by the residues exhibiting the largest ${ }^{15} \mathrm{~N}$ and ${ }^{1} \mathrm{H}$ chemical shift changes upon the NMR titration.

\subsubsection{Determination of protein-ligand dissociation constants}

Chemical shift changes observed in a series of ${ }^{15} \mathrm{~N}-{ }^{1} \mathrm{H}-\mathrm{HSQC}$ spectra during the titration of a ligand to a protein can be used to calculate the dissociation constant $\mathrm{K}_{d}$ if the exchange rate is much faster than the difference between the chemical shifts of the free and bound protein. In this case of fast exchange on the NMR time scale, the $\mathrm{K}_{d}$ is larger than $10^{-3} \mathrm{M}$.

Considering a complex with 1:1 stoichiometry composed of a protein $(\mathrm{P})$ which binds a DNA ligand (L), the complex formation and dissociation is described as follows: and $\mathrm{K}_{d}$ is defined as: 


$$
\begin{gathered}
P+L \frac{k_{o n}}{k_{o f f}} P L \\
K_{d}=\frac{k_{o f f}}{k_{o n}}=\frac{[P][L]}{[P L]}=\frac{\left([P]_{0}-X_{P L}[P]_{0}\right)\left([L]_{0}-X_{P L}[P]_{0}\right)}{X_{P L}[P]_{0}}
\end{gathered}
$$

with $[\mathrm{P}]_{0}=[\mathrm{P}]+[\mathrm{PL}]$ and $\mathrm{X}_{P L}+\mathrm{X}_{P}=1$.

$\mathrm{k}_{\text {off }}$ : rate of dissociation

$\mathrm{k}_{\text {on }}$ : $\quad$ rate of association

$[\mathrm{P}]_{0}$ : total or starting concentration of the protein

$[\mathrm{P}]: \quad$ concentration of the protein

$[\mathrm{L}]_{0}$ : total or starting concentration of the ligand

[L]: $\quad$ concentration of the ligand

[PL]: concentration of the complex

$\mathrm{X}_{P}: \quad$ fraction of the free protein

$\mathrm{X}_{P L}$ : fraction of the bound protein

The observed chemical shift $\delta_{o b s}$ can be written as:

$$
\delta_{o b s}=X_{P} \delta_{P}+X_{P L} \delta_{P L}
$$

and with $\mathrm{r}=\frac{[L]_{0}}{[P]_{0}}$ the following equation can be derived:

$$
\delta_{o b s}=\delta_{P}+\left(\delta_{P L}-\delta_{P}\right) \frac{\left(K_{d}+(1+r)[P]_{0}\right)-\sqrt{\left(K_{d}+(1+r)[P]_{0}\right)^{2}-4[P]_{0}^{2} r}}{2[P]_{0}}
$$

$\delta_{P}: \quad$ chemical shift of the protein

$\delta_{P L}: \quad$ chemical shift of the complex

Fitting the experimental data to equation 3.14 yields the $\mathrm{K}_{d}$ [86].

\subsubsection{Long-range distances from paramagnetic relaxation en- hancement (PRE)}

The distance-dependent transverse relaxation enhancements of resonances in protein samples containing paramagnetic centers were used to derive long-range distances. 
Peak intensites of cross-peaks for amide proton-nitrogen pairs in the ${ }^{15} \mathrm{~N}-\mathrm{HSQC}$ spectrum of the paramagnetic $\left(\mathrm{I}_{\text {para }}\right)$ and of the diamagnetic $\left(\mathrm{I}_{\text {dia }}\right)$ state of a protein were measured. Peak intensity ratios $\mathrm{I}_{\text {para }} / \mathrm{I}_{\text {dia }}$ were calculated to linearly fit for the enhancement of the transverse relaxation rate by the unpaired electron $\left(\mathrm{R}_{2}^{\text {para }}\right)$ [87]:

$$
\frac{I_{\text {para }}}{I_{\text {dia }}}=\frac{R_{2} \exp \left(-R_{2}^{\text {para }} t\right)}{R_{2}+R_{2}^{\text {para }}}
$$

where $t$ is the total INEPT evolution time of the ${ }^{15} \mathrm{~N}-\mathrm{HSQC}(\sim 11.3 \mathrm{~ms})$ and amide proton $R_{2}$ values were approximated by experimental amide nitrogen $R_{2}$ values (see 3.5.4.2). The distances $r$ between the unpaired electron and the amide protons have been determined according to:

$$
r=\left[\frac{K}{R_{2}^{\text {para }}}\left(4 \tau+\frac{3 \tau}{1+\omega_{h}^{2} \tau^{2}}\right)\right]^{1 / 6},
$$

in which $\mathrm{K}$ is $1.23 \times 10^{-32} \mathrm{~cm}^{6} \mathrm{~s}^{-2}$ [88], $\tau$ is the correlation time for the electronnuclear interaction and $\omega_{h}$ is the Larmor frequency of the proton. For calculating the distances, $\tau$ was assumed to be equal to the global correlation time $\tau_{c}$ of the protein. $\tau_{c}$ was determined using the Stokes' law [5]:

$$
\tau_{c}=\frac{4 \pi \eta_{W} r_{H}^{3}}{3 k_{B} T}
$$

$\eta_{W}: \quad$ viscosity of the solvent

$\mathrm{r}_{H}$ : effective hydrodynamic radius

$\mathrm{k}_{B}: \quad$ Boltzmann's constant

T: $\quad$ temperature

Under the assumption that the specific volume of the protein is $\bar{V}=0.73 \mathrm{~cm}^{3} \mathrm{~g}^{-1}$ and that a hydration layer of $\mathrm{r}_{W}=1.6-3.2 \AA$ surrounds the protein, a rough estimate of $\mathrm{r}_{H}$ can be made [5]:

$$
r_{H}=\left[\frac{3 \bar{V} M_{r}}{4 \pi N_{A}}\right]^{1 / 3}+r_{W}
$$

$\mathrm{N}_{A}: \quad$ Avogadro's number 


\subsection{Structure calculation}

\subsubsection{Monomer structure calculation}

The monomer structure of CylR2 was calculated using simulated annealing and torsion angle dynamics within the program CYANA [40]. Structure calculation was combined with automated NOE assignment for seven cycles calculating 100 structures each and using the 20 structures with the lowest target function for the next cycle. The final 20 structures with the lowest target function were refined with HNRDCs in explicit solvent using a XPLOR-NIH protocol [53] which is provided in the Appendix D.

\subsubsection{Dimer structure calculation}

The CylR2 homodimer structure was calculated on the basis of HN-RDCs and intermolecular distances using a XPLOR-NIH protocol [89]. This rigid-body docking protocol is composed of three rounds of rigid-body minimization and a final simulated annealing. In the first round of the rigid-body minimization the force constant for dipolar couplings $\mathrm{k}_{\text {dip }}$ was set to $0.001 \mathrm{kcal} \mathrm{mol}^{-1} \mathrm{~Hz}^{-2}$ and the force constant for the intermonomer distances $\mathrm{k}_{\text {inter }}$ was set to $0.01 \mathrm{kcal} \mathrm{mol}^{-1} \AA^{-2}$. In the second round $\mathrm{k}_{\text {dip }}$ was slowly increased from $0.001 \mathrm{kcal} \mathrm{mol}^{-1} \mathrm{~Hz}^{-2}$ to $0.1 \mathrm{kcal} \mathrm{mol}^{-1} \mathrm{~Hz}^{-2}$ and $\mathrm{k}_{\text {inter }}$ was slowly increased from $0.01 \mathrm{kcal} \mathrm{mol}^{-1} \AA^{-2}$ to $30 \mathrm{kcal} \mathrm{mol}^{-1} \AA^{-2}$ and in the third round $\mathrm{k}_{\text {dip }}$ and $\mathrm{k}_{\text {inter }}$ were set to $0.1 \mathrm{kcal} \mathrm{mol}^{-1} \mathrm{~Hz}^{-2}$ and $60 \mathrm{kcal} \mathrm{mol}{ }^{-1} \AA^{-2}$, respectively. 100 structures were calculated during the final simulated annealing applying a standard protocol with inter- and intramonomer distances and with all coordinates fixed with the exception of the side chain atoms of residues contributing to the dimer interface. The 20 lowest energy structures after the simulated annealing were refined with the same protocol as the monomer ensemble.

\subsubsection{Ab initio docking}

For ab initio docking with restricted symmetry the ClusPro protein-protein docking Web server (http://nrc.bu.edu/cluster) was used [37, 38]. Refined monomer struc- 
tures were docked with the standard settings, but with ZDOCK chosen as the docking program and the symmetry restricted to $\mathrm{C}_{2}$ [39]. 15 structures were received as the output.

\subsubsection{Protein/DNA docking}

The structure of the CylR2/DNA complex was modeled by Monte Carlo docking using the program Monty [90, 91]. DNA bending in Monty is achieved by a two-step procedure. Firstly a regular helix is fitted through the B-DNA backbone which is randomly over- or underwound. The straight starting double helix is subsequently bent by shifting each base pair, which is not in van der Waals contact with the protein. These base pairs are shifted along an intermolecular vector toward the protein by a distance smaller or equal to the so-called bending parameter. The outer base pairs are shifted by a larger amount than the inner ones, which is determined by a quadratic function of base pair numbering. A larger value for the bending parameter thus will result in a stronger shifting of base pairs toward the protein, i.e., a stronger bending of the DNA [90, 91]. Here, docking simulations were performed with DNA bending parameters varying from 0.0 to 0.6. Corresponding to NMR titration results, side chains were randomised and allowed to rotate freely during the entire simulation and energy bonuses of 30 and $40 \mathrm{kcal} / \mathrm{mol}$ were given to complex structures where the backbone amides are in close contact with the DNA. The square-well depths for hydrogen bonds and van der Waals interactions were 5 and $0.5 \mathrm{kcal} / \mathrm{mol}$, respectively. The value of $\mathrm{kT}$ was set to $7.7 \mathrm{kcal} / \mathrm{mol}$. For each DNA bending parameter 64 Monte Carlo simulations of 200000 attempted moves were performed.

\subsection{Structure analysis}

References for the utilized softwares can be found in Table 2.9. For the analyses of the X-ray structure of CylR2 PROCHECK and the CCP4 suite of programs were employed. Structure figures were prepared with MOLSCRIPT, BOBSCRIPT, RASTER3D, MSMS, DINO, PyMOL and MOLMOL. NMR structures were analysed with MOLMOL, AQUA and PROCHECK-NMR. Energies were evaluated with 
XPLOR-NIH.

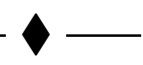




\section{4}

\section{Structure and DNA-binding properties of the cytolysin regulator CyIR2}

\subsection{Introduction}

Enterococcus faecalis is one of the major causes for hospital-acquired antibioticresistent infections [92, 93]. It produces an exotoxin, called cytolysin [94], which is lethal for a wide range of Gram-positive bacteria and is toxic to higher organisms. The toxicity of strains expressing cytolysin was found to be at least 10-times higher than for isogenic, non-cytolytic mutants [95, 96] and the virulence of enterococcal infections is five-fold increased because of cytolysin secretion [97]. Cytolysin is a heterodimer consisting of a large subunit $\mathrm{CylL}_{L}$ and a small subunit $\mathrm{CylL}_{S}$, which are post-translationally modified by CylM. CylM introduces the modified amino acids lanthionine , $\beta$-methyllanthionine, dehydroalanine and dehydrobutyrine which are characteristic of the lantibiotic class of bacteriocins. After modification, both subunits interact with CylB. This ATP-binding cassette (ABC) transporter has a dual function: it removes a leader sequence from the modified subunits and secreted them into the extracellular space [94, 98]. In the extracellular space the subtilisin-class serine protease, CylA, activates cytolysin by cleaving the precursors and releasing the mature subunits $\mathrm{CylL}_{L} "$ and $\mathrm{CylL}_{S} "$ [99]. These subunits interact to cause lysis

of erythrocytes, to destroy neural tissue and to kill a wide range of Gram-positive bacteria [100, 101]. The cytolysin-producing cell is protected from the bactericidal activity by the specific immunity protein CylI [102]. The genes for all these proteins 
including those of cytolysin were found on a collinear gene cluster [103] (Figure 4.1]. As cytolysin is lethal for strains that lack CylI [105], regulation of cytolysin ex-

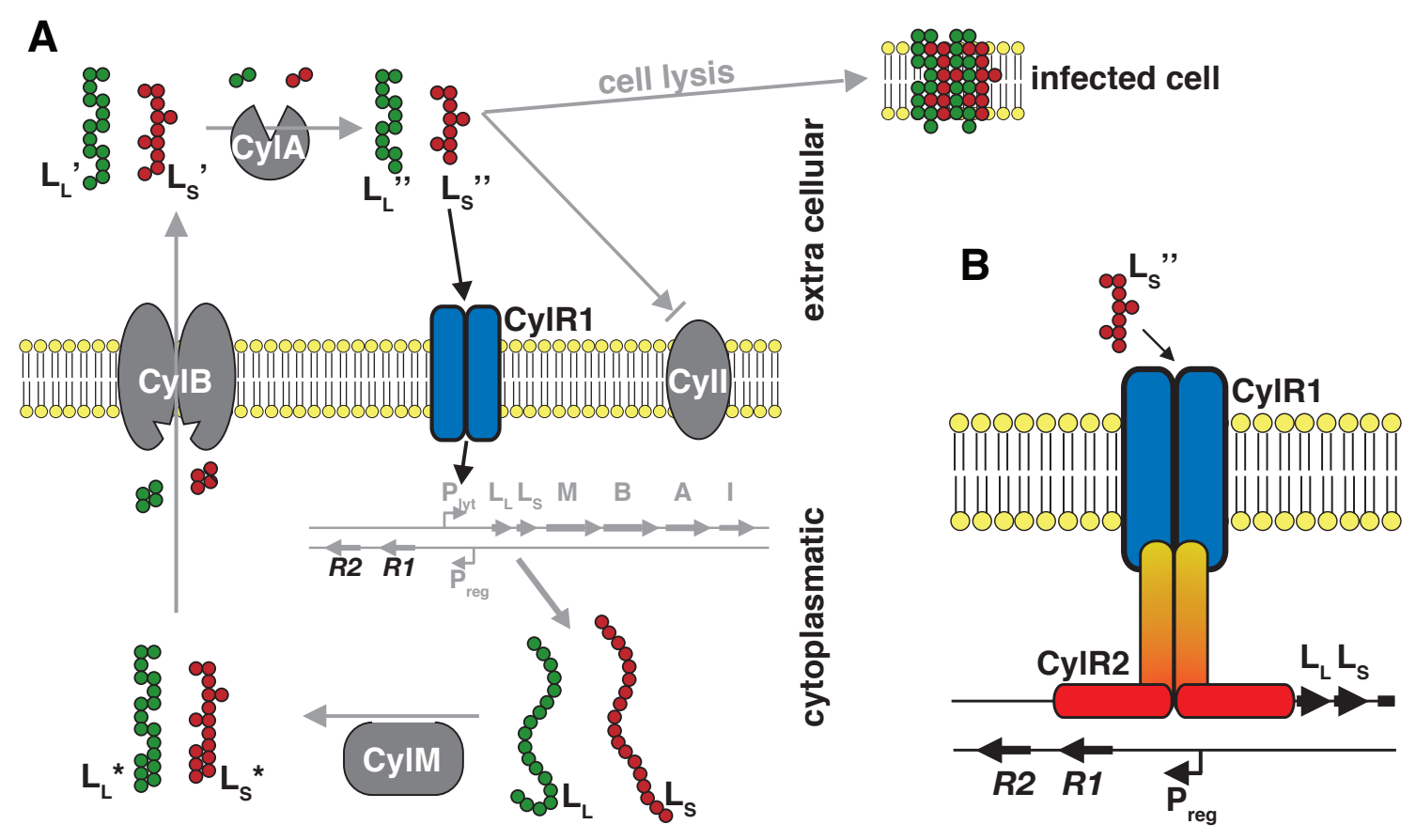

Figure 4.1: (A) Model of cytolysin maturation. The subunits $\mathrm{CylL}_{L}$ and $\mathrm{CylL}_{S}$ are ribosomally synthesized, modified post-translationally by CylM, proteolytically cleaved, secreted and extracellularly activated by CylA. The fully active subunits $\mathrm{CylL}_{L}$ " and $\mathrm{CylL}_{S}$ " interact to cause cell lysis. The cytolysin-producing cell is protected by CylI. The box indicates the part of the scheme which is enlarged in (B): Model of the regulation of cytolysin production. CylR1 and CylR2 act in concert to repress expression of the cytolysin operon with CylR2 binding to the promotor $\mathrm{P}_{l y t}$. Interaction between CylL $_{S}$ " and CylR1 induces transcription from $\mathrm{P}_{l y t}[104]$.

pression has to be carefully orchestrated with regulation of immunity. Regulation of cytolysin production involves two proteins, CylR1 and CylR2, whose genes were identified upstream of the collinear gene cluster in opposite orientation [104]. Both, CylR1 and CylR2, were shown to be essential for the regulation. This CylR1/CylR2 two-component regulatory system was found to be connected to autoinduction by a quorum sensing mechanism [104]. Quorum sensing is generally a bacterial mechanism of bacteria for communication and coordinated behaviour at the level of cell density and the signalling molecules are also referred to as autoinducers [106]. In most cases of cell-cell communication between bacteria, binding of a signalling peptide to a histidine kinase receptor on the cell surface results in autophosphorylation of the 
kinase. The phosphate is then transferred to a response regulator converting it to an active transcription factor. Alternatively, the peptide signal is imported into the cell by an oligopeptide permease where it interacts with an intracellular receptor [107]. However, the quorum sensing mechanism involving the CylR1/CylR2 two-component regulatory system is different from the previously described two-component quorum sensing signal transduction systems and neither CylR1 nor CylR2 are related to the family of bacterial two-component regulators [104]. Repression of the cytolysin gene is overcome in a cell density-dependent fashion as a result of the accumulation of one of the posttranslationally modified subunits of the toxin, $\mathrm{CylL}_{S}$ ", but the mechanism by which this accumulation is sensed by the cell is presently unknown. It is predicted that CylR2 may contain a helix-turn-helix motif, and that CylR1 would likely be a membrane-bound protein. Thus, a model was proposed by Haas et al. 104 in which CylR2, interacting with membrane-bound CylR1, binds and represses the cytolysin promoter until $\mathrm{CylL}_{S}$ " is accumulated to a threshold level to trigger a change that alters or releases the promoter, allowing for transcription (Figure 4.1).

Aside from the demonstration that mutations in either CylR1 or CylR2 lead to derepression of the operon [104], precise details of the mechanism of how quorum sensing autoinduction of cytolysin expression is achieved remain to be determined. The binding of CylR2 to either of the two inverted repeats (IR1 and IR2) within the cytolysin promotor (Figure 4.2) has been determined by gel shift analysis [108]. The absence or

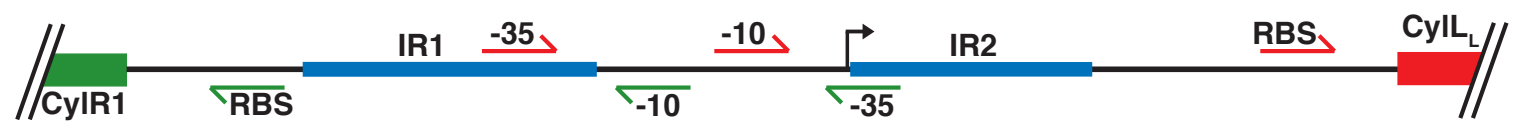

Figure 4.2: Intergenic region between $\operatorname{cylL}_{L}$ and cylR1, containing divergent promotors, $\mathrm{P}_{\text {reg }}$ and $\mathrm{P}_{l y t}$, involved in cytolysin expression and regulation. The two palindromic sequences, IR1 and IR2, within the promotor region are indicated and the transcriptional start site is indicated as a bent arrow. Promotor elements (-10: -10 box, $-35:-35$ box) for $\mathrm{P}_{r e g}$ and $\mathrm{P}_{l y t}$ are shown in green and red, respectively.

presence of band shifts revealed that CylR2 binds only to the 25-bp double-stranded (ds) IR1 and neither to single-stranded (ss) IR1, nor ds IR2 or ss IR2. Furthermore, the CylR2-binding was unchanged for 25-bp ds DNA with up to 4 nucleotides missing 
at the 5'or 3'end of IR1. Having this information on-hand, it is of interest to determine the high-resolution structure of CylR2 and its DNA-binding properties as an important step towards unravelling the mechanism of regulation of the enterococcal virulence factor cytolysin and towards approving the proposed mechanism.

\subsection{Materials and methods}

\subsubsection{Expression and purification}

Cell cultures for the expression of CylR2, wildtype or mutants, were switched to $28{ }^{\circ} \mathrm{C}$ at an $\mathrm{OD}_{600}$ of $0.4-0.5$ and were harvested $6-7 \mathrm{~h}$ after induction (see 3.2.1). The cell lysis was performed under native conditions with DNase I supplemented in order to degrade DNA (see 3.2.2). After the native cell lysis, the supernatant was filtrated through a $0.20 \mu \mathrm{m}$ sterile filter and loaded on a 5 -ml anion exchange column to remove the DNA (see 3.3.2). The flow-through and 5 column volumes of wash were pooled and loaded on a 10-ml cation exchange column equilibrated with lysis buffer. Elution was performed at a flow rate of $3.0 \mathrm{ml} / \mathrm{min}$ with a linear gradient of $0-100 \%$ of ion exchange buffer B for CylR2 (see 3.3.2). Fractions containing CylR2 were pooled, shock-frozen in liquid nitrogen and dried by lyophilisation. The lyophilised protein was dissolved in $\sim 3 \mathrm{ml}$ water and further purified by RP-HPLC (see 3.3.4). The purity of the eluted CylR2 was confirmed by ESI-MS (Figure A.2). The purified CylR2 was stored in lyophilised form at $-20{ }^{\circ} \mathrm{C}$. The yield is $\sim 25 \mathrm{mg}$ CylR2 per liter of expression culture.

\subsubsection{Preparation of ds DNA}

Complementary oligonucleotides (Table 2.3) were resuspended in $50 \mathrm{mM}$ HEPES pH 7.0 and $350 \mathrm{mM} \mathrm{NaCl}$, mixed to a final 1:1 molar ratio and annealed by heating to $100{ }^{\circ} \mathrm{C}$ for $5 \mathrm{~min}$ in a heatblock and cooling down slowly to room temperature. The annealed DNA was used for NMR titration experiments or in the case of the ds DNA comprising IR1 (Figure 4.3) for the preparation of CylR2/DNA complex samples. 


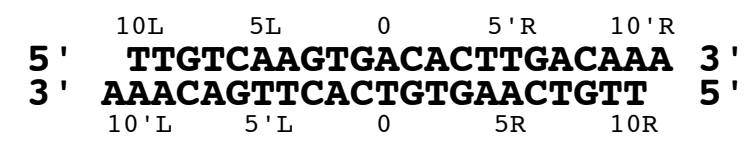

Figure 4.3: The 22-mer DNA sequence of IR1 used for the NMR experiments.

\subsubsection{NMR experiments}

About $1 \mathrm{mM}$ CylR2 samples for resonance assignment were prepared by dissolving lyophilised CylR2 directly in $250 \mu \mathrm{l}$ NMR buffer with $600 \mathrm{mM} \mathrm{NaCl}$. At lower salt concentrations CylR2 is less soluble and tends to crystallize. Therefore, for NMR samples with lower salt concentrations CylR2 was first dissolved in $250 \mu \mathrm{l} 50 \mathrm{mM}$ HEPES pH 7.0, 1.5 M NaCl followed by dialysis against 50 mM HEPES pH 7.0 with 250 to $350 \mathrm{mM} \mathrm{NaCl}$. Finally $5 \%(\mathrm{v} / \mathrm{v}) \mathrm{D}_{2} \mathrm{O}$ were added.

For CylR2/DNA complex samples, the protein in $50 \mathrm{mM}$ HEPES pH 7.0 and $1.5 \mathrm{M}$ $\mathrm{NaCl}$ was slowly mixed with the DNA (see 4.2.2) up to a 1:1 molar ratio. Afterwards the complex was dialysed against 50 mM HEPES pH 7.0 and $350 \mathrm{mM} \mathrm{NaCl}$ and $5 \%$ $(\mathrm{v} / \mathrm{v}) \mathrm{D}_{2} \mathrm{O}$ were added. NMR samples contained $1 \mathrm{mM}$ protein/DNA complex.

Anisotropic samples for the measurement of HN-RDCs contained $12 \mathrm{mg} / \mathrm{ml} \mathrm{Pf1}$. To lower the the electrostatic attraction between CylR2 and the highly negatively charged Pf1, HN-RDCs for free CylR2 were measured at a NaCl concentration of 0.5 M. IPAP${ }^{15} \mathrm{~N}-\mathrm{HSQC}$ spectra of CylR2 in complex with the DNA under anisotropic conditions were acquired at $\mathrm{NaCl}$ concentrations of $0 \mathrm{M}, 0.025 \mathrm{M}, 0.05 \mathrm{M}, 0.1 \mathrm{M}, 0.2 \mathrm{M}, 0.3 \mathrm{M}$ and 0.5 M. HN-RDCs for DNA-bound CylR2 at $0.5 \mathrm{M} \mathrm{NaCl}$ were measured for $12 \mathrm{mg} / \mathrm{ml}$ and $14 \mathrm{mg} / \mathrm{ml}$ Pf1.

All NMR experiments were carried out at $298 \mathrm{~K}$ at a field strength of 600,700 or $800 \mathrm{MHz}$ and are summarized in the Appendix Table B.1.

\subsubsection{DNA titration}

The CylR2/DNA complex formation was monitored by recording a series of $2 \mathrm{D}{ }^{15} \mathrm{~N}$ ${ }^{1} \mathrm{H}-\mathrm{HSQC}$ spectra of a $100 \mu \mathrm{M}{ }^{15} \mathrm{~N}$-labeled CylR2 NMR sample with a stepwise increasing DNA concentration. DNA was added from a $1.4 \mathrm{M}$ stock solution and final DNA concentrations were 10, 20, 30, 50, 60 and $100 \mu \mathrm{M}$. The combined chemical shift 
changes for a particular residue upon DNA-binding were calculated according to:

$$
\Delta \delta_{t o t}=\sqrt{\left(\Delta \delta_{H N}\right)^{2}+\left(f_{C \alpha} \Delta \delta_{C \alpha}\right)^{2}+\left(f_{C \beta} \Delta \delta_{C \beta}\right)^{2}+\left(f_{C^{\prime}} \Delta \delta_{C^{\prime}}\right)^{2}}
$$

with $\Delta \delta_{H N}$ calculated following equation 3.11 .

$$
\begin{gathered}
\Delta \delta_{C \alpha, \beta}=\sqrt{\left(f_{C \alpha} \Delta \delta_{C \alpha}\right)^{2}+\left(f_{C \beta} \Delta \delta_{C \beta}\right)^{2}} \\
\Delta \delta_{C^{\prime \star}}=\sqrt{\left(f_{C^{\prime}} \Delta \delta_{C^{\prime}}\right)^{2}}
\end{gathered}
$$

and for amide side chains:

$$
\begin{gathered}
\Delta \delta_{\text {side }}=\sqrt{\left(f_{H \epsilon} \Delta \delta_{H \epsilon}\right)^{2}+\left(f_{N \epsilon} \Delta \delta_{N \epsilon}\right)^{2}+\left(f_{C \gamma / \delta} \Delta \delta_{C \gamma / \delta}\right)^{2}} \\
\Delta \delta_{\text {sideC }}=\sqrt{\left(f_{C \gamma / \delta} \Delta \delta_{C \gamma / \delta}\right)^{2}}
\end{gathered}
$$

$\Delta \delta_{i}$ are the differences in chemical shifts between the free and bound protein and $f_{i}$ denotes the weight factor of nucleus $\mathrm{i} ; \mathrm{f}_{H \epsilon}=1, \mathrm{f}_{N \epsilon}=0.154, \mathrm{f}_{C \alpha}=\mathrm{f}_{C \beta}=0.256$ and $\mathrm{f}_{C \gamma / \delta}=\mathrm{f}_{C^{\prime}}=0.341[109]$.

\subsubsection{Model of the CyIR2/DNA complex}

Initially, a starting model for the CylR2/DNA complex was built by superimposing the backbone atoms of residues 1 to 66 of the CylR2 dimer on corresponding atoms of residues 1 to 66 of the $\mathrm{N}$-terminal domain of repressor of phage 434 in complex with its DNA operator (PDB code: 1rpe) [110]. The 434 repressor DNA was replaced by a 22 bp B-DNA, which was adjusted to the IR1 inverted repeat sequence using InsightII, by positioning bp $9 \mathrm{~L}$ to $9 \mathrm{R}$ of the B-DNA on bp $2 \mathrm{R}$ to $3 \mathrm{~L}$ of the 434 repressor DNA. The program Monty was used for the refinement of this start structure (see 3.6.4). Further docking trials were performed starting from initial models, where the DNA operator in complex with the 434 repressor (PDB code: 1rpe) or the lambda repressor (PDB code: 1lmb) [111] was used instead of regular B-DNA. No DNA flexibility was allowed in these cases and the DNA sequence was adjusted prior to docking to IR1 using InsightII. Prolonged simulations with 600000 Monte Carlo moves showed virtually no improvement. From the 64 final solutions, those in which the DNA was 
detached from the protein were removed, leaving a total of 41 CylR2/DNA complexes. Since the number of possible side chain conformers is extremely large and sampling is computationally limited, it is unlikely that one can obtain complexes with all correct protein-DNA interactions simultaneously present. Therefore, important CylR2/DNA interactions were identified based on their presence in more than $20 \%$ (van der Waals) or more than $30 \%$ (H-bonds) of the successful docking solutions.

\subsection{Results and discussion}

\subsubsection{Three dimensional structure of CyIR2}

The crystal structure of CylR2 was determined at $1.9 \AA$ resolution in the group of Stefan Becker by Adelia Razeto. The model of CylR2 consists of 132 residues, corresponding to two molecules in the asymmetric unit, 15 iodide ions, a sodium ion and 194 water molecules and is deposited with the PDB (accession code: 1utx). The structure of CylR2 consists of five helices $(\alpha 1-\alpha 5)$ and an antiparallel $\beta$-sheet, which is formed by strands $\beta 1$ and $\beta 2$, at the $\mathrm{N}$ - and C-terminus of CylR2, respectively (Figure 4.4). Helices $\alpha 2$ and $\alpha 3$ make up the HTH DNA-binding motif and are defined as the 'scaffold' and 'recognition' helix, respectively [112. Helices $\alpha 1, \alpha 2$, and $\alpha 3$ pack against each other and the architecture of their fold is supported by hydrophobic and hydrophilic interactions, reflecting their amphipathic nature. The hydrophobic residues are oriented towards the centre of the molecule, while hydrophilic residues, on the surface of the molecule, protect the hydrophobic core. A buried salt-bridge between Arg10 and Glu35 connects helix $\alpha 1$ to $\alpha 3$. The salt-bridge has already been indicated as an important feature in HTH DNA-binding proteins with the function of stabilising the fold and determining the relative orientation of the scaffold and recognition helices [113]. In HTH DNA-binding proteins the tight turns between the helices are usually sterically favoured by glycine [114]. However, in CylR2 other residues replace these glycines in sharp bends: Lys14 ( $\alpha 1-\alpha 2)$, Glu25 ( $\alpha 2-\alpha 3)$, Asn37 ( $\alpha 3-\alpha 4)$ and Asn54 ( $\alpha 4-\alpha 5)$. All of these have main-chain dihedral angles lying in the left-handed $\alpha$-helical region of the Ramachandran plot, which is usually occupied by 


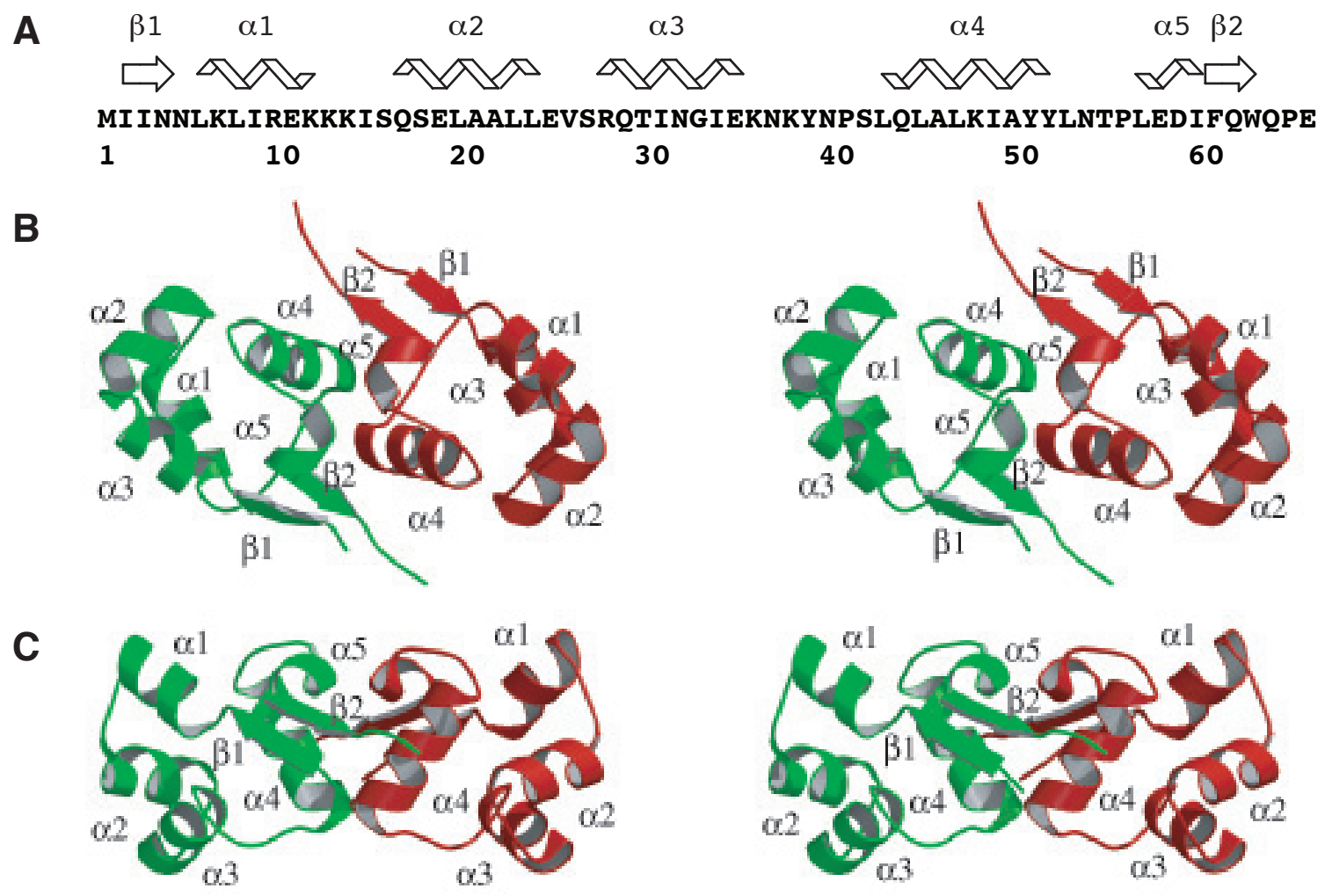

Figure 4.4: Sequence of CylR2 (A) and stereo view of the crystal structure of CylR2 (B,C). Helices are labeled with $\alpha 1-\alpha 5$ and $\beta$-strands are labeled with $\beta 1$ and $\beta 2$. (B,C) Subunit A and B are shown in red and green. (B) Stereo view of the homodimer looking down the dimer twofold axis. (C) CylR2 is rotated by $90^{\circ}$ about the horizontal axis.

glycines. Their main-chain conformations are mostly stabilised by H-bonds between their amino groups and carbonyl groups of residues (n-3), a typical feature of $\beta$-turns. Helices $\alpha 4$ and $\alpha 5$ are predominantly hydrophobic, contribute to the hydrophobic core of the molecule and, more importantly, mediate the dimeric contacts (see 4.3.2).

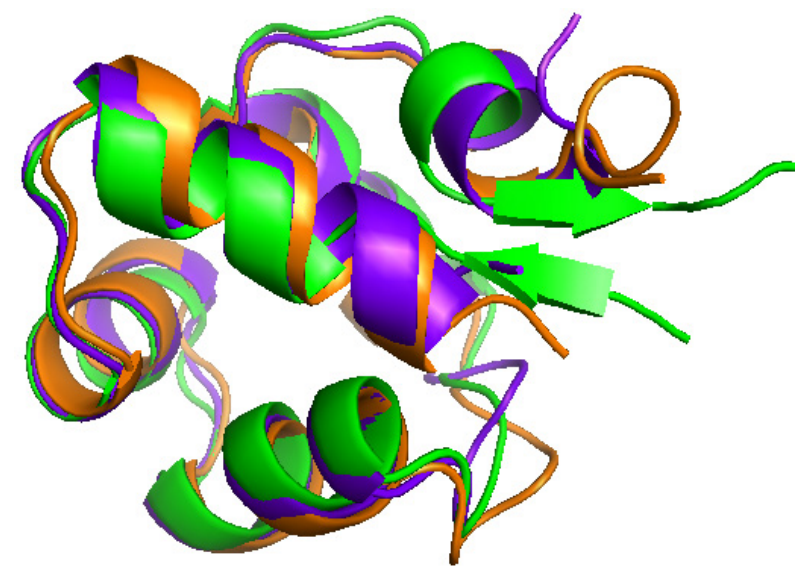

Figure 4.5: Superposition of the CylR2 monomer with SinR and 434 repressor. CylR2 is shown in green, SinR in orange and 434 repressor in violet. 
A DALI [115] search identified Bacillus subtilis SinR [116] and the N-terminal domain of 434 repressor as the structures having the closest structural similarity to CylR2 dimer with rmsd values of 2.2 and $1.7 \AA$, respectively (alignment of $62 \mathrm{C} \alpha$ atoms of SinR and $59 \mathrm{C} \alpha$ atoms of 434 repressor with structurally equivalent atoms of CylR2). The $\alpha$-helical portion of the structure superimposes quite well on both, SinR [116] and 434 repressor [114. The main divergences are in the turn between $\alpha 3$ and $\alpha 4$ and in the $\mathrm{N}$ - and C-terminal regions (Figure 4.5). In CylR2 the N-and C-terminal helices $\alpha 1$ and $\alpha 5$ are shorter than in SinR and 434 repressor and are preceded and followed by $\beta$-strands, which form an antiparallel $\beta$-sheet (Figure 4.5). SinR and 434 repressor lack this $\beta$-sheet.

\subsubsection{Dimerisation}

In the crystal structure CylR2 forms a dimer with overall dimensions of about $18 \AA \mathrm{x}$ $36 \AA$ x $44 \AA$. The two subunits, which are related by a twofold non-crystallographic symmetry, are very similar: the rmsd is $0.7 \AA$ by superposition of $\mathrm{C} \alpha$ atoms (Figure 4.4). The N- and C-terminal residues have the highest rms deviations of about $2 \AA$. Helices $\alpha 4$ and $\alpha 5$ contribute extensively to the dimeric interface (Figure 4.6). Helices $\alpha 4$ are very close to one another at their N-termini (the distance between
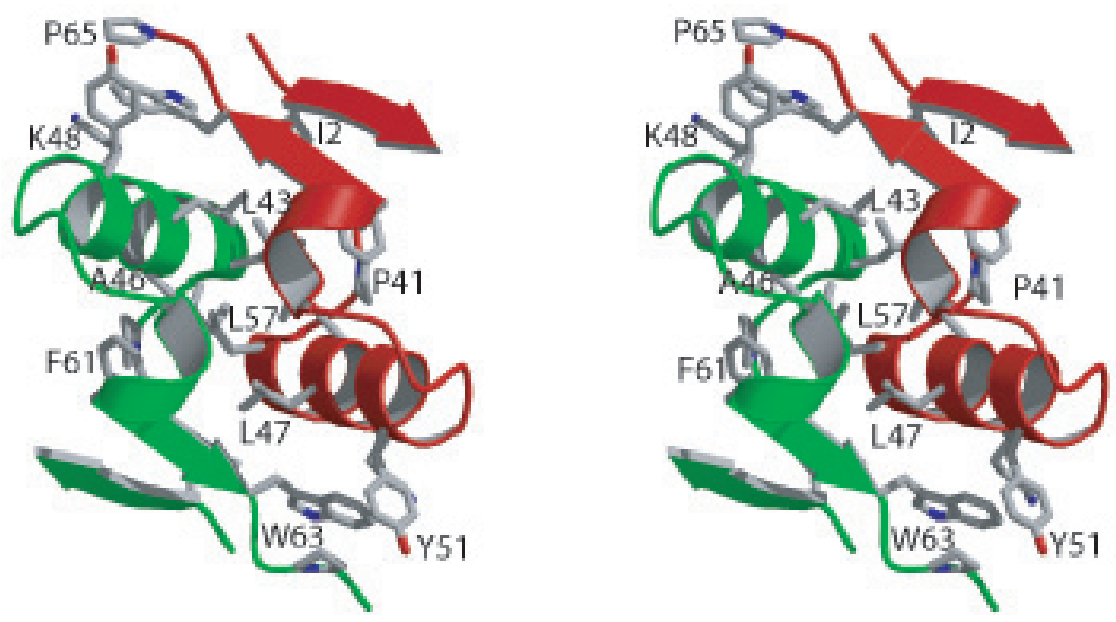

Figure 4.6: Dimer interface of CylR2. Residues 1 to 5 and 40 to 65 of subunit A and $\mathrm{B}$ are colored red and green. Side chains of the predominantly hydrophobic residues Ile2, Pro41, Leu43, Ala46, Leu47, Lys48, Tyr51, Leu57, Phe61, Trp63 and Pro65 are shown. The orientation corresponds to Figure 4.4A. 
the Leu43 $\mathrm{C} \alpha \mathrm{s}$ is $4.5 \AA$ ) and diverge at their C-termini. C-terminally of $\alpha 4$ the two CylR2 subunits come again closer by means of helices $\alpha 5$, whose N-termini contact one another. Strands $\beta 1$ and $\beta 2$ mediate also intersubunit contacts by sandwiching helices $\alpha 4$ and $\alpha 5$. In general, the side chains along the dimeric interface have the same conformations and superimpose well. The interface is predominantly hydrophobic, consisting of residues: Ile2, Pro41, Leu43, Ala46, Leu47 and the aliphatic part of Lys48, Tyr51, Leu57, Phe61, Trp63, Pro65 (Figure 4.6). The hydrophilic interactions between the subunits are mostly water-mediated. There are only four direct hydrogen bonds from the side chains of Gln44 and Glu58 of one subunit to the main chain nitrogens of Met1 and Leu57 of the other subunit, respectively. The side chains of the two Glu58 come close to one another. A sodium ion between the Glu58 side chains neutralises the local negative potential.

A similar dimerisation mode is also observed for the HTH DNA-binding protein GerE [117]. However, helices $\alpha 5$ and strands $\beta 1$ and $\beta 2$ are missing and the angle between helices $\alpha 4$ is $20^{\circ}$ narrower than in CylR2 (Figure 4.7). In CylR2 helices

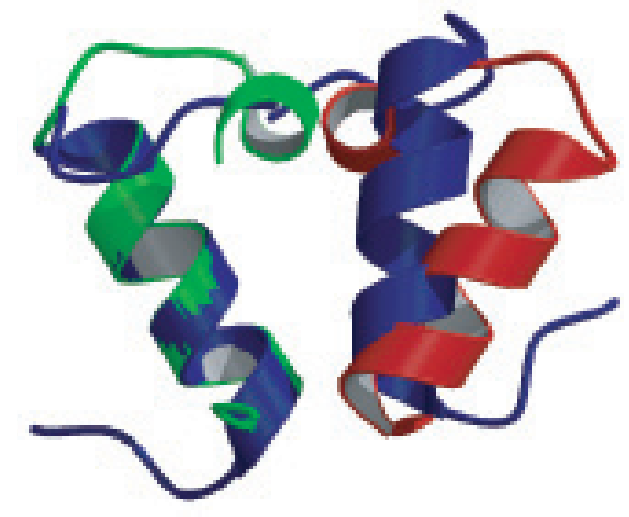

Figure 4.7: Residues 40 to 60 of CylR2 (red/green) are superposed on residues 55 to 74 of GerE (blue). The orientation corresponds to Figure 4.4B.

$\alpha 5$ are pushing helices $\alpha 4$ apart (Figure 4.6). Dimerisation of CylR2 is achieved by formation of an extensive hydrophobic interface, which is shielded from the solvent (Figure 4.6). It buries a total of $1600 \AA^{2}$ of the solvent accessible surface, accounting for about $18 \%$ of the total accessible area of each subunit. This is significantly more than in GerE and the N-terminal domain of 434 repressor, where $12 \%$ [117] and $9 \%$ [118] of the accessible area of each subunit are buried upon dimerisation, respectively. Especially for the 434 repressor, dimerisation is energetically more favourable 
in the DNA complex structure as charged residues at the dimer interface are neutralised upon DNA-binding [118]. The relative orientations of the two monomers in CylR2 and the N-terminal domain of 434 repressor bound to DNA are quite different. If only one monomer of CylR2 is superimposed on one 434 repressor monomer, the other monomers are related by a rotation of about $20^{\circ}$ around the axis connecting the CylR2 Ser42 C $\alpha$ s (Figure 4.8). In addition, the two recognition helices are about

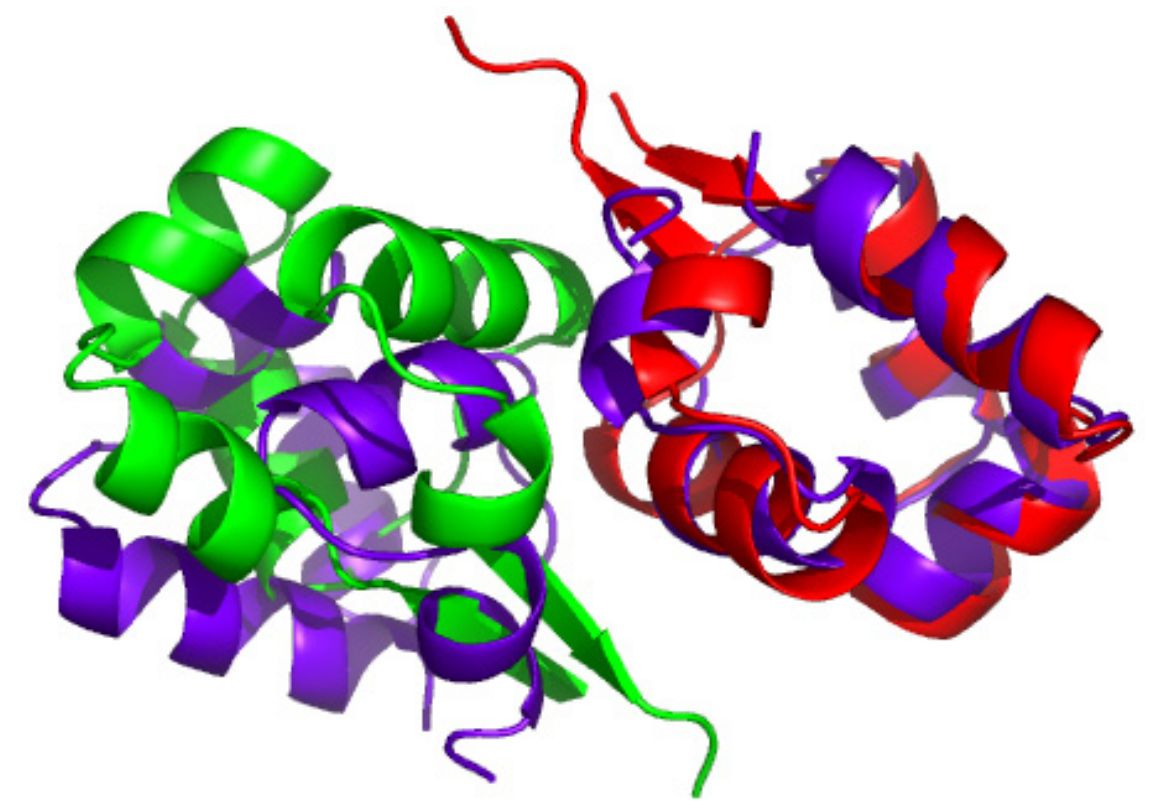

Figure 4.8: Superposition of one subunit of CylR2 dimer to 434 repressor dimer. The subunits of CylR2 are colored red/green and 434 repressor is colored violet. The orientation corresponds to Figure 4.4A.

$2 \AA$ further apart in CylR2 than in the N-terminal domain of 434 repressor bound to DNA.

\subsubsection{Solution NMR studies of CyIR2 and CyIR2/DNA complex}

Analysis of triple resonance NMR spectra allowed identification and sequential assignment of all backbone amide resonances and of all $\mathrm{C} \alpha$ and $\mathrm{C} \beta$ chemical shifts (see 3.5.1). The assignment of C' (except of Pro65), $\mathrm{H} \alpha$ and the aliphatic proton and carbon side chain resonances is complete for all residues with exception of the carboxyterminal Glu66. Protonated ${ }^{15} \mathrm{~N}$ resonances have been found for the side chains of all five glutamine residues, of five out of six asparagines and of the tryptophan side 
chain. More than $80 \%$ of the aromatic proton and carbon ${ }^{1} \mathrm{H}$ and ${ }^{13} \mathrm{C}$ side chain resonances have been unambiguously assigned. Assigned ${ }^{1} \mathrm{H},{ }^{13} \mathrm{C}$ and ${ }^{15} \mathrm{~N}$ chemical shifts have been deposited in the BMRB (http://www.bmrb.wisc.edu), accession code 6317. This $98.8 \%$ complete assignment, was used in Chapter 5 for calculating the monomer solution structure of CylR2.

NMR relaxation times indicate that CylR2 is dimeric in solution for concentrations between $10 \mu \mathrm{M}$ and $2 \mathrm{mM}$ (Appendix Figure B.1). In order to detect differences between crystal and solution structure of CylR2 HN-RDCs were measured (see 3.5.3.1). Comparison of experimental ${ }^{1} \mathrm{D}_{H N}$ couplings measured at $0.5 \mathrm{M} \mathrm{NaCl}$ with values back-calculated from the CylR2 crystal structure using SVD resulted in a correlation coefficient of 0.97 and a Q-factor of 0.19 for 57 out of 62 backbone amide protons (Figure 4.9A). The experimental HN-RDCs of free CylR2 can be found in the Appendix Table C.1. This proves that the backbone structure of CylR2 in solution

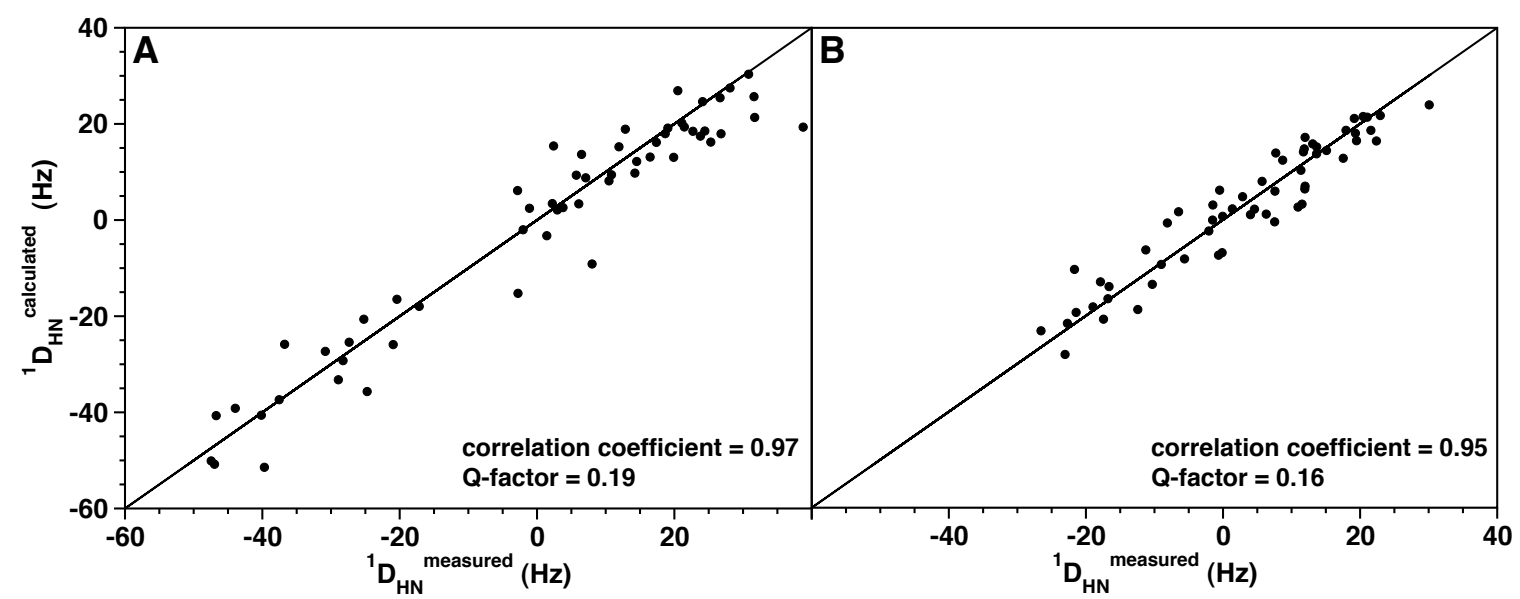

Figure 4.9: HN-RDC of CylR2. Correlation between experimental backbone ${ }^{1} \mathrm{D}_{H N}$ couplings and values back-calculated from the crystal structure. (A) Correlation for free CylR2 at $0.5 \mathrm{M} \mathrm{NaCl}$ and (B) correlation for DNA-bound CylR2 at $0 \mathrm{M} \mathrm{NaCl}$.

agrees well with that in the crystalline state. The magnitude $\mathrm{D}_{a}$ and the rhombicity $\mathrm{R}$ of the alignment tensor were back-calculated as $\mathrm{D}_{a}=-26.0 \mathrm{~Hz}$ and $\mathrm{R}=0.16$.

In order to evaluate if DNA-binding is accompanied by changes in the backbone conformation of CylR2, HN-RDCs were measured for CylR2 when bound to DNA (see 3.5.3.1). Best-fitting ${ }^{1} \mathrm{D}_{H N}$ couplings observed at $0 \mathrm{M} \mathrm{NaCl}$ for CylR2 in complex with DNA to the crystal structure of free CylR2 results in a correlation coefficient 
of 0.94 and a Q-factor of 0.16 between experimental and back-calculated RDCs for 57 out of 62 backbone amide protons (Figure 4.9B). The back-calculated alignment tensor is characterized by $\mathrm{D}_{a}=-22.1 \mathrm{~Hz}$ and $\mathrm{R}=0.07$ for the protein/DNA complex at $0 \mathrm{M} \mathrm{NaCl}$. Due to a significantly reduced signal-to-noise ratio of the IPAP- ${ }^{15} \mathrm{~N}-\mathrm{HSQC}$ spectrum acquired at $0.5 \mathrm{M} \mathrm{NaCl}$ for DNA-bound CylR2, experimental HN-RDCs could only be determined accurately for 49 out of 62 backbone amides. These HNRDCs resulted in a correlation coefficient of 0.94 and a Q-factor of 0.19. The SVD results for DNA-bound CylR2 at the different $\mathrm{NaCl}$ concentrations are summarized in Table 4.1 and the experimental HN-RDCs are listed in the Appendix Table C.2. The

Table 4.1: Best-fit of HN-RDCs for DNA-bound CylR2 to the X-ray structure of free CylR2.

\begin{tabular}{ccccccc}
\hline \hline $\mathrm{NaCl}$ & $\mathrm{Pf1}$ & $\begin{array}{c}\text { correlation } \\
\text { coefficient }\end{array}$ & Q-factor & $\mathrm{D}_{a}$ & $\mathrm{R}$ & $\begin{array}{c}\text { number of } \\
\text { HN-RDCs }\end{array}$ \\
\hline $0 \mathrm{M}$ & $12 \mathrm{mg} / \mathrm{ml}$ & 0.951 & 0.158 & $-22.1 \mathrm{~Hz}$ & 0.067 & 57 \\
$0.025 \mathrm{M}$ & $12 \mathrm{mg} / \mathrm{ml}$ & 0.941 & 0.177 & $-20.5 \mathrm{~Hz}$ & 0.057 & 57 \\
$0.05 \mathrm{M}$ & $12 \mathrm{mg} / \mathrm{ml}$ & 0.937 & 0.182 & $-19.2 \mathrm{~Hz}$ & 0.075 & 57 \\
$0.1 \mathrm{M}$ & $12 \mathrm{mg} / \mathrm{ml}$ & 0.939 & 0.184 & $-17.6 \mathrm{~Hz}$ & 0.184 & 57 \\
$0.2 \mathrm{M}$ & $12 \mathrm{mg} / \mathrm{ml}$ & 0.942 & 0.176 & $-15.1 \mathrm{~Hz}$ & 0.176 & 57 \\
$0.3 \mathrm{M}$ & $12 \mathrm{mg} / \mathrm{ml}$ & 0.941 & 0.189 & $-13.4 \mathrm{~Hz}$ & 0.121 & 52 \\
$0.5 \mathrm{M}$ & $14 \mathrm{mg} / \mathrm{ml}$ & 0.943 & 0.189 & $-19.2 \mathrm{~Hz}$ & 0.189 & 49 \\
\hline \hline
\end{tabular}

very good correlation between the experimental HN-RDCs for DNA-bound CylR2 and the X-ray structure of free CylR2 at all salt concentrations shows that there are no major changes in the backbone structure of CylR2 upon binding to DNA.

Dynamics of free and DNA-bound CylR2 in solution were probed by heteronuclear NMR spectroscopy to reveal flexible regions and mobility changes upon DNA-binding. Steady state heteronuclear ${ }^{15} \mathrm{~N}-{ }^{1} \mathrm{H}-\mathrm{NOE}$ values (see 3.5.4.3) for most of the CylR2 backbone amides in the free and bound form are above 0.7 indicating that the backbone of CylR2 is well ordered in solution (Figure 4.10). Only Ser42 in the loop connecting $\alpha$-helices 3 and 4 is flexible with a ${ }^{15} \mathrm{~N}-{ }^{1} \mathrm{H}-\mathrm{NOE}$ of 0.4 and the four $\mathrm{N}$ - and C-terminal residues are slightly affected by motion. Fluctuations of ${ }^{15} \mathrm{~N}-{ }^{1} \mathrm{H}-\mathrm{NOE}$ values in the $\beta$-strands and $\alpha$-helix 2 for bound CylR2 are due to a bad signal to 


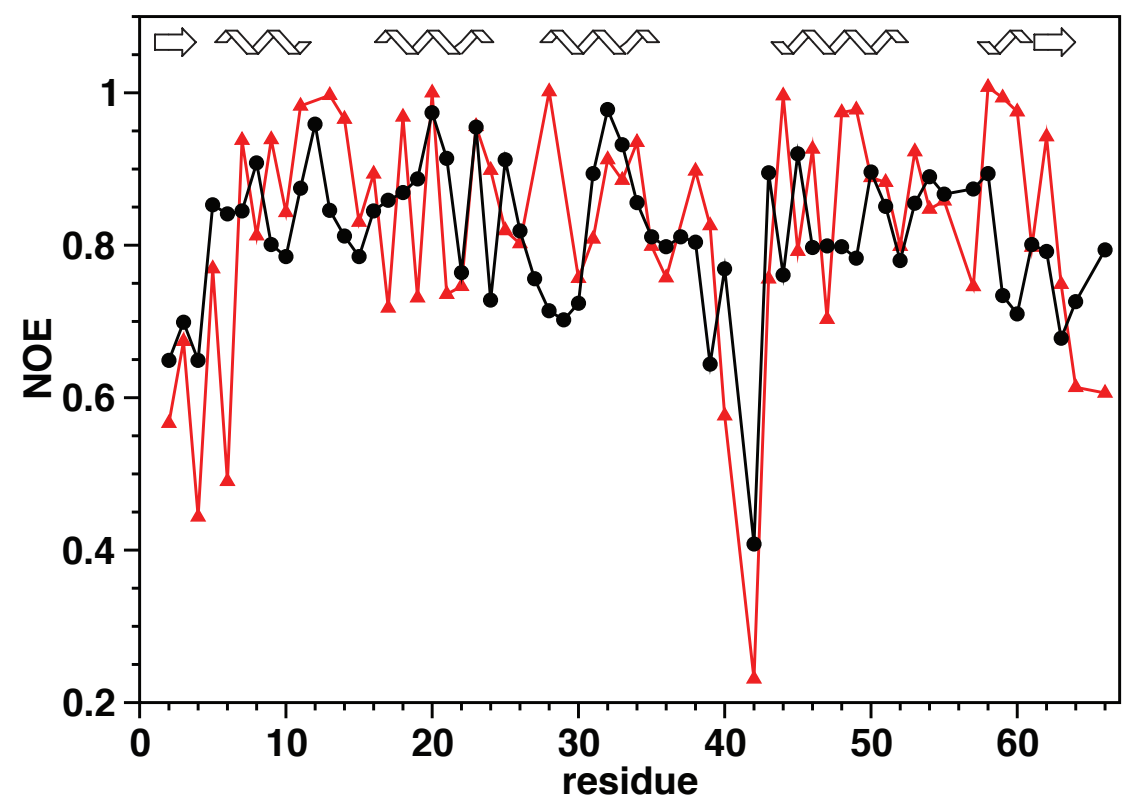

Figure 4.10: The ${ }^{15} \mathrm{~N}-{ }^{1} \mathrm{H}-\mathrm{NOE}$ plotted against the residue number. Values for free CylR2 are indicated as black circles and values for CylR2 in complex with its DNA are shown as red triangles. Secondary structure elements are indicated.

noise ratio.

\subsubsection{Mapping of the DNA-binding site}

The DNA interaction surface of CylR2 was determined using the NMR chemical shift perturbation method (see 3.5.5), in which ${ }^{15} \mathrm{~N}-\mathrm{HSQC}$ spectra were recorded with successive addition of the 22-bp DNA comprising IR1 (see 4.2.4). The spectra for the free CylR2 and the DNA-bound CylR2 are shown in Figure 4.11. Upon DNA-binding, resonances of unbound CylR2 weaken continuously and new resonances appear with significant chemical shift differences. This indicates that the free protein is in slow exchange on the NMR time scale with its DNA-bound form. The apparent $\mathrm{K}_{d}$ of $2.2 \mathrm{nM}$ was determined by gel shift analysis by Chris M. Pillar [108]. A similarly strong DNA-binding with a $\mathrm{K}_{d}$ of about $3.3 \mathrm{nM}$ was observed for the repressor from bacteriophage 434 [119, 120]. For both, free and bound protein, only a single set of resonances was observed. In order to allow quantification of the observed chemical shift changes, backbone resonances of CylR2 in complex with its DNA were reassigned. Assigned chemical shifts of CylR2 in the complex can be found in the Appendix B.7. 


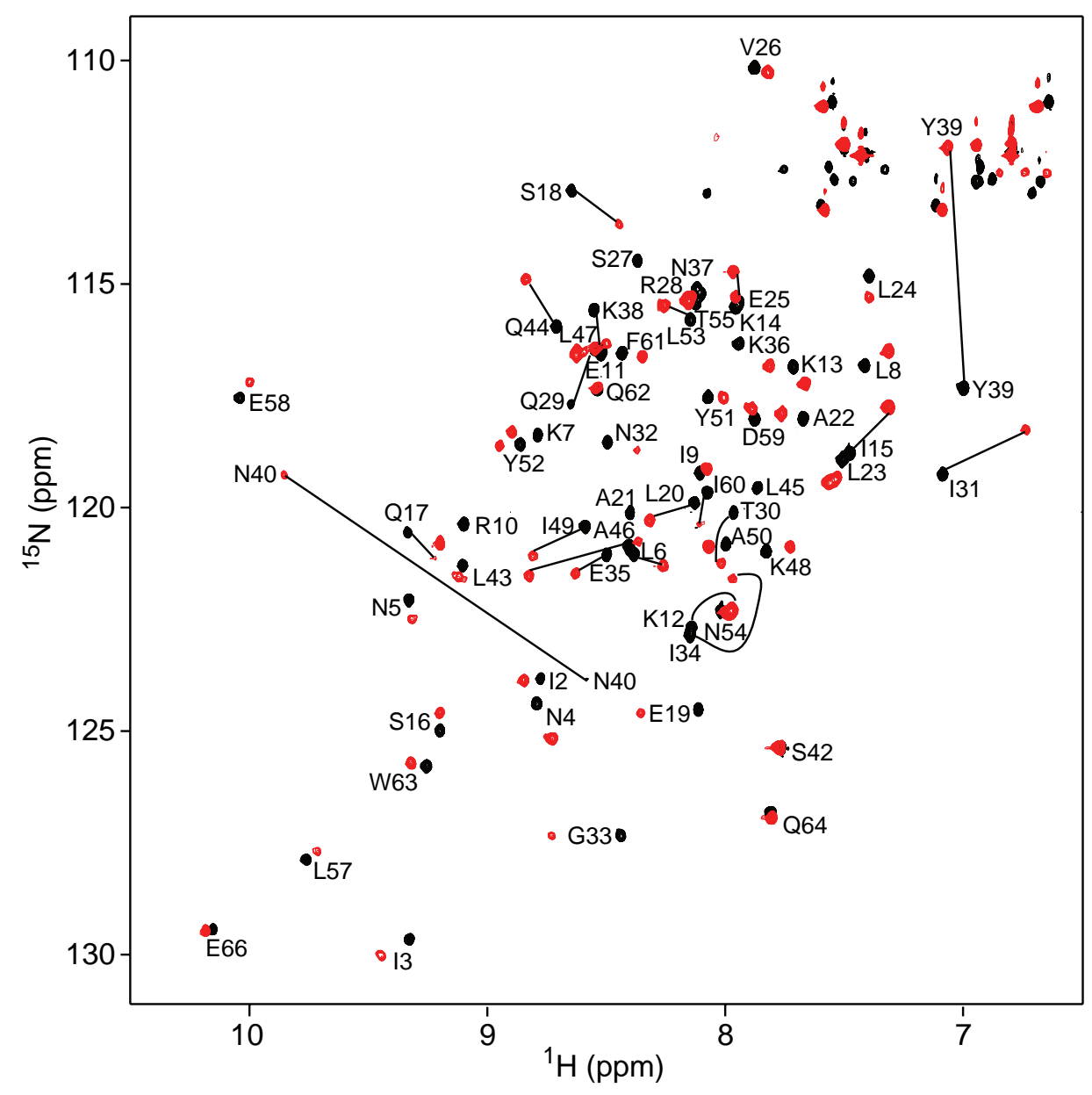

Figure 4.11: Overlay of ${ }^{15} \mathrm{~N}-\mathrm{HSQC}$ spectra of CylR2 (black) and CylR2/DNA complex (red). Black line indicate strongly shifted resonances of free and complexed CylR2.

The result of the mapping of the DNA-binding surface of CylR2 for the amide resonances is shown in Figure 4.12A. Largest chemical shift changes were observed for Tyr39 and Asn40. In addition to the amide chemical shift changes, the chemical shift perturbation was analysed for $\mathrm{C}^{\alpha}, \mathrm{C}^{\beta}, \mathrm{C}^{\prime}$ and the side chain amides (see 4.2.4 to give more detailed information about the DNA-binding. The side chain amides of Gln29, Asn40 and Gln44 are strongly affected and large chemical shift changes are found for residue 16-20, 28-34 and 44-46. This indicates that especially the recognition helix ( $\alpha 3$ ), the loop connecting helices $\alpha 3$ and $\alpha 4$ and the N-terminal halves of helices $\alpha 2$ and $\alpha 4$ are involved in IR1 binding. Residues of CylR2 form a positively charged surface for DNA-binding and residues strongly affected by DNA-binding are colored on the surface of CylR2 (Figure 4.13). A similar DNA-binding mode was found for 


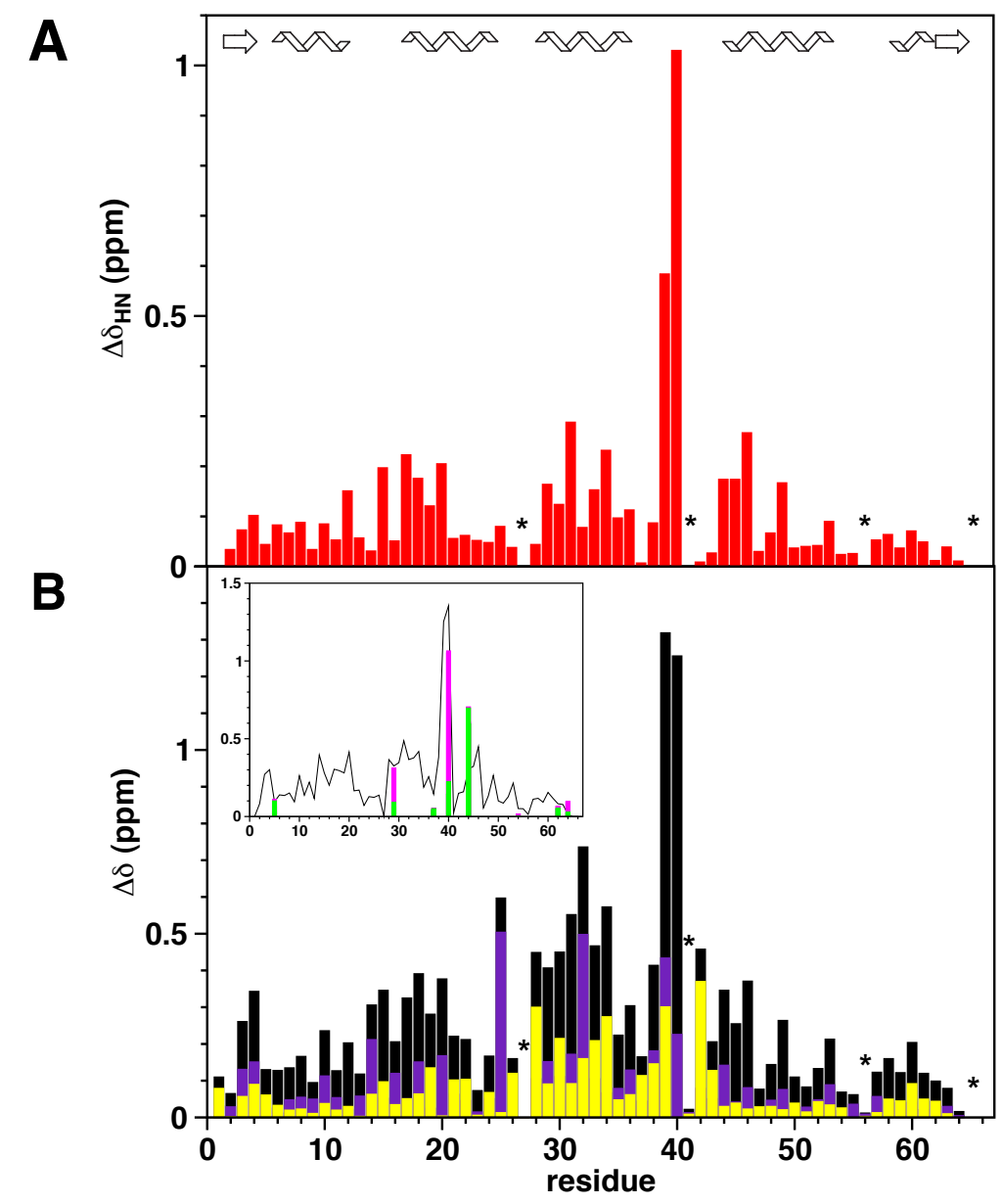

Figure 4.12: Chemical shift changes upon CylR2/DNA complex formation. The for DNA-bound CylR2 unassigned residue Ser27 and proline residues are labeled with an asterisk. (A) Amide chemical shift changes and (B) Combined chemical shift changes ( $\Delta \delta_{\text {tot }}$ in black, $\Delta \delta_{C \alpha C \beta}$ in violet and $\Delta \delta_{C^{\prime \star}}$ in yellow). The inset displays $\Delta \delta_{\text {tot }}$ as a solid line and the chemical shift changes for the complete amide side chain $\Delta \delta_{\text {side }}$ in magenta and for the amide $\mathrm{C}^{\gamma}$ or $\mathrm{C}^{\delta} \Delta \delta_{\text {sideC }}$ in green.

the N-terminal domain of the 434 repressor [110, 118]. The DNA titration was in addition performed for an 18-bp DNA comprising IR1, but no chemical shift changes could be observed for CylR2. This proves that ClyR2/DNA interactions also occur outside of the 18-bp core and the minimum length of an IR1 fragment required for binding to CylR2 is 20 or $22 \mathrm{bp}$ long.

The NMR chemical shift perturbation studies implicated an important role of Asn40 of CylR2 in binding the cytolysin promoter. Based on this result, the consequence on cytolysin expression upon mutating this residue to Ala was examined by Chris 

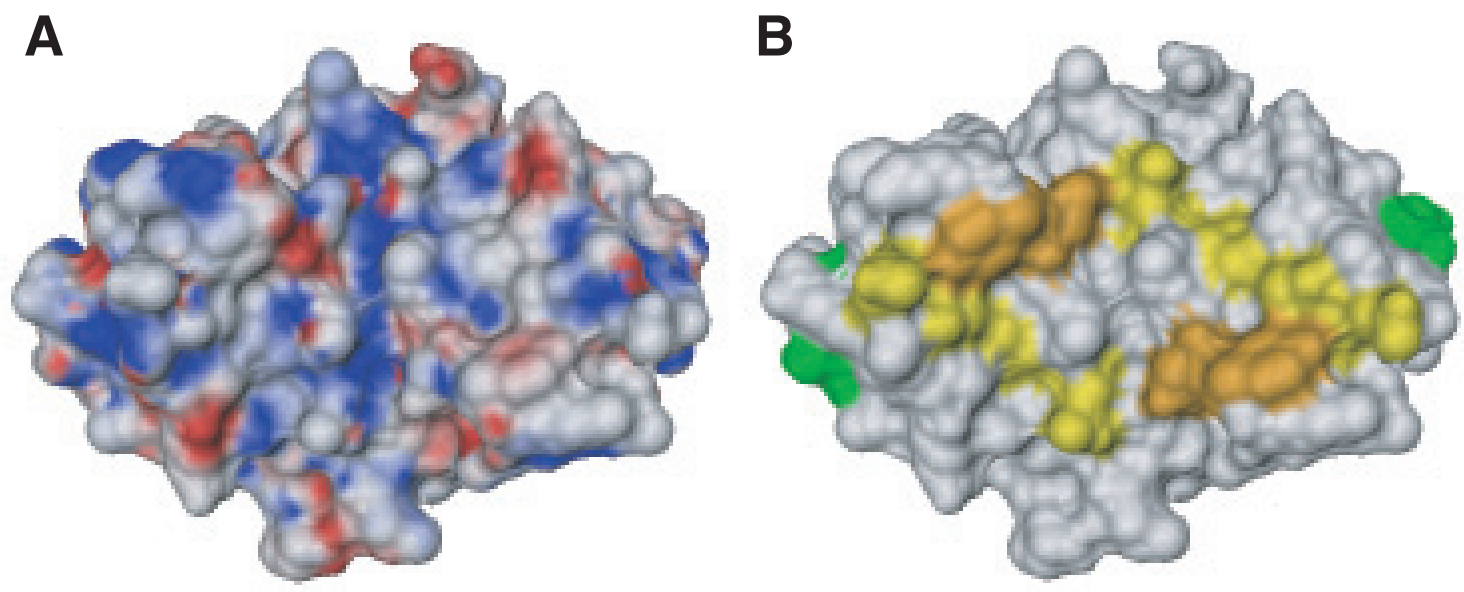

Figure 4.13: (A) Electrostatic surface of CylR2 with positive and negative potentials colored blue and red, respectively. (B) Chemical shift changes upon DNA-binding mapped to the CylR2 surface. Residues with very strong (Tyr39, Asn40), strong (Gln29, Thr30, Ile31, Gln44, Leu45, Ala46) and intermediate chemical shift changes (Gln17, Ser18, Glu19, Leu20) are colored orange, yellow and green, respectively.

M. Pillar in vivo with a LacZ reporter system, in which the LacZ transcription is driven by the cytolysin promotor. The LacZ activity was repressed in the presence of wild-type CylR2 ans was 10-fold increased only after addition of the autoinducer $\mathrm{CylL}_{S} "$. However, for an identical construct in which the Asn40 of CylR2 was mutated to an Ala the LacZ activity was high whether the operon was induced or not, revealing a derepression of the cytolysin promoter [108]. These results obtained from experiments, which were performed due to the NMR chemical shift perturbation, are consistent with the proposed role of CylR2 as a repressor of cytolysin expression from $\mathrm{P}_{l y t}$ via its interaction with cytolysin promoter DNA (see 4.1).

\subsubsection{Model for the CyIR2/DNA complex}

Chris M. Pillar has shown that CylR2 binds specifically to the 25-bp IR1 inverted repeat that exists within the cytolysin promoter [108]. The NMR shift perturbation results of CylR2 with a 22-bp fragment of the IR1 repeat sequence (Figures 4.11, 4.12) further demonstrated that similar regions in dimeric CylR2 are interacting with DNA as compared to the N-terminal domain of 434 repressor. Furthermore, HN-RDCs for CylR2 when bound to DNA showed that there are no major changes in the backbone structure of CylR2 upon binding to DNA (Figure 4.9. 
Based on the combined information from crystallographic (see 4.3.1) and solution NMR studies (see 4.3.3), a model of the CylR2/DNA complex was constructed. In agreement with the absence of major conformational changes in CylR2 upon binding to DNA, a starting structure for the complex was built by superposition of the structure of the unbound CylR2 dimer onto the N-terminal domain of 434 repressor and replacement of the 434 operator by standard linear B-DNA. This initial model was refined using the protein-DNA docking program Monty [90, 91] guided by information obtained from the NMR chemical shift perturbation studies: Side chains of residues 10, 16-20, 24-32 and 34-45 were allowed to rotate freely in accordance with the chemical shift differences (Figure 4.12). In addition, energy bonuses were given during the simulation to complex structures where the backbone amides of Tyr39, Asn40, Gln44, Leu45 and Ala46 are in close contact to the DNA and when the $\mathrm{NH}_{2}$ of Gln29 contacts the DNA reflecting the strong chemical shift changes upon addition of DNA. Figure 4.14 shows the model for the CylR2/DNA complex obtained from this docking approach. It agrees with established dimeric HTH/DNA interactions [112, 121]; binding occurs with dyad symmetry on two adjacent major grooves through their recognition helix. In a similar manner as observed for the 434 repressor $/ \mathrm{O}_{R} 1$ complex structure, side chains of Ser27 and Gln29 form a van der Waals pocket to receive the methyl group of thymine 4' (Figures 4.12 and 4.14B). Moreover, complex models indicate a bidentate hydrogen bonding for the $\mathrm{NH}_{2}$ of Arg28 to either G8, T7 or C6 and a hydrogen bond between the side chain of Gln29 and bp 4. The hydrogen bond of Gln29 to bp 4 is likely to be formed with $\mathrm{O}^{\epsilon}$ of Gln29 as the hydrogen bond acceptor, because the $\mathrm{C}^{\delta}$ resonance showed a strong downfield shift of $\sim 0.9 \mathrm{ppm}$ while $\mathrm{N}^{\epsilon}$ and $\mathrm{H}^{\epsilon}$ remained nearly unchanged (Figures 4.12 and $4.14 \mathrm{~B}$ ). For the side chain of Arg28 only $\mathrm{C}^{\alpha}$ and $\mathrm{C}^{\beta}$ could be assigned, whereas both backbone and side chain resonances were missing for Ser27 in the complex. The disappearance or strong perturbation of these resonances upon binding to DNA supports their importance for complex formation. At the N-terminus of helix 2, Gln17 forms van der Waals contacts and a hydrogen bond with the phosphate of T9, in agreement with the disappearance of the side chain resonances of the Gln17 carbonyl group upon DNA-binding. Docking re- 


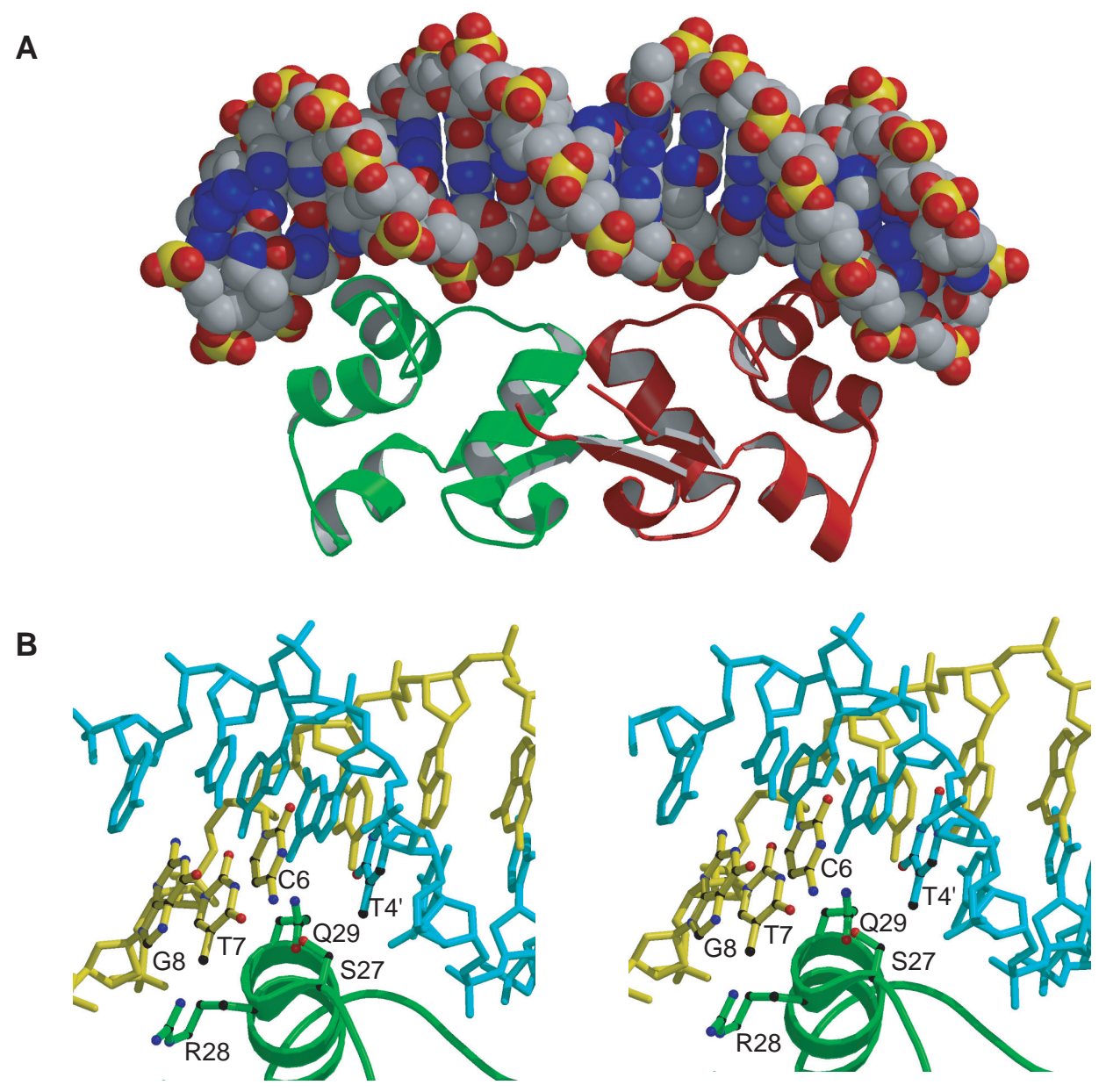

Figure 4.14: (A) Overall model of the CylR2/DNA complex structure. (B) Detailed stereo view indicating important protein-DNA interactions in the major groove. The DNA is shown with the sense strand in yellow and the antisense strand in cyan. Side chains of CylR 2 and nucleobases interacting by van der Waals contacts and/or hydrogen bonds are indicated.

sults und chemical shift changes also suggest that bp 6 accepts a hydrogen bond from Asn32. NMR resonances from the carbonyl side chain of Asn32 were neither found for free nor complexed CylR2, but the $\mathrm{C}^{\beta}$ and $\mathrm{C}^{\alpha}$ of Asn32 were shifted by $2.8 \mathrm{ppm}$ and $1.1 \mathrm{ppm}$ downfield, respectively (Figure 4.14B). Very important for stabilizing the CylR2/DNA complex is the $(\alpha 3-\alpha 4)$ loop: for both CylR2 molecules a hydrogen bond is formed from the main chain amide group of Asn40 to the phosphate of $\mathrm{C} 1$ in more than $30 \%$ of docking solutions, in agreement with a downfield shift of $1.1 \mathrm{ppm}$ of its amide proton resonance.

The best-fit of DNA to the dimeric structure of CylR2 was obtained for a DNA 
bending parameter of 0.4. For a more extended DNA, formation of protein/DNA interactions, which were expected on the basis of the NMR chemical shift perturbation studies, was not possible for both molecules of the CylR2 dimer simultaneously. When the DNA was more strongly bent, on the other hand, no docking solutions could be obtained that provided the necessary space to accommodate both CylR2 molecules in the orientation and position that were observed for the uncomplexed CylR2 structure. This orientation and position were experimentally indicated by the very good agreement of the experimental HN-RDCs for DNA-bound CylR2 with the X-ray structure of free CylR2 (Figure 4.9B). Similar to the 434 repressor/DNA complex, the DNA is relatively straight in the middle of the operator and bends towards the ends to enable interactions with the side chains of Arg28 and Gln29 (Figure 4.14B). The helical axes of individual bp, when projected onto the mean plane of bending, lie on a circle with a radius of $65 \AA$ (Figure $4.14 \mathrm{~A}$ ). A specific feature of the 434/DNA complex is a strong compression of the minor groove due to insertion of the side chains of Arg43 of both molecules of the dimeric 434 repressor: the two positively charged side chains allow a closer approach of the negatively charged phosphates [118]. In CylR2, on the other hand, Arg43 is replaced by a serine; hence, the positive side chains are absent within the minor groove and no compression of the minor groove is required to dock DNA onto CylR2.

\subsubsection{Specificity of CyIR2/DNA interactions}

The DNA-binding site of CylR2 had been revealed by gel shift analysis as the inverted repeat IR1 of the intergenic region of the cytolysin operon (Figure 4.2). In contrast, no DNA band shift was observed for the inverted repeat IR2 in the presence of CylR2 [108]. Nevertheless, NMR titration experiments were performed for IR2. ${ }^{15} \mathrm{~N}-\mathrm{HSQC}$ spectra recorded at different protein-to-IR2 ratios showed chemical shift changes for residues which were already found to be affected by IR1-binding (Figure 4.15). However, CylR2 signals shift continuously until a $\sim 7$-fold excess of IR2 is reached. This indicates fast exchange between CylR2 and IR2 and agrees with the absence of retardation in the gel shift analysis. ${ }^{15} \mathrm{~N}-\mathrm{HSQC}$ spectra measured for 


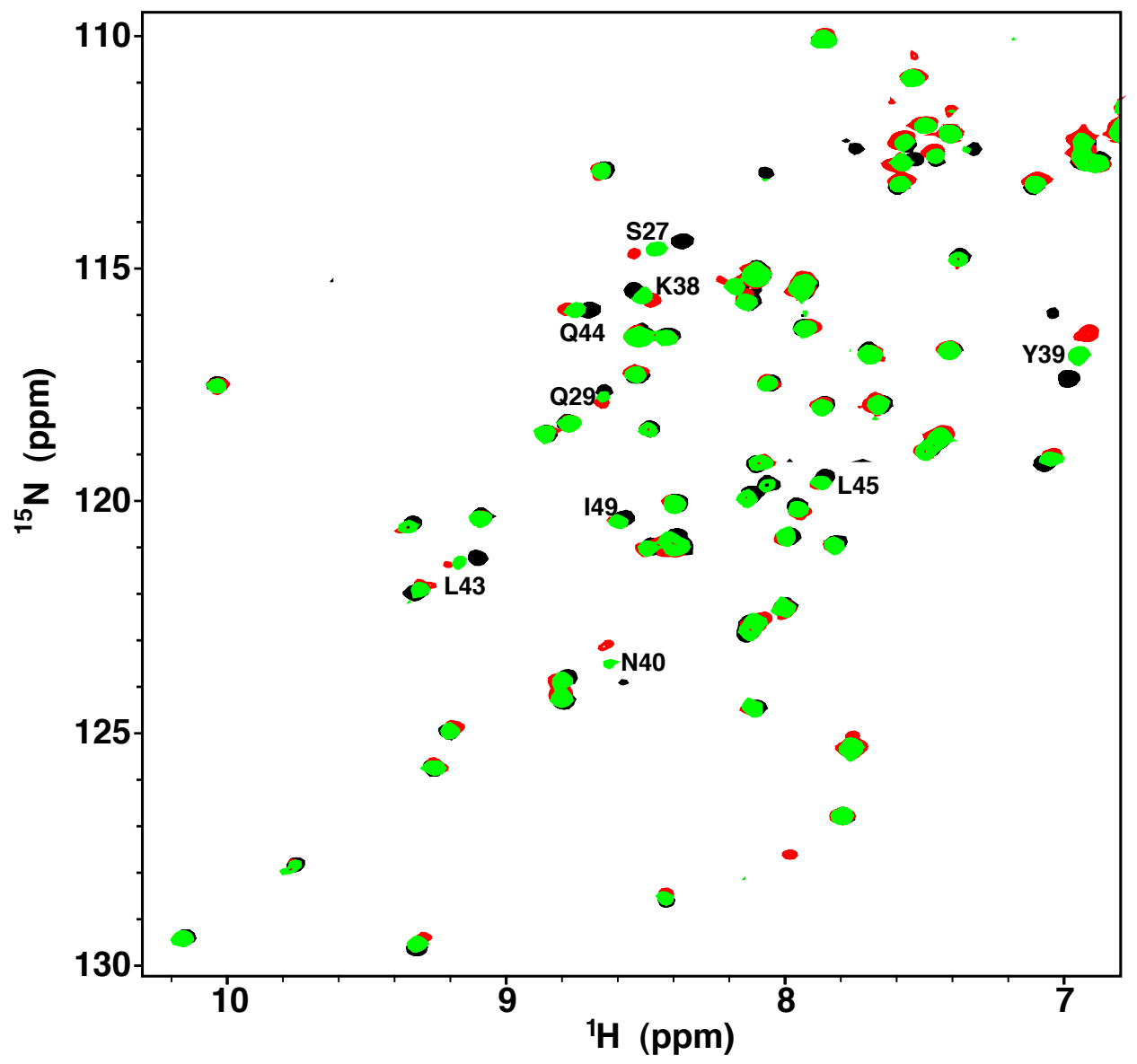

Figure 4.15: Overlay of ${ }^{15} \mathrm{~N}-\mathrm{HSQC}$ spectra of CylR2 at a molar protein to IR2 ratio of 1 to 0 (black), of 1 to 1 (green) and of 1 to 5 (red). Shifted resonances are labeled with the residue number.

a titration of CylR2 with a random ds DNA comprising a 22-bp long ACTG-repeat displayed almost identical continuous shifts as IR2. From these data a $\mathrm{K}_{d}$ of $\sim 2 \mathrm{mM}$ was calculated (see 3.5.6). Thus, the affinity of CylR2 for a non-specific DNA is by six orders of magnitude reduced. Figure 4.16 shows the amide chemical shift changes upon binding of CylR2 to unspecific DNA. At a $\sim 7$-fold excess of the unspecific DNA the chemical shift changes for the mainly affected region around residue 40 are very similar to the chemical shift changes of CylR2 upon binding to its specific IR1 DNA while the regions around residue 17 and 45 are less affected by the unspecific DNA. This suggests an important function of the regions around residue 17 and 45 of CylR2 for the specificity to IR1 DNA. 


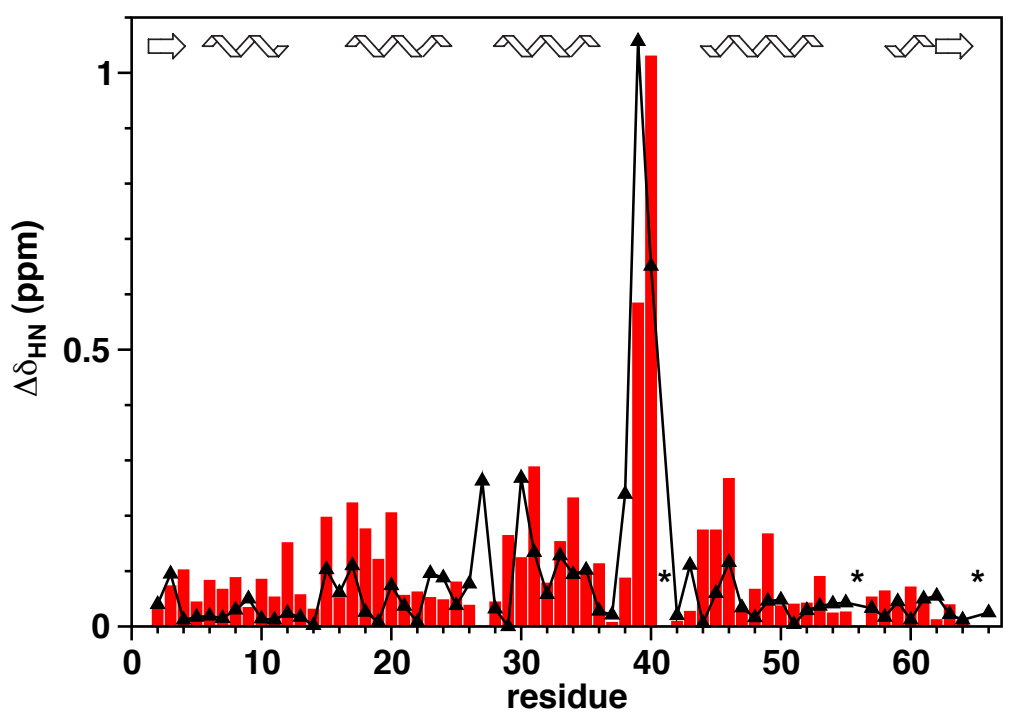

Figure 4.16: Amide chemical shift changes upon binding of CylR2 to unspecific DNA (black triangles). The red bars show the amide chemical shift changes upon binding CylR2 to its specific IR1 DNA for comparison. Proline residues are labeled with an asterisk.

\subsubsection{Interaction between CyIR2 and CyIR1}

In addition to CylR2, the 94-residue protein CylR1 has been demonstrated to be essential for repression of cytolysin production [104]. Using TMHMM Server v. 2.0 for the prediction of transmembrane helices, $20 \mathrm{~N}$-terminal residues, residues 40 to 60 and residues 68 to 89 of CylR1 were found to probably form transmembrane helices [122](Figure 4.17). Furthermore, intracelluar loop regions were predicted for residues 20 to 40 and the C-terminal residues therewith suggesting an interaction of these residues with CylR2. As all trials to recombinantly prepare CylR1 failed, unlabeled peptides comprising the predicted intracellular loop regions were synthesized (see 3.3.11 and Table 2.4) for NMR-titration experiments with ${ }^{15} \mathrm{~N}$-CylR2. Even at a 20 -fold excess of the peptide, the recorded ${ }^{15} \mathrm{~N}-\mathrm{HSQC}$ spectra did not show chemical shift changes so that the interaction between CylR2 and CylR1 cannot be investigated with the peptides constituting the predicted intracellular loop regions. These data propose the absence of a direct interaction between CylR2 and CylR1, the binding of CylR2 to two or all three intracellular loops in the correct 3D arrangement or the interaction of CylR2 with transmembrane regions of CylR1. To distinguish these 


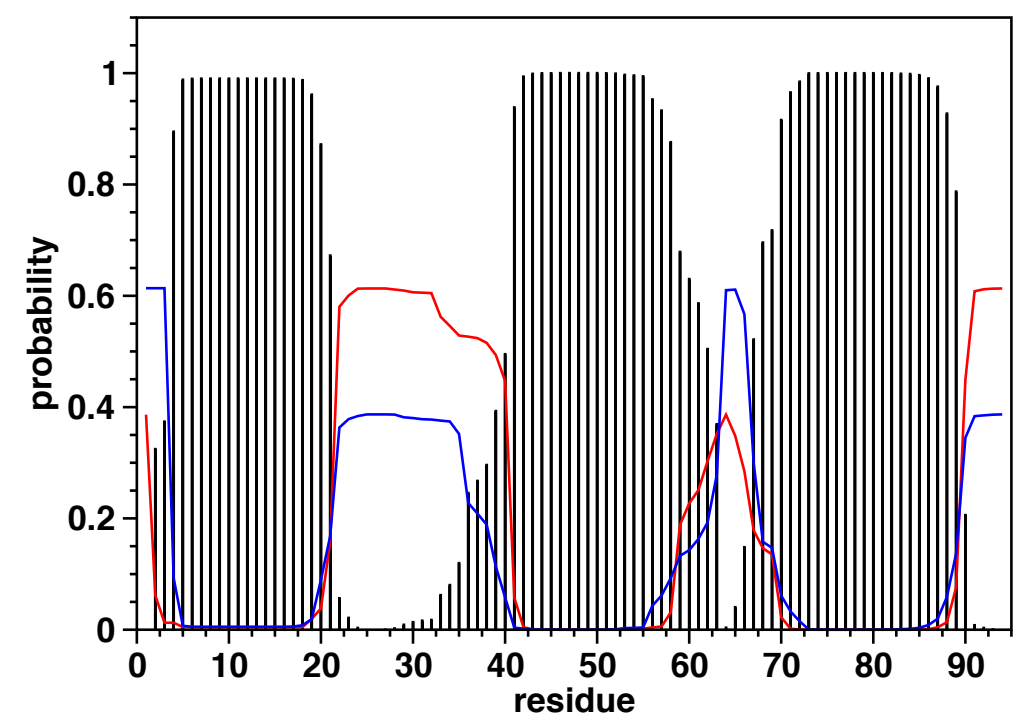

Figure 4.17: Prediction of transmembrane helices for CylR1. Probabilities for helical, inside and outside regions are indicated in black, red and blue, respectively.

possibilities intact CylR1 is required. However, this is a demanding task as reconstitution of CylR1 in micelles is necessary and so far there is no generally recommended refolding protocol for $\alpha$-helical membrane proteins available [15].

\subsection{Conclusions}

The performed studies show that CylR2 binds as a preformed dimer to a 22-bp fragment of the cytolysin promoter region. The promoter binding is highly specific and is mediated through a complex network of CylR2/DNA interactions. In particular, the very strong chemical shift change of Asn40 upon DNA-binding motivated an in vivo activity test in the group of Michael S. Gilmore. This showed significantly reduced strength for the CylR2-operator binding when Asn40 was mutated to Ala. Based on these results a mechanism was proposed where repression is achieved by CylR2 obstruction of the promoter preventing transcription of the cytolysin operon.

CylR2 works together with CylR1 in order to repress the transcription of cytolysin genes [104]. To get an idea on how CylR1 influences the CylR2-operator interaction, comparison with the repressor of phage 434 is particularly interesting. In the case of the 434 repressor, it is the intricate combination of direct protein-DNA interactions and DNA conformational effects that enable differential binding to different oper- 
ator binding sites. This very well orchestrated, differential binding determines the developmental fate of phage 434. By analogy, it can therefore be speculated that in the presence of the autoinducer $\mathrm{CylL}_{S}$ ", CylR1 shifts the DNA-binding specificity of CylR2 to sequences adjacent to the IR1 repeat. The three-dimensional structure of CylR2 and its DNA-binding properties presented here provide first steps in understanding the molecular basis of cytolysin repression by the CylR1/CylR2 twocomponent regulatory system. 


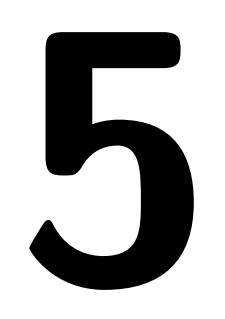

\section{Using dipolar couplings and intermonomer paramagnetic relaxation enhancement to determine the structure of the CyIR2 homodimer}

\subsection{Introduction}

The majority of structures in the PDB represents monomers, but most of the soluble and membrane proteins form homodimers or higher oligomers. Therefore oligomerization plays an important role in protein function, and improved methods for structure determination of homooligomers are required.

Solving the NMR solution structure of homodimeric proteins is especially complicated as inter- and intramonomer contacts are difficult to distinguish. The standard method to determine intermonomer distances in homodimeric proteins combines isotope-filtered and -edited NOE experiments conducted on samples formed by labeled and unlabeled protein [123]. An alternative method uses a dimer mixed from a completely deuterated ${ }^{15} \mathrm{~N}$-labeled and an unlabeled protonated protein to measure a 3D $\left[{ }^{15} \mathrm{~N}-{ }^{1} \mathrm{H}\right]$ NOESY-HSQC, which shows in the aliphatic region only intermonomer contacts between HN and aliphatic protons [124]. In addition, computational methods exist which restrain the symmetry during structure calculation with ambiguous NOE data [125]. All of these methods require experimental observation of a significant number of side chain to side chain or HN to side chain intermolecular NOEs. How- 
ever, deuteration is necessary for large homodimers to reduce the linewidths which increase with molecular weight. Due to deuteration intermolecular NOE information is very limited.

As an alternative or a complement to NOE restraints, which are limited to distances up to $\sim 6 \AA$, long-range distance information may be derived from paramagnetic relaxation enhancement (PRE) [88]. As paramagnetic metalloproteins represent only a small class of proteins in nature, approaches to introduce paramagnetic centers into proteins have been developed to determine PRE. These approaches include ions bound to engineered metal-binding sites [126, 127], chelating tags [128, 129] and nitroxide groups [60].

The interaction between a specifically attached paramagnetic center and protons within a distance of up to 25-30 $\AA$ causes broadening of their NMR signal due to an increase in transverse relaxation rate [88. This effect has an $\mathrm{r}^{-6}$ dependence on the electron-proton distance and thus allows the detection of long-range interactions in proteins. PRE-derived distances have already been used in monomer structure calculations [128, 130, 131] and are particularly useful for the characterization of highly flexible systems, such as intrinsically disordered proteins [132]. Moreover, they can be used to detect transient intermediates in macromolecular binding processes [133]. PRE-derived distances might also be used to overcome the difficulties in detection of intermolecular NOEs in large homodimers. By adding a paramagnetic probe substoichiometrically, the homodimer symmetry is broken and monomer specific RDCs and pseudocontact shifts (PCSs) are measured [134]. However, due to the broken symmetry and the pseudocontact shifts, the number of signals is increased significantly, limiting the use of this approach especially for larger proteins.

This chapter introduces a method for structure determination of homodimeric proteins, which is based on intermonomer distances derived from paramagnetic relaxation enhancement and residual dipolar couplings. Using this method, the high-resolution solution structure of the $15.4 \mathrm{kDa}$ homodimer of CylR2 was determined. In order to derive intermonomer distances, two paramagnetic labels have been applied. Firstly, site-directed spin-labeling (SDSL) of a single cysteine residue with the nitroxide spin 
label methanethiosulfonate (MTSL) [60] and secondly, prepending the three-residue amino terminal $\mathrm{Cu}^{2+}\left(\mathrm{Ni}^{2+}\right)$-binding (ATCUN) motif to the N-terminus [130]. In addition, it is shown that only HN-RDCs can be used to rank homodimeric models of CylR2 obtained from ab initio docking.

\subsection{Materials and methods}

\subsubsection{Construction of CyIR2-mutants}

The plasmid pET32a-CylR2 provided by Karin Giller (Max Planck Institute for Biophysical Chemistry, Göttingen) was the template for mutagenesis using the QuikChange $^{T M}$ Site-Directed Mutagenesis Kit (see 3.1.5). The plasmid was amplified with the mutagenic primers CylR2_N40C5' and CylR2_N40C3' to generate pET32a$\mathrm{CylR}_{N 40 \mathrm{C}}$ and with the mutagenic primers CylR2_T55C5' and CylR2_T55C3' to generate pET32a-CylR2 $2_{T 55 C}$ (see Tables 2.3 and 2.2.

To attach an ATCUN-motif to CylR2, pET32a-CylR2 was amplified by PCR (see 3.1.4 with the primers MGSHG-CylR2_5' and MGSHG-CylR2_3' (see Table 2.3). The purified PCR-product was ligated between the NdeI and XhoI sites of the pET32a expression vector to construct pET32a-MGSHG-CylR2 (see Table 2.2). The codon for serine within MGSHG was deleted by mutagenesis (see 3.1.5 with the primers ATCUN-CylR2_5' and ATCUN-CylR2_3' to generate pET32a-MGHG-CylR2 (see Tables 2.3 and 2.2.

\subsubsection{NMR sample preparation}

Wild type CylR2 and CylR2 mutants were purified as described in 4.2.1. Samples

to determine intermonomer distances were prepared by dissolving ${ }^{15} \mathrm{~N}$-labeled wild type $\left({ }^{15} \mathrm{~N}\right.$-wt) and unlabeled mutant CylR2 (mut) in $8 \mathrm{M}$ urea and mixing them in a 1:1 molar ratio. Samples containing single cysteine mutants were refolded by dialysis against NMR buffer with $600 \mathrm{mM} \mathrm{NaCl}$ and $5 \mathrm{mM}$ DTT whereas samples containing the ATCUN-motif were refolded by dialysis against $50 \mathrm{mM}$ MOPS pH 7.0 and $600 \mathrm{mM}$ $\mathrm{NaCl}$. Directly before labeling with MTSL (see 3.3.12.1), DTT was removed via a 
PD-10 column from the buffer for single cysteine mutants. MTSL-labeled samples to measure intermonomer distances are referred to as 1:1-mixed ${ }^{15} \mathrm{~N}$-wt/mut* sample. Following NMR analysis of the paramagnetic oxidized state of the proteins, a 2-3 molar excess of $200 \mathrm{mM}$ ascorbic acid dissolved in NMR buffer was added and NMR spectra were acquired for the resulting diamagnetic reduced state of the proteins $(1: 1-$ mixed ${ }^{15} \mathrm{~N}-\mathrm{wt} / \mathrm{mut}$ ). For ATCUN-CylR2 (see 3.3.12.2), NMR spectra were measured in the absence and presence of $\mathrm{Cu}^{2+}$.

\subsubsection{NMR spectroscopy}

$2 \mathrm{D}{ }^{15} \mathrm{~N}-{ }^{1} \mathrm{H}-\mathrm{HSQC}$ and ${ }^{15} \mathrm{~N} \mathrm{~T}_{2}$ experiments (see 3.5.4.2) for site-directed spin-labeling studies were performed at $298 \mathrm{~K}$ and $700 \mathrm{MHz}$ with NMR samples containing 0.4$0.8 \mathrm{mM}$ CylR2. For structure determination, a 3D $\left[{ }^{15} \mathrm{~N},{ }^{1} \mathrm{H}\right]$ NOESY-HSQC and a $\left[{ }^{13} \mathrm{C},{ }^{1} \mathrm{H}\right]$ NOESY-HSQC with a mixing time of $120 \mathrm{~ms}$ were measured at $600 \mathrm{MHz}$. All experiments for the solution structure determination of CylR2 are summarized in the Appendix Table B.1.

\subsubsection{Monomer structure calculation}

The monomer NMR structure ensemble was calculated as described in 3.6.1. The $98.8 \%$ complete resonance assignment (see 4.3.3), torsion angle restraints as predicted from chemical shifts with TALOS [52] and the peaklists of the $3 \mathrm{D}\left[{ }^{15} \mathrm{~N},{ }^{1} \mathrm{H}\right]$ and the $\left[{ }^{13} \mathrm{C},{ }^{1} \mathrm{H}\right]$ NOESY-HSQC were used as input for CYANA.

A monomeric MTSL-modified CylR2-mutant structure was calculated as the wild type monomer structure. Thereby the wild type structure was used as the starting structure and intramolecular distances derived from PRE plus $5 \AA$ (see 3.5.7) were used as additional upper limit restraints. For peaks broadened beyond detection for the paramagnetic sample, the upper distance limit was assigned to $12 \AA$. To avoid inclusion of wrong distances into the structure calculation, intramolecular PRE distances have only been used for those residues without an intensity difference in the experiments to determine intermonomer distances. 


\subsubsection{Intermonomer distances}

Intermonomer distances were calculated from PRE (see 3.5.7). For peaks broadened beyond detection for the paramagnetic sample, distances were assigned to $7 \pm 5 \AA$. To calculate intermonomer distances for peaks that did not show a chemical shift difference between homodimeric wild type and heterodimeric wild type/paramagneticmutant, intensities of the paramagnetic heterodimeric molecule $\mathrm{I}_{\text {para }}$ have been calculated using:

$$
I_{\text {para }}=2\left(I_{\text {para }}-\frac{I_{d i a}}{2}\right),
$$

where $\mathrm{I}_{\text {para }}$ is the intensity in the spectrum of the paramagnetic state and $\mathrm{I}_{d i a}$ is the intensity in the spectrum of the diamagnetic state. Upper and lower long-range distances were set to $\pm 5 \AA$ of the calculated distances. For residues neighboring residues without a PRE effect, no upper limit was used and for residues without a PRE effect, the lower limit was set to $25 \AA$.

Intermolecular NOEs have been manually assigned for 20 peaks of the $\left[{ }^{13} \mathrm{C},{ }^{1} \mathrm{H}\right]$ NOESY-HSQC spectrum which have not been automatically assigned during the monomer structure calculation with CYANA (Appendix Table C.9). For NOE data upper and lower distances were set to $\pm 2 \AA$ of the calculated distances.

\subsubsection{Homodimer structure calculation}

All homodimer calculations (see 3.6.2 were performed with the lowest energy structure of the monomer ensemble. In the starting coordinates, one monomer was translated by $50 \AA$ and oriented differently with respect to the other monomer. Seven different sets of restraints have been used: (a) NOE and RDCs, (b) PRE and RDCs, (c) PRE, NOE and RDCs, (d) PRE, RDCs and symmetry (e) PRE, NOE, RDCs and symmetry, (f) PRE and symmetry and (g) PRE, RDCs and symmetry. Twofold symmetry was implied by distance difference restraints [135]. With the exception of (a) all rigid-body dockings were performed with two monomers which contain MTSL at position N40C and T55C. The intermonomer PRE distances in the rigid-body docking were restrained from the nitrogen of the MTSL ring in one monomer to the amide 
protons of the other monomer. Each monomer in the homodimer structure resulting from the rigid-body minimization was replaced by the refined wild type monomer structure. Intermolecular HN-HN distances $\pm 2 \AA$ from N40 and Thr55 to the other subunit were extracted using MOLMOL and used for the simulated annealing. The rigid-body docking as well as the simulated annealing protocol are provided in the Appendix D.

\subsubsection{Ranking of homodimer models from ab initio docking}

The lowest energy monomer structure was docked to itself using the ClusPro Web server (see 3.6.3). Ranking of the docking results without experimental data about the dimer interface was carried out via prediction of RDCs with PALES (see 3.5.3.3).

\subsection{Results}

\subsubsection{Monomer solution structure}

The solution monomer structure of CylR2 was solved based on a $98.8 \%$ complete chemical shift assignment, 987 interproton distances, 86 dihedral angle restraints and 57 HN-RDCs. The chemical shift assignments can be found in the BMRB under the accession code 6317 and the structural restraints are provided in the Appendix C. Nine medium- and seven long-range ${ }^{13} \mathrm{C}$-distances have been manually assigned to avoid wrong assignments during the automated NOE assignment within CYANA. Thereby convergence was ensured even in the presence of intermolecular NOEs in the peak list. The ensemble of 20 structures determined by CYANA has a backbone rmsd (residues 3-63) of $0.45 \AA$ and an average CYANA target function of $0.84 \AA^{2}$. After the final water refinement with XPLOR-NIH including 57 HN-RDCs, the backbone rmsd (residues 3-63) is slightly increased to $0.60 \AA$, but all restraints are well satisfied (Table 5.1). The structural characteristics have been described in the previous chapter (see 4.3.1). Highest rmsd values within the 20 structure bundle were found for the $\mathrm{N}$ - and C-terminal residues (Figure 5.1B), which also have the highest 
Table 5.1: Statistics for CylR2 monomer solution structure determination.

\begin{tabular}{|c|c|}
\hline \multicolumn{2}{|l|}{ NOE distance restraints: } \\
\hline total & 987 \\
\hline short range $(|i-j| \leq 1)$ & 594 \\
\hline medium range $(1 \leq|\mathrm{i}-\mathrm{j}| \leq 5)$ & 180 \\
\hline long range $(|\mathrm{i}-\mathrm{j}| \geq 5)$ & 213 \\
\hline Dihedral angle restraints & 86 \\
\hline${ }^{15} \mathrm{~N}-{ }^{1} \mathrm{H}$ residual dipolar couplings & 57 \\
\hline \multicolumn{2}{|l|}{ Mean rmsd from experimental restraints } \\
\hline NOE & $0.0048 \AA$ \\
\hline dihedral angles & $0.743 \mathrm{deg}$ \\
\hline \multicolumn{2}{|l|}{ Average number of } \\
\hline NOE violations $\geq 0.5 \AA$ & 0 \\
\hline Dihedral angle violations $\geq 5^{\circ}$ & 0 \\
\hline \multicolumn{2}{|l|}{ Mean rmsd from idealized covalent geometry } \\
\hline Bonds & $0.0097 \AA$ \\
\hline Angles & $1.35 \mathrm{deg}$ \\
\hline Impropers & $1.62 \mathrm{deg}$ \\
\hline \multicolumn{2}{|l|}{ Ramachandran analysis } \\
\hline Most-favored region & $88.5 \%$ \\
\hline Additionally allowed region & $10.3 \%$ \\
\hline Generously allowed region & $1.0 \%$ \\
\hline Disfavoured region & $0.3 \%$ \\
\hline \multicolumn{2}{|l|}{ Rmsd from the mear ${ }^{a}$} \\
\hline Backbone atoms & $0.60 \pm 0.14 \AA$ \\
\hline All heavy atoms & $1.06 \pm 0.12 \AA$ \\
\hline \multicolumn{2}{|l|}{ Rmsd from the X-ray structure ${ }^{a}$} \\
\hline Backbone atoms & $0.89 \AA$ \\
\hline All heavy atoms & $1.88 \AA$ \\
\hline
\end{tabular}

${ }^{a}$ determined for residues 3-63

rmsd values when superimposing the two subunits of the X-ray structure. Overall, there is a good correlation between the rmsd values within the ensemble of 20 NMR structures and the ${ }^{15} \mathrm{~N}$ transverse relaxation times $\left(\mathrm{T}_{2}\right)$ (Figure $5.1 \mathrm{C}$ ). There are no major differences between the X-ray structure (Figure 4.4) and the NMR structure ensemble. A superposition of the mean NMR structure and the X-ray structure is shown in Figure 5.1A. The average backbone rmsd (residues 3-63) between the mean NMR-structure and the X-ray structure is $0.89 \AA$. Differences within the ensemble and to the X-ray structure are similar with the exception of the region around residue Ser42 and residue Glu11, where the backbone rmsd to the X-ray structure is around $1 \AA$ higher (Figure 5.1B). For the region around residue Ser42, this can be explained by the results from the steady-state heteronuclear ${ }^{15} \mathrm{~N}-{ }^{1} \mathrm{H}$ NOE (Figure 4.10) and by the $\mathrm{T}_{2}$ times (Figure $5.1 \mathrm{C}$ ), which reveal Ser42 as the most flexible residue. 


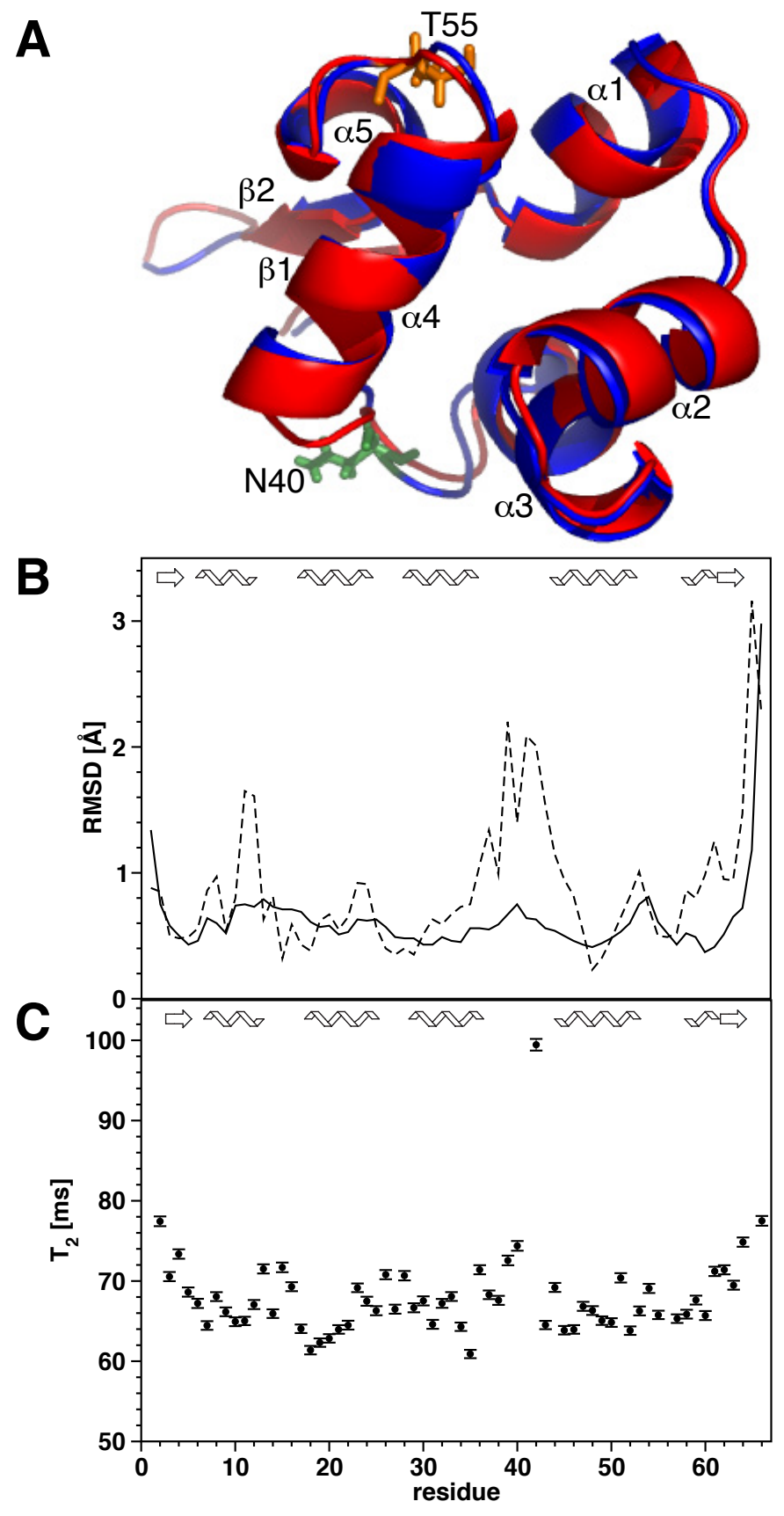

Figure 5.1: Monomer solution structure of CylR2. (A) Mean structure of the NMR ensemble (blue) superimposed on a monomer from the X-ray structure (red). Side chains of Asn40 and Thr55 which are mutated to cysteine for dimer structure determination are shown in green and orange, respectively. (B) Average backbone rmsd per residue between the mean NMR-structure and the 20 NMR-structures (solid line) and the X-ray structure (dashed line). (C) Measured $\mathrm{T}_{2}$ values versus the residue number. Secondary structure elements are indicated.

\subsubsection{Mutagenesis and spin-labeling}

SDSL with MTSL was applied to determine distances from one subunit of CylR2 to the other (Figure 5.2). Therefore single-cysteine residues were introduced into the cysteine-free wild type CylR2 (see 5.2.1). Conservative mutation sites were chosen at position Asn40 and Thr55 in the middle of loop regions on opposite sites of the monomer structure. By comparing amide proton and nitrogen chemical shifts as well 


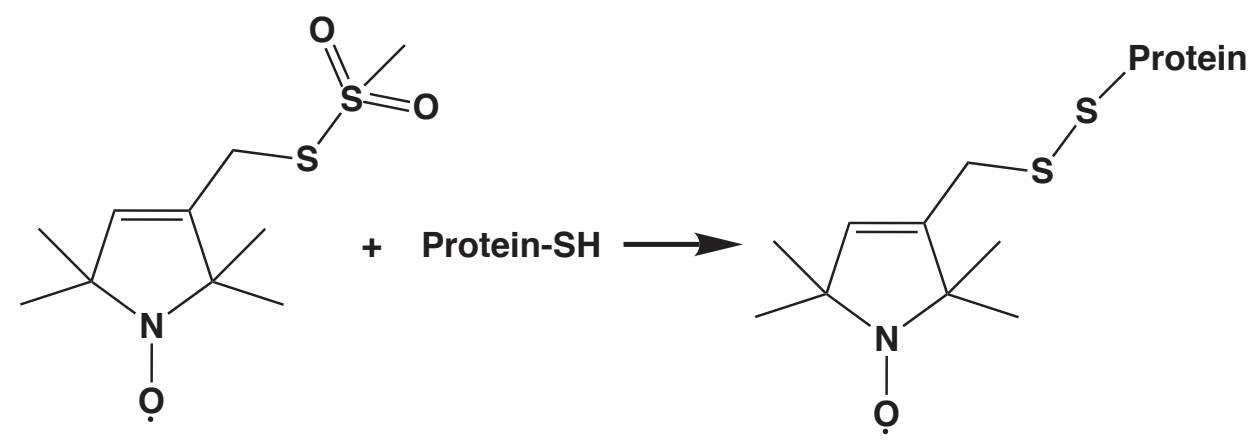

Figure 5.2: Structure of the MTSL spin label and reaction with the -SH group of a protein.

as rotating frame relaxation times $\left(\mathrm{T}_{1 \rho}\right)$ between wild type and mutant, it was confirmed that introduction of a Cys residue at these two positions does not significantly affect the protein structure (Figure 5.3). Chemical shift differences are smaller than

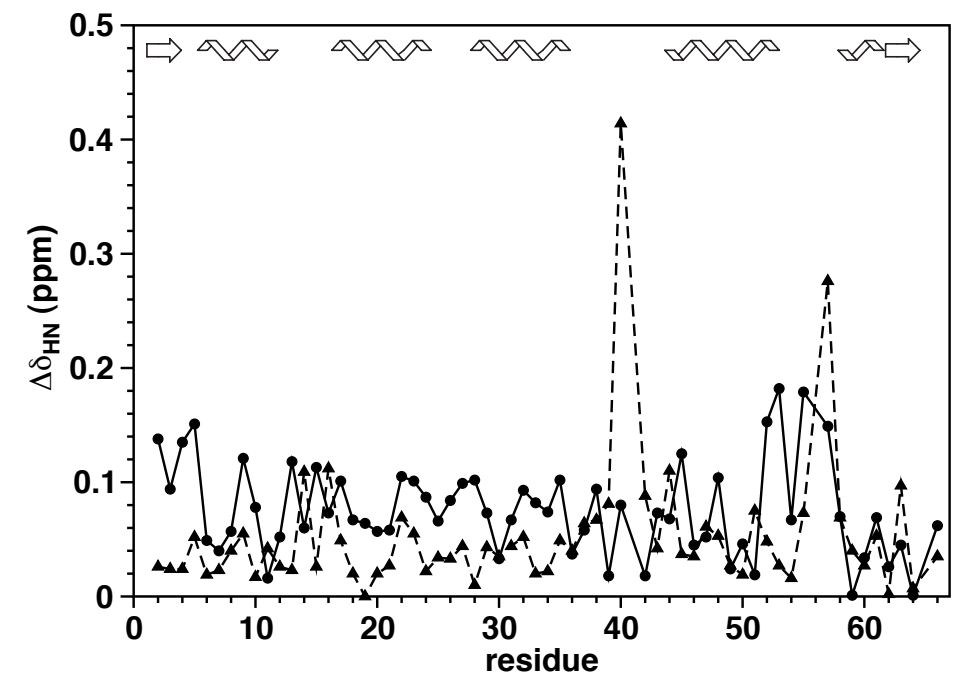

Figure 5.3: Chemical shift changes of backbone amides due to mutation of Asn40 (triangles and dashed line) and Thr55 (circles and solid line) to cysteine. Secondary structure elements are indicated.

$0.1 \mathrm{ppm}$ with the exception of Leu57 for CylR $2_{N 40 C}$ and residues around the mutation site. Residues in the $\mathrm{N}$-terminal region of CylR2 $2_{T 55 C}$ show $\Delta \delta_{H N}$ values between 0.1 and $0.16 \mathrm{ppm}$. Additionally, unchanged $\mathrm{T}_{1 \rho}$ values indicate an unaltered oligomerization state. Subsequent modification of the single cysteine mutants with the spin label MTSL (Figure 5.2 allows to measure distances within a range of $\leq 25-30 \AA$ from the spin label.

${ }^{15} \mathrm{~N}-\mathrm{HSQC}$ spectra have been recorded for the para- and diamagnetic form of each CylR2-mutant. These spectra were measured for two different samples of each CylR2mutant: a pure ${ }^{15} \mathrm{~N}$ - and spin-labeled mutant $\left({ }^{15} \mathrm{~N}\right.$-mut $\left.\left({ }^{\star}\right)\right)$ and a 1:1 mixture of 
${ }^{15} \mathrm{~N}$-labeled wild type and spin-labeled mutant $\left(1: 1\right.$-mixed ${ }^{15} \mathrm{~N}$-wt/mut $\left.\left({ }^{\star}\right)\right)$. Paramagnetic states are indicated by a star. Each 1:1-mixed ${ }^{15} \mathrm{~N}$-wt/mut $\left({ }^{\star}\right)$ sample is composed of three different dimers: $50 \%{ }^{15} \mathrm{~N}$-wild type/spin-labeled mutant $\left({ }^{15} \mathrm{~N}\right.$ wt/mut( $\left.\left(^{\star}\right)\right), 25 \%{ }^{15} \mathrm{~N}$-wild type $\left({ }^{15} \mathrm{~N}\right.$-wt $\left./{ }^{15} \mathrm{~N}-\mathrm{wt}\right)$ and $25 \%$ spin-labeled mutant $\left(\operatorname{mut}\left(^{\star}\right) / \operatorname{mut}\left({ }^{\star}\right)\right)\left(\right.$ Figure 5.4). Both, ${ }^{15} \mathrm{~N}-\mathrm{wt} / \mathrm{mut}^{\star}$ heterodimer and ${ }^{15} \mathrm{~N}-\mathrm{wt} /{ }^{15} \mathrm{~N}-\mathrm{wt}$

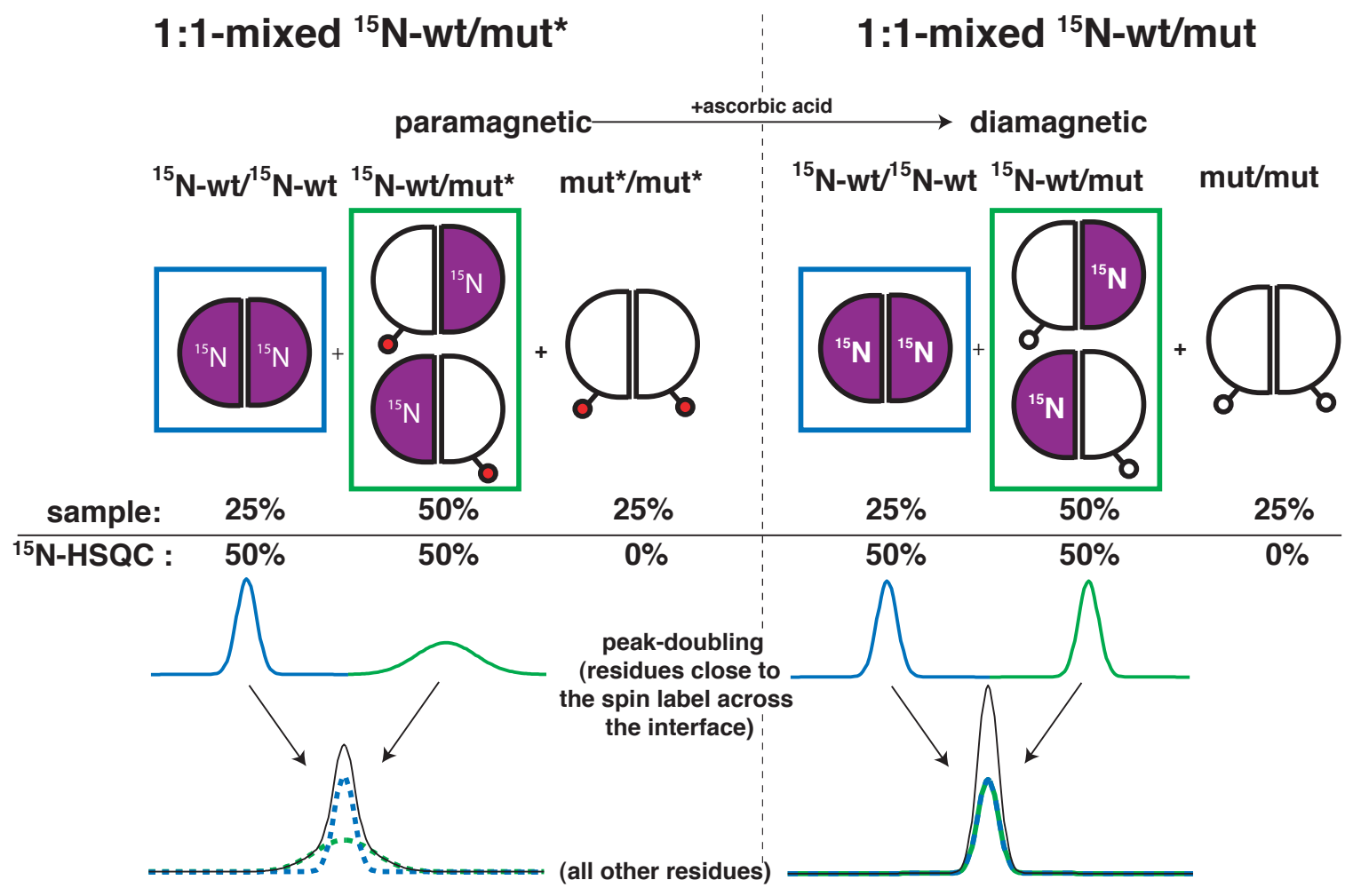

Figure 5.4: Overall strategy to derive intermonomer distances from PRE in homodimers. The paramagnetic $\left({ }^{\star}\right)$ and the diamagnetic sample $\left(\right.$ no $\left.{ }^{*}\right)$ are shown on the left and right hand side of the figure, respectively. The 1:1-mixed samples are composed of equal amounts of ${ }^{15} \mathrm{~N}$-labeled wild type $\left({ }^{15} \mathrm{~N}-\mathrm{wt}\right.$, violet) and of paramagnetic mutant $\left(\operatorname{mut}\left(^{\star}\right)\right.$, white) monomers. The monomers combine into three distinct dimerization pairs: $25 \%{ }^{15} \mathrm{~N}$-wt $/{ }^{15} \mathrm{~N}$-wt (blue), $50 \%{ }^{15} \mathrm{~N}$-wt $/$ mut $\left({ }^{\star}\right.$ )(green) and $25 \%$ $\operatorname{mut}\left({ }^{\star}\right) / \operatorname{mut}\left(^{\star}\right)$. The former two species contribute equally to the NMR signal while the latter is undetected. For a few residues close to the para- or diamagnetic tag across the dimer interface, the chemical shift can be distinguished (peak-doubling), while for all other residues, the ${ }^{15} \mathrm{~N}$-wt $/{ }^{15} \mathrm{~N}-\mathrm{wt}$ and ${ }^{15} \mathrm{~N}-\mathrm{wt} / \mathrm{mut}\left({ }^{\star}\right)$ peaks overlap. The PRE distance is derived from the peak intensity ratio $\left(\mathrm{I}_{\text {para }} / \mathrm{I}_{\text {dia }}\right)$ obtained from the paramagnetic and diamagnetic lines (green lines). For the overlapped case, $\mathrm{I}_{\text {para }}$ can be obtained by substracting $\mathrm{I}_{d i a} / 2$ according to formula 5.1. For MTSL, the diamagnetic sample can easily be obtained from the paramagnetic sample by ascorbic acid reduction.

homodimer, contribute $50 \%$ to the NMR signal. For CylR2 $2_{T 55 C}$, an overlay of the ${ }^{15} \mathrm{~N}-\mathrm{HSQC}$ spectra of the para- and diamagnetic state for ${ }^{15} \mathrm{~N}-\mathrm{mut}\left({ }^{\star}\right)$ and for 1:1- 
mixed ${ }^{15} \mathrm{~N}-\mathrm{wt} / \operatorname{mut}\left({ }^{\star}\right)$ are shown in Figure 5.5A and in Figure 5.5B, respectively. ${ }^{15} \mathrm{~N}-\mathrm{HSQC}$ experiments measured for ${ }^{15} \mathrm{~N}-\mathrm{mut}\left({ }^{\star}\right)$ were used to accurately define the

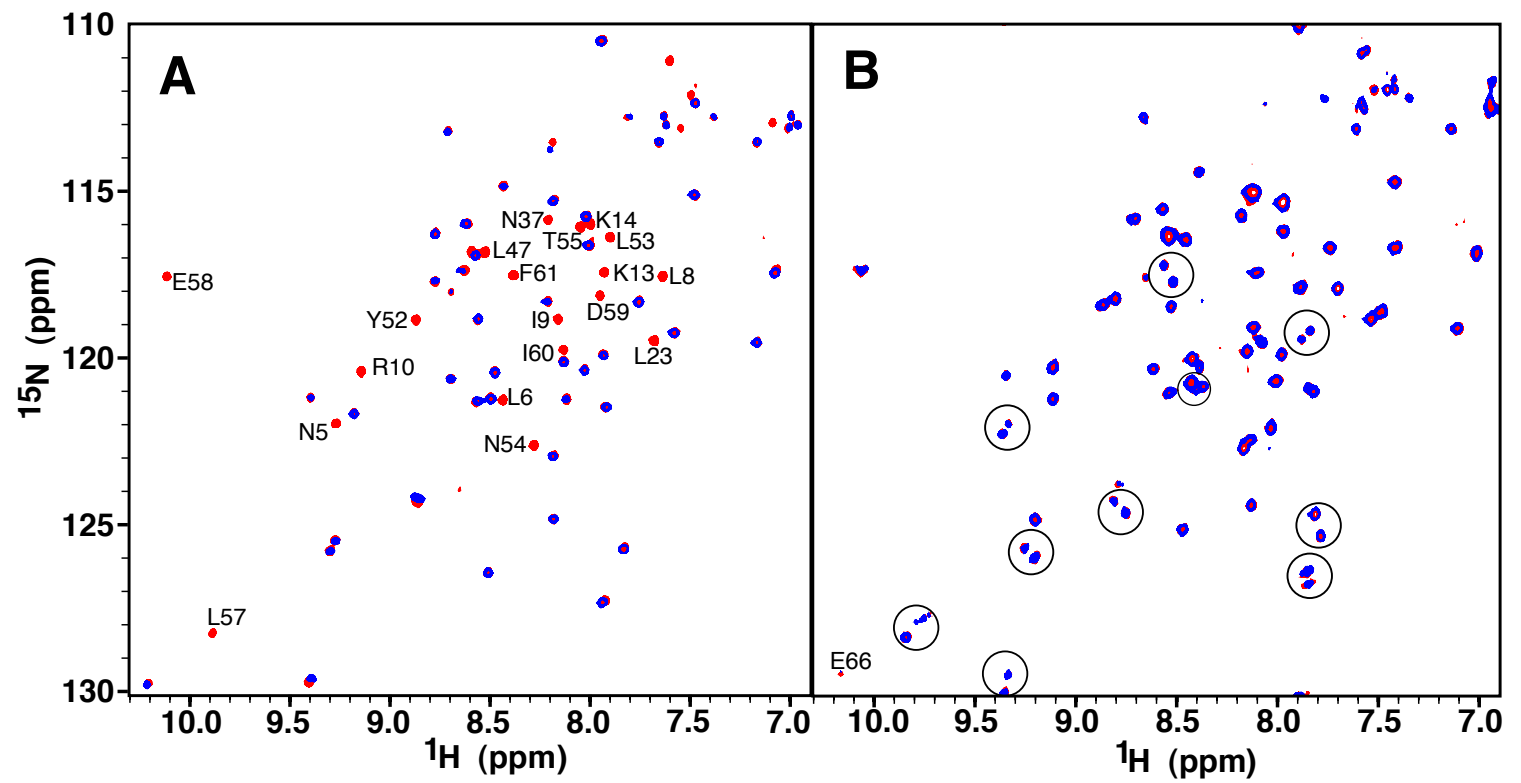

Figure 5.5: Overlay of ${ }^{15} \mathrm{~N}-\mathrm{HSQC}$ spectra of paramagnetic (blue) and diamagnetic (red) forms of ${ }^{15} \mathrm{~N}-\mathrm{CylR} 2{ }_{T 55 C}(\mathrm{~A})$ and the $1: 1$ mixture of ${ }^{15} \mathrm{~N}-\mathrm{CylR} 2$ and CylR2 $2_{T 55 C}$ (B). Residues that disappeared in the paramagnetic state are labeled and doubled peaks in (B) are indicated by ellipses.

two positions of the spin label MTSL within the monomer structure.

Intermonomer distances are derived from peak intensities of HSQC spectra for the paramagnetic and diamagnetic 1:1-mixed ${ }^{15} \mathrm{~N}-\mathrm{wt} / \mathrm{mut}\left({ }^{\star}\right)$ sample. Overall reduction of signal intensities for the 1:1-mixed ${ }^{15} \mathrm{~N}-\mathrm{wt} / \mathrm{mut}^{\star}$ sample is significantly less important than for the ${ }^{15} \mathrm{~N}$-mut ${ }^{\star}$ sample due to larger distances of the backbone amide $\mathrm{HN}$ group to the spin label. Indeed, whereas around one third of all backbone amide signals disappeared for the ${ }^{15} \mathrm{~N}-$ mut $^{\star}$ sample, only a single signal (Glu66) was not observed for the 1:1-mixed ${ }^{15} \mathrm{~N}$-wt $/$ mut* sample. For the 1:1-mixed ${ }^{15} \mathrm{~N}$-wt/mut( $\left(^{\star}\right)$ sample, peak-doubling is observed for 10 of the 62 backbone amide signals because of slightly different chemical shifts for the wild type monomer residues within the homodimer and the wild type monomer residues that are close to the mutation site within the heterodimer (Figure $5.5 \mathrm{~B})$. 


\subsubsection{Long-range distances from PRE}

The approach by Battiste and Wagner was used to derive long-range distances from PRE [87] and has been described in 3.5.7 and 5.2.5. For CylR2, the correlation time $\tau$ for the electron-nuclear interaction was estimated as 6 ns from Stokes' law 3.17 under the assumption that $\tau$ is equal to $\tau_{c}$. Intramonomer distances were determined for residues whose signals were not broadened for the $1: 1$-mixed ${ }^{15} \mathrm{~N}$-wt/mut ${ }^{\star}$ sample. The resulting 24 and 31 intramonomer distances for CylR $2_{N 40 C}$ and $\mathrm{CylR} 2_{T 55 C}$, respectively, were used to calculate the average position of the spin label MTSL at position N40C and T55C within the monomer structure. These distances were added as restraints to the protocol described in paragraph 5.2.4 and the monomer structure containing MTSL at position N40C and T55C was calculated using the wild type structure as the start structure. After the water refinement, the mean monomer structure of the 20 lowest energy structures was calculated using MOLMOL and used for the PRE-based rigid-body docking (see 5.3.4).

Intermonomer distances for the residues with peak-doubling for the $1: 1$-mixed ${ }^{15} \mathrm{~N}$ $\mathrm{wt} / \operatorname{mut}\left(^{\star}\right)$ sample were derived like the intramonomer distances in the ${ }^{15} \mathrm{~N}$-mut $\left({ }^{\star}\right)$ sample. The peak corresponding to the heterodimeric ${ }^{15} \mathrm{~N}-\mathrm{wt} / \mathrm{mut}\left({ }^{\star}\right)$ mutant was identified as the signal which is shifted compared to the ${ }^{15} \mathrm{~N}-\mathrm{HSQC}$ of wild type CylR2. For the residues without peak-doubling, the intensity corresponding to PRE across the dimer interface $\left(\mathrm{I}_{\text {para }}\right)$ for all molecules of the $1: 1$-mixed ${ }^{15} \mathrm{~N}$-wt/mut $\left({ }^{\star}\right)$ sample was calculated according to formula 5.1. This formula is valid under the assumption that the sample contains $50 \%{ }^{15} \mathrm{~N}$-wt $/$ mut $\left(^{\star}\right)$ and $25 \%{ }^{15} \mathrm{~N}-w t /{ }^{15} \mathrm{~N}-w t$. From the ${ }^{15} \mathrm{~N}-\mathrm{HSQC}$ peak intensities $\left(\mathrm{I}_{\text {para }}\right.$ ) is subtracted half the reference ${ }^{15} \mathrm{~N}-\mathrm{HSQC}$ peak intensity $\left(\mathrm{I}_{\text {dia }}\right)$, the latter corresponding to the contribution of the ${ }^{15} \mathrm{~N}-\mathrm{wt} /{ }^{15} \mathrm{~N}-\mathrm{wt}$ (Figure 5.4). The calculated intermonomer distances for the spin label at residue 40 and 55 are listed in the Appendix Table C.8.

The accuracy of the intermonomer PRE distances was assessed by comparing the calculated distances with the theoretically expected distances for the X-ray structure (Figure 5.6A). Only 6 out of 103 experimental PRE distances for each subunit are 


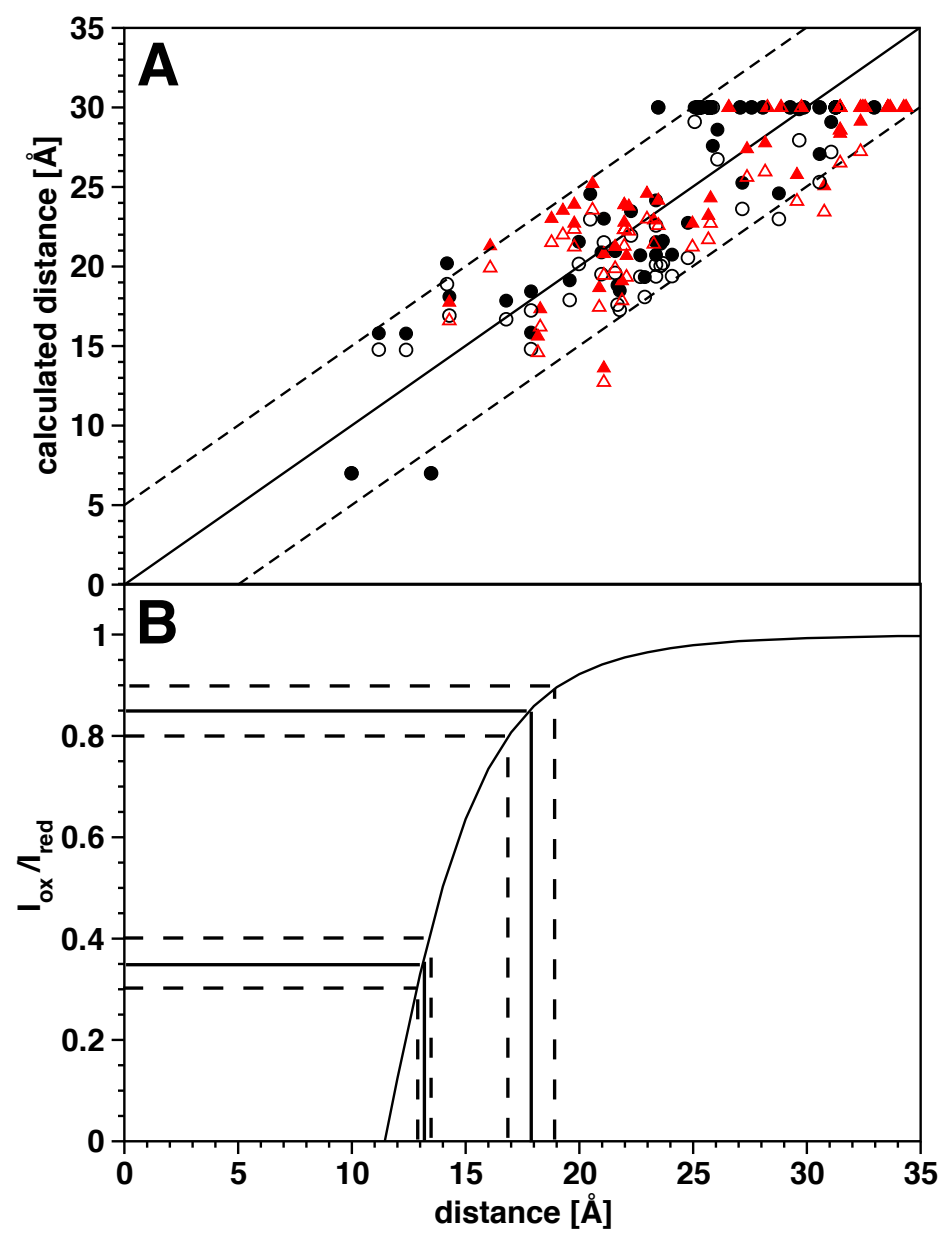

Figure 5.6: (A) Theoretically expected distances from the X-ray structure versus distances calculated from PRE data. The solid line indicates optimal correlation between experimental and expected distances and the dashed lines mark the $\pm 5 \AA$ error bounds. Distances calculated with the spin label at position N40C and T55C are shown as black circles and red triangles, respectively. Distances calculated with a $\tau$ of $6 \mathrm{~ns}$ and $4 \mathrm{~ns}$ are indicated as filled and empty symbols, respectively. (B) Measured intensity ratio plotted as a function of the calculated distance. The dashed lines show that for an intensity ratio of $0.85 \pm 0.05$ the uncertainty of the distance is approximately four times larger than for an intensity ratio of $0.35 \pm 0.05$.

found to deviate by more than $\pm 5 \AA$ from the theoretically expected distances. The $\pm 5 \AA$ error bounds are based on different assumptions and sources of experimental errors. Amide proton $\mathrm{T}_{2}$ relaxation times were approximated by the experimental amide nitrogen $\mathrm{T}_{2}$ relaxation times (Figure 5.1 $\mathrm{C}$ ) and $\tau$ was assumed as essentially equal to $\tau_{c}$. Because of the $\mathrm{r}^{-6}$ dependence of the PRE, errors in the $\mathrm{T}_{2}$ relaxation time and $\tau$ (Figure 5.6A) have a very small effect on the calculated distance. Positional averaging of the flexible nitroxide side chain of MTSL is another source of errors. However, these errors were minimized by calculating the average position of the spin label (see 5.2.4). Errors in the determination of protein concentration and interference of MTSL during refolding the dimer from $8 \mathrm{M}$ urea (see 5.2.2) may result in a deviation from the $50 \%$ contribution of the ${ }^{15} \mathrm{~N}-\mathrm{wt} / \mathrm{mut}\left({ }^{\star}\right)$ and the ${ }^{15} \mathrm{~N}-\mathrm{wt} /{ }^{15} \mathrm{~N}-$ wt to the ${ }^{15} \mathrm{~N}-\mathrm{HSQC}$ signal which was assumed to calculate intermonomer distances 
for non-doubled peaks. In addition, the error is larger for intermonomer than for intramonomer distances as the measured distances are in general larger (Figure 5.6B). MTSL attached to N40C is slightly closer to the dimer interface than MTSL attached to T55C (Figure 5.7). The average theoretical distance from the nitrogen of the MTSL ring to amide protons across the interface is $\sim 3.5 \AA$ smaller for the spin-labeled N40C than for spin-labeled T55C.

\subsubsection{Homodimeric solution structure}

Accurate docking of two proteins requires both orientational and translational information. Measurement of HN-RDCs in a single alignment medium, leads to four possible orientations of one subunit of CylR2 with respect to the second subunit, when the alignment tensor is not axially symmetric. The four-fold degeneracy is resolved by intermolecular PRE distances or intermolecular NOE data. PRE distances have the same distance dependence as NOE data and so the XPLOR-protocol for rapid protein-protein docking on the basis of RDCs and intermolecular NOEs and PRE data can be used with the same standard energy function [89].

The performance of this PRE-based rigid-body docking has been tested for the 57 HN-RDCs for each monomer in combination with two different sets of experimental intermolecular restraints: PRE data (Table 5.2a) and PRE together with NOE data (Table 5.2b). In addition, the docking was tested with symmetry distance restraints to imply twofold symmetry [135]. These symmetry distance restraints were either used together with RDC data (Table $5.2 \mathrm{c}$ and d) or used to replace RDC data (Table 5.2 and $\mathrm{f}$ ). For comparison, the same rigid-body protocol was run with RDCs and intermolecular NOEs as the only experimental restraints (Table 5.2 $\mathrm{g}$ ). These seven rigid-body dockings with different sets of restraints resulted in structures with the same monomer-to-monomer orientation. After simulated annealing with all coordinates fixed with the exception of the side-chain atoms of residues contributing to the dimer interface and refinement in explicit solvent, the seven ensembles, composed of 20 structures, have a backbone rmsd (residues 3-63) between $0.54 \AA$ and 
Table 5.2: Statistics for CylR2 dimer solution structure determination.

\begin{tabular}{|c|c|c|c|c|c|c|c|}
\hline & (a) & (b) & (c) & (d) & (e) & (f) & $(\mathrm{g})$ \\
\hline \multicolumn{8}{|l|}{ intermolecular distance restraints: } \\
\hline symmetry & - & - & yes & yes & yes & yes & - \\
\hline PRE & 206 & 206 & 206 & 206 & 206 & 206 & - \\
\hline NOE & - & 48 & - & 48 & - & 48 & 48 \\
\hline HN-RDCs & 114 & 114 & - & - & 114 & 114 & 114 \\
\hline \multicolumn{8}{|l|}{ Ramachandran analysis: } \\
\hline Most-favored region (\%) & 88.3 & 88.0 & 88.0 & 88.2 & 88.5 & 88.7 & 87.7 \\
\hline Additionally allowed region (\%) & 10.4 & 10.8 & 10.5 & 10.3 & 9.4 & 9.9 & 11.2 \\
\hline Generously allowed region (\%) & 1.0 & 1.0 & 1.2 & 1.3 & 2.0 & 1.3 & 1.0 \\
\hline Disfavoured region (\%) & 0.2 & 0.2 & 0.3 & 0.2 & 0.2 & 0.2 & 0.1 \\
\hline \multicolumn{8}{|l|}{ Rmsd from the mear ${ }^{b}$} \\
\hline Backbone atoms (A) & $\begin{array}{c}0.59 \pm \\
0.11\end{array}$ & $\begin{array}{c}0.59 \pm \\
0.08\end{array}$ & $\begin{array}{c}0.59 \pm \\
0.11\end{array}$ & $\begin{array}{c}0.61 \pm \\
0.07\end{array}$ & $\begin{array}{c}0.54 \pm \\
0.09\end{array}$ & $\begin{array}{c}0.59 \pm \\
0.10\end{array}$ & $\begin{array}{c}0.65 \pm \\
0.13\end{array}$ \\
\hline All heavy atoms $(\AA)$ & $\begin{array}{c}0.90 \pm \\
0.10\end{array}$ & $\begin{array}{c}0.89 \pm \\
0.09\end{array}$ & $\begin{array}{c}0.88 \pm \\
0.11\end{array}$ & $\begin{array}{c}0.91 \pm \\
0.09\end{array}$ & $\begin{array}{c}0.86 \pm \\
0.08\end{array}$ & $\begin{array}{c}0.89 \pm \\
0.10\end{array}$ & $\begin{array}{c}0.94 \pm \\
0.13\end{array}$ \\
\hline \multicolumn{8}{|l|}{ Rmsd from the X-ray structure ${ }^{b}$ : } \\
\hline Backbone atoms $(\AA)$ & 1.15 & 1.14 & 1.18 & 1.22 & 1.99 & 1.23 & 2.14 \\
\hline All heavy atoms $(\AA)$ & 2.08 & 2.01 & 2.10 & 2.07 & 2.79 & 2.10 & 2.92 \\
\hline \multicolumn{8}{|l|}{$\begin{array}{l}\text { intermonomer rmsd: } \\
\text { mean NMR structure: }\end{array}$} \\
\hline Backbone atoms $(\AA)$ & 0.29 & 0.37 & 0.34 & 0.25 & 0.52 & 0.33 & 0.33 \\
\hline All heavy atoms $(\AA)$ & 0.72 & 0.75 & 0.66 & 0.88 & 0.81 & 0.78 & 0.76 \\
\hline \multicolumn{8}{|l|}{ NMR ensemble (20 structures): } \\
\hline Backbone atoms $(\AA)$ & $\begin{array}{c}0.88 \pm \\
0.28\end{array}$ & $\begin{array}{c}0.79 \pm \\
0.27\end{array}$ & $\begin{array}{c}0.84 \pm \\
0.36\end{array}$ & $\begin{array}{c}0.94 \pm \\
0.28\end{array}$ & $\begin{array}{c}0.73 \pm \\
0.22\end{array}$ & $\begin{array}{c}0.92 \pm \\
0.34\end{array}$ & $\begin{array}{c}0.83 \pm \\
0.42\end{array}$ \\
\hline All heavy atoms $(\AA)$ & $\begin{array}{c}1.49 \pm \\
0.25\end{array}$ & $\begin{array}{c}1.44 \pm \\
0.26\end{array}$ & $\begin{array}{c}1.47 \pm \\
0.4\end{array}$ & $\begin{array}{c}1.54 \pm \\
0.3\end{array}$ & $\begin{array}{c}1.34 \pm \\
0.3\end{array}$ & $\begin{array}{c}1.54 \pm \\
0.32\end{array}$ & $\begin{array}{c}1.43 \pm \\
0.4\end{array}$ \\
\hline intermolecular energy $(\mathrm{kcal} / \mathrm{mol})$ & -1609.6 & -1343.5 & -1662.2 & -1309.7 & -1527.9 & -1422.1 & -1436.4 \\
\hline
\end{tabular}

${ }^{a}$ Restrains distance differences to imply twofold symmetry [135]

${ }^{b}$ determined for residues 3-63 of each monomer

$0.65 \AA$ (Table 5.2). The 10 lowest energy structures of the NMR ensemble calculated with HN-RDCs and PREs is shown in Figure 5.7. The small backbone intermonomer

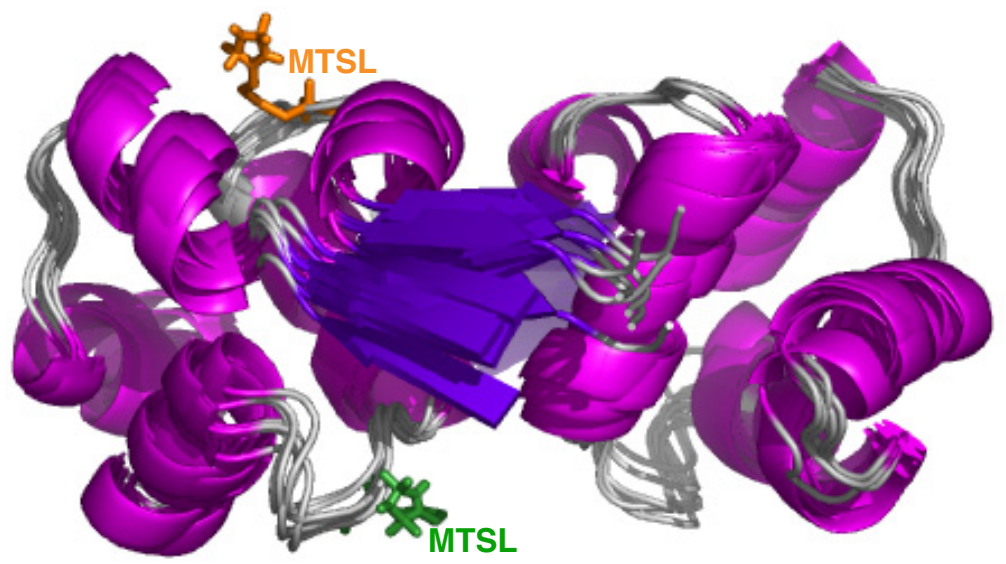

Figure 5.7: Homodimeric solution structure of CylR2. Superposition of the 10 NMR structures with the lowest energy. Helices and $\beta$-strands are shown in magenta and violet, respectively. The calculated average position of MTSL attached to either position N40C (green) or position T55C (orange) is indicated for the left subunit. 
rmsd values for the mean structure $(<0.6 \AA)$ and all structures of the NMR ensemble $(<1 \AA)$ indicate the high symmetry of the homodimers and are similar to the backbone intermonomer rmsd value of $0.75 \AA$ between the monomers of the X-ray structures. As expected, the orientation of the monomers within the homodimer can be restrained by experimental RDCs or a symmetry function [135] (Table 5.2). Therefore, in this case, the experimental RDCs were not absolutely required. However, considering the backbone rmsd (residues 3-63) to the X-ray structure, the experimental HN-RDCs perform significantly better in the absence of intermolecular NOEs $(1.15 \AA$ versus $1.99 \AA)$ and slightly better in the presence of intermolecular NOEs $(1.14 \AA$ versus $1.22 \AA)$. This can be explained by the fact that with the HN-RDCs, one of the principal axis of the alignment tensor must be parallel and the other two orthogonal to the twofold axis [136] while the symmetry function restrains symmetry-related intermonomer distance differences to 0 without restraining the two axis. As the HN-RDCs restrain the symmetry more stringently than the symmetry function, the docking results are not improved when HN-RDCs are used together with the symmetry function. This is demonstrated by an almost unchanged backbone rmsd (residues 3-63) to the X-ray structure independent of the use of intermolecular NOEs for the calculations (Table 5.2a,b,e and f).

Decreasing the error bounds to $\pm 4 \AA$ resulted in an increased rmsd and in a larger number of violated intermolecular restraints. Changing $\tau$ from 4 ns to 6 ns did not change the docking results because of the small difference in the calculated distances (Figure 5.6). Due to the different sources of errors for the PRE-derived distances (see 5.3.3), the docking did not converge to a unique solution when only intermolecular distances for one spin label position together with the HN-RDCs were used.

The backbone rmsd (residues 3-63) between the mean NMR dimer structure generated using intermolecular PRE distances as the only intermolecular restraints together with HN-RDCs and the X-ray structure is $1.15 \AA$ (Table 5.2a). The precision of the structure was only insignificantly improved by addition of 24 manually assigned intermolecular NOEs as indicated by the backbone rmsd (residues 3-63) to the X-ray structure of $1.14 \AA$ (Table 5.2b). Moreover, the combination of HN-RDCs and inter- 
molecular NOEs is not sufficient to define the dimer structure as accurately as the intermolecular PREs in combination with HN-RDCs. This is indicated by a backbone rmsd of $2.14 \AA$ relative to the X-ray structure (Table $5.2 \mathrm{~g}$ ).

There are small differences between the orientation of the two monomers in the NMR and the X-ray structure as the rmsd values are overall higher for the dimer than for the monomer (Figures $5.1 \mathrm{~B}$ and $5.8 \mathrm{~B}$ ). Most notable are the differences for the

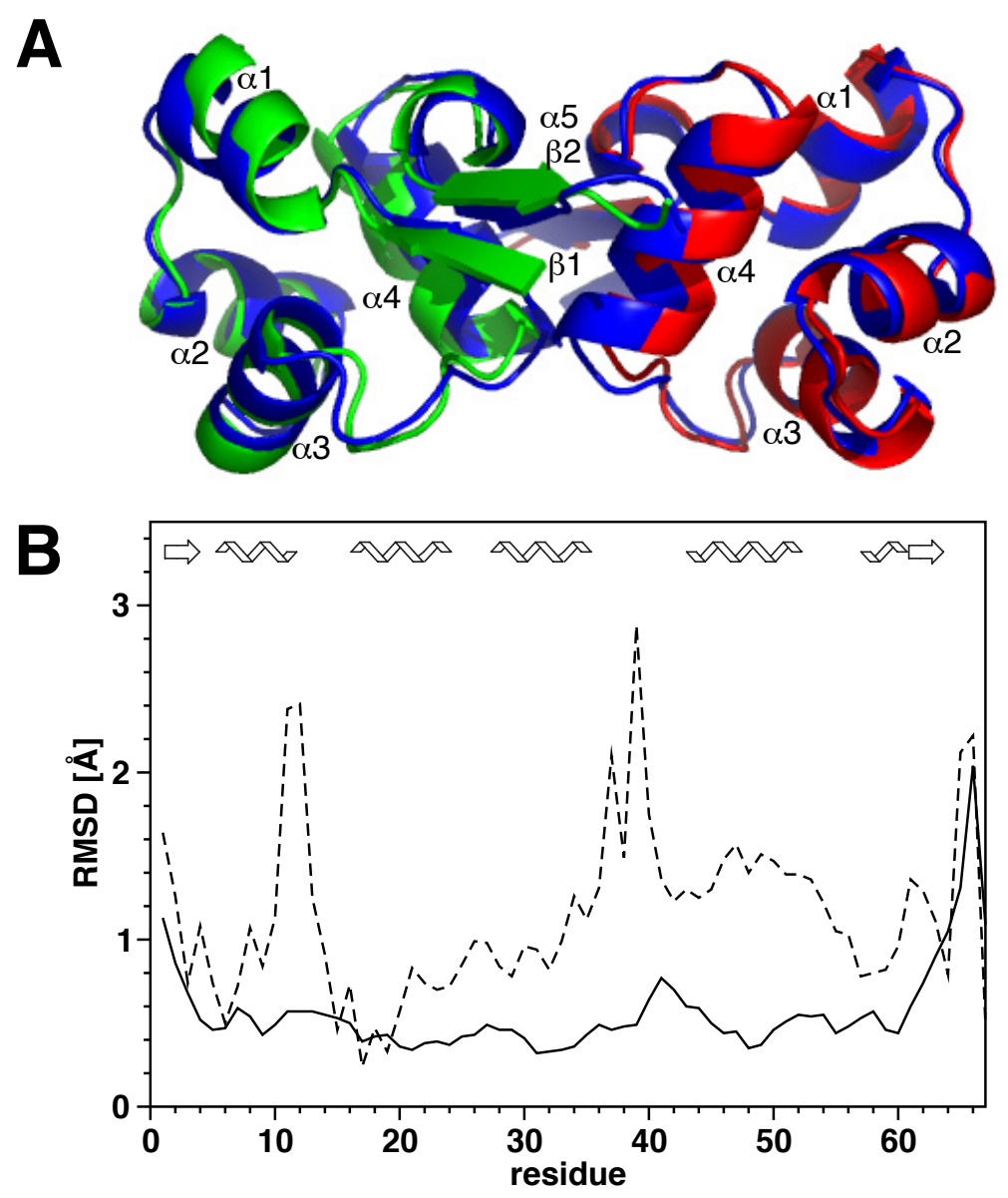

Figure 5.8: Comparison of the solution and crystal structure of CylR2. Secondary structure elements are indicated. (A) Mean structure of the NMR ensemble (blue) superimposed on the X-ray structure (green/red). (B) Average backbone rmsd per residue between the mean NMR-structure and the 20 NMR-structures (solid line) and the X-ray structure (dashed line).

longest helix $\alpha 4$ (residues 43-52) that contributes strongly to the dimer interface and the loop connecting helix $\alpha 3$ and $\alpha 4$ involved in the DNA binding. Within this loop the flexible residue Ser42 is found. Structural flexibility in this region is probably important for DNA binding (see 4.3.4 and 4.3.5). 


\subsubsection{The ATCUN-motif as a paramagnetic position}

In order to obtain a paramagnetic probe at a third position and to use another paramagnetic probe, the three-residue $\mathrm{Cu}^{2+}$-binding ATCUN-motif was attached via a glycine-linker to the N-terminal residue of wild type CylR2 (see 5.2.1) [130]. Because a histidine residue at position three is essential for the high affinity $\mathrm{Cu}^{2+}$ binding, the endogenous cleavage of the N-terminal translation initiator methionine in E. coli is determinant in choosing the length of the ATCUN-motif insert for CylR2. This Nterminal methionine has been shown to be cleaved more efficiently by the methionylaminopeptidase in E. coli with small side chains at the second residue position [137]. Since MGSHG-CylR2 contains glycine at the second position, it was most reasonable to produce GSHG-CylR2 with the required histidine at position three from $E$. coli BL21(DE3)pET32a-MGSHG-CylR2. But ESI-MS (see 3.4.3) revealed the Nterminal methionine to be present in $100 \%$ of the purified protein. Therefore, the serine was deleted and the expected molecular weight for MGHG-CylR2 (ATCUNCylR2) was confirmed by ESI-MS.

Intermonomer distances derived with the help of this third paramagnetic position were additionally included in the docking or replaced intermonomer distances for $\mathrm{N} 40 \mathrm{C}$ or T55C. However, the rmsd to the X-ray was increased by $\sim 1.5 \AA$ when intermonomer distances for all three paramagnetic positions were used. When intermonomer distances for $\mathrm{N} 40 \mathrm{C}$ or $\mathrm{T} 55 \mathrm{C}$ were replaced by the intermonomer distances derived with the help of the ATCUN-motif, solutions from rigid-body docking did not converge. Hence, the intermonomer distances determined via the ATCUN-motif are too imprecise. The reason for the inaccuracy may be the presence of different conformations for the terminal regions of ATCUN-CylR2 as suggested by the ${ }^{15} \mathrm{~N}$ HSQC spectra (Figure 5.9). The ${ }^{15} \mathrm{~N}-\mathrm{HSQC}$ spectrum exhibited peak-doubling for $\mathrm{N}$ - and C-terminal residues and Leu24, Ala46 and Leu47 (Figure 5.9A). After $\mathrm{Cu}^{2+}$ binding to ATCUN-CylR2 (see 5.2.2), paramagnetic peak broadening was observed and in addition, the extent of peak-doubling was increased compared to ATCUNCylR2 (Figure 5.9B). Most noticeable is the effect on Leu57 and Glu58 which have 


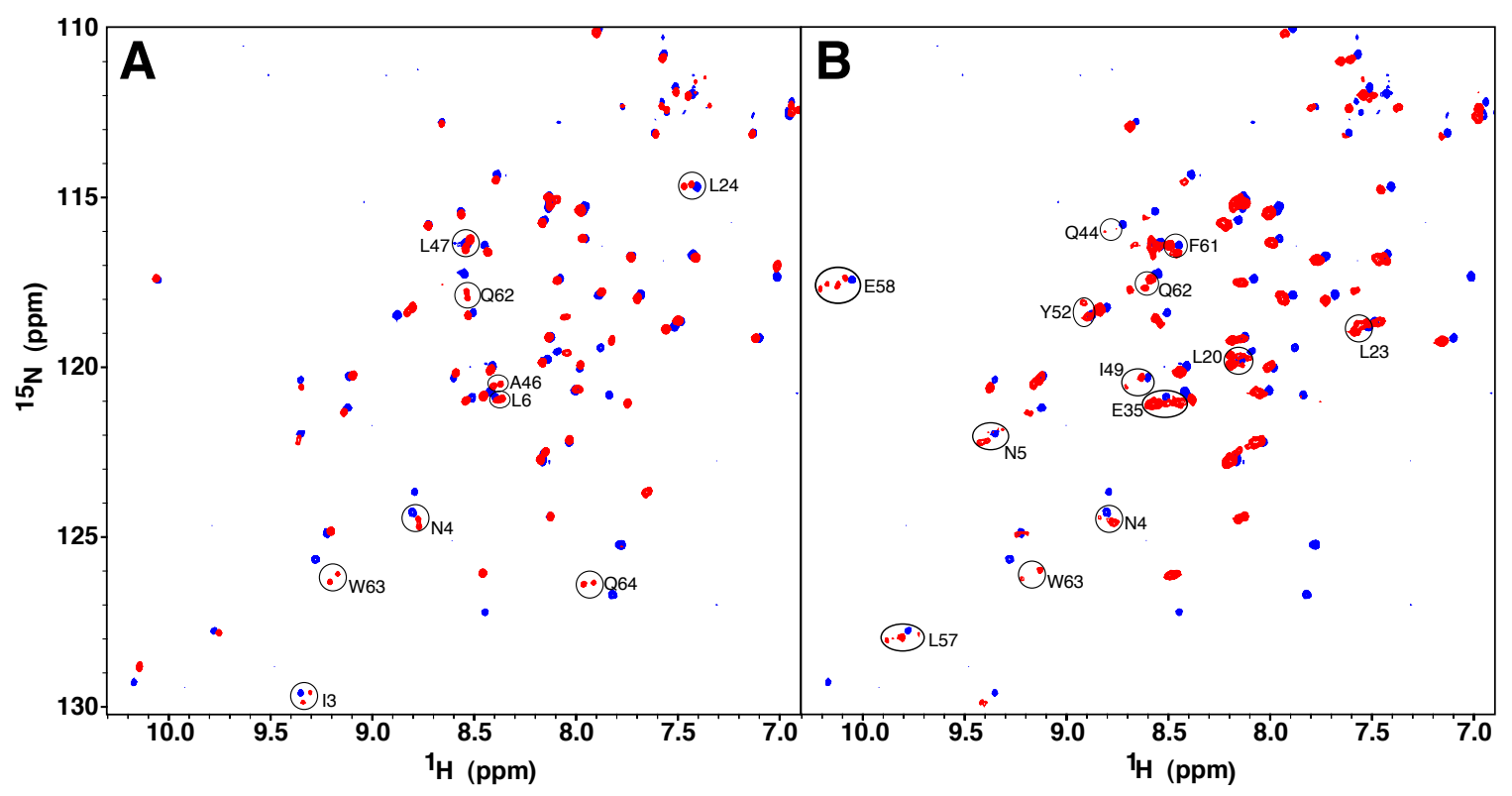

Figure 5.9: Overlay of the ${ }^{15} \mathrm{~N}-\mathrm{HSQC}$ spectrum of CylR2 (blue) on the ${ }^{15} \mathrm{~N}-\mathrm{HSQC}$ spectrum of ${ }^{15} \mathrm{~N}-\mathrm{ATCUN}-\mathrm{CylR} 2$ (red) (A) and on the ${ }^{15} \mathrm{~N}-\mathrm{HSQC}$ spectrum of ${ }^{15} \mathrm{~N}$ $\mathrm{Cu}^{2+}$-ATCUN-CylR2 (red) (B). Doubled or quadrupled peaks are indicated by ellipses.

quadrupled peaks. Hence, the distances derived with the help of the ATCUN-motif were not used for the solution NMR structure determination of CylR2.

\subsubsection{Ranking of homodimer structures from $a b$ initio docking}

Good progress has been made in the ab initio docking of protein complexes and homooligomeric proteins [18]. For many systems, an ensemble of low energy docking solutions can be calculated, which contains a structural model with an rmsd of 2-5 to the real structure. RDCs are highly useful to evaluate the quality of structures of biomolecules [78]. Previously, it was shown that it is possible to filter docking solutions using RDCs, chemical shift perturbation data and one or two distances from biological data [138]. To evaluate the feasibility of a structure determination of homodimers with HN-RDCs as the only experimental information, ab initio docking was performed for the lowest energy structure of the monomer NMR ensemble with the ClusPro Web server (http://nrc.bu.edu/cluster). The obtained ab initio docking results were then ranked according to the agreement of experimental RDCs with values predicted by the electrostatic module of PALES (see 3.5.3.3). The highest 
correlation between predicted and experimental RDCs was 0.73 and was found for the homodimer structure with the lowest rmsd to the X-ray structure (Figure 5.10). This docking solution has a a backbone rmsd (residues 3-63) to the X-ray structure

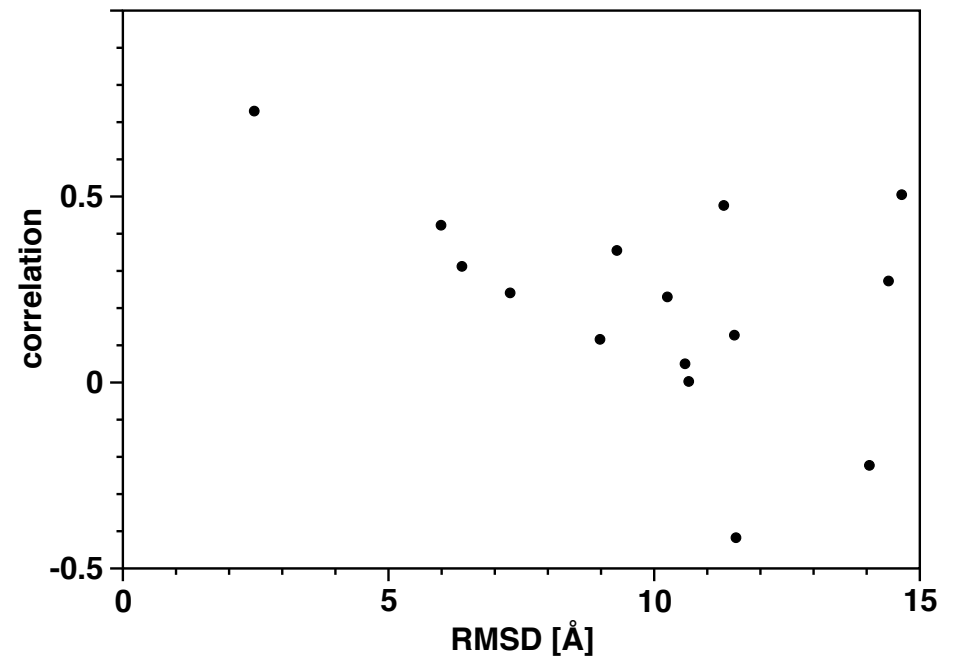

Figure 5.10: NMR-based ranking of structural models obtained from $a b$ initio docking. Correlation between measured and charge-shape predicted RDCs multiplied by the RDC correlation obtained from best-fitting RDCs to the docking solution. Values are compared to the backbone rmsd (residues 3-63) of the $a b$ initio model to the X-ray structure.

of $2.47 \AA$ and is thus only slightly worse than the NOE-only structure of CylR2, but $\sim 1.3 \AA$ worse than the structure obtained from intermonomer PREs and NOEs. For all other docking solutions, the correlation was at least 0.22 worse (Figure 5.10). Thus, the correct monomer-to-monomer orientation was identified without any experimental data about the dimer interface only using a small number of easily accessible HNRDCs.

\subsection{Discussion}

The structure determination of homooligomeric proteins by NMR is problematic because of the difficulty to distinguish inter- and intramonomer contacts. To overcome this problem, backbone HN-RDCs have been combined with long-range distances derived from PRE in a rigid-body docking protocol. Application of this method to CylR2, the regulator of cytolysin production from Enterococcus faecalis, resulted in a homodimeric structure that deviated by $1.15 \AA$ from the X-ray structure (Figure 5.8). The distance information derived from PREs has four advantages: (i) it is not limited to the dimer interface, (ii) it is long-range, (iii) it can be used in the case of 
fully deuterated proteins or for proteins for which no side chain assignment can be obtained and (iv) the number of long-range distances can be increased as required by using additional paramagnetic positions. The quality of structures determined using distances from PRE depends on the number and accuracy of the calculated distances. Compared to intramolecular long-range distances, the ratio of residues with strong and medium PRE to residues with weak PRE is decreased (Figures 5.5 and 5.6). PRE-derived distances from two mutation sites were required to unambiguously define the monomer-to-monomer orientation of CylR2. Due to the fact that hydrophobic interactions determine the dimer interface of CylR2 almost exclusively, it is especially difficult to unambiguously define it. The small differences between the NMR and the X-ray structure of CylR2 may be due to crystal packing artefacts.

For the measurement of intermolecular PRE distances, sample preparation by refolding or mixing spin-labeled and ${ }^{15} \mathrm{~N}$-labeled protein is required. HSQC spectra of the 1:1-mixed ${ }^{15} \mathrm{~N}$-wt $\left./ \operatorname{mut}^{\star}\right)$ sample may show two peaks for residues close to the interface (Figure 5.5B), but peak-doubling is limited to residues close to the mutation site. Hence, the number of residues with doubled peaks will not depend on the molecular weight. This method is complementary to a previously reported approach, in which $\mathrm{Co}^{2+}$ was introduced as a paramagnetic probe sub-stoichiometrically into a homodimer to break the symmetry and measure RDCs and PCSs [134]. The approach by Gaponenko et al. abrogates degeneracy more efficiently then the approach reported here. Due to the PCSs, paramagnetic effects across the dimer interface can be determined directly from NMR spectra and must not be calculated. However, the triplicated signals and thus the increased peak overlap required a 3D HNCO to identify the signals of a medium sized protein $(\sim 30 \mathrm{kDa})$. Therefore, a ${ }^{13} \mathrm{C}-$ and ${ }^{15} \mathrm{~N}-$ labeled sample is necessary to determine the intermolecular distances. In contrast, the presented approach needs ${ }^{13} \mathrm{C}$ - and ${ }^{15} \mathrm{~N}$-labeling only for the sequential assignment. This promises to render the novel method superior for larger homodimers.

For proteins with multiple structurally important cysteines, PRE distances may be derived using other paramagnetic probes, e.g. the ATCUN-motif [130]. Application of PRE distances determined with the help of the ATCUN-motif was not possible 
for CylR2 because an attached ATCUN-motif resulted in multiple conformations of CylR2 (Figure 5.9).

For successful rigid-body minimization, the position of the spin label has to be defined by intramolecular PRE distances prior to the docking. In addition, the intramolecular PRE distances represent an easy check on the specificity of MTSL binding. By comparing experimental intramolecular PRE distances with the theoretical distances within the monomer, unspecific binding of MTSL, which is a problem for some proteins [139], can be excluded.

Experimental data about the dimer interface may not be needed at all to identify the correct monomer-to-monomer orientation when ab initio docking results are filtered according to the correlation between experimental RDCs and values predicted from the $3 \mathrm{D}$ charge distribution and shape of the $a b$ initio structural models. In case of CylR2, RDCs predicted from the docking solution, which was closest to the native structure, correlated best with experimental values (Figure 5.10). Previously, Zweckstetter et al. have used charge-shape predicted RDCs to distinguish between parallel and antiparallel orientations of helices in coiled-coil proteins [140]. In that case, the two possible structural assemblies had very different electrostatic properties, and distinction between the two assemblies was unambiguous. On the other hand, ab initio docking of protein complexes produces in general many different solutions. Some of these solutions are quite similar, at least with respect to their global electrostatic properties. In addition, PALES is based on a strongly simplified electrostatic model. Therefore, the 15 - $\AA$ dimer model of CylR2 had a similar RDC correlation as a $6 \AA$ model (Figure 5.10B). Thus, charge-shape predicted RDCs may fail to rank ab initio docking solutions in other cases, but they have the advantage that no mutations have to be introduced into the protein and experimental data about the dimer interface are not required.

\subsection{Conclusions}

In this chapter, it was demonstrated that a combination of long-range distances derived from paramagnetic relaxation enhancement induced by a spin label and a small 
number of ${ }^{1} \mathrm{H}_{-}{ }^{15} \mathrm{~N}$ residual dipolar couplings enables a high-resolution structure determination of a symmetric homodimer in solution. Here, the structure of the CylR2 monomer was determined by conventional NOE-based methods. For larger homodimers, which require deuteration, the structure determination of the monomeric unit will be more difficult. However, assignment of side chain resonances is not required as the distances and RDCs are solely determined from HSQC spectra. Therefore, the method is particularly useful for large homodimers or higher oligomers, for which a backbone assignment can be obtained, but for which the essential deuteration limits the availability of NOE data. In these cases, the intramolecular PREs, which were only used for positioning the spin label in case of CylR2, will also be highly valuable for the structure determination of the monomer [87]. In addition, the NMR-based rigid-body docking might be combined with X-ray crystallography for complexes, for which the dimer interface is not certain from the electron density map. Only based on a backbone assignment and a small number of HN-RDCs revealing similar solution and crystal structures, the dimer interface can be defined by measurement of PRE broadenings for a complex composed of spin-labeled and ${ }^{15} \mathrm{~N}$-labeled protein. $\mathrm{Al}-$ ternatively, charge-shape predicted RDCs, whose value for ranking ab initio docking solutions has been demonstrated in paragraph 5.3.6 of this chapter, can be applied to unravel uncertainties from X-ray crystallography. An example for this application of charge-shape predicted RDCs is presented for the $35.4 \mathrm{kDa}$ homodimer CesT in paragraph 6.3.4 of the following chapter. 


\section{6}

\section{Structural studies of the function of the chaperone CesT in protein secretion via the type three secretion machinery of enteropathogenic $E$.}

coli

\subsection{Introduction}

A large number of Gram-negative bacterial pathogens of plants and animals utilize a type three secretion system (TTSS) to deliver virulence effector proteins into eukaryotic cells. These TTSSs are composed of $\sim 25$ proteins, which assemble to form a needle-like secretion complex spanning the bacterial inner and outer membrane and comprising an inner pore with a diameter of 2-3 nm (Figure 6.1). Through this pore, bacterial proteins are secreted and translocated into the eukaryotic cell.

Amongst the Gram-negative bacteria, which utilize a TTSS, are the human pathogens enteropathogenic Escherichia coli (EPEC) and enterohemorrhagic Escherichia coli (EHEC) O157:H7. They have evolved from commensal E. coli, the most abundant facultative anaerobic Gram-negative bacterium of the intestinal microflora, by horizontal gene transfer. These pathogenic bacteria form lesions on the intestinal surface by attaching and effacing $(\mathrm{A} / \mathrm{E})$ on host epithelial cells. Both, EPEC and EHEC, use a related translocation mechanism [141]. EPEC is the major cause of diarrhoea in young children and EHEC causes hemorrhagic colitis [142]. Figure 6.1 depicts the 


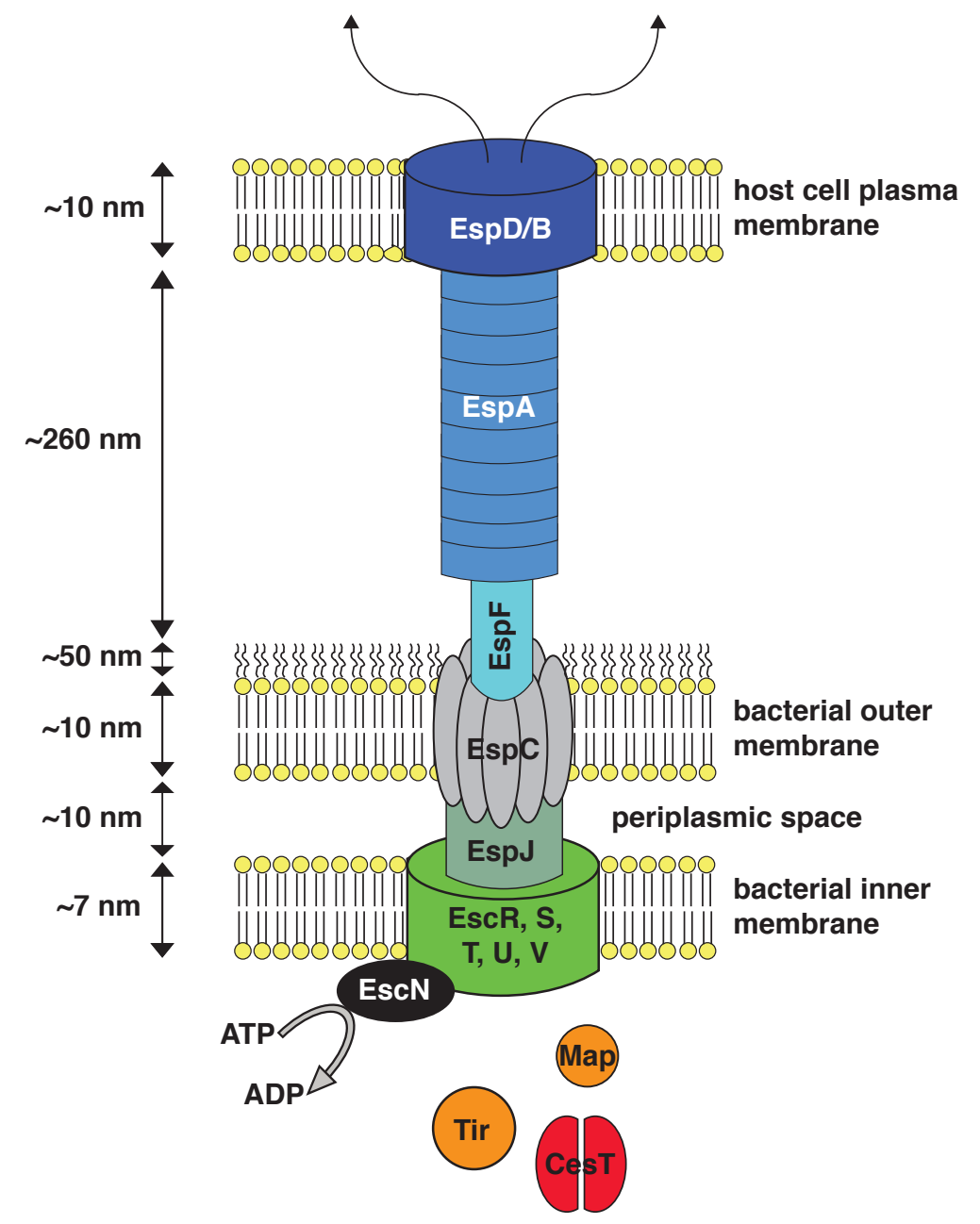

Figure 6.1: Schematic representation of the EPEC/EHEC type three secretion system. The basal body of the TTSS is composed of the outer membrane protein EscN, the inner membrane proteins EscR, EscS, EscT, EscU and EscV and the lipoprotein EscJ, which connects the inner and outer membrane ring structures. The needle structure is composed of EscF and EspA filaments. The translocation pore in the host cell plasma membrane is formed by EspB and EspD. By ATP hydrolyses, the ATPase EscN provides energy to the system. The chaperone CesT and the effectors Tir and Map are indicated as cytoplasmic components of the TTSS [141].

TTSS of EPEC and EHEC including the proteins composing the needle complex, proteins involved in the secretion process in the bacterial cytoplasm and proteins translocated from the bacterial cytoplasm into the eukaryotic host cell. The translocated proteins are called TTSS effectors and have a variety of activities that damage host cells. Two of the TTSS effectors of EPEC and EHEC are the translocated intimin receptor (Tir) and the mitochondrial associated protein (Map). 
Map was given the name mitochondrial associated protein because it targets mitochondria via an N-terminal targeting sequence. The N-terminal 44 residues were shown to be sufficient to target Map to mitochondria where it is imported into the mitochondrial matrix [143]. Map mediates filopodia formation, causes mitochondrial dysfunction and may have an anti-apoptotic function [144], 145]. Recently, the biological function of Map was demonstrated to be the subversion of small G protein signaling pathways through molecular mimicry of the activated forms of distinct Rho family small G proteins [146]. However, the relevance of Map to the pathogenesis of EPEC-induced disease is uncertain.

Tir is inserted into the host cell plasma membrane forming a hairpin-like structure composed of two transmembrane domains. The extracellular loop between the two transmembrane domains interacts with intimin, a bacterial adhesion molecule. The C- and N-terminus, which are located within the host cell, interact with focal adhesion and cytoskeletal proteins. Thus, the bacterium is linked to the host cell cytoskeleton and actin-rich pedestals beneath adherent bacteria are formed [141].

As many other TTSS effectors, Tir and Map have a specific chaperone which is important for their secretion. The TTSS chaperones are a family of proteins which share only limited sequence similarity, tend to form homodimers, are highly acidic and are predicted to contain a C-terminal amphipathic $\alpha$-helix [147]. A classification scheme of these chaperones has been proposed based on the function of the substrates [148]: Class I are effector binding chaperones whereas class II are chaperones binding the translocators, which form the TTSS channel. Class I was divided into two subclasses based on the number of substrates and the location of the genes encoding the chaperone and its substrates: class IA chaperones bind one effector and are encoded in the vicinity of their substrate gene, whilst class IB chaperones bind several effectors and are encoded within the operons for the TTSS components. Class III is composed of the chaperones of the flagellar export system which is the evolutionary predecessor of TTSSs [148, 149]. In general, the function of chaperones is to assist other proteins to achieve a proper folding. Though the function of TTSS chaperones is not clearly understood and seems to be versatile. Most widely accepted is their role in prevent- 
ing aggregation or degradation of effector molecules. There is also evidence for a regulatory role in the release of effectors [150] and an involvement in the feedback regulation of the TTSS-associated gene expression [151].

The TTSS chaperone specific for Tir and Map is called CesT. CesT stands for chaperone for efficient secretion of Tir as it was originally discovered to bind only Tir [30] and was grouped to class IA of TTSS chaperones [148]. CesT is not absolutely required for the secretion of Tir [152]. In 2003, it was found to also bind Map [30] and in 2005 its binding to five effectors, NleA, NleH, NleF, EspH and SepZ, in addition to Tir and Map was revealed [153]. Therefore, Thomas et al. [153] redefined CesT as a chaperone with broad specificity for multiple TTSS effectors.

At present, nine X-ray structures of class IA TTSS chaperones are solved, including the structure of CesT from EHEC O157:H7 [154], SycE from Yersinia pseudotuberculosis [155], SycH from Yersinia pestis [156], SycT from Yersinia enterocolitica [157], [158], SicP [159] and SigE [154] from Salmonella enterica as well as the AvrPphFOrf1 from the plant pathogen Pseudomonas syringae (Figure 6.2). The X-ray structures of Spa15 from Shigella flexneri [160] and InvB from Salmonella typhimurium [161] are the only solved structures of a class IB chaperone. In spite of their limited sequence similarity, the TTSS chaperones exhibit similar structures. A structure-based sequence alignment of the nine TTSS chaperones with known X-ray structures is displayed in Figure 6.20. Each monomer is composed of five $\beta$ strands and three $\alpha$ helices. CesT contains an additional $\beta$ strand while SycT lacks the dimerization helix $\alpha 2$ (Figure 6.2). The hydrophobic side of the predicted amphipathic C-terminal helix $\alpha 3$ is buried in the core of the chaperone. Each homodimeric chaperone possesses two pairs of hydrophobic patches on the surface [162].

For CesT, the structure shown in Figure 6.2 E is a model constructed due to structural homology. However, the crystal structure of CesT from EHEC O157:H7 reveals a domain-swapped homodimer (Figure 6.3A) [154], where the N- and C-terminal regions lie on the adjacent molecule. As all other solved crystal structures of TTSS chaperones do not show a 3D domain swap, the question arises whether it is a crystallographic artefact or facilitates the chaperone-effector interaction [154]. 
A

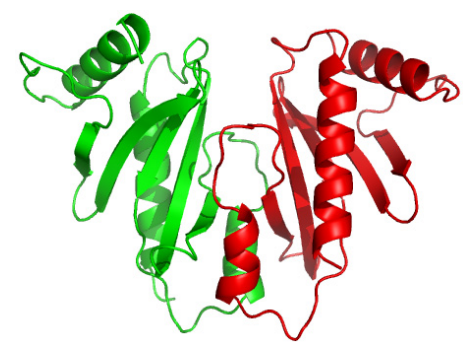

D

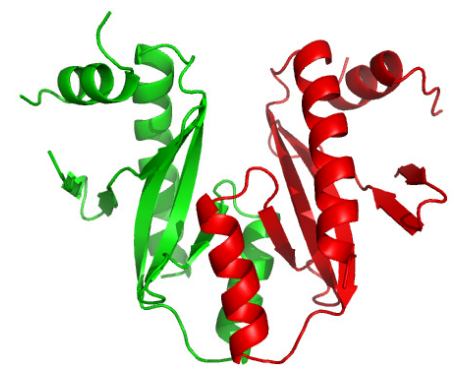

B

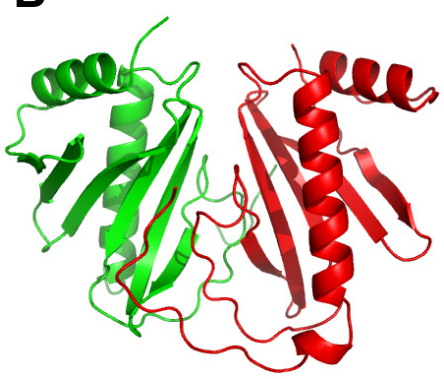

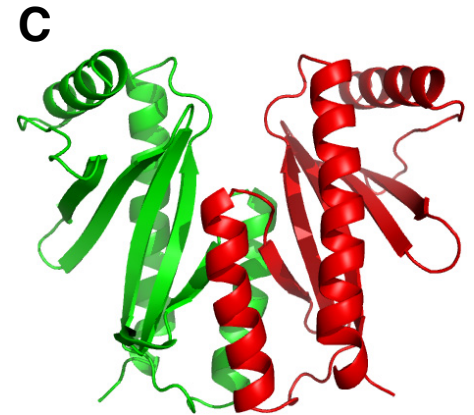

E

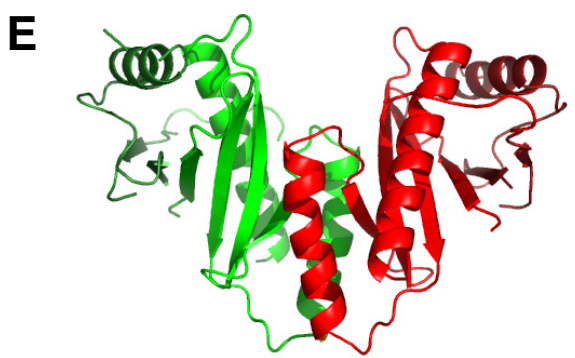

Figure 6.2: X-ray structures of TTSS chaperones as ribbon models with one monomer shown in red and the other in green: (A) SycE (PDB access code: 1L2W), (B) SycT (PDB access code: 2BSJ), (C) SicP (PDB access code: 1JYO), (D) SigE (PDB access code: 1K3S) and (E) the CesT model structure (PDB access code: 1K3E) with an analogous dimerization interface and the amino- and carboxy-terminal regions of each monomer in dark red and dark green.

With the help of recent structural data, progress has been made towards understanding the key elements of this chaperone-effector interaction. Four crystal structures of complexes of the N-terminal chaperone binding domain (CBD) of the effector and its cognate class IA chaperone have been solved: the Salmonella typhimurium chaperone SicP with its virulence factor SptP [159], the Yersinia pseudotuberculosis chaperone SycE with its effector YopE [162](Figure 6.4), the Yersinia pestis chaperone SycH and YSCM2, a negative regulator of type three secretion [156] and the heterodimeric chaperone SycN-YscB from Yersinia pestis with its substrate YopN [163]. Very recently, the X-ray structure of the class IB chaperone InvB with SipA from Salmonella typhimurium was published [161]. This structure is an exception as the interaction between InvB and SipA occurs only with one molecule of the homodimeric InvB [161]. All other known chaperone/effector structures show effector interactions with both chaperone monomers. These structures have revealed the N-terminal CBDs of the effectors as extended, nonglobular polypeptides which are wrapped around the 
A

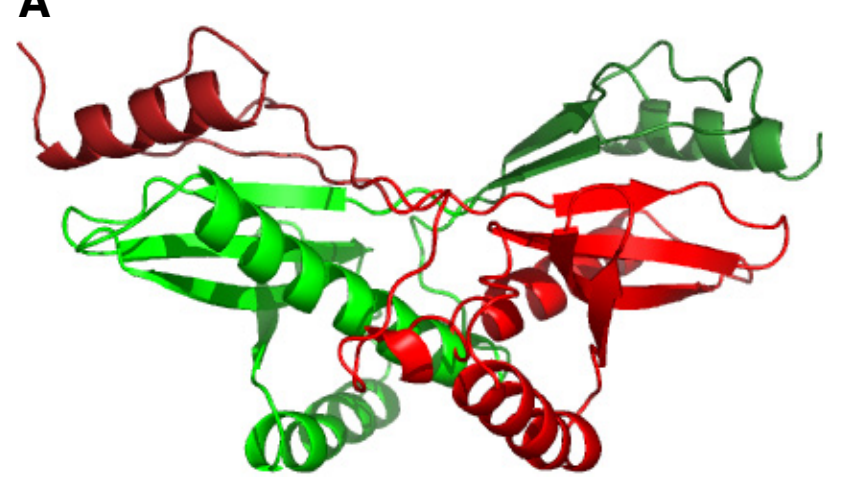

B

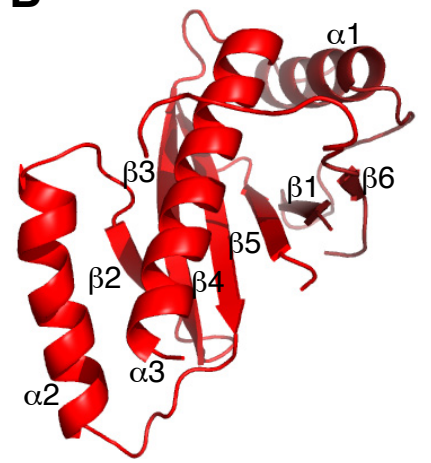

C

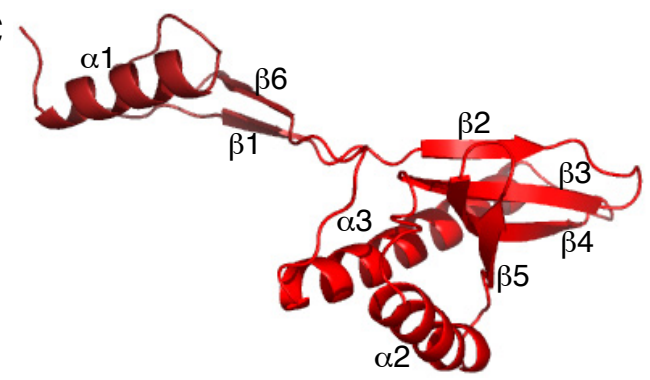

D

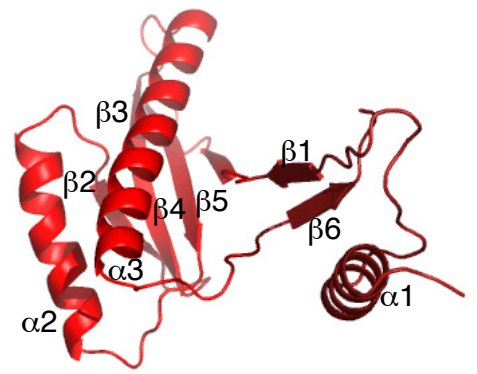

Figure 6.3: Structure of CesT. Dimeric X-ray structure (A), one monomeric subunit of the model structure (B) and one monomeric subunit of the X-ray structure (C,D) of CesT. The orientation of the monomeric subunit of the X-ray structure in (C) and (D) corresponds to the orientation of (A) and (B), respectively. One subunit is colored red and the other green. The small domain of each monomer (residues 1-34 and 139-146 forming $\alpha 1, \beta 1$ and $\beta 6$ ) are colored in dark red and dark green.

dimeric chaperones (Figure 6.4). Chaperone/effector interactions occur mainly between the hydrophobic patches on the chaperone surface and secondary structure elements of the effector. As different parts of the effector bind to identical regions of the chaperone, the specificity of the chaperone may arise from two complementary contributions [148]. Based on the X-ray structures of chaperone/effector complexes (Figure 6.4), the N-terminal CBDs can be roughly superimposed indicating a general three-dimensional fold [164]. The only conserved structural element in all five solved complex structures is a $\beta$-motif and hence an adaptor-targeting model was proposed by Lilic et al. [161]: the $\beta$-motif of the CBD functions as an adaptor element, which targets the effector to the chaperone. Then, the chaperone docks the effector to the membrane-associated ATPase of the TTS machinery. Only a homology-based nonswapped model structure of CesT is consistent with the key binding elements of the chaperone/effector interaction [164]. 
A

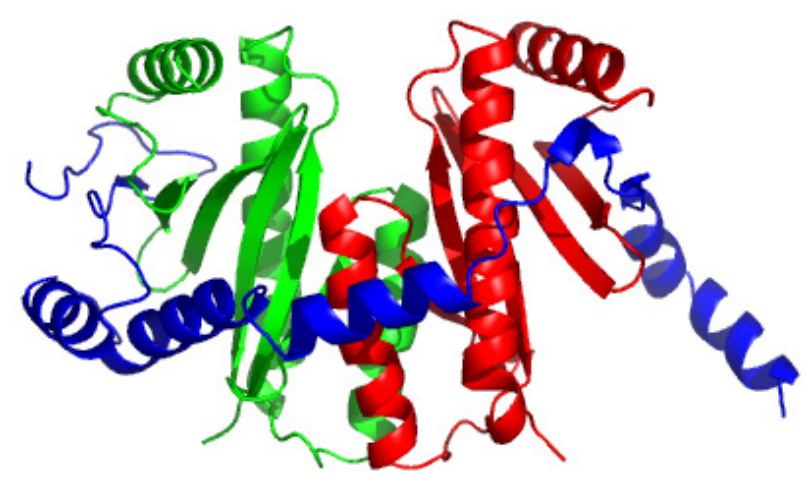

C

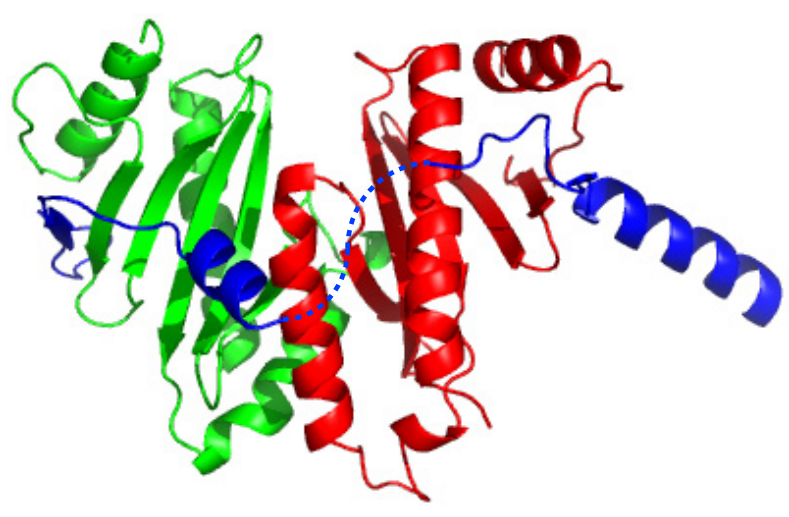

B

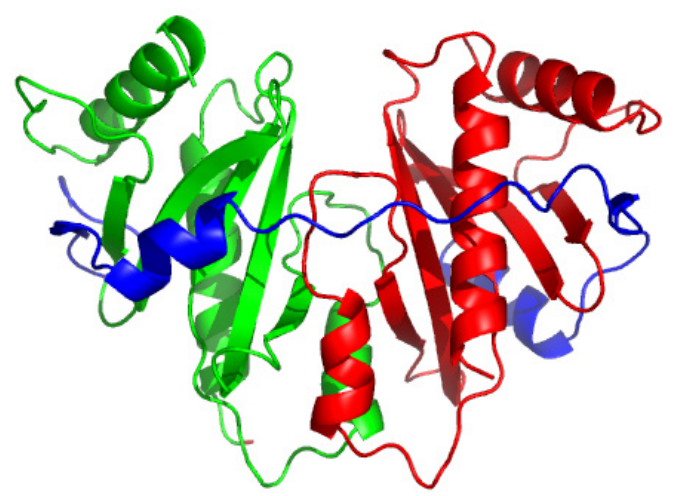

D

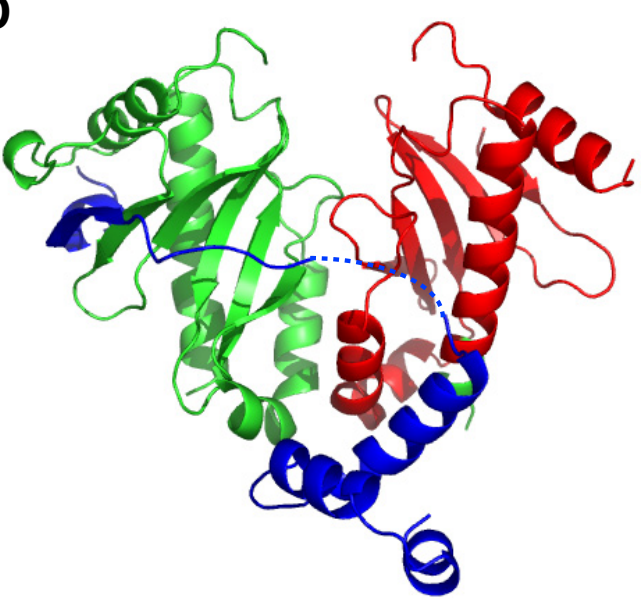

Figure 6.4: Ribbon diagrams of crystal structures of chaperone/effector complexes. The two subunits of the chaperones are shown in red and green and the effectors in blue with disordered regions as dotted lines. (A) The chaperone SicP with residues 35-139 of SptP (PDB access code:1JYO), (B) the chaperone SycE with residues 17-85 of YopE (PDB access code: $1 \mathrm{~L} 2 \mathrm{~W}),(\mathrm{C})$ the heterodimeric chaperone $\mathrm{SycN}-\mathrm{YscB}$ with residues 32-92 of YopN (PDB access code:1XKP) and (D) the chaperone InvB with residues 23-93 of SipA (PDB access code:2FM8).

Little is known about the structure of the free CBDs of TTSS effectors. The X-ray structure of the CBD of SipA comprising residues 48-158 is the only known structure of a CBD of a TTSS effector. However, SipA ${ }^{48-264}$ is not able to form a stable complex and SipA ${ }^{22-264}$ was used to solve the complex structure of the InvB/SipA complex [161].

At this stage, different experimental data exist which render a global unfolding activity of the chaperone on the effector unlikely. Specifically, experimental data about the TTSS effector, e.g. for Tir and SigD, demonstrate similar physical or enzymatic 
properties of the effector in the absence and presence of their chaperone [154]. On the other hand, an unfolding activity of the TTSS-associated ATPase InvC in the presence of ATP for the chaperone/effector complex SicP/SptP from Salmonella enterica was demonstrated [165].

Overall, the secretion mechanism is only partly understood. Especially intriguing is the question how the passage of the effector proteins through the $\sim 3 \mathrm{~nm}$ narrow pore is achieved and what the role of the specific chaperones is. Disassembly of the chaperone/effector complex prior to and during secretion may occur partly or entirely through domain swapping [164]. Further structural insights, particularly of the chaperone and chaperone/effector complexes are prerequisite for a better understanding of the TTSS mechanism which is crucial for many medically important bacterial pathogens. To answer these questions, the chaperone CesT and its complexes may prove to be extremely valuable. Firstly, as the CesT X-ray structure differs from the other X-ray structures of other TTSS chaperones, further structural studies of CesT may improve our understanding of the TTSS mechanism. Secondly, in recent experiments five effector proteins in addition to Map and Tir, which bind CesT, were found and CesT was shown to specifically deliver multiple effectors to the TTSS [153]. Furthermore, it was demonstrated that CesT interacts not only with TTSS effectors but also with the membrane-associated ATPase EscN [166].

During this project, a contribution to the understanding of how the TTSS chaperone CesT from EPEC E2348/69 aids in the injection of the TTSS effector proteins Tir and Map was made. In the first part of this chapter, the backbone assignment of the $35.4 \mathrm{kDa}$ homodimer CesT and its properties in solution are depicted as a fundamental step for all further NMR studies. Without calculating a NMR solution structure of CesT experimental RDCs and charge-shape predicted RDCs with PALES were applied to investigate whether the solution structure is the domain-swapped (Figure 6.3) or the non swapped structure (Figure 6.2 E).

In the second part of this chapter, steps towards a CesT/effector complex structure are presented. These structural studies are the starting point to further understand the mechanism of the TTS machinery in general and in particular the TTSS of EPEC 
and EHEC. This knowledge is important to answer the question how the translocation and unfolding of TTSS effectors is triggered. An answer to these questions may render the introduction of engineered proteins into almost any cultured cell via a TTSS possible. Eventually the design of drugs that can combat a broad range of diseases should be possible.

\subsection{Materials and methods}

\subsubsection{Cloning}

Initially, the NdeI cleavage site within the gene for CesT from EPEC E2348/69 in pQE30-His ${ }_{6}$ CesT [29] was eliminated through a silent mutation using the QuikChange $^{T M}$ Site-directed mutagenesis Kit (see 3.1.5). Afterwards the gene coding for CesT from EPEC E2348/69 was amplified by PCR and cloned into the NdeI and BamHI restriction sites of pET16bTEV.

Template plasmids containing genes coding for Tir and Map are specified in Table 2.2. The genes coding for Tir and Map as well as different C-terminally truncated genes were cloned into the $N d e \mathrm{I}$ and XhoI restriction sites of pET16bTEV (Figure 2.1A). For coexpression of CesT/effector complexes, the gene coding for the effector was inserted into the $N d e \mathrm{I}$ and $X h o \mathrm{I}$ restriction sites of the multiple cloning site 2 (MCS2) of pETDuet1 and the gene coding for CesT was inserted into the NcoI and BamHI restriction sites of the MCS1 of pETDuet1 (Figure 2.1B). The different constructs are summarized in Table 2.2.

\subsubsection{Protein expression}

Details of protein expression are described in 3.2.1. For coexpression of CesT and of CesT/Map complexes in M9-based minimal medium the temperature was reduced to $17^{\circ} \mathrm{C}$ at an $\mathrm{OD}_{600}$ of $0.4-0.5$ and cells were harvested $16-20 \mathrm{~h}$ after induction. Cells producing CesT and growing in M9-based minimal medium prepared with $99.9 \%$ $\mathrm{D}_{2} \mathrm{O}$ were harvested 40 - $48 \mathrm{~h}$ after induction. 


\subsubsection{Purification}

Images of SDS-PAGEs documenting the different protein purifications as well as ESIMS spectra confirming the purity and molecular weight of the proteins can be found in Appendix A.

\subsubsection{Purification of CesT}

The cell lysis was performed under native conditions for cells grown in LB and under denaturing conditions for cells grown in M9-based minimal medium (see 3.2.2). Recombinant, His-tagged CesT was initially purified via affinity chromatography on a Ni-NTA Agarose resin under native or denaturing conditions (see 3.3.1). After removal of the His-tag by cleavage with the TEV-protease (see 3.3.5), CesT was further purified by anion exchange chromatography on a 5-ml HiTrap Q-Sepharose column (see 3.3.2). The protein solution was diluted to $120 \mathrm{ml}$ with anion exchange buffer A and was supplemented with DTT and EDTA to a final concentration of $1 \mathrm{mM}$ and $0.5 \mathrm{mM}$, respectively. The protein was eluted by linearly increasing the percentage of anion exchange buffer B from 0 - $100 \%$ over a volume of $100 \mathrm{ml}$ at a flow rate of $3.0 \mathrm{ml} / \mathrm{min}$. Fractions containing CesT were pooled, dialysed extensively against $\mathrm{H}_{2} \mathrm{O}$ and stored in lyophilised form at $-20^{\circ} \mathrm{C}$. One litre of culture medium yielded $\sim 40 \mathrm{mg}$ of unlabeled CesT, $\sim 30 \mathrm{mg}$ of ${ }^{15} \mathrm{~N}$ or ${ }^{15} \mathrm{~N} /{ }^{13} \mathrm{C}$ labeled CesT and $\sim 20 \mathrm{mg}$ of ${ }^{2} \mathrm{H},{ }^{2} \mathrm{H} /{ }^{15} \mathrm{~N}$ or ${ }^{2} \mathrm{H} /{ }^{15} \mathrm{~N} /{ }^{13} \mathrm{C}$ labeled CesT.

\subsubsection{Purification of Map}

At first, cells were ruptured under native conditions. After centrifugation the pellet was applied to cell lysis under denaturing conditions (see 3.2.2). Then the His-tagged Map was purified via a $\mathrm{Ni}^{2+}$-column (see 3.3.1). Finally the His-tagged Map was used for the preparation of CesT/Map complexes or dialysed directly against NMR buffer. 


\subsubsection{Purification of Tir}

Cell pellets containing recombinantly expressed Tir were ruptured under native conditions (see 3.2.2, the His-tagged Tir was purified by affinity chromatography on a Ni-NTA Agarose resin (see 3.3.1) and the His-tag was cleaved with the TEV-protease (see 3.3.5). After removal of the TEV-protease Tir was further purified by RP-HPLC (see 3.3.4). The purity of Tir was confirmed by ESI-MS (see 3.4.3). The purified Tir was stored in lyophilised form at $-20{ }^{\circ} \mathrm{C}$. The yield is $\sim 5 \mathrm{mg} / \mathrm{l}$.

\subsubsection{Purification of CesT/effector complexes}

After native cell rupture (see 3.2.2 coexpressed CesT/effector complexes were bound via the N-terminal His-tag of CesT to Ni-NTA Agarose resin and eluted by increasing the imidazole concentration. For the preparation of selectively labeled CesT/Map complexes, both complex components were purified under denaturing conditions via a $\mathrm{Ni}^{2+}$-column (see 3.3.1). Protein concentrations were determined by measuring the $\mathrm{A}_{280}$ (see 3.4.1). Sample volumes of CesT and Map were mixed with a three- to fourfold molar excess of CesT. The CesT/Map complex was formed during dialysis against 2 times 51 TEV-buffer.

All His-tags were cleaved using the TEV-protease (see 3.3.5). For further purification and removal of the excess of CesT, CesT/Map complexes were applied to anion exchange chromatography on a 5-ml HiTrap Q-Sepharose column performed as described for CesT (see 3.3.2 and 6.2.3.1). To achieve an optimal separation of CesT and the CesT/Map complex, the flow rate was $1.0 \mathrm{ml} / \mathrm{min}$ during the elution. Fractions containing the CesT/Map complex were pooled and dialysed against the NMR buffer for CesT without $\mathrm{D}_{2} \mathrm{O}$ (Table 2.6).

For crystallization the CesT/Map complex was dialysed after anion exchange chromatography into gel filtration buffer (Table 2.6) and concentrated to $\sim 10 \mathrm{mg} / \mathrm{ml}$. The gel filtration was performed as described in 3.3.3. 


\subsubsection{NMR experiments}

Lyophilised CesT and Tir108 were dissolved directly in NMR buffer (Table 2.4). CesT/effector samples and refolded $\mathrm{His}_{7}$-Map84 were concentrated by ultrafiltration (see 3.3.6) to a final volume of $\sim 200 \mu \mathrm{l}$ and $20-25 \mu \mathrm{l} \mathrm{D}_{2} \mathrm{O}$ were added.

All NMR spectra were acquired at $303 \mathrm{~K}$ with the exception of the spectra used for the backbone assignment of Tir108 which were acquired at $293 \mathrm{~K}$. Triple resonance experiments measured for the backbone assignments are listed in the Appendix B.1. Backbone assignments of CesT and Tir108 as well as the reassignment of the CesT backbone in CesT/effector complexes were derived from triple resonance NMR spectra as summarized in the Appendix B.2. $\mathrm{HN}, \mathrm{C}^{\prime} \mathrm{N}$ and $\mathrm{C}^{\prime} \mathrm{C}_{\alpha}$ RDCs were measured for a concentration of $12 \mathrm{mg} / \mathrm{ml} \mathrm{Pf1}$ in the NMR buffer (see 3.5.3). The oligomerization state was estimated as described in 3.5.4.1 and the ${ }^{15} \mathrm{~N}-{ }^{1} \mathrm{H}$ steady-state NOE of free and bound CesT were measured and analysed as described in 3.5.4.3.

A series of $2 \mathrm{D}{ }^{15} \mathrm{~N}-{ }^{1} \mathrm{H}-\mathrm{HSQC}$ spectra were measured to record the formation of CesT/effector complexes. To monitor the chemical shift changes of CesT upon complex formation $100 \mu \mathrm{M}{ }^{2} \mathrm{H},{ }^{15} \mathrm{~N}$-labeled CesT was titrated with $100 \mu \mathrm{M} \mathrm{His}$ H-Map84 or $300 \mu \mathrm{M}$ Tir108. 2D ${ }^{15} \mathrm{~N}^{1}{ }^{1} \mathrm{H}-\mathrm{TROSY}-\mathrm{HSQC}$ spectra were recorded at a CesT to effector ratio of 1:0.1, 1:0.2, 1:0.4 and 1:1. For CesT/Tir108, an additional 2D ${ }^{15} \mathrm{~N}-{ }^{1} \mathrm{H}-$ TROSY-HSQC spectrum was acquired for a twofold excess of Tir108. To determine the chemical shift changes of the effectors upon complex formation, CesT was added from a $1-\mathrm{mM}$ stock solution to a $100-\mu \mathrm{M}{ }^{15} \mathrm{~N}$-labeled effector sample. $2 \mathrm{D}{ }^{15} \mathrm{~N}-{ }^{1} \mathrm{H}-$ HSQC spectra were measured at Tir108:CesT ratios of 1:0.25, 1:0.5, 1:1, 1:2, 1:4 and 1:8, at TirN:CesT ratios of 1:0.05, 1:0.1, 1:0.2, 1:0.5, 1:1 and 1:2 and at His $7^{-}$ Map84:CesT ratios of 1:0.2, 1:0.4, 1:1 and 1:2.

\subsection{Results and discussion}

\subsubsection{Experimental difficulties}

The plasmid pQE30-His ${ }_{6}$-CesT was kindly provided by the group of Gad Frankel (Imperial College London) and was initially used to prepare His-tagged CesT expressed 
in E. coli BL21. However, the ${ }^{15} \mathrm{~N}-\mathrm{HSQC}$ and the ${ }^{15} \mathrm{~N}-\mathrm{TROSY}-\mathrm{HSQC}$ spectrum recorded for ${ }^{15} \mathrm{~N}^{-H_{i s}}{ }_{6}$-CesT suggested aggregation of the sample (Figure 6.5). NMR

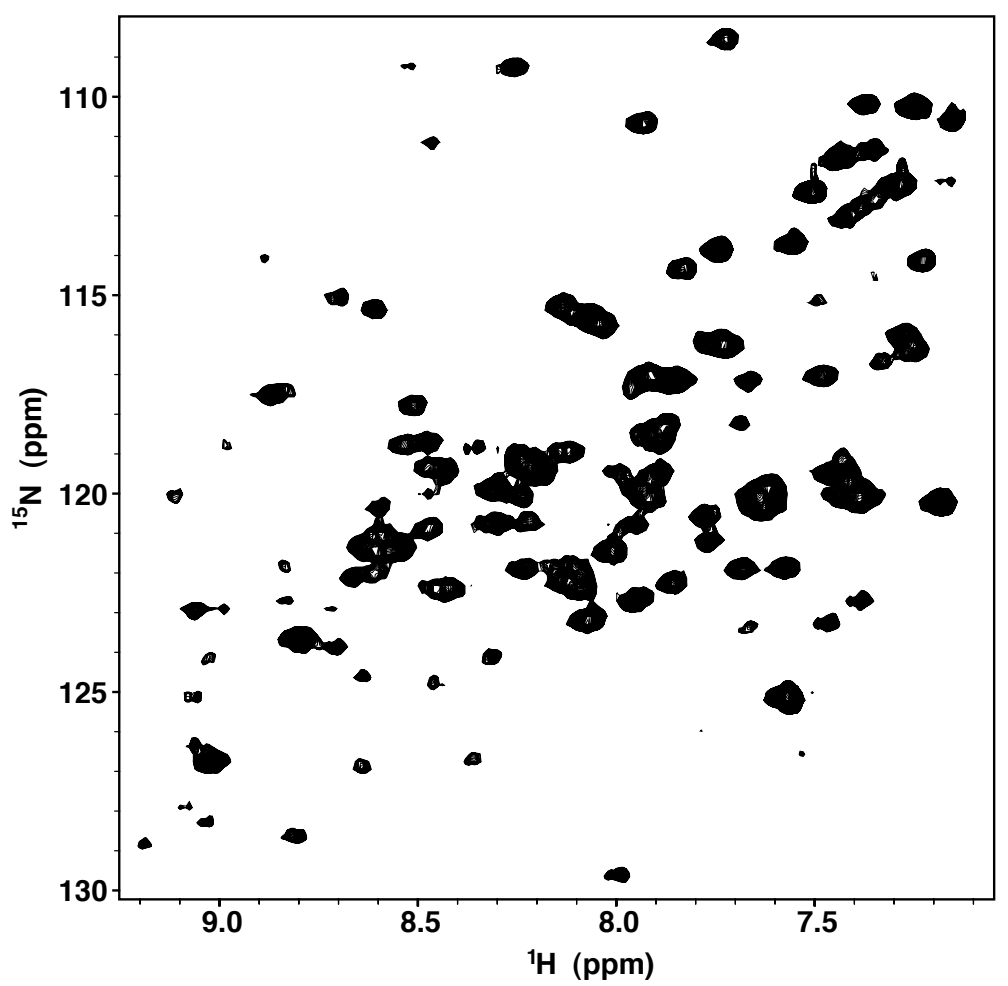

Figure 6.5: $800 \mathrm{MHz}{ }^{15} \mathrm{~N}-\mathrm{HSQC}$ spectrum of $\mathrm{His}_{6}$-CesT at $303 \mathrm{~K}$.

relaxation times and results from dynamic light scattering indicated a CesT trimer which is in disaggreement with previous results showing a CesT dimer [154]. Furthermore, His $_{6}$-CesT was not soluble at concentrations above $300 \mu \mathrm{M}$ and the construct did not contain a protease cleavage site for the removal of the $\mathrm{His}_{6}$-tag. Therefore pET16bTEV-CesT was constructed to enable the preparation of CesT without a Histag. The ${ }^{15} \mathrm{~N}-\mathrm{HSQC}$ and the ${ }^{15} \mathrm{~N}-\mathrm{TROSY}-\mathrm{HSQC}$ spectrum for CesT without His-tag were significantly improved (Figure 6.6). Intensities were almost identical for all signals and no strong line broadening was observed. CesT without His-tag is soluble to concentrations up to $1 \mathrm{mM}$ and stable for a few months if $0.5 \mathrm{mM}$ EDTA is present in the NMR buffer. 


\subsubsection{Extent of the assignment of CesT}

3D NMR experiments acquired for the backbone assignment of CesT are summarized in the Appendix Table B.2. The analysis of the triple resonance spectra (see 3.5.1.1) allowed identification and sequential assignment of $97 \%$ of the backbone amide resonances, not including the ${ }^{15} \mathrm{~N}$ of the five proline residues. Five residues could not be found in the HSQC due to overlap (Figure 6.6).

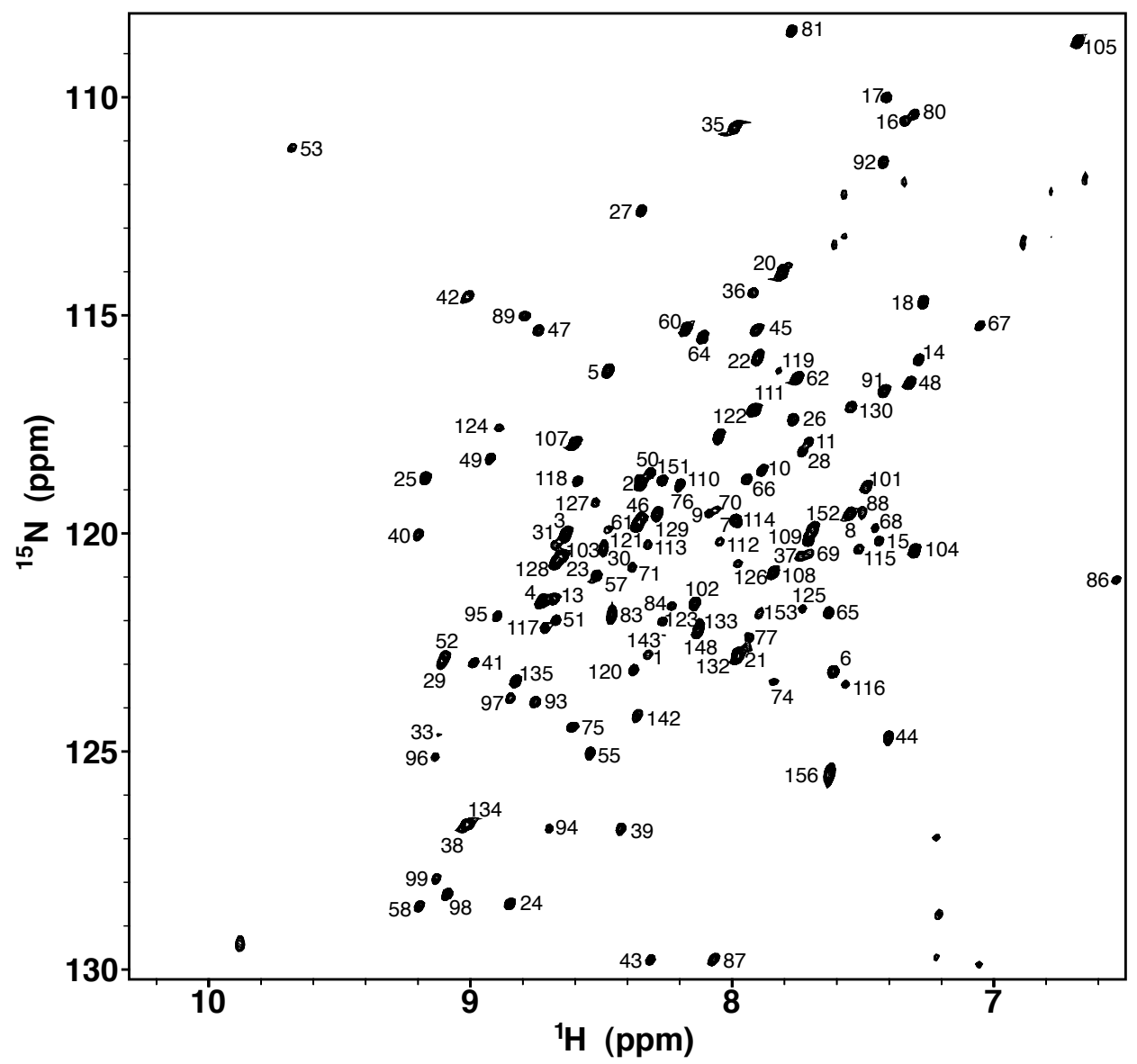

Figure 6.6: $900 \mathrm{MHz}{ }^{15} \mathrm{~N}-\mathrm{TROSY}-\mathrm{HSQC}$ spectrum of CesT at $303 \mathrm{~K}$. Resonance assignments are indicated with residue numbers.

Of the C' resonances $98.7 \%$, of the $\mathrm{C}_{\alpha}$ resonances $98 \%$ and of the $\mathrm{C}_{\beta} 98 \%$ resonances have been assigned unambiguously. The chemical shift data have been deposited in the BMRB (http://www.bmrb.wisc.edu) under accession number 6451. 


\subsubsection{Secondary structure and dynamics of CesT in solution}

Secondary structure elements were determined from the deviation of the $\mathrm{C}_{\alpha}, \mathrm{C}_{\beta}$ and C' chemical shifts from the random coil values (see 3.5.2) and are shown in Figure 6.7. Regions of secondary structure identified by the combined secondary chemical shifts

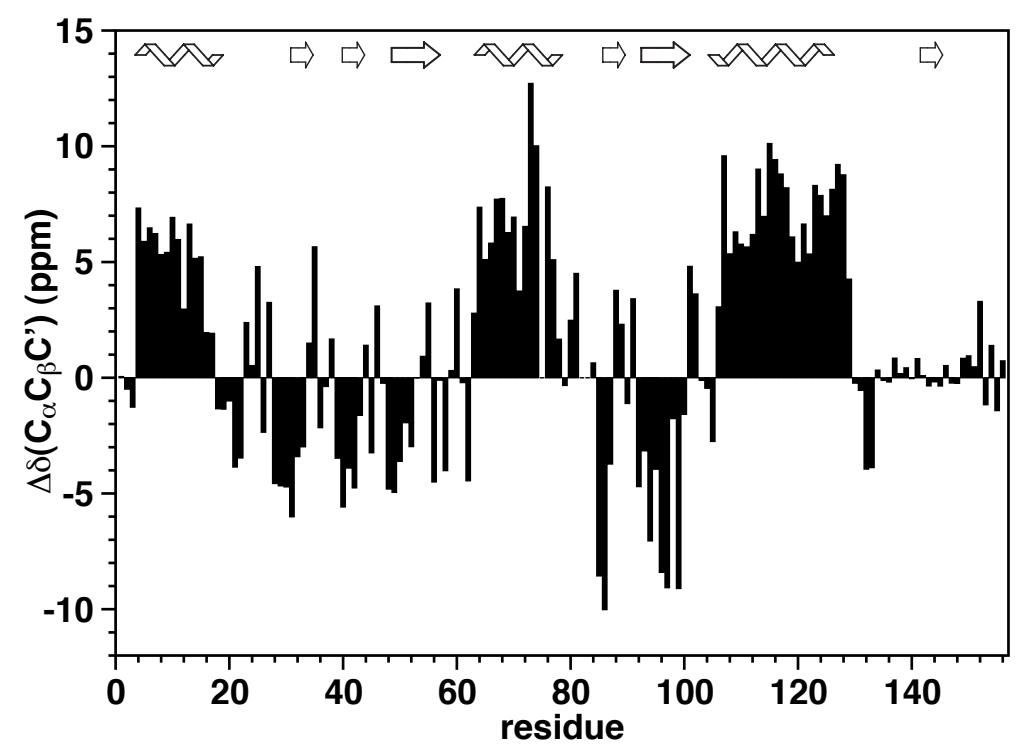

Figure 6.7: Combined secondary chemical shifts for CesT plotted against the residue number. Secondary structure elements of the X-ray structure are indicated.

were confirmed by the characteristic NOE patterns [7] in the $3 \mathrm{D}\left[{ }^{15} \mathrm{~N}-{ }^{1} \mathrm{H}\right]$ NOESYHSQC. The X-ray structure reveals three $\alpha$ helices and six $\beta$ strands: $\alpha 1$ from residue 4 to $16, \alpha 2$ from residue 63 to 78, $\alpha 3$ from residue 106 to 128, $\beta 1$ from residue 30 to $33, \beta 2$ from residue 38 to $42, \beta 3$ from residue 30 to $33, \beta 4$ from residue 83 to 87, $\beta 5$ from residue 92 to 100 and $\beta 6$ from residue 141 to 144 . These regions are identical to the regions found via the secondary chemical shifts with the exception of the C-terminal part containing residues 130 to 156 where no secondary structure is indicated by the secondary chemical shifts. Therewith the additional strand $\beta 6$ of the CesT X-ray structure which is not found in the other X-ray structures of TTSS chaperones, is also not present for CesT in solution.

The amplitude of motions in the pico- to nanosecond time-scale was investigated with the steady-state heteronuclear ${ }^{15} \mathrm{~N}-{ }^{1} \mathrm{H}-\mathrm{NOE}$ (see 3.5.4.3). Heteronuclear amide NOE values larger than 0.6 for most of the residues of CesT indicate rigidity of CesT 
especially for the secondary structure regions (Figure 6.23A). Heteronuclear amide NOE values are slightly decreased for the $\mathrm{N}$-terminal residues, the C-terminal region of $\alpha 2$ and in loop regions connecting secondary structure elements. In agreement with the secondary chemical shifts a highly flexible, unstructured region is indicated by the negative heteronuclear amide NOE values for the C-terminal region comprising residues 138 to 156 . Thus the 20 C-terminal residues are unfolded in solution.

\subsubsection{Solution structure of CesT}

The main question that arises about the $2.8 \AA$ resolution $\mathrm{X}$-ray structure of CesT in comparison to the other X-ray structures of TTSS chaperones regards the relevance of the 3D domain-swap. The CesT dimer in the crystal is created by domain-swapping of the small domain comprising residues 1-34 and 139-156 and $\sim 4550 \AA^{2}$ of the surface are buried at the swapped dimer interface. This is significantly more than the $\sim 2000 \AA^{2}$ surface area which is buried in the CesT model structure. The overall shape of both CesT structures and their charge distribution is depicted in Figure 6.8. The shape and the charge distribution of the two structures is very different. Thus,

A

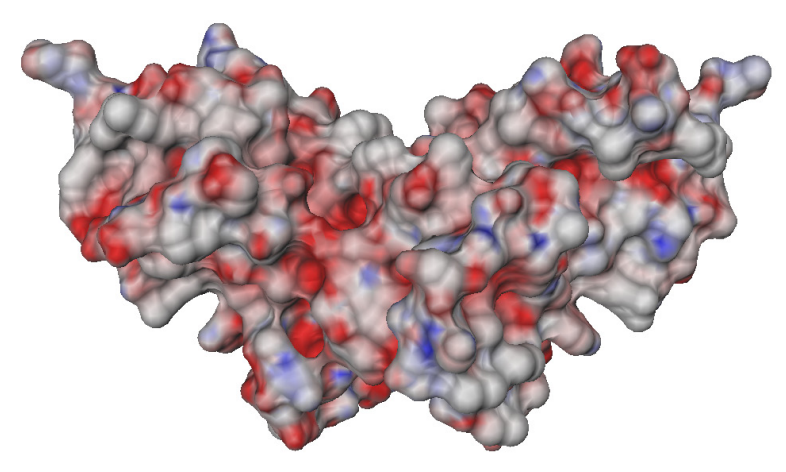

B

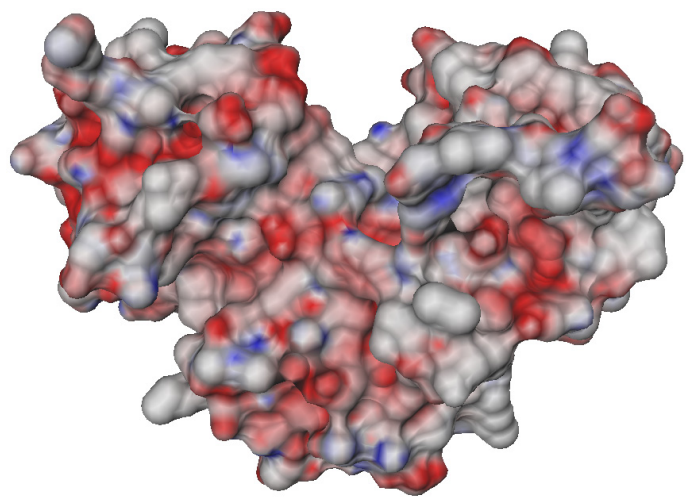

Figure 6.8: Electrostatic surface of the X-ray structure (A) and the model structure (B ) of CesT with positive and negative potentials colored blue and red, respectively.

the orientation of the alignment tensor predicted using the electrostatic module of the software PALES should be very different for the two structures. Comparison of this predicted RDCs with RDCs measured in Pf1 medium can then be used to identify the structure present under solution NMR conditions. 100 backbone amide RDCs, 96 C'N 
RDCs and $71 \mathrm{C}^{\prime} \mathrm{C}_{\alpha}$ RDCs were derived experimentally for each monomer (see 3.5.3). All backbone RDCs that could be determined accurately were used. A few RDCs had to be excluded due to peak overlap and some outlying RDCs in loop regions were excluded. RDCs for the $20 \mathrm{C}$-terminal residues could not be derived as the peaks were missing in the TROSY-based experiments. Best-fitting the observed RDCs to the crystal structure (Figure 6.9A) and to the model structure of CesT (Figure 6.9 $\mathrm{C}$ ) results in a correlation coefficient of 0.772 and 0.930 between experimental and backcalculated RDCs, respectively.

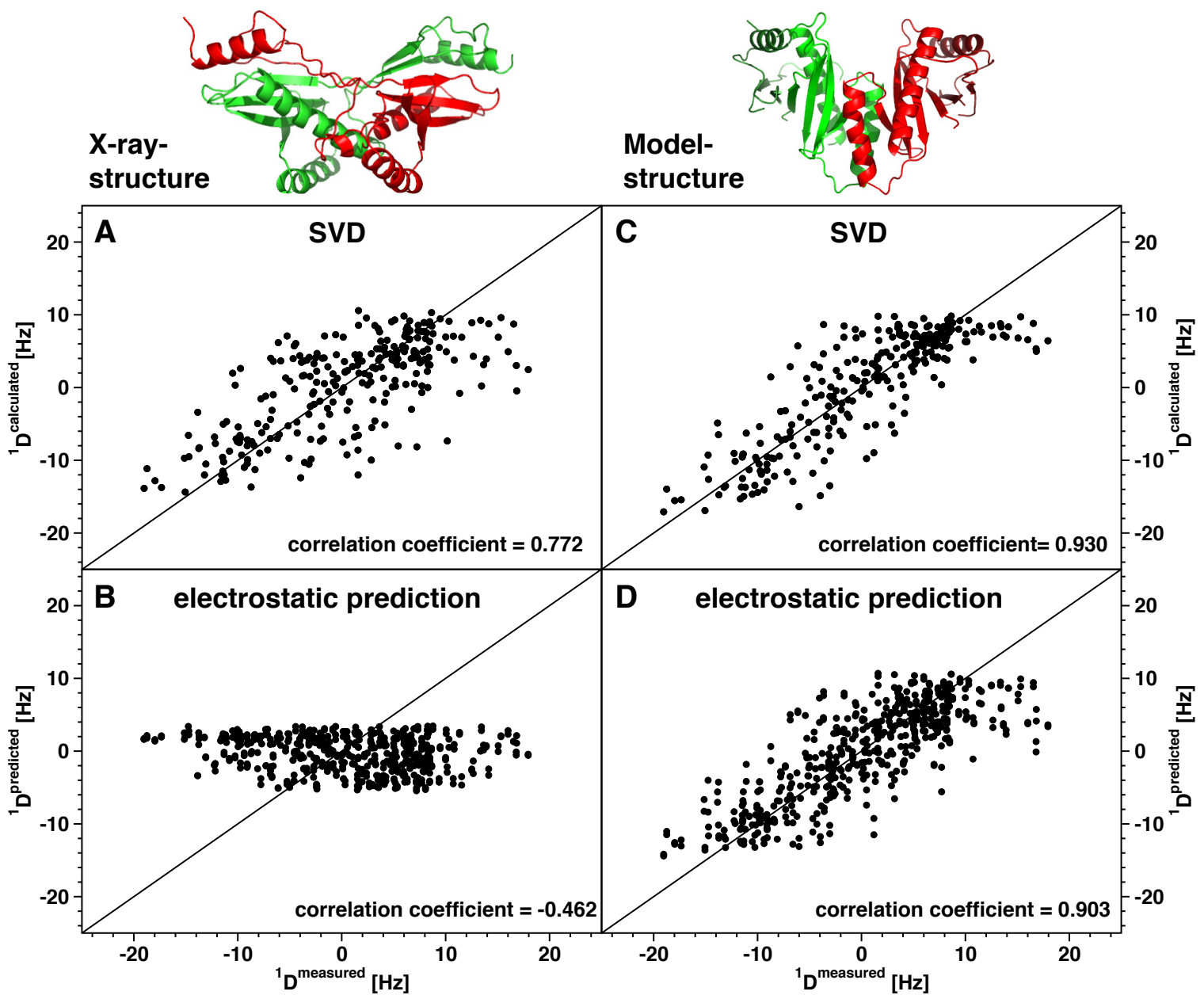

Figure 6.9: Correlation between experimental and predicted or back-calculated HN, C'N and C'C $\mathrm{C}_{\alpha}$ RDCs of CesT: For the X-ray structure and back-calculated RDCs (A) and charge-shape predicted RDCs (B), for the model structure and back-calculated RDCs (C) and charge-shape predicted RDCs (D). C'N and $\mathrm{C}^{\prime} \mathrm{C}_{\alpha}$ RDCs are normalized to those of HN using the appropriate factor $\gamma_{H} \gamma_{N} \mathrm{r}_{H N}^{-3} / \gamma_{A} \gamma_{B} \mathrm{r}_{A B}^{-3}$ where $\mathrm{r}_{A B}$ is the bond distance between $\mathrm{A}$ and $\mathrm{B}$ and $\gamma_{i}$ is the gyromagnetic ratio of $\mathrm{i}$. 
The back-calculated alignment tensor is characterized by $\mathrm{D}_{a}=-5.4 \mathrm{~Hz}$ and $\mathrm{R}=0.09$ for the X-ray structure and by $\mathrm{D}_{a}=-7.5 \mathrm{~Hz}$ and $\mathrm{R}=0.52$ for the model structure. The correlation coefficient from best-fitting for the X-ray structure is 0.16 less than for the model structure. These best-fit results point slightly at the non-swapped model structure as the structure which is present under solution NMR conditions. However the X-ray structure cannot be excluded based on these results.

Therefore, RDCs were predicted using the electrostatic module of PALES with the default charges placed at the positions of the ionizable side chains (see 3.5.3.3). Even though the $10 \mathrm{C}$-terminal residues of the native CesT sequence are missing in the X-ray and model structure, the net charge of the structures used for the prediction is identical to the native situation as two positive and negative charges are present among the $10 \mathrm{C}$-terminal residues. In Figure 6.9B and D, the RDCs predicted with PALES via the charge-shape model for a $\mathrm{NaCl}$ concentration of $0.1 \mathrm{M}$ are plotted against the experimental RDCs for the two structures. With a correlation coefficient of -0.462, the RDCs predicted from the X-ray structure do not fit to the experimental values (Figure 6.9B). As opposed to the X-ray structure, the sign and size of the predicted and experimental RDCs correlate very well for the model structure with a correlation coefficient of 0.9 (Figure 6.9D).

Consequently, a non-swapped structure which is best represented by the model structure exists under solution NMR condition and the 3D domain-swap is most likely a crystallographic artefact.

\subsubsection{Ces $\mathbf{T}_{V 116 R}$ aggregates}

The C-terminal amphipathic $\alpha$-helix is the only conserved feature among TTSS chaperones. Mutation of the central residue Val116 of this $\alpha$-helix of CesT to Arg was demonstrated to abrogate the interaction with Tir [31] and Map [30] raising the question whether the effects of this point mutation are a direct consequence of impaired inter- or intra-helical interactions or a indirect consequence from associated conformational changes elsewhere in CesT.

To address this question, the structure of CesT $T_{V 116 R}$ should be determined and com- 
pared with the CesT structure. However, the ${ }^{15} \mathrm{~N}-\mathrm{HSQC}$ spectrum of $\mathrm{CesT}_{V 116 R}$ reveals a strongly aggregated protein and $\mathrm{T}_{2}$ as well as $\mathrm{T}_{1 \rho}$ relaxation times are halved compared to CesT. Changing the buffer and decreasing the concentration of CesT $_{V 116 R}$ to $100 \mu \mathrm{M}$ did not reduce the level of aggregation. These in vitro results indicate a strong effect of the point mutation V116R on the stability and probably the structure of CesT. Therefore, it is unlikely that $\operatorname{CesT}_{V 116 R}$ can provide insights into the TTSS mechanism. It was therefore not used for further studies.

\subsubsection{Structural characterisation of Tir and Map}

As for most TTSS effectors, the CBD of Map and Tir has been shown to be located within the first $\sim 100$ amino-terminal residues. For Tir, it was demonstrated by Abe et al. [167] that residues 50-100 are essential for CesT binding. For Map it was demonstrated by Creasey et al. [30] that CesT interacts within the 101 amino-terminal residues and that neither residues 1-51 nor 51-101 are sufficient for the interaction. Therefore, the C-terminally truncated fragments of Tir and Map were mainly studied here.

A degradation product of Map101, coexpressed and copurified with CesT, was detected by SDS-PAGE (see 3.3.8). Analysis of this CesT/Map101 sample by ESI-MS (see 3.4.3) pointed at a mass of $9157.71 \pm 0.60$ for the degradation product (Figure 6.10. Further analysis by Edman degradation (see 3.3.9) revealed only two sequences with the intact N-termini of CesT and Map. Combining these experimental results, the degration product of Map101 was assessed as residues 1-84 of Map (Map84). This shortened fragment was used for further studies.

All differently expressed Map constructs are insoluble in aqueous buffers. Therefore, the protein was prepared in the presence of $8 \mathrm{M}$ urea via a single $\mathrm{Ni}^{2+}$-column and refolded by dialysis against NMR-buffer (see 6.2.3.2). In this way, it was possible to produce His ${ }_{7}$-Map84 at a concentration of $\sim 100 \mu \mathrm{M}$ in the CesT NMR-buffer (Table 2.6 with $\sim 5 \%$ impurities.

In order to estimate the secondary structure content of the N-terminal regions of Tir and Map, far-UV CD spectra of $\mathrm{His}_{7}$-Map84 and Tir108 in NMR buffer were ac- 


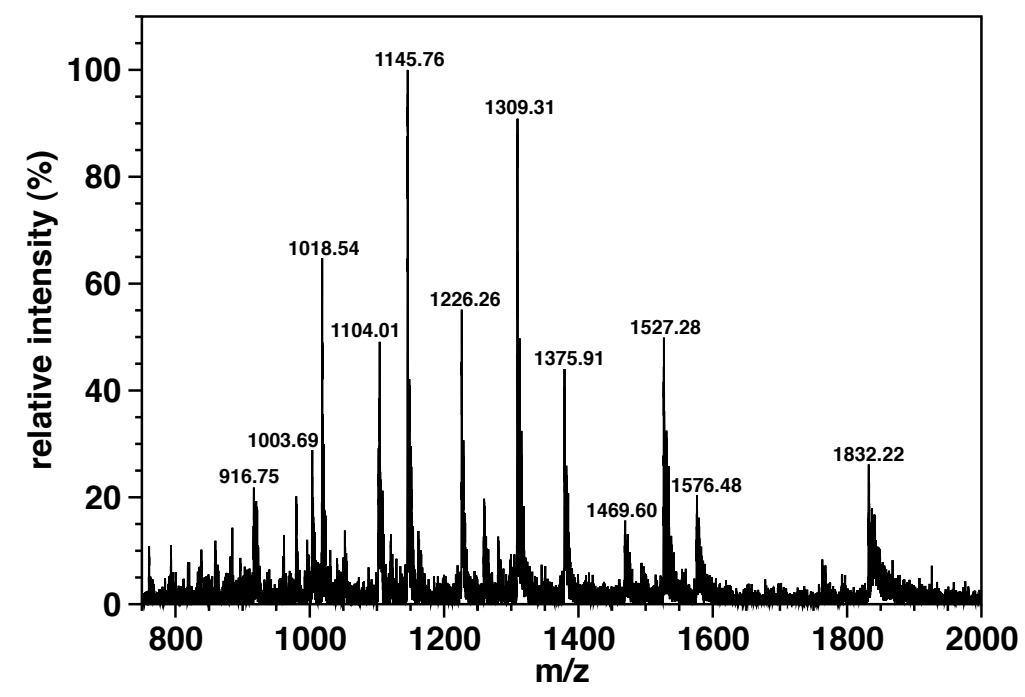

Figure 6.10: ESI-mass spectrum of coexpressed and copurified CesT/Map101 in $20 \mathrm{mM} \mathrm{NH} 4 \mathrm{OAc}$. The signals at a $\mathrm{m} / \mathrm{z}$-ratio of 1832.22, 1527.28, 1309.31, 1145.76, 1018.54 and 916.75 correspond to a 5 - to 10-fold positively charged degradation product of Map101. The less intense signals at a m/z-ratio of 1576.76, 1375.91, 1226.26, 1104.01 and 1003.69 belong to 7- to 11-fold positively charged Map101.

quired (Figure 6.11). Both proteins show far-UV CD spectra typical of an unfolded

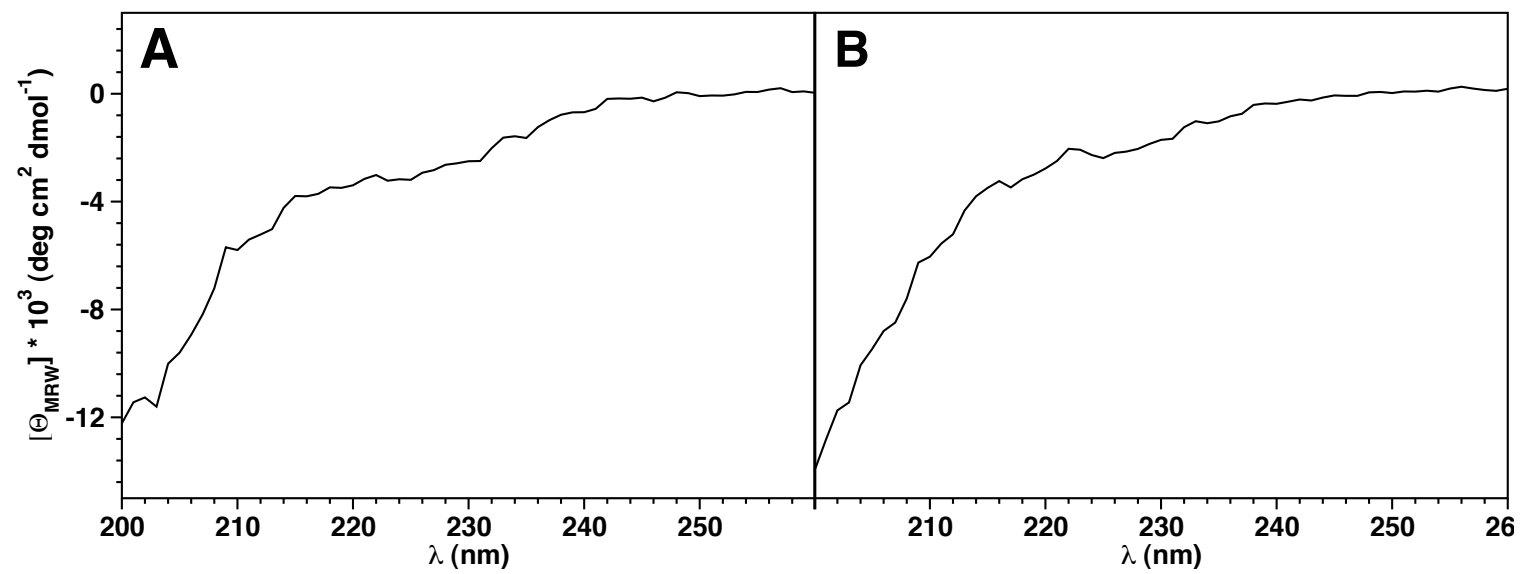

Figure 6.11: Far-UV-CD spectra in NMR buffer at $303 \mathrm{~K}$ of His7-Map84 (A) and Tir108 (B).

polypeptide chain with a largely negative ellipticity around $200 \mathrm{~nm}$ and a negligible ellipticity at $222 \mathrm{~nm}$ [168]. The unfolded nature of His ${ }_{7}$-Map84 and Tir108 is additionally evidenced by the absence of dispersion in the proton dimension of the ${ }^{15}$ N-HSQC spectra of free His 7 -Map84 (Figure 6.15) and Tir108 (Figure 6.13). 


\subsubsection{Crystallisation of the CesT/Map complex}

Limited proteolysis (see 3.3.10) was performed in order to detect the proteolytically stable core of Map84 which is protected by CesT. This approach has already been successfully applied to reveal the proteolytically stable core for the homologous chaperone/effector complexes of SptP when bound to SicP [159], of YopE when bound to SycE [162], of YopN when bound to SycN-YscB [163] and of SipA when bound to InvB [161]. However, in the case of the CesT/Map84 complex the chaperone was proteolysed even at low protease concentrations and short reaction times. Only a CesT fragment of about $15 \mathrm{kDa}$ proved stable when digested with elastase. The digestion was completed at a elastase:protein ratio of 1:1000 after a reaction time of $2 \mathrm{~h}$. Since Map84 was revealed as the shortest N-terminal fragment of Map which binds CesT, crystallisation of Map84 in complex with full length CesT was attempted.

Coexpressed and copurified CesT/Map84 complex after gel filtration (see 3.3.3) was concentrated to $\sim 10 \mathrm{mg} / \mathrm{ml}$ for crystallisation trials using 372 different conditions from 6 crystallisation screens (see 2.5). As the C-terminal residues of CesT were detected as highly flexible by NMR (Figure 6.23) and in vivo studies of CesT $\mathrm{T}_{E 142 G}$ demonstrated unchanged effector binding [153], crystallisation trials were also performed with a Ces $\mathrm{T}_{1-135} / \mathrm{Map} 84$ complex. Small crystals were obtained at $0.2 \mathrm{M}$ $\mathrm{NH}_{4} \mathrm{OAc}, 0.1 \mathrm{M} \mathrm{Na}_{3}$-citrate $\mathrm{pH} 5.6$ and 30 \% PEG 4000 and were improved by a grid screen and increasing the complex concentration to $20 \mathrm{mg} / \mathrm{ml}$. Crystals grown in hanging drops at the final conditions, $0.2 \mathrm{M} \mathrm{NH}_{4} \mathrm{OAc}, 0.1 \mathrm{M} \mathrm{Na}_{3}$-citrate $\mathrm{pH} 5.7$ and $26 \%$ PEG 4000, were washed and shown to contain both complex components by SDS-PAGE. Nevertheless the crystals did not diffract. Trials to improve them were not successful so far.

\subsubsection{Backbone assignment and secondary structure of Tir108}

Backbone and $\mathrm{C}_{\beta}$ resonances of Tir108 were assigned. The assignment is complete with the exception of Pro17 and Pro20 which are followed by a proline residue, of the amide ${ }^{1} \mathrm{H}$ and ${ }^{15} \mathrm{~N}$ of His14 and the carbon resonances of Met1 (Table B.10). The completeness and confirmation of the assignment was achieved with the help of a 3D 
HNN experiment which establishes correlations between amide protons and amide nitrogen atoms of both neighbouring residues [169].

The secondary chemical shifts of the $\mathrm{C}_{\alpha}$ and C' resonances, derived as described in 3.5.2, is another evidence for the unfolded state of Tir108 as all secondary chemical shift values are small (Figure 6.12).

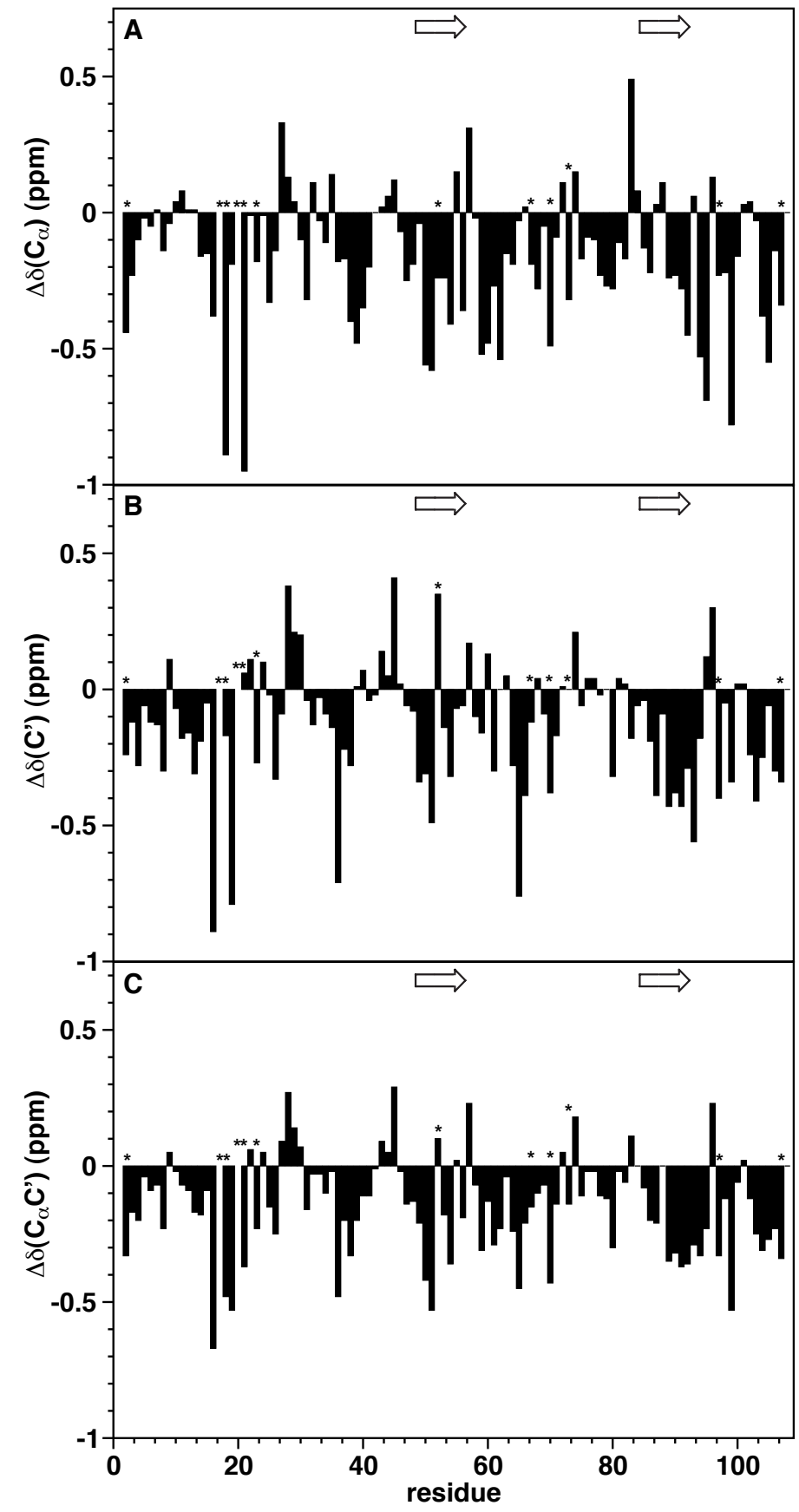

Figure 6.12: Secondary chemical shifts of Tir108 in $50 \mathrm{mM}$ potassium phosphate buffer $\mathrm{pH}$ $6.8,100 \mathrm{mM} \mathrm{NaCl}$ and $5 \mathrm{mM}$ DTT at $293 \mathrm{~K}$. Secondary chemical shifts for $\Delta \mathrm{C}_{\alpha}(\mathrm{A}), \Delta \mathrm{C}^{\prime} \quad(\mathrm{B})$ and averaged for $\Delta \mathrm{C}_{\alpha}$ and $\Delta \mathrm{C}^{\prime}(\mathrm{C})$ plotted against the residue number. The average shifts in $(\mathrm{C})$ were calculated as $\left[3 \Delta \delta\left(\mathrm{C}^{\alpha}\right)\right.$ $\left.+4 \Delta \delta\left(\mathrm{C}^{\prime}\right)\right] / 7$. The two proposed $\beta$-motifs for Tir108 are indicated [161] and proline residues are labeled with an asterisk.

A rapid exchange between extended and $\beta$-structures is suggested by the negative 
secondary chemical shift values for 85 out of the 104 residues. In agreement with a $\beta$-motif which is proposed as the only conserved targeting motif in type three secretion [161] and with secretion of Tir in the absence of CesT [29], the $\beta$-structure tendency indicates a preformed secretion signal for free Tir.

\subsubsection{Effects of CesT binding on Tir and Map}

To monitor chemical shift changes and potentially induced secondary structure formation of Tir and Map upon CesT binding, NMR titration experiments were performed (see 6.2.4). The start and end ${ }^{15} \mathrm{~N}-\mathrm{HSQC}$ spectra for the titration of ${ }^{15} \mathrm{~N}$-Tir108 with CesT are shown in Figure 6.13. Around $30 \%$ of the peaks of Tir108 disappear

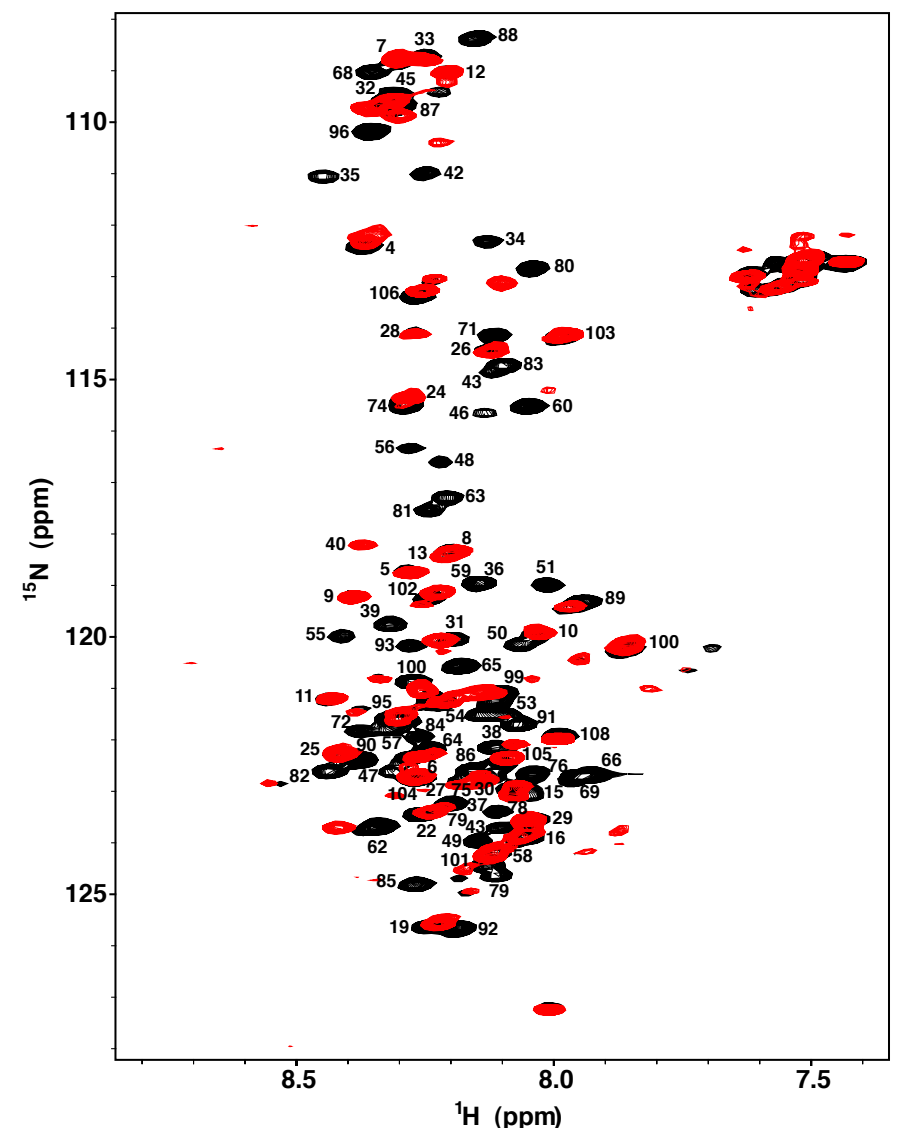

Figure 6.13: Overlay of ${ }^{15} \mathrm{~N}-\mathrm{HSQC}$ spectra of free Tir108 (black) and Tir108 bound to CesT (red) measured at $800 \mathrm{MHz}$ and $293 \mathrm{~K}$. Resonance assignments for free Tir108 are indicated with residue numbers.

upon complex formation. Changing the temperature alone or in combination with a decreased $\mathrm{pH}$ did not increase the number of detectable signals even with a TROSY 
pulse sequence. Nevertheless, the average amide chemical shift perturbation of Tir108 upon CesT binding was calculated (see 3.5.5) and $\Delta \delta_{H N}$ values of the disappeared residues were assigned to 0.5 (Figure 6.14).

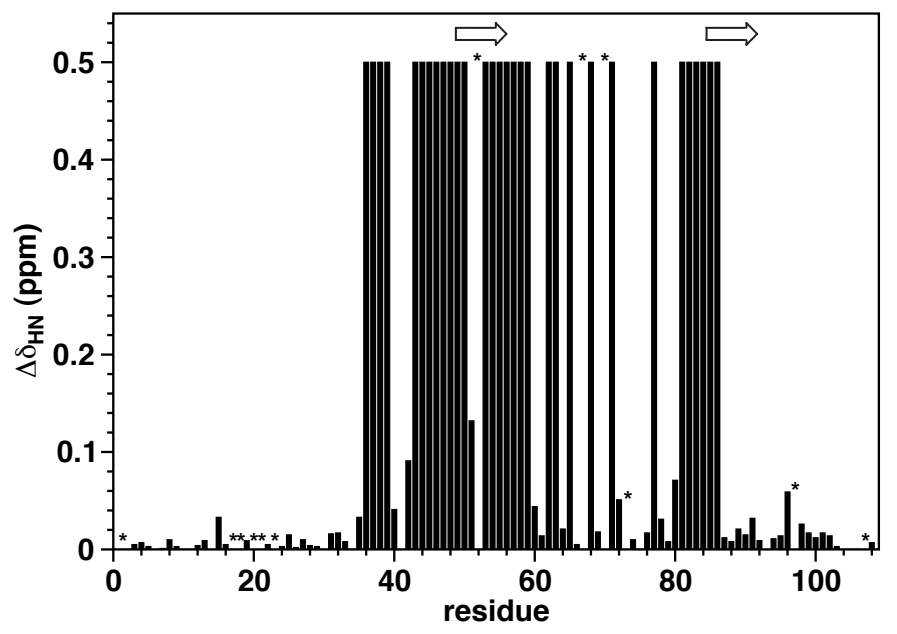

Figure 6.14: Chemical shift changes of Tir108 upon formation of the CesT/Tir108 complex. For vanished peaks $\Delta \delta_{H N}$ values were set to 0.5 . Proposed $\beta$-motifs for Tir108 are indicated [161] and proline residues are labeled with an asterisk.

This way the region comprising residues 35-86 could be identified as most affected by interaction with CesT and composes most likely the CBD of Tir. In Lilic et al. [161], the $\beta$-motif was proposed for residues 49-56 and 85-92. The $\beta$-motif for residues 49-56 is supported by the changes of chemical shift changes of Tir108 upon CesT/Tir108 complex formation (Figure 6.14). For residues 85-92, which were proposed to form the other $\beta$-motif, strong effects could only be found for residues 85 and 86 . In this region, residues 80-86 were most strongly affected by CesT-binding (Figure 6.14) and may form the second $\beta$-motif.

${ }^{15} \mathrm{~N}-\mathrm{HSQC}$ spectra of free His 7 -Map84 and bound Map84 are depicted in Figure6.15. Like in the ${ }^{15} \mathrm{~N}-\mathrm{HSQC}$ of free Tir108 and characteristic for unfolded proteins, amide proton signals of free Map84 are limited to a region between 7.75 and 8.5 ppm. However, unlike for CesT-bound Tir108 (Figures 6.13), only 12\% of the Map84 signals are missing, four amide proton signals appear lowfield to $8.5 \mathrm{ppm}$ and six signals appear highfield to $7.75 \mathrm{ppm}$ (Figures 6.15). Altogether the dispersion of signals is increased by 2 ppm upon CesT/Map84 complex formation therewith suggesting ter- 


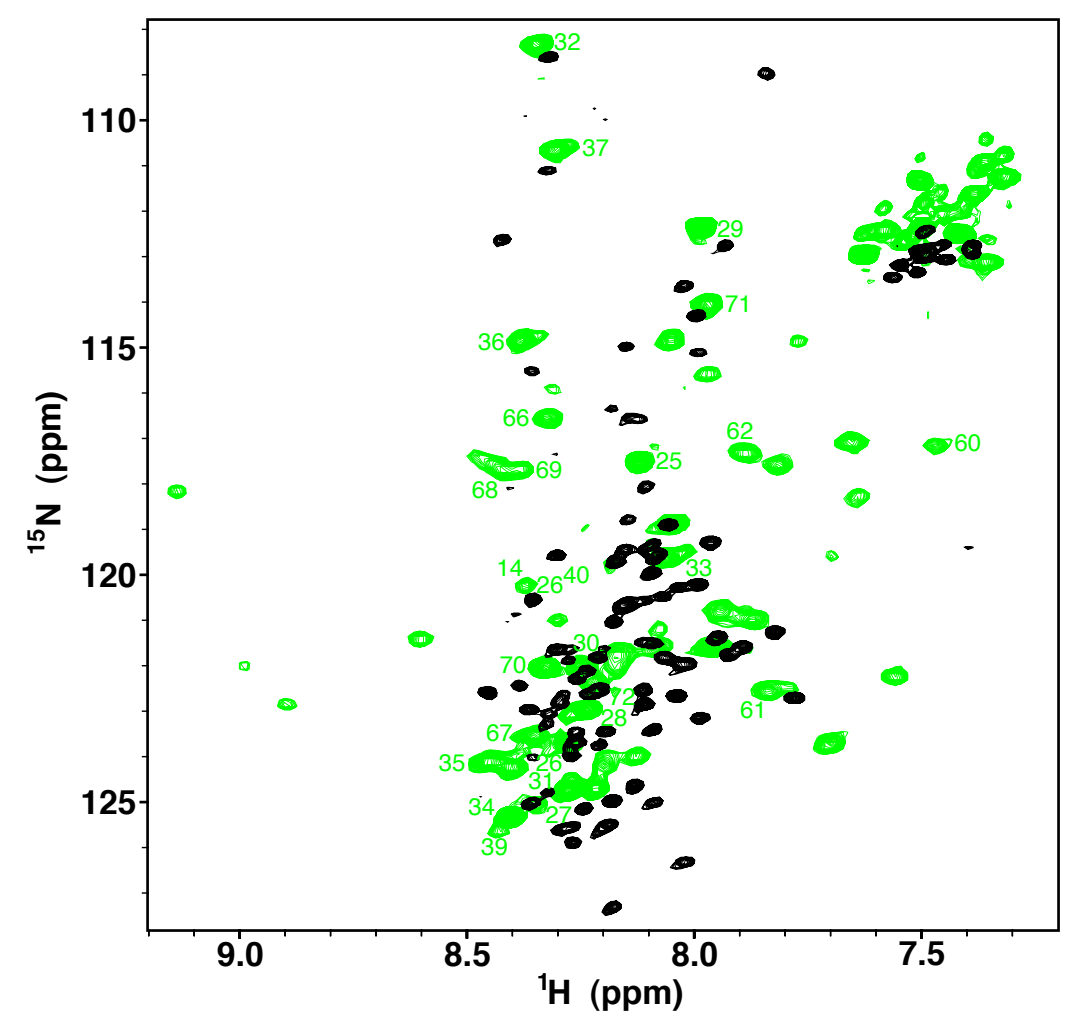

Figure 6.15: Overlay of ${ }^{15} \mathrm{~N}-\mathrm{HSQC}$ spectra of free His7-Map84 (black) and Map84 bound to CesT (green) at $303 \mathrm{~K}$. Assigned residues in the bound state are indicated by green numbers.

tiary structure formation for a part of the CBD of Map.

Map84 in complex with CesT was partially assigned. Assigned resonances are indicated in Figure 6.15 by green numbers and the assigned chemical shifts are tabulated in the Appendix Table B.11.

Broad signals of bound Map84 (Figure 6.15) and disappearing signals upon CesT binding especially for Tir108 (Figure 6.13) point at motions in the intermediate exchange regime. This disagrees with the $\mathrm{K}_{d}$ of $18.51 \pm 1.97 \mathrm{nM}$ for the CesT/Tir and the $\mathrm{K}_{d}$ of $16.89 \pm 6.62 \mathrm{nM}$ for the CesT/Map complex which were determined using an enzyme-linked immunosorbent assay (ELISA)-based approach [30]. As the full length effector proteins were used for the $\mathrm{K}_{d}$ determination, missing interactions between the C-terminal effector regions and the chaperone may explain the decreased affinity. This is suggested by signals outside the CBD of Tir which are affected upon CesT binding (Figure 6.16). However, a decreased affinity due to missing interac- 
tions between the C-terminal effector regions and the chaperone is unlikely for the CesT/Map84 complex because its copurification including a gel filtration step is possible. A decreased affinity between CesT and Map84 would probably lead to their separation.

\subsubsection{Effect of CesT on effector regions outside the CBD}

The full N-terminal cytoplasmic part of Tir, referred to as TirN, and full length Map were prepared to unravel a potential unfolding activity of CesT on effector regions outside the CBD. As already observed for the C-terminally truncated proteins, only TirN is solubly expressed and can be concentrated to $\sim 1 \mathrm{mM}$. All peaks which are present in the ${ }^{15} \mathrm{~N}-\mathrm{HSQC}$ spectrum of Tir108 overlay well with peaks of TirN. Additional signals in the ${ }^{15} \mathrm{~N}-\mathrm{HSQC}$ spectrum of TirN are highly dispersed (Figure 6.16). The signals low field to $8.5 \mathrm{ppm}$ show that the C-terminal part of TirN is folded. The dispersion of TirN signals remains unchanged upon CesT binding (Figure 6.16) contradicting an unfolding activity of CesT on residues outside the CBD. This finding agrees with results from isothermal titration calorimetry which show a retained intimin binding activity in the presence of CesT [154]. However, upon CesT binding, there are at least six residues outside the CBD of Tir which disappear or show significantly decreased intensity and two residue signals which are shifted. This suggests that residues of Tir outside the CBD may contribute to the interaction with CesT.

\subsubsection{Effects of Tir and Map binding on CesT}

In order to explore the effector binding region on the surface of CesT, ${ }^{2} \mathrm{H}^{15} \mathrm{~N}$-labeled CesT was titrated with Tir108 and His 7 -Map84 (see 6.2.4). This approach allows for the identification of the binding surface of CesT specific for Tir and Map based on chemical shift perturbation. Start and end ${ }^{15} \mathrm{~N}-\mathrm{TROSY}-\mathrm{HSQC}$ spectra for the CesT/Tir108 and the CesT/His ${ }_{7}$-Map84 titration are shown in Figure 6.17.For both complexes, slow exchange is observed between free and bound CesT and almost all peak positions are changed. In order to reassign the backbone resonances of CesT in 


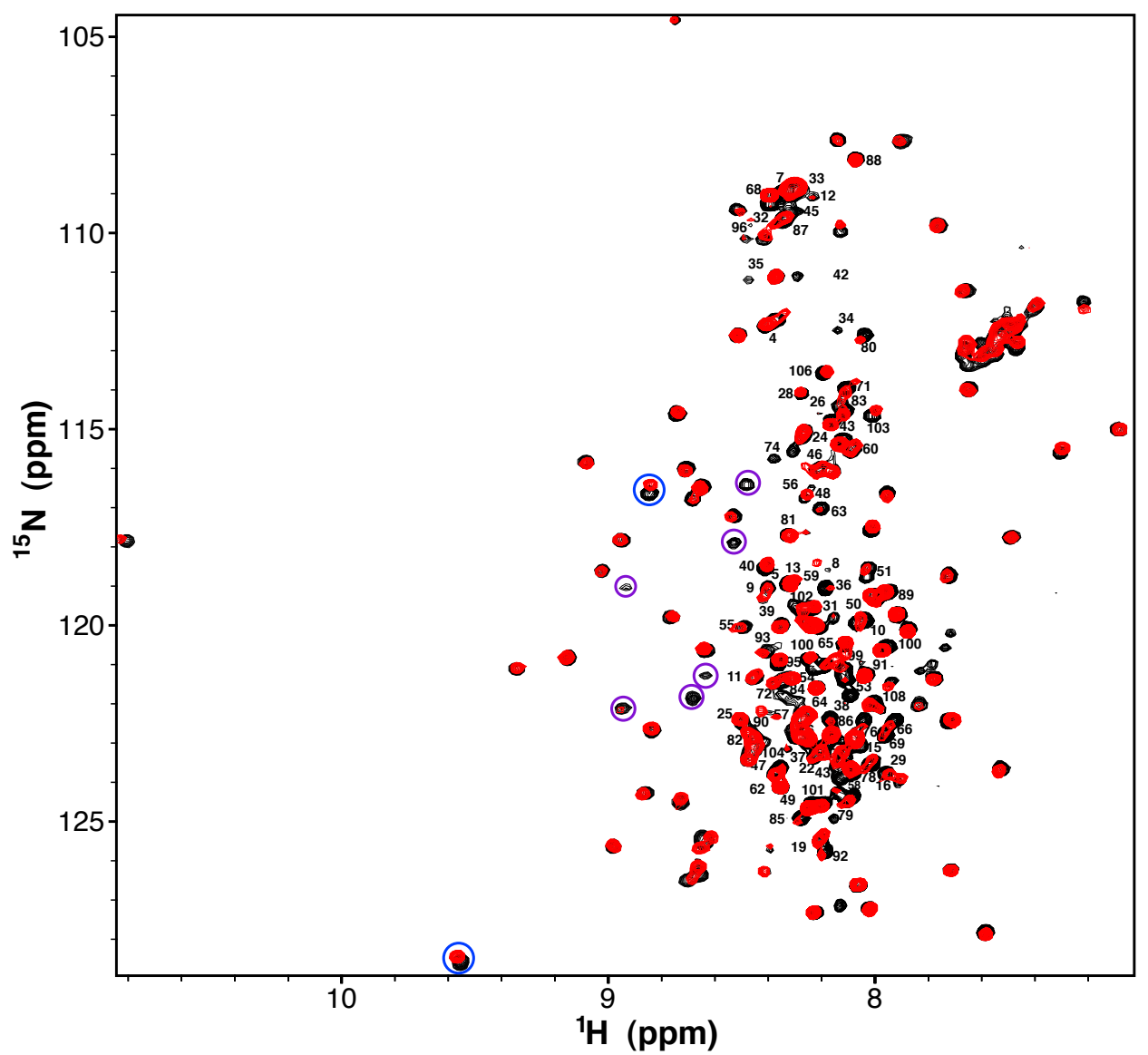

Figure 6.16: Overlay of ${ }^{15} \mathrm{~N}-\mathrm{HSQC}$ spectra of TirN and TirN bound to CesT at $303 \mathrm{~K}$. Spectra were measured at $800 \mathrm{MHz}$. Peaks of Tir108 superimpose well with TirN and are labeled with the residue number. Signals which do not belong to Tir108 and are affected upon addition of CesT are indicated by circles: Violet circles mark peaks which show significantly decreased intensity or which vanished and blue circles mark shifted peaks.

complex with Tir108 as well as in complex with Map84, 3D triple resonance spectra were acquired for the two different ${ }^{2} \mathrm{H},{ }^{15} \mathrm{~N},{ }^{13} \mathrm{C}$-CesT/effector complex samples (Appendix Table B.5 and B.4. $93 \%$ of the backbone amide resonances, $96 \%$ of the $\mathrm{C}_{\alpha}$ and $\mathrm{C}^{\prime}$ resonances and $95 \%$ of the $\mathrm{C}_{\beta}$ resonances could be reassigned for CesT in complex with Map84. To analyse the structural changes of CesT upon Map84 binding, the secondary chemical shifts were calculated and subtracted from the secondary chemical shift of free CesT (Figure 6.18). No secondary structure changes were observed for CesT upon Map binding. Furthermore, averaged amide chemical shift perturbations were determined (see 3.5.5) in order to map the binding surface 


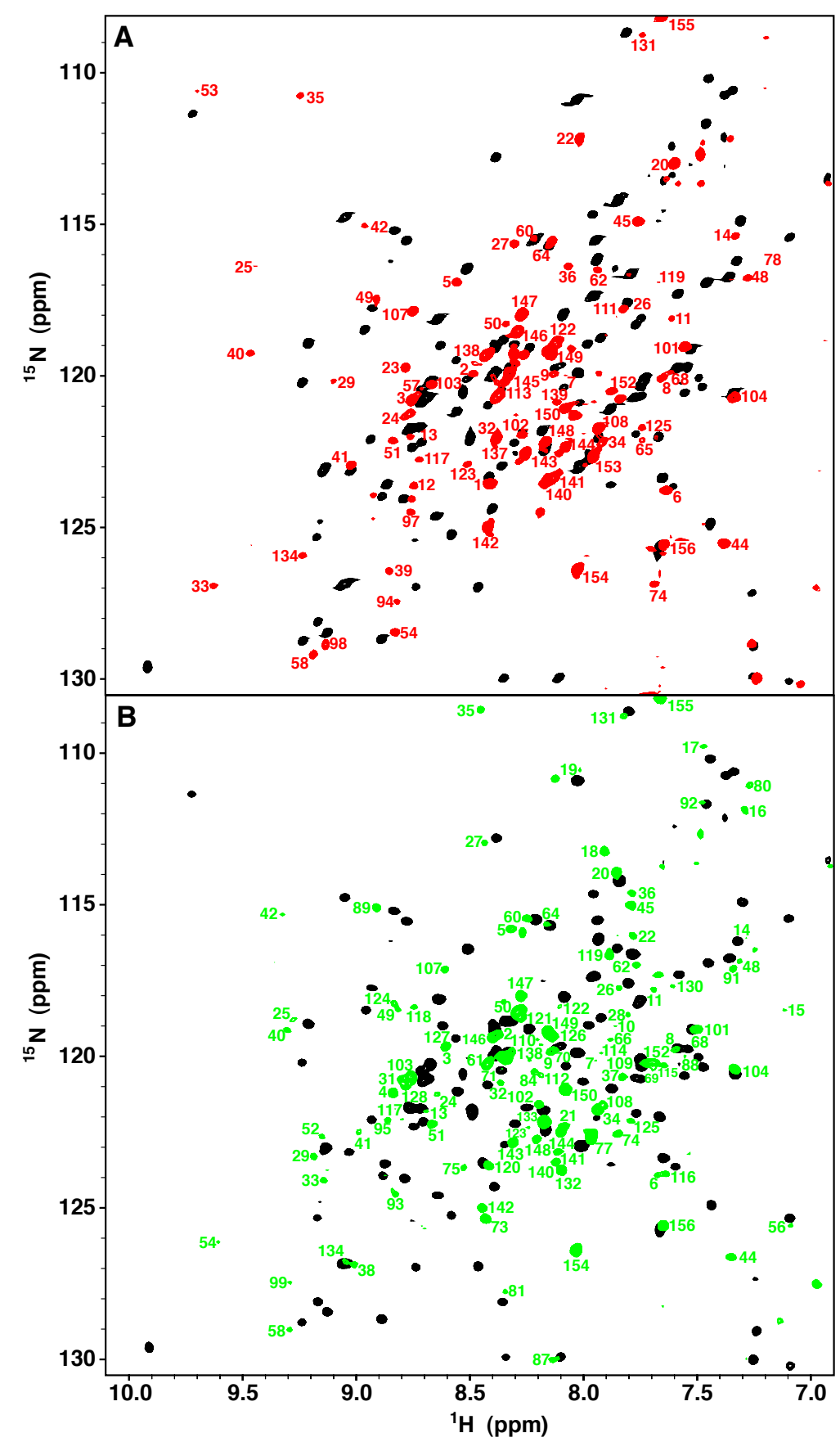

Figure 6.17: Overlay of ${ }^{15} \mathrm{~N}-\mathrm{TROSY}-\mathrm{HSQC}$ spectra of free CesT (black) and effectorbound CesT: (A) Red peaks correspond to CesT in complex with Tir108 and (B) green peaks correspond to CesT in complex with Map84. Assigned peaks are indicated by the sequence number.

for Map84 on CesT (Figure 6.19). Strongly shifted residues in the chemical shift perturbation plot (Figure 6.19), in the structure-based sequence alignment of TTSS chaperone (Figure 6.20) and on the surface of CesT (Figure 6.21) are colored identi- 


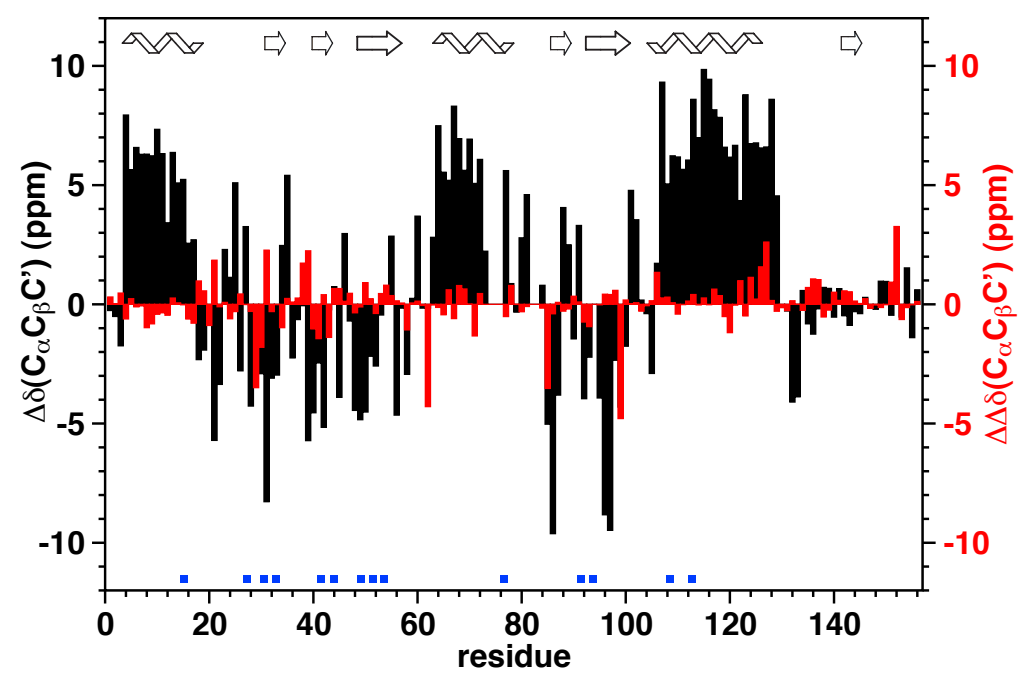

Figure 6.18: Secondary structure of CesT bound to Map84. Secondary chemical shifts $\Delta \mathrm{C}_{\alpha}-\Delta \mathrm{C}_{\beta}+\Delta \mathrm{C}^{\prime}$ of CesT bound to Map84 (black) and secondary chemical shift difference for $\Delta \mathrm{C}_{\alpha}-\Delta \mathrm{C}_{\beta}+\Delta \mathrm{C}^{\prime}$ between free and bound CesT (red) plotted against the residue number. Secondary structure regions of the X-ray structure are indicated. Residues proposed to form hydrophobic patches are labeled by blue squares [162].

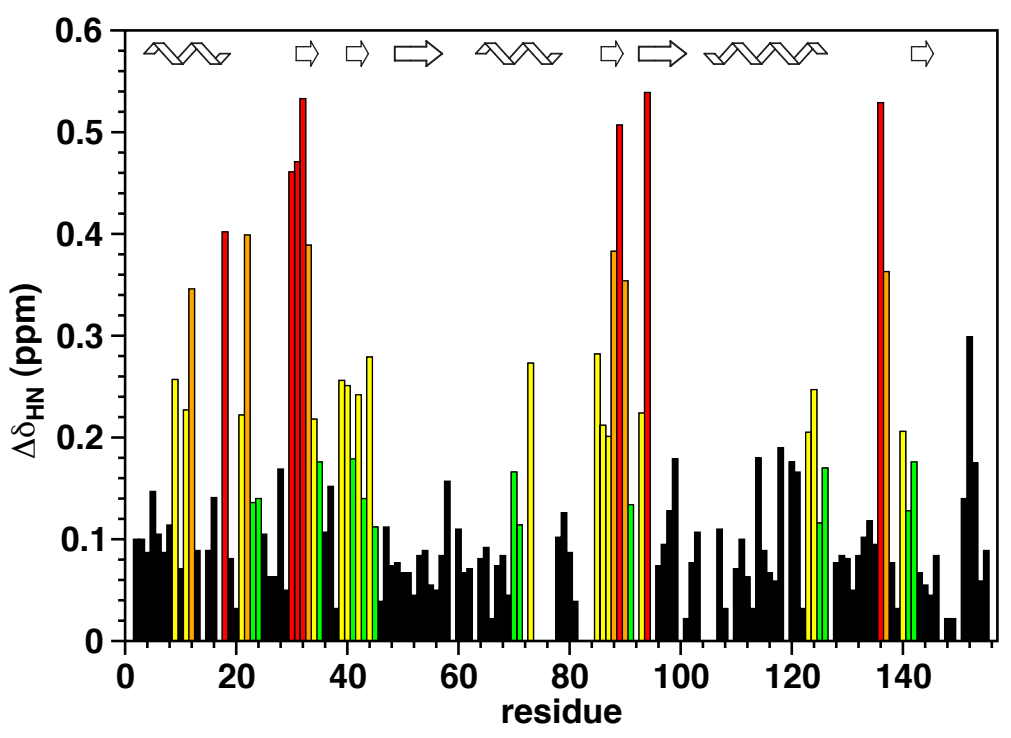

Figure 6.19: Chemical shift changes of CesT upon formation of the CesT/Map84 complex. Residues shifted $>0.4 \mathrm{ppm},>0.3 \mathrm{ppm}$ and $>0.2 \mathrm{ppm}$ are colored red, orange, yellow and green, respectively, and residues shifted $>0.1 \mathrm{ppm}$ are colored green if an adjacent residue is also shifted $>0.1 \mathrm{ppm}$. Secondary structure regions of the X-ray structure are indicated.

cally with red for shifts $>0.4 \mathrm{ppm}$, orange for shifts $>0.3 \mathrm{ppm}$ and yellow for shifts $>0.2 \mathrm{ppm}$. Green indicates residues with shifts $>0.1 \mathrm{ppm}$ that have at least one adjacent residue shifted by $>0.1 \mathrm{ppm}$. All strongly shifted peaks $(>0.3 \mathrm{ppm})$ are 


CesT
SigE
SicP
SycE
Sych
SycT
InvB
Spa15
AvrPphFORF 1

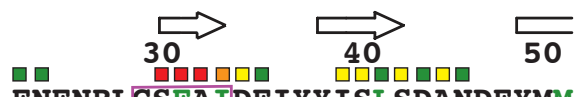
FNENRLCSFAIDEIYYISLSDANDEYMM PLLIID DGIQVYFNE SDHTLE QCLLLIDSDIFTSIEAK DDIWL VIGVKVG EFACHITEHPVGQIL NELGHGAVT IDKIWVVHLAP INEKELV DVYTFEVDG HIQVLIACYHQQWV MOTTFTELMQQLFLKLGLNHQVNEN MOHLDIAELVRSALEVSGCDPSLIGGIDSHSTIVLDI FALPSICISVKDDDVW MSNINLVQLVRDSLFTIGCPPSIITDLDSHSAITISID SMPAINIALVNEQVM MKNSFDRLIDGLAKDYGMPGFPEKKHEHEVYCFEEKEV S IRIYQDKFKWV

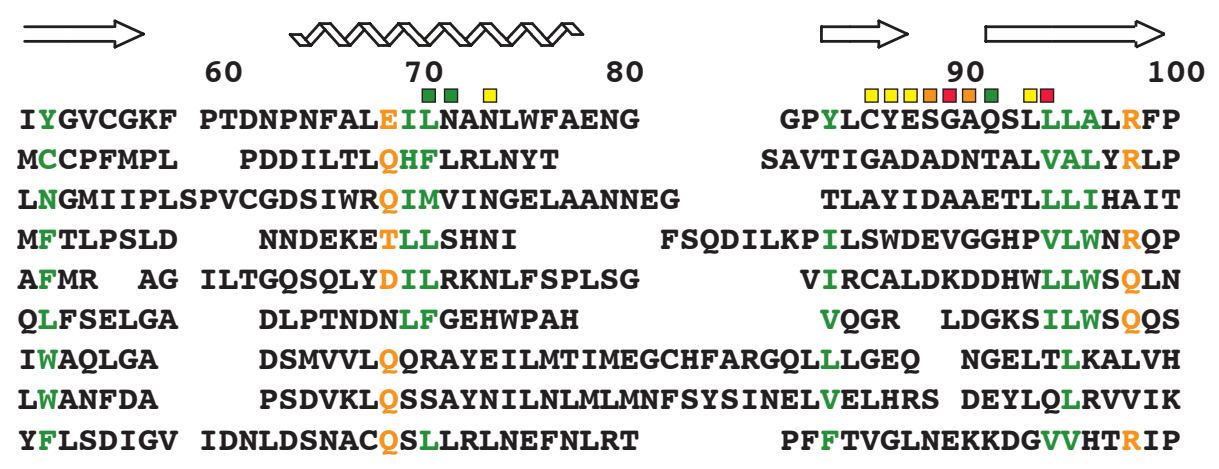
CesT
SigE
SicP
SYCE
SYCH
SycT
InvB
Spa15
LDDAT PEKLENETEVVVKSMENLYLVLHNQGITLENEHMKIEEISSSDNKHYYAGR
QT ST EEEALTGFELFISNVKQLKEHYA
D LTN TYHIISQLESFYNQQEALKNILQEYAKV
LNSLD NNSLYTQLEMLVQGAERLQTSSLISPPRSFS
INDTS GTQLASVLTSLVDKAVTLSCEPTMKKEEDDHRPSSSH
SLVGLD IDEMQAWLERF IDDIEQRKEPQNTKFQPNSTSPILFI
HPDFLSDGEKF STALNGS Y YNYLEVF SRSLMR
DDYVHDGIVF AEILHEF YQRMEILNGVL
AvrPphFORF 1
LLNLD NVEMRRVFEALINLSGEV
KKTFGFV

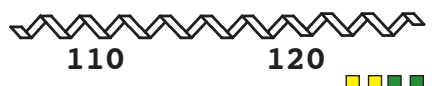

Figure 6.20: Structure-based sequence alignment of nine TTSS chaperones. Conserved residues are highlighted: green for hydrophobic and orange for polar residues. Differently colored squares above the sequence indicate residues as in Figure 6.19. The magenta boxes point at the two regions which are assumed to interact with the $\beta$-motif of the TTSS effector [161]. Secondary structure elements of CesT in the X-ray structure are displayed above the sequence.

hydrophobic, thereby indicating the hydrophobic character of the CesT/ Map84 interaction. The only exceptions are the strongly shifted residues Asn136 and Glu137. These shifts suggest a contribution of electrostatic interactions between Map84 and Glu137 of CesT to the complex formation. The $\sim 0.2 \mathrm{ppm}$ shifted residues 151-153 were not colored in Figures 6.19, 6.20 and 6.21 because they are not present in the X-ray structure. Altogether, residues exhibiting strong shifts correspond well to the 
A

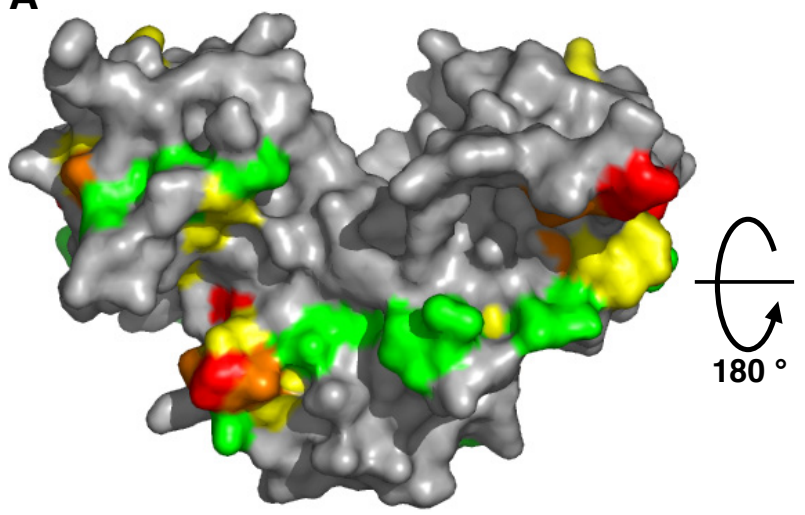

C

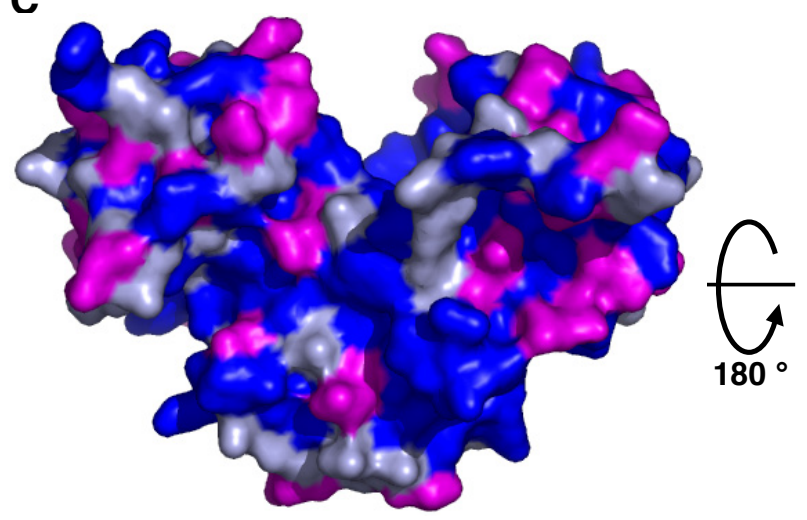

B

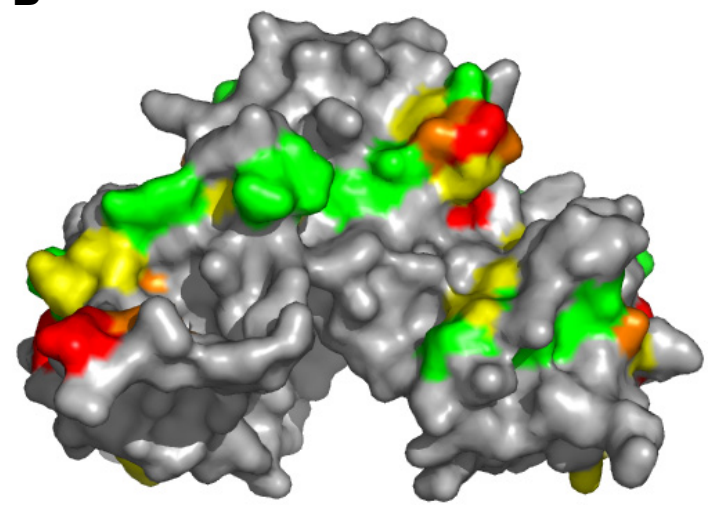

D

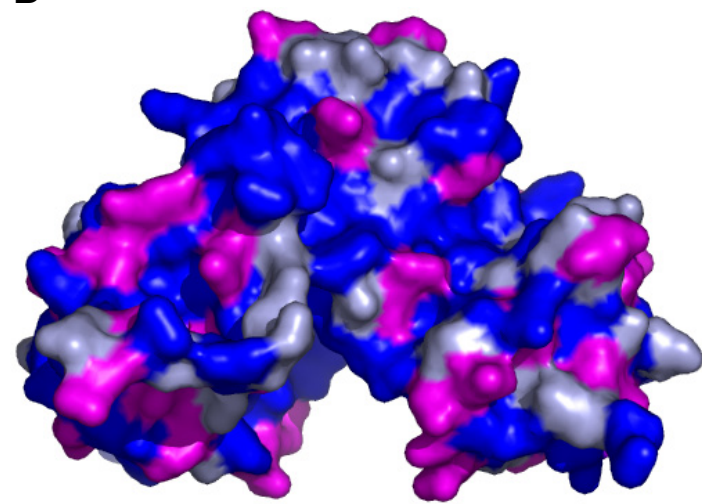

Figure 6.21: Side views of the surface representation of CesT. (A,B) Chemical shift changes upon Map84 binding mapped to the surface of CesT. Residues colored green, yellow, orange and red are identical to Figure 6.19. (C,D) Residue properties mapped to the surface of CesT with charged, polar and hydrophobic residues colored magenta, lightblue and blue. (A,C) are rotated by $180^{\circ}$ around the x-axis to generate (B,D).

hydrophobic patches proposed by Birtalan et al. [162]. The binding mode seems to be mainly identical to the homologous chaperone/effector complexes with known structure. Large hydrophobic surfaces are buried in these homologous chaperone/effector complexes and CesT achieves the effector recognition in a similar way through numerous hydrophobic interactions. The mapping of the shifted residues onto the 3D surface reveals a narrow band of contact around the circumference of the chaperone. This path involves almost exclusively hydrophobic residues, revealing that the interaction is largely hydrophobic in nature. A few additional auxiliary points are also provided by polar or charged interactions and are shifted $<0.3 \mathrm{ppm}$ (Figure 6.21). For residues 29-33 and 113-117, which are indicated by magenta boxes in Figure 6.20, the formation of an intermolecular $\beta$-sheet with the effector has been proposed [161]. 
This could be experimentally verified by the chemical shift analysis for residues 29-33, which are most affected upon Map binding. However, the chemical shift perturbation for residues 113-117 is not more significant than for other regions of CesT.

For CesT in complex with Tir108, $\sim 67 \%$ of the backbone amide resonances were reassigned (Appendix Table B.9). A reason for the missing reassignments is the quality of the $3 \mathrm{D}$ spectra which is inferior to the $3 \mathrm{D}$ spectra recorded for CesT in complex with Map84. An almost complete reassignment may be achieved with a better CesT/Tir108 sample and with additional 3D spectra. Among the $\sim 67 \%$ assigned backbone amide resonances, residues with the largest shifts also presented large shifts for CesT in complex with Map84 (Figure 6.22). Therefore, it is likely that the

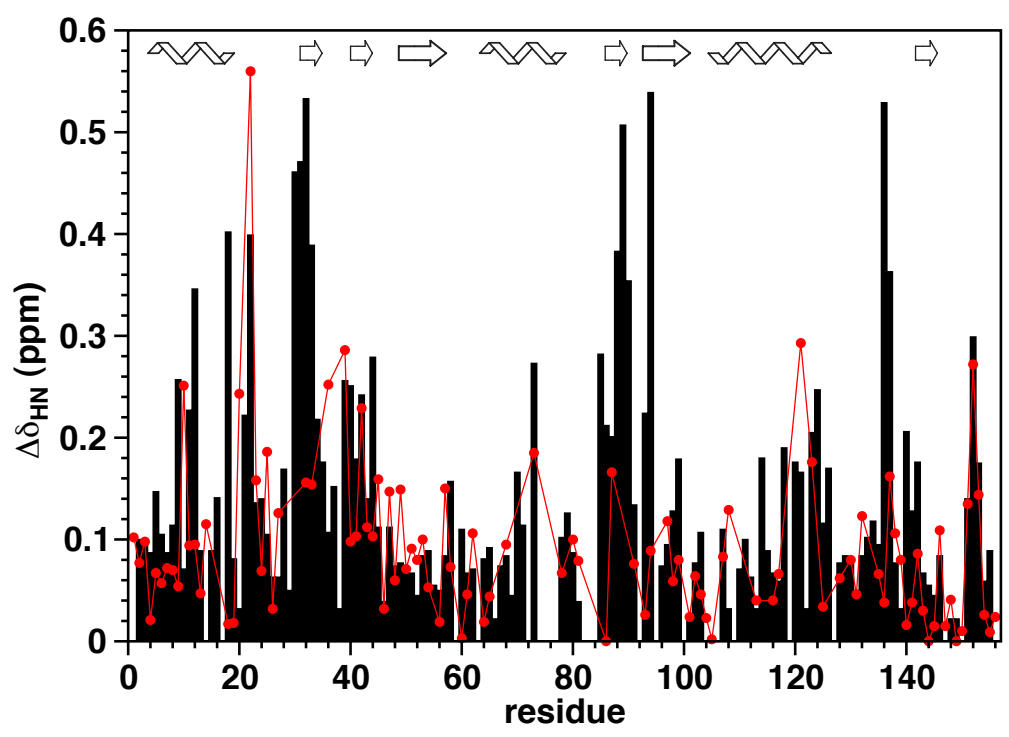

Figure 6.22: Chemical shift changes of CesT upon formation of the CesT/Tir108 complex (red circles). The black bars show the chemical shift changes of CesT upon formation of the CesT/Map84 complex for comparison. Secondary structure regions of the X-ray structure are indicated.

CesT/Map and the CesT/Tir interaction involve the same surface regions of CesT. This agrees with previous ELISA and yeast three hybrid results which demonstrate a competition between Map and Tir for CesT binding [30]. 


\subsubsection{Properties of the CesT/Map84 complex in solution}

To detect dynamic regions of the CesT/Map84 complex and differences in dynamics between free and effector-bound CesT, the amplitude of motions were determined via the steady-state heteronuclear ${ }^{15} \mathrm{~N}^{1} \mathrm{H}-\mathrm{NOE}$ (see 3.5.4.3 measured for a uniformly ${ }^{15} \mathrm{~N}$-labeled CesT/Map84 sample (Figure 6.23). ${ }^{15} \mathrm{~N}-{ }^{1} \mathrm{H}-\mathrm{NOE}$ values suggest overall

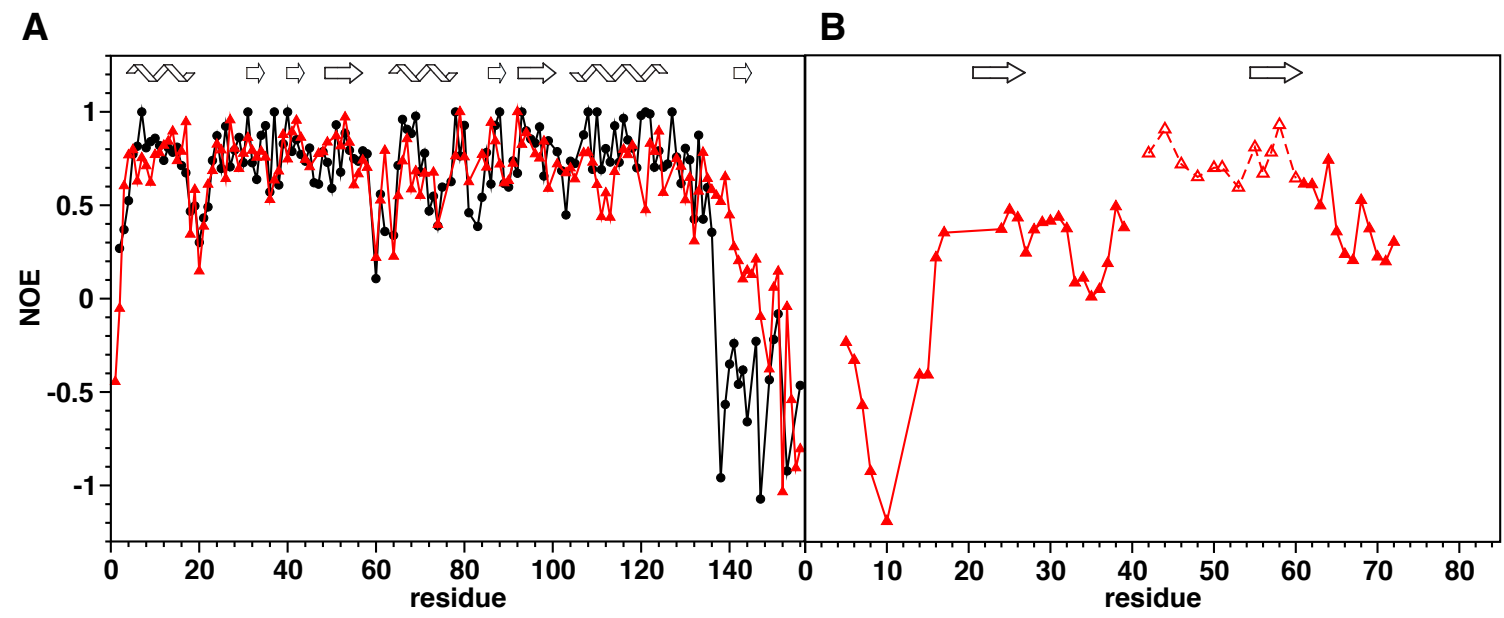

Figure 6.23: ${ }^{15} \mathrm{~N}-{ }^{1} \mathrm{H}-\mathrm{NOE}$ values are plotted against the residue number. Values for free CesT are indicated as black circles. Values for CesT (A) and Map84 (B) in complex with each other are shown as red filled triangles and are determined for a coexpressed and copurified ${ }^{15} \mathrm{~N}-\mathrm{CesT} / \mathrm{Map} 84$ complex sample. ${ }^{15} \mathrm{~N}-{ }^{1} \mathrm{H}-\mathrm{NOE}$ values without assignment in (B) are indicated by empty triangles and connected by a dashed line. Secondary structure elements of the X-ray structure are indicated for CesT and proposed $\beta$-motifs for Map84 [161].

unchanged dynamics of CesT upon complex formation (Figure 6.23A). Only ${ }^{15} \mathrm{~N}-$ ${ }^{1} \mathrm{H}-\mathrm{NOE}$ values for residues $138-144$ of CesT in complex are increased. This region includes the additional $\beta$-strand of the X-ray structure of free CesT which was not found under solution NMR conditions. However, ${ }^{15} \mathrm{~N}-{ }^{1} \mathrm{H}-\mathrm{NOE}$ values for residues 138-144 for CesT in the complex are smaller than for the secondary structure regions and secondary chemical shifts do not indicate structure (Figure 6.18). Thus, reduced flexibility but no additional secondary structure formation is indicated for residues 138-144 of CesT in complex with Map84 suggesting a conformation for Map84-bound CesT which is between the conformation of free CesT in the crystal and in solution. A conformational change of C-terminal residues upon complex formation is also indicated by chemical shift changes $\sim 0.2 \mathrm{ppm}$ for residues 151-153 (Figure 6.19). 
For Map84 in complex only $\sim 50 \%$ of the backbone is assigned. The longest stretches with missing assignments are residues 39-58 and 10 C-terminal residues. For 12 non-assigned peaks ${ }^{15} \mathrm{~N}-{ }^{1} \mathrm{H}-\mathrm{NOE}$ values between 0.6 and 0.8 were found. Overall ${ }^{15} \mathrm{~N}-{ }^{1} \mathrm{H}-\mathrm{NOE}$ values indicate flexibility for most residues of Map84 in complex with CesT (Figure 6.23B). Only for residue 36 and around residue $60{ }^{15} \mathrm{~N}-{ }^{1} \mathrm{H}-\mathrm{NOE}$ values $>0.5$ and $>0.6$ are found, respectively. Therefore the ${ }^{15} \mathrm{~N}-{ }^{1} \mathrm{H}-\mathrm{NOE}$ values without assignment were assumed to belong to residues 39-58. Under this assumption residues 40-64 have ${ }^{15} \mathrm{~N}-{ }^{1} \mathrm{H}-\mathrm{NOE}$ values above 0.6 and are thus suggested to directly interact with CesT. Due to homology to chaperone/effector complexes with known structures, $\beta$-motifs forming an intermolecular $\beta$-sheet were proposed for residues 20-27 and 5461 of Map [161]. ${ }^{15} \mathrm{~N}-{ }^{1} \mathrm{H}-\mathrm{NOE}$ values $\sim 0.4$ are found thereby pointing at restricted flexibility but not complete rigidity. Combined with the fact that the $\beta$-motif was inferred from a chaperone/effector-complex structure containing only one $\beta$-motif, one $\beta$-motif is concluded as sufficient to provide the specific recognition between CesT and its effectors. This $\beta$-motif is most likely composed of residues 54-61 of Map. For Tir this $\beta$-motif is probably formed by residues 49-56 because the other proposed $\beta$-motif is only partly affected by CesT binding (Figure 6.13). Through interactions with the second $\beta$-motif of the effector, CesT may confer a hierarchy to the secretion of its seven known effectors via the TTSS of EPEC. To confirm this, at least for one of the CesT/effector complexes, the effector has to be assigned in complex with CesT. For Map84 in complex with CesT the assignment has to completed but $\sim 15$-20 of the Map84 signals are weak or missing completely in the ${ }^{15} \mathrm{~N}$-TROSYHSQC spectrum of Map84 after refolding the CesT/Map84 complex (Figure 6.15). But in the ${ }^{15} \mathrm{~N}$-TROSY-HSQC spectrum of coexpressed and copurified CesT/Map84 complex (Figure 6.24) there are 10 sharp peaks of Map84 which are weak in the spectrum of the CesT/Map84 complex after refolding (compare Figures 6.24 and 6.15. Furthermore, there are $\sim 10$ additional peaks for the coexpressed CesT/Map84 complex sample (Figure 6.24) which are not found in the spectra of complex samples after refolding. This finding is in agreement with the fact that the coexpressed sample is stable for more than a year while the refolded sample shows degradation after two 


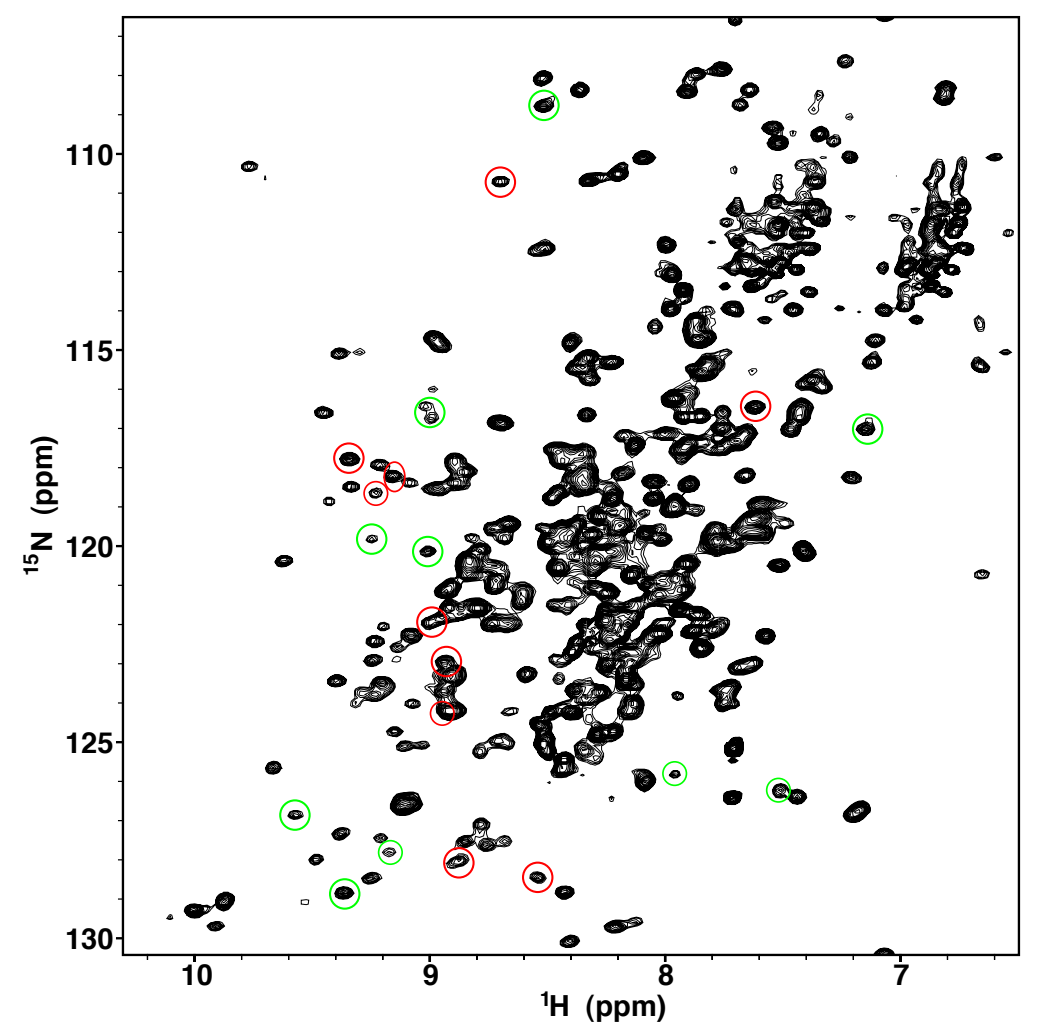

Figure 6.24: ${ }^{15} \mathrm{~N}-\mathrm{TROSY}-\mathrm{HSQC}$ spectrum of uniformly ${ }^{15} \mathrm{~N}$-labeled CesT/Map84 complex. Red circles indicate signals from Map84 which are weak in the HSQC spectrum of the refolded complex (Figure 6.15) and green circles indicate peaks which are absent in both HSQC spectra with one of the components of the refolded complex labeled.

weaks. Thus, TROSY-based triple resonance spectra measured for a coexpressed and copurified, ${ }^{2} \mathrm{H}(75 \%),{ }^{13} \mathrm{C},{ }^{15} \mathrm{~N}$-labeled CesT/Map84 sample should be most suitable to complete the assignment of Map84 in complex with CesT.

As yet, $\sim 30 \%$ of the expected Tir108 signals could not be detected in complex with CesT (Figure 6.13) and a stable coexpressed and copurified CesT/Tir108 complex could not be prepared. Since NMR-titration experiments with TirN and CesT suggested a role of residues outside the N-terminal 100 residues of Tir on CesT-binding (Figure 6.16), a coexpressed and copurified CesT/TirN complex should be prepared and may turn out to be best for NMR experiments and assignment of Tir in complex with CesT. 


\subsubsection{Conclusions}

In this chapter, the role of the chaperone CesT and its chaperone/effector complexes for the TTSS mechanism were investigated using mainly NMR spectroscopy. Structural data about TTSS chaperones and their complexes from solution NMR spectroscopy are not yet available while many X-ray structures have already been solved. In the first part of this chapter, the solution structure of CesT was revealed to be the non-swapped model structure based on experimental and charge-shape predicted RDCs. Results presented in the second part of this chapter demonstrate the relevance of models and conclusions derived for homologous chaperones and chaperone/effector complexes for CesT and its complexes. Firstly, the wrapping of the effector around the

chaperone dimer is indicated by a shift of almost all CesT signals in the ${ }^{15} \mathrm{~N}-\mathrm{HSQC}$ upon effector binding whereas no peaks are doubled due to a broken homodimer symmetry thus hinting at a pseudo-symmetric chaperone/effector complex in which effector residues with similar properties mediate the interaction with both monomers (Figure 6.17). Secondly, an extended, non-globular N-terminal structure of the effector in the chaperone/effector complex is consistent with the small number of signals low field to $8.5 \mathrm{ppm}$ for Map84 in complex with CesT (Figure 6.15). Thirdly, the insolubility of Map in the absence of CesT agrees with the suggested chaperone function of preventing inappropriate interactions. The solubility of Tir without the chaperone agrees with experiments showing a CesT-independent secretion of Tir [29] thereby implying a pre-formed secretion signal for free Tir. Finally, the hydrophobic properties of the residues of CesT with the strongest chemical shift perturbations upon complex formation reveal the CesT/effector complex to be mediated by many hydrophobic interactions (Figure 6.19).

However, the absence of an effector core protected by the chaperone and the additional flexible $\sim 20$ C-terminal residues point at differences in the effector binding mode with CesT compared to the homologous chaperones and the function for the TTSS mechanism in general. The depicted results agree with and extend the current view of the role of the chaperone in the effector targeting to the TTSS. Figure 6.25 
summarizes the overall extended effector targeting model, including additional chaperone/effector interactions proposed based on the results presented in this chapter.

(I) CesT recognizes an effector in the bacterial cytoplasm via hydrophobic surface

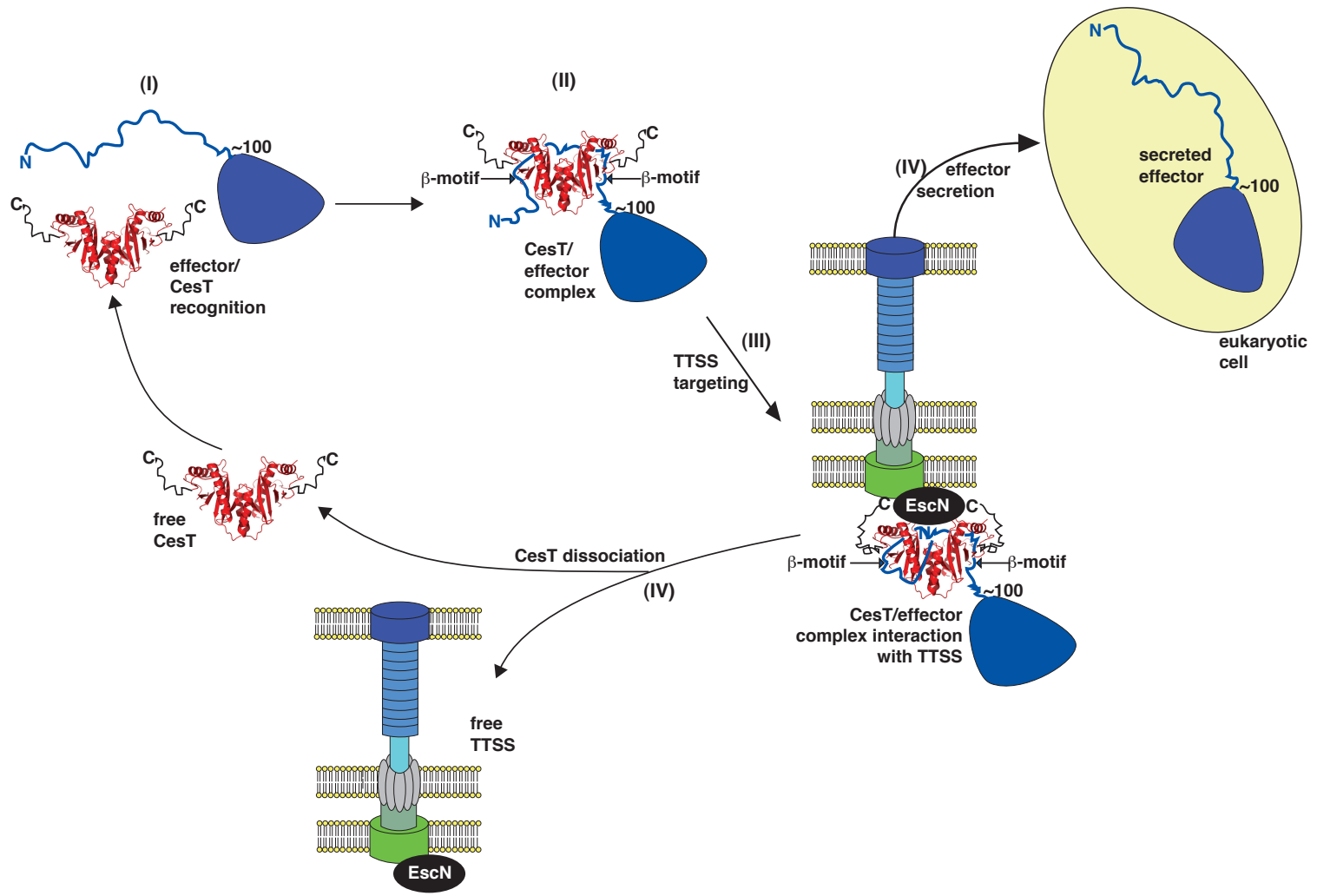

Figure 6.25: Model of effector targeting to the TTSS through CesT in pathogenic E. coli. CesT is shown as a red ribbon diagram with the C-terminus added as a black line and the effector in blue with a globular C-terminal and extended or unfolded $\sim 100$ $\mathrm{N}$-terminal residues. In the bacterial cytoplasm, the effector is recognized by CesT (I) and the CesT/effector complex is formed via $\beta$-motifs (II). (III) Mediated by the unfolded C-termini of the CesT dimer and the unfolded N-terminal $\sim 15 \mathrm{~N}$-terminal residues of the effector the CesT/effector complex interacts with the ATPase EscN of the TTSS. (IV) Conformational change catalyzed by EscN leads to CesT dissociation and translocation of the effector into the eukaryotic cell.

patches. (II) Upon formation of manifold hydrophobic CesT/effector interactions, a part of the N-terminal $\sim 100$ residues wraps around the chaperone. In the complex, the most important new tertiary structure is formed between two $\beta$-motif regions of the tertiary structure lacking effector and identical regions of the CesT monomers. (III) The two unfolded C-terminal 20 residues of CesT and the $\sim 15$ N-terminal residues of the effector [170] direct the complex to the TTSS and mediate the association with 
the ATPase EscN. (IV) Using the unfolded regions as starting points, EscN catalyses conformational changes which lead to dissociation of CesT and translocation of the unfolded effector into the eukaryotic cell. 


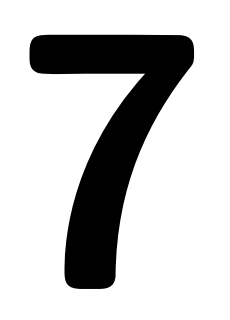

\section{Summary and future considerations}

This thesis makes a contribution to the understanding of two mechanisms of bacterial pathogenicity: A new transcription regulation mechanism of the pathogen E. faecalis and the type three protein secretion mechanism of enteropathogenic E. coli. In addition a novel method for the improvement of high-resolution structure determination of symmetric oligomers by NMR is presented.

The research projects aimed mainly at the determination of unknown structures of proteins, protein-DNA and protein-protein complexes in order to unravel protein functions and mechanisms. Methods based on recent advances in NMR spectroscopy concerning sample preparation, NMR experiments as well as structure calculation have been applied. In the initial sample preparation stages, plasmids were constructed or mutations introduced to allow for the production and purification of the desired isotopically-labeled or unlabeled proteins. The second stage consisted of the application of well-established and novel NMR experiments. Finally the experimental data were analysed to generate a model for a protein-DNA complex, were utilized to develop a novel method for NMR structure determination of homodimeric proteins and were interpreted to structurally characterize a $\sim 36 \mathrm{kDa}$ homodimeric chaperone and two of its complexes. The major results of Chapter 46 are summarized and future experiments are proposed in the following paragraphs.

In 2002, Haas et al. showed that CylR2 regulates together with CylR1 the production of the highly toxic cytolysin via an autoinduction quorum sensing mechanism [104]. 
The results presented in Chapter 4 , entitled "Structure and DNA-binding properties of the cytolysin regulator CylR2", are the first structural data about this new quorum sensing mechanism. The structure of CylR2 reveals a rigid dimer containing a helix-turn-helix DNA-binding motif as part of a five helix-bundle that is extended by an antiparallel $\beta$-sheet. NMR chemical shift perturbation experiments identify surfaces involved in DNA-binding and are in agreement with a model for the CylR2/DNA complex that attributes binding specificity through a complex network of CylR2/DNA interactions. The results reveal about the new quorum sensing mechanism that CylR2 acts as a repressor of cytolysin transcription by binding to the inverted repeat 1 within the promotor region of the cytolysin operon.

Next steps towards a complete understanding of the molecular basis of cytolysin repression by the CylR1/CylR2 two-component regulatory system rely on the preparation of the putative transmembrane protein CylR1. If its production is not possible by recombinant expression and reconstitution in micelles, the recently developed cellfree expression should be attempted [171]. The proposed binding of CylR1 to CylR2 may involve the $\mathrm{N}$ - and $\mathrm{C}$-terminal $\beta$-strands which are not found in the structurally homologous proteins $\operatorname{SinR}$ and 434 repressor (see 4.3.1). Once the regulation of cytolysin expression is understood, a remedy for hospital-acquired antibiotic-resistent infections caused by E. faecalis can be developed.

Oligomerization plays an important role in protein function. The structure determination of homooligomeric proteins by NMR spectroscopy, however, is problematic due to the difficulty of distinguishing inter- and intramonomer contacts. To attack this challenging problem, the protein CylR2 was used to develop a novel method for high-resolution structure determination of symmetric homodimers in solution which is introduced in Chapter 5, "Using dipolar couplings and intermonomer paramagnetic relaxation enhancement to determine the structure of the CylR2 homodimer". Based on the structure of the monomer of CylR2, which was obtained by conventional NOE-based methods, the high-resolution structure of the CylR2 homodimer was determined by rigid-body docking. The rigid-body docking was driven 
by a few backbone HN residual dipolar couplings and intermolecular long-range distances derived from relaxation enhancement observed for two paramagnetic spin label positions. The final NMR ensemble of the $15.4 \mathrm{kDa}$ CylR2 homodimer deviates by $1.15 \AA$ from the previously determined X-ray structure. In addition, it is shown that charge-shape predicted residual dipolar couplings can be used to select a structural model from an ensemble of ab initio docking solutions that is closest to the native structure. This method does not require intermolecular NOEs or assignment of side chain resonances, making it particularly useful for the structure determination of large, symmetric homooligomers.

In the next step this novel method should be applied for the solution NMR structure calculation of the $35.4 \mathrm{kDa}$ homodimer CesT which is investigated in Chapter 6 . Thereby it could be shown that the method is more generally valuable. Moreover, due to its size CesT is an ideal test protein for the applicability of the method en route to NMR structure determination of large oligomeric proteins up to $80 \mathrm{kDa}$.

The superordinate aim of Chapter 6, called "Structural studies of the function of the chaperone CesT in protein secretion via the type three secretion machinery of enteropathogenic $\boldsymbol{E}$. coli", is the understanding of the type three secretion mechanism. This is a controversially discussed topic with very recent and important findings published only this or last year [146, 153, 161].

In the first half of Chapter 6, the non-swapped homology-based model structure of CesT is revealed as the solution structure solely based on the backbone assignment and backbone residual dipolar couplings. The method described in Chapter 5 can be applied for the NMR structure determination of the homodimer CesT to replace the non-swapped model structure by a solution NMR structure. The second part of the Chapter 6 presents experimental data about the complexes between CesT and its effectors Tir and Map. Hydrophobic patches on the surface of the chaperone CesT which are important for the chaperone/effector interaction were identified via backbone amide chemical shift changes and correspond to important regions of homologue chaperones. Further results are consistent with the function of CesT to keep effectors 
in an unfolded, secretion competent state and in targeting its effectors to the type three secretion system.

A CesT/effector structure is required for a detailed understanding of the differences and conserved features between the CesT/effector complexes and the homologue chaperone/effector complexes. Structure determination trials made so far have been focussed on the CesT/Map complex. However, it was not possible to prepare crystals suitable for X-ray diffraction experiments. The first steps towards a solution NMR structure of the CesT/Map84 complex have already been made but more experimental data are needed to generate a model structure. Due to stability problems of the refolded CesT/Map84 sample a uniformly ${ }^{2} \mathrm{H},{ }^{15} \mathrm{~N},{ }^{13} \mathrm{C}$-CesT/Map84 sample should be used to acquire TROSY-based triple resonance spectra to complete the assignment of Map84 in complex with CesT and to derive secondary structure information. Intermolecular distance information will be best determined via site-directed spin-labeling for CesT/Map84 samples with one complex component ${ }^{15} \mathrm{~N}$-labeled and the spin label attached to the other complex component. These intermolecular distances could then be used together with the model structure of CesT and secondary structure information about Map84 to generate a model structure of the CesT/Map84 complex. Regarding the translocation mechanism, it will be of particular interest to perform NMR titration experiments with the chaperone CesT, the effector Tir or Map and the ATPase EscN. Since it was shown that EscN binds CesT as well as the CesT/Tir complex the effector is probably directed towards the ATPase by CesT and unfolded by the ATPase [153, 166]. Even for the entire trimeric and more than $100 \mathrm{kDa}$ large complex, ${ }^{15} \mathrm{~N}-\mathrm{HSQC}$ spectra will be accessible and will not suffer from severe signal overlap if the chaperone or the effector are ${ }^{15} \mathrm{~N}$-labeled. Thus, the interaction region and recognition between the ATPase and the chaperone could be revealed. Furthermore, very important information about changes in the fold of the effector and insights into the unfolding mechanism can be gained especially upon addition of ATP to the trimeric complex.

It is the author's hope, that the conclusions reached in this work will be a fruitful 
source for further experiments finally leading to a complete understanding of these mechanisms of bacterial pathogenicity allowing for drug design to fight against the related diseases. The author also aimed for a general contribution to approaches attacking protein oligomerization and therewith its biological functions especially in homodimerization and homooligomerization.

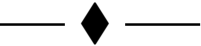




\section{References}

[1] T. M. Fuchs, Molecular mechanisms of bacterial pathogenicity, Naturwissenschaften 85: 99-108 (1998).

[2] J. W. Wilson, M. J. Schurr, C. L. LeBlanc, R. Ramamurthy, K. L. Buchanan, and C. A. Nickerson, Mechanisms of bacterial pathogenicity, Postgrad Med J 78: 216-224 (2002).

[3] H. Remaut and G. Waksman, Structural biology of bacterial pathogenesis, Curr Opin Struct Biol 14: 161-170 (2004).

[4] A. Abragam, The principles of nuclear magnetism, Oxford University Press, London (1961).

[5] J. Cavanagh, W. J. Fairbrother, A. G. Palmer III, and N. J. Skelton, Protein NMR spectroscopy: Principles and Practice, Academic Press Inc., San Diego (1996).

[6] M. H. Lewitt, Spin Dynamics - Basics of Nuclear Magnetic Resonance, Wiley, Chichester (2001).

[7] K. Wüthrich, NMR of proteins and nucleic acids, Wiley-Interscience, New York (1986).

[8] F. Bloch, W. W. Hansen, and M. Packard, Nuclear induction., Physical Review 69: $127(1946)$. 
[9] E. Purcell, H. C. Torrey, and R. V. Pound, Nuclear induction., Physical Review 69: $37-38$ (1946).

[10] R. R. Ernst, Nobel Lecture. Nuclear magnetic resonance Fourier transform spectroscopy, Biosci Rep 12: 143-187 (1992).

[11] K. Wüthrich, NMR studies of structure and function of biological macromolecules (Nobel lecture), Angew Chem Int Ed Engl 42: 3340-3363 (2003).

[12] A. E. Ferentz and G. Wagner, NMR spectroscopy: a multifaceted approach to macromolecular structure, Q Rev Biophys 33: 29-65 (2000).

[13] K. H. Gardner and L. E. Kay, The use of ${ }^{2} \mathrm{H},{ }^{13} \mathrm{C},{ }^{15} \mathrm{~N}$ multidimensional NMR to study the structure and dynamics of proteins, Annu Rev Biophys Biomol Struc 27: 357-406 (1998).

[14] K. Pervushin, R. Riek, G. Wider, and K. Wüthrich, Attenuated $\mathrm{T}_{2}$ relaxation by mutual cancellation of dipole-sdipole coupling and chemical shift anisotropy indicates an avenue to NMR structures of very large biological macromolecules in solution, Proc Natl Acad Sci USA 94: 12366-12371 (1997).

[15] C. Fernández and G. Wider, TROSY in NMR studies of the structure and function of large biological macromolecules, Curr Opin Struct Biol 13: 570$580(2003)$.

[16] P. Güntert, Structure calculation of biological macromolecules from NMR data, Q Rev Biophys 31: 145-237 (1998).

[17] G. M. Clore and C. D. Schwieters, Theoretical and computational advances in biomolecular NMR spectroscopy, Curr Opin Struct Biol 12: 146-153 (2002).

[18] J. J. Gray, High-resolution protein-protein docking, Curr Opin Struct Biol 16: 183-193 (2006). 
[19] R. B. Russell, F. Alber, P. Aloy, F. P. Davis, D. Korkin, M. Pichaud, M. Topf, and A. Sali, A structural perspective on protein-protein interactions, Curr Opin Struct Biol 14: 313-24 (2004).

[20] J. H. Prestegard, H. M. al Hashimi, and J. R. Tolman, NMR structures of biomolecules using field oriented media and residual dipolar couplings, $Q$ Rev Biophys 33: 371-424 (2000).

[21] A. Bax, G. Kontaxis, and N. Tjandra, Dipolar couplings in macromolecular structure determination, Meth Enzymol 339: 127-174 (2001).

[22] R. S. Lipsitz and N. Tjandra, Residual dipolar couplings in NMR structure analysis, Annu Rev Biophys Biomol Struct 33: 387-413 (2004).

[23] M. Blackledge, Recent progress in the study of biomolecular structure and dynamics in solution from residual dipolar couplings, Prog Nucl Magn Reson Spectros 46: 23-61 (2005).

[24] D. S. Goodsell and A. J. Olson, Structural symmetry and protein function, Annu Rev Biophys Biomol Struct 29: 105-153 (2000).

[25] Y. Liu and D. Eisenberg, 3D domain swapping: as domains continue to swap, Protein Sci 11: 1285-1299 (2002).

[26] M. J. Bennett, M. P. Schlunegger, and D. Eisenberg, 3D domain swapping: a mechanism for oligomer assembly, Protein Sci 4: 2455-2468 (1995).

[27] M. J. Bennett, M. R. Sawaya, and D. Eisenberg, Deposition diseases and 3D domain swapping, Structure 14: 811-824 (2006).

[28] J. Grodberg and J. J. Dunn, ompT encodes the Escherichia coli outer membrane protease that cleaves T7 RNA polymerase during purification, J Bacteriol 170: $1245-1253$ (1988).

[29] S. J. Elliott, S. W. Hutcheson, M. S. Dubois, J. L. Mellies, L. A. Wainwright, M. Batchelor, G. Frankel, S. Knutton, and J. B. Kaper, Identification of CesT, 
a chaperone for the type III secretion of Tir in enteropathogenic Escherichia coli, Mol Microbiol 33: 1176-1189 (1999).

[30] E. A. Creasey, R. M. Delahay, A. A. Bishop, R. K. Shaw, B. Kenny, S. Knutton, and G. Frankel, CesT is a bivalent enteropathogenic Escherichia coli chaperone required for translocation of both Tir and Map, Mol Microbiol 47: 209-221 (2003).

[31] R. M. Delahay, R. K. Shaw, S. J. Elliott, J. B. Kaper, S. Knutton, and G. Frankel, Functional analysis of the enteropathogenic Escherichia coli type III secretion system chaperone CesT identifies domains that mediate substrate interactions, Mol Microbiol 43: 61-73 (2002).

[32] E. L. Hartland, M. Batchelor, R. M. Delahay, C. Hale, S. Matthews, G. Dougan, S. Knutton, I. Connerton, and G. Frankel, Binding of intimin from enteropathogenic Escherichia coli to Tir and to host cells, Mol Microbiol 32: $151-158(1999)$.

[33] R. A. Laskowski, J. A. Rullmannn, M. W. MacArthur, R. Kaptein, and J. M. Thornton, AQUA and PROCHECK-NMR: programs for checking the quality of protein structures solved by NMR, J Biomol NMR 8: 477-486 (1996).

[34] R. M. Esnouf, Further additions to MolScript version 1.4, including reading and contouring of electron-density maps, Acta Crystallogr D Biol Crystallogr 55: 938-940 (1999).

[35] M. D. Winn, A. W. Ashton, P. J. Briggs, C. C. Ballard, and P. Patel, Ongoing developments in CCP4 for high-throughput structure determination, Acta Crystallogr D Biol Crystallogr 58: 1929-1936 (2002).

[36] E. Potterton, P. Briggs, M. Turkenburg, and E. Dodson, A graphical user interface to the CCP4 program suite, Acta Crystallogr Sect D-Biol Crystallogr 59: 1131-1137 (2003). 
[37] S. R. Comeau, D. W. Gatchell, S. Vajda, and C. J. Camacho, ClusPro: An automated docking and discrimination method for the prediction of protein complexes, Bioinformatics 20: 45-50 (2004).

[38] S. R. Comeau, D. W. Gatchell, S. Vajda, and C. J. Camacho, ClusPro: a fully automated algorithm for protein-protein docking, Nucleic Acids Res 32: W96-9 (2004).

[39] S. R. Comeau and C. J. Camacho, Predicting oligomeric assemblies: N-mers a primer, J Struct Biol 150: 233-44 (2005).

[40] P. Güntert, Automated NMR structure calculation with CYANA, Methods Mol Biol 278: 353-378 (2004).

[41] A. Phillippsen, DINO: Visualizing Strucutral Biology, http://www.dino3d.org (2003).

[42] Y. S. Jung and M. Zweckstetter, Mars - robust automatic backbone assignment of proteins, J Biomol NMR 30: 11-23 (2004).

[43] R. Koradi, M. Billeter, and K. Wüthrich, MOLMOL: a program for display and analysis of macromolecular structures, J Mol Graph 14: 51-5, 29-32 (1996).

[44] P. J. Kraulis, Molscript - a program to produce both detailed and schematic plots of protein structures, J Appl Crystallogr 24: 946-950 (1991).

[45] M. F. Sanner, A. J. Olson, and J. C. Spehner, Reduced surface: An efficient way to compute molecular surfaces, Biopolymers 38: 305-320 (1996).

[46] F. Delaglio, S. Grzesiek, G. W. Vuister, G. Zhu, J. Pfeifer, and A. Bax, NMRPipe: a multidimensional spectral processing system based on UNIX pipes, $J$ Biomol NMR 6: 277-93 (1995).

[47] B. A. Johnson and R. A. Blevins, NMR View: A computer program for the visualization and analysis of NMR data, J Biomol NMR 4: 603-614 (1994). 
[48] M. Zweckstetter and A. Bax, Prediction of sterically induced alignment in a dilute liquid crystalline phase: Aid to protein structure determination by NMR, J Am Chem Soc 122: 3791-3792 (2000).

[49] R. A. Laskowski, M. W. Macarthur, D. S. Moss, and J. M. Thornton, Procheck - a program to check the stereochemical quality of protein structures, $J A p p l$ Crystallogr 26: 283-291 (1993).

[50] W. L. DeLano, The PyMOL molecular graphics system, http://www.pymol.org (2002).

[51] E. A. Merritt and D. J. Bacon, Raster3D: Photorealistic molecular graphics, Macromol Crystallogr, Pt B 277: 505-524 (1997).

[52] G. Cornilescu, F. Delaglio, and A. Bax, Protein backbone angle restraints from searching a database for chemical shift and sequence homology, J Biomol NMR 13: 289-302 (1999).

[53] C. D. Schwieters, J. J. Kuszewski, N. Tjandra, and G. M. Clore, The Xplor-NIH NMR molecular structure determination package, J Magn Reson 160: 65-73 (2003).

[54] K. Mullis, F. Faloona, S. Scharf, R. Saiki, G. Horn, and H. Erlich, Specific enzymatic amplification of DNA in vitro: the polymerase chain reaction, Cold Spring Harb Symp Quant Biol 51 Pt 1: 263-273 (1986).

[55] J. Sambrook, F. F. Fritsch, and M. Maniatis, Molecular Cloning: A laboratory manual, Cold Spring Harbor Laboratory Press, Cold Spring Harbor (1989).

[56] U. K. Laemmli, Cleavage of structural proteins during assembly of the head of bacteriophage T4, Nature 227: 680-685 (1970).

[57] T. Okajima, T. Tanabe, and T. Yasuda, Nonurea sodium dodecyl sulfatepolyacrylamide gel electrophoresis with high-molarity buffers for the separation of proteins and peptides, Anal Biochem 211: 293-300 (1993). 
[58] P. Edman, Method for determination of the amino acid sequence in peptides, Acta Chem Scand 4: 283-293 (1950).

[59] A. Fontana, P. P. de Laureto, B. Spolaore, E. Frare, P. Picotti, and M. Zambonin, Probing protein structure by limited proteolysis, Acta Biochim Pol 51: 299-321 (2004).

[60] W. L. Hubbell, A. Gross, R. Langen, and M. A. Lietzow, Recent advances in site-directed spin labeling of proteins, Curr Opin Struct Biol 8: 649-656 (1998).

[61] H. E. Mash, Y. P. Chin, L. Sigg, R. Hari, and H. Xue, Complexation of copper by zwitterionic aminosulfonic (good) buffers, Anal Chem 75: 671-677 (2003).

[62] F. X. Schmid, Spectral methods of characterizing protein conformation and conformational changes., in T. E. Creighton, editor, Protein structure: A practical approach., IRL Press Oxford, Oxford (1989).

[63] A. Bax and S. Grzesiek, Methodological advances in protein NMR, Accounts Chem Res 26: 131-138 (1993).

[64] M. Salzmann, K. Pervushin, G. Wider, H. Senn, and K. Wüthrich, TROSY in triple-resonance experiments: new perspectives for sequential NMR assignment of large proteins, Proc Natl Acad Sci U S A 95: 13585-13590 (1998).

[65] M. Salzmann, G. Wider, K. Pervushin, H. Senn, and K. Wüthrich, TROSY-type triple-resonance experiments for sequential NMR assignments of large proteins, J Am Chem Soc 121: 844-848 (1999).

[66] A. Eletsky, A. Kienhofer, and K. Pervushin, TROSY NMR with partially deuterated proteins, J Biomol NMR 20: 177-180 (2001).

[67] T. Yamazaki, W. Lee, C. H. Arrowsmith, D. R. Muhandiram, and L. E. Kay, A suite of triple-resonance NMR experiments for the backbone assignment of N-15, C-13, H-2 labeled proteins with high-sensitivity, J Am Chem Soc 116: 11655-11666 (1994). 
[68] W. Peti, C. Griesinger, and W. Bermel, Adiabatic TOCSY for C,C and H,H J-transfer, J Biomol NMR 18: 199-205 (2000).

[69] M. Zweckstetter and T. A. Holak, Robust refocusing of C-13 magnetization in multidimensional NMR experiments by adiabatic fast passage pulses, J Biomol NMR 15: 331-334 (1999).

[70] T. Yamazaki, J. D. Forman-Kay, and L. E. Kay, 2-dimensional NMR experiments for correlating ${ }^{13} \mathrm{C} \beta$ and ${ }^{1} \mathrm{H} \delta / \epsilon$ chemical shifts of aromatic residues in ${ }^{13}$ C-labeled proteins via scalar couplings, J Am Chem Soc 115: 11054-11055 (1993).

[71] S. Schwarzinger, G. J. Kroon, T. R. Foss, J. Chung, P. E. Wright, and H. J. Dyson, Sequence-dependent correction of random coil NMR chemical shifts, $J$ Am Chem Soc 123: 2970-2978 (2001).

[72] D. S. Wishart and B. D. Sykes, Chemical shifts as a tool for structure determination, Methods Enzymol 239: 363-392 (1994).

[73] W. J. Metzler, K. L. Constantine, M. S. Friedrichs, A. J. Bell, E. G. Ernst, T. B. Lavoie, and L. Mueller, Characterization of the three-dimensional solution structure of human profilin: $1 \mathrm{H}, 13 \mathrm{C}$, and $15 \mathrm{~N}$ NMR assignments and global folding pattern, Biochemistry 32: 13818-13829 (1993).

[74] M. R. Hansen, L. Mueller, and A. Pardi, Tunable alignment of macromolecules by filamentous phage yields dipolar coupling interactions, Nat Struct Biol 5: 1065-1074 (1998).

[75] M. Zweckstetter and A. Bax, Characterization of molecular alignment in aqueous suspensions of Pf1 bacteriophage, J Biomol NMR 20: 365-377 (2001).

[76] G. Cornilescu, J. L. Marquardt, M. Ottiger, and A. Bax, Validation of protein structure from anisotropic carbonyl chemical shifts in a dilute liquid crystalline phase, J Am Chem Soc 120: 6836-6837 (1998). 
[77] M. Ottiger, F. Delaglio, and A. Bax, Measurement of J and dipolar couplings from simplified two-dimensional NMR spectra, J Magn Reson 131: 373-378 (1998).

[78] N. Tjandra and A. Bax, Direct measurement of distances and angles in biomolecules by NMR in a dilute liquid crystalline medium, Science 278: 11111114 (1997).

[79] V. Vijayan and M. Zweckstetter, Simultaneous measurement of protein onebond residual dipolar couplings without increased resonance overlap, J Magn Reson 174: 245-253 (2005).

[80] J. J. Chou, F. Delaglio, and A. Bax, Measurement of one-bond ${ }^{15} \mathrm{~N}-{ }^{13} \mathrm{C}$ ' dipolar couplings in medium sized proteins, J Biomol NMR 18: 101-105 (2000).

[81] C. P. Jaroniec, T. S. Ulmer, and A. Bax, Quantitative J correlation methods for the accurate measurement of ${ }^{13} \mathrm{C}^{\prime}-{ }^{13} \mathrm{C} \alpha$ dipolar couplings in proteins, J Biomol NMR 30: 181-194 (2004).

[82] M. Zweckstetter, G. Hummer, and A. Bax, Prediction of charge-induced molecular alignment of biomolecules dissolved in dilute liquid-crystalline phases, Biophys J 86: 3444-60 (2004).

[83] V. Sklenar and A. Bax, Two-dimensional heteronuclear chemical-shift correlation of proteins at natural abundance ${ }^{15} \mathrm{~N}$ and ${ }^{13} \mathrm{C}$ levels, J Magn Reson 71: 379-383 (1987).

[84] J. W. Peng and G. Wagner, Investigation of protein motions via relaxation measurements, Methods Enzymol 239: 563-596 (1994).

[85] D. J. Craik and J. A. Wilce, Studies of protein-ligand interactions by NMR, Methods Mol Biol 60: 195-232 (1997).

[86] L. Fielding, NMR methods for the determination of protein-ligand dissociation constants, Curr Top Med Chem 3: 39-53 (2003). 
[87] J. L. Battiste and G. Wagner, Utilization of site-directed spin labeling and highresolution heteronuclear nuclear magnetic resonance for global fold determination of large proteins with limited nuclear overhauser effect data, Biochemistry 39: 5355-5365 (2000).

[88] P. A. Kosen, Spin labeling of proteins, Methods Enzymol 177: 86-121 (1989).

[89] G. M. Clore, Accurate and rapid docking of protein-protein complexes on the basis of intermolecular nuclear Overhauser enhancement data and dipolar couplings by rigid body minimization, Proc Natl Acad Sci USA 97: 9021-9025 (2000).

[90] R. M. A. Knegtel, R. Boelens, and R. Kaptein, Monte-Carlo docking of proteinDNA complexes - incorporation of DNA flexibility and experimental data, Protein Eng 7: 761-767 (1994).

[91] R. M. A. Knegtel, J. Antoon, C. Rullmann, R. Boelens, and R. Kaptein, Monty a Monte-Carlo approach to protein-DNA recognition, J Mol Biol 235: 318-324 (1994).

[92] B. D. Jett, M. M. Huycke, and M. S. Gilmore, Virulence of Enterococci, Clin Microbiol Rev 7: 462-78 (1994).

[93] B. E. Murray, The life and times of the Enterococcus, Clin Microbiol Rev 3: 46-65 (1990).

[94] M. S. Gilmore, R. A. Segarra, and M. C. Booth, An Hlyb-type function is required for expression of the Enterococcus faecalis hemolysin/bacteriocin, Infect Immun 58: 3914-3923 (1990).

[95] Y. Ike, H. Hashimoto, and D. B. Clewell, Hemolysin of Streptococcus faecalis subspecies zymogenes contributes to virulence in mice, Infect Immun 45: 528$530(1984)$. 
[96] K. V. Singh, X. Qin, G. M. Weinstock, and B. E. Murray, Generation and testing of mutants of Enterococcus faecalis in a mouse peritonitis model, J Infect Dis 178: $1416-1420$ (1998).

[97] B. D. Shepard and M. S. Gilmore, Antibiotic-resistant enterococci: the mechanisms and dynamics of drug introduction and resistance, Microbes Infect 4: $215-224(2002)$.

[98] L. S. Havarstein, D. B. Diep, and I. F. Nes, A family of bacteriocin ABC transporters carry out proteolytic processing of their substrates concomitant with export, Mol Microbiol 16: 229-240 (1995).

[99] M. C. Booth, C. P. Bogie, H. G. Sahl, R. J. Siezen, K. L. Hatter, and M. S. Gilmore, Structural analysis and proteolytic activation of Enterococcus faecalis cytolysin, a novel lantibiotic, Mol Microbiol 21: 1175-84 (1996).

[100] P. S. Coburn and M. S. Gilmore, The Enterococcus faecalis cytolysin: a novel toxin active against eukaryotic and prokaryotic cells, Cell Microbiol 5: 661-669 (2003).

[101] B. D. Jett and M. S. Gilmore, The growth-inhibitory effect of the Enterococcus faecalis bacteriocin encoded by pAD1 extends to the oral streptococci, J Dent Res 69: 1640-5 (1990).

[102] W. Haas and M. S. Gilmore, Molecular nature of a novel bacterial toxin: the cytolysin of Enterococcus faecalis, Med Microbiol Immunol (Berl) 187: 183-90 (1999).

[103] M. S. Gilmore, R. A. Segarra, M. C. Booth, C. P. Bogie, L. R. Hall, and D. B. Clewell, Genetic structure of the Enterococcus faecalis plasmid pAD1encoded cytolytic toxin system and its relationship to lantibiotic determinants, J Bacteriol 176: 7335-44 (1994). 
[104] W. Haas, B. D. Shepard, and M. S. Gilmore, Two-component regulator of Enterococcus faecalis cytolysin responds to quorum-sensing autoinduction, Nature 415: 84-87 (2002).

[105] P. S. Coburn, L. E. Hancock, M. C. Booth, and M. S. Gilmore, A novel means of self-protection, unrelated to toxin activation, confers immunity to the bactericidal effects of the Enterococcus faecalis cytolysin, Infect Immun 67: 3339-47 (1999).

[106] L. Keller and M. G. Surette, Communication in bacteria: an ecological and evolutionary perspective, Nat Rev Microbiol 4: 249-258 (2006).

[107] J. A. Hoch, Two-component and phosphorelay signal transduction, Curr Opin Microbiol 3: 165-70 (2000).

[108] S. Rumpel, A. Razeto, C. M. Pillar, V. Vijayan, A. Taylor, K. Giller, M. S. Gilmore, S. Becker, and M. Zweckstetter, Structure and DNA-binding properties of the cytolysin regulator CylR2 from Enterococcus faecalis, EMBO J 23: 3632-42 (2004).

[109] F. A. Mulder, D. Schipper, R. Bott, and R. Boelens, Altered flexibility in the substrate-binding site of related native and engineered high-alkaline Bacillus subtilisins, J Mol Biol 292: 111-123 (1999).

[110] L. J. W. Shimon and S. C. Harrison, The phage $434 \mathrm{O}(\mathrm{R}) 2 / \mathrm{R} 1-69$ complex at $2.5 \AA$ resolution, J Mol Biol 232: 826-838 (1993).

[111] L. J. Beamer and C. O. Pabo, Refined $1.8 \AA$ crystal structure of the lambda repressor-operator complex, J Mol Biol 227: 177-196 (1992).

[112] S. C. Harrison and A. K. Aggarwal, DNA recognition by proteins with the Helix-Turn-Helix motif, Annu Rev Biochem 59: 933-969 (1990).

[113] K. Pervushin, M. Billeter, G. Siegal, and K. Wüthrich, Structural role of a buried salt bridge in the 434 repressor DNA-binding domain, J Mol Biol 264: 1002-1012 (1996). 
[114] A. Mondragon, S. Subbiah, S. C. Almo, M. Drottar, and S. C. Harrison, Structure of the amino-terminal domain of phage- 434 repressor at $2.0 \AA$ resolution, J Mol Biol 205: 189-200 (1989).

[115] L. Holm and C. Sander, Protein structure comparison by alignment of distance matrices, J Mol Biol 233: 123-138 (1993).

[116] R. J. Lewis, J. A. Brannigan, W. A. Offen, I. Smith, and A. J. Wilkinson, An evolutionary link between sporulation and prophage induction in the structure of a repressor:anti-repressor complex, J Mol Biol 283: 907-912 (1998).

[117] V. M. A. Ducros, R. J. Lewis, C. S. Verma, E. J. Dodson, G. Leonard, J. P. Turkenburg, G. N. Murshudov, A. J. Wilkinson, and J. A. Brannigan, Crystal structure of GerE, the ultimate transcriptional regulator of spore formation in Bacillus subtilis, Journal of Molecular Biology 306: 759-771 (2001).

[118] A. K. Aggarwal, D. W. Rodgers, M. Drottar, M. Ptashne, and S. C. Harrison, Recognition of a DNA operator by the repressor of phage 434 - a view at highresolution, Science 242: 899-907 (1988).

[119] A. C. Bell and G. B. Koudelka, Operator sequence context influences amino acid-base-pair interactions in 434 repressor-operator complexes, J Mol Biol 234: 542-553 (1993).

[120] A. Simoncsits, J. Chen, P. Percipalle, S. Wang, I. Toro, and S. Pongor, Singlechain repressors containing engineered DNA-binding domains of the phage 434 repressor recognize symmetric or asymmetric DNA operators, J Mol Biol 267: 118-131 (1997).

[121] C. Wolberger, Y. Dong, M. Ptashne, and S. C. Harrison, Structure of a phage 434 Cro/DNA complex, Nature 335: 789-795 (1988).

[122] E. L. Sonnhammer, G. von Heijne, and A. Krogh, A hidden Markov model for predicting transmembrane helices in protein sequences, Proc Int Conf Intell Syst Mol Biol 6: 175-182 (1998). 
[123] A. L. Breeze, Isotope-filtered NMR methods for the study of biomolecular structure and interactions, Prog Nucl Magn Reson Spectros 36: 323-372 (2000).

[124] K. J. Walters, H. Matsuo, and G. Wagner, A simple method to distinguish intermonomer nuclear overhauser effects in homodimeric proteins with C-2 symmetry, J Am Chem Soc 119: 5958-5959 (1997).

[125] F. K. Junius, S. I. O’Donoghue, M. Nilges, A. S. Weiss, and G. F. King, High resolution NMR solution structure of the leucine zipper domain of the c-Jun homodimer, J Biol Chem 271: 13663-13667 (1996).

[126] J. Voss, L. Salwinski, H. R. Kaback, and W. L. Hubbell, A method for distance determination in proteins using a designed metal ion binding site and sitedirected spin labeling: evaluation with T4 lysozyme, Proc Natl Acad Sci U S A 92: 12295-12299 (1995).

[127] C. Ma and S. J. Opella, Lanthanide ions bind specifically to an added "EFhand" and orient a membrane protein in micelles for solution NMR spectroscopy, J Magn Reson 146: 381-384 (2000).

[128] V. Gaponenko, J. W. Howarth, L. Columbus, G. Gasmi-Seabrook, J. Yuan, W. L. Hubbell, and P. R. Rosevear, Protein global fold determination using site-directed spin and isotope labeling, Protein Sci 9: 302-309 (2000).

[129] A. Leonov, B. Voigt, F. Rodriguez-Castaneda, P. Sakhaii, and C. Griesinger, Convenient synthesis of multifunctional EDTA-based chiral metal chelates substituted with an S-mesylcysteine, Chemistry 11: 3342-3348 (2005).

[130] L. W. Donaldson, N. R. Skrynnikov, W. Y. Choy, D. R. Muhandiram, B. Sarkar, J. D. Forman-Kay, and L. E. Kay, Structural characterization of proteins with an attached ATCUN motif by paramagnetic relaxation enhancement NMR spectroscopy, J Am Chem Soc 123: 9843-9847 (2001).

[131] J. Feeney, B. Birdsall, A. F. Bradbury, R. R. Biekofsky, and P. M. Bayley, Calmodulin tagging provides a general method of using lanthanide induced 
magnetic field orientation to observe residual dipolar couplings in proteins in solution, J Biomol NMR 21: 41-48 (2001).

[132] H. J. Dyson and P. E. Wright, Equilibrium NMR studies of unfolded and partially folded proteins, Nat Struct Biol 5 Suppl: 499-503 (1998).

[133] J. Iwahara and G. M. Clore, Detecting transient intermediates in macromolecular binding by paramagnetic NMR, Nature 440: 1227-1230 (2006).

[134] V. Gaponenko, A. S. Altieri, J. Li, and R. A. Byrd, Breaking symmetry in the structure determination of (large) symmetric protein dimers, J Biomol NMR 24: 143-148 (2002).

[135] M. Nilges, A calculation strategy for the structure determination of symmetric dimers by 1H NMR, Proteins 17: 297-309 (1993).

[136] C. A. Bewley and G. M. Clore, Determination of the relative orientation of the two halves of the domain-swapped dimer of cyanovirin- $\mathrm{N}$ in solution using dipolar couplings and rigid body minimization, J Am Chem Soc 122: 60096016 (2000).

[137] P. H. Hirel, J. M. Schmitter, P. Dessen, G. Fayat, and S. Blanquet, Extent of N-terminal methionine excision from Escherichia coli proteins Is governed by the side chain length of the penultimate amino acid, Proc Nat Acad Sci USA 86: 8247-8251 (1989).

[138] A. Dobrodumov and A. M. Gronenborn, Filtering and selection of structural models: combining docking and NMR, Proteins 53: 18-32 (2003).

[139] P. B. Card, P. J. Erbel, and K. H. Gardner, Structural basis of ARNT PAS-B dimerization: use of a common $\beta$-sheet interface for hetero- and homodimerization, J Mol Biol 353: 664-77 (2005).

[140] M. Zweckstetter, J. R. Schnell, and J. J. Chou, Determination of the packing mode of the coiled-coil domain of cGMP-dependent protein kinase Ialpha in 
solution using charge-predicted dipolar couplings, J Am Chem Soc 127: 11918$9(2005)$.

[141] J. Garmendia, G. Frankel, and V. F. Crepin, Enteropathogenic and enterohemorrhagic Escherichia coli infections: translocation, translocation, translocation, Infect Immun 73: 2573-2585 (2005).

[142] J. P. Nataro and J. B. Kaper, Diarrheagenic Escherichia coli, Clin Microbiol Rev 11: 142-201 (1998).

[143] P. Papatheodorou, G. Domanska, M. Oxle, J. Mathieu, O. Selchow, B. Kenny, and J. Rassow, The enteropathogenic Escherichia coli (EPEC) Map effector is imported into the mitochondrial matrix by the TOM/Hsp70 system and alters organelle morphology, Cell Microbiol 8: 677-689 (2006).

[144] B. Kenny and M. Jepson, Targeting of an enteropathogenic Escherichia coli (EPEC) effector protein to host mitochondria, Cell Microbiol 2: 579-590 (2000).

[145] B. Kenny, Mechanism of action of EPEC Type III effector molecules, Int J Med Microbiol 291: 469-477 (2002).

[146] N. M. Alto, F. Shao, C. S. Lazar, R. L. Brost, G. Chua, S. Mattoo, S. A. McMahon, P. Ghosh, T. R. Hughes, C. Boone, and J. E. Dixon, Identification of a bacterial type III effector family with G protein mimicry functions, Cell 124: $133-145$ (2006).

[147] P. Wattiau, S. Woestyn, and G. R. Cornelis, Customized secretion chaperones in pathogenic bacteria, Mol Microbiol 20: 255-262 (1996).

[148] C. Parsot, C. Hamiaux, and A. L. Page, The various and varying roles of specific chaperones in type III secretion systems, Curr Opin Microbiol 6: 7-14 (2003).

[149] R. M. Macnab, The bacterial flagellum: reversible rotary propellor and type III export apparatus, J Bacteriol 181: 7149-7153 (1999). 
[150] R. R. Isberg and G. Dumenil, Delivering dangerous cargoes, Nat Struct Biol 8: 1006-1008 (2001).

[151] A. L. Page and C. Parsot, Chaperones of the type III secretion pathway: jacks of all trades, Mol Microbiol 46: 1-11 (2002).

[152] J. A. Crawford and J. B. Kaper, The N-terminus of enteropathogenic Escherichia coli (EPEC) Tir mediates transport across bacterial and eukaryotic cell membranes, Mol Microbiol 46: 855-868 (2002).

[153] N. A. Thomas, W. Deng, J. L. Puente, E. A. Frey, C. K. Yip, N. C. Strynadka, and B. B. Finlay, CesT is a multi-effector chaperone and recruitment factor required for the efficient type III secretion of both LEE- and non-LEE-encoded effectors of enteropathogenic Escherichia coli, Mol Microbiol 57: 1762-79 (2005).

[154] Y. Luo, M. G. Bertero, E. A. Frey, R. A. Pfuetzner, M. R. Wenk, L. Creagh, S. L. Marcus, D. Lim, F. Sicheri, C. Kay, C. Haynes, B. B. Finlay, and N. C. J. Strynadka, Structural and biochemical characterization of the type III secretion chaperones CesT and SigE, Nat Struct Biol 8: 1031-1036 (2001).

[155] S. Birtalan and P. Ghosh, Structure of the Yersinia type III secretory system chaperone SycE, Nat Struct Biol 8: 974-978 (2001).

[156] J. Phan, J. E. Tropea, and D. S. Waugh, Structure of the Yersinia pestis type III secretion chaperone $\mathrm{SycH}$ in complex with a stable fragment of YscM2, Acta Crystallogr D Biol Crystallogr 60: 1591-1599 (2004).

[157] C. R. Buttner, G. R. Cornelis, D. W. Heinz, and H. H. Niemann, Crystal structure of Yersinia enterocolitica type III secretion chaperone SycT, Protein Sci 14: 1993-2002 (2005).

[158] M. Locher, B. Lehnert, K. Krauss, J. Heesemann, M. Groll, and G. Wilharm, Crystal structure of the Yersinia enterocolitica type III secretion chaperone SycT, J Biol Chem 280: 31149-31155 (2005). 
[159] C. E. Stebbins and J. E. Galan, Maintenance of an unfolded polypeptide by a cognate chaperone in bacterial type III secretion, Nature 414: 77-81 (2001).

[160] A. van Eerde, C. Hamiaux, J. Perez, C. Parsot, and B. W. Dijkstra, Structure of Spa15, a type III secretion chaperone from Shigella flexneri with broad specificity, EMBO Reports 5: 477-483 (2004).

[161] M. Lilic, M. Vujanac, and C. E. Stebbins, A common structural motif in the binding of virulence factors to bacterial secretion chaperones, Mol Cell 21: 653-664 (2006).

[162] S. C. Birtalan, R. M. Phillips, and P. Ghosh, Three-dimensional secretion signals in chaperone-effector complexes of bacterial pathogens, Mol Cell 9: 971980 (2002).

[163] F. D. Schubot, M. W. Jackson, K. J. Penrose, S. Cherry, J. E. Tropea, G. V. Plano, and D. S. Waugh, Three-dimensional structure of a macromolecular assembly that regulates type III secretion in Yersinia pestis, J Mol Biol 346: $1147-61(2005)$.

[164] C. E. Stebbins and J. E. Galan, Priming virulence factors for delivery into the host, Nat Rev Mol Cell Biol 4: 738-743 (2003).

[165] Y. Akeda and J. E. Galan, Chaperone release and unfolding of substrates in type III secretion, Nature 437: 911-5 (2005).

[166] A. Gauthier and B. B. Finlay, Translocated intimin receptor and its chaperone interact with ATPase of the type III secretion apparatus of enteropathogenic Escherichia coli, J Bacteriol 185: 6747-6755 (2003).

[167] A. Abe, M. de Grado, R. A. Pfuetzner, C. Sanchez-SanMartin, R. DeVinney, J. L. Puente, N. C. J. Strynadka, and B. B. Finlay, Enteropathogenic Escherichia coli translocated intimin receptor, Tir, requires a specific chaperone for stable secretion, Mol Microbiol 33: 1162-1175 (1999). 
[168] V. Receveur-Brechot, J. M. Bourhis, V. N. Uversky, B. Canard, and S. Longhi, Assessing protein disorder and induced folding, Proteins 62: 24-45 (2006).

[169] S. C. Panchal, N. S. Bhavesh, and R. V. Hosur, Improved 3D triple resonance experiments, $\mathrm{HNN}$ and $\mathrm{HN}(\mathrm{C}) \mathrm{N}$, for $\mathrm{HN}$ and ${ }^{15} \mathrm{~N}$ sequential correlations in $\left({ }^{13} \mathrm{C},{ }^{15} \mathrm{~N}\right)$ labeled proteins: application to unfolded proteins, J Biomol NMR 20: 135-47 (2001).

[170] P. Ghosh, Process of protein transport by the type III secretion system, Microbiol Mol Biol Rev 68: 771-95 (2004).

[171] C. Klammt, F. Löhr, B. Schafer, W. Haase, V. Dötsch, H. Rüterjans, C. Glaubitz, and F. Bernhard, High level cell-free expression and specific labeling of integral membrane proteins, Eur J Biochem 271: 568-580 (2004).

[172] S. B. Nabuurs, A. J. Nederveen, W. Vranken, J. F. Doreleijers, A. M. Bonvin, G. W. Vuister, G. Vriend, and C. A. Spronk, DRESS: a database of REfined solution NMR structures, Proteins 55: 483-6 (2004).

[173] J. P. Linge, M. A. Williams, C. Spronk, A. Bonvin, and M. Nilges, Refinement of protein structures in explicit solvent, Prot Struct Funct Genet 50: 496-506 (2003). 


\section{A}

\section{Appendix: Sample preparation}

This appendix shows SDS-gels to document the stages of the different protein purification protocols and ESI-MS spectra to verify the purified proteins.

\section{A.1 CyIR2}

E. coli BL21(DE3) cells harbouring pET32a-vectors with an inserted wild type or mutant CylR2 gene were cultivated at $28^{\circ} \mathrm{C}$ and the expressed proteins were purified after cell rupture via three chromatographic steps. The SDS-PAGE in Figure A.1 presents an exemplary preparation.

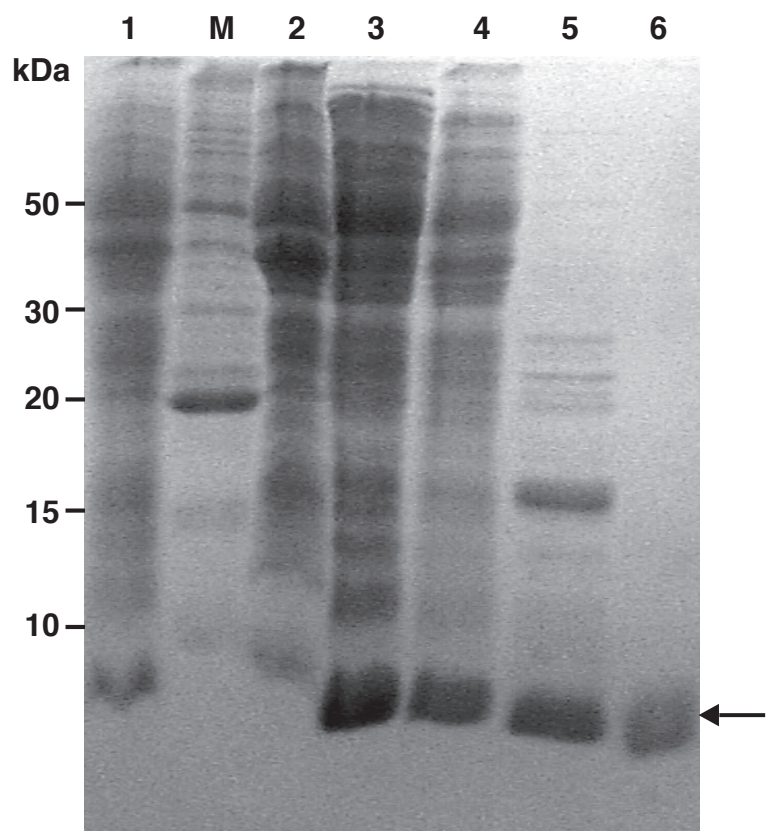

Figure A.1: Coomassie bluestained SDS-PAGE documenting the expression and purification of CylR2 in E. coli BL21(DE3). The arrow indicates the CylR2 band. Lane 1, induced crude cell extract. Lane 2, pellet proteins of the sonicated cells. Lane 3, supernatant proteins of the sonicated cells. Lane 4, flow through and wash of the anion exchange chromatography. Lane 5, after cation exchange chromatography. Lane 6, purified CylR2 after RP-HPLC. Lane M, molecular weight standard.

The result of the ESI-MS analysis of the final sample after RP-HPLC is shown for CylR $2_{T 55 C}$ in Figure A.2. 


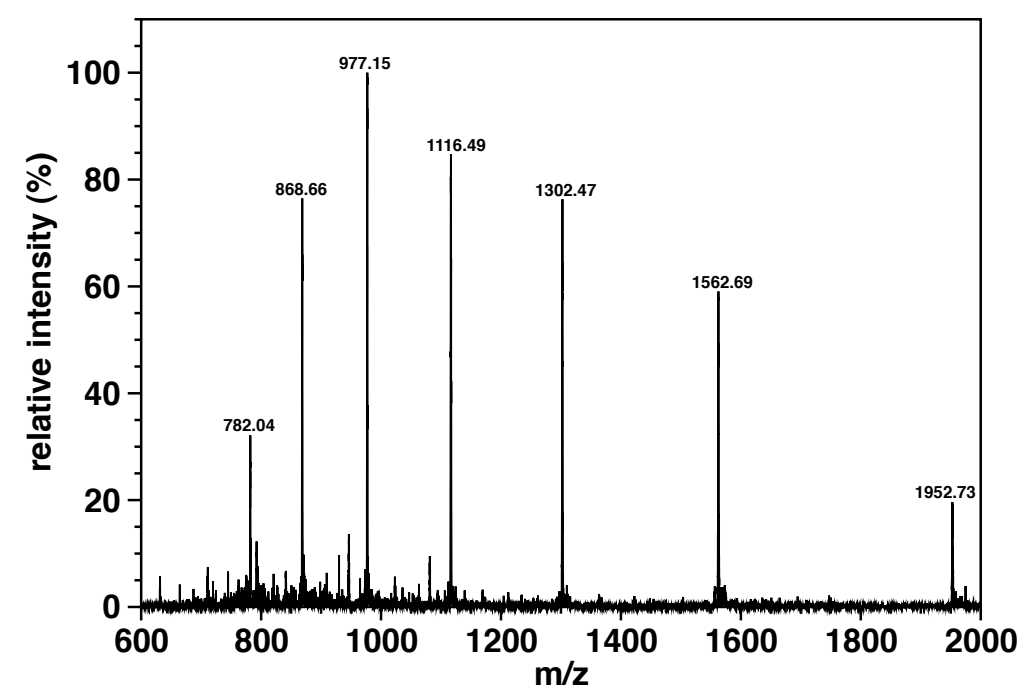

Figure A.2: ESI-MS spectrum of CylR2 $2_{T 55 C}$ after RP-HPLC. The indicated $\mathrm{m} / \mathrm{z}$ ratios belong to 4 - to 10 -fold positively charged CylR $2_{T 55} \mathrm{C}$ molecule ions.

\section{A.2 CesT}

The expression and purification of CesT is only shown together with Map84 (Figure A.8). The ESI-MS spectrum documents simultaneously the molecular weight and deuteration degree of a perdeuterated His7-CesT sample (Figure A.3.).

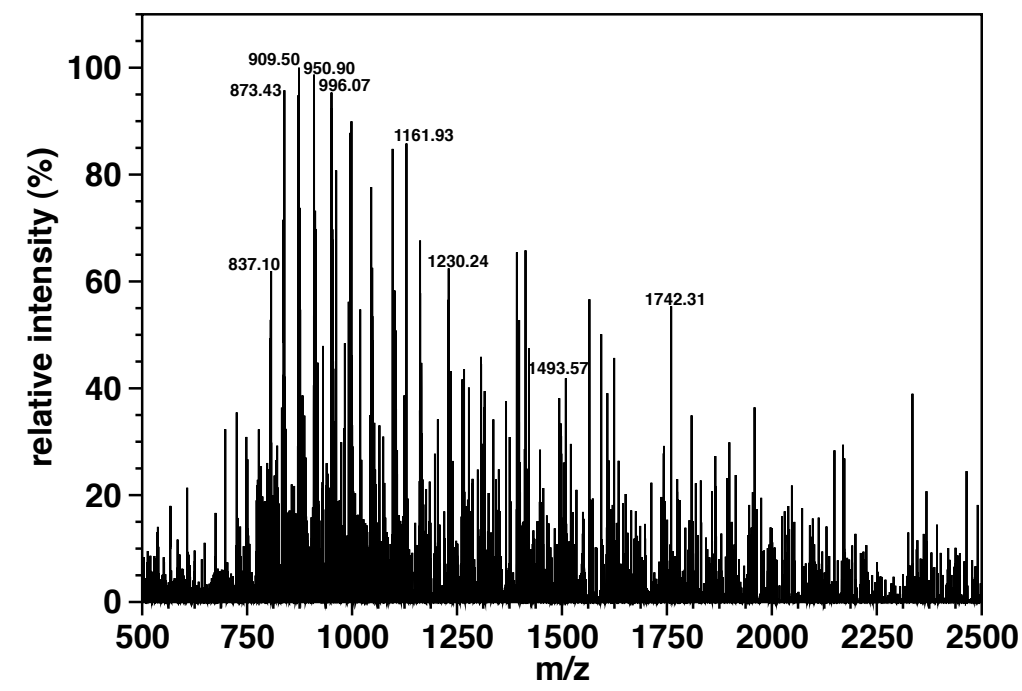

Figure A.3: ESI-MS spectrum of perdeuterated ${ }^{2} \mathrm{H}$-His 7 -CesT in $20 \mathrm{mM} \mathrm{NH}_{4} \mathrm{OAc}$. The $\mathrm{m} / \mathrm{z}$-ratio is explicitly indicated for peaks corresponding to $99 \%$ deuterated His7CesT with the number of positive charges decreasing from left to right from 25 to 12. 


\section{A.3 Map}

Figure A.4 displays the expression of His7-Map84 at $37{ }^{\circ} \mathrm{C}$ in E. coli BL21(DE3) and its purification.

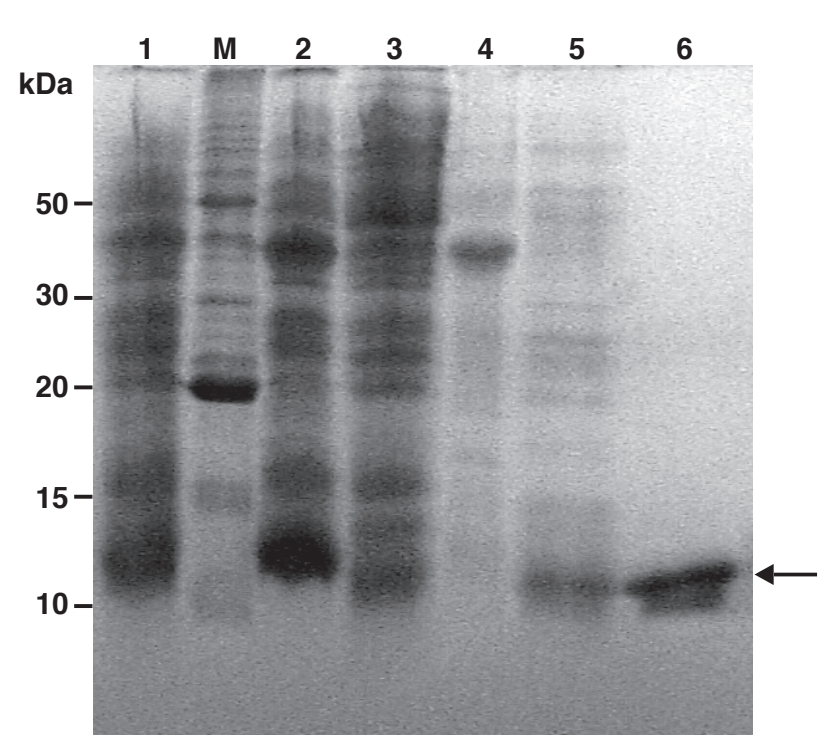

Figure A.4: Coomassie bluestained SDS-PAGE illustrates expression and purification of $\mathrm{His}_{7}-$ Map84 in E. coli BL21(DE3). The arrow indicates His7-Map84. Lane 1 , induced crude cell extract. Lane 2 , pellet proteins of the sonicated cells. Lane 3, supernatant proteins of the sonicated cells. Lane 4, pellet proteins after denaturing treatment of the pellet after sonication. Lane 5 , supernatant proteins after denaturing treatment of the pellet after sonication. Lane 6, after Ni-NTAagarose column. Lane M, molecular weight standard.

\section{A.4 Tir}

The expression and purification for different Tir-constructs is presented exemplarily for Tir108 in Figure A.5. ESI-MS spectra of Tir108 and TirN are shown in Figures A.6 and A.7. respectively.

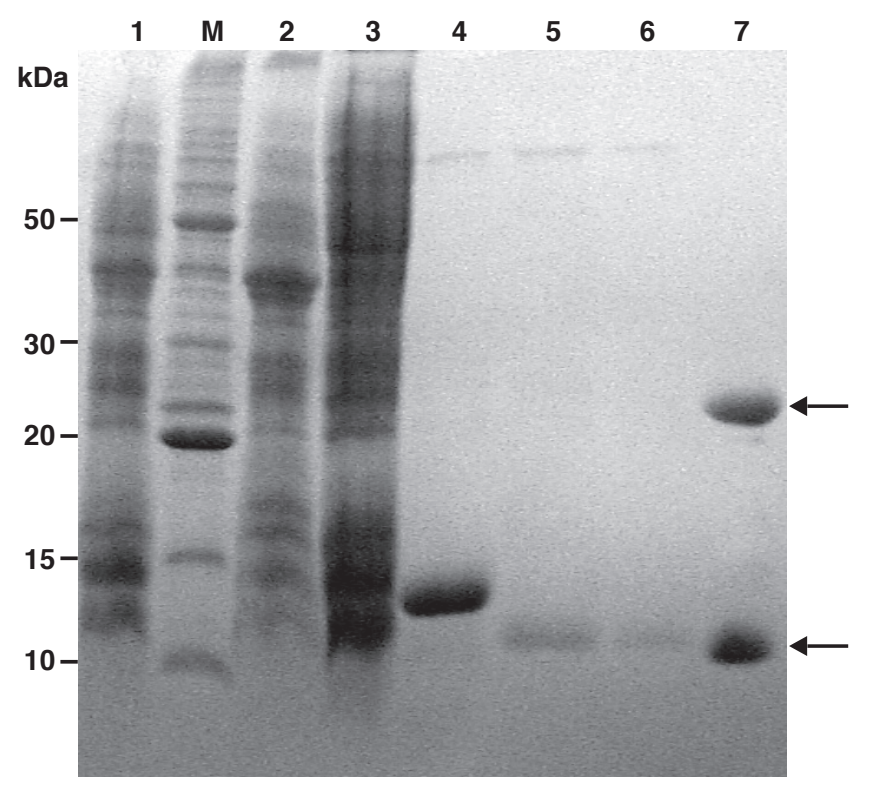

Figure A.5: Coomassie bluestained SDS-PAGE documenting the expression and purification of Tir108 in E. coli BL21(DE3). The arrows indicate monomeric and dimeric Tir108. Lane 1, induced crude cell extract. Lane 2, pellet proteins of the sonicated cells. Lane 3, supernatant proteins of the sonicated cells. Lane 4, after first Ni-NTA-agarose column. Lane 5, after TEV-cleavage. Lane 6, after second Ni-NTA-agarose column. Lane 7, purified Tir108 after RP-HPLC. Lane M, molecular weight standard. 


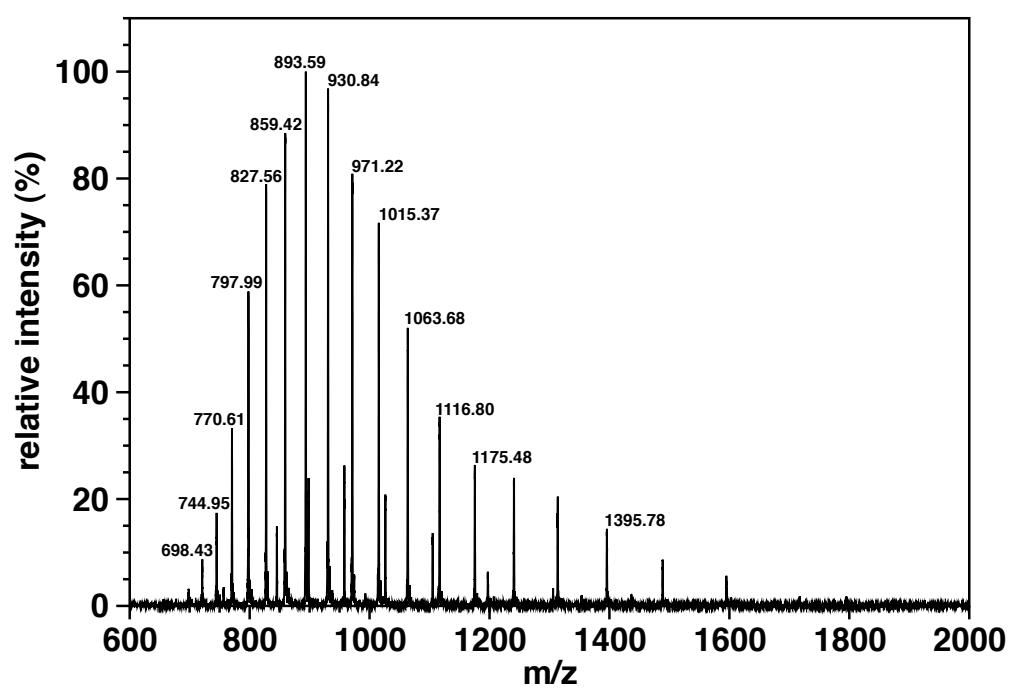

Figure A.6: ESI-MS spectrum of Tir108 after RP-HPLC. The $\mathrm{m} / \mathrm{z}$-ratio is shown the 16- and the 19- to 32-fold positively charged molecule ions of Tir108. The small peaks belong to dimeric Tir108. Two Tir108 are covalently bond via a disulfide bridge between the unique cysteine residue in each chain.

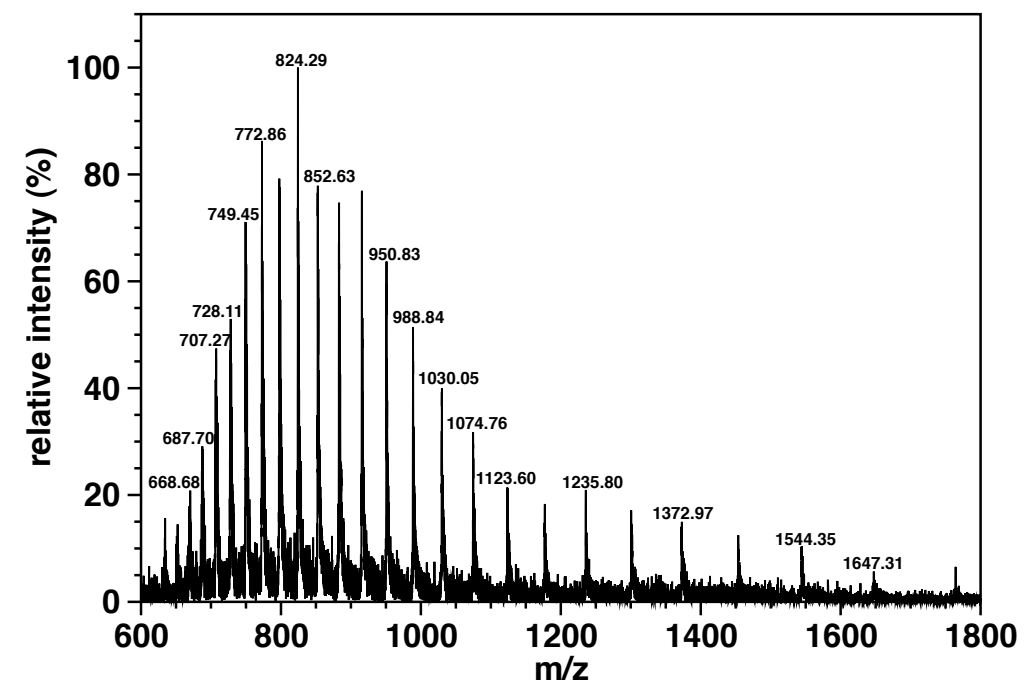

Figure A.7: ESI-MS spectrum of TirN in $20 \mathrm{mM} \mathrm{NH}_{4} \mathrm{OAc}$. The found $\mathrm{m} / \mathrm{z}$-ratios belong to TirN molecule ions with a different number of positive charges. The detected molecular is $24698.46 \pm 7.87$ what deviates by $0.032 \%$ from the expected mass for TirN. 


\section{A.5 CesT/effector-complexes}

The coexpression and copurification of the CesT/Map84 complex is demonstrated in Figure A.8. The ESI-MS spectra exhibit the molecular weights of coexpressed and copurified complex samples of CesT/Map84 (Figure A.9) and CesT/Tir108 (Figure A.10).

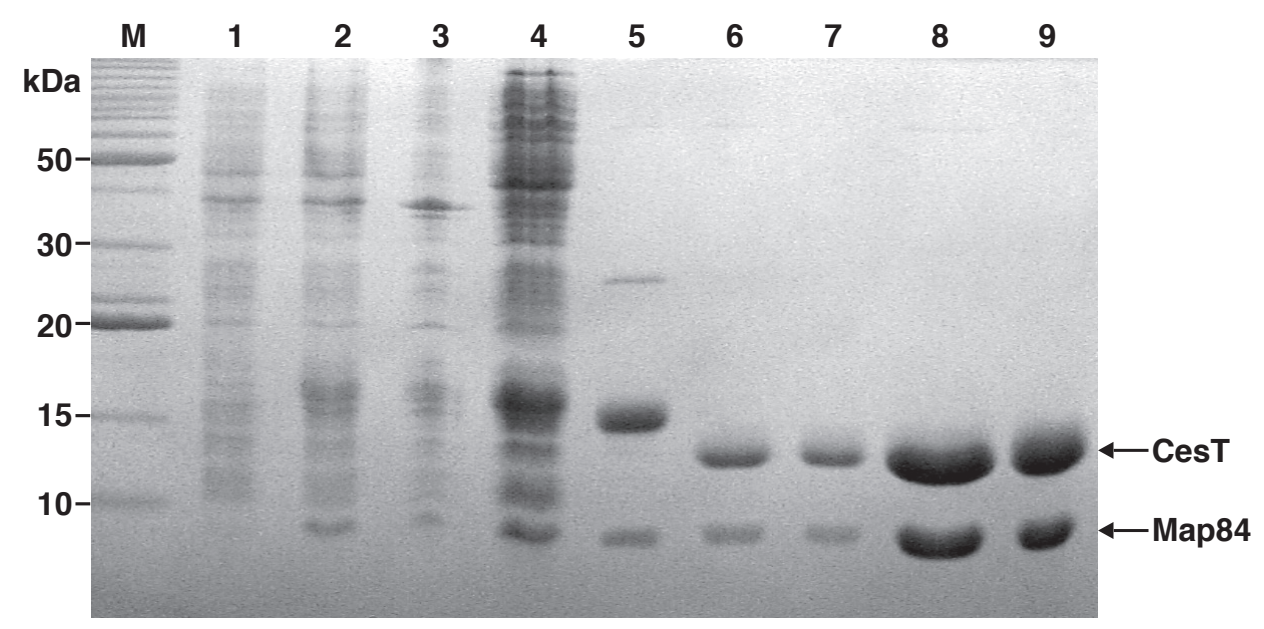

Figure A.8: Coomassie blue-stained SDS-PAGE documenting the coexpression and copurification of CesT/Map84 in E. coli BL21(DE3). CesT and Map84 bands are indicated. Lane M, molecular weight standard. Lane 1, uninduced crude cell extract. Lane 2, induced crude cell extract. Lane 3, pellet proteins of the sonicated cells. Lane 4, supernatant proteins of the sonicated cells. Lane 5, after first Ni-NTA-Agarose column. Lane 6, after TEV-cleavage. Lane 7, after second Ni-NTA-Agarose column. Lane 8, after anion exchange chromatography. Lane 9, purified CesT/Map84 after gel filtration.

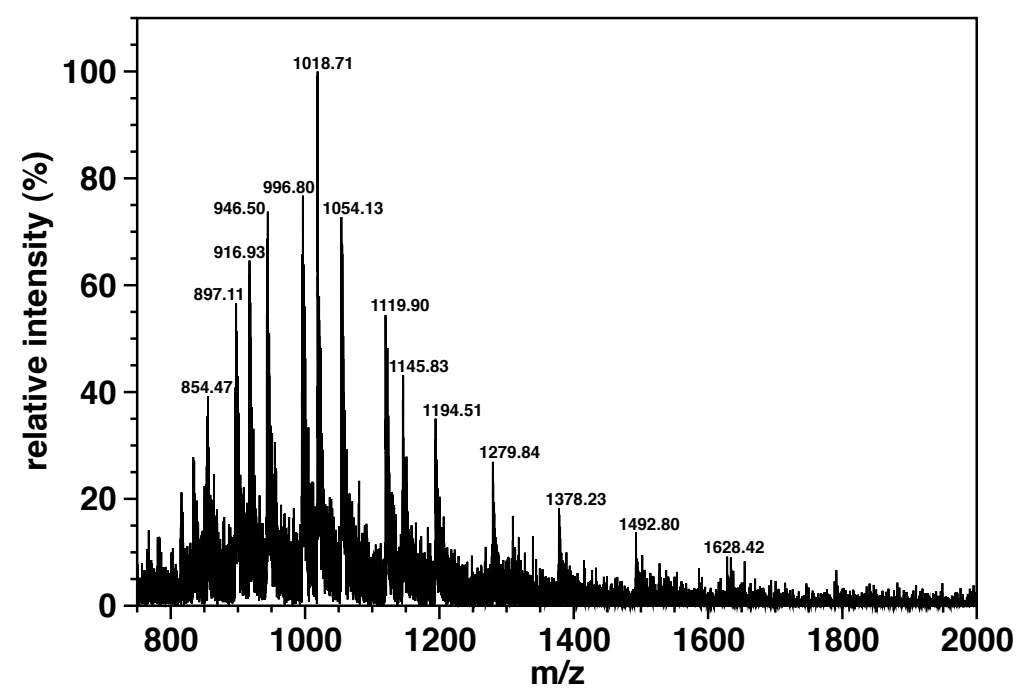

Figure A.9: ESI-MS spectrum of coexpressed and copurified CesT/Map84 in $20 \mathrm{mM}$ $\mathrm{NH}_{4} \mathrm{OAc}$. The $\mathrm{m} / \mathrm{z}$-ratio is displayed for the 8- to 10-fold positively charged molecule ions of Map84 and for the 11- to 21-fold positively charged molecule ions of CesT. 


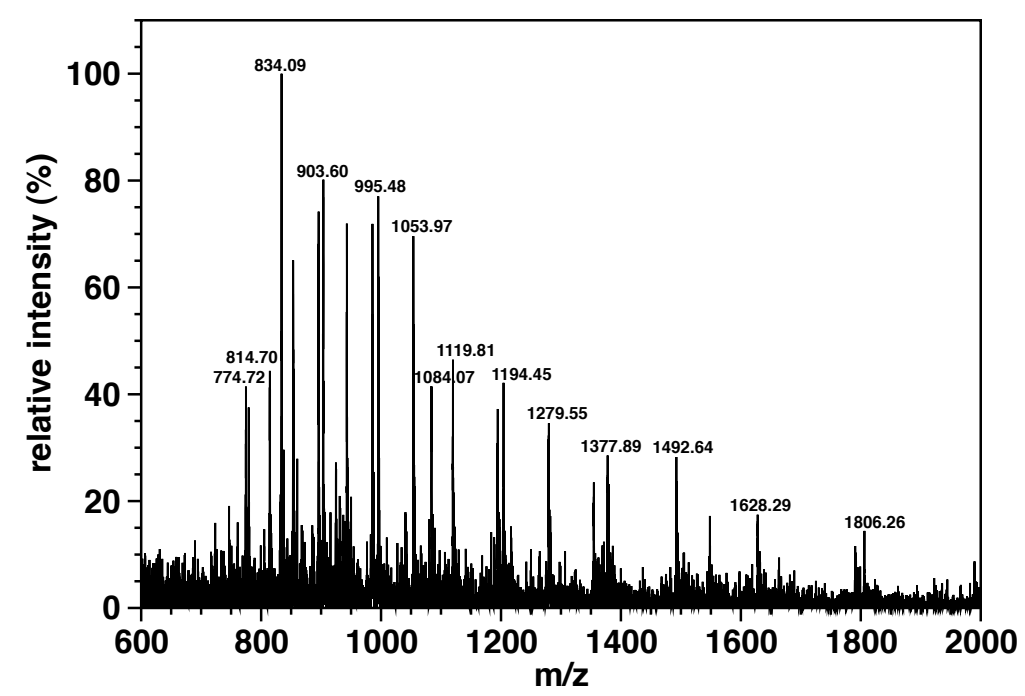

Figure A.10: ESI-MS spectrum of coexpressed and copurified CesT/Tir108 in $20 \mathrm{mM}$ $\mathrm{NH}_{4}$ OAc. The $\mathrm{m} / \mathrm{z}$-ratio is indicated for the 6-, 10-, 12-, 13- and 14-fold positively charged molecule ions of Tir108 and the 11- to 18- and 22-fold positively charged molecule ions of CesT. 


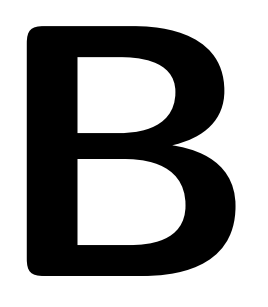

\section{Appendix: NMR experiments and chemical shift assignments}

In the following the NMR experiments measured for resonance assignments and structure calculation (B.1) as well as the obtained chemical shift assignments (B.2) which have not been deposited with the BMRB are summarized.

\section{B.1 NMR experiments}

Figure B.1 shows an overlay of the 1D NMR experiments measured with two different relaxation delays for a $10 \mu \mathrm{M}$ CylR2 sample at $298 \mathrm{~K}$ to estimate $\mathrm{T}_{2}$. The intensity ratio of 1.26 between the two spectra corresponds to an experimental molecular weight of $\sim 17 \mathrm{kDa}$ which indicates the concentration-independent dimeric state of CylR2.

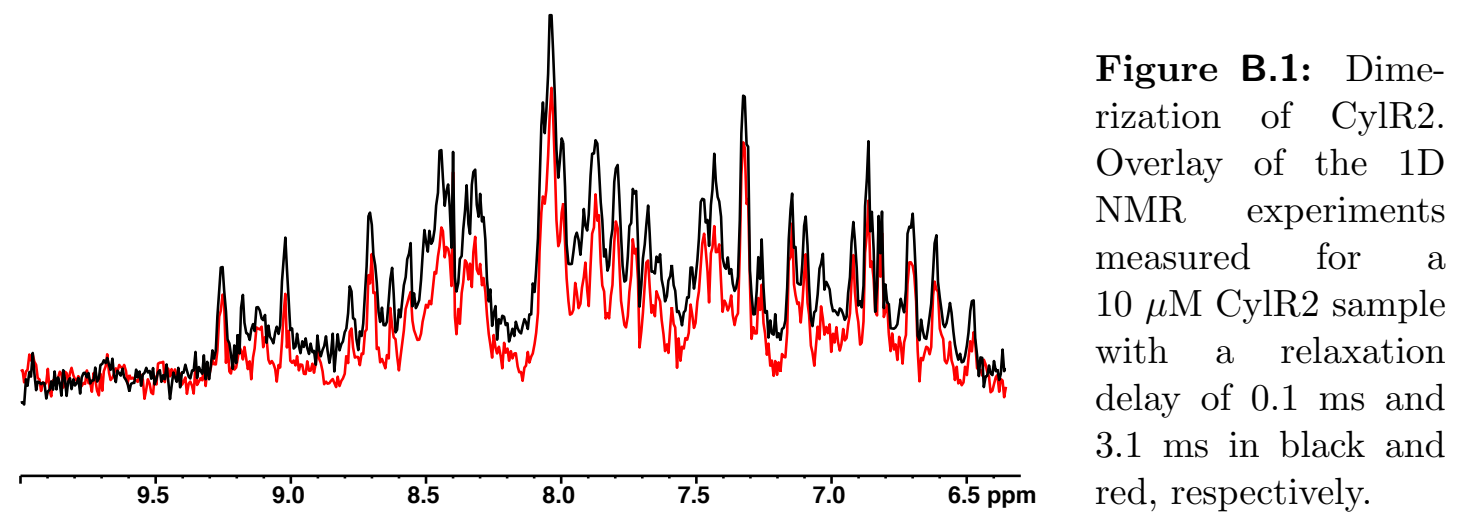

NMR spectra acquired for the assignment and structure determination of CylR2 (Table B.1), for the backbone assignment of CesT (Table B.2) and Tir108 (Table B.3), the reassignment of the CesT backbone in complex with Map84 (Table B.4) and in complex with Tir108 (Table B.5) and the accomplished backbone assignments of Map84 in complex with CesT (Table B.6 are provided in the following: 
Table B.1: NMR spectra for assignment and structure determination of CylR2 measured at $298 \mathrm{~K}$ and with a ${ }^{13} \mathrm{C} /{ }^{15} \mathrm{~N}$-labeled CylR2 sample if not indicated differently.

\begin{tabular}{|c|c|c|c|}
\hline & experiment & spectrometer & comment/pulse program (pp) \\
\hline \multirow{6}{*}{$\begin{array}{l}\text { backbone } \\
\text { assignment }\end{array}$} & $2 \mathrm{D}{ }^{15} \mathrm{~N} \mathrm{HSQC}$ & AVANCE 600 , cryogenic probe & hsqc-ge.mz, reference experiment \\
\hline & 3D HNCA & AVANCE 600 , cryogenic probe & hnca-ge.mz \\
\hline & 3D $\mathrm{HNCO}$ & AVANCE 600 , cryogenic probe & hnco-cpd-fb.mz \\
\hline & $3 \mathrm{D} \mathrm{HN}(\mathrm{CA}) \mathrm{CO}$ & AVANCE 600 , cryogenic probe & Bruker pp hncacogp3d \\
\hline & 3D HNCACB & AVANCE 600 , cryogenic probe & hncacb-cpd.mz \\
\hline & 3D CBCACONH & AVANCE 600 , cryogenic probe & cbcaconh-J.mz \\
\hline aliphatic & 3D HCCH-TOCSY & DRX 800 & \multirow{3}{*}{$\begin{array}{l}{ }^{13} \mathrm{C} \text { refocusing and mixing } \\
\text { by adiabatic pulses } 69,68\end{array}$} \\
\hline sidechain & 3D CCONH-TOCSY & AVANCE 600 , cryogenic probe & \\
\hline assignment & 3D HCCONH-TOCSY & AVANCE 600 , cryogenic probe & \\
\hline aromatic & $2 \mathrm{D}{ }^{13} \mathrm{C} \mathrm{HSQC}$ & AVANCE 900 & Bruker pulse program hsqcetgpsp \\
\hline sidechain & $2 \mathrm{D}(\mathrm{H} \beta) \mathrm{C} \beta(\mathrm{C} \gamma \mathrm{C} \delta) \mathrm{H} \delta$ & AVANCE 700 & hbcbcgaro.vivi.txt, 70 \\
\hline assignment & $2 \mathrm{D}(\mathrm{H} \beta) \mathrm{C} \beta(\mathrm{C} \gamma \mathrm{C} \delta \mathrm{C} \epsilon) \mathrm{H} \epsilon$ & AVANCE 700 & hbcbcgcearo.vivi.txt, 70 \\
\hline \multirow{4}{*}{$\begin{array}{l}\text { distance } \\
\text { determination }\end{array}$} & $3 \mathrm{D}{ }^{15} \mathrm{~N}$ NOESY-HSQC & AVANCE 600 , cryogenic probe & ${ }^{15}$ N-labeled, noesy3d.mz \\
\hline & $3 \mathrm{D}{ }^{13} \mathrm{C}$ NOESY-HSQC & DRX 600 & c13noesy_hsqc.vivi.txt \\
\hline & $2 \mathrm{D}{ }^{15} \mathrm{~N}$ HSQC & AVANCE 700 & ${ }^{15} \mathrm{~N}$-labeled, hsqc-ge.mz, PRE \\
\hline & $2 \mathrm{D}{ }^{15} \mathrm{~N} \mathrm{~T}_{2}$ & AVANCE 700 & ${ }^{15}$ N-labeled, T2N.sr.txt \\
\hline
\end{tabular}

Table B.2: NMR spectra for the backbone assignment of CesT measured at $303 \mathrm{~K}$.

\begin{tabular}{|c|c|c|c|}
\hline labeling & experiment & spectrometer & comment/pulse program (pp) \\
\hline${ }^{13} \mathrm{C} /{ }^{15} \mathrm{~N}$ & $2 \mathrm{D}^{15} \mathrm{~N}$ HSQC & AVANCE 900 & hsqc-ge.mz, reference spectrum \\
\hline${ }^{2} \mathrm{H}(75 \%) /{ }^{15} \mathrm{~N}$ & $2 \mathrm{D}{ }^{15} \mathrm{~N}$ Trosy-HSQC & AVANCE 900 & trosyfb.mz, reference spectrum \\
\hline${ }^{2} \mathrm{H}(75 \%) /{ }^{13} \mathrm{C} /{ }^{15} \mathrm{~N}$ & $3 \mathrm{D}$ HNCA & AVANCE 700 & hnca-ge-2H.sr.txt \\
\hline${ }^{2} \mathrm{H}(75 \%) /{ }^{13} \mathrm{C} /{ }^{15} \mathrm{~N}$ & 3D Trosy-HN $(\mathrm{CO}) \mathrm{CA}$ & $\begin{array}{l}\text { AVANCE } 600, \\
\text { cryogenic probe }\end{array}$ & trhncoca3d-QJ.cpj.txt, 81 \\
\hline${ }^{2} \mathrm{H}(75 \%) /{ }^{13} \mathrm{C} /{ }^{15} \mathrm{~N}$ & 3D $\mathrm{HN}(\mathrm{CO}) \mathrm{CACB}$ & $\begin{array}{l}\text { AVANCE 600, } \\
\text { cryogenic probe }\end{array}$ & Bruker pp hncocacbgp2h3d, $\Delta\left(\mathrm{C}_{\alpha} \mathrm{C}_{\beta}\right)=3.6 \mathrm{~ms}$ \\
\hline${ }^{2} \mathrm{H}(75 \%) /{ }^{13} \mathrm{C} /{ }^{15} \mathrm{~N}$ & 3D $\mathrm{HN}(\mathrm{COCA}) \mathrm{CB}$ & $\begin{array}{l}\text { AVANCE } 600, \\
\text { cryogenic probe }\end{array}$ & Bruker pp hncocacbgp $2 \mathrm{~h} 3 \mathrm{~d}, \Delta\left(\mathrm{C}_{\alpha} \mathrm{C}_{\beta}\right)=7 \mathrm{~ms}$ \\
\hline${ }^{2} \mathrm{H}(75 \%) /{ }^{13} \mathrm{C} /{ }^{15} \mathrm{~N}$ & 3D Trosy-HN(CA)CB & AVANCE 900 & hncacb-tr-h2.sr, $\Delta\left(\mathrm{C}_{\alpha} \mathrm{C}_{\beta}\right)=7.1 \mathrm{~ms}$ \\
\hline${ }^{2} \mathrm{H}(75 \%) /{ }^{13} \mathrm{C} /{ }^{15} \mathrm{~N}$ & 3D HNCO & AVANCE 700 & hnco-cpd-viv.txt \\
\hline${ }^{2} \mathrm{H}(75 \%) /{ }^{13} \mathrm{C} /{ }^{15} \mathrm{~N}$ & $3 \mathrm{D} \mathrm{HN}(\mathrm{CA}) \mathrm{CO}$ & $\begin{array}{l}\text { AVANCE } 600, \\
\text { cryogenic probe }\end{array}$ & Bruker pp hncacogp2h3d \\
\hline${ }^{2} \mathrm{H}(75 \%) /{ }^{15} \mathrm{~N}$ & $3 \mathrm{D}^{15} \mathrm{~N}$ NOESY-HSQC & $\begin{array}{l}\text { AVANCE } 900, \\
\text { cryogenic probe }\end{array}$ & noesy3d.sr \\
\hline
\end{tabular}

Table B.3: NMR spectra for the backbone assignment of Tir108 measured at $293 \mathrm{~K}$ with a ${ }^{13} \mathrm{C} /{ }^{15} \mathrm{~N}$-labeled sample.

\begin{tabular}{llc}
\hline \hline experiment & spectrometer & comment/pulse program (pp) \\
\hline 2D ${ }^{15} \mathrm{~N}$ HSQC & AVANCE 600, cryogenic probe & hsqc-ge.mz, reference experiment \\
3D HNCO & AVANCE 600, cryogenic probe & hnco-cpd-fb.mz \\
3D HN(CA)CO & AVANCE 600, cryogenic probe & Bruker pp hncacogp3d \\
3D HNCACB & AVANCE 600, cryogenic probe & hncacb-cpd.mz \\
3D CBCACONH & AVANCE 600, cryogenic probe & hnn.sr.txt, [169] \\
3D HNN & AVANCE 600, cryogenic probe \\
\hline \hline
\end{tabular}


Table B.4: NMR spectra for the backbone assignment of CesT in complex with Map84 measured at $303 \mathrm{~K}$ for a ${ }^{2} \mathrm{H}(75 \%) /{ }^{13} \mathrm{C} /{ }^{15} \mathrm{~N}$ CesT/His 7 Map84 complex sample.

\begin{tabular}{llc}
\hline \hline experiment & spectrometer & comment/pulse program (pp) \\
\hline 2D ${ }^{15}$ N Trosy-HSQC & AVANCE 900, cryogenic probe & trosyetf3gpsi, ${ }^{13} C$ decoupling added \\
3D Trosy-HNCA & AVANCE 900, cryogenic probe & trhnca_tr_d.rtf \\
3D Trosy-HN(COCA)CB & AVANCE 600, cryogenic probe & Ehncocacbtrosywgrt, $\Delta\left(\mathrm{C}_{\alpha} \mathrm{C}_{\beta}\right)=7 \mathrm{~ms}$ \\
3D Trosy-HN(CA)CB & AVANCE 900, cryogenic probe & hncacb-tr-h2.sr, $\Delta\left(\mathrm{C}_{\alpha} \mathrm{C}_{\beta}\right)=7.1 \mathrm{~ms}$ \\
3D Trosy-HNCO & AVANCE 900, cryogenic probe & Bruker pp trhncogp2h3d \\
3D Trosy-HN(CA)CO & AVANCE 600, cryogenic probe & trhncacoetgp3d, deuterium decoupling added \\
3D ${ }^{15}$ N NOESY-Trosy-HSQC & AVANCE 900, cryogenic probe & Bruker pp noesytretf3gp3d \\
\hline \hline
\end{tabular}

Table B.5: NMR spectra for the backbone assignment of CesT in complex with Tir108 measured at $303 \mathrm{~K}$ with a ${ }^{2} \mathrm{H}(75 \%) /{ }^{13} \mathrm{C} /{ }^{15} \mathrm{~N}$ CesT/Tir108 complex sample.

\begin{tabular}{llc}
\hline \hline experiment & spectrometer & comment/pulse program (pp) \\
\hline 2D ${ }^{15}$ N Trosy-HSQC & AVANCE 900, cryogenic probe & trosyetf3gpsi, ${ }^{13} C$ decoupling added \\
3D Trosy-HNCA & AVANCE 900, cryogenic probe & trhnca_tr_d.rtf \\
3D Trosy-HN(COCA)CB & AVANCE 600, cryogenic probe & Ehncocacbtrosywgrt, $\Delta\left(\mathrm{C}_{\alpha} \mathrm{C}_{\beta}\right)=7 \mathrm{~ms}$ \\
3D Trosy-HN(CA)CB & AVANCE 900, cryogenic probe & hncacb-tr-h2.sr, $\Delta\left(\mathrm{C}_{\alpha} \mathrm{C}_{\beta}\right)=7.1 \mathrm{~ms}$ \\
3D Trosy-HNCO & AVANCE 600, cryogenic probe & Bruker pp trhncogp2h3d \\
3D Trosy-HN(CA)CO & AVANCE 600, cryogenic probe & trhncacoetgp3d, deuterium decoupling added \\
3D ${ }^{15}$ N NOESY-Trosy-HSQC & AVANCE 900, cryogenic probe & Bruker pp noesytretf3gp3d \\
\hline \hline
\end{tabular}

Table B.6: NMR spectra for the backbone assignment of Map84 in complex with CesT measured at $303 \mathrm{~K}$ for a ${ }^{2} \mathrm{H}(99 \%) \mathrm{CesT} /{ }^{13} \mathrm{C} /{ }^{15} \mathrm{~N}$ Map84 complex sample.

\begin{tabular}{llc}
\hline \hline experiment & spectrometer & comment/pulse program (pp) \\
\hline 2D ${ }^{15}$ N Trosy-HSQC & AVANCE 900, cryogenic probe & trosyetf3gpsi, ${ }^{13} C$ decoupling added \\
2D ${ }^{15}$ N HSC & AVANCE 900, cryogenic probe & hsqc-ge.mz, reference experiment \\
3D HNCA & AVANCE 900, cryogenic probe & hnca-ge.mz \\
3D Trosy-HNCA & AVANCE 900, cryogenic probe & trhnca_tr_d.rtf \\
3D HNCACB & AVANCE 600, cryogenic probe & hncacb-cpd.mz \\
3D CBCACONH & AVANCE 600, cryogenic probe & cbcaconh-J.mz \\
3D Trosy-HNCO & AVANCE 900, cryogenic probe & tr-hnco.vivi \\
3D HN $(\mathrm{CA}) \mathrm{CO}$ & AVANCE 600, cryogenic probe & Bruker pp hncacogp3d \\
\hline \hline
\end{tabular}

\section{B.2 Chemical shift assignments}

Resonances assigned during this thesis, which are not deposited in the BMRB, are specified in the following Tables. Table B.7 gives the assigned backbone, $\mathrm{C}_{\beta}$ and amide side chain resonances of CylR2 in complex with its DNA. The assigned backbone and $\mathrm{C}_{\beta}$ resonances of CesT in complex with Map84 and in complex with Tir108 are indicated in Table B.8 and Table B.9, respectively. Assignments obtained for backbone and $\mathrm{C}_{\beta}$ resonances of Tir108 are stated in Table B.10. Table B.11 contains the assignments for Map84 in complex with CesT. 
Table B.7: Assigned chemical shifts of CylR2 in complex with its DNA at $298 \mathrm{~K}$ based on 3D HNCO, CBCA $(\mathrm{CO}) \mathrm{NH}, \mathrm{HNCA}$ and 2D HSQC experiments.

\begin{tabular}{|c|c|c|c|c|c|c|c|c|c|c|}
\hline & Res. & $\mathrm{C}^{\prime}$ & $\mathrm{C}_{\alpha}$ & $\mathrm{C}_{\beta}$ & $\mathrm{N}$ & $\mathrm{H}_{N}$ & $\mathrm{~N}_{\delta}$ & $\mathrm{H}_{\delta}$ & $\mathrm{N}_{\epsilon}$ & $\mathrm{H}_{\epsilon}$ \\
\hline 1 & MET & 181.67 & 55.18 & 33.33 & - & - & & & & \\
\hline 2 & ILE & 175.83 & 60.16 & 39.65 & 123.782 & 8.830 & & & & \\
\hline 3 & ILE & 174.29 & 60.58 & 38.11 & 129.925 & 9.426 & & & & \\
\hline 4 & ASN & 174.81 & 51.38 & 40.44 & 125.099 & 8.716 & 112.911 & $8.063 / 6.698$ & & \\
\hline 5 & ASN & 175.50 & 51.88 & 39.77 & 122.382 & 9.292 & 112.647 & $6.915 / 6.662$ & & \\
\hline 6 & LEU & 176.23 & 59.92 & 41.22 & 121.181 & 8.233 & & & & \\
\hline 7 & LYS & 177.43 & 60.51 & 32.50 & 118.245 & 8.881 & & & & \\
\hline 8 & LEU & 180.29 & 57.86 & 42.50 & 116.423 & 7.297 & & & & \\
\hline 9 & ILE & 178.01 & 63.05 & 36.74 & 119.068 & 8.058 & & & & \\
\hline 10 & ARG & 179.19 & 61.02 & 29.73 & 120.751 & 9.181 & & & & \\
\hline 11 & GLU & 181.61 & 59.88 & 29.04 & 116.461 & 8.607 & & & & \\
\hline 12 & LYS & 178.66 & 59.50 & 32.32 & 122.190 & 7.933 & & & & \\
\hline 13 & LYS & 175.32 & 55.41 & 31.61 & 117.130 & 7.640 & & & & \\
\hline 14 & LYS & 174.88 & 57.06 & 28.66 & 115.194 & 7.933 & & & & \\
\hline 15 & ILE & 175.45 & 59.45 & 41.43 & 117.674 & 7.284 & & & & \\
\hline 16 & SER & 175.36 & 58.14 & 65.23 & 124.513 & 9.185 & & & & \\
\hline 17 & GLN & 178.59 & 61.21 & 29.20 & 121.519 & 9.083 & & & 112.364 & 7.739/7.314 \\
\hline 18 & SER & 177.18 & 62.98 & - & 113.589 & 8.417 & & & & \\
\hline 19 & GLU & 179.56 & 59.11 & 30.19 & 124.496 & 8.337 & & & & \\
\hline 20 & LEU & 177.70 & 57.70 & 42.50 & 120.681 & 8.356 & & & & \\
\hline 21 & ALA & 178.62 & 55.56 & 17.47 & 120.227 & 8.296 & & & & \\
\hline 22 & ALA & 181.83 & 54.87 & 17.90 & 117.791 & 7.739 & & & & \\
\hline 23 & LEU & 178.21 & 57.51 & 41.83 & 119.294 & 7.509 & & & & \\
\hline 24 & LEU & 175.10 & 54.93 & 43.58 & 115.218 & 7.364 & & & & \\
\hline 25 & GLU & 175.97 & 57.64 & 31.16 & 114.644 & 7.938 & & & & \\
\hline 26 & VAL & 174.32 & 58.55 & - & 110.175 & 7.794 & & & & \\
\hline 27 & SER & 176.82 & 58.09 & - & 120.966 & 9.196 & & & & \\
\hline 28 & $\mathrm{ARG}$ & 179.21 & 60.29 & 30.28 & 115.330 & 8.151 & & & & \\
\hline 29 & GLN & 178.66 & 58.74 & 27.43 & 116.400 & 8.563 & & & 112.311 & $7.552 / 6.914$ \\
\hline 30 & THR & 176.46 & 66.08 & 68.04 & 121.166 & 7.990 & & & & \\
\hline 31 & ILE & 177.24 & 61.31 & 34.90 & 118.166 & 6.707 & & & & \\
\hline 32 & ASN & 176.52 & 58.62 & 41.93 & 118.617 & 8.343 & & & & \\
\hline 33 & GLY & 175.97 & 46.90 & - & 105.072 & 8.710 & & & & \\
\hline 34 & ILE & 181.44 & 64.60 & 38.90 & 121.474 & 7.946 & & & & \\
\hline 35 & GLU & 178.53 & 60.61 & 29.42 & 121.379 & 8.603 & & & & \\
\hline 36 & LYS & 175.41 & 55.14 & 31.75 & 116.762 & 7.791 & & & & \\
\hline 37 & ASN & 175.29 & 54.82 & 37.01 & 115.132 & 8.096 & 113.177 & $7.583 / 7.100$ & & \\
\hline 38 & LYS & 176.32 & 57.91 & 33.19 & 116.326 & 8.524 & & & & \\
\hline 39 & TYR & 173.07 & 55.34 & 36.95 & 111.877 & 7.040 & & & & \\
\hline 40 & ASN & - & 51.14 & - & 119.177 & 9.839 & 112.588 & $7.529 / 6.863$ & & \\
\hline 41 & $\mathrm{PRO}$ & 175.99 & 62.69 & 31.9 & - & - & & & & \\
\hline 42 & SER & 172.82 & 57.87 & 65.06 & 125.303 & 7.761 & & & & \\
\hline 43 & LEU & 178.09 & 59.04 & 41.00 & 121.439 & 9.120 & & & & \\
\hline 44 & GLN & 176.87 & 60.13 & 28.18 & 114.805 & 8.819 & & & 112.640 & $7.449 / 6.927$ \\
\hline 45 & LEU & 178.43 & 57.74 & 40.77 & 119.374 & 7.536 & & & & \\
\hline 46 & ALA & 178.93 & 55.74 & 19.34 & 121.438 & 8.803 & & & & \\
\hline 47 & LEU & 180.58 & 57.81 & 42.54 & 116.257 & 8.481 & & & & \\
\hline 48 & LYS & 178.15 & 60.24 & 32.76 & 120.796 & 7.702 & & & & \\
\hline 49 & ILE & 176.76 & 67.40 & 38.29 & 120.994 & 8.791 & & & & \\
\hline 50 & ALA & 179.06 & 55.79 & 18.36 & 120.794 & 8.048 & & & & \\
\hline 51 & TYR & 180.39 & 61.73 & 38.51 & 117.457 & 7.980 & & & & \\
\hline 52 & TYR & 177.97 & 63.24 & 37.83 & 118.551 & 8.929 & & & & \\
\hline 53 & LEU & 176.53 & 54.42 & 39.93 & 115.427 & 8.233 & & & & \\
\hline 54 & ASN & 174.03 & 53.92 & 38.00 & 122.388 & 7.969 & 111.898 & $7.486 / 6.772$ & & \\
\hline 55 & THR & - & 60.31 & - & 115.260 & 8.126 & & & & \\
\hline 56 & $\mathrm{PRO}$ & 178.46 & 62.85 & 32.36 & - & - & & & & \\
\hline 57 & LEU & 179.89 & 60.26 & 41.82 & 127.612 & 9.699 & & & & \\
\hline 58 & GLU & 177.01 & 58.87 & 29.78 & 117.102 & 9.980 & & & & \\
\hline 59 & ASP & 176.86 & 55.85 & 40.35 & 117.663 & 7.863 & & & & \\
\hline 60 & ILE & 174.04 & 64.09 & 39.64 & 120.354 & 8.067 & & & & \\
\hline 61 & PHE & 172.61 & 55.72 & 42.66 & 116.521 & 8.319 & & & & \\
\hline
\end{tabular}




\begin{tabular}{|c|c|c|c|c|c|c|c|c|c|c|}
\hline & Res. & $\overline{\mathrm{C}^{\prime}}$ & $\overline{\overline{C_{\alpha}}}$ & $\overline{\mathrm{C}_{\beta}}$ & $\overline{\mathrm{N}}$ & $\overline{\mathrm{H}_{N}}$ & $\overline{\mathrm{N}_{\delta}}$ & $\overline{\mathrm{H}_{\delta}}$ & $\overline{\mathrm{N}_{\epsilon}}$ & $\overline{\mathrm{H}_{\epsilon}}$ \\
\hline 62 & GLN & 175.09 & 54.20 & 32.34 & 117.231 & 8.517 & & & 110.852 & $7.535 / 6.624$ \\
\hline 63 & TRP & 174.60 & 57.00 & 31.09 & 125.665 & 9.309 & & & & \\
\hline 64 & GLN & - & 51.76 & - & 126.861 & 7.782 & & & 111.997 & $7.394 / 6.783$ \\
\hline 65 & $\mathrm{PRO}$ & - & - & - & - & - & & & & \\
\hline 66 & GLU & - & - & - & - & - & & & & \\
\hline
\end{tabular}

Table B.8: Assigned chemical shifts of CesT bound to Map84 at $303 \mathrm{~K}$ based on TROSY versions of 3D HNCO, $\mathrm{HN}(\mathrm{CA}) \mathrm{CO}, \mathrm{HNCA}, \mathrm{HN}(\mathrm{COCA}) \mathrm{CB}, \mathrm{HN}(\mathrm{CA}) \mathrm{CB}$ and 2D HSQC experiments.

\begin{tabular}{|c|c|c|c|c|c|c|c|c|c|c|c|c|c|}
\hline & Res. & $\mathrm{C}^{\prime}$ & $\overline{\mathrm{C}_{\alpha}}$ & $\overline{\mathrm{C}_{\beta}}$ & $\mathrm{N}$ & $\overline{\mathrm{H}_{N}}$ & & Res. & $\mathrm{C}^{\prime}$ & $\overline{\mathrm{C}_{\alpha}}$ & $\overline{\mathrm{C}_{\beta}}$ & $\mathrm{N}$ & $\overline{\mathrm{H}_{N}}$ \\
\hline 1 & MET & 54.63 & 32.02 & 175.77 & & & 79 & ASN & 52.59 & 38.80 & 175.33 & 112.724 & 6.781 \\
\hline 2 & SER & 58.49 & 63.43 & 174.05 & 119.263 & 8.405 & 80 & GLY & 46.42 & - & 175.39 & 110.894 & 7.255 \\
\hline 3 & SER & 57.03 & 64.19 & 175.08 & 120.525 & 8.748 & 81 & GLY & 43.75 & - & 104.82 & 105.066 & 8.320 \\
\hline 4 & ARG & 60.00 & 29.99 & 180.03 & 121.088 & 8.829 & 82 & PRO & 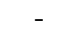 & - & - & - & - \\
\hline 5 & SER & 61.50 & 62.56 & 176.04 & 117.103 & 8.601 & 83 & TYR & - & - & - & - & - \\
\hline 6 & GLU & 59.42 & 28.64 & 179.15 & 123.840 & 7.667 & 84 & LEU & 53.65 & 40.60 & 177.29 & - & - \\
\hline 7 & LEU & 57.34 & 40.90 & 179.59 & 119.733 & 8.128 & 85 & CYS & 56.30 & 29.87 & 172.70 & 123.442 & 8.908 \\
\hline 8 & LEU & 57.33 & 39.95 & 178.56 & 120.230 & 7.630 & 86 & TYR & 54.91 & 42.81 & 172.69 & 120.221 & 6.295 \\
\hline 9 & LEU & 57.79 & 40.13 & 178.52 & 120.804 & .361 & 87 & GLU & 55.51 & 31.43 & 175.00 & 130.370 & 8.337 \\
\hline 10 & ASP & 57.15 & 40.08 & 179.69 & 118.543 & 7.799 & 88 & SER & 60.04 & 62.65 & 175.58 & 118.570 & 7.006 \\
\hline 11 & ARG & 58.17 & 29.90 & 179.91 & 119.308 & 7.863 & 89 & GLY & 46.84 & & 175.40 & 116.881 & 9.415 \\
\hline 12 & PHE & 60.73 & 38.98 & 175.63 & 123.752 & 9.125 & 90 & ALA & 50.55 & 18.22 & 177.56 & 123.714 & 9.289 \\
\hline 13 & ALA & 54.57 & 17.63 & 179.99 & 121.686 & 8.814 & 91 & GLN & 56.44 & 25.12 & 175.08 & 116.420 & 7.249 \\
\hline 14 & GLU & 58.27 & 28.91 & 178.87 & 115.951 & 7.277 & 92 & SER & 57.88 & 65.49 & 173.31 & 111.498 & 7.442 \\
\hline 15 & LYS & 58.38 & 31.26 & 178.74 & 120.737 & 7.425 & 93 & LEU & 54.32 & 41.50 & 174.46 & 124.375 & 9.066 \\
\hline 16 & ILE & 61.60 & 36.66 & 176.88 & 109.696 & 7.464 & 94 & LEU & - & - & & 124.463 & 9.322 \\
\hline 17 & GLY & 46.62 & & 175.65 & 110.018 & 7.428 & 95 & LEU & 53.32 & 43.60 & 173.48 & - & - \\
\hline 18 & VAL & 59.92 & 32.06 & 175.38 & 111.824 & 7.299 & 96 & ALA & 50.27 & 23.35 & 174.59 & 125.161 & 9.041 \\
\hline 19 & GLY & 44.60 & & 172.84 & 110.764 & 8.120 & 97 & LEU & 53.77 & 47.31 & 176.08 & 124.418 & 8.845 \\
\hline 20 & SER & 58.19 & 62.62 & 174.53 & 113.825 & 7.850 & 98 & ARG & 55.52 & 30.95 & 175.37 & 128.481 & 9.278 \\
\hline 21 & ILE & 59.68 & 40.90 & 173.97 & & 8.190 & 99 & PHE & 53.62 & 40.36 & 177.02 & 126.747 & 9.038 \\
\hline 22 & SER & 56.68 & 65.13 & 173 & & 896 & 100 & $\mathrm{PRO}$ & 62.10 & 31 & 173.84 & - & - \\
\hline 23 & PHE & 58.55 & 39.20 & 176 & 19 & 01 & 101 & LEU & & 1 & 175.07 & 19.039 & .490 \\
\hline 24 & ASN & 51.02 & 37.91 & 17 & 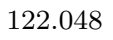 & 55 & 10 & ASP & 2 & 39. & 6 & 22.094 & 8.191 \\
\hline 25 & GLU & 58.71 & 27.65 & 177 & 074 & .313 & 10 & ASP & 4.50 & 39. & 178.85 & 120.702 & 8.777 \\
\hline 26 & ASN & 52.62 & 38.47 & 173.32 & 755 & .826 & 10 & ALA & 53.52 & 20.57 & 176.64 & 120.327 & 7.332 \\
\hline 27 & ARG & 55.29 & 23.79 & 173.84 & 2.841 & 8.427 & 10 & THR & 57.75 & 70.05 & 177.47 & 108.720 & 6.706 \\
\hline 28 & LEU & 53.63 & 45.77 & 177.74 & 5.901 & 7.757 & 10 & PRO & 5.14 & 31.20 & 175.26 & - & - \\
\hline 29 & CYS & 58.78 & 28.44 & 174.11 & 3.279 & 9.182 & 107 & GLU & 60.16 & 27.94 & 177.47 & 118.302 & 8.749 \\
\hline 30 & SER & 58.50 & 64.33 & 172.25 & 3.568 & 8.521 & 108 & LYS & 59.28 & 32. & 176.84 & 120.692 & 7.827 \\
\hline 31 & PHE & 56.14 & 44.10 & 173.89 & 3.705 & 9.280 & 109 & LEU & 38 & 39. & 178.64 & 120.210 & 7.733 \\
\hline 32 & ALA & 50.34 & 19.86 & 177.17 & 2.490 & 8.979 & 110 & GLU & 60.29 & 29.09 & 178.81 & 119.369 & 8.195 \\
\hline 33 & ILE & 58.67 & 37.76 & 175.65 & 6.023 & 9.604 & 111 & ASN & 56.11 & 38.17 & 177.54 & 116.413 & 7.875 \\
\hline 34 & ASP & 55.56 & 38.09 & 175.37 & .494 & 9.289 & 112 & GLU & 58.25 & 28.17 & 177.92 & 120.375 & 8.138 \\
\hline 35 & GLU & 60.20 & 28.30 & 176.81 & & 8.441 & 113 & ILE & 66.16 & & 177.88 & 120.331 & 8.358 \\
\hline 36 & ILE & 61.83 & 39.93 & 174. & & 7.781 & 114 & GLU & & & 179.06 & 120.404 & 8.195 \\
\hline 37 & TYR & 56.94 & 38.26 & & & & 115 & VAL & & & 178.07 & 9.705 & 7.524 \\
\hline 38 & TYR & 5 & & & & & 116 & VAL & & & 94 & 008 & 7.541 \\
\hline 39 & $\mathrm{E}$ & 56.70 & 40 & & & 88 & 117 & VAL & 8 & & 179.98 & .735 & 8.693 \\
\hline 40 & SER & 12 & 66. & 17 & & 46 & 11 & LYS & 0 & 30 & 177.31 & 118.775 & 8.873 \\
\hline 41 & LEU & 52.55 & 42.23 & 176 & 78 & 9.138 & 119 & SER & 62.58 & 63 & 177.61 & - & - \\
\hline 42 & SER & 54.51 & 65.46 & 175 & 77 & 9.328 & 120 & MET & 60.30 & 31 & 176.59 & 122.397 & 8.243 \\
\hline 43 & ASP & 51.90 & 37.59 & 175. & 22 & 8.130 & 121 & GLU & 59.25 & 28.74 & 179.38 & 120.988 & 8.561 \\
\hline 44 & ALA & 54.17 & 19.48 & 176. & 6.589 & 7.339 & 122 & ASN & 55.68 & 37.82 & 176.74 & 117.386 & 7.922 \\
\hline 45 & ASN & 52.45 & 39.83 & 173. & 4.925 & 7 . & 123 & LEU & 57.88 & 38. & 178.61 & 120.595 & 8.189 \\
\hline 46 & ASP & 56.35 & 40.61 & 176 & 35 & 8 & 124 & TYR & 58.78 & 36. & 179.08 & 117.960 & 9.244 \\
\hline 47 & GLU & 56.45 & 30.79 & 176.90 & 4.993 & 8.898 & 125 & LEU & 58.44 & 40.98 & 178.66 & 121.520 & 7.905 \\
\hline 48 & TYR & 56.74 & 38.82 & 172.50 & 6.991 & 7.337 & 126 & VAL & 65.22 & 31.62 & 179.63 & 120.436 & 8.216 \\
\hline 49 & MET & 53.95 & 36.92 & 176.33 & 118.246 & 8.827 & 127 & LEU & 58.98 & - & 180.13 & - & - \\
\hline 50 & MET & 52.00 & 32.46 & 174.36 & 118.133 & 8.341 & 128 & HIS & 59.63 & 29.26 & 179.63 & 121.005 & 8.779 \\
\hline 51 & ILE & 60.20 & 38.37 & 174.24 & 121.979 & 8.782 & 129 & ASN & 55.27 & 37.60 & 176.63 & 120.009 & 8.236 \\
\hline
\end{tabular}




\begin{tabular}{|c|c|c|c|c|c|c|c|c|c|c|c|c|c|}
\hline & Res. & $\mathrm{C}^{\prime}$ & $\overline{\mathrm{C}_{\alpha}}$ & $\overline{\mathrm{C}_{\beta}}$ & $\mathrm{N}$ & $\overline{\mathrm{H}_{N}}$ & & Res. & $\mathrm{C}^{\prime}$ & $\overline{\mathrm{C}_{\alpha}}$ & $\overline{\mathrm{C}_{\beta}}$ & $\overline{\mathrm{N}}$ & $\overline{\mathrm{H}_{N}}$ \\
\hline 52 & TYR & 54.74 & 40.04 & 176.03 & 122.569 & 9.143 & 130 & GLN & 55.06 & 28.69 & 175.81 & 117.223 & 7.666 \\
\hline 53 & GLY & 45.45 & & 173.50 & 110.542 & 9.692 & 131 & GLY & 45.49 & & 174.30 & 108.675 & 7.821 \\
\hline 54 & VAL & 61.89 & 30.14 & 175.24 & 127.955 & 8.784 & 132 & ILE & 60.16 & 38.51 & 174.41 & 123.084 & 8.103 \\
\hline 55 & CYS & 60.93 & 27.63 & 175.44 & 125.168 & 8.626 & 133 & THR & 60.50 & 69.74 & 174.21 & 122.695 & 8.200 \\
\hline 56 & GLY & 43.58 & & 171.20 & 102.972 & 7.074 & 134 & LEU & 53.65 & 40.38 & 177.00 & 127.142 & 9.161 \\
\hline 57 & LYS & 56.17 & 32.66 & 176.54 & 121.154 & 8.641 & 135 & GLU & 56.21 & 29.99 & 175.96 & 124.000 & 8.870 \\
\hline 58 & PHE & 56.33 & 39.66 & 174.29 & 128.202 & 9.422 & 136 & ASN & 52.11 & 38.73 & 175.57 & 122.157 & 8.652 \\
\hline 59 & PRO & 61.90 & 30.02 & 176.43 & - & - & 137 & GLU & 55.51 & 29.32 & 176.67 & 118.545 & 8.189 \\
\hline 60 & THR & 62.38 & 68.71 & 174.91 & 115.930 & 8.274 & 138 & HIS & 56.25 & 28.94 & 175.02 & 118.509 & 8.254 \\
\hline 61 & ASP & 53.84 & 39.62 & 175.22 & 120.219 & 8.423 & 139 & MET & 55.77 & 31.87 & 175.40 & 121.232 & 8.000 \\
\hline 62 & ASN & 49.79 & 38.27 & 116.27 & 116.517 & 7.867 & 140 & LYS & 55.60 & 31.99 & 176.12 & 122.556 & 7.858 \\
\hline 63 & PRO & 64.35 & 31.33 & 178.23 & - & - & 141 & ILE & 60.79 & 37.77 & 176.25 & 123.765 & 8.103 \\
\hline 64 & ASN & 55.15 & 37.99 & 177.21 & 115.376 & 8.240 & 142 & GLU & 56.12 & 29.67 & 176.18 & 125.376 & 8.428 \\
\hline 65 & PHE & 61.08 & 38.14 & 176.92 & 122.088 & 7.773 & 143 & GLU & 56.14 & 29.57 & 176.49 & 122.775 & 8.298 \\
\hline 66 & ALA & 55.18 & 18.07 & 178.74 & 118.689 & 7.993 & 144 & ILE & 60.89 & 37.86 & 176.39 & 122.409 & 8.091 \\
\hline 67 & LEU & 57.07 & 38.75 & 179.58 & 114.871 & 6.985 & 145 & SER & 57.88 & 63.31 & 174.68 & 119.909 & 8.323 \\
\hline 68 & GLU & 58.82 & 28.27 & 179.65 & 119.875 & 7.584 & 146 & SER & 58.06 & 63.34 & 174.89 & 118.469 & 8.285 \\
\hline 69 & ILE & 60.92 & 34.16 & 177.42 & 120.179 & 7.690 & 147 & SER & 58.31 & 63.27 & 174.34 & 117.908 & 8.267 \\
\hline 70 & LEU & 57.82 & 42.85 & 180.87 & 118.275 & 8.093 & 148 & ASP & 54.14 & 40.49 & 176.00 & 122.070 & 8.160 \\
\hline 71 & ASN & 57.31 & 40.17 & 178.22 & 119.941 & 8.416 & 149 & ASN & 53.10 & 38.06 & 175.36 & 119.109 & 8.147 \\
\hline 72 & ALA & 54.72 & 18.00 & 180.12 & - & - & 150 & LYS & 56.26 & 31.64 & 176.41 & 121.013 & 8.071 \\
\hline 73 & ASN & 55.92 & - & 177.27 & 117.721 & 7.604 & 151 & HIS & 55.44 & 29.21 & 174.58 & 119.210 & 8.116 \\
\hline 74 & LEU & - & - & - & - & - & 152 & TYR & 57.61 & 38.15 & 175.18 & 121.672 & 7.930 \\
\hline 75 & TRP & - & - & - & - & - & 153 & TYR & 57.20 & 38.17 & 175.06 & 122.663 & 7.958 \\
\hline 76 & PHE & - & - & - & - & - & 154 & ALA & 52.07 & 18.47 & 177.78 & 126.360 & 8.026 \\
\hline 77 & ALA & 55.35 & 18.13 & 179.03 & - & - & 155 & GLY & 45.00 & & 173.09 & 108.141 & 7.656 \\
\hline 78 & GLU & 57.06 & 28.75 & 176.09 & 115.488 & 7.014 & 156 & ARG & 56.89 & 30.49 & 171.00 & 125.509 & 7.638 \\
\hline
\end{tabular}

Table B.9: Assigned chemical shifts of CesT bound to Tir108 at $303 \mathrm{~K}$ based on TROSY versions of 3D HNCO, HN(CA)CO, HNCA, HN(COCA)CB, HN(CA)CB and 2D HSQC experiments.

\begin{tabular}{|c|c|c|c|c|c|c|c|c|c|c|c|c|c|}
\hline & Res. & $\mathrm{C}^{\prime}$ & $\overline{\mathrm{C}_{\alpha}}$ & $\mathrm{C}_{\beta}$ & $\mathrm{N}$ & $\mathrm{H}_{N}$ & & Res. & $\mathrm{C}^{\prime}$ & $\mathrm{C}_{\alpha}$ & $\mathrm{C}_{\beta}$ & $\mathrm{N}$ & $\mathrm{H}_{N}$ \\
\hline 1 & MET & 54.58 & 32.01 & 175.79 & 122.732 & 8.368 & 79 & ASN & - & - & 1 & - & 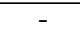 \\
\hline 2 & SER & 58.40 & 63.39 & 174.18 & 118.669 & 8.334 & 80 & GLY & 46.08 & - & 175.33 & 110.566 & 7.201 \\
\hline 3 & SER & 57.11 & 64.33 & 175.17 & 120.275 & 8.665 & 81 & GLY & 43.76 & - & 180.57 & 105.593 & 8.420 \\
\hline 4 & ARG & 59.55 & 29.79 & 179.70 & 121.230 & 8.759 & 82 & PRO & - & - & - & - & - \\
\hline 5 & SER & 61.31 & - & 176.08 & 116.874 & 8.556 & 83 & TYR & - & - & - & - & - \\
\hline 6 & GLU & 59.30 & - & 179.17 & 123.730 & 7.622 & 84 & LEU & - & - & - & - & - \\
\hline 7 & LEU & 57.25 & 40.94 & 179.43 & 119.999 & 8.075 & 85 & CYS & - & - & - & - & - \\
\hline 8 & LEU & 57.38 & 40.41 & 178.04 & 120.083 & 7.657 & 86 & TYR & 55.91 & - & 172.18 & 121.261 & 6.571 \\
\hline 9 & LEU & 57.67 & - & 178.61 & 119.929 & 8.129 & 87 & GLU & 55.60 & - & - & 130.760 & 8.278 \\
\hline 10 & ASP & 56.70 & - & - & 118.120 & 7.612 & 88 & SER & - & - & - & - & - \\
\hline 11 & ARG & 58.25 & - & 180.04 & 118.808 & 7.687 & 89 & GLY & - & - & - & - & - \\
\hline 12 & PHE & 60.22 & - & 175.35 & 123.645 & 8.746 & 90 & ALA & - & - & - & - & - \\
\hline 13 & ALA & 54.34 & - & 179.68 & 121.973 & 8.762 & 91 & GLN & - & - & - & 115.410 & 7.552 \\
\hline 14 & GLU & 57.99 & - & - & 116.774 & 7.276 & 92 & SER & - & - & - & - & - \\
\hline 15 & LYS & - & - & - & - & - & 93 & LEU & 54.00 & - & 174.20 & 124.546 & 8.759 \\
\hline 16 & ILE & - & - & - & - & - & 94 & LEU & 52.42 & - & 171.08 & 127.453 & 8.817 \\
\hline 17 & GLY & - & - & - & - & - & 95 & LEU & - & - & - & - & - \\
\hline 18 & VAL & - & - & - & 115.417 & 7.328 & 96 & ALA & - & - & - & - & - \\
\hline 19 & GLY & 44.29 & - & 172.11 & 112.215 & 8.017 & 97 & LEU & 53.83 & - & 173.63 & 124.763 & 8.924 \\
\hline 20 & SER & 58.18 & 62.82 & 174.59 & 114.897 & 7.759 & 98 & ARG & 55.67 & - & 175.20 & 128.875 & 9.136 \\
\hline 21 & ILE & 59.81 & - & 173.74 & 122.922 & 7.990 & 99 & PHE & 53.80 & - & - & 128.541 & 9.093 \\
\hline 22 & SER & 56.88 & - & 174.23 & 116.371 & 8.061 & 100 & PRO & 62.13 & 31.25 & 176.07 & - & - \\
\hline 23 & PHE & 58.23 & - & 176.67 & 119.719 & 8.783 & 101 & LEU & 54.93 & - & 179.01 & 119.028 & 7.554 \\
\hline 24 & ASN & 50.84 & - & 177.73 & 121.424 & 8.799 & 102 & ASP & 56.23 & - & 176.70 & 121.833 & 8.189 \\
\hline 25 & GLU & 58.68 & - & 177.49 & 116.378 & 9.441 & 103 & ASP & 54.13 & - & 175.40 & 120.819 & 8.594 \\
\hline 26 & ASN & 52.77 & - & 173.14 & 119.250 & 9.466 & 104 & ALA & 53.43 & 20.41 & 177.42 & 120.705 & 7.319 \\
\hline 27 & ARG & 55.24 & - & - & 117.802 & 7.816 & 105 & THR & 57.68 & - & 177.37 & 108.927 & 6.714 \\
\hline 28 & LEU & 53.07 & - & 177.85 & 115.694 & 8.299 & 106 & PRO & 65.35 & - & 177.22 & - & - \\
\hline 29 & CYS & 48.84 & - & 173.65 & 118.444 & 7.801 & 107 & GLU & 59.82 & - & 178.72 & 117.872 & 8.746 \\
\hline
\end{tabular}




\begin{tabular}{|c|c|c|c|c|c|c|c|c|c|c|c|c|c|}
\hline & Res. & $\mathrm{C}^{\prime}$ & $\overline{\mathrm{C}_{\alpha}}$ & $\overline{\mathrm{C}_{\beta}}$ & $\mathrm{N}$ & $\overline{\mathrm{H}_{N}}$ & & Res. & $\overline{\mathrm{C}^{\prime}}$ & $\overline{\overline{\mathrm{C}_{\alpha}}}$ & $\overline{\mathrm{C}_{\beta}}$ & $\overline{\mathrm{N}}$ & $\overline{\mathrm{H}_{N}}$ \\
\hline 30 & SER & - & - & - & - & - & 108 & LYS & 59.30 & - & - & 121.759 & 7.934 \\
\hline 31 & PHE & - & - & - & - & - & 109 & LEU & - & - & - & - & - \\
\hline 32 & ALA & 49.83 & - & 177.80 & 122.094 & 8.379 & 110 & GLU & - & - & - & - & - \\
\hline 33 & ILE & - & - & - & - & - & 111 & ASN & - & - & - & - & - \\
\hline 34 & $\mathrm{ASP}$ & - & - & - & - & - & 112 & GLU & - & - & - & - & - \\
\hline 35 & GLU & - & - & - & - & - & 113 & ILE & - & - & - & 120.627 & 8.368 \\
\hline 36 & ILE & - & - & - & 115.545 & 8.133 & 114 & GLU & _- & - & - & - & - \\
\hline 37 & TYR & - & - & - & - & - & 115 & VAL & 65.94 & - & 180.14 & 120.758 & 7.667 \\
\hline 38 & TYR & - & - & - & - & - & 116 & VAL & 67.81 & - & 177.13 & 123.786 & 7.655 \\
\hline 39 & ILE & 56.14 & - & 175.08 & 126.443 & 8.854 & 117 & VAL & 67.16 & - & - & 122.178 & 8.834 \\
\hline 40 & SER & 57.89 & - & 172.95 & 120.228 & 9.099 & 118 & LYS & - & - & - & - & - \\
\hline 41 & LEU & 52.16 & - & 176.47 & 122.943 & 9.022 & 119 & SER & - & - & - & - & - \\
\hline 42 & SER & 54.67 & - & 175.83 & 115.049 & 8.963 & 120 & MET & - & - & - & - & - \\
\hline 43 & ASP & 51.79 & - & 175.68 & 130.758 & 8.361 & 121 & GLU & - & - & - & 119.023 & 8.305 \\
\hline 44 & ALA & 54.24 & - & 177.14 & 125.531 & 7.377 & 122 & ASN & _- & - & - & - & - \\
\hline 45 & ASN & 52.27 & - & 173.43 & 115.675 & 7.924 & 123 & LEU & - & - & - & 122.571 & 8.254 \\
\hline 46 & ASP & 56.14 & - & 176.63 & 120.088 & 8.395 & 124 & TYR & - & - & - & - & - \\
\hline 47 & GLU & 56.35 & - & 177.05 & 117.475 & 8.910 & 125 & $\mathrm{LEU}$ & - & - & 178.94 & 121.717 & 7.739 \\
\hline 48 & TYR & 56.61 & - & 172.22 & 116.814 & 7.351 & 126 & VAL & - & - & - & - & - \\
\hline 49 & MET & 53.98 & 37.75 & 176.18 & 118.607 & 8.981 & 127 & $\mathrm{LEU}$ & - & - & - & - & - \\
\hline 50 & MET & 51.83 & - & 174.57 & 118.559 & 8.288 & 128 & HIS & 59.62 & - & - & 120.787 & 8.755 \\
\hline 51 & ILE & 60.36 & - & 174.13 & 122.782 & 8.717 & 129 & $\mathrm{ASN}$ & - & - & - & - & - \\
\hline 52 & TYR & 54.98 & - & 175.82 & 123.864 & 9.034 & 130 & GLN & - & - & 176.09 & 118.633 & 7.899 \\
\hline 53 & GLY & 44.87 & - & 173.61 & 110.652 & 9.701 & 131 & GLY & 45.28 & - & 174.12 & 108.183 & 7.654 \\
\hline 54 & VAL & 62.25 & - & 175.24 & 128.483 & 8.824 & 132 & ILE & - & - & - & 121.309 & 8.040 \\
\hline 55 & CYS & - & - & - & - & - & 133 & THR & - & - & - & - & - \\
\hline 56 & GLY & - & - & - & 102.898 & 7.117 & 134 & LEU & - & - & - & - & - \\
\hline 57 & LYS & - & - & 176.62 & 121.307 & 8.555 & 135 & GLU & 55.77 & 29.74 & 176.09 & 123.950 & 8.924 \\
\hline 58 & PHE & 56.33 & - & 173.82 & 129.203 & 9.187 & 136 & ASN & 52.94 & 38.41 & 175.45 & 119.302 & 8.305 \\
\hline 59 & PRO & 61.82 & 29.98 & 176.53 & - & - & 137 & GLU & 56.74 & 29.40 & 176.49 & 122.418 & 8.391 \\
\hline 60 & THR & 62.56 & - & 174.86 & 115.482 & 8.217 & 138 & HIS & 55.18 & 28.43 & 174.66 & 119.317 & 8.258 \\
\hline 61 & $\mathrm{ASP}$ & 53.25 & - & 175.25 & 120.294 & 8.373 & 139 & MET & 55.28 & 32.14 & 175.82 & 121.150 & 8.095 \\
\hline 62 & $\mathrm{ASN}$ & 49.99 & - & 177.88 & 116.664 & 7.798 & 140 & LYS & 55.79 & 32.02 & 176.44 & 123.366 & 8.137 \\
\hline 63 & PRO & 64.29 & - & - & - & - & 141 & ILE & 60.81 & 37.59 & 176.34 & 123.178 & 8.107 \\
\hline 64 & ASN & 54.85 & - & 177.09 & 115.737 & 8.155 & 142 & GLU & 56.13 & 29.44 & 176.31 & 124.978 & 8.414 \\
\hline 65 & PHE & 60.57 & - & 176.59 & 122.134 & 7.736 & 143 & GLU & 56.09 & 29.45 & 176.49 & 122.849 & 8.279 \\
\hline 66 & ALA & - & - & - & - & - & 144 & ILE & 60.85 & 37.77 & 176.42 & 122.300 & 8.074 \\
\hline 67 & LEU & - & - & - & - & - & 145 & SER & 57.83 & 63.30 & 174.69 & 120.185 & 8.341 \\
\hline 68 & GLU & 58.52 & - & 179.38 & 119.655 & 7.507 & 146 & SER & 58.01 & 63.31 & 174.91 & 119.315 & 8.426 \\
\hline 69 & ILE & - & - & - & - & - & 147 & SER & 58.26 & 63.20 & 174.40 & 118.069 & 8.273 \\
\hline 70 & LEU & - & - & - & - & - & 148 & ASP & 54.04 & 40.44 & 176.04 & 122.425 & 8.175 \\
\hline 71 & ASN & - & - & - & - & - & 149 & ASN & 53.08 & 38.05 & 175.36 & 119.323 & 8.133 \\
\hline 72 & ALA & - & - & - & - & - & 150 & LYS & 56.13 & 31.63 & 176.42 & 121.054 & 8.066 \\
\hline 73 & ASN & 54.44 & - & 174.33 & 120.832 & 8.391 & 151 & HIS & 54.99 & 28.61 & 174.20 & 119.191 & 8.159 \\
\hline 74 & LEU & - & - & - & - & - & 152 & TYR & 57.63 & 38.15 & 175.21 & 120.514 & 7.867 \\
\hline 75 & TRP & - & - & - & - & - & 153 & TYR & 57.09 & 38.20 & 175.05 & 122.665 & 7.957 \\
\hline 76 & PHE & - & - & - & - & - & 154 & ALA & 51.99 & 18.50 & 177.74 & 126.384 & 8.027 \\
\hline 77 & ALA & - & - & - & - & - & 155 & GLY & 44.97 & - & 173.10 & 122.037 & 7.683 \\
\hline 78 & GLU & 57.72 & - & 176.20 & 122.476 & 7.937 & 156 & ARG & 56.83 & 30.48 & 170.96 & 125.561 & 7.646 \\
\hline
\end{tabular}

Table B.10: Assigned chemical shifts of Tir108 at $283 \mathrm{~K}$ based on 3D HNCO, $\mathrm{HN}(\mathrm{CA}) \mathrm{CO}, \mathrm{CBCA}(\mathrm{CO}) \mathrm{NH}, \mathrm{HNCACB}$ and 2D HSQC experiments.

\begin{tabular}{ccccccc||ccccccc}
\hline \hline & Res. & $\mathrm{C}^{\prime}$ & $\mathrm{C}_{\alpha}$ & $\mathrm{C}_{\beta}$ & $\mathrm{N}$ & $\mathrm{H}_{N}$ & & Res. & $\mathrm{C}^{\prime}$ & $\mathrm{C}_{\alpha}$ & $\mathrm{C}_{\beta}$ & $\mathrm{N}$ & $\mathrm{H}_{N}$ \\
\hline 1 & MET & - & - & - & - & - & 55 & ASN & 175.96 & 53.28 & 38.79 & 8.411 & 119.985 \\
2 & PRO & 176.84 & 62.89 & 31.91 & - & - & 56 & SER & 175.30 & 58.53 & 63.68 & 8.280 & 116.330 \\
3 & ILE & 176.72 & 61.30 & 38.82 & 8.223 & 121.327 & 57 & MET & 174.74 & 55.93 & 32.87 & 8.315 & 121.799 \\
4 & GLY & 176.84 & 45.14 & & 8.373 & 112.411 & 58 & ALA & 176.25 & 52.63 & 19.10 & 8.115 & 124.277 \\
5 & ASN & 173.72 & 53.10 & 38.88 & 8.285 & 118.749 & 59 & ASP & 177.49 & 54.36 & 41.10 & 8.150 & 119.005 \\
6 & LEU & 175.39 & 55.54 & 42.06 & 8.282 & 122.423 & 60 & SER & 176.23 & 58.12 & 63.85 & 8.054 & 115.550 \\
7 & GLY & 177.93 & 45.29 & & 8.310 & 108.855 & 61 & VAL & 174.67 & 62.36 & 32.65 & 8.104 & 121.517 \\
8 & ASN & 173.88 & 53.05 & 38.74 & 8.206 & 118.377 & 62 & ASP & 175.88 & 54.23 & 41.31 & 8.344 & 123.742 \\
\hline
\end{tabular}




\begin{tabular}{|c|c|c|c|c|c|c|c|c|c|c|c|c|c|}
\hline & Res. & $\mathrm{C}^{\prime}$ & $\mathrm{C}_{\alpha}$ & $\mathrm{C}_{\beta}$ & $\mathrm{N}$ & $\mathrm{H}_{N}$ & & Res. & $\mathrm{C}^{\prime}$ & $\mathrm{C}_{\alpha}$ & $\mathrm{C}_{\beta}$ & $\mathrm{N}$ & $\mathrm{H}_{N}$ \\
\hline 9 & ASN & 174.93 & 53.20 & 38.72 & 8.392 & 119.244 & 63 & SER & 176.41 & 58.59 & 63.47 & 8.211 & 117.286 \\
\hline 10 & VAL & 175.39 & 62.74 & 32.41 & 8.040 & 119.993 & 64 & ARG & 174.76 & 56.31 & 30.65 & 8.244 & 122.322 \\
\hline 11 & ASN & 176.08 & 53.28 & 38.87 & 8.441 & 121.238 & 65 & ASP & 176.05 & 54.26 & 41.01 & 8.188 & 120.591 \\
\hline 12 & GLY & 175.62 & 45.49 & & 8.209 & 109.026 & 66 & ILE & 175.63 & 58.61 & 38.57 & 7.933 & 122.719 \\
\hline 13 & ASN & 173.94 & 53.14 & 38.93 & 8.215 & 118.403 & 67 & $\mathrm{PRO}$ & 173.81 & 63.39 & 32.05 & - & - \\
\hline 14 & HIS & 176.79 & 56.03 & 30.08 & - & - & 68 & GLY & 177.39 & 44.92 & & 8.355 & 109.019 \\
\hline 15 & LEU & 174.75 & 55.03 & 42.11 & 8.047 & 123.038 & 69 & LEU & 173.82 & 52.94 & 41.72 & 7.961 & 122.747 \\
\hline 16 & ILE & 176.79 & 58.21 & 38.44 & 8.060 & 123.940 & 70 & PRO & 174.11 & 63.09 & 31.92 & - & - \\
\hline 17 & RO & - & - & - & - & - & 71 & THR & 177.00 & 61.80 & 69.86 & 8.119 & 114.176 \\
\hline 18 & RO & 175.05 & 62.55 & 31.93 & - & - & 72 & ASN & 174.12 & 51.30 & 38.79 & 8.378 & 121.881 \\
\hline 19 & ALA & 176.22 & 50.20 & 18.04 & 8.248 & 125.646 & 73 & $\mathrm{PRO}$ & 174.87 & 63.42 & 31.96 & - & \\
\hline 20 & RO & - & - & - & - & - & 74 & SER & 177.24 & 58.66 & 63.63 & 8.293 & 115.482 \\
\hline 21 & RO & 175.65 & 62.56 & 32.14 & - & - & 75 & ARG & 174.84 & 56.23 & 30.50 & 8.151 & 122.760 \\
\hline 22 & LEU & 176.70 & 52.98 & 41.61 & 8.240 & 123.471 & 76 & LEU & 176.30 & 55.09 & 42.16 & 8.044 & 122.708 \\
\hline 23 & PRO & 174.84 & 63.36 & 32.00 & - & - & 77 & ALA & 177.22 & 52.47 & 19.04 & 8.116 & 124.662 \\
\hline 24 & SER & 176.94 & 58.51 & 63.55 & 8.289 & 115.438 & 78 & ALA & 177.45 & 52.37 & 19.12 & 8.090 & 122.996 \\
\hline 25 & GLN & 174.84 & 55.87 & 29.42 & 8.421 & 122.360 & 79 & ALA & 177.57 & 52.46 & 19.17 & 8.203 & 123.284 \\
\hline 26 & THR & 176.28 & 61.80 & 69.87 & 8.130 & 114.461 & 80 & THR & 178.04 & 61.60 & 69.90 & 8.044 & 112.838 \\
\hline 27 & ASP & 174.46 & 54.45 & 41.12 & 8.267 & 122.766 & 81 & SER & 174.68 & 58.49 & 63.66 & 8.245 & 117.550 \\
\hline 28 & GLY & 176.24 & 45.49 & & 8.271 & 114.034 & 82 & GLU & 174.66 & & 30.14 & 8.439 & 122.658 \\
\hline 29 & ALA & 174.32 & 52.58 & 19.01 & 8.054 & 123.606 & 83 & THR & 76.85 & 37 & 69.61 & 8.098 & 114.740 \\
\hline 30 & ALA & 177.81 & 52.60 & 18.95 & 8.117 & 122.722 & 84 & CYS & 57 & 62 & 28.03 & 8.267 & 121.945 \\
\hline 31 & ARG & 177.98 & 56.11 & 30.66 & 8.198 & 120.102 & 85 & LEU & 174.50 & 23 & 42.12 & 8.270 & 124.801 \\
\hline 32 & GLY & 176.91 & 45.38 & & 8.326 & 109.630 & 86 & LEU & 177.33 & 55.17 & 42.27 & 8.162 & 122.648 \\
\hline 33 & GLY & 174.66 & 45.21 & & 8.250 & 108.718 & 87 & GLY & 177.78 & 45.34 & & 8.309 & 109.700 \\
\hline 34 & THR & 174.57 & 61.79 & 69.68 & 8.129 & 112.341 & 88 & GLY & 174.45 & 45.16 & & 8.155 & 108.389 \\
\hline 35 & GLY & 175.46 & 45.45 & & 8.450 & 111.077 & 89 & PHE & 173.88 & 57.66 & 39.66 & 7.949 & 119.363 \\
\hline 36 & HIS & 173.90 & 56.09 & 30.42 & 8.139 & 118.905 & 90 & GLU & 175.56 & 56.48 & 30.27 & 8.383 & 122.419 \\
\hline 37 & LEU & 175.03 & 55.01 & 42.19 & 8.110 & 123.423 & 91 & VAL & 175.99 & 62.17 & 32.59 & 8.074 & 121.751 \\
\hline 38 & ILE & 177.14 & 61.06 & 38.62 & 8.117 & 122.193 & 92 & LEU & 175.80 & 54.80 & 42.22 & 8.193 & 125.728 \\
\hline 39 & SER & 176.22 & 57.99 & 63.79 & 8.321 & 119.799 & 93 & HIS & 176.95 & 55.61 & 30.14 & 8.279 & 120.230 \\
\hline 40 & SER & 174.75 & 58.30 & 63.68 & 8.411 & 118.448 & 94 & ASP & 174.61 & 54.27 & 41.16 & 8.292 & 121.691 \\
\hline 41 & THR & 174.97 & 61.83 & 69.82 & 8.117 & 114.827 & 95 & LYS & 176.08 & 56.16 & 32.88 & 8.318 & 121.639 \\
\hline 42 & GLY & 175.29 & 45.23 & & 8.253 & 111.010 & 96 & GLY & 176.81 & 44.62 & & 8.361 & 110.203 \\
\hline 43 & ALA & 173.94 & 52.63 & 19.20 & 8.108 & 123.757 & 97 & PRO & 177.35 & 63.38 & 32.07 & - & - \\
\hline 44 & LEU & 178.04 & 55.48 & 42.13 & 8.240 & 121.229 & 98 & LEU & 177.38 & & 42.12 & 8.275 & 120.895 \\
\hline 45 & GLY & 178.19 & 45.35 & & 8.320 & 10 & 99 & ASP & 33 & & 41.09 & 8.108 & 121.150 \\
\hline 46 & SER & 174.37 & 58.42 & 63.71 & 8.137 & 115.643 & 100 & ILE & 176.43 & & 38.57 & 7.869 & 120.254 \\
\hline 47 & ARG & 174.81 & 56.17 & 30.59 & 8.313 & 122.658 & 10 & LEU & 176.40 & & 42.09 & 8.133 & 124.460 \\
\hline 48 & SER & 176.35 & 58.26 & 63.70 & 8.222 & 116.696 & 10 & ASN & 177.24 & 53.25 & 38.70 & 8.247 & 119.293 \\
\hline 49 & LEU & 174.48 & 55.22 & 42.17 & 8.149 & 124.030 & 103 & THR & 175.44 & 62.04 & 69.58 & 7.987 & 114.191 \\
\hline 50 & PHE & 176.84 & 57.37 & 39.64 & 8.068 & 120.179 & 104 & GLN & 174.47 & 55.65 & 29.16 & 8.275 & 122.757 \\
\hline 51 & SER & 175.24 & 55.71 & 63.59 & 8.017 & 119.038 & 105 & ILE & 175.66 & 61.00 & 38.70 & 8.109 & 122.517 \\
\hline 52 & RO & 177.43 & 3.26 & 32.05 & - & 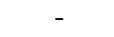 & 106 & GLY & 176.32 & 44.36 & & 8.269 & 113.408 \\
\hline 53 & LEU & 176.80 & 55. & 42.07 & 8.117 & 121.285 & 107 & PRO & 178.72 & 63.25 & 32.09 & - & - \\
\hline 54 & ARG & 177.44 & 55.96 & 30.74 & 8.142 & 121.538 & 108 & SER & 176.50 & 59.93 & 64.82 & 7.993 & 121.962 \\
\hline
\end{tabular}

Table B.11: Assigned chemical shifts of Map84 in complex with CesT at $303 \mathrm{~K}$ based on 3D HNCO, HN(CA)CO, CBCA(CO)NH, HNCACB, HNCA and 2D HSQC experiments.

\begin{tabular}{|c|c|c|c|c|c|c|c|c|c|c|c|c|c|}
\hline & Res. & $\mathrm{C}^{\prime}$ & $\overline{\mathrm{C}_{\alpha}}$ & $\overline{\mathrm{C}_{\beta}}$ & $\overline{\mathrm{N}}$ & $\overline{\mathrm{H}_{N}}$ & & Res. & $\overline{\mathrm{C}^{\prime}}$ & $\overline{\mathrm{C}_{\alpha}}$ & $\overline{\mathrm{C}_{\beta}}$ & $\mathrm{N}$ & $\overline{\mathrm{H}_{N}}$ \\
\hline 1 & MET & - & - & - & - & - & 43 & MET & - & - & - & - & - \\
\hline 2 & PHE & - & - & - & - & - & 44 & GLN & - & - & - & - & - \\
\hline 3 & SER & - & - & - & - & - & 45 & SER & - & - & - & - & - \\
\hline 4 & PRO & 63.56 & 32.15 & 177.94 & - & - & 46 & ASN & - & - & - & - & - \\
\hline 5 & THR & 61.95 & 69.89 & 174.38 & 113.008 & 8.053 & 47 & LEU & - & - & - & - & - \\
\hline 6 & ALA & 52.61 & 19.36 & 177.55 & 125.985 & 8.132 & 48 & MET & - & - & - & - & - \\
\hline 7 & MET & 55.53 & 32.87 & 176.07 & 119.740 & 8.286 & 49 & ILE & - & - & - & - & - \\
\hline 8 & VAL & 62.59 & 32.75 & 176.54 & 121.220 & 8.040 & 50 & $\mathrm{ASN}$ & - & - & - & - & - \\
\hline 9 & GLY & 45.44 & & 173.16 & 113.116 & 8.508 & 51 & HIS & - & - & - & - & - \\
\hline 10 & ARG & 57.26 & 31.63 & 170.99 & 125.569 & 7.789 & 52 & GLY & - & - & - & - & - \\
\hline
\end{tabular}




\begin{tabular}{|c|c|c|c|c|c|c|c|c|c|c|c|c|c|}
\hline & Res. & $\overline{\mathrm{C}^{\prime}}$ & $\overline{\mathrm{C}_{\alpha}}$ & $\overline{\mathrm{C}_{\beta}}$ & $\mathrm{N}$ & $\overline{\mathrm{H}_{N}}$ & & Res. & $\overline{\mathrm{C}^{\prime}}$ & $\overline{\mathrm{C}_{\alpha}}$ & $\overline{\overline{\mathrm{C}_{\beta}}}$ & $\overline{\mathrm{N}}$ & $\overline{\overline{\mathrm{H}_{N}}}$ \\
\hline 11 & ALA & - & - & - & - & - & 53 & LYS & - & - & - & - & - \\
\hline 12 & LEU & - & - & - & - & - & 54 & LEU & - & - & - & - & - \\
\hline 13 & $\mathrm{ALA}$ & 52.36 & 19.17 & 178.98 & - & - & 55 & THR & - & - & - & - & - \\
\hline 14 & GLN & 55.55 & - & 175.44 & 120.273 & 8.411 & 56 & THR & - & - & - & - & - \\
\hline 15 & ALA & 52.49 & 19.39 & 177.75 & 125.660 & 8.365 & 57 & GLN & - & - & - & - & - \\
\hline 16 & VAL & 62.25 & 32.92 & 176.25 & 119.431 & 8.151 & 58 & LEU & - & - & - & - & - \\
\hline 17 & THR & 61.85 & 69.91 & 173.46 & 118.895 & 8.278 & 59 & LEU & 55.07 & - & 179.82 & - & - \\
\hline 18 & GLN & 57.45 & 30.57 & 180.35 & 127.704 & 8.035 & 60 & GLN & 58.00 & - & 177.63 & 117.187 & 7.498 \\
\hline 19 & THR & - & - & - & - & - & 61 & ALA & 54.50 & - & - & 122.607 & 7.880 \\
\hline 20 & LEU & - & - & - & - & - & 62 & VAL & 64.41 & - & 177.38 & 117.385 & 7.9 \\
\hline 21 & ARG & - & - & - & - & - & 63 & ALA & 53.83 & - & 178.50 & 123.767 & 7.741 \\
\hline 22 & PRO & 63.08 & 32.08 & 176.44 & - & - & 64 & LYS & 56.53 & 32.72 & 176.81 & 117.125 & 7.6 \\
\hline 23 & ALA & 52.42 & 19.25 & 177.56 & 124.602 & 8.402 & 65 & GLN & 56.32 & 29.48 & 176.29 & 121.041 & 7.915 \\
\hline 24 & VAL & 62.46 & 32.92 & 176.34 & 120.130 & 8.268 & 66 & THR & 62.25 & 69.91 & 174.57 & 116.612 & 8.3 \\
\hline 25 & THR & 61.86 & 69.87 & 174.51 & 117.568 & 8.161 & 67 & ARG & 55.97 & 31.13 & 176.36 & 123.499 & 8.375 \\
\hline 26 & LYS & 56.54 & 32.92 & 176.19 & 123.753 & 8.317 & 68 & SER & 58.58 & 63.86 & 174.78 & 117.587 & 8.4 \\
\hline 27 & ALA & 52.54 & - & 177.63 & 125.458 & 8.329 & 69 & SER & 58.64 & 63.81 & 174.34 & 117.745 & 8.4 \\
\hline 28 & ALA & 52.74 & 19.19 & 178.16 & 123.028 & 8.277 & 70 & ASP & 54.53 & 41.17 & 176.30 & 122.094 & 8.370 \\
\hline 29 & THR & 62.03 & 69.86 & 174.70 & 112.416 & 8.026 & 71 & THR & 62.28 & 69.59 & 174.49 & 114.142 & 8.014 \\
\hline 30 & GLN & 55.99 & 29.55 & 175.66 & - & - & 72 & GLN & 56.22 & 32.91 & 175.65 & 122.305 & 8.266 \\
\hline 31 & ALA & 52.91 & 19.22 & 178.23 & 124.797 & 8.319 & 73 & GLN & - & - & - & - & - \\
\hline 32 & GLY & 45.47 & & 174.22 & 108.358 & 8.386 & 74 & TRP & - & - & - & - & - \\
\hline 33 & MET & 55.58 & 33.38 & 175.98 & 119.683 & 8.105 & 75 & PHE & - & - & - & - & - \\
\hline 34 & ALA & 52.46 & 19.37 & 177.69 & 125.388 & 8.445 & 76 & LYS & - & - & - & - & - \\
\hline 35 & ALA & 52.70 & 19.33 & 178.05 & 124.166 & 8.494 & 77 & GLN & - & - & - & - & - \\
\hline 36 & SER & 58.98 & 64.10 & 175.22 & 114.813 & 8.414 & 78 & GLU & - & - & - & - & - \\
\hline 37 & GLY & 45.31 & & 173.99 & 110.657 & 8.343 & 79 & GLN & - & - & - & - & - \\
\hline 38 & MET & 55.40 & 29.29 & 175.74 & 119.993 & 8.248 & 80 & ILE & - & - & - & - & - \\
\hline 39 & $\mathrm{ARG}$ & 52.04 & 19.98 & 177.50 & 125.967 & 8.523 & 81 & THR & - & - & - & - & - \\
\hline 40 & PHE & - & - & - & - & - & 82 & PHE & - & - & - & - & - \\
\hline 41 & THR & - & - & - & - & - & 83 & LEU & 55.10 & 42.62 & 176.17 & - & - \\
\hline 42 & PRO & - & - & - & - & - & 84 & SER & 59.94 & 64.88 & 178.56 & 122.015 & 7.808 \\
\hline
\end{tabular}




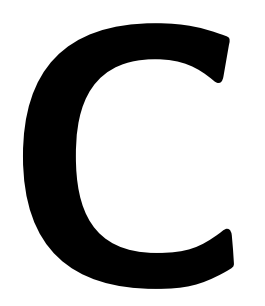

\section{Appendix: NMR restraints}

In this appendix the experimentally in Pf1 medium determined RDCs (C.1) and the

distance restraints (C.2) as well as the dihedral angle restraints (C.3) for the CylR2 solution structure determination are tabulated.

\section{C.1 Experimental residual dipolar couplings}

Table C.1: HN-RDCs of CylR2 measured at $298 \mathrm{~K}, 800 \mathrm{MHz}$, a Pf1 concentration of $\sim 12 \mathrm{mg} / \mathrm{ml}$ and at $0.5 \mathrm{M} \mathrm{NaCl}$.

\begin{tabular}{|c|c|c|c|c|c|c|c|c|c|}
\hline Residue 1 & Atom 1 & Residue 2 & Atom 2 & $\mathrm{D}[\mathrm{Hz}]$ & Residue 1 & Atom 1 & Residue 2 & Atom 2 & $\mathrm{D}[\mathrm{Hz}]$ \\
\hline 2 & $\mathrm{HN}$ & 2 & $\mathrm{~N}$ & 17.46 & 33 & $\mathrm{HN}$ & 33 & $\mathrm{~N}$ & -37.35 \\
\hline 3 & $\mathrm{HN}$ & 3 & $\mathrm{~N}$ & 17.93 & 34 & $\mathrm{HN}$ & 34 & $\mathrm{~N}$ & -51.42 \\
\hline 4 & $\mathrm{HN}$ & 4 & $\mathrm{~N}$ & 15.39 & 35 & $\mathrm{HN}$ & 35 & $\mathrm{~N}$ & -40.61 \\
\hline 5 & $\mathrm{HN}$ & 5 & $\mathrm{~N}$ & 16.20 & 36 & $\mathrm{HN}$ & 36 & $\mathrm{~N}$ & -20.63 \\
\hline 6 & $\mathrm{HN}$ & 6 & $\mathrm{~N}$ & -3.24 & 37 & $\mathrm{HN}$ & 37 & $\mathrm{~N}$ & -29.24 \\
\hline 7 & $\mathrm{HN}$ & 7 & $\mathrm{~N}$ & -25.41 & 38 & $\mathrm{HN}$ & 38 & $\mathrm{~N}$ & -33.25 \\
\hline 8 & $\mathrm{HN}$ & 8 & $\mathrm{~N}$ & -16.49 & 39 & $\mathrm{HN}$ & 39 & $\mathrm{~N}$ & 15.26 \\
\hline 9 & $\mathrm{HN}$ & 9 & $\mathrm{~N}$ & 2.58 & 43 & $\mathrm{HN}$ & 43 & $\mathrm{~N}$ & 17.97 \\
\hline 10 & $\mathrm{HN}$ & 10 & $\mathrm{~N}$ & -17.96 & 44 & $\mathrm{HN}$ & 44 & $\mathrm{~N}$ & 19.35 \\
\hline 11 & $\mathrm{HN}$ & 11 & $\mathrm{~N}$ & -35.67 & 45 & $\mathrm{HN}$ & 45 & $\mathrm{~N}$ & -2.01 \\
\hline 12 & $\mathrm{HN}$ & 12 & $\mathrm{~N}$ & -9.13 & 46 & $\mathrm{HN}$ & 46 & $\mathrm{~N}$ & 9.43 \\
\hline 13 & $\mathrm{HN}$ & 13 & $\mathrm{~N}$ & 3.45 & 47 & $\mathrm{HN}$ & 47 & $\mathrm{~N}$ & 24.63 \\
\hline 14 & $\mathrm{HN}$ & 14 & $\mathrm{~N}$ & -50.81 & 48 & $\mathrm{HN}$ & 48 & $\mathrm{~N}$ & 12.21 \\
\hline 15 & $\mathrm{HN}$ & 15 & $\mathrm{~N}$ & -39.17 & 49 & $\mathrm{HN}$ & 49 & $\mathrm{~N}$ & 2.43 \\
\hline 16 & $\mathrm{HN}$ & 16 & $\mathrm{~N}$ & -50.14 & 50 & $\mathrm{HN}$ & 50 & $\mathrm{~N}$ & 18.90 \\
\hline 17 & $\mathrm{HN}$ & 17 & $\mathrm{~N}$ & 18.52 & 51 & $\mathrm{HN}$ & 51 & $\mathrm{~N}$ & 20.20 \\
\hline 18 & $\mathrm{HN}$ & 18 & $\mathrm{~N}$ & 30.33 & 52 & $\mathrm{HN}$ & 52 & $\mathrm{~N}$ & 8.15 \\
\hline 19 & $\mathrm{HN}$ & 19 & $\mathrm{~N}$ & 8.79 & 53 & $\mathrm{HN}$ & 53 & $\mathrm{~N}$ & 9.33 \\
\hline 20 & $\mathrm{HN}$ & 20 & $\mathrm{~N}$ & 3.35 & 54 & $\mathrm{HN}$ & 54 & $\mathrm{~N}$ & 9.76 \\
\hline 21 & $\mathrm{HN}$ & 21 & $\mathrm{~N}$ & 25.43 & 55 & $\mathrm{HN}$ & 55 & $\mathrm{~N}$ & 19.34 \\
\hline 22 & $\mathrm{HN}$ & 22 & $\mathrm{~N}$ & 27.48 & 57 & $\mathrm{HN}$ & 57 & $\mathrm{~N}$ & 13.65 \\
\hline 23 & $\mathrm{HN}$ & 23 & $\mathrm{~N}$ & 6.11 & 58 & $\mathrm{HN}$ & 58 & $\mathrm{~N}$ & 13.07 \\
\hline 24 & $\mathrm{HN}$ & 24 & $\mathrm{~N}$ & 19.13 & 59 & $\mathrm{HN}$ & 59 & $\mathrm{~N}$ & -25.85 \\
\hline 25 & $\mathrm{HN}$ & 25 & $\mathrm{~N}$ & 16.17 & 60 & $\mathrm{HN}$ & 60 & $\mathrm{~N}$ & 21.35 \\
\hline 26 & $\mathrm{HN}$ & 26 & $\mathrm{~N}$ & 26.91 & 61 & $\mathrm{HN}$ & 61 & $\mathrm{~N}$ & 13.11 \\
\hline 27 & $\mathrm{HN}$ & 27 & $\mathrm{~N}$ & 2.08 & 62 & $\mathrm{HN}$ & 62 & $\mathrm{~N}$ & 25.69 \\
\hline 29 & $\mathrm{HN}$ & 29 & $\mathrm{~N}$ & -25.88 & 64 & $\mathrm{HN}$ & 64 & $\mathrm{~N}$ & 18.48 \\
\hline 31 & $\mathrm{HN}$ & 31 & $\mathrm{~N}$ & -40.69 & 66 & $\mathrm{HN}$ & 66 & $\mathrm{~N}$ & -15.25 \\
\hline 32 & $\mathrm{HN}$ & 32 & $\mathrm{~N}$ & -27.32 & & & & & \\
\hline
\end{tabular}


Table C.2: HN-RDCs of DNA-bound CylR2 measured at $298 \mathrm{~K}, 700 \mathrm{MHz}$ and at a Pf1 concentration of $\sim 12 \mathrm{mg} / \mathrm{ml}$. The IPAP- ${ }^{15} \mathrm{~N}-\mathrm{HSQC}$ spectrum at $0.5 \mathrm{M} \mathrm{NaCl}$ was acquired at $800 \mathrm{MHz}$ and at a Pf1 concentration of $14 \mathrm{mg} / \mathrm{ml}$.

\begin{tabular}{|c|c|c|c|c|c|c|c|c|c|c|c|c|c|}
\hline \multicolumn{2}{|c|}{$0 \mathrm{M} \mathrm{NaCl}$} & \multicolumn{2}{|c|}{$0.025 \mathrm{M} \mathrm{NaCl}$} & \multicolumn{2}{|c|}{$0.05 \mathrm{M} \mathrm{NaCl}$} & \multicolumn{2}{|c|}{$\overline{0.1 \mathrm{M} \mathrm{NaCl}}$} & \multicolumn{2}{|c|}{$\overline{0.2 \mathrm{M} \mathrm{NaCl}}$} & \multicolumn{2}{|c|}{ 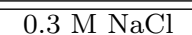 } & \multicolumn{2}{|c|}{$0.5 \mathrm{M} \mathrm{NaCl}$} \\
\hline Res. & $\mathrm{D}[\mathrm{Hz}]$ & Res. & $\mathrm{D}[\mathrm{Hz}]$ & Res. & $\mathrm{D}[\mathrm{Hz}]$ & Res. & $\mathrm{D}[\mathrm{Hz}]$ & Res. & $\mathrm{D}[\mathrm{Hz}]$ & Res. & $\mathrm{D}[\mathrm{Hz}]$ & Res. & $\mathrm{D}[\mathrm{Hz}]$ \\
\hline 2 & 11.38 & 2 & 10.68 & 2 & 10.44 & 2 & 9.96 & 2 & 9.14 & 2 & 7.98 & 2 & -8.37 \\
\hline 3 & -16.64 & 3 & -16.50 & 3 & -16.62 & 3 & -14.80 & 3 & -9.12 & 3 & -10.06 & 3 & 8.41 \\
\hline 4 & 10.94 & 4 & 8.40 & 4 & 8.86 & 4 & 6.22 & 4 & 6.92 & 4 & 5.80 & 4 & -10.06 \\
\hline 5 & -21.68 & 5 & -23.26 & 5 & -19.02 & 5 & -19.72 & 5 & -17.36 & 6 & 0.80 & 5 & 13.37 \\
\hline 6 & -0.50 & 6 & 2.34 & 6 & 2.52 & 6 & 3.14 & 6 & 1.74 & 7 & 6.24 & 6 & -1.66 \\
\hline 7 & 11.92 & 7 & 11.06 & 7 & 9.00 & 7 & 7.66 & 7 & 7.38 & 8 & -9.12 & 7 & -14.19 \\
\hline 8 & -12.44 & 8 & -12.30 & 8 & -11.90 & 8 & -10.82 & 8 & -8.12 & 9 & -0.20 & 8 & 12.00 \\
\hline 9 & -0.12 & 9 & 0.16 & 9 & 0.60 & 9 & -0.18 & 9 & 0.26 & 10 & 9.38 & 10 & -9.98 \\
\hline 10 & 11.96 & 10 & 11.68 & 10 & 15.48 & 10 & 12.66 & 10 & 8.24 & 12 & -10.60 & 11 & -0.35 \\
\hline 11 & 1.36 & 11 & 0.58 & 11 & -0.38 & 11 & -0.90 & 11 & 0.54 & 13 & 5.18 & 13 & -3.75 \\
\hline 12 & -21.42 & 12 & -20.70 & 12 & -18.64 & 12 & -17.06 & 12 & -15.98 & 14 & 12.36 & 14 & -19.41 \\
\hline 13 & 7.52 & 13 & 10.80 & 13 & 9.92 & 13 & 8.22 & 13 & 5.78 & 15 & -1.44 & 15 & -4.16 \\
\hline 14 & 19.14 & 14 & 14.04 & 14 & 14.66 & 14 & 10.96 & 14 & 9.50 & 16 & 9.40 & 16 & -14.20 \\
\hline 15 & -2.06 & 15 & -0.78 & 15 & -1.76 & 15 & -2.66 & 15 & -2.52 & 17 & -18.16 & 18 & 23.60 \\
\hline 16 & 13.66 & 16 & 9.40 & 16 & 11.32 & 16 & 8.44 & 16 & 10.42 & 18 & -18.26 & 20 & -7.54 \\
\hline 17 & -17.88 & 17 & -18.54 & 17 & -16.28 & 17 & -18.60 & 17 & -8.74 & 19 & 0.62 & 21 & 21.02 \\
\hline 18 & -23.04 & 18 & -29.28 & 18 & -22.04 & 18 & -28.64 & 18 & -21.32 & 20 & -3.90 & 22 & 18.70 \\
\hline 19 & 4.00 & 19 & 3.44 & 19 & -2.22 & 19 & 1.86 & 19 & -5.16 & 21 & -14.92 & 23 & -7.94 \\
\hline 20 & 4.60 & 20 & 1.18 & 20 & 4.98 & 20 & -1.04 & 20 & -0.44 & 22 & -12.84 & 24 & 17.30 \\
\hline 21 & -22.70 & 21 & -20.58 & 21 & -19.22 & 21 & -17.46 & 21 & -18.52 & 24 & -8.42 & 25 & 23.33 \\
\hline 22 & -18.96 & 22 & -18.94 & 22 & -15.34 & 22 & -17.40 & 22 & -14.66 & 25 & -14.16 & 26 & 8.76 \\
\hline 23 & 11.54 & 23 & 15.52 & 23 & 13.26 & 23 & 10.08 & 23 & 11.40 & 26 & -3.78 & 29 & -23.22 \\
\hline 24 & -10.32 & 24 & -10.62 & 24 & -16.42 & 24 & -12.52 & 24 & -10.88 & 29 & 3.60 & 31 & -23.12 \\
\hline 25 & -26.56 & 25 & -22.72 & 25 & -17.94 & 25 & -20.08 & 25 & -15.38 & 30 & 12.38 & 32 & -17.60 \\
\hline 26 & -6.48 & 26 & -5.90 & 26 & -5.14 & 26 & -4.10 & 26 & -1.90 & 32 & 8.96 & 34 & -14.06 \\
\hline 29 & 19.46 & 29 & 10.26 & 29 & 7.42 & 29 & 14.42 & 29 & 6.18 & 33 & 12.14 & 35 & -14.61 \\
\hline 30 & 21.56 & 30 & 18.30 & 30 & 14.74 & 30 & 11.82 & 30 & 10.24 & 34 & 12.10 & 36 & -27.32 \\
\hline 31 & -1.48 & 31 & -2.90 & 31 & -2.44 & 31 & -5.24 & 31 & -1.00 & 35 & 9.42 & 37 & -25.07 \\
\hline 32 & 17.54 & 32 & 15.84 & 32 & 17.70 & 32 & 11.30 & 32 & 14.14 & 36 & 13.50 & 38 & -5.36 \\
\hline 33 & 22.94 & 33 & 22.66 & 33 & 21.32 & 33 & 17.70 & 33 & 13.24 & 37 & 21.32 & 39 & -4.08 \\
\hline 34 & 11.98 & 34 & 16.66 & 34 & 15.64 & 34 & 8.44 & 34 & 8.02 & 38 & 6.44 & 40 & 7.17 \\
\hline 35 & 5.70 & 35 & 8.10 & 35 & 10.10 & 35 & 6.82 & 35 & 7.96 & 39 & 5.22 & 42 & 1.97 \\
\hline 36 & 20.44 & 36 & 20.58 & 36 & 18.00 & 36 & 15.54 & 36 & 12.76 & 40 & -0.96 & 43 & 15.03 \\
\hline 37 & 30.08 & 37 & 25.12 & 37 & 27.84 & 37 & 21.52 & 37 & 17.82 & 43 & -1.76 & 44 & -15.25 \\
\hline 38 & -1.52 & 38 & -1.80 & 38 & -0.02 & 38 & 1.14 & 38 & 1.42 & 44 & 7.14 & 45 & -10.20 \\
\hline 39 & 6.30 & 39 & 5.74 & 39 & 5.78 & 39 & 2.00 & 39 & 5.58 & 45 & 0.28 & 46 & 11.39 \\
\hline 40 & -0.66 & 40 & -0.52 & 40 & -2.50 & 40 & 1.28 & 40 & -0.64 & 47 & 1.26 & 47 & -1.08 \\
\hline 43 & -11.26 & 43 & -1.98 & 43 & -5.92 & 43 & -4.46 & 43 & -2.54 & 48 & 13.12 & 49 & 0.21 \\
\hline 44 & 11.74 & 44 & 8.48 & 44 & 9.06 & 44 & 7.16 & 44 & 8.90 & 49 & 1.04 & 50 & 4.99 \\
\hline 45 & 7.68 & 45 & 6.96 & 45 & 6.84 & 45 & 3.96 & 45 & 3.88 & 50 & -7.34 & 51 & -8.33 \\
\hline 46 & -8.12 & 46 & -8.42 & 46 & -6.00 & 46 & -6.68 & 46 & -6.26 & 51 & 7.72 & 52 & -15.24 \\
\hline 47 & 7.58 & 47 & 4.46 & 47 & 6.04 & 47 & 7.12 & 47 & 6.74 & 52 & 7.74 & 54 & -2.10 \\
\hline 48 & 19.32 & 48 & 15.92 & 48 & 15.72 & 48 & 16.22 & 48 & 13.64 & 54 & 7.14 & 57 & 0.97 \\
\hline 49 & 2.86 & 49 & 2.84 & 49 & 2.86 & 49 & 7.28 & 49 & 2.34 & 55 & 15.90 & 59 & -25.78 \\
\hline 50 & -9.00 & 50 & -9.98 & 50 & -10.82 & 50 & -7.62 & 50 & -6.62 & 57 & -3.72 & 61 & -5.11 \\
\hline 51 & 13.08 & 51 & 12.66 & 51 & 12.06 & 51 & 10.60 & 51 & 9.96 & 59 & 15.90 & 62 & 12.29 \\
\hline 52 & 17.94 & 52 & 14.52 & 52 & 13.00 & 52 & 12.78 & 52 & 12.68 & 60 & -13.54 & 63 & -10.05 \\
\hline 54 & 15.10 & 54 & 14.40 & 54 & 14.88 & 54 & 13.94 & 54 & 11.94 & 61 & -1.98 & 64 & -9.02 \\
\hline 55 & 22.38 & 55 & 21.08 & 55 & 20.22 & 55 & 19.50 & 55 & 17.70 & 62 & -11.24 & 66 & -9.63 \\
\hline 57 & -5.62 & 57 & -6.34 & 57 & -3.40 & 57 & -3.18 & 57 & -5.20 & 63 & 5.16 & & \\
\hline 59 & 21.04 & 59 & 20.04 & 59 & 19.06 & 59 & 13.98 & 59 & 14.90 & 64 & 9.66 & & \\
\hline 60 & -16.80 & 60 & -15.94 & 60 & -19.08 & 60 & -19.30 & 60 & -13.70 & 66 & 7.38 & & \\
\hline 61 & -0.06 & 61 & 1.06 & 61 & 0.98 & 61 & -1.26 & 61 & -1.58 & & & & \\
\hline 62 & -17.44 & 62 & -15.88 & 62 & -12.08 & 62 & -12.10 & 62 & -14.08 & & & & \\
\hline 63 & 8.70 & 63 & 9.14 & 63 & 7.32 & 63 & 6.80 & 63 & 7.54 & & & & \\
\hline 64 & 13.64 & 64 & 13.22 & 64 & 13.22 & 64 & 12.48 & 64 & 10.68 & & & & \\
\hline 66 & 11.86 & 66 & 11.16 & 66 & 9.82 & 66 & 9.18 & 66 & 8.16 & & & & \\
\hline
\end{tabular}


Table C.3: HN-RDCs of CesT measured at $303 \mathrm{~K}, 700 \mathrm{MHz}$ and at a Pf1 concentration of $\sim 10 \mathrm{mg} / \mathrm{ml}$.

\begin{tabular}{|c|c|c|c|c|c|c|c|c|c|}
\hline 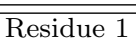 & Atom 1 & Residue 2 & Atom 2 & $\overline{\mathrm{D}[\mathrm{Hz}]}$ & Residue 1 & Atom 1 & "Residue 2 & Atom 2 & $\overline{\overline{\mathrm{D}}[\mathrm{Hz}]}$ \\
\hline 5 & $\mathrm{HN}$ & 5 & $\mathrm{~N}$ & 6.71 & 67 & $\mathrm{HN}$ & 67 & $\mathrm{~N}$ & 8.47 \\
\hline 6 & $\mathrm{HN}$ & 6 & $\mathrm{~N}$ & 2.48 & 68 & $\mathrm{HN}$ & 68 & $\mathrm{~N}$ & 0.55 \\
\hline 7 & $\mathrm{HN}$ & 7 & $\mathrm{~N}$ & -6.59 & 70 & $\mathrm{HN}$ & 70 & $\mathrm{~N}$ & 13.37 \\
\hline 8 & $\mathrm{HN}$ & 8 & $\mathrm{~N}$ & -3.88 & 71 & $\mathrm{HN}$ & 71 & $\mathrm{~N}$ & 4.83 \\
\hline 9 & HN & 9 & $\mathrm{~N}$ & -0.43 & 72 & $\mathrm{HN}$ & 72 & $\mathrm{~N}$ & 6.68 \\
\hline 10 & HN & 10 & $\mathrm{~N}$ & 4.02 & 74 & $\mathrm{HN}$ & 74 & $\mathrm{~N}$ & 7.91 \\
\hline 11 & $\mathrm{HN}$ & 11 & $\mathrm{~N}$ & -2.68 & 75 & $\mathrm{HN}$ & 75 & $\mathrm{~N}$ & 4.36 \\
\hline 13 & HN & 13 & $\mathrm{~N}$ & 4.85 & 77 & $\mathrm{HN}$ & 77 & $\mathrm{~N}$ & 8.67 \\
\hline 14 & HN & 14 & $\mathrm{~N}$ & -0.81 & 78 & $\mathrm{HN}$ & 78 & $\mathrm{~N}$ & 5.03 \\
\hline 15 & $\mathrm{HN}$ & 15 & $\mathrm{~N}$ & -5.87 & 80 & $\mathrm{HN}$ & 80 & $\mathrm{~N}$ & 0.02 \\
\hline 16 & HN & 16 & $\mathrm{~N}$ & 7.69 & 86 & $\mathrm{HN}$ & 86 & $\mathrm{~N}$ & -6.87 \\
\hline 17 & HN & 17 & $\mathrm{~N}$ & 2.99 & 87 & $\mathrm{HN}$ & 87 & $\mathrm{~N}$ & -2.71 \\
\hline 18 & $\mathrm{HN}$ & 18 & $\mathrm{~N}$ & -8.44 & 89 & $\mathrm{HN}$ & 89 & $\mathrm{~N}$ & 1.52 \\
\hline 20 & $\mathrm{HN}$ & 20 & $\mathrm{~N}$ & 4.80 & 92 & $\mathrm{HN}$ & 92 & $\mathrm{~N}$ & 7.17 \\
\hline 23 & $\mathrm{HN}$ & 23 & $\mathrm{~N}$ & 6.07 & 93 & $\mathrm{HN}$ & 93 & $\mathrm{~N}$ & -12.25 \\
\hline 24 & HN & 24 & $\mathrm{~N}$ & 1.56 & 94 & $\mathrm{HN}$ & 94 & $\mathrm{~N}$ & -10.17 \\
\hline 26 & $\mathrm{HN}$ & 26 & $\mathrm{~N}$ & -3.36 & 95 & $\mathrm{HN}$ & 95 & $\mathrm{~N}$ & -13.22 \\
\hline 27 & $\mathrm{HN}$ & 27 & $\mathrm{~N}$ & -9.64 & 96 & $\mathrm{HN}$ & 96 & $\mathrm{~N}$ & -3.00 \\
\hline 28 & $\mathrm{HN}$ & 28 & $\mathrm{~N}$ & -5.08 & 97 & $\mathrm{HN}$ & 97 & $\mathrm{~N}$ & -9.81 \\
\hline 29 & $\mathrm{HN}$ & 29 & $\mathrm{~N}$ & -11.37 & 98 & $\mathrm{HN}$ & 98 & $\mathrm{~N}$ & -8.36 \\
\hline 30 & $\mathrm{HN}$ & 30 & $\mathrm{~N}$ & -2.95 & 99 & $\mathrm{HN}$ & 99 & $\mathrm{~N}$ & 5.93 \\
\hline 31 & $\mathrm{HN}$ & 31 & $\mathrm{~N}$ & -11.41 & 101 & $\mathrm{HN}$ & 101 & $\mathrm{~N}$ & -2.81 \\
\hline 33 & HN & 33 & $\mathrm{~N}$ & 1.26 & 102 & $\mathrm{HN}$ & 102 & $\mathrm{~N}$ & -7.33 \\
\hline 34 & $\mathrm{HN}$ & 34 & $\mathrm{~N}$ & 6.90 & 103 & $\mathrm{HN}$ & 103 & $\mathrm{~N}$ & 6.16 \\
\hline 36 & HN & 36 & $\mathrm{~N}$ & 9.99 & 104 & $\mathrm{HN}$ & 104 & $\mathrm{~N}$ & 8.35 \\
\hline 37 & HN & 37 & $\mathrm{~N}$ & -2.67 & 105 & $\mathrm{HN}$ & 105 & $\mathrm{~N}$ & 1.03 \\
\hline 38 & HN & 38 & $\mathrm{~N}$ & -1.69 & 107 & $\mathrm{HN}$ & 107 & $\mathrm{~N}$ & 3.49 \\
\hline 39 & HN & 39 & $\mathrm{~N}$ & -18.74 & 108 & $\mathrm{HN}$ & 108 & $\mathrm{~N}$ & 5.94 \\
\hline 40 & HN & 40 & $\mathrm{~N}$ & -11.45 & 109 & $\mathrm{HN}$ & 109 & $\mathrm{~N}$ & 2.19 \\
\hline 41 & HN & 41 & $\mathrm{~N}$ & -11.65 & 110 & $\mathrm{HN}$ & 110 & $\mathrm{~N}$ & 6.53 \\
\hline 42 & HN & 42 & $\mathrm{~N}$ & -3.18 & 111 & $\mathrm{HN}$ & 111 & $\mathrm{~N}$ & 7.70 \\
\hline 43 & $\mathrm{HN}$ & 43 & $\mathrm{~N}$ & -1.18 & 112 & $\mathrm{HN}$ & 112 & $\mathrm{~N}$ & 5.84 \\
\hline 44 & $\mathrm{HN}$ & 44 & $\mathrm{~N}$ & 5.01 & 113 & $\mathrm{HN}$ & 113 & $\mathrm{~N}$ & 2.84 \\
\hline 45 & HN & 45 & $\mathrm{~N}$ & 2.79 & 114 & $\mathrm{HN}$ & 114 & $\mathrm{~N}$ & 12.51 \\
\hline 46 & HN & 46 & $\mathrm{~N}$ & -4.29 & 115 & $\mathrm{HN}$ & 115 & $\mathrm{~N}$ & 3.65 \\
\hline 47 & $\mathrm{HN}$ & 47 & $\mathrm{~N}$ & 6.20 & 116 & $\mathrm{HN}$ & 116 & $\mathrm{~N}$ & 3.26 \\
\hline 48 & HN & 48 & $\mathrm{~N}$ & 10.05 & 117 & $\mathrm{HN}$ & 117 & $\mathrm{~N}$ & 7.43 \\
\hline 49 & HN & 49 & $\mathrm{~N}$ & 5.93 & 118 & $\mathrm{HN}$ & 118 & $\mathrm{~N}$ & 6.30 \\
\hline 51 & HN & 51 & $\mathrm{~N}$ & -9.74 & 119 & $\mathrm{HN}$ & 119 & $\mathrm{~N}$ & 5.39 \\
\hline 52 & HN & 52 & $\mathrm{~N}$ & -9.10 & 120 & $\mathrm{HN}$ & 120 & $\mathrm{~N}$ & 7.97 \\
\hline 53 & $\mathrm{HN}$ & 53 & $\mathrm{~N}$ & -17.98 & 121 & $\mathrm{HN}$ & 121 & $\mathrm{~N}$ & 14.14 \\
\hline 54 & HN & 54 & $\mathrm{~N}$ & -11.09 & 122 & $\mathrm{HN}$ & 122 & $\mathrm{~N}$ & 11.54 \\
\hline 55 & HN & 55 & $\mathrm{~N}$ & -8.66 & 123 & $\mathrm{HN}$ & 123 & $\mathrm{~N}$ & 7.83 \\
\hline 56 & HN & 56 & $\mathrm{~N}$ & 1.94 & 124 & HN & 124 & $\mathrm{~N}$ & 5.26 \\
\hline 57 & HN & 57 & $\mathrm{~N}$ & -8.10 & 125 & $\mathrm{HN}$ & 125 & $\mathrm{~N}$ & 7.36 \\
\hline 58 & $\mathrm{HN}$ & 58 & $\mathrm{~N}$ & 12.66 & 126 & $\mathrm{HN}$ & 126 & $\mathrm{~N}$ & 8.46 \\
\hline 60 & $\mathrm{HN}$ & 60 & $\mathrm{~N}$ & -11.47 & 127 & $\mathrm{HN}$ & 127 & $\mathrm{~N}$ & 4.67 \\
\hline 61 & HN & 61 & $\mathrm{~N}$ & -6.21 & 128 & $\mathrm{HN}$ & 128 & $\mathrm{~N}$ & 6.57 \\
\hline 65 & $\mathrm{HN}$ & 65 & $\mathrm{~N}$ & 4.10 & 133 & $\mathrm{HN}$ & 133 & $\mathrm{~N}$ & 8.38 \\
\hline 66 & $\mathrm{HN}$ & 66 & $\mathrm{~N}$ & 6.53 & 134 & $\mathrm{HN}$ & 134 & $\mathrm{~N}$ & 6.17 \\
\hline
\end{tabular}

Table C.4: NC'-RDCs of CesT measured at $303 \mathrm{~K}, 700 \mathrm{MHz}$ and at a Pf1 concentration of $\sim 10 \mathrm{mg} / \mathrm{ml}$.

\begin{tabular}{|c|c|c|c|c|c|c|c|c|c|}
\hline Residue 1 & Atom 1 & Residue 2 & Atom 2 & $\mathrm{D}[\mathrm{Hz}]$ & Residue 1 & Atom 1 & Residue 2 & Atom 2 & $\mathrm{D}[\mathrm{Hz}]$ \\
\hline 4 & $\mathrm{~N}$ & 3 & $\mathrm{C}^{\prime}$ & -1.06 & 69 & $\mathrm{~N}$ & 68 & $\mathrm{C}^{\prime}$ & -0.22 \\
\hline 5 & $\mathrm{~N}$ & 4 & $\mathrm{C}^{\prime}$ & 0.34 & 71 & $\mathrm{~N}$ & 70 & $\mathrm{C}^{\prime}$ & 1.00 \\
\hline
\end{tabular}




\begin{tabular}{|c|c|c|c|c|c|c|c|c|c|}
\hline Residue 1 & Atom 1 & Residue 2 & Atom 2 & $\overline{\mathrm{D}[\mathrm{Hz}]}$ & Residue 1 & Atom 1 & Residue 2 & Atom 2 & $\mathrm{D}[\mathrm{Hz}]$ \\
\hline 7 & $\mathrm{~N}$ & 6 & $\mathrm{C}^{\prime}$ & 0.25 & 73 & $\mathrm{~N}$ & 72 & $\mathrm{C}^{\prime}$ & 1.79 \\
\hline 8 & $\mathrm{~N}$ & 7 & $\mathrm{C}^{\prime}$ & -1.47 & 74 & $\mathrm{~N}$ & 73 & $\mathrm{C}^{\prime}$ & -0.32 \\
\hline 9 & $\mathrm{~N}$ & 8 & $\mathrm{C}^{\prime}$ & 0.89 & 77 & $\mathrm{~N}$ & 76 & $\mathrm{C}^{\prime}$ & -1.57 \\
\hline 10 & $\mathrm{~N}$ & 9 & $\mathrm{C}^{\prime}$ & 0.17 & 79 & $\mathrm{~N}$ & 78 & $\mathrm{C}^{\prime}$ & -0.42 \\
\hline 11 & $\mathrm{~N}$ & 10 & $\mathrm{C}^{\prime}$ & -0.20 & 81 & $\mathrm{~N}$ & 80 & $\mathrm{C}^{\prime}$ & 0.46 \\
\hline 13 & $\mathrm{~N}$ & 12 & $\mathrm{C}^{\prime}$ & 0.30 & 86 & $\mathrm{~N}$ & 85 & $\mathrm{C}^{\prime}$ & 1.71 \\
\hline 14 & $\mathrm{~N}$ & 13 & $\mathrm{C}^{\prime}$ & -0.39 & 87 & $\mathrm{~N}$ & 86 & $\mathrm{C}^{\prime}$ & -1.23 \\
\hline 15 & $\mathrm{~N}$ & 14 & $\mathrm{C}^{\prime}$ & -1.47 & 88 & $\mathrm{~N}$ & 87 & $\mathrm{C}^{\prime}$ & 0.82 \\
\hline 16 & $\mathrm{~N}$ & 15 & $\mathrm{C}^{\prime}$ & 1.64 & 89 & $\mathrm{~N}$ & 88 & $\mathrm{C}^{\prime}$ & -0.39 \\
\hline 17 & $\mathrm{~N}$ & 16 & $\mathrm{C}^{\prime}$ & -0.34 & 90 & $\mathrm{~N}$ & 89 & $\mathrm{C}^{\prime}$ & 0.74 \\
\hline 19 & $\mathrm{~N}$ & 18 & $\mathrm{C}^{\prime}$ & 0.17 & 91 & $\mathrm{~N}$ & 90 & $\mathrm{C}^{\prime}$ & -2.03 \\
\hline 20 & $\mathrm{~N}$ & 19 & $\mathrm{C}^{\prime}$ & 0.28 & 92 & $\mathrm{~N}$ & 91 & $\mathrm{C}^{\prime}$ & 0.17 \\
\hline 21 & $\mathrm{~N}$ & 20 & $\mathrm{C}^{\prime}$ & -0.70 & 93 & $\mathrm{~N}$ & 92 & $\mathrm{C}^{\prime}$ & 0.87 \\
\hline 22 & $\mathrm{~N}$ & 21 & $\mathrm{C}^{\prime}$ & 0.78 & 95 & $\mathrm{~N}$ & 94 & $\mathrm{C}^{\prime}$ & 0.14 \\
\hline 23 & $\mathrm{~N}$ & 22 & $\mathrm{C}^{\prime}$ & -0.50 & 96 & $\mathrm{~N}$ & 95 & $\mathrm{C}^{\prime}$ & -0.97 \\
\hline 25 & $\mathrm{~N}$ & 24 & $\mathrm{C}^{\prime}$ & 1.21 & 97 & $\mathrm{~N}$ & 96 & $\mathrm{C}^{\prime}$ & 0.03 \\
\hline 26 & $\mathrm{~N}$ & 25 & $\mathrm{C}^{\prime}$ & 1.08 & 98 & $\mathrm{~N}$ & 97 & $\mathrm{C}^{\prime}$ & -0.72 \\
\hline 28 & $\mathrm{~N}$ & 27 & $\mathrm{C}^{\prime}$ & -0.49 & 99 & $\mathrm{~N}$ & 98 & $\mathrm{C}^{\prime}$ & 0.08 \\
\hline 30 & $\mathrm{~N}$ & 29 & $\mathrm{C}^{\prime}$ & 1.15 & 101 & $\mathrm{~N}$ & 100 & $\mathrm{C}^{\prime}$ & 0.80 \\
\hline 33 & $\mathrm{~N}$ & 32 & $\mathrm{C}^{\prime}$ & -0.93 & 102 & $\mathrm{~N}$ & 101 & $\mathrm{C}^{\prime}$ & -1.21 \\
\hline 38 & $\mathrm{~N}$ & 37 & $\mathrm{C}^{\prime}$ & 0.03 & 103 & $\mathrm{~N}$ & 102 & $\mathrm{C}^{\prime}$ & 0.05 \\
\hline 39 & $\mathrm{~N}$ & 38 & $\mathrm{C}^{\prime}$ & 0.87 & 104 & $\mathrm{~N}$ & 103 & $\mathrm{C}^{\prime}$ & 0.57 \\
\hline 40 & $\mathrm{~N}$ & 39 & $\mathrm{C}^{\prime}$ & -0.78 & 105 & $\mathrm{~N}$ & 104 & $\mathrm{C}^{\prime}$ & 0.18 \\
\hline 41 & $\mathrm{~N}$ & 40 & $\mathrm{C}^{\prime}$ & -0.26 & 107 & $\mathrm{~N}$ & 106 & $\mathrm{C}^{\prime}$ & 0.40 \\
\hline 42 & $\mathrm{~N}$ & 41 & $\mathrm{C}^{\prime}$ & -1.40 & 108 & $\mathrm{~N}$ & 107 & $\mathrm{C}^{\prime}$ & -0.15 \\
\hline 43 & $\mathrm{~N}$ & 42 & $\mathrm{C}^{\prime}$ & 0.55 & 109 & $\mathrm{~N}$ & 108 & $\mathrm{C}^{\prime}$ & -0.22 \\
\hline 45 & $\mathrm{~N}$ & 44 & $\mathrm{C}^{\prime}$ & 0.32 & 110 & $\mathrm{~N}$ & 109 & $\mathrm{C}^{\prime}$ & -0.09 \\
\hline 46 & $\mathrm{~N}$ & 45 & $\mathrm{C}^{\prime}$ & 0.86 & 111 & $\mathrm{~N}$ & 110 & $\mathrm{C}^{\prime}$ & -0.46 \\
\hline 47 & $\mathrm{~N}$ & 46 & $\mathrm{C}^{\prime}$ & -0.73 & 112 & $\mathrm{~N}$ & 111 & $\mathrm{C}^{\prime}$ & 0.91 \\
\hline 48 & $\mathrm{~N}$ & 47 & $\mathrm{C}^{\prime}$ & 0.54 & 113 & $\mathrm{~N}$ & 112 & $\mathrm{C}^{\prime}$ & -1.85 \\
\hline 49 & $\mathrm{~N}$ & 48 & $\mathrm{C}^{\prime}$ & 0.21 & 114 & $\mathrm{~N}$ & 113 & $\mathrm{C}^{\prime}$ & 0.94 \\
\hline 50 & $\mathrm{~N}$ & 49 & $\mathrm{C}^{\prime}$ & 0.43 & 115 & $\mathrm{~N}$ & 114 & $\mathrm{C}^{\prime}$ & -1.30 \\
\hline 51 & $\mathrm{~N}$ & 50 & $\mathrm{C}^{\prime}$ & 1.46 & 116 & $\mathrm{~N}$ & 115 & $\mathrm{C}^{\prime}$ & -0.06 \\
\hline 52 & $\mathrm{~N}$ & 51 & $\mathrm{C}^{\prime}$ & -0.14 & 117 & $\mathrm{~N}$ & 116 & $\mathrm{C}^{\prime}$ & -1.05 \\
\hline 54 & $\mathrm{~N}$ & 53 & $\mathrm{C}^{\prime}$ & -0.84 & 118 & $\mathrm{~N}$ & 117 & $\mathrm{C}^{\prime}$ & -0.14 \\
\hline 55 & $\mathrm{~N}$ & 54 & $\mathrm{C}^{\prime}$ & -0.82 & 119 & $\mathrm{~N}$ & 118 & $\mathrm{C}^{\prime}$ & 0.75 \\
\hline 56 & $\mathrm{~N}$ & 55 & $\mathrm{C}^{\prime}$ & 0.88 & 120 & $\mathrm{~N}$ & 119 & $\mathrm{C}^{\prime}$ & -0.38 \\
\hline 57 & $\mathrm{~N}$ & 56 & $\mathrm{C}^{\prime}$ & 0.17 & 121 & $\mathrm{~N}$ & 120 & $\mathrm{C}^{\prime}$ & -0.06 \\
\hline 60 & $\mathrm{~N}$ & 59 & $\mathrm{C}^{\prime}$ & 0.39 & 122 & $\mathrm{~N}$ & 121 & $\mathrm{C}^{\prime}$ & -1.15 \\
\hline 61 & $\mathrm{~N}$ & 60 & $\mathrm{C}^{\prime}$ & 0.16 & 123 & $\mathrm{~N}$ & 122 & $\mathrm{C}^{\prime}$ & 1.16 \\
\hline 62 & $\mathrm{~N}$ & 61 & $\mathrm{C}^{\prime}$ & 0.39 & 124 & $\mathrm{~N}$ & 123 & $\mathrm{C}^{\prime}$ & -1.61 \\
\hline 64 & $\mathrm{~N}$ & 63 & $\mathrm{C}^{\prime}$ & 0.78 & 125 & $\mathrm{~N}$ & 124 & $\mathrm{C}^{\prime}$ & -0.18 \\
\hline 65 & $\mathrm{~N}$ & 64 & $\mathrm{C}^{\prime}$ & -1.19 & 126 & $\mathrm{~N}$ & 125 & $\mathrm{C}^{\prime}$ & 0.13 \\
\hline 66 & $\mathrm{~N}$ & 65 & $\mathrm{C}^{\prime}$ & 1.49 & 128 & $\mathrm{~N}$ & 127 & $\mathrm{C}^{\prime}$ & -1.58 \\
\hline 67 & $\mathrm{~N}$ & 66 & $\mathrm{C}^{\prime}$ & -0.09 & 133 & $\mathrm{~N}$ & 132 & $\mathrm{C}^{\prime}$ & -0.33 \\
\hline 68 & $\mathrm{~N}$ & 67 & $\mathrm{C}^{\prime}$ & -0.97 & 134 & $\mathrm{~N}$ & 133 & $\mathrm{C}^{\prime}$ & 0.36 \\
\hline
\end{tabular}

Table C.5: C $\alpha$ C'-RDCs of CesT measured at $303 \mathrm{~K}, 600 \mathrm{MHz}$ and at a Pf1 concentration of $\sim 10 \mathrm{mg} / \mathrm{ml}$.

\begin{tabular}{|c|c|c|c|c|c|c|c|c|c|}
\hline Residue 1 & Atom 1 & Residue 2 & Atom 2 & $\mathrm{D}[\mathrm{Hz}]$ & Residue 1 & Atom 1 & Residue 2 & Atom 2 & $\mathrm{D}[\mathrm{Hz}]$ \\
\hline 4 & $\mathrm{C} \alpha$ & 4 & $\mathrm{C}^{\prime}$ & 0.92 & 57 & $\mathrm{C} \alpha$ & 57 & $\mathrm{C}^{\prime}$ & 0.09 \\
\hline 6 & $\mathrm{C} \alpha$ & 6 & $\mathrm{C}^{\prime}$ & 1.30 & 59 & $\mathrm{C} \alpha$ & 59 & $\mathrm{C}^{\prime}$ & -1.61 \\
\hline 7 & $\mathrm{C} \alpha$ & 7 & $\mathrm{C}^{\prime}$ & 1.28 & 60 & $\mathrm{C} \alpha$ & 60 & $\mathrm{C}^{\prime}$ & 1.07 \\
\hline 8 & $\mathrm{C} \alpha$ & 8 & $\mathrm{C}^{\prime}$ & -0.93 & 61 & $\mathrm{C} \alpha$ & 61 & $\mathrm{C}^{\prime}$ & -0.35 \\
\hline 9 & $\mathrm{C} \alpha$ & 9 & $\mathrm{C}^{\prime}$ & -1.86 & 64 & $\mathrm{C} \alpha$ & 64 & $\mathrm{C}^{\prime}$ & 0.89 \\
\hline 10 & $\mathrm{C} \alpha$ & 10 & $\mathrm{C}^{\prime}$ & 1.47 & 65 & $\mathrm{C} \alpha$ & 65 & $\mathrm{C}^{\prime}$ & 1.28 \\
\hline 12 & $\mathrm{C} \alpha$ & 12 & $\mathrm{C}^{\prime}$ & -1.73 & 66 & $\mathrm{C} \alpha$ & 66 & $\mathrm{C}^{\prime}$ & 0.51 \\
\hline 13 & $\mathrm{C} \alpha$ & 13 & $\mathrm{C}^{\prime}$ & -0.79 & 68 & $\mathrm{C} \alpha$ & 68 & $\mathrm{C}^{\prime}$ & 1.15 \\
\hline 14 & $\mathrm{C} \alpha$ & 14 & $\mathrm{C}^{\prime}$ & 0.96 & 76 & $\mathrm{C} \alpha$ & 76 & $\mathrm{C}^{\prime}$ & -1.38 \\
\hline 15 & $\mathrm{C} \alpha$ & 15 & $\mathrm{C}^{\prime}$ & 0.63 & 80 & $\mathrm{C} \alpha$ & 80 & $\mathrm{C}^{\prime}$ & 1.09 \\
\hline 16 & $\mathrm{C} \alpha$ & 16 & $\mathrm{C}^{\prime}$ & -1.82 & 86 & $\mathrm{C} \alpha$ & 86 & $\mathrm{C}^{\prime}$ & 1.27 \\
\hline
\end{tabular}




\begin{tabular}{|c|c|c|c|c|c|c|c|c|c|}
\hline Residue 1 & Atom 1 & Residue 2 & Atom 2 & $\overline{\mathrm{D}[\mathrm{Hz}]}$ & Residue 1 & Atom 1 & Residue 2 & Atom 2 & $\overline{\mathrm{D}[\mathrm{Hz}]}$ \\
\hline 18 & $\mathrm{C} \alpha$ & 18 & $\mathrm{C}^{\prime}$ & 0.73 & 87 & $\mathrm{C} \alpha$ & 87 & $\mathrm{C}^{\prime}$ & 2.94 \\
\hline 20 & $\mathrm{C} \alpha$ & 20 & $\mathrm{C}^{\prime}$ & -0.56 & 90 & $\mathrm{C} \alpha$ & 90 & $\mathrm{C}^{\prime}$ & 0.91 \\
\hline 22 & $\mathrm{C} \alpha$ & 22 & $\mathrm{C}^{\prime}$ & 0.03 & 91 & $\mathrm{C} \alpha$ & 91 & $\mathrm{C}^{\prime}$ & 0.30 \\
\hline 25 & $\mathrm{C} \alpha$ & 25 & $\mathrm{C}^{\prime}$ & -1.07 & 92 & $\mathrm{C} \alpha$ & 92 & $\mathrm{C}^{\prime}$ & 0.24 \\
\hline 26 & $\mathrm{C} \alpha$ & 26 & $\mathrm{C}^{\prime}$ & 2.99 & 93 & $\mathrm{C} \alpha$ & 93 & $\mathrm{C}^{\prime}$ & 1.48 \\
\hline 29 & $\mathrm{C} \alpha$ & 29 & $\mathrm{C}^{\prime}$ & 0.02 & 96 & $\mathrm{C} \alpha$ & 96 & $\mathrm{C}^{\prime}$ & 2.67 \\
\hline 30 & $\mathrm{C} \alpha$ & 30 & $\mathrm{C}^{\prime}$ & -0.45 & 97 & $\mathrm{C} \alpha$ & 97 & $C^{\prime}$ & -0.09 \\
\hline 32 & $\mathrm{C} \alpha$ & 32 & $\mathrm{C}^{\prime}$ & 2.38 & 100 & $\mathrm{C} \alpha$ & 100 & $\mathrm{C}^{\prime}$ & -0.92 \\
\hline 36 & $\mathrm{C} \alpha$ & 36 & $\mathrm{C}^{\prime}$ & -1.66 & 103 & $\mathrm{C} \alpha$ & 103 & $\mathrm{C}^{\prime}$ & -0.43 \\
\hline 39 & $\mathrm{C} \alpha$ & 39 & $\mathrm{C}^{\prime}$ & 3.18 & 104 & $\mathrm{C} \alpha$ & 104 & $\mathrm{C}^{\prime}$ & -1.27 \\
\hline 40 & $\mathrm{C} \alpha$ & 40 & $\mathrm{C}^{\prime}$ & 0.67 & 107 & $\mathrm{C} \alpha$ & 107 & $\mathrm{C}^{\prime}$ & -1.78 \\
\hline 41 & $\mathrm{C} \alpha$ & 41 & $\mathrm{C}^{\prime}$ & -0.30 & 108 & $\mathrm{C} \alpha$ & 108 & $\mathrm{C}^{\prime}$ & 1.68 \\
\hline 42 & $\mathrm{C} \alpha$ & 42 & $\mathrm{C}^{\prime}$ & -1.09 & 109 & $\mathrm{C} \alpha$ & 109 & $\mathrm{C}^{\prime}$ & 0.95 \\
\hline 44 & $\mathrm{C} \alpha$ & 44 & $\mathrm{C}^{\prime}$ & -0.42 & 110 & $\mathrm{C} \alpha$ & 110 & $\mathrm{C}^{\prime}$ & 0.73 \\
\hline 45 & $\mathrm{C} \alpha$ & 45 & $C^{\prime}$ & -0.72 & 112 & $\mathrm{C} \alpha$ & 112 & $\mathrm{C}^{\prime}$ & -1.22 \\
\hline 46 & $\mathrm{C} \alpha$ & 46 & $\mathrm{C}^{\prime}$ & 1.46 & 114 & $\mathrm{C} \alpha$ & 114 & $\mathrm{C}^{\prime}$ & -2.69 \\
\hline 47 & $\mathrm{C} \alpha$ & 47 & $\mathrm{C}^{\prime}$ & 0.75 & 115 & $\mathrm{C} \alpha$ & 115 & $\mathrm{C}^{\prime}$ & 1.06 \\
\hline 48 & $\mathrm{C} \alpha$ & 48 & $\mathrm{C}^{\prime}$ & 0.56 & 116 & $\mathrm{C} \alpha$ & 116 & $\mathrm{C}^{\prime}$ & -0.89 \\
\hline 49 & $\mathrm{C} \alpha$ & 49 & $\mathrm{C}^{\prime}$ & 1.24 & 117 & $\mathrm{C} \alpha$ & 117 & $\mathrm{C}^{\prime}$ & -0.81 \\
\hline 50 & $\mathrm{C} \alpha$ & 50 & $\mathrm{C}^{\prime}$ & -2.46 & 121 & $\mathrm{C} \alpha$ & 121 & $\mathrm{C}^{\prime}$ & -0.31 \\
\hline 51 & $\mathrm{C} \alpha$ & 51 & $\mathrm{C}^{\prime}$ & 1.40 & 123 & $\mathrm{C} \alpha$ & 123 & $\mathrm{C}^{\prime}$ & 0.06 \\
\hline 52 & $\mathrm{C} \alpha$ & 52 & $\mathrm{C}^{\prime}$ & 0.03 & 124 & $\mathrm{C} \alpha$ & 124 & $\mathrm{C}^{\prime}$ & 1.35 \\
\hline 53 & $\mathrm{C} \alpha$ & 53 & $\mathrm{C}^{\prime}$ & 1.53 & 126 & $\mathrm{C} \alpha$ & 126 & $\mathrm{C}^{\prime}$ & 1.83 \\
\hline 55 & $\mathrm{C} \alpha$ & 55 & $\mathrm{C}^{\prime}$ & 1.13 & 130 & $\mathrm{C} \alpha$ & 130 & $\mathrm{C}^{\prime}$ & -0.96 \\
\hline 56 & $\mathrm{C} \alpha$ & 56 & $\mathrm{C}^{\prime}$ & -0.67 & & & & & \\
\hline
\end{tabular}

Table C.6: HN-RDCs of Tir108 measured at $293 \mathrm{~K}, 700 \mathrm{MHz}$ and at a Pf1 concentration of $\sim 10 \mathrm{mg} / \mathrm{ml}$.

\begin{tabular}{|c|c|c|c|c|c|c|c|c|c|}
\hline Residue 1 & Atom 1 & Residue 2 & Atom 2 & $\mathrm{D}[\mathrm{Hz}]$ & Residue 1 & Atom 1 & Residue 2 & Atom 2 & $\mathrm{D}[\mathrm{Hz}]$ \\
\hline 3 & $\mathrm{HN}$ & 3 & $\mathrm{~N}$ & 0.14 & 57 & $\mathrm{HN}$ & 57 & $\mathrm{~N}$ & 3.41 \\
\hline 4 & $\mathrm{HN}$ & 4 & $\mathrm{~N}$ & 1.06 & 58 & $\mathrm{HN}$ & 58 & $\mathrm{~N}$ & 2.50 \\
\hline 6 & $\mathrm{HN}$ & 6 & $\mathrm{~N}$ & 1.07 & 60 & $\mathrm{HN}$ & 60 & $\mathrm{~N}$ & 0.21 \\
\hline 7 & HN & 7 & $\mathrm{~N}$ & -3.34 & 61 & HN & 61 & $\mathrm{~N}$ & -0.52 \\
\hline 8 & HN & 8 & $\mathrm{~N}$ & 4.25 & 62 & HN & 62 & $\mathrm{~N}$ & 1.21 \\
\hline 9 & HN & 9 & $\mathrm{~N}$ & 1.20 & 63 & $\mathrm{HN}$ & 63 & $\mathrm{~N}$ & 0.56 \\
\hline 10 & $\mathrm{HN}$ & 10 & $\mathrm{~N}$ & 3.55 & 64 & $\mathrm{HN}$ & 64 & $\mathrm{~N}$ & -0.22 \\
\hline 11 & $\mathrm{HN}$ & 11 & $\mathrm{~N}$ & -0.93 & 65 & $\mathrm{HN}$ & 65 & $\mathrm{~N}$ & 0.57 \\
\hline 12 & $\mathrm{HN}$ & 12 & $\mathrm{~N}$ & 0.50 & 66 & $\mathrm{HN}$ & 66 & $\mathrm{~N}$ & 1.34 \\
\hline 15 & $\mathrm{HN}$ & 15 & $\mathrm{~N}$ & 1.33 & 68 & $\mathrm{HN}$ & 68 & $\mathrm{~N}$ & 1.42 \\
\hline 16 & $\mathrm{HN}$ & 16 & $\mathrm{~N}$ & 4.97 & 69 & $\mathrm{HN}$ & 69 & $\mathrm{~N}$ & 0.78 \\
\hline 19 & $\mathrm{HN}$ & 19 & $\mathrm{~N}$ & 3.05 & 71 & $\mathrm{HN}$ & 71 & $\mathrm{~N}$ & 2.21 \\
\hline 22 & HN & 22 & $\mathrm{~N}$ & 1.77 & 72 & $\mathrm{HN}$ & 72 & $\mathrm{~N}$ & 0.62 \\
\hline 24 & $\mathrm{HN}$ & 24 & $\mathrm{~N}$ & 0.58 & 74 & $\mathrm{HN}$ & 74 & $\mathrm{~N}$ & 1.02 \\
\hline 25 & $\mathrm{HN}$ & 25 & $\mathrm{~N}$ & 1.08 & 75 & $\mathrm{HN}$ & 75 & $\mathrm{~N}$ & -0.20 \\
\hline 26 & $\mathrm{HN}$ & 26 & $\mathrm{~N}$ & -0.81 & 76 & $\mathrm{HN}$ & 76 & $\mathrm{~N}$ & 0.15 \\
\hline 27 & $\mathrm{HN}$ & 27 & $\mathrm{~N}$ & 0.06 & 77 & $\mathrm{HN}$ & 77 & $\mathrm{~N}$ & 0.43 \\
\hline 28 & $\mathrm{HN}$ & 28 & $\mathrm{~N}$ & 5.86 & 78 & HN & 78 & $\mathrm{~N}$ & 1.76 \\
\hline 29 & $\mathrm{HN}$ & 29 & $\mathrm{~N}$ & 1.07 & 79 & $\mathrm{HN}$ & 79 & $\mathrm{~N}$ & 2.68 \\
\hline 30 & $\mathrm{HN}$ & 30 & $\mathrm{~N}$ & -2.58 & 80 & $\mathrm{HN}$ & 80 & $\mathrm{~N}$ & 4.09 \\
\hline 31 & $\mathrm{HN}$ & 31 & $\mathrm{~N}$ & 2.14 & 81 & $\mathrm{HN}$ & 81 & $\mathrm{~N}$ & 4.83 \\
\hline 32 & $\mathrm{HN}$ & 32 & $\mathrm{~N}$ & 2.28 & 82 & $\mathrm{HN}$ & 82 & $\mathrm{~N}$ & 0.22 \\
\hline 33 & $\mathrm{HN}$ & 33 & $\mathrm{~N}$ & 0.70 & 86 & $\mathrm{HN}$ & 86 & $\mathrm{~N}$ & 5.84 \\
\hline 34 & $\mathrm{HN}$ & 34 & $\mathrm{~N}$ & -0.83 & 87 & $\mathrm{HN}$ & 87 & $\mathrm{~N}$ & 0.71 \\
\hline 35 & $\mathrm{HN}$ & 35 & $\mathrm{~N}$ & 1.63 & 88 & $\mathrm{HN}$ & 88 & $\mathrm{~N}$ & 4.12 \\
\hline 36 & $\mathrm{HN}$ & 36 & $\mathrm{~N}$ & 3.65 & 89 & $\mathrm{HN}$ & 89 & $\mathrm{~N}$ & 4.64 \\
\hline 37 & $\mathrm{HN}$ & 37 & $\mathrm{~N}$ & -0.05 & 90 & $\mathrm{HN}$ & 90 & $\mathrm{~N}$ & 2.14 \\
\hline 38 & HN & 38 & $\mathrm{~N}$ & 0.93 & 91 & $\mathrm{HN}$ & 91 & $\mathrm{~N}$ & 3.98 \\
\hline 39 & $\mathrm{HN}$ & 39 & $\mathrm{~N}$ & 4.26 & 92 & $\mathrm{HN}$ & 92 & $\mathrm{~N}$ & 2.62 \\
\hline 41 & $\mathrm{HN}$ & 41 & $\mathrm{~N}$ & 3.48 & 93 & $\mathrm{HN}$ & 93 & $\mathrm{~N}$ & -0.60 \\
\hline 42 & $\mathrm{HN}$ & 42 & $\mathrm{~N}$ & 2.84 & 94 & $\mathrm{HN}$ & 94 & $\mathrm{~N}$ & 5.00 \\
\hline 43 & $\mathrm{HN}$ & 43 & $\mathrm{~N}$ & 2.97 & 95 & $\mathrm{HN}$ & 95 & $\mathrm{~N}$ & 0.43 \\
\hline \multicolumn{10}{|c|}{ continued on next page } \\
\hline
\end{tabular}




\begin{tabular}{ccccc||ccccc}
\hline \hline Residue 1 & Atom 1 & Residue 2 & Atom 2 & D [Hz] & Residue 1 & Atom 1 & Residue 2 & Atom 2 & D [Hz] \\
\hline 44 & HN & 44 & N & -0.39 & 96 & HN & 96 & N & -0.50 \\
45 & HN & 45 & N & -2.75 & 98 & HN & 98 & N & -0.14 \\
46 & HN & 46 & N & -0.15 & 99 & HN & 99 & N & -0.83 \\
47 & HN & 47 & N & 6.87 & 100 & HN & 100 & N & 1.07 \\
48 & HN & 48 & N & 0.64 & 101 & HN & 101 & N & 0.78 \\
49 & HN & 49 & N & -0.25 & 102 & HN & 102 & N & -1.75 \\
50 & HN & 50 & N & 0.49 & 103 & HN & 103 & N & 2.70 \\
51 & HN & 51 & N & -0.52 & 104 & HN & 104 & N & -0.71 \\
53 & HN & 53 & N & -0.91 & 105 & HN & 105 & N & 0.35 \\
54 & HN & 54 & N & 2.33 & 106 & HN & 106 & N & 0.36 \\
56 & HN & 56 & N & 3.41 & 108 & HN & 108 & N & 0.64 \\
\hline \hline
\end{tabular}

\section{C.2 Distance restraints for CyIR2}

Automatically assigned intramonomer proton distances are listed in Table C.7 and intermonomer distances from PRE and NOE can be found in Table C.8 and C.9. respectively.

Table C.7: Intramolecular NOE distances of CylR2 which were automatically assigned using Cyana 2.0.

\begin{tabular}{|c|c|c|c|c|c|c|c|c|c|}
\hline Residue 1 & H1 & Residue 2 & $\mathrm{H} 2$ & distance $[\AA]$ & Residue 1 & H1 & Residue 2 & $\mathrm{H} 2$ & distance $[\AA]$ \\
\hline 43 & HN & 43 & 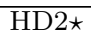 & 4.0 & 33 & HA2 & 36 & HB2 & 4.0 \\
\hline 36 & $\mathrm{HN}$ & 36 & HG1 & 4.0 & 47 & HB1 & 47 & HD1* & 4.0 \\
\hline 43 & $\mathrm{HD} 2 \star$ & 63 & $\mathrm{HH} 2$ & 4.0 & 47 & HB1 & 48 & HB1 & 4.0 \\
\hline 36 & $\mathrm{HA}$ & 36 & HG1 & 4.0 & 60 & $\mathrm{HB}$ & 61 & HB2 & 4.0 \\
\hline 36 & HA & 36 & $\mathrm{HG} 2$ & 4.0 & 10 & HA & 15 & $\mathrm{HB}$ & 4.0 \\
\hline 43 & HA & 43 & $\mathrm{HD} 2 \star$ & 4.0 & 6 & HB1 & 60 & $\mathrm{HG} 2 \star$ & 4.0 \\
\hline 43 & HB2 & 43 & HD2 & 4.0 & 53 & HB2 & 60 & HD1* & 4.0 \\
\hline 34 & HA & 38 & $\mathrm{HN}$ & 4.0 & 29 & HA & 32 & HB1 & 2.0 \\
\hline 14 & $\mathrm{HN}$ & 14 & HG2 & 4.0 & 30 & $\mathrm{HN}$ & 32 & HB1 & 4.0 \\
\hline 14 & $\mathrm{HN}$ & 14 & HG1 & 4.0 & 57 & HB2 & 58 & $\mathrm{HG} 2$ & 4.0 \\
\hline 13 & HN & 13 & HG2 & 4.0 & 58 & HA & 58 & HG2 & 4.0 \\
\hline 8 & $\mathrm{HN}$ & 8 & $\mathrm{HD} 2 \star$ & 4.0 & 9 & HB & 55 & $\mathrm{HG} 2 \star$ & 4.0 \\
\hline 34 & HA & 39 & $\mathrm{HN}$ & 4.0 & 62 & HG1 & 63 & HN & 4.0 \\
\hline 5 & HA & 8 & HD2 & 4.0 & 17 & $\mathrm{HN}$ & 17 & HG1 & 4.0 \\
\hline 33 & HA2 & 34 & $\mathrm{HA}$ & 4.0 & 49 & $\mathrm{HG} 2 \star$ & 49 & HG11 & 3.0 \\
\hline 34 & HA & 39 & HB1 & 4.0 & 36 & HB1 & 36 & HD2 & 2.0 \\
\hline 34 & HA & 34 & $\mathrm{HG} 2 \star$ & 4.0 & 41 & HB1 & 42 & $\mathrm{HN}$ & 4.0 \\
\hline 38 & $\mathrm{HN}$ & 38 & HG2 & 4.0 & 36 & HB2 & 38 & $\mathrm{HN}$ & 3.0 \\
\hline 38 & $\mathrm{HN}$ & 38 & $\mathrm{HG} 1$ & 4.0 & 62 & $\mathrm{HN}$ & 62 & HB2 & 4.0 \\
\hline 8 & HD2 $2 \star$ & 12 & $\mathrm{HN}$ & 4.0 & 63 & $\mathrm{HN}$ & 63 & HB1 & 3.0 \\
\hline 38 & HA & 38 & $\mathrm{HG} 2$ & 4.0 & 36 & $\mathrm{HN}$ & 36 & HB2 & 2.0 \\
\hline 38 & HA & 38 & HG1 & 4.0 & 45 & HG & 48 & HD2 & 4.0 \\
\hline 13 & HA & 14 & HG2 & 4.0 & 21 & $\mathrm{HN}$ & 31 & HG12 & 4.0 \\
\hline 8 & HB1 & 8 & HD2 & 3.0 & 53 & HN & 53 & $\mathrm{HD} 2 \star$ & 4.0 \\
\hline 23 & HB1 & 23 & HD1* & 4.0 & 43 & HD1ऋ & 57 & $\mathrm{HD} 2 \star$ & 4.0 \\
\hline 42 & HB2 & 43 & $\mathrm{HN}$ & 4.0 & 29 & HB2 & 30 & $\mathrm{HN}$ & 4.0 \\
\hline 42 & HB1 & 43 & HN & 4.0 & 31 & $\mathrm{HN}$ & 31 & HG12 & 4.0 \\
\hline 8 & HD1* & 10 & $\mathrm{HN}$ & 4.0 & 23 & $\mathrm{HN}$ & 23 & HG & 4.0 \\
\hline 8 & HD1* & 11 & HN & 4.0 & 12 & HN & 13 & HG2 & 4.0 \\
\hline 8 & HD1* & 9 & HN & 4.0 & 5 & HB1 & 60 & $\mathrm{HG} 2 \star$ & 4.0 \\
\hline 8 & HN & 8 & HD1* & 4.0 & 9 & $\mathrm{HG} 2 \star$ & 60 & $\mathrm{HG} 2 \star$ & 4.0 \\
\hline 8 & HA & 8 & HD1* & 4.0 & 2 & HD1* & 63 & HA & 2.0 \\
\hline 8 & HD1* & 12 & $\mathrm{HE} 2$ & 4.0 & 15 & HD1* & 16 & $\mathrm{HN}$ & 4.0 \\
\hline 8 & HD1* & 12 & HE1 & 4.0 & 3 & HD1* & 64 & $\mathrm{HN}$ & 4.0 \\
\hline 5 & HB2 & 8 & HD1* & 4.0 & 51 & $\mathrm{HN}$ & 52 & HD2 & 4.0 \\
\hline 8 & HD1* & 11 & HB1 & 4.0 & 34 & $\mathrm{HN}$ & 39 & HD1 & 4.0 \\
\hline 8 & HB2 & 8 & HD1* & 4.0 & 34 & $\mathrm{HN}$ & 39 & HD2 & 4.0 \\
\hline 45 & HB2 & 45 & $\mathrm{HD} 2 \star$ & 3.0 & 38 & $\mathrm{HN}$ & 39 & HD1 & 4.0 \\
\hline 5 & HN & 60 & HA & 4.0 & 39 & HD1 & 40 & $\mathrm{HN}$ & 4.0 \\
\hline
\end{tabular}




\begin{tabular}{|c|c|c|c|c|c|c|c|c|c|}
\hline Residue 1 & H1 & Residue 2 & $\mathrm{H} 2$ & distance $[\check{A}]$ & Residue 1 & H1 & Residue 2 & $\mathrm{H} 2$ & distance $[\AA]$ \\
\hline 24 & 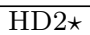 & 49 & $\mathrm{HN}$ & 4.0 & 39 & HD2 & 40 & $\mathrm{HN}$ & 4.0 \\
\hline 45 & HD2 $2 \star$ & 46 & $\mathrm{HN}$ & 4.0 & 49 & HA & 52 & HE2 & 4.0 \\
\hline 6 & HD2* & 9 & $\mathrm{HN}$ & 4.0 & 58 & HG2 & 63 & HZ2 & 4.0 \\
\hline 45 & $\mathrm{HN}$ & 45 & $\mathrm{HD} 2 \star$ & 4.0 & 3 & HG12 & 4 & $\mathrm{HN}$ & 4.0 \\
\hline 24 & $\mathrm{HN}$ & 24 & $\mathrm{HD} 2 \star$ & 4.0 & 2 & HA & 3 & HA & 4.0 \\
\hline 24 & HA & 24 & $\mathrm{HD} 2 \star$ & 3.0 & 3 & HA & 4 & HA & 4.0 \\
\hline 6 & HA & 60 & HA & 4.0 & 24 & HG & 49 & HA & 4.0 \\
\hline 6 & HA & 6 & HD2 & 3.0 & 51 & HE1 & 52 & HA & 4.0 \\
\hline 24 & HD2 $2 \star$ & 49 & HA & 4.0 & 50 & $\mathrm{HB} \star$ & 51 & HD1 & 4.0 \\
\hline 5 & HB1 & 60 & HA & 4.0 & 24 & $\mathrm{HD} 2 \star$ & 51 & HD1 & 4.0 \\
\hline 5 & HB2 & 60 & HA & 4.0 & 60 & $\mathrm{HN}$ & 61 & HD2 & 4.0 \\
\hline 24 & HD2 & 48 & HE1 & 4.0 & 51 & HD1 & 52 & HD2 & 4.0 \\
\hline 24 & $\mathrm{HD} 2 \star$ & 48 & $\mathrm{HE} 2$ & 4.0 & 30 & HB & 31 & $\mathrm{HN}$ & 4.0 \\
\hline 24 & $\mathrm{HD} 2 \star$ & 49 & HG12 & 3.0 & 30 & HB & 31 & HB & 4.0 \\
\hline 6 & HD2 & 9 & HB & 3.0 & 30 & $\mathrm{HN}$ & 30 & HG $2 \star$ & 4.0 \\
\hline 55 & HG $2 \star$ & 60 & HA & 4.0 & 24 & $\mathrm{HN}$ & 26 & HG1ऋ & 4.0 \\
\hline 60 & HA & 60 & HD1* & 4.0 & 20 & HB2 & 20 & HG & 2.0 \\
\hline 24 & $\mathrm{HD} 2 \star$ & 48 & HG1 & 3.0 & 48 & HG1 & 49 & HG12 & 4.0 \\
\hline 24 & HD2* & 48 & HG2 & 4.0 & 60 & HG11 & 61 & HN & 4.0 \\
\hline 53 & HN & 53 & $\mathrm{HG}$ & 4.0 & 2 & HG12 & 3 & $\mathrm{HN}$ & 4.0 \\
\hline 48 & HG1 & 49 & HA & 4.0 & 59 & $\mathrm{HN}$ & 60 & HG11 & 4.0 \\
\hline 47 & HG & 48 & $\mathrm{HN}$ & 4.0 & 50 & HA & 60 & HG11 & 4.0 \\
\hline 53 & HA & 53 & $\mathrm{HG}$ & 3.0 & 56 & HD2 & 60 & HG11 & 4.0 \\
\hline 44 & HA & 47 & HG & 4.0 & 57 & HA & 60 & HG11 & 4.0 \\
\hline 43 & $\mathrm{HN}$ & 43 & HD1* & 4.0 & 2 & HG12 & 63 & HB2 & 4.0 \\
\hline 9 & HA & 12 & $\mathrm{HN}$ & 4.0 & 43 & $\mathrm{HD} 2 \star$ & 57 & HD1* & 4.0 \\
\hline 52 & HA & 52 & HD1 & 4.0 & 48 & HA & 52 & HD2 & 4.0 \\
\hline 43 & HA & 43 & HD1* & 3.0 & 47 & HB1 & 48 & HE1 & 4.0 \\
\hline 43 & HB2 & 43 & HD1* & 4.0 & 48 & HE1 & 49 & $\mathrm{HN}$ & 4.0 \\
\hline 23 & HD1* & 52 & HA & 4.0 & 50 & $\mathrm{HB} \star$ & 51 & HB2 & 4.0 \\
\hline 23 & HD2 2 & 52 & HA & 4.0 & 50 & $\mathrm{HB} \star$ & 51 & HB1 & 4.0 \\
\hline 9 & HA & 9 & HG11 & 2.0 & 56 & HB2 & 58 & HG1 & 3.0 \\
\hline 20 & $\mathrm{HN}$ & 20 & $\mathrm{HG}$ & 4.0 & 43 & $\mathrm{HD} 2 \star$ & 61 & HB2 & 4.0 \\
\hline 20 & HA & 20 & HG & 4.0 & 63 & HB2 & 64 & $\mathrm{HN}$ & 4.0 \\
\hline 23 & HB1 & 23 & HD2 & 4.0 & 63 & HB1 & 64 & $\mathrm{HN}$ & 4.0 \\
\hline 20 & HG & 21 & $\mathrm{HN}$ & 4.0 & 63 & HB2 & 64 & HB2 & 4.0 \\
\hline 50 & $\mathrm{HB} \star$ & 51 & HA & 4.0 & 2 & HG11 & 63 & HB2 & 4.0 \\
\hline 31 & HA & 31 & $\mathrm{HG} 2 \star$ & 3.0 & 63 & HB1 & 63 & HZ3 & 4.0 \\
\hline 26 & $\mathrm{HN}$ & 26 & HG1* & 4.0 & 62 & HN & 63 & HB1 & 4.0 \\
\hline 51 & HA & 51 & HD2 & 4.0 & 57 & HB2 & 63 & HZ2 & 4.0 \\
\hline 26 & HA & 26 & HG1ऋ & 4.0 & 52 & $\mathrm{HN}$ & 52 & HE2 & 4.0 \\
\hline 24 & HB2 & 26 & 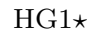 & 4.0 & 63 & $\mathrm{HN}$ & 63 & HE3 & 4.0 \\
\hline 24 & HB1 & 26 & HG1* & 4.0 & 33 & HA1 & 39 & HD1 & 4.0 \\
\hline 10 & HA & 12 & $\mathrm{HN}$ & 4.0 & 11 & HB1 & 11 & HG2 & 3.0 \\
\hline 17 & HA & 20 & HN & 4.0 & 40 & HA & 41 & HB1 & 4.0 \\
\hline 3 & $\mathrm{HA}$ & 4 & $\mathrm{HN}$ & 3.0 & 41 & HD2 & 42 & $\mathrm{HN}$ & 4.0 \\
\hline 3 & HA & 4 & HB2 & 4.0 & 41 & HD1 & 42 & $\mathrm{HN}$ & 4.0 \\
\hline 48 & HA & 52 & $\mathrm{HN}$ & 4.0 & 61 & HB2 & 63 & $\mathrm{HH} 2$ & 4.0 \\
\hline 48 & HA & 51 & $\mathrm{HN}$ & 4.0 & 48 & $\mathrm{HN}$ & 48 & HG2 & 4.0 \\
\hline 55 & HG $2 \star$ & 60 & $\mathrm{HN}$ & 4.0 & 2 & 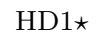 & 63 & $\mathrm{HN}$ & 4.0 \\
\hline 48 & HA & 52 & HE2 & 4.0 & 2 & HA & 64 & $\mathrm{HN}$ & 4.0 \\
\hline 4 & HD21 & 35 & HA & 4.0 & 2 & HA & 63 & HA & 3.0 \\
\hline 48 & HA & 51 & HD1 & 4.0 & 1 & HA & 2 & HG12 & 4.0 \\
\hline 55 & HA & 55 & HG $2 \star$ & 4.0 & 2 & $\mathrm{HG} 2 \star$ & 63 & HA & 4.0 \\
\hline 55 & HG $2 \star$ & 59 & HA & 4.0 & 3 & HA & 4 & HB1 & 4.0 \\
\hline 48 & HA & 51 & HB1 & 4.0 & 3 & $\mathrm{HB}$ & 4 & HA & 4.0 \\
\hline 55 & HG $2 \star$ & 59 & HB1 & 4.0 & 2 & $\mathrm{HN}$ & 3 & HG11 & 4.0 \\
\hline 55 & HG $2 \star$ & 60 & HG11 & 4.0 & 2 & HA & 3 & HG11 & 4.0 \\
\hline 48 & HA & 48 & HB1 & 2.0 & 3 & HD1* & 63 & HA & 4.0 \\
\hline 57 & HA & 57 & HG & 4.0 & 4 & HA & 62 & $\mathrm{HN}$ & 4.0 \\
\hline 57 & HA & 60 & HD1* & 4.0 & 4 & HA & 4 & HD21 & 4.0 \\
\hline 55 & HG $2 \star$ & 60 & HD1* & 4.0 & 4 & HA & 60 & HA & 4.0 \\
\hline 55 & HG $2 \star$ & 60 & HG $2 \star$ & 4.0 & 41 & HB2 & 61 & HE1 & 4.0 \\
\hline 48 & HA & 48 & HG2 & 4.0 & 63 & HA & 63 & HE3 & 4.0 \\
\hline 7 & $\mathrm{HN}$ & 35 & HA & 4.0 & 2 & HG11 & 63 & HA & 4.0 \\
\hline 21 & $\mathrm{HN}$ & 28 & HA & 4.0 & 63 & HA & 64 & HA & 4.0 \\
\hline
\end{tabular}




\begin{tabular}{|c|c|c|c|c|c|c|c|c|c|}
\hline Residue 1 & $\mathrm{H} 1$ & Residue 2 & $\mathrm{H} 2$ & distance $[\AA]$ & Residue 1 & $\mathrm{H} 1$ & Residue 2 & $\mathrm{H} 2$ & distance $[\AA]$ \\
\hline 28 & HA & 31 & $\mathrm{HN}$ & 4.0 & 2 & HA & 63 & HB1 & 4.0 \\
\hline 2 & HA & 2 & HG11 & 4.0 & 62 & HA & 63 & HB1 & 4.0 \\
\hline 43 & $\mathrm{HN}$ & 46 & $\mathrm{HB} \star$ & 4.0 & 2 & HA & 63 & HB2 & 4.0 \\
\hline 29 & HA & 32 & $\mathrm{HN}$ & 4.0 & 63 & HA & 64 & HB2 & 4.0 \\
\hline 45 & $\mathrm{HN}$ & 46 & $\mathrm{HB} \star$ & 4.0 & 3 & $\mathrm{HN}$ & 64 & HG2 & 4.0 \\
\hline 46 & $\mathrm{HB} \star$ & 61 & HE2 & 4.0 & 3 & $\mathrm{HN}$ & 64 & HG1 & 4.0 \\
\hline 46 & $\mathrm{HB} \star$ & 61 & HD2 & 4.0 & 43 & HA & 46 & $\mathrm{HN}$ & 4.0 \\
\hline 11 & HA & 11 & HG2 & 4.0 & 43 & HG & 44 & $\mathrm{HN}$ & 4.0 \\
\hline 46 & $\mathrm{HB} \star$ & 57 & HD1* & 4.0 & 31 & HG12 & 32 & HA & 4.0 \\
\hline 45 & HB1 & 46 & $\mathrm{HB} \star$ & 4.0 & 13 & HG2 & 14 & HN & 4.0 \\
\hline 46 & $\mathrm{HB} \star$ & 60 & HB & 4.0 & 13 & HG1 & 14 & $\mathrm{HN}$ & 4.0 \\
\hline 45 & HB2 & 46 & $\mathrm{HB} \star$ & 4.0 & 10 & HA & 13 & HG1 & 4.0 \\
\hline 43 & HD1* & 46 & $\mathrm{HB} \star$ & 3.0 & 5 & HA & 8 & HD1* & 4.0 \\
\hline 43 & HD2 & 46 & $\mathrm{HB} \star$ & 4.0 & 8 & HD1* & 9 & HA & 4.0 \\
\hline 29 & HA & 31 & $\mathrm{HN}$ & 4.0 & 43 & HB1 & 43 & HD1* & 4.0 \\
\hline 29 & HA & 30 & HA & 4.0 & 9 & HA & 11 & HN & 4.0 \\
\hline 19 & HA & 22 & $\mathrm{HN}$ & 4.0 & 30 & HA & 31 & HA & 4.0 \\
\hline 49 & HD1* & 61 & HE2 & 4.0 & 31 & HA & 34 & HA & 4.0 \\
\hline 49 & HA & 49 & HD1* & 4.0 & 21 & $\mathrm{HB} \star$ & 31 & HA & 4.0 \\
\hline 49 & HB & 49 & HD1* & 4.0 & 10 & HA & 13 & $\mathrm{HN}$ & 4.0 \\
\hline 45 & HB1 & 49 & HD1* & 3.0 & 35 & $\mathrm{HN}$ & 35 & HA & 3.0 \\
\hline 19 & HA & 22 & $\mathrm{HB} \star$ & 4.0 & 15 & HA & 15 & HD1* & 4.0 \\
\hline 49 & HD1* & 60 & $\mathrm{HG} 2 \star$ & 4.0 & 31 & HG $2 \star$ & 32 & HB2 & 3.0 \\
\hline 43 & HA & 46 & $\mathrm{HB} \star$ & 4.0 & 47 & HB1 & 48 & HN & 4.0 \\
\hline 5 & $\mathrm{HN}$ & 60 & 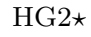 & 4.0 & 47 & HB2 & 48 & $\mathrm{HN}$ & 4.0 \\
\hline 21 & $\mathrm{HN}$ & 26 & $\mathrm{HG} 2 \star$ & 4.0 & 39 & $\mathrm{HN}$ & 39 & HB1 & 3.0 \\
\hline 60 & HG $2 \star$ & 61 & $\mathrm{HN}$ & 4.0 & 39 & $\mathrm{HN}$ & 39 & HB2 & 3.0 \\
\hline 60 & $\mathrm{HN}$ & 60 & $\mathrm{HG} 2 \star$ & 4.0 & 56 & HB1 & 59 & HN & 4.0 \\
\hline 24 & $\mathrm{HN}$ & 26 & HG2 & 4.0 & 3 & HN & 3 & HB & 4.0 \\
\hline 60 & HG $2 \star$ & 61 & HE2 & 4.0 & 2 & HA & 3 & $\mathrm{HN}$ & 4.0 \\
\hline 60 & HG $2 \star$ & 61 & HD2 & 4.0 & 57 & $\mathrm{HN}$ & 57 & HB2 & 4.0 \\
\hline 26 & HG $2 \star$ & 31 & $\mathrm{HN}$ & 4.0 & 57 & $\mathrm{HN}$ & 58 & $\mathrm{HN}$ & 4.0 \\
\hline 4 & HA & 60 & $\mathrm{HG} 2 \star$ & 4.0 & 3 & $\mathrm{HN}$ & 64 & $\mathrm{HN}$ & 4.0 \\
\hline 60 & HG $2 \star$ & 61 & HA & 4.0 & 41 & HA & 42 & $\mathrm{HN}$ & 3.0 \\
\hline 26 & HA & 26 & HG $2 \star$ & 4.0 & 41 & HB2 & 42 & $\mathrm{HN}$ & 3.0 \\
\hline 26 & HG $2 \star$ & 30 & HA & 4.0 & 2 & 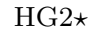 & 63 & $\mathrm{HN}$ & 4.0 \\
\hline 25 & HA & 26 & $\mathrm{HG} 2 \star$ & 4.0 & 3 & HB & 63 & $\mathrm{HN}$ & 4.0 \\
\hline 46 & HA & 60 & $\mathrm{HG} 2 \star$ & 4.0 & 18 & $\mathrm{HN}$ & 19 & HN & 4.0 \\
\hline 6 & HA & 60 & 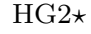 & 3.0 & 16 & HA & 19 & HN & 4.0 \\
\hline 60 & HA & 60 & $\mathrm{HG} 2 \star$ & 4.0 & 15 & HG $2 \star$ & 19 & $\mathrm{HN}$ & 4.0 \\
\hline 5 & HB2 & 60 & $\mathrm{HG} 2 \star$ & 4.0 & 3 & $\mathrm{HN}$ & 4 & $\mathrm{HN}$ & 4.0 \\
\hline 6 & HD2 & 60 & $\mathrm{HG} 2 \star$ & 3.0 & 4 & $\mathrm{HN}$ & 5 & HN & 4.0 \\
\hline 60 & HG $2 \star$ & 60 & HG12 & 4.0 & 4 & $\mathrm{HN}$ & 4 & HD22 & 4.0 \\
\hline 50 & $\mathrm{HB} \star$ & 57 & $\mathrm{HN}$ & 4.0 & 2 & $\mathrm{HN}$ & 2 & HG12 & 4.0 \\
\hline 49 & $\mathrm{HN}$ & 50 & $\mathrm{HB} \star$ & 4.0 & 34 & $\mathrm{HN}$ & 36 & HN & 4.0 \\
\hline 34 & $\mathrm{HG} 2 \star$ & 35 & $\mathrm{HN}$ & 4.0 & 11 & $\mathrm{HN}$ & 12 & HN & 3.0 \\
\hline 31 & HG $2 \star$ & 32 & $\mathrm{HN}$ & 4.0 & 34 & HN & 35 & $\mathrm{HN}$ & 4.0 \\
\hline 34 & HG $2 \star$ & 37 & $\mathrm{HN}$ & 4.0 & 31 & HA & 34 & HN & 4.0 \\
\hline 9 & $\mathrm{HN}$ & 9 & HG2 & 4.0 & 8 & HD1* & 12 & HN & 4.0 \\
\hline 17 & HE21 & 31 & 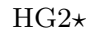 & 4.0 & 9 & HG2 & 12 & $\mathrm{HN}$ & 4.0 \\
\hline 9 & HG $2 \star$ & 13 & $\mathrm{HN}$ & 4.0 & 12 & HN & 12 & HA & 3.0 \\
\hline 17 & HE22 & 31 & $\mathrm{HG} 2 \star$ & 4.0 & 34 & $\mathrm{HN}$ & 34 & HG $2 \star$ & 4.0 \\
\hline 34 & HG $2 \star$ & 61 & HE1 & 4.0 & 12 & $\mathrm{HN}$ & 13 & HN & 2.0 \\
\hline 31 & $\mathrm{HN}$ & 31 & $\mathrm{HG} 2 \star$ & 4.0 & 5 & $\mathrm{HN}$ & 6 & $\mathrm{HN}$ & 4.0 \\
\hline 34 & HG $2 \star$ & 61 & $\mathrm{HZ}$ & 4.0 & 4 & HA & 5 & $\mathrm{HN}$ & 3.0 \\
\hline 4 & HD21 & 34 & 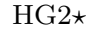 & 4.0 & 4 & HB1 & 5 & $\mathrm{HN}$ & 4.0 \\
\hline 34 & HG $2 \star$ & 40 & HA & 4.0 & 43 & $\mathrm{HN}$ & 43 & HB2 & 4.0 \\
\hline 50 & $\mathrm{HB} \star$ & 56 & HA & 4.0 & 43 & $\mathrm{HN}$ & 43 & HB1 & 4.0 \\
\hline 31 & $\mathrm{HG} 2 \star$ & 32 & HA & 4.0 & 6 & $\mathrm{HN}$ & 7 & HN & 4.0 \\
\hline 9 & HA & 9 & 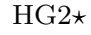 & 2.0 & 35 & $\mathrm{HN}$ & 37 & HN & 4.0 \\
\hline 17 & HA & 31 & $\mathrm{HG} 2 \star$ & 3.0 & 35 & HN & 35 & HG2 & 4.0 \\
\hline 28 & HA & 31 & $\mathrm{HG} 2 \star$ & 3.0 & 48 & HN & 48 & HB1 & 4.0 \\
\hline 48 & HA & 50 & $\mathrm{HB} \star$ & 4.0 & 6 & $\mathrm{HN}$ & 6 & HD1* & 4.0 \\
\hline 31 & $\mathrm{HG} 2 \star$ & 32 & HB1 & 4.0 & 48 & $\mathrm{HN}$ & 48 & HG1 & 4.0 \\
\hline 8 & HA & 11 & HG2 & 4.0 & 48 & HA & 50 & $\mathrm{HN}$ & 4.0 \\
\hline 34 & HG $2 \star$ & 41 & HB1 & 3.0 & 49 & HB & 50 & HN & 3.0 \\
\hline
\end{tabular}




\begin{tabular}{|c|c|c|c|c|c|c|c|c|c|}
\hline Residue 1 & H1 & Residue 2 & $\mathrm{H} 2$ & distance $[\check{A}]$ & Residue 1 & H1 & Residue 2 & $\mathrm{H} 2$ & distance $[\AA]$ \\
\hline 9 & 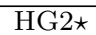 & 9 & HG12 & 2.0 & 6 & $\mathrm{HN}$ & 6 & HB1 & 2.0 \\
\hline 9 & $\mathrm{HG} 2 \star$ & 55 & $\mathrm{HG} 2 \star$ & 3.0 & 45 & $\mathrm{HG}$ & 46 & $\mathrm{HN}$ & 4.0 \\
\hline 50 & $\mathrm{HB} \star$ & 60 & HD1* & 3.0 & 46 & HN & 47 & HB1 & 4.0 \\
\hline 31 & $\mathrm{HG} 2 \star$ & 31 & HD1* & 3.0 & 46 & $\mathrm{HN}$ & 46 & $\mathrm{HB} \star$ & 4.0 \\
\hline 34 & $\mathrm{HG} 2 \star$ & 60 & $\mathrm{HG} 2 \star$ & 4.0 & 50 & $\mathrm{HN}$ & 50 & $\mathrm{HB} \star$ & 3.0 \\
\hline 8 & HA & 11 & $\mathrm{HN}$ & 4.0 & 6 & $\mathrm{HN}$ & 9 & 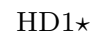 & 4.0 \\
\hline 3 & $\mathrm{HG} 2 \star$ & 62 & $\mathrm{HN}$ & 4.0 & 16 & HA & 17 & $\mathrm{HN}$ & 4.0 \\
\hline 21 & $\mathrm{HN}$ & 22 & $\mathrm{HB} \star$ & 4.0 & 49 & $\mathrm{HN}$ & 49 & HD1ᄎ & 4.0 \\
\hline 20 & $\mathrm{HN}$ & 22 & $\mathrm{HB} \star$ & 4.0 & 48 & $\mathrm{HN}$ & 49 & HN & 4.0 \\
\hline 22 & $\mathrm{HB} \star$ & 25 & $\mathrm{HN}$ & 4.0 & 45 & HA & 49 & $\mathrm{HN}$ & 4.0 \\
\hline 22 & $\mathrm{HN}$ & 22 & $\mathrm{HB} \star$ & 3.0 & 49 & $\mathrm{HN}$ & 49 & HB & 4.0 \\
\hline 47 & HA & 47 & HG & 2.0 & 49 & $\mathrm{HN}$ & 49 & HG12 & 4.0 \\
\hline 8 & HA & 8 & $\mathrm{HD} 2 \star$ & 2.0 & 10 & $\mathrm{HN}$ & 10 & HB2 & 4.0 \\
\hline 45 & HA & 45 & $\mathrm{HD} 2 \star$ & 4.0 & 9 & HB & 10 & $\mathrm{HN}$ & 4.0 \\
\hline 15 & $\mathrm{HG} 2 \star$ & 22 & $\mathrm{HB} \star$ & 4.0 & 10 & $\mathrm{HN}$ & 10 & HB1 & 4.0 \\
\hline 3 & $\mathrm{HN}$ & 3 & $\mathrm{HG} 2 \star$ & 4.0 & 49 & $\mathrm{HN}$ & 49 & HG11 & 4.0 \\
\hline 3 & $\mathrm{HG} 2 \star$ & 5 & HN & 4.0 & 6 & $\mathrm{HD} 2 \star$ & 10 & HN & 4.0 \\
\hline 3 & $\mathrm{HG} 2 \star$ & 4 & $\mathrm{HN}$ & 4.0 & 9 & $\mathrm{HG} 2 \star$ & 10 & $\mathrm{HN}$ & 4.0 \\
\hline 3 & $\mathrm{HG} 2 \star$ & 5 & HD21 & 4.0 & 46 & $\mathrm{HB} \star$ & 49 & HN & 4.0 \\
\hline 3 & $\mathrm{HG} 2 \star$ & 5 & HD22 & 4.0 & 30 & $\mathrm{HN}$ & 31 & $\mathrm{HN}$ & 4.0 \\
\hline 3 & $\mathrm{HG} 2 \star$ & 4 & $\mathrm{HA}$ & 4.0 & 30 & $\mathrm{HN}$ & 30 & HB & 4.0 \\
\hline 2 & HA & 3 & HG $2 \star$ & 4.0 & 21 & $\mathrm{HN}$ & 22 & $\mathrm{HN}$ & 4.0 \\
\hline 3 & HA & 3 & HG $2 \star$ & 4.0 & 21 & $\mathrm{HN}$ & 31 & 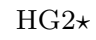 & 4.0 \\
\hline 20 & HA & 20 & 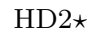 & 4.0 & 20 & $\mathrm{HN}$ & 21 & HN & 2.0 \\
\hline 2 & $\mathrm{HG} 2 \star$ & 3 & $\mathrm{HN}$ & 3.0 & 20 & $\mathrm{HN}$ & 20 & HB2 & 2.0 \\
\hline 2 & $\mathrm{HN}$ & 2 & HG $2 \star$ & 4.0 & 20 & $\mathrm{HN}$ & 20 & HB1 & 4.0 \\
\hline 2 & $\mathrm{HG} 2 \star$ & 62 & $\mathrm{HN}$ & 4.0 & 60 & $\mathrm{HN}$ & 61 & $\mathrm{HN}$ & 4.0 \\
\hline 32 & HA & 34 & $\mathrm{HN}$ & 4.0 & 60 & $\mathrm{HN}$ & 60 & HG12 & 3.0 \\
\hline 20 & $\mathrm{HN}$ & 21 & $\mathrm{HB} \star$ & 4.0 & 44 & HB2 & 45 & HN & 4.0 \\
\hline 15 & $\mathrm{HG} 2 \star$ & 20 & HN & 4.0 & 44 & HB1 & 45 & $\mathrm{HN}$ & 4.0 \\
\hline 2 & $\mathrm{HG} 2 \star$ & 64 & $\mathrm{HN}$ & 4.0 & 45 & HN & 45 & HB1 & 4.0 \\
\hline 14 & HA & 15 & $\mathrm{HN}$ & 3.0 & 45 & $\mathrm{HN}$ & 45 & $\mathrm{HG}$ & 4.0 \\
\hline 15 & $\mathrm{HN}$ & 15 & HG $2 \star$ & 4.0 & 45 & $\mathrm{HN}$ & 45 & HD1* & 4.0 \\
\hline 2 & $\mathrm{HG} 2 \star$ & 61 & HD1 & 4.0 & 8 & HB1 & 9 & HN & 2.0 \\
\hline 2 & HA & 2 & $\mathrm{HG} 2 \star$ & 4.0 & 31 & $\mathrm{HN}$ & 31 & HG11 & 4.0 \\
\hline 15 & HA & 15 & $\mathrm{HG} 2 \star$ & 4.0 & 9 & $\mathrm{HN}$ & 10 & HN & 4.0 \\
\hline 22 & HA & 25 & $\mathrm{HA}$ & 4.0 & 9 & $\mathrm{HN}$ & 9 & HG12 & 4.0 \\
\hline 15 & $\mathrm{HG} 2 \star$ & 19 & HA & 4.0 & 30 & $\mathrm{HG} 2 \star$ & 31 & $\mathrm{HN}$ & 4.0 \\
\hline 2 & $\mathrm{HG} 2 \star$ & 61 & HB1 & 4.0 & 9 & $\mathrm{HN}$ & 9 & HD1* & 3.0 \\
\hline 15 & $\mathrm{HG} 2 \star$ & 19 & HB2 & 3.0 & 52 & $\mathrm{HN}$ & 52 & HD2 & 4.0 \\
\hline 15 & $\mathrm{HG} 2 \star$ & 19 & HB1 & 3.0 & 51 & $\mathrm{HN}$ & 52 & $\mathrm{HN}$ & 3.0 \\
\hline 3 & $\mathrm{HN}$ & 63 & HA & 4.0 & 51 & HD1 & 52 & $\mathrm{HN}$ & 4.0 \\
\hline 18 & $\mathrm{HN}$ & 21 & $\mathrm{HB} \star$ & 4.0 & 51 & HB2 & 52 & $\mathrm{HN}$ & 4.0 \\
\hline 21 & $\mathrm{HN}$ & 21 & $\mathrm{HB} \star$ & 3.0 & 31 & $\mathrm{HN}$ & 32 & $\mathrm{HN}$ & 4.0 \\
\hline 63 & HA & 64 & HN & 3.0 & 32 & $\mathrm{HN}$ & 32 & HB1 & 2.0 \\
\hline 21 & $\mathrm{HB} \star$ & 22 & $\mathrm{HN}$ & 2.0 & 7 & $\mathrm{HN}$ & 8 & HN & 4.0 \\
\hline 21 & $\mathrm{HB} \star$ & 31 & $\mathrm{HN}$ & 4.0 & 6 & HG & 7 & $\mathrm{HN}$ & 4.0 \\
\hline 63 & HA & 63 & HD1 & 4.0 & 6 & HB2 & 7 & $\mathrm{HN}$ & 4.0 \\
\hline 37 & HA & 38 & HA & 4.0 & 59 & $\mathrm{HN}$ & 60 & $\mathrm{HN}$ & 4.0 \\
\hline 21 & $\mathrm{HB} \star$ & 31 & HB & 3.0 & 22 & $\mathrm{HN}$ & 23 & $\mathrm{HN}$ & 4.0 \\
\hline 21 & $\mathrm{HB} \star$ & 26 & HG $2 \star$ & 3.0 & 56 & HA & 59 & $\mathrm{HN}$ & 4.0 \\
\hline 5 & $\mathrm{HN}$ & 61 & HA & 4.0 & 59 & $\mathrm{HN}$ & 59 & HB2 & 4.0 \\
\hline 36 & HA & 38 & $\mathrm{HN}$ & 4.0 & 59 & $\mathrm{HN}$ & 59 & HB1 & 4.0 \\
\hline 50 & HA & 55 & $\mathrm{HN}$ & 4.0 & 55 & HG $2 \star$ & 59 & HN & 4.0 \\
\hline 61 & HA & 61 & HD1 & 4.0 & 58 & $\mathrm{HN}$ & 59 & HN & 4.0 \\
\hline 61 & HA & 61 & HD2 & 4.0 & 51 & $\mathrm{HN}$ & 51 & HB1 & 2.0 \\
\hline 46 & HA & 61 & HE2 & 4.0 & 58 & $\mathrm{HN}$ & 58 & $\mathrm{HG} 2$ & 3.0 \\
\hline 46 & HA & 49 & HB & 4.0 & 58 & $\mathrm{HN}$ & 58 & HG1 & 4.0 \\
\hline 36 & HA & 36 & HD1 & 4.0 & 57 & HB2 & 58 & $\mathrm{HN}$ & 4.0 \\
\hline 2 & $\mathrm{HG} 2 \star$ & 61 & HA & 4.0 & 50 & $\mathrm{HB} \star$ & 51 & $\mathrm{HN}$ & 4.0 \\
\hline 21 & HA & 26 & $\mathrm{HN}$ & 4.0 & 51 & $\mathrm{HN}$ & 51 & HB2 & 3.0 \\
\hline 21 & HA & 24 & $\mathrm{HN}$ & 4.0 & 38 & HB2 & 39 & $\mathrm{HN}$ & 4.0 \\
\hline 50 & HA & 55 & HB & 4.0 & 57 & $\mathrm{HD} 2 \star$ & 58 & $\mathrm{HN}$ & 3.0 \\
\hline 13 & HA & 13 & HG2 & 3.0 & 61 & HA & 62 & $\mathrm{HN}$ & 3.0 \\
\hline 21 & HA & 26 & HG $2 \star$ & 3.0 & 61 & HB1 & 62 & HN & 4.0 \\
\hline 1 & HA & 2 & HA & 4.0 & 13 & $\mathrm{HN}$ & 14 & HN & 4.0 \\
\hline
\end{tabular}




\begin{tabular}{|c|c|c|c|c|c|c|c|c|c|}
\hline Residue 1 & H1 & Residue 2 & $\mathrm{H} 2$ & distance $[\AA]$ & Residue 1 & H1 & Residue 2 & $\mathrm{H} 2$ & 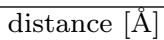 \\
\hline 13 & HA & 14 & HG1 & 4.0 & 13 & HN & 13 & HG1 & 4.0 \\
\hline 9 & HG2 $2 \star$ & 13 & HA & 4.0 & 8 & HN & 9 & HN & 4.0 \\
\hline 60 & $\mathrm{HN}$ & 60 & HD1* & 4.0 & 11 & HA & 13 & $\mathrm{HN}$ & 4.0 \\
\hline 60 & HD1* & 61 & HE2 & 4.0 & 13 & $\mathrm{HN}$ & 13 & HB2 & 4.0 \\
\hline 50 & HA & 60 & HD1* & 3.0 & 8 & $\mathrm{HN}$ & 8 & HB1 & 3.0 \\
\hline 34 & HD1 & 41 & HB2 & 4.0 & 8 & HN & 8 & HB2 & 3.0 \\
\hline 60 & $\mathrm{HG} 2 \star$ & 60 & HD1* & 4.0 & 61 & $\mathrm{HN}$ & 61 & HD2 & 4.0 \\
\hline 34 & HD1* & 35 & $\mathrm{HN}$ & 4.0 & 11 & $\mathrm{HN}$ & 11 & HG1 & 2.0 \\
\hline 51 & HN & 51 & HD1 & 4.0 & 60 & HB & 61 & HN & 4.0 \\
\hline 34 & HD1* & 39 & HD2 & 4.0 & 47 & $\mathrm{HN}$ & 47 & HB1 & 4.0 \\
\hline 34 & HD1* & 39 & HA & 4.0 & 10 & HN & 11 & HN & 4.0 \\
\hline 51 & HD1 & 52 & HA & 4.0 & 47 & $\mathrm{HN}$ & 48 & $\mathrm{HN}$ & 4.0 \\
\hline 34 & HA & 34 & HD1* & 4.0 & 61 & $\mathrm{HN}$ & 61 & HB2 & 3.0 \\
\hline 6 & HA & 60 & HD1* & 4.0 & 47 & $\mathrm{HN}$ & 47 & HB2 & 3.0 \\
\hline 31 & HA & 34 & HD1* & 4.0 & 47 & HN & 47 & HD $2 \star$ & 4.0 \\
\hline 34 & 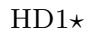 & 41 & HB1 & 3.0 & 46 & $\mathrm{HB} \star$ & 47 & HN & 4.0 \\
\hline 23 & HG & 52 & HD1 & 4.0 & 47 & $\mathrm{HN}$ & 47 & HD1 $\star$ & 4.0 \\
\hline 24 & $\mathrm{HD} 2 \star$ & 52 & HD2 & 4.0 & 36 & $\mathrm{HN}$ & 37 & HN & 4.0 \\
\hline 48 & HG1 & 52 & HD2 & 4.0 & 36 & $\mathrm{HN}$ & 36 & HD2 & 4.0 \\
\hline 54 & HA & 55 & $\mathrm{HN}$ & 3.0 & 47 & $\mathrm{HN}$ & 57 & $\mathrm{HG}$ & 4.0 \\
\hline 54 & HA & 54 & HD21 & 4.0 & 36 & $\mathrm{HN}$ & 36 & HG2 & 2.0 \\
\hline 39 & HA & 39 & HD1 & 4.0 & 43 & $\mathrm{HN}$ & 44 & $\mathrm{HN}$ & 3.0 \\
\hline 33 & HA2 & 39 & HD1 & 4.0 & 22 & HN & 25 & HN & 4.0 \\
\hline 2 & HB & 61 & HD1 & 4.0 & 44 & HN & 46 & $\mathrm{HB} \star$ & 4.0 \\
\hline 41 & HB2 & 61 & HD1 & 4.0 & 44 & $\mathrm{HN}$ & 45 & $\mathrm{HN}$ & 4.0 \\
\hline 39 & HD2 & 45 & $\mathrm{HG}$ & 4.0 & 34 & HB & 36 & $\mathrm{HN}$ & 4.0 \\
\hline 30 & HG2 $2 \star$ & 39 & HD2 & 4.0 & 43 & $\mathrm{HD} 2 \star$ & 44 & $\mathrm{HN}$ & 4.0 \\
\hline 49 & HN & 49 & HG $2 \star$ & 4.0 & 52 & HN & 53 & $\mathrm{HN}$ & 4.0 \\
\hline 49 & HG $2 \star$ & 50 & $\mathrm{HN}$ & 4.0 & 53 & $\mathrm{HN}$ & 53 & HB2 & 3.0 \\
\hline 13 & HN & 15 & HD1* & 4.0 & 37 & HN & 38 & $\mathrm{HN}$ & 4.0 \\
\hline 49 & HG $2 \star$ & 61 & HE2 & 4.0 & 38 & HN & 39 & HN & 3.0 \\
\hline 49 & HG $2 \star$ & 50 & HA & 4.0 & 22 & HA & 25 & $\mathrm{HN}$ & 4.0 \\
\hline 10 & HA & 15 & HD1* & 4.0 & 55 & $\mathrm{HN}$ & 55 & $\mathrm{HG} 2 \star$ & 4.0 \\
\hline 13 & HB2 & 15 & HD1* & 4.0 & 37 & $\mathrm{HN}$ & 37 & HB2 & 4.0 \\
\hline 2 & HD1* & 3 & HN & 4.0 & 26 & $\mathrm{HG} 2 \star$ & 28 & $\mathrm{HN}$ & 4.0 \\
\hline 2 & HD1* & 62 & $\mathrm{HN}$ & 4.0 & 26 & HG1* & 27 & $\mathrm{HN}$ & 4.0 \\
\hline 2 & HD1* & 61 & HD1 & 4.0 & 17 & $\mathrm{HN}$ & 18 & $\mathrm{HN}$ & 3.0 \\
\hline 2 & HA & 2 & HD1* & 4.0 & 16 & HA & 18 & HN & 4.0 \\
\hline 2 & HD1* & 3 & HA & 4.0 & 18 & HN & 18 & HB2 & 3.0 \\
\hline 2 & HD1* & 63 & HB1 & 4.0 & 18 & $\mathrm{HN}$ & 18 & HB1 & 3.0 \\
\hline 3 & HN & 3 & HD1* & 4.0 & 5 & HD21 & 9 & HD1* & 4.0 \\
\hline 2 & $\mathrm{HN}$ & 3 & HD1* & 4.0 & 21 & $\mathrm{HB} \star$ & 26 & HN & 4.0 \\
\hline 3 & HD1* & 4 & $\mathrm{HN}$ & 4.0 & 26 & $\mathrm{HN}$ & 26 & $\mathrm{HG} 2 \star$ & 4.0 \\
\hline 3 & HD1* & 64 & HE21 & 4.0 & 25 & HB2 & 26 & $\mathrm{HN}$ & 4.0 \\
\hline 3 & HD1ऋ & 64 & HE22 & 4.0 & 33 & $\mathrm{HN}$ & 34 & HN & 4.0 \\
\hline 2 & HA & 3 & HD1* & 4.0 & 49 & $\mathrm{HN}$ & 50 & HN & 4.0 \\
\hline 3 & HA & 3 & HD1* & 4.0 & 48 & HB2 & 49 & $\mathrm{HN}$ & 4.0 \\
\hline 3 & HD1* & 64 & HG2 & 4.0 & 47 & HB1 & 49 & $\mathrm{HN}$ & 4.0 \\
\hline 3 & HD1* & 64 & HG1 & 4.0 & 15 & $\mathrm{HN}$ & 15 & HD1* & 4.0 \\
\hline 5 & HA & 8 & $\mathrm{HN}$ & 4.0 & 15 & $\mathrm{HN}$ & 15 & HB & 3.0 \\
\hline 5 & HA & 60 & HA & 4.0 & 50 & $\mathrm{HB} \star$ & 52 & $\mathrm{HN}$ & 4.0 \\
\hline 5 & HA & 7 & $\mathrm{HN}$ & 4.0 & 35 & $\mathrm{HN}$ & 36 & $\mathrm{HN}$ & 4.0 \\
\hline 4 & HA & 61 & HD1 & 4.0 & 37 & HA & 38 & $\mathrm{HN}$ & 3.0 \\
\hline 56 & HD2 & 59 & HA & 4.0 & 5 & HB1 & 5 & HD21 & 3.0 \\
\hline 56 & HD2 & 59 & HB1 & 4.0 & 1 & HA & 1 & $\mathrm{HG} \star$ & 4.0 \\
\hline 5 & HA & 8 & HB1 & 4.0 & 1 & $\mathrm{HB} \star$ & 2 & $\mathrm{HN}$ & 4.0 \\
\hline 55 & HG $2 \star$ & 56 & HD2 & 4.0 & 1 & $\mathrm{HG} \star$ & 64 & HB2 & 4.0 \\
\hline 55 & HG $2 \star$ & 56 & HD1 & 4.0 & 1 & $\mathrm{HG} \star$ & 64 & HB1 & 4.0 \\
\hline 2 & 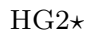 & 4 & HA & 4.0 & 2 & HA & 64 & $\mathrm{HG} \star$ & 4.0 \\
\hline 9 & HD1* & 10 & $\mathrm{HN}$ & 4.0 & 2 & HD1* & 65 & $H G \star$ & 4.0 \\
\hline 9 & HD1* & 11 & $\mathrm{HN}$ & 4.0 & 2 & 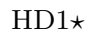 & 65 & $\mathrm{HD} \star$ & 4.0 \\
\hline 9 & HD1* & 59 & HA & 4.0 & 3 & HB & 64 & $\mathrm{HE} 2 \star$ & 4.0 \\
\hline 6 & HA & 9 & HD1* & 4.0 & 3 & $\mathrm{HG} 2 \star$ & 4 & $\mathrm{HB} \star$ & 4.0 \\
\hline 9 & HA & 9 & HD1* & 4.0 & 3 & $\mathrm{HG} 2 \star$ & 62 & $\mathrm{HE} 2 \star$ & 4.0 \\
\hline 9 & HD1* & 60 & HA & 4.0 & 3 & HD1* & 62 & $\mathrm{HG} \star$ & 4.0 \\
\hline 9 & HD1* & 59 & HB1 & 4.0 & 3 & HD1* & 62 & HE2 $2 \star$ & 4.0 \\
\hline
\end{tabular}




\begin{tabular}{|c|c|c|c|c|c|c|c|c|c|}
\hline Residue 1 & H1 & Residue 2 & $\mathrm{H} 2$ & distance $[\check{A}]$ & Residue 1 & H1 & Residue 2 & $\mathrm{H} 2$ & distance $[\AA$ \\
\hline 8 & HD2 & 9 & HD1ऋ & 4.0 & 3 & HD1ऋ & 64 & $\mathrm{HG} \star$ & 3.0 \\
\hline 9 & HD1* & 60 & $\mathrm{HG} 2 \star$ & 4.0 & 3 & HD1* & 64 & HE2 & 4.0 \\
\hline 37 & HA & 40 & HA & 4.0 & 4 & $\mathrm{HB} \star$ & 4 & HD21 & 2.0 \\
\hline 31 & HD1* & 33 & $\mathrm{HN}$ & 4.0 & 4 & $\mathrm{HB} \star$ & 4 & HD22 & 3.0 \\
\hline 31 & HD1* & 34 & $\mathrm{HN}$ & 4.0 & 4 & $\mathrm{HB} \star$ & 5 & $\mathrm{HN}$ & 4.0 \\
\hline 31 & HN & 31 & HD1* & 4.0 & 4 & $\mathrm{HB} \star$ & 6 & $\mathrm{HN}$ & 4.0 \\
\hline 31 & HD1* & 32 & HA & 4.0 & 4 & $\mathrm{HB} \star$ & 34 & 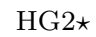 & 4.0 \\
\hline 31 & HA & 31 & HD1* & 3.0 & 4 & $\mathrm{HB} \star$ & 61 & HE1 & 4.0 \\
\hline 31 & HB & 31 & 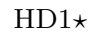 & 4.0 & 4 & $\mathrm{HB} \star$ & 62 & HN & 4.0 \\
\hline 20 & HB2 & 31 & HD1* & 4.0 & 6 & HD1* & 10 & $\mathrm{HB} \star$ & 4.0 \\
\hline 20 & $\mathrm{HG}$ & 31 & HD1* & 4.0 & 6 & $\mathrm{HD} 1 \star$ & 35 & $\mathrm{HG} \star$ & 3.0 \\
\hline 33 & HA2 & 35 & $\mathrm{HN}$ & 4.0 & 7 & $\mathrm{HN}$ & 7 & $\mathrm{HB} \star$ & 3.0 \\
\hline 33 & HA1 & 39 & HB2 & 4.0 & 7 & $\mathrm{HN}$ & 7 & $\mathrm{HD} \star$ & 4.0 \\
\hline 33 & HA1 & 36 & HB2 & 4.0 & 7 & $\mathrm{HN}$ & 7 & $\mathrm{HE} \star$ & 4.0 \\
\hline 33 & HA2 & 36 & HG1 & 4.0 & 7 & HA & 7 & $\mathrm{HG} \star$ & 4.0 \\
\hline 61 & HB1 & 61 & HD1 & 4.0 & 7 & HA & 7 & $\mathrm{HD} \star$ & 4.0 \\
\hline 47 & HB2 & 47 & 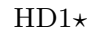 & 4.0 & 7 & $\mathrm{HB} \star$ & 8 & $\mathrm{HN}$ & 4.0 \\
\hline 8 & HB2 & 9 & $\mathrm{HN}$ & 4.0 & 7 & $H G \star$ & 7 & $\mathrm{HD} \star$ & 2.0 \\
\hline 5 & HA & 8 & HB2 & 4.0 & 7 & $H G \star$ & 7 & $\mathrm{HE} \star$ & 3.0 \\
\hline 5 & HB1 & 8 & HB2 & 4.0 & 7 & $\mathrm{HD} \star$ & 7 & $\mathrm{HE} \star$ & 2.0 \\
\hline 48 & $\mathrm{HN}$ & 48 & HE1 & 4.0 & 7 & $\mathrm{HD \star}$ & 8 & $\mathrm{HN}$ & 4.0 \\
\hline 48 & $\mathrm{HE} 2$ & 52 & HE2 & 4.0 & 7 & $\mathrm{HD \star}$ & 8 & HA & 4.0 \\
\hline 57 & HB2 & 58 & HG1 & 4.0 & 7 & $\mathrm{HE} \star$ & 8 & $\mathrm{HN}$ & 4.0 \\
\hline 23 & HB2 & 23 & 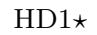 & 4.0 & 8 & HD1* & 12 & $\mathrm{HD} \star$ & 3.0 \\
\hline 23 & HB2 & 23 & $\mathrm{HD} 2 \star$ & 4.0 & 8 & HD1* & 12 & $\mathrm{HE \star}$ & 4.0 \\
\hline 4 & HB2 & 5 & $\mathrm{HN}$ & 4.0 & 9 & $\mathrm{HA}$ & 12 & $\mathrm{HB} \star$ & 3.0 \\
\hline 4 & $\mathrm{HN}$ & 4 & HB2 & 4.0 & 9 & HA & 12 & $\mathrm{HD} \star$ & 4.0 \\
\hline 4 & $\mathrm{HN}$ & 4 & HB1 & 4.0 & 9 & $\mathrm{HG} 2 \star$ & 13 & $\mathrm{HE} \star$ & 4.0 \\
\hline 20 & HB2 & 21 & $\mathrm{HN}$ & 3.0 & 10 & HA & 10 & $\mathrm{HG \star}$ & 3.0 \\
\hline 20 & HB1 & 21 & $\mathrm{HN}$ & 4.0 & 10 & $\mathrm{HB} \star$ & 10 & $\mathrm{HD} \star$ & 3.0 \\
\hline 6 & HB1 & 6 & 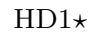 & 3.0 & 10 & $\mathrm{HB} \star$ & 11 & HN & 4.0 \\
\hline 20 & HB1 & 31 & HG $2 \star$ & 4.0 & 10 & $\mathrm{HG \star}$ & 20 & HG & 4.0 \\
\hline 45 & HB1 & 46 & $\mathrm{HN}$ & 3.0 & 10 & $\mathrm{HD} \star$ & 20 & HB1 & 4.0 \\
\hline 6 & $\mathrm{HN}$ & 6 & HB2 & 4.0 & 10 & $\mathrm{HD} \star$ & 20 & $\mathrm{HG}$ & 4.0 \\
\hline 43 & HB1 & 43 & HD2 & 4.0 & 10 & $\mathrm{HD \star}$ & 20 & $\mathrm{HD} \star$ & 3.0 \\
\hline 6 & HB2 & 60 & $\mathrm{HG} 2 \star$ & 4.0 & 12 & $\mathrm{HN}$ & 12 & $\mathrm{HB} \star$ & 3.0 \\
\hline 34 & $\mathrm{HN}$ & 39 & HB2 & 4.0 & 12 & $\mathrm{HN}$ & 12 & $\mathrm{HG} \star$ & 4.0 \\
\hline 59 & HB1 & 60 & HG11 & 4.0 & 12 & $\mathrm{HN}$ & 12 & $\mathrm{HD} \star$ & 4.0 \\
\hline 55 & $\mathrm{HG} 2 \star$ & 59 & HB2 & 4.0 & 12 & HA & 12 & $\mathrm{HB} \star$ & 2.0 \\
\hline 39 & HB1 & 40 & $\mathrm{HN}$ & 4.0 & 12 & HA & 12 & $\mathrm{HG} \star$ & 2.0 \\
\hline 39 & HB2 & 40 & $\mathrm{HN}$ & 4.0 & 12 & HA & 12 & $\mathrm{HD} \star$ & 4.0 \\
\hline 34 & $\mathrm{HN}$ & 39 & HB1 & 4.0 & 12 & $\mathrm{HB} \star$ & 12 & $\mathrm{HD} \star$ & 3.0 \\
\hline 39 & HB1 & 39 & HD2 & 3.0 & 12 & $\mathrm{HB} \star$ & 13 & HN & 4.0 \\
\hline 39 & HB2 & 39 & HD1 & 3.0 & 12 & $H G \star$ & 12 & $\mathrm{HE} \star$ & 3.0 \\
\hline 5 & HB2 & 5 & HD22 & 4.0 & 12 & $\mathrm{HG \star}$ & 13 & $\mathrm{HN}$ & 4.0 \\
\hline 5 & HB1 & 5 & HD22 & 4.0 & 13 & HN & 13 & $\mathrm{HD} \star$ & 4.0 \\
\hline 39 & HA & 39 & HB1 & 3.0 & 13 & HA & 13 & $\mathrm{HD} \star$ & 3.0 \\
\hline 34 & HG11 & 39 & HB1 & 4.0 & 13 & HA & 13 & $\mathrm{HE} \star$ & 4.0 \\
\hline 56 & HB2 & 59 & HB2 & 4.0 & 13 & HA & 14 & $\mathrm{HG} \star$ & 4.0 \\
\hline 5 & HB1 & 8 & HB1 & 4.0 & 13 & HB1 & 13 & $\mathrm{HE} \star$ & 4.0 \\
\hline 5 & HB2 & 8 & HB1 & 4.0 & 13 & HB1 & 14 & $\mathrm{HG} \star$ & 4.0 \\
\hline 5 & HB1 & 8 & HD1* & 4.0 & 13 & HG2 & 13 & $\mathrm{HE} \star$ & 3.0 \\
\hline 30 & $\mathrm{HG} 2 \star$ & 39 & HB1 & 4.0 & 14 & $\mathrm{HN}$ & 14 & $\mathrm{HG} \star$ & 4.0 \\
\hline 60 & $\mathrm{HN}$ & 60 & HB & 3.0 & 14 & $\mathrm{HN}$ & 14 & $\mathrm{HD} \star$ & 4.0 \\
\hline 60 & HB & 61 & HD2 & 4.0 & 14 & HA & 14 & $\mathrm{HG} \star$ & 3.0 \\
\hline 6 & HB2 & 60 & HB & 4.0 & 14 & HA & 14 & $\mathrm{HD} \star$ & 4.0 \\
\hline 2 & $\mathrm{HN}$ & 2 & HB & 4.0 & 14 & $\mathrm{HG}_{\star}$ & 14 & $\mathrm{HD} \star$ & 2.0 \\
\hline 31 & $\mathrm{HN}$ & 32 & HB2 & 4.0 & 15 & $\mathrm{HN}$ & 15 & HG1ऋ & 4.0 \\
\hline 31 & $\mathrm{HN}$ & 32 & HB1 & 4.0 & 15 & HA & 15 & HG1* & 4.0 \\
\hline 32 & HA & 32 & HB2 & 3.0 & 15 & HA & 16 & $\mathrm{HB} \star$ & 4.0 \\
\hline 29 & HA & 32 & HB2 & 3.0 & 15 & HA & 19 & $\mathrm{HB} \star$ & 4.0 \\
\hline 3 & HB & 4 & $\mathrm{HN}$ & 4.0 & 15 & HB & 19 & $\mathrm{HB} \star$ & 4.0 \\
\hline 3 & HB & 62 & $\mathrm{HN}$ & 4.0 & 15 & $\mathrm{HG} 2 \star$ & 19 & $\mathrm{HB} \star$ & 3.0 \\
\hline 2 & HA & 3 & HB & 4.0 & 15 & $\mathrm{HG} 2 \star$ & 19 & $\mathrm{HG} \star$ & 4.0 \\
\hline 3 & HB & 62 & HA & 4.0 & 15 & HG1* & 19 & $\mathrm{HB} \star$ & 4.0 \\
\hline 58 & HA & 58 & HG1 & 4.0 & 16 & $\mathrm{HN}$ & 16 & $\mathrm{HB} \star$ & 3.0 \\
\hline
\end{tabular}




\begin{tabular}{|c|c|c|c|c|c|c|c|c|c|}
\hline Residue 1 & H1 & Residue 2 & $\mathrm{H} 2$ & distance $[\AA]$ & Residue 1 & H1 & Residue 2 & $\mathrm{H} 2$ & 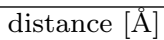 \\
\hline 51 & HB1 & 52 & $\mathrm{HN}$ & 4.0 & 16 & HN & 19 & $\mathrm{HB} \star$ & 3.0 \\
\hline 34 & HB & 35 & HN & 4.0 & 16 & $\mathrm{HB} \star$ & 17 & HN & 4.0 \\
\hline 34 & HN & 34 & HB & 4.0 & 16 & $\mathrm{HB} \star$ & 17 & HA & 4.0 \\
\hline 31 & HA & 34 & HB & 4.0 & 16 & $\mathrm{HB} \star$ & 17 & $\mathrm{HB} \star$ & 4.0 \\
\hline 52 & $\mathrm{HN}$ & 52 & HB2 & 3.0 & 16 & $\mathrm{HB} \star$ & 17 & $\mathrm{HG} \star$ & 4.0 \\
\hline 52 & $\mathrm{HN}$ & 52 & HB1 & 4.0 & 16 & $\mathrm{HB} \star$ & 18 & HN & 4.0 \\
\hline 11 & $\mathrm{HN}$ & 11 & HG2 & 2.0 & 16 & $\mathrm{HB} \star$ & 19 & $\mathrm{HN}$ & 4.0 \\
\hline 11 & HG2 & 12 & HN & 4.0 & 16 & $\mathrm{HB} \star$ & 19 & $\mathrm{HB} \star$ & 4.0 \\
\hline 52 & HB2 & 52 & HD2 & 3.0 & 17 & $\mathrm{HN}$ & 17 & $\mathrm{HB} \star$ & 4.0 \\
\hline 10 & HA & 11 & HG2 & 4.0 & 17 & HA & 17 & $\mathrm{HG} \star$ & 4.0 \\
\hline 23 & HG & 52 & HB1 & 4.0 & 17 & $\mathrm{HB} \star$ & 18 & $\mathrm{HB} \star$ & 4.0 \\
\hline 24 & HD2 & 52 & HB2 & 4.0 & 17 & $\mathrm{HG \star}$ & 17 & $\mathrm{HE} 2 \star$ & 3.0 \\
\hline 35 & HN & 35 & HG1 & 4.0 & 17 & $\mathrm{HG} \star$ & 31 & $\mathrm{HG} 2 \star$ & 4.0 \\
\hline 51 & HE1 & 52 & HD1 & 4.0 & 17 & $\mathrm{HG} \star$ & 32 & HA & 4.0 \\
\hline 51 & HE1 & 52 & HD2 & 4.0 & 17 & HE2 & 31 & $\mathrm{HG} 2 \star$ & 4.0 \\
\hline 39 & HA & 39 & HE1 & 4.0 & 17 & HE2 $2 \star$ & 32 & HA & 4.0 \\
\hline 51 & HB1 & 51 & HE1 & 4.0 & 18 & HN & 18 & $\mathrm{HB} \star$ & 2.0 \\
\hline 48 & HB2 & 52 & $\mathrm{HE} 2$ & 3.0 & 18 & $\mathrm{HN}$ & 28 & $\mathrm{HD} \star$ & 4.0 \\
\hline 30 & HG $2 \star$ & 39 & HE2 & 3.0 & 18 & $\mathrm{HB} \star$ & 19 & $\mathrm{HN}$ & 4.0 \\
\hline 24 & $\mathrm{HD} 2 \star$ & 52 & $\mathrm{HE} 2$ & 3.0 & 18 & $\mathrm{HB} \star$ & 20 & $\mathrm{HN}$ & 4.0 \\
\hline 37 & $\mathrm{HN}$ & 37 & HB1 & 4.0 & 18 & $\mathrm{HB} \star$ & 21 & $\mathrm{HN}$ & 4.0 \\
\hline 9 & $\mathrm{HN}$ & 9 & HB & 4.0 & 18 & $\mathrm{HB} \star$ & 28 & $\mathrm{HB} \star$ & 4.0 \\
\hline 6 & HA & 9 & HB & 4.0 & 18 & $\mathrm{HB} \star$ & 28 & $\mathrm{HD} \star$ & 2.0 \\
\hline 31 & HB & 32 & HN & 4.0 & 19 & $\mathrm{HN}$ & 19 & $\mathrm{HB} \star$ & 3.0 \\
\hline 26 & HB & 27 & $\mathrm{HN}$ & 4.0 & 19 & HA & 19 & $\mathrm{HG} \star$ & 2.0 \\
\hline 31 & $\mathrm{HN}$ & 31 & HB & 3.0 & 19 & $\mathrm{HB} \star$ & 20 & $\mathrm{HN}$ & 3.0 \\
\hline 20 & HB1 & 31 & HB & 4.0 & 19 & $\mathrm{HG \star}$ & 22 & $\mathrm{HB} \star$ & 4.0 \\
\hline 26 & HB & 30 & HG $2 \star$ & 4.0 & 20 & $\mathrm{HN}$ & 20 & $\mathrm{HD} \star$ & 4.0 \\
\hline 55 & $\mathrm{HN}$ & 55 & HB & 4.0 & 20 & $\mathrm{HN}$ & 23 & $\mathrm{HD} \star$ & 4.0 \\
\hline 55 & HB & 56 & HD2 & 3.0 & 20 & HA & 20 & $\mathrm{HD} \star$ & 3.0 \\
\hline 55 & HB & 60 & 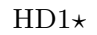 & 4.0 & 20 & $\mathrm{HD} \star$ & 31 & HD1* & 2.0 \\
\hline 44 & $\mathrm{HN}$ & 44 & HG2 & 4.0 & 21 & HA & 24 & $\mathrm{HB} \star$ & 4.0 \\
\hline 44 & $\mathrm{HN}$ & 44 & HG1 & 4.0 & 21 & $\mathrm{HB} \star$ & 28 & $\mathrm{HD} \star$ & 4.0 \\
\hline 44 & HG2 & 45 & $\mathrm{HN}$ & 4.0 & 22 & $\mathrm{HB} \star$ & 23 & $\mathrm{HD} \star$ & 4.0 \\
\hline 44 & HG1 & 45 & $\mathrm{HN}$ & 4.0 & 23 & HA & 23 & $\mathrm{HD} \star$ & 3.0 \\
\hline 44 & HA & 44 & HG2 & 4.0 & 23 & $\mathrm{HB} \star$ & 23 & $\mathrm{HD} \star$ & 2.0 \\
\hline 44 & HA & 44 & HG1 & 4.0 & 23 & $\mathrm{HB} \star$ & 52 & HE1 & 4.0 \\
\hline 17 & HN & 17 & HG2 & 4.0 & 23 & $\mathrm{HB} \star$ & 52 & HE2 & 4.0 \\
\hline 62 & HG2 & 63 & HN & 4.0 & 23 & $\mathrm{HD} \star$ & 52 & HA & 4.0 \\
\hline 38 & HB1 & 39 & $\mathrm{HN}$ & 4.0 & 23 & $\mathrm{HD} \star$ & 52 & HB2 & 4.0 \\
\hline 64 & HA & 64 & HG2 & 4.0 & 23 & $\mathrm{HD} \star$ & 52 & HE1 & 4.0 \\
\hline 64 & HA & 64 & HG1 & 4.0 & 23 & $\mathrm{HD} \star$ & 53 & HN & 4.0 \\
\hline 3 & HG12 & 64 & HG2 & 4.0 & 24 & $\mathrm{HB} \star$ & 24 & HG & 2.0 \\
\hline 3 & HG12 & 64 & HG1 & 4.0 & 24 & $\mathrm{HB} \star$ & 24 & HD1* & 3.0 \\
\hline 48 & HB1 & 49 & HN & 4.0 & 24 & $\mathrm{HB} \star$ & 24 & HD $2 \star$ & 2.0 \\
\hline 45 & HA & 48 & HB2 & 4.0 & 24 & $\mathrm{HB} \star$ & 26 & 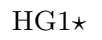 & 4.0 \\
\hline 48 & HB2 & 48 & HE1 & 4.0 & 24 & $\mathrm{HB} \star$ & 26 & $\mathrm{HG} 2 \star$ & 2.0 \\
\hline 48 & HB2 & 48 & HE2 & 4.0 & 24 & 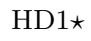 & 45 & $\mathrm{HD} \star$ & 4.0 \\
\hline 24 & HD2 & 48 & HB1 & 4.0 & 25 & $\mathrm{HN}$ & 25 & $\mathrm{HG} \star$ & 2.0 \\
\hline 24 & HD2 $2 \star$ & 48 & HB2 & 4.0 & 27 & $\mathrm{HB} \star$ & 28 & $\mathrm{HN}$ & 4.0 \\
\hline 48 & HB2 & 48 & HG1 & 2.0 & 28 & HA & 28 & $\mathrm{HB} \star$ & 2.0 \\
\hline 7 & $\mathrm{HN}$ & 7 & HB2 & 4.0 & 28 & HA & 28 & $\mathrm{HG} \star$ & 4.0 \\
\hline 7 & $\mathrm{HN}$ & 7 & HB1 & 4.0 & 28 & HA & 28 & $\mathrm{HD} \star$ & 4.0 \\
\hline 7 & HB2 & 8 & $\mathrm{HN}$ & 4.0 & 28 & $\mathrm{HB} \star$ & 28 & $\mathrm{HD} \star$ & 3.0 \\
\hline 7 & HB1 & 8 & $\mathrm{HN}$ & 4.0 & 28 & $\mathrm{HB} \star$ & 29 & $\mathrm{HN}$ & 4.0 \\
\hline 62 & $\mathrm{HN}$ & 62 & HB1 & 4.0 & 29 & $\mathrm{HN}$ & 29 & $\mathrm{HB} \star$ & 2.0 \\
\hline 36 & $\mathrm{HN}$ & 36 & HB1 & 4.0 & 29 & $\mathrm{HN}$ & 29 & $\mathrm{HG} \star$ & 4.0 \\
\hline 56 & HB2 & 59 & $\mathrm{HN}$ & 4.0 & 29 & HA & 29 & $H G \star$ & 3.0 \\
\hline 41 & HB1 & 61 & HE1 & 4.0 & 29 & $\mathrm{HB} \star$ & 30 & $\mathrm{HN}$ & 3.0 \\
\hline 49 & HA & 49 & HG12 & 4.0 & 29 & $\mathrm{HB} \star$ & 32 & HB1 & 4.0 \\
\hline 49 & 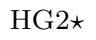 & 49 & HG12 & 2.0 & 31 & 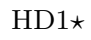 & 35 & $\mathrm{HG} \star$ & 4.0 \\
\hline 63 & $\mathrm{HN}$ & 63 & HB2 & 4.0 & 34 & $\mathrm{HG} 2 \star$ & 35 & $\mathrm{HG} \star$ & 4.0 \\
\hline 64 & $\mathrm{HN}$ & 64 & HB2 & 3.0 & 34 & HD1ऋ & 41 & $\mathrm{HG} \star$ & 3.0 \\
\hline 63 & HB1 & 63 & HE3 & 3.0 & 35 & HA & 35 & $\mathrm{HG} \star$ & 2.0 \\
\hline 3 & HD1* & 64 & HB1 & 4.0 & 35 & $\mathrm{HB} \star$ & 36 & $\mathrm{HN}$ & 3.0 \\
\hline 16 & $\mathrm{HN}$ & 19 & HB2 & 4.0 & 36 & $\mathrm{HN}$ & 36 & $\mathrm{HE} \star$ & 4.0 \\
\hline
\end{tabular}




\begin{tabular}{|c|c|c|c|c|c|c|c|c|c|}
\hline Residue 1 & $\overline{\mathrm{H} 1}$ & Residue 2 & $\overline{\mathrm{H} 2}$ & 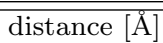 & Residue 1 & H1 & Residue 2 & $\mathrm{H} 2$ & distance $[\AA]$ \\
\hline 16 & $\mathrm{HN}$ & 19 & HB1 & 4.0 & 36 & HA & 36 & $\mathrm{HE} \star$ & 4.0 \\
\hline 19 & HB2 & 20 & HN & 3.0 & 36 & HB2 & 36 & $\mathrm{HE} \star$ & 4.0 \\
\hline 19 & HB1 & 20 & HN & 3.0 & 36 & HB1 & 36 & $\mathrm{HE} \star$ & 3.0 \\
\hline 34 & HG11 & 35 & $\mathrm{HN}$ & 4.0 & 36 & HD1 & 36 & $\mathrm{HE} \star \star$ & 2.0 \\
\hline 11 & $\mathrm{HN}$ & 11 & HB2 & 4.0 & 37 & $\mathrm{HN}$ & 37 & $\mathrm{HB} \star$ & 3.0 \\
\hline 11 & $\mathrm{HN}$ & 11 & HB1 & 4.0 & 37 & $\mathrm{HB} \star$ & 37 & HD21 & 2.0 \\
\hline 11 & HB2 & 12 & HN & 2.0 & 37 & $\mathrm{HB} \star$ & 38 & HA & 4.0 \\
\hline 34 & HN & 34 & HG11 & 4.0 & 38 & $\mathrm{HN}$ & 38 & $\mathrm{HB} \star$ & 2.0 \\
\hline 24 & HG & 25 & HN & 4.0 & 38 & HN & 38 & $\mathrm{HG} \star$ & 4.0 \\
\hline 34 & HG11 & 39 & HD2 & 4.0 & 38 & $\mathrm{HB} \star$ & 39 & $\mathrm{HN}$ & 4.0 \\
\hline 11 & HA & 11 & HB1 & 2.0 & 38 & $\mathrm{HD} \star$ & 38 & $\mathrm{HE} \star$ & 2.0 \\
\hline 36 & HA & 36 & HD2 & 3.0 & 39 & HA & 40 & $\mathrm{HB} \star$ & 4.0 \\
\hline 36 & HB2 & 36 & HD2 & 4.0 & 39 & HE2 & 45 & $\mathrm{HD} \star$ & 4.0 \\
\hline 6 & HD1* & 10 & HB1 & 4.0 & 40 & $\mathrm{HB} \star$ & 40 & $\mathrm{HD} 2 \star$ & 3.0 \\
\hline 9 & HN & 9 & HG11 & 4.0 & 40 & $\mathrm{HB} \star$ & 41 & HA & 4.0 \\
\hline 26 & HB & 30 & HA & 4.0 & 41 & $\mathrm{HG} \star$ & 42 & $\mathrm{HN}$ & 4.0 \\
\hline 30 & HA & 30 & $\mathrm{HG} 2 \star$ & 3.0 & 41 & $\mathrm{HG} \star$ & 61 & HD1 & 4.0 \\
\hline 2 & $\mathrm{HN}$ & 2 & HG11 & 4.0 & 41 & $\mathrm{HG} \star$ & 61 & $\mathrm{HZ}$ & 4.0 \\
\hline 29 & HB1 & 30 & $\mathrm{HN}$ & 4.0 & 42 & $\mathrm{HB} \star$ & 43 & $\mathrm{HN}$ & 4.0 \\
\hline 24 & HA & 24 & HD1ऋ & 4.0 & 42 & $\mathrm{HB} \star$ & 43 & $\mathrm{HB} \star$ & 4.0 \\
\hline 24 & HD1* & 49 & HG12 & 4.0 & 43 & $\mathrm{HB} \star$ & 43 & HD1* & 3.0 \\
\hline 3 & HG2 $2 \star$ & 3 & HG12 & 3.0 & 43 & $\mathrm{HB} \star$ & 43 & $\mathrm{HD} 2 \star$ & 3.0 \\
\hline 57 & $\mathrm{HN}$ & 57 & $\mathrm{HG}$ & 4.0 & 43 & $\mathrm{HB} \star$ & 44 & $\mathrm{HN}$ & 4.0 \\
\hline 3 & $\mathrm{HN}$ & 3 & HG11 & 4.0 & 44 & HN & 44 & $\mathrm{HB} \star$ & 2.0 \\
\hline 3 & $\mathrm{HN}$ & 3 & HG12 & 4.0 & 44 & $\mathrm{HN}$ & 44 & $\mathrm{HG} \star$ & 4.0 \\
\hline 3 & HA & 3 & HG11 & 3.0 & 44 & HA & 44 & $\mathrm{HG} \star$ & 3.0 \\
\hline 6 & HD1ऋ & 35 & HA & 4.0 & 44 & $\mathrm{HB} \star$ & 45 & $\mathrm{HN}$ & 4.0 \\
\hline 6 & HD1* & 10 & HB2 & 4.0 & 44 & $\mathrm{HG} \star$ & 45 & $\mathrm{HN}$ & 4.0 \\
\hline 55 & $\mathrm{HG} 2 \star$ & 60 & HG12 & 4.0 & 45 & $\mathrm{HN}$ & 45 & $\mathrm{HD} \star$ & 3.0 \\
\hline 49 & HA & 49 & $\mathrm{HG} 2 \star$ & 3.0 & 45 & HA & 45 & $\mathrm{HD} \star$ & 3.0 \\
\hline 49 & HA & 49 & HG11 & 4.0 & 45 & HB2 & 45 & $\mathrm{HD} \star$ & 2.0 \\
\hline 47 & HD1* & 57 & HG & 2.0 & 45 & $\mathrm{HD} \star$ & 46 & $\mathrm{HN}$ & 4.0 \\
\hline 6 & HD1* & 60 & $\mathrm{HG} 2 \star$ & 4.0 & 45 & $\mathrm{HD} \star$ & 48 & HG2 & 4.0 \\
\hline 31 & HG11 & 32 & $\mathrm{HN}$ & 4.0 & 45 & $\mathrm{HD} \star$ & 48 & HG1 & 4.0 \\
\hline 25 & HN & 25 & HB1 & 4.0 & 45 & $\mathrm{HD} \star$ & 48 & HD2 & 4.0 \\
\hline 28 & HA & 28 & HG2 & 4.0 & 45 & $\mathrm{HD} \star$ & 48 & HE2 & 4.0 \\
\hline 31 & HA & 31 & HG11 & 4.0 & 45 & $\mathrm{HD} \star$ & 48 & HE1 & 4.0 \\
\hline 28 & HA & 28 & HG1 & 4.0 & 45 & $\mathrm{HD} \star$ & 49 & HG12 & 4.0 \\
\hline 47 & HD1* & 48 & $\mathrm{HN}$ & 4.0 & 49 & HA & 53 & $\mathrm{HD} \star$ & 4.0 \\
\hline 20 & HA & 20 & 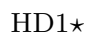 & 4.0 & 53 & $\mathrm{HD} \star$ & 60 & $\mathrm{HG} 2 \star$ & 4.0 \\
\hline 47 & HA & 47 & 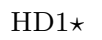 & 4.0 & 53 & $\mathrm{HD} \star$ & 60 & HG12 & 4.0 \\
\hline 24 & HA & 25 & HB2 & 4.0 & 54 & $\mathrm{HN}$ & 54 & $\mathrm{HB} \star$ & 4.0 \\
\hline 44 & HA & 47 & HD1* & 4.0 & 54 & $\mathrm{HB} \star$ & 54 & HD21 & 3.0 \\
\hline 43 & HD2 2 & 47 & 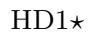 & 4.0 & 55 & HA & 56 & $\mathrm{HG} \star$ & 4.0 \\
\hline 30 & HB & 32 & HN & 4.0 & 56 & $\mathrm{HG} \star$ & 59 & HA & 4.0 \\
\hline 45 & HD1* & 46 & $\mathrm{HN}$ & 4.0 & 56 & $\mathrm{HG} \star$ & 59 & HB2 & 4.0 \\
\hline 53 & HN & 53 & HD1* & 4.0 & 58 & $\mathrm{HB} \star$ & 63 & HH2 & 4.0 \\
\hline 57 & $\mathrm{HD} 2 \star$ & 63 & HH2 & 4.0 & 61 & HA & 62 & $\mathrm{HB} \star$ & 4.0 \\
\hline 47 & HA & 47 & $\mathrm{HD} 2 \star$ & 4.0 & 62 & $\mathrm{HN}$ & 62 & $\mathrm{HB} \star$ & 3.0 \\
\hline 57 & HA & 57 & HD $2 \star$ & 4.0 & 62 & $\mathrm{HN}$ & 62 & $\mathrm{HG} \star$ & 4.0 \\
\hline 45 & HA & 45 & HD1* & 4.0 & 62 & HA & 62 & $\mathrm{HG} \star$ & 4.0 \\
\hline 57 & HB2 & 57 & $\mathrm{HD} 2 \star$ & 3.0 & 62 & $\mathrm{HB} \star$ & 62 & $\mathrm{HG} \star$ & 2.0 \\
\hline 45 & HB2 & 45 & HD1* & 3.0 & 62 & $\mathrm{HB} \star$ & 62 & $\mathrm{HE} 2 \star$ & 4.0 \\
\hline 57 & HN & 57 & $\mathrm{HD} 2 \star$ & 3.0 & 62 & $\mathrm{HG} \star$ & 62 & $\mathrm{HE} 2 \star$ & 3.0 \\
\hline 24 & $\mathrm{HN}$ & 24 & 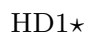 & 4.0 & 63 & HA & 65 & $\mathrm{HD} \star$ & 4.0 \\
\hline 24 & HD1* & 49 & HD1ऋ & 4.0 & 63 & HB2 & 65 & $\mathrm{HB} \star$ & 4.0 \\
\hline 43 & HD2 & 57 & $\mathrm{HG}$ & 4.0 & 63 & HB2 & 65 & $\mathrm{HG} \star$ & 4.0 \\
\hline 31 & $\mathrm{HG} 2 \star$ & 31 & HG12 & 3.0 & 63 & HB1 & 64 & $\mathrm{HG} \star$ & 4.0 \\
\hline 31 & HB & 31 & HG12 & 2.0 & 63 & HB1 & 65 & $\mathrm{HG} \star$ & 4.0 \\
\hline 55 & HB & 60 & HG11 & 4.0 & 64 & $\mathrm{HN}$ & 64 & $H G \star$ & 4.0 \\
\hline 60 & $\mathrm{HN}$ & 60 & HG11 & 3.0 & 64 & $\mathrm{HN}$ & 65 & $\mathrm{HG} \star$ & 4.0 \\
\hline 33 & HA2 & 39 & HB2 & 4.0 & 64 & HA & 64 & $H G \star$ & 4.0 \\
\hline
\end{tabular}


Table C.8: Intermolecular distances of CylR2 from PRE

\begin{tabular}{|c|c|c|c|c|c|c|c|c|c|}
\hline Residue 1 & Atom 1 & Residue 2 & Atom 2 & distance $[\AA]$ & Residue 1 & Atom 1 & Residue 2 & Atom 2 & distance $[\AA]$ \\
\hline 40 & NZ & 68 & HN & 30.00 & 55 & NZ & 68 & $\mathrm{HN}$ & 22.71 \\
\hline 40 & NZ & 69 & HN & 30.00 & 55 & NZ & 69 & $\mathrm{HN}$ & 24.59 \\
\hline 40 & NZ & 70 & $\mathrm{HN}$ & 28.60 & 55 & NZ & 70 & $\mathrm{HN}$ & 24.30 \\
\hline 40 & $\mathrm{NZ}$ & 71 & $\mathrm{HN}$ & 30.00 & 55 & $\mathrm{NZ}$ & 71 & $\mathrm{HN}$ & 24.15 \\
\hline 40 & $\mathrm{NZ}$ & 72 & HN & 30.00 & 55 & NZ & 72 & $\mathrm{HN}$ & 23.20 \\
\hline 40 & $\mathrm{NZ}$ & 73 & HN & 30.00 & 55 & NZ & 73 & $\mathrm{HN}$ & 27.76 \\
\hline 40 & NZ & 74 & HN & 29.89 & 55 & NZ & 74 & $\mathrm{HN}$ & 30.00 \\
\hline 40 & NZ & 75 & HN & 24.60 & 55 & NZ & 75 & $\mathrm{HN}$ & 30.00 \\
\hline 40 & $\mathrm{NZ}$ & 76 & $\mathrm{HN}$ & 30.00 & 55 & $\mathrm{NZ}$ & 76 & $\mathrm{HN}$ & 27.39 \\
\hline 40 & NZ & 77 & $\mathrm{HN}$ & 30.00 & 55 & NZ & 78 & $\mathrm{HN}$ & 25.77 \\
\hline 40 & NZ & 79 & $\mathrm{HN}$ & 30.00 & 55 & NZ & 79 & $\mathrm{HN}$ & 30.00 \\
\hline 40 & NZ & 80 & $\mathrm{HN}$ & 30.00 & 55 & NZ & 80 & $\mathrm{HN}$ & 30.00 \\
\hline 40 & $\mathrm{NZ}$ & 81 & $\mathrm{HN}$ & 30.00 & 55 & $\mathrm{NZ}$ & 81 & $\mathrm{HN}$ & 30.00 \\
\hline 40 & $\mathrm{NZ}$ & 82 & HN & 29.09 & 55 & NZ & 82 & HN & 30.00 \\
\hline 40 & $\mathrm{NZ}$ & 83 & HN & 27.07 & 55 & NZ & 83 & $\mathrm{HN}$ & 25.31 \\
\hline 40 & NZ & 84 & $\mathrm{HN}$ & 30.00 & 55 & $\mathrm{NZ}$ & 84 & $\mathrm{HN}$ & 30.00 \\
\hline 40 & NZ & 85 & $\mathrm{HN}$ & 30.00 & 55 & NZ & 85 & $\mathrm{HN}$ & 23.71 \\
\hline 40 & NZ & 86 & $\mathrm{HN}$ & 30.00 & 55 & NZ & 86 & $\mathrm{HN}$ & 30.00 \\
\hline 40 & NZ & 87 & $\mathrm{HN}$ & 30.00 & 55 & $\mathrm{NZ}$ & 87 & $\mathrm{HN}$ & 25.92 \\
\hline 40 & NZ & 89 & HN & 20.75 & 55 & NZ & 88 & $\mathrm{HN}$ & 30.00 \\
\hline 40 & NZ & 90 & $\mathrm{HN}$ & 20.97 & 55 & NZ & 89 & $\mathrm{HN}$ & 28.36 \\
\hline 40 & NZ & 92 & HN & 24.56 & 55 & $\mathrm{NZ}$ & 90 & $\mathrm{HN}$ & 30.00 \\
\hline 40 & NZ & 93 & HN & 20.89 & 55 & NZ & 92 & $\mathrm{HN}$ & 28.58 \\
\hline 40 & NZ & 95 & $\mathrm{HN}$ & 27.59 & 55 & NZ & 95 & $\mathrm{HN}$ & 30.00 \\
\hline 40 & NZ & 96 & $\mathrm{HN}$ & 21.53 & 55 & $\mathrm{NZ}$ & 96 & $\mathrm{HN}$ & 30.00 \\
\hline 40 & NZ & 97 & $\mathrm{HN}$ & 24.16 & 55 & NZ & 97 & $\mathrm{HN}$ & 30.00 \\
\hline 40 & NZ & 98 & $\mathrm{HN}$ & 30.00 & 55 & $\mathrm{NZ}$ & 98 & $\mathrm{HN}$ & 30.00 \\
\hline 40 & NZ & 99 & $\mathrm{HN}$ & 30.00 & 55 & NZ & 99 & $\mathrm{HN}$ & 30.00 \\
\hline 40 & NZ & 101 & HN & 30.00 & 55 & NZ & 102 & $\mathrm{HN}$ & 30.00 \\
\hline 40 & NZ & 102 & $\mathrm{HN}$ & 25.26 & 55 & $\mathrm{NZ}$ & 104 & $\mathrm{HN}$ & 29.12 \\
\hline 40 & NZ & 104 & $\mathrm{HN}$ & 30.00 & 55 & NZ & 105 & $\mathrm{HN}$ & 25.06 \\
\hline 40 & NZ & 105 & $\mathrm{HN}$ & 21.61 & 55 & NZ & 109 & $\mathrm{HN}$ & 23.78 \\
\hline 40 & $\mathrm{NZ}$ & 109 & $\mathrm{HN}$ & 15.81 & 55 & NZ & 110 & $\mathrm{HN}$ & 22.77 \\
\hline 40 & NZ & 110 & $\mathrm{HN}$ & 7.00 & 55 & $\mathrm{NZ}$ & 111 & $\mathrm{HN}$ & 22.91 \\
\hline 40 & NZ & 111 & $\mathrm{HN}$ & 15.79 & 55 & NZ & 112 & $\mathrm{HN}$ & 20.68 \\
\hline 40 & NZ & 112 & HN & 20.20 & 55 & NZ & 113 & $\mathrm{HN}$ & 23.90 \\
\hline 40 & NZ & 113 & HN & 7.00 & 55 & NZ & 114 & $\mathrm{HN}$ & 20.80 \\
\hline 40 & NZ & 114 & HN & 18.10 & 55 & NZ & 115 & $\mathrm{HN}$ & 23.89 \\
\hline 40 & NZ & 115 & $\mathrm{HN}$ & 17.85 & 55 & NZ & 116 & $\mathrm{HN}$ & 22.71 \\
\hline 40 & NZ & 116 & $\mathrm{HN}$ & 18.44 & 55 & NZ & 117 & $\mathrm{HN}$ & 23.52 \\
\hline 40 & NZ & 117 & $\mathrm{HN}$ & 15.84 & 55 & NZ & 118 & $\mathrm{HN}$ & 21.25 \\
\hline 40 & $\mathrm{NZ}$ & 118 & $\mathrm{HN}$ & 19.13 & 55 & NZ & 119 & $\mathrm{HN}$ & 23.87 \\
\hline 40 & NZ & 119 & $\mathrm{HN}$ & 18.82 & 55 & NZ & 120 & $\mathrm{HN}$ & 25.20 \\
\hline 40 & NZ & 120 & HN & 20.71 & 55 & NZ & 124 & $\mathrm{HN}$ & 17.74 \\
\hline 40 & NZ & 123 & $\mathrm{HN}$ & 21.56 & 55 & NZ & 125 & $\mathrm{HN}$ & 21.29 \\
\hline 40 & NZ & 124 & HN & 23.01 & 55 & NZ & 126 & $\mathrm{HN}$ & 17.33 \\
\hline 40 & NZ & 125 & $\mathrm{HN}$ & 20.73 & 55 & NZ & 127 & $\mathrm{HN}$ & 23.01 \\
\hline 40 & NZ & 126 & $\mathrm{HN}$ & 19.34 & 55 & NZ & 128 & $\mathrm{HN}$ & 18.66 \\
\hline 40 & NZ & 127 & $\mathrm{HN}$ & 18.49 & 55 & NZ & 129 & $\mathrm{HN}$ & 15.61 \\
\hline 40 & NZ & 128 & $\mathrm{HN}$ & 21.43 & 55 & NZ & 130 & $\mathrm{HN}$ & 19.09 \\
\hline 40 & NZ & 129 & $\mathrm{HN}$ & 23.48 & 55 & NZ & 132 & $\mathrm{HN}$ & 13.61 \\
\hline 40 & NZ & 130 & $\mathrm{HN}$ & 22.74 & 2 & $\mathrm{HN}$ & 121 & NZ & 22.71 \\
\hline 2 & $\mathrm{HN}$ & 106 & NZ & 30.00 & 3 & $\mathrm{HN}$ & 121 & NZ & 24.59 \\
\hline 3 & $\mathrm{HN}$ & 106 & NZ & 30.00 & 4 & $\mathrm{HN}$ & 121 & NZ & 24.30 \\
\hline 4 & $\mathrm{HN}$ & 106 & $\mathrm{NZ}$ & 28.60 & 5 & $\mathrm{HN}$ & 121 & NZ & 24.15 \\
\hline 5 & $\mathrm{HN}$ & 106 & NZ & 30.00 & 6 & $\mathrm{HN}$ & 121 & NZ & 23.20 \\
\hline 6 & $\mathrm{HN}$ & 106 & $\mathrm{NZ}$ & 30.00 & 7 & $\mathrm{HN}$ & 121 & NZ & 27.76 \\
\hline 7 & $\mathrm{HN}$ & 106 & NZ & 30.00 & 8 & $\mathrm{HN}$ & 121 & NZ & 30.00 \\
\hline 8 & HN & 106 & NZ & 29.89 & 9 & $\mathrm{HN}$ & 121 & NZ & 30.00 \\
\hline 9 & $\mathrm{HN}$ & 106 & $\mathrm{NZ}$ & 24.60 & 10 & $\mathrm{HN}$ & 121 & NZ & 27.39 \\
\hline 10 & $\mathrm{HN}$ & 106 & NZ & 30.00 & 12 & $\mathrm{HN}$ & 121 & NZ & 25.77 \\
\hline 11 & $\mathrm{HN}$ & 106 & NZ & 30.00 & 13 & $\mathrm{HN}$ & 121 & NZ & 30.00 \\
\hline
\end{tabular}




\begin{tabular}{|c|c|c|c|c|c|c|c|c|c|}
\hline Residue 1 & H1 & Residue 2 & $\mathrm{H} 2$ & distance $[\AA]$ & Residue 1 & H1 & Residue 2 & $\mathrm{H} 2$ & distance $[\AA]$ \\
\hline 13 & HN & 106 & $\mathrm{NZ}$ & 30.00 & 14 & $\mathrm{HN}$ & 121 & NZ & 30.00 \\
\hline 14 & $\mathrm{HN}$ & 106 & NZ & 30.00 & 15 & $\mathrm{HN}$ & 121 & NZ & 30.00 \\
\hline 15 & $\mathrm{HN}$ & 106 & $\mathrm{NZ}$ & 30.00 & 16 & $\mathrm{HN}$ & 121 & $\mathrm{NZ}$ & 30.00 \\
\hline 16 & $\mathrm{HN}$ & 106 & NZ & 29.09 & 17 & $\mathrm{HN}$ & 121 & NZ & 25.31 \\
\hline 17 & $\mathrm{HN}$ & 106 & $\mathrm{NZ}$ & 27.07 & 18 & $\mathrm{HN}$ & 121 & NZ & 30.00 \\
\hline 18 & $\mathrm{HN}$ & 106 & $\mathrm{NZ}$ & 30.00 & 19 & $\mathrm{HN}$ & 121 & NZ & 23.71 \\
\hline 19 & $\mathrm{HN}$ & 106 & NZ & 30.00 & 20 & $\mathrm{HN}$ & 121 & NZ & 30.00 \\
\hline 20 & HN & 106 & $\mathrm{NZ}$ & 30.00 & 21 & $\mathrm{HN}$ & 121 & NZ & 25.92 \\
\hline 21 & $\mathrm{HN}$ & 106 & NZ & 30.00 & 22 & $\mathrm{HN}$ & 121 & NZ & 30.00 \\
\hline 23 & $\mathrm{HN}$ & 106 & NZ & 20.75 & 23 & $\mathrm{HN}$ & 121 & NZ & 28.36 \\
\hline 24 & $\mathrm{HN}$ & 106 & NZ & 20.97 & 24 & $\mathrm{HN}$ & 121 & $\mathrm{NZ}$ & 30.00 \\
\hline 26 & $\mathrm{HN}$ & 106 & NZ & 24.56 & 26 & $\mathrm{HN}$ & 121 & NZ & 28.58 \\
\hline 27 & $\mathrm{HN}$ & 106 & $\mathrm{NZ}$ & 20.89 & 29 & $\mathrm{HN}$ & 121 & $\mathrm{NZ}$ & 30.00 \\
\hline 29 & $\mathrm{HN}$ & 106 & $\mathrm{NZ}$ & 27.59 & 30 & $\mathrm{HN}$ & 121 & NZ & 30.00 \\
\hline 30 & HN & 106 & $\mathrm{NZ}$ & 21.53 & 31 & $\mathrm{HN}$ & 121 & NZ & 30.00 \\
\hline 31 & $\mathrm{HN}$ & 106 & NZ & 24.16 & 32 & $\mathrm{HN}$ & 121 & NZ & 30.00 \\
\hline 32 & $\mathrm{HN}$ & 106 & NZ & 30.00 & 33 & $\mathrm{HN}$ & 121 & NZ & 30.00 \\
\hline 33 & $\mathrm{HN}$ & 106 & NZ & 30.00 & 36 & $\mathrm{HN}$ & 121 & NZ & 30.00 \\
\hline 35 & $\mathrm{HN}$ & 106 & NZ & 30.00 & 38 & $\mathrm{HN}$ & 121 & NZ & 29.12 \\
\hline 36 & $\mathrm{HN}$ & 106 & $\mathrm{NZ}$ & 25.26 & 39 & $\mathrm{HN}$ & 121 & $\mathrm{NZ}$ & 25.06 \\
\hline 38 & $\mathrm{HN}$ & 106 & NZ & 30.00 & 43 & $\mathrm{HN}$ & 121 & NZ & 23.78 \\
\hline 39 & $\mathrm{HN}$ & 106 & NZ & 21.61 & 44 & $\mathrm{HN}$ & 121 & NZ & 22.77 \\
\hline 43 & $\mathrm{HN}$ & 106 & $\mathrm{NZ}$ & 15.81 & 45 & $\mathrm{HN}$ & 121 & $\mathrm{NZ}$ & 22.91 \\
\hline 44 & $\mathrm{HN}$ & 106 & NZ & 7.00 & 46 & $\mathrm{HN}$ & 121 & NZ & 20.68 \\
\hline 45 & $\mathrm{HN}$ & 106 & NZ & 15.79 & 47 & $\mathrm{HN}$ & 121 & NZ & 23.90 \\
\hline 46 & $\mathrm{HN}$ & 106 & $\mathrm{NZ}$ & 20.20 & 48 & $\mathrm{HN}$ & 121 & NZ & 20.80 \\
\hline 47 & $\mathrm{HN}$ & 106 & $\mathrm{NZ}$ & 7.00 & 49 & $\mathrm{HN}$ & 121 & NZ & 23.89 \\
\hline 48 & $\mathrm{HN}$ & 106 & $\mathrm{NZ}$ & 18.10 & 50 & $\mathrm{HN}$ & 121 & NZ & 22.71 \\
\hline 49 & $\mathrm{HN}$ & 106 & $\mathrm{NZ}$ & 17.85 & 51 & $\mathrm{HN}$ & 121 & NZ & 23.52 \\
\hline 50 & $\mathrm{HN}$ & 106 & $\mathrm{NZ}$ & 18.44 & 52 & $\mathrm{HN}$ & 121 & $\mathrm{NZ}$ & 21.25 \\
\hline 51 & $\mathrm{HN}$ & 106 & NZ & 15.84 & 53 & $\mathrm{HN}$ & 121 & NZ & 23.87 \\
\hline 52 & $\mathrm{HN}$ & 106 & NZ & 19.13 & 54 & $\mathrm{HN}$ & 121 & NZ & 25.20 \\
\hline 53 & $\mathrm{HN}$ & 106 & $\mathrm{NZ}$ & 18.82 & 58 & $\mathrm{HN}$ & 121 & NZ & 17.74 \\
\hline 54 & $\mathrm{HN}$ & 106 & NZ & 20.71 & 59 & $\mathrm{HN}$ & 121 & NZ & 21.29 \\
\hline 57 & $\mathrm{HN}$ & 106 & NZ & 21.56 & 60 & $\mathrm{HN}$ & 121 & NZ & 17.33 \\
\hline 58 & $\mathrm{HN}$ & 106 & $\mathrm{NZ}$ & 23.01 & 61 & $\mathrm{HN}$ & 121 & NZ & 23.01 \\
\hline 59 & $\mathrm{HN}$ & 106 & $\mathrm{NZ}$ & 20.73 & 62 & $\mathrm{HN}$ & 121 & $\mathrm{NZ}$ & 18.66 \\
\hline 60 & $\mathrm{HN}$ & 106 & $\mathrm{NZ}$ & 19.34 & 63 & $\mathrm{HN}$ & 121 & $\mathrm{NZ}$ & 15.61 \\
\hline 61 & $\mathrm{HN}$ & 106 & NZ & 18.49 & 64 & $\mathrm{HN}$ & 121 & NZ & 19.09 \\
\hline 62 & $\mathrm{HN}$ & 106 & NZ & 21.43 & 66 & $\mathrm{HN}$ & 121 & NZ & 13.61 \\
\hline 63 & $\mathrm{HN}$ & 106 & NZ & 23.48 & & & & & \\
\hline 64 & $\mathrm{HN}$ & 106 & $\mathrm{NZ}$ & 22.74 & & & & & \\
\hline
\end{tabular}

NZ is the nitrogen of the MTSL ring.

Table C.9: Intermolecular NOE distances of CylR2 which were manually assigned.

\begin{tabular}{|c|c|c|c|c|c|c|c|c|c|}
\hline Residue 1 & H1 & Residue 2 & $\mathrm{H} 2$ & distance $[\AA]$ & Residue 1 & H1 & Residue 2 & $\mathrm{H} 2$ & distance $[\AA]$ \\
\hline 47 & HG & 127 & $\mathrm{HB} \star$ & 4.0 & 114 & HA & 63 & HE3 & 4.0 \\
\hline 113 & HG & 61 & $\mathrm{HB} \star$ & 4.0 & 48 & HA & 129 & HE3 & 4.0 \\
\hline 47 & HG & 124 & HG2 & 4.0 & 114 & $\mathrm{HE \star}$ & 63 & HH2 & 4.0 \\
\hline 113 & HG & 58 & HG2 & 4.0 & 48 & 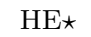 & 129 & HH2 & 4.0 \\
\hline 47 & $\mathrm{HG}$ & 124 & HG1 & 4.0 & 114 & HG2 & 63 & HH2 & 4.0 \\
\hline 113 & HG & 58 & HG1 & 4.0 & 48 & HG2 & 129 & HH2 & 4.0 \\
\hline 44 & $\mathrm{HA}$ & 68 & $\mathrm{HG} 2 \star$ & 4.0 & 117 & HD1 & 63 & HE3 & 4.0 \\
\hline 110 & HA & 2 & $\mathrm{HG} 2 \star$ & 4.0 & 51 & HD1 & 129 & HE3 & 4.0 \\
\hline 41 & HG2 & 109 & HB1 & 4.0 & 117 & HD1 & 63 & HZ2 & 4.0 \\
\hline 107 & HG2 & 43 & HB1 & 4.0 & 51 & HD1 & 129 & HZ2 & 4.0 \\
\hline 47 & HD1* & 129 & HB1 & 4.0 & 117 & $\mathrm{HB} \star$ & 63 & HZ2 & 4.0 \\
\hline 113 & HD1* & 63 & HB1 & 4.0 & 51 & $\mathrm{HB} \star$ & 129 & HZ2 & 4.0 \\
\hline 48 & HG2 & 129 & HZ2 & 4.0 & 110 & HA & 63 & HD1 & 4.0 \\
\hline 114 & HG2 & 63 & HZ2 & 4.0 & 44 & HA & 129 & HD1 & 4.0 \\
\hline 47 & HA & 129 & HB1 & 4.0 & 113 & $\mathrm{HD} 2 \star$ & 63 & HB2 & 4.0 \\
\hline 113 & HA & 63 & HB1 & 4.0 & 47 & HD2 $2 \star$ & 129 & HB2 & 4.0 \\
\hline
\end{tabular}




\begin{tabular}{|c|c|c|c|c|c|c|c|c|c|}
\hline Residue 1 & H1 & Residue 2 & $\mathrm{H} 2$ & distance $[\AA]$ & Residue 1 & H1 & Residue 2 & $\mathrm{H} 2$ & distance $[\check{A}]$ \\
\hline 48 & HA & 129 & HE3 & 4.0 & 113 & HB2 & 63 & HB2 & 4.0 \\
\hline 114 & HA & 63 & HE3 & 4.0 & 47 & HB2 & 129 & HB2 & 4.0 \\
\hline 47 & HB1 & 129 & HB1 & 4.0 & 113 & HB1 & 63 & HB2 & 4.0 \\
\hline 113 & HB1 & 63 & HB1 & 4.0 & 47 & HB1 & 129 & HB2 & 4.0 \\
\hline 44 & $\mathrm{HG} \star$ & 129 & $\mathrm{HB} \star$ & 4.0 & 113 & $\mathrm{HD} 2 \star$ & 63 & HB1 & 4.0 \\
\hline 110 & $\mathrm{HG} \star$ & 63 & $\mathrm{HB} \star$ & 4.0 & 47 & $\mathrm{HD} 2 \star$ & 129 & HB1 & 4.0 \\
\hline 114 & $\mathrm{HE} \star$ & 63 & $\mathrm{HZ2}$ & 4.0 & 113 & $\mathrm{HB} \star$ & 63 & HB1 & 4.0 \\
\hline 48 & $\mathrm{HE} \star$ & 129 & $\mathrm{HZ2}$ & 4.0 & 47 & $\mathrm{HB} \star$ & 129 & HB1 & 4.0 \\
\hline
\end{tabular}

\section{C.3 Dihedral angle restraints for CyIR2}

Table C.10: Dihedral angle restraints for CylR2 derived from the chemical shifts with TALOS.

\begin{tabular}{|c|c|c|c|c|c|c|c|}
\hline Residue & PHI & lower angle & upper angle & Residue & PSI & lower angle & upper angle \\
\hline 2 & -140.0 & -100.0 & 2 & 127.3 & 156.5 & & \\
\hline 3 & -140.0 & -99.5 & 3 & 112.0 & 129.2 & & \\
\hline 4 & -140.0 & -99.6 & 4 & 124.0 & 144.7 & & \\
\hline 6 & -70.0 & -40.0 & 6 & -60.0 & -20.0 & & \\
\hline 7 & -66.9 & -54.7 & 7 & -50.6 & -40.4 & & \\
\hline 8 & -67.9 & -56.3 & 8 & -45.2 & -35.9 & & \\
\hline 9 & -76.2 & -58.2 & 9 & -51.4 & -38.7 & & \\
\hline 10 & -66.4 & -56.2 & 10 & -45.7 & -33.8 & & \\
\hline 11 & -68.5 & -61.5 & 11 & -44.4 & -34.6 & & \\
\hline 12 & -67.5 & -59.1 & 12 & -37.2 & -19.8 & & \\
\hline 17 & -63.3 & -57.2 & 17 & -45.0 & -30.5 & & \\
\hline 18 & -67.5 & -59.2 & 18 & -48.3 & -28.7 & & \\
\hline 19 & -71.0 & -59.6 & 19 & -49.3 & -38.1 & & \\
\hline 20 & -72.3 & -59.4 & 20 & -43.3 & -33.1 & & \\
\hline 21 & -70.1 & -58.9 & 21 & -49.1 & -33.6 & & \\
\hline 22 & -70.7 & -60.7 & 22 & -45.1 & -36.7 & & \\
\hline 23 & -72.3 & -61.3 & 23 & -38.8 & -21.4 & & \\
\hline 24 & -104.4 & -85.2 & 24 & -4.6 & 14.6 & & \\
\hline 28 & -60.9 & -52.7 & 28 & -45.9 & -28.9 & & \\
\hline 29 & -73.4 & -59.8 & 29 & -49.9 & -29.1 & & \\
\hline 30 & -71.6 & -56.1 & 30 & -50.8 & -28.0 & & \\
\hline 31 & -72.8 & -60.0 & 31 & -44.5 & -31.5 & & \\
\hline 32 & -69.5 & -52.3 & 32 & -49.4 & -38.2 & & \\
\hline 33 & -66.0 & -60.4 & 33 & -45.4 & -32.6 & & \\
\hline 34 & -71.6 & -59.5 & 34 & -47.4 & -36.6 & & \\
\hline 35 & -71.4 & -60.0 & 35 & -35.5 & -18.8 & & \\
\hline 43 & -70.0 & -40.0 & 43 & -60.0 & -20.0 & & \\
\hline 44 & -62.6 & -56.6 & 44 & -49.9 & -36.3 & & \\
\hline 45 & -69.3 & -57.9 & 45 & -48.3 & -33.6 & & \\
\hline 46 & -71.9 & -60.6 & 46 & -48.3 & -40.3 & & \\
\hline 47 & -70.3 & -61.7 & 47 & -45.1 & -34.9 & & \\
\hline 48 & -68.4 & -60.5 & 48 & -47.9 & -35.5 & & \\
\hline 49 & -69.1 & -61.9 & 49 & -44.4 & -37.9 & & \\
\hline 50 & -63.9 & -54.9 & 50 & -45.8 & -33.7 & & \\
\hline 51 & -70.0 & -58.8 & 51 & -51.3 & -35.1 & & \\
\hline 52 & -72.8 & -57.9 & 52 & -41.7 & -29.3 & & \\
\hline 57 & -66.9 & -54.9 & 57 & -40.6 & -21.3 & & \\
\hline 58 & -71.2 & -59.6 & 58 & -44.0 & -28.4 & & \\
\hline 59 & -70.0 & -30.0 & 59 & -60.0 & -20.0 & & \\
\hline 60 & -70.0 & -30.0 & 60 & -60.0 & -20.0 & & \\
\hline 61 & -142.9 & -112.9 & 61 & 142.3 & 162.7 & & \\
\hline 62 & -146.4 & -105.6 & 62 & 126.0 & 147.8 & & \\
\hline 63 & -123.2 & -90.2 & 63 & 118.4 & 135.1 & & \\
\hline
\end{tabular}

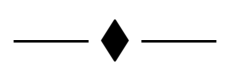




\section{Appendix: Xplor input files}

Xplor input files used for water refinement, rigid-body docking and simulated annealing in chapter 5 are specified in the following.

\section{D.1 Water refinement}

The protocol used for water refinement is given below. It is slightly different from the one in literature [172], [173].

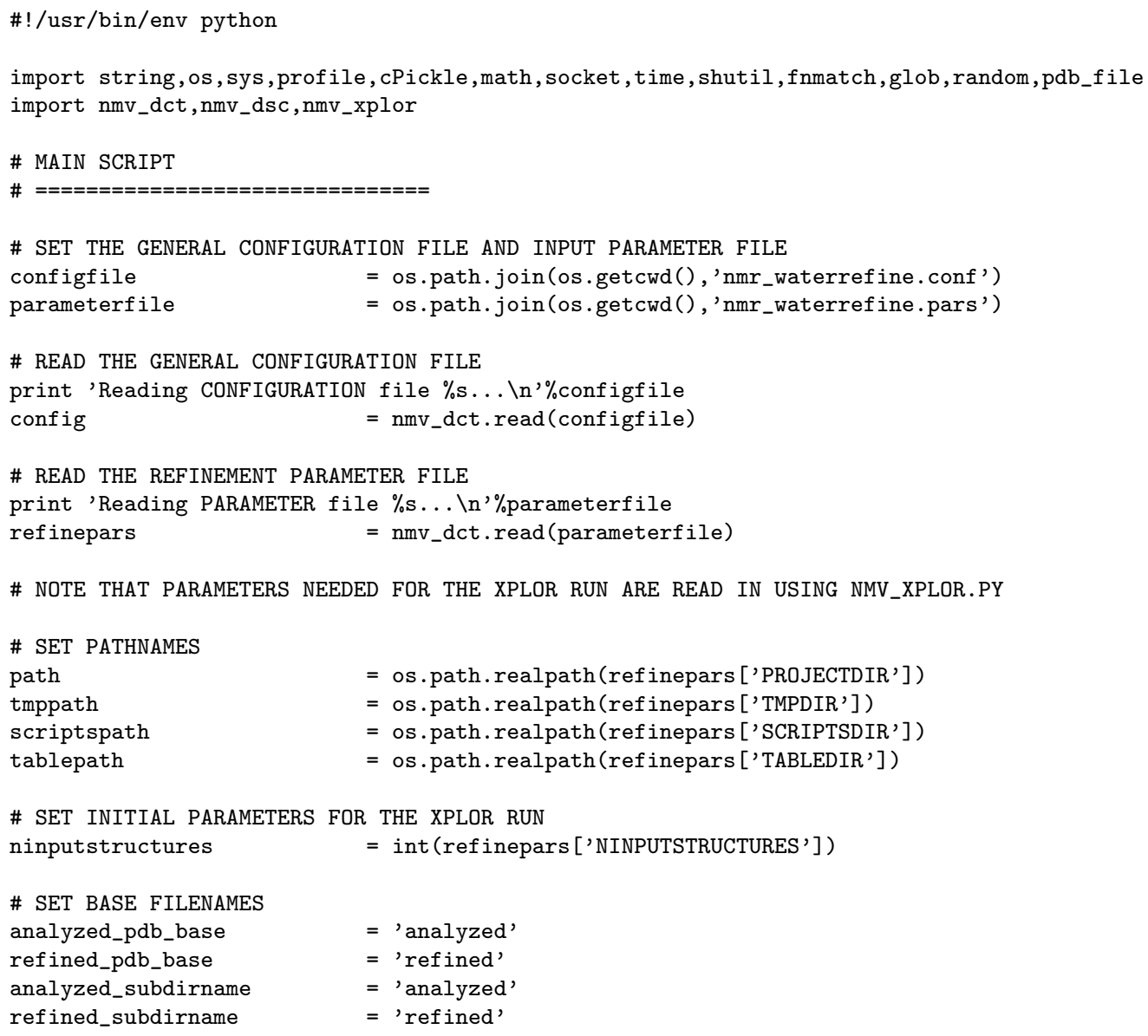




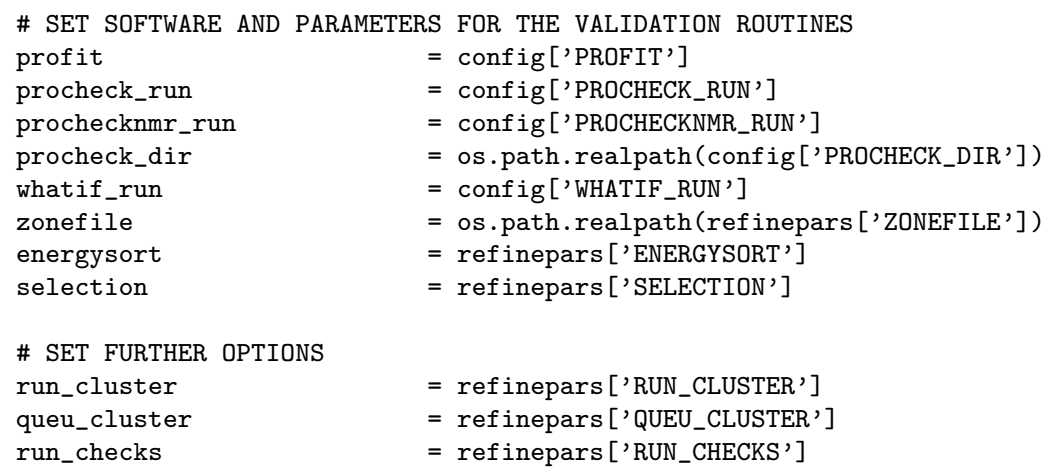

\#\#\#\#\#\#\#\#\#\#\#\#\#\#\#\#\#\#\#\#\#\#\#\#\#\#

\# ANALYSIS INPUT STRUCTURES \#

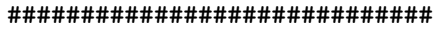

\# CHECK WHETHER ANALYSIS WAS DONE

\# SET THE OUTPUTCOORDINATESPATH FOR THE ANALYSIS OF THE INPUT STRUCTURES

outputcoordinatespath = os.path.join(path, analyzed_subdirname)

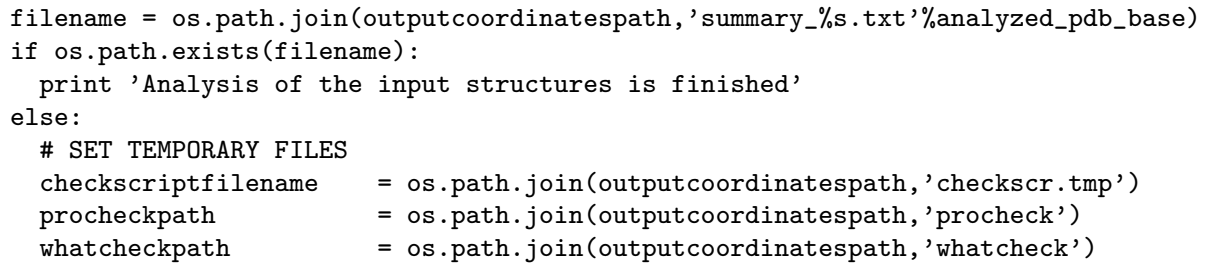

\# RE-CREATE ANALYSIS DIRECTORY/CREATE ANALYSIS DIRECTORY IF NOT PRESENT:

\# REMOVE ALL PREVIOUS TEMPORARY FILES FOR CLEAN START

if os.path.exists (outputcoordinatespath):

print 'Cleaning directory $\% \mathrm{~s} \%$ outputcoordinatespath

nmv_dsc.removedir (outputcoordinatespath)

os.mkdir (outputcoordinatespath)

else:

print 'Creating directory \%s\%outputcoordinatespath

os.mkdir (outputcoordinatespath)

\# ANALYZE THE INPUT STRUCTURES IN THE XPLOR FORCEFIELD USED FOR THE WATERREFINEMENT

\# INCLUDES VIOLATION AND ENERGY ANALYSIS

\# THE NMV_XPLOR MODULE CREATES THE SCRIPTS NEEDED TO CALCULATE STRUCTURES ON A CLUSTER

\# USING THE PARAMETER AND CONFIG FILES

\# AND GENERATES A 'jobs' SUBDIRECTORY(=RUNPATH) IN WHICH THE XPLOR RUN IS PERFORMED

nmv_xplor.analyze (parameterfile, configfile)

\# CHECK EVERY 10 SECS WHETHER ALL STRUCTURES ARE ANALYZED (NEEDED WHEN RUNNING ON A CLUSTER)

\# todo: check acceptance criteria and loop until we have enough accepted structures

done $=0$

while not done:

time.sleep (10)

if len (glob.glob(os.path.join (outputcoordinatespath, analyzed_pdb_base+' *.pdb')) )==ninputstructures : done $=1$

\# RUN THE OTHER CHECKS

\# CREATE INPUT SCRIPTS FOR THE CHECKING ROUTINES

print 'Preparing for protein structure checks of the analyzed input structures'

checkscript=open (checkscriptfilename, 'w')

checkscript.write('\#!/bin/tcsh\n')

\# SET ENVIRONMENT VARIABLES

checkscript.write('setenv prodir $\%$ s $\backslash n$ \% $\%$ procheck_dir)

\# WRITE A XPLOR FILE-LIST CONTAINING THE INFORMATION ABOUT THE ANALYZED INPUT STRUCTURES 
\# FILES ARE SORTED ON RESTRAINT ENERGY (SET ENERGYSORT), THE FUNCTION USES THE PDB-HEADER INFORMATION checkscript.write( $\%$ s -writexplorfilelist $\%$ s $\%$ s \%s $\backslash n$ '\%(os.path.join(scriptspath, 'nmv_nmrcheck.py'), outputcoordinatespath, $\backslash$ analyzed_pdb_base, 1 energysort))

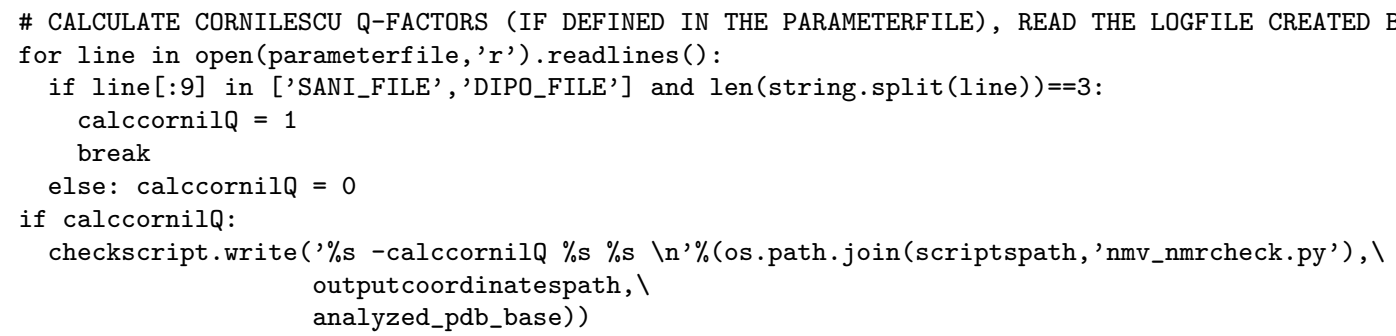

\# AUTOMATICALLY CHECK THE ANALYZED INPUT STRUCTURES WITH PROCHECK AND WHATIF \# PROFIT IS USED FOR RMSD CALCULATIONS

if run_checks=='y':

checkscript.write( $\%$ s -checkall $\%$ s $\%$ s $\%$ s $\%$ s $\%$ s $\%$ s $\%$ s $\%$ s $\%$ s $\%$ s $\backslash$ n $\%$ (os.path.join(scriptspath, 'nmv_nmrcheck.py'), profit, $\backslash$

procheck_run, I prochecknmr_run, $\backslash$ procheck_dir,l

whatif_run, $\backslash$

outputcoordinatespath, $\backslash$

analyzed_pdb_base, $\backslash$

zonefile,

selection,

tmppath))

elif run_checks=='n':

print 'Skipping WHATIF, PROCHECK protein structure checks, and PROFIT rmsd calculations'

\# CREATE A SUMMARY FILE THAT CONTAINS ALL INFORMATION

checkscript.write( $\%$ s - summary \%s \% \n'\%(os.path.join(scriptspath, 'nmv_nmrcheck.py'), । outputcoordinatespath, $\backslash$ analyzed_pdb_base))

\# CLOSE THE SCRIPTFILE

checkscript.close()

\# SUBMIT THE SCRIPT EITHER TO THE CLUSTER OR ON A SINGLE PROCESSOR MACHINE

os.system('/bin/chmod $+\mathrm{x} \% \mathrm{~s} \%$ checkscriptfilename)

print 'Starting structure checks of the analyzed input structures'

if run_cluster==' $y$ ':

os.system( $\%$ s \% \& $\%$ (queu_cluster, checkscriptfilename))

else:

os.system( $\%$ s $\%$ checkscriptfilename)

\# CHECK EVERY 10 SECS WHETHER THE CHECKING ROUTINES ARE FINISHED (NEEDED WHEN RUNNING ON A CLUSTER) done $=0$

while not done:

time.sleep (10)

if os.path.exists(os.path.join(outputcoordinatespath, 'summary_\%.txt \%analyzed_pdb_base)): done $=1$

\# COMPRESS JOBS, PROCHECK AND WHATCHECK SUBDIRECTORIES

print 'Compressing files...'

current_location=os.getcwd()

os.chdir (outputcoordinatespath)

os.system('tar cf - jobs | gzip > jobs.tgz')

if run_checks=='y':

os.system('tar cf - procheck | gzip > procheck.tgz')

os.system('tar cf - whatcheck | gzip > whatcheck.tgz')

os.chdir(current_location)

\# REMOVE THE SCRIPTFILES AND PROCHECK AND WHATCHECK DIRECTORIES 


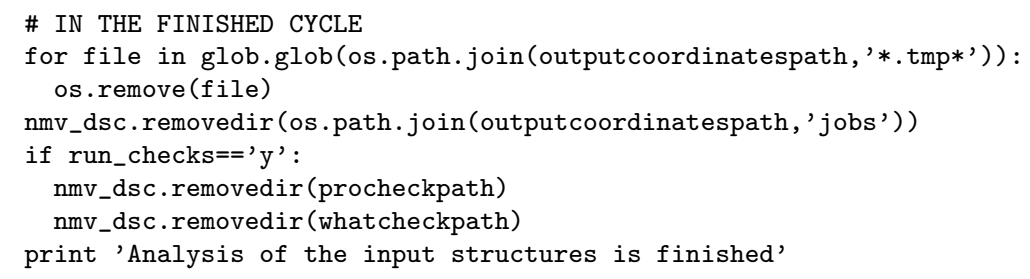

\#\#\#\#\#\#\#\#\#\#\#\#\#\#\#\#\#\#\#\#\#\#\#\#\#\#\#\#\#\#\#

\# REFINEMENT OF INPUT STRUCTURES \#

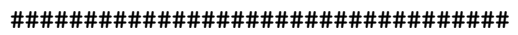

\# SET THE OUTPUTCOORDINATESPATH FOR THE ACTUAL REFINEMENT OF THE INPUT STRUCTURES

outputcoordinatespath = os.path.join(path,refined_subdirname)

\# CHECK WHETHER ANALYSIS WAS DONE

filename = os.path.join (outputcoordinatespath, 'summary_\%s.txt,\%refined_pdb_base)

if os.path.exists (filename):

print 'Analysis of refined structures is finished'

else:

\# SET TEMPORARY FILES

$\begin{array}{ll}\text { checkscriptfilename } & \text { os.path.join (outputcoordinatespath, 'checkscr.tmp') } \\ \text { procheckpath } & =\text { os.path.join (outputcoordinatespath, 'procheck') }\end{array}$

whatcheckpath $\quad$ os.path.join (outputcoordinatespath, 'whatcheck')

\# CREATE REFINED STRUCTURES DIRECTORY IF NOT PRESENT:

if not os.path.exists (outputcoordinatespath):

print 'Creating directory $\%$ s\%outputcoordinatespath

os.mkdir (outputcoordinatespath)

\# DO THE ACTUAL WATERREFINEMENT

nmv_xplor. waterrefine (parameterfile, configfile)

\# CHECK EVERY 10 SECS WHETHER ALl STRUCTURES ARE CALCULATED (NEEDED WHEN RUNNing ON A CLUSTER)

\# check acceptance criteria and loop until we have enough accepted structures

done $=0$

while not done:

time.sleep (10)

if len (glob.glob (os.path.join (outputcoordinatespath, refined_pdb_base+' *.pdb*')) ) ==ninputstructures: done $=1$

\# RUN THE OTHER CHECKS

\# CREATE INPUT SCRIPTS FOR THE CHECKING ROUTINES

print 'Preparing for protein structure checks of the refined input structures'

checkscript=open (checkscriptfilename, ' $w$ ')

checkscript.write('\#!/bin/tcsh \n')

\# SET ENVIRONMENT VARIABLES

checkscript.write('setenv prodir $\%$ s $\backslash n$ \% $\%$ procheck_dir)

\# WRITE A XPLOR FILE-LIST CONTAINING THE INFORMATION ABOUT THE ANALYZED INPUT STRUCTURES

\# FILES ARE SORTED ON RESTRAINT ENERGY (SET ENERGYSORT), THE FUNCTION USES THE PDB-HEADER INFORMATION

checkscript.write( $\%$ s -writexplorfilelist \%s \%s \%s $\backslash n$ \% (os.path.join (scriptspath, 'nmv_nmrcheck.py'), । outputcoordinatespath, 1 refined_pdb_base, 1 energysort))

\# CALCULATE CORNILESCU Q-FACTORS (IF DEFINED IN THE PARAMETERFILE), READ THE LOGFILE CREATED BY XPLOR for line in open(parameterfile, ' $r$ ').readlines():

if line [:9] in ['SANI_FILE','DIPO_FILE'] and len(string.split(line))==3:

calccornilQ $=1$

break

else: calccornilQ $=0$

if calccornilQ:

checkscript.write('\%s -calccornilQ \%s \%s \n\% (os.path.join(scriptspath, 'nmv_nmrcheck.py'), outputcoordinatespath, $\backslash$ 
refined_pdb_base))

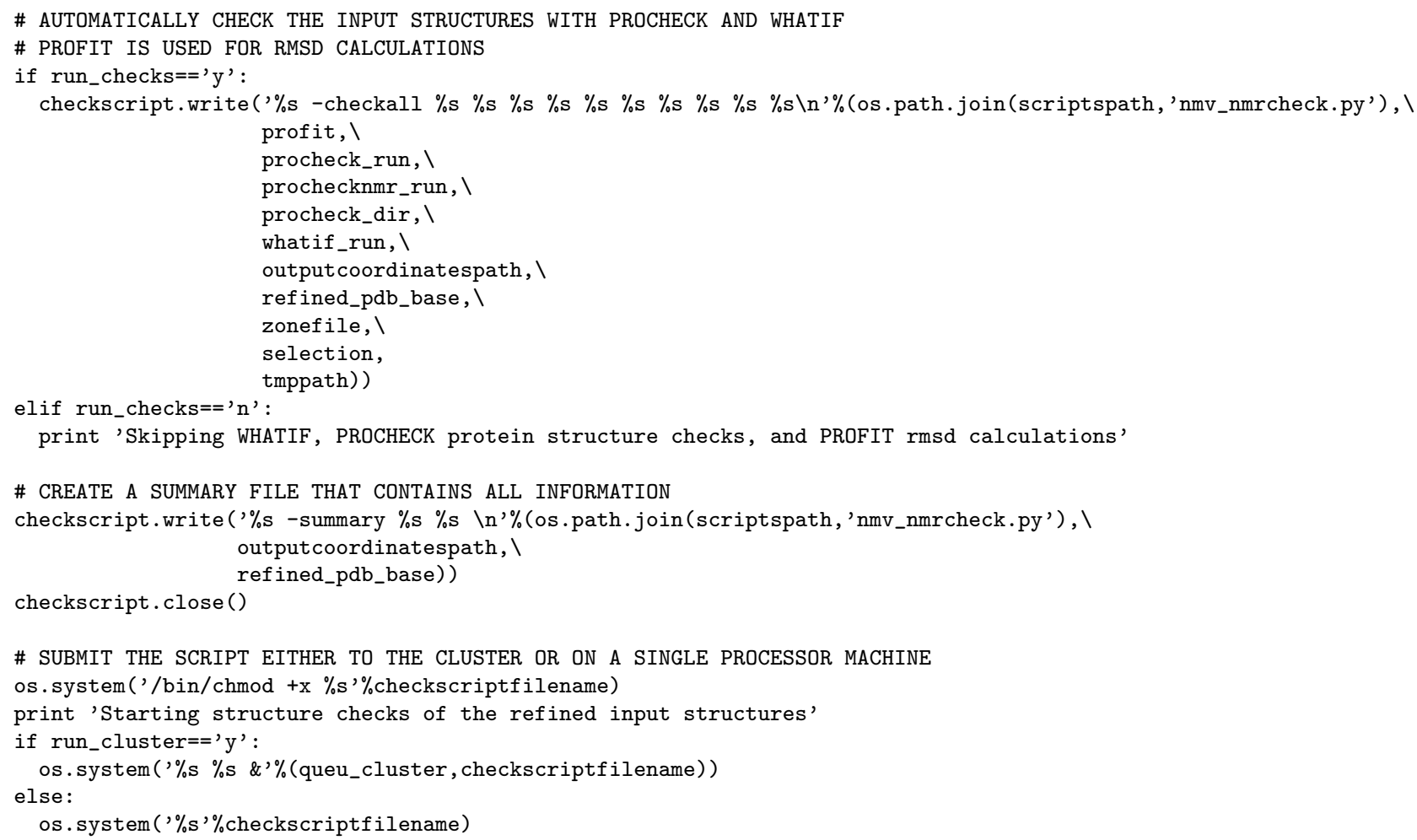


An example parameter file as used for the refinement of monomeric CylR2:

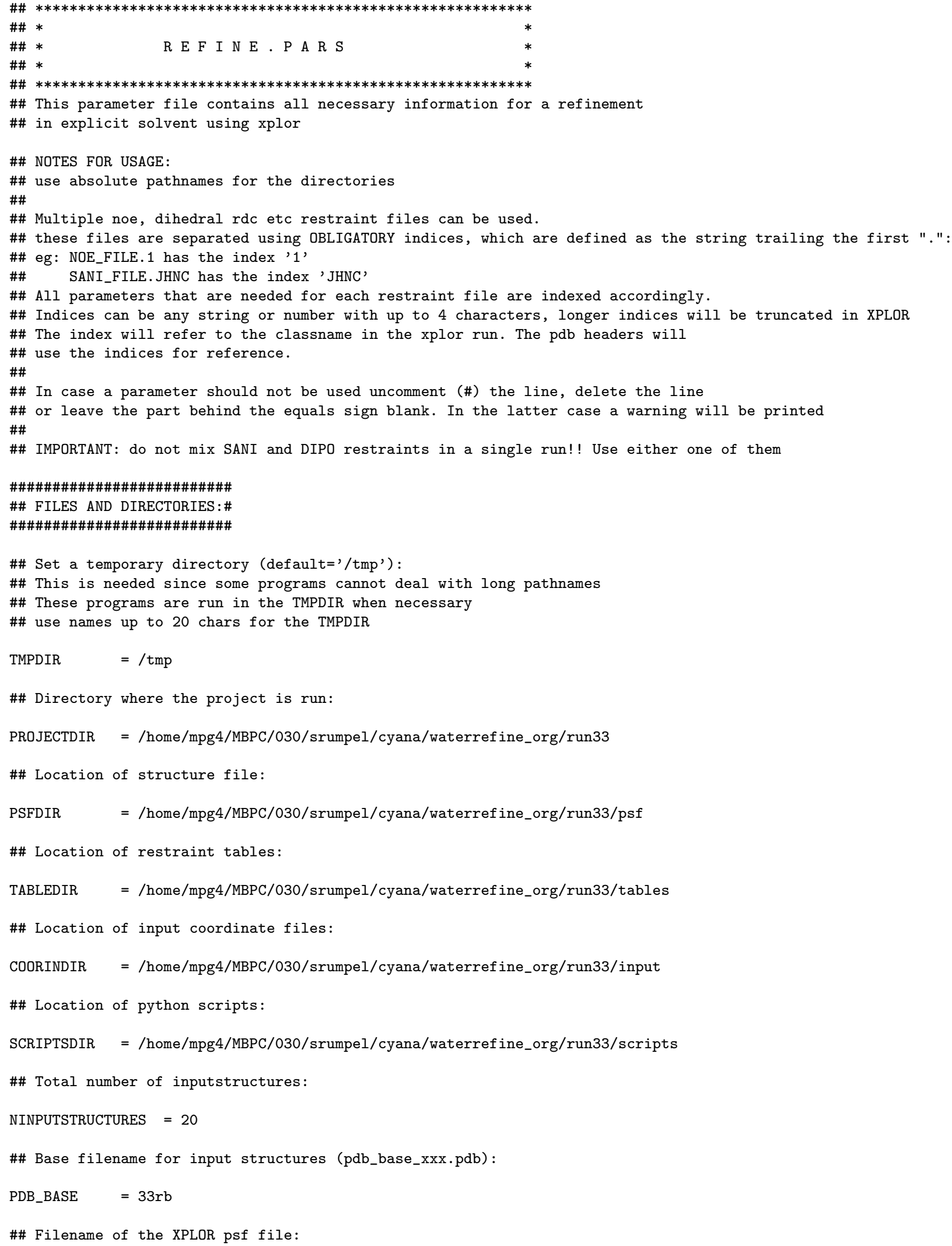




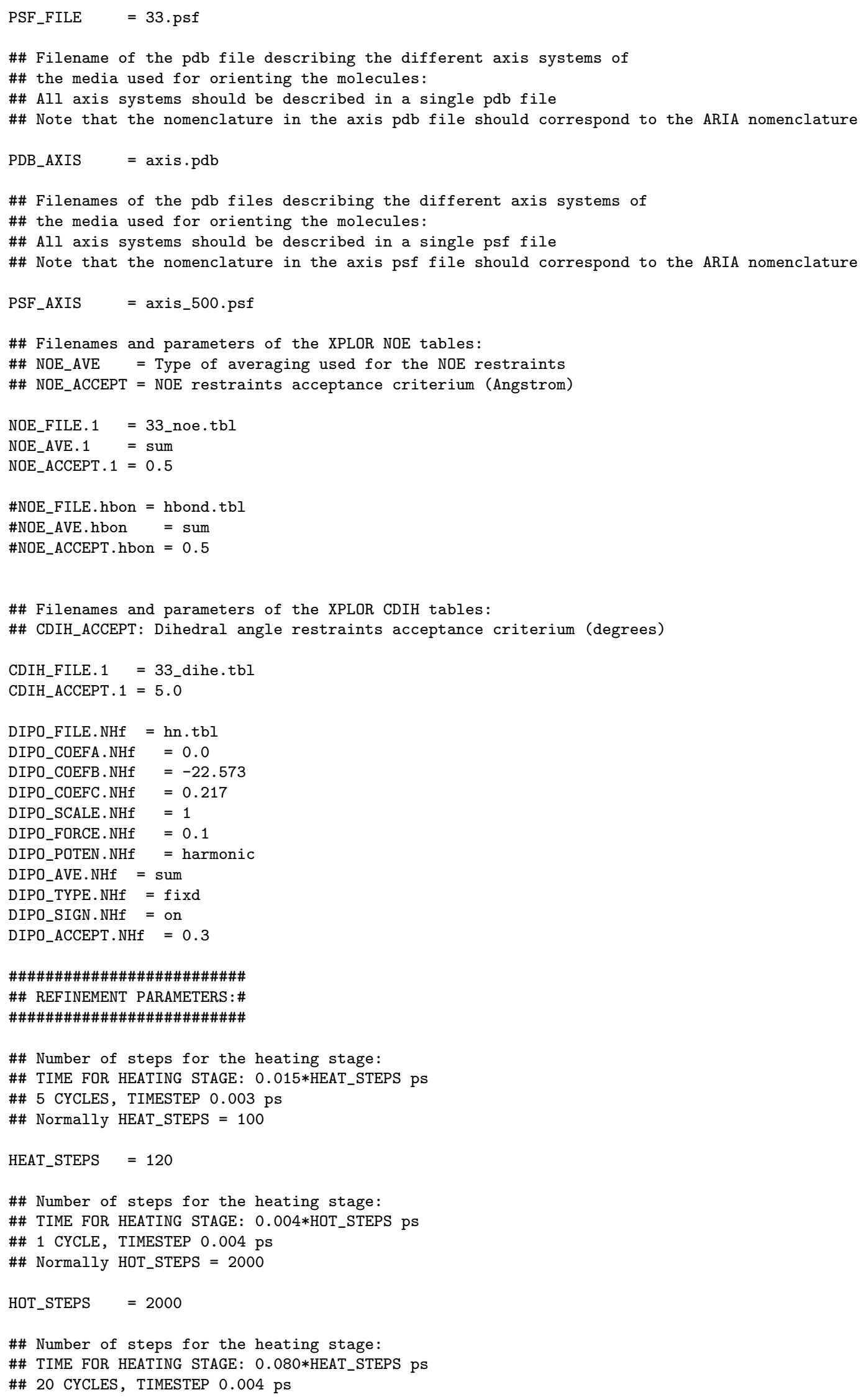

\#\# Number of steps for the heating stage:

\#\# TIME FOR HEATING STAGE: $0.015 *$ HEAT_STEPS ps

\#\# 5 CYCLES, TIMESTEP 0.003 ps

\#\# Normally HEAT_STEPS $=100$

HEAT_STEPS $=120$

\#\# Number of steps for the heating stage:

\#\# TIME FOR HEATING STAGE: $0.004 *$ HOT_STEPS ps

\#\# 1 CYCLE, TIMESTEP $0.004 \mathrm{ps}$

\#\# Normally HOT_STEPS $=2000$

HOT_STEPS $\quad=2000$

\#\# Number of steps for the heating stage:

\#\# TIME FOR HEATING STAGE: $0.080 *$ HEAT_STEPS ps

\#\# 20 CYCLES, TIMESTEP $0.004 \mathrm{ps}$ 


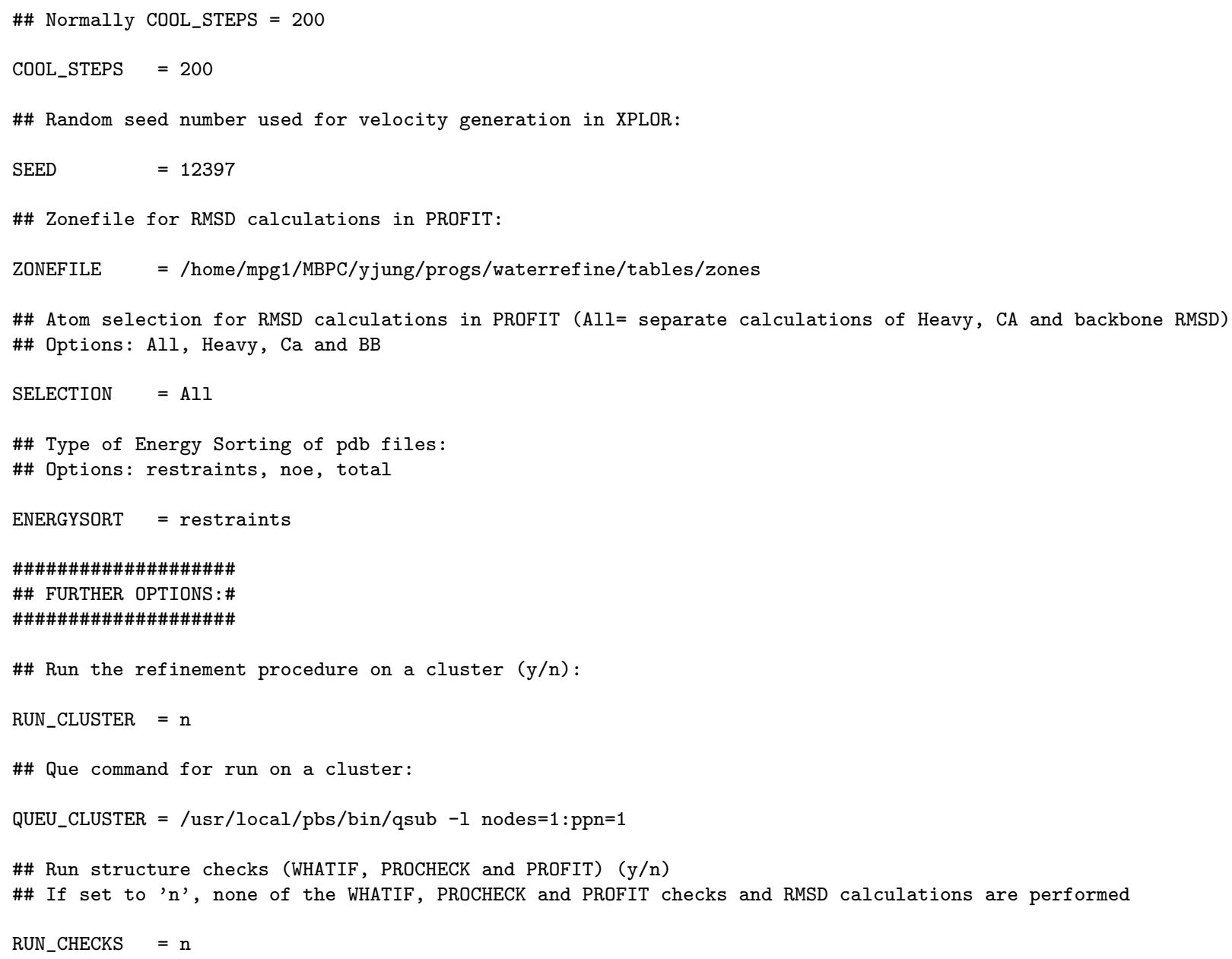

For other refinements the filenames and paths as well as the dipolar coupling coefficients were changed.

\section{D.2 Rigid body docking}

The Xplor input file for rigid-body docking described by Clore [89] was slightly changed. The used input file is indicated.

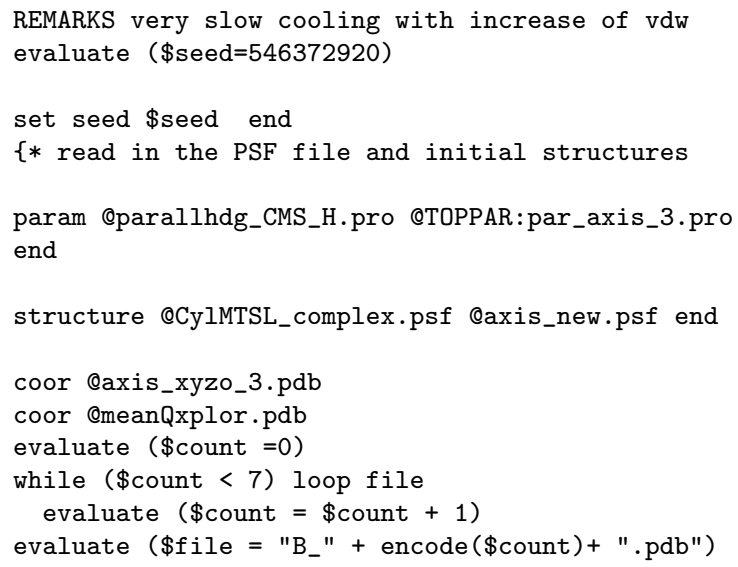




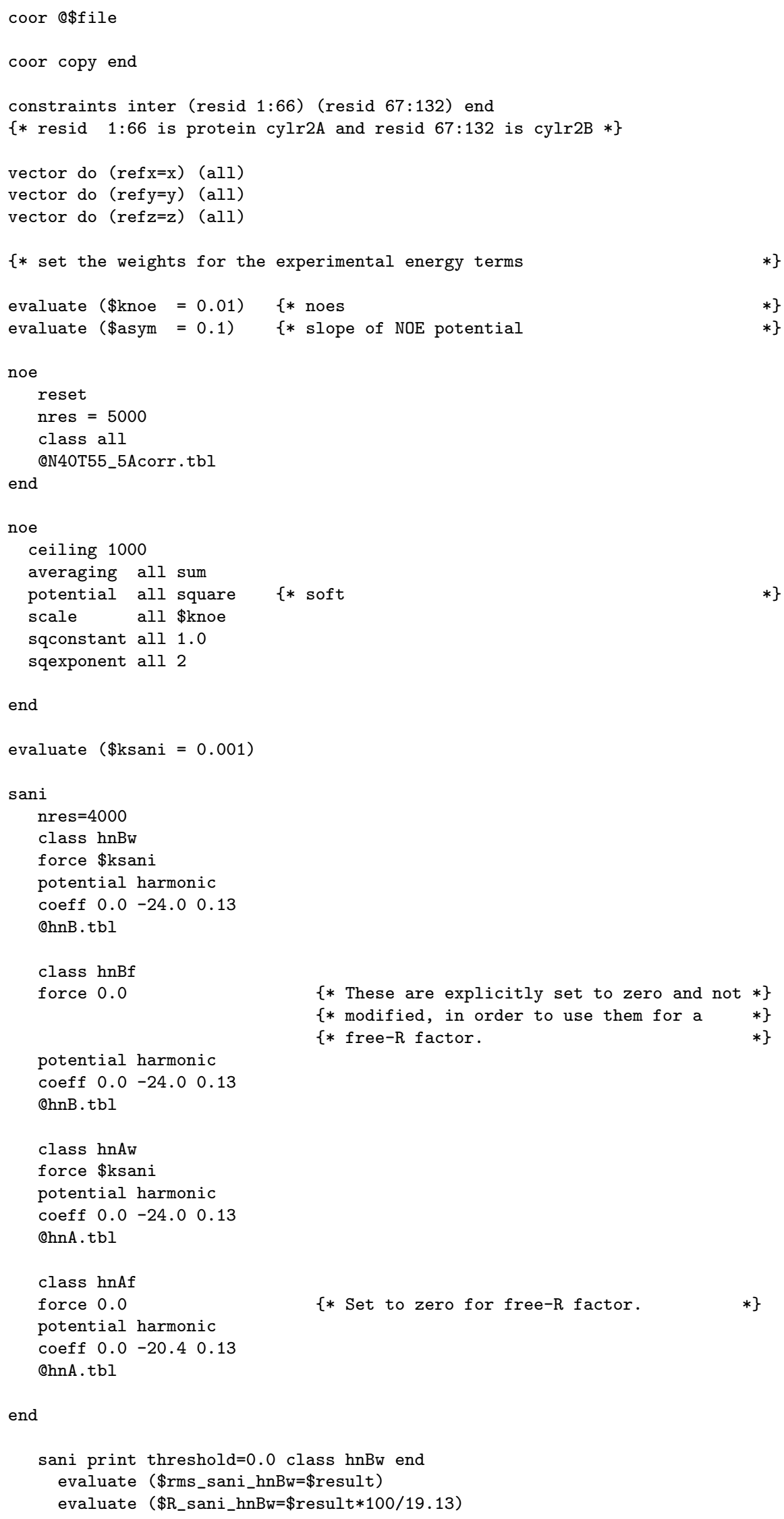




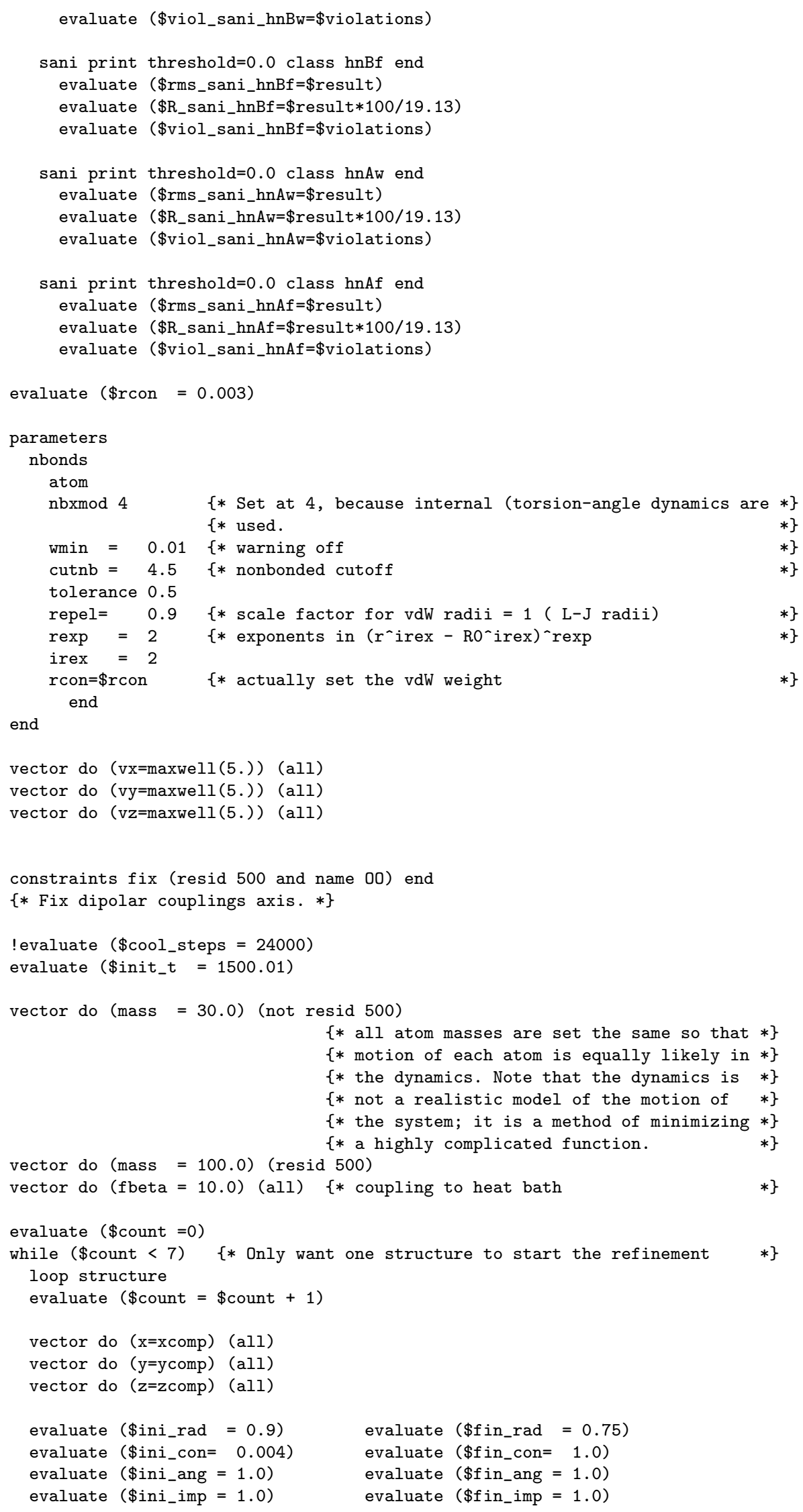




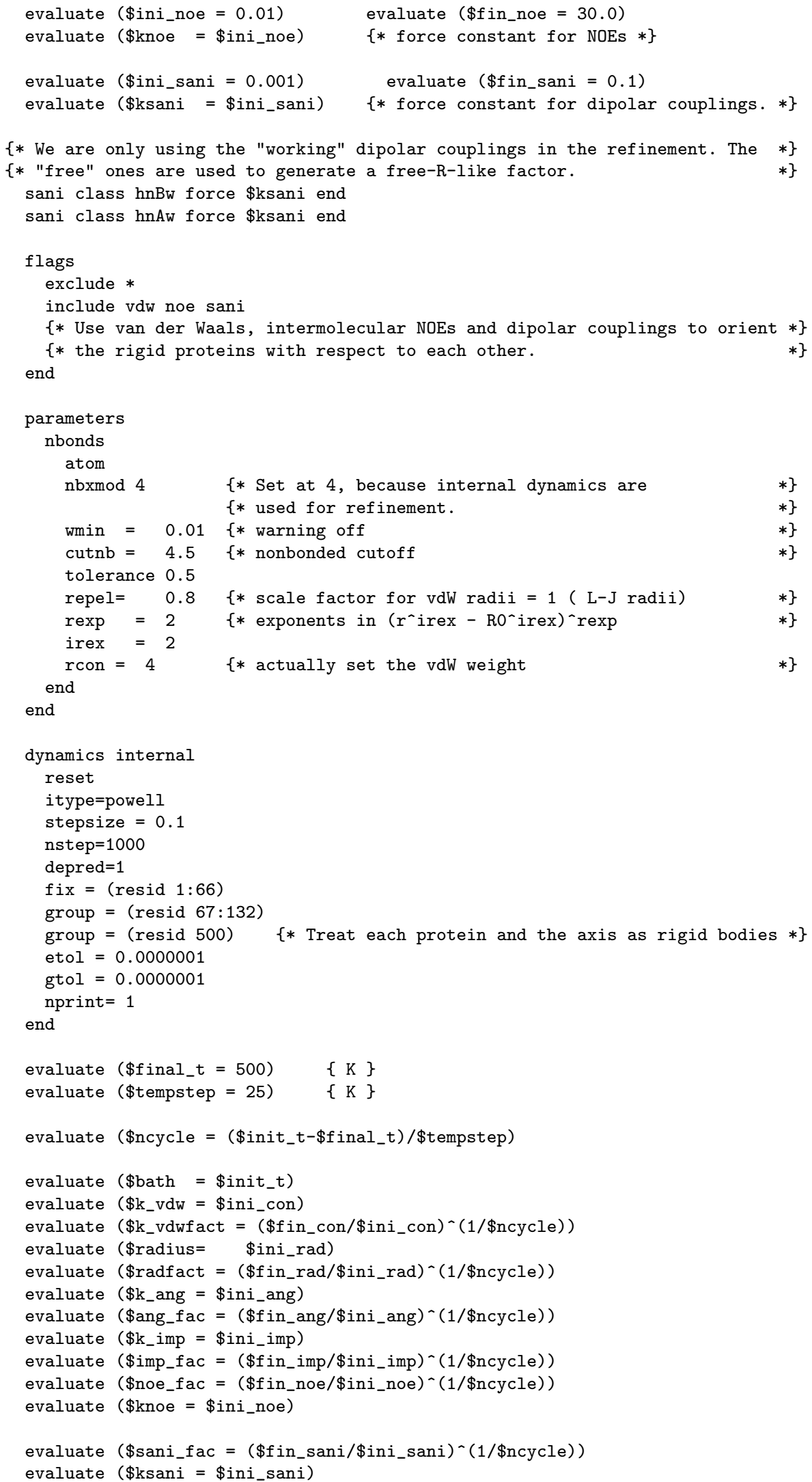




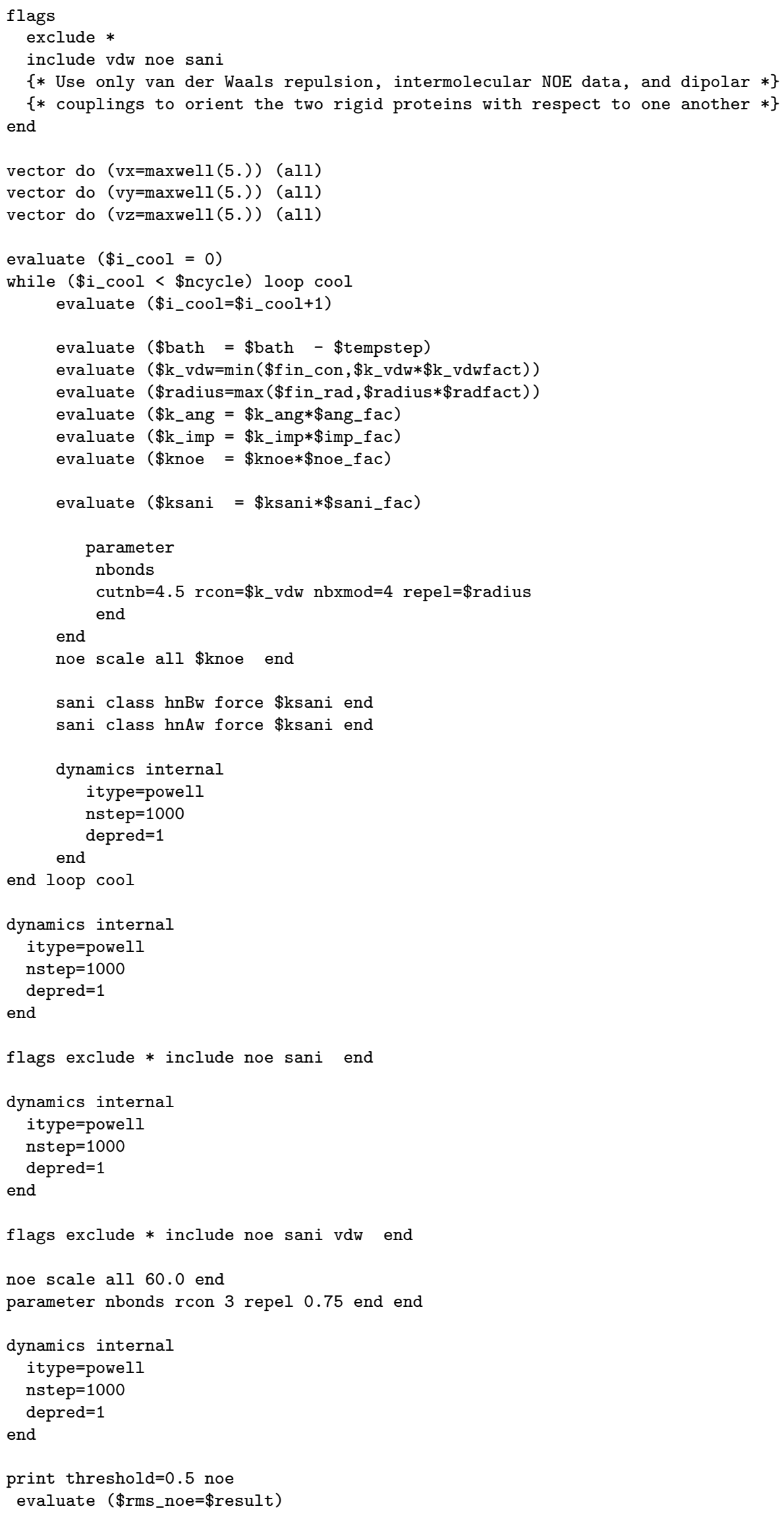


evaluate (\$violations_noe=\$violations)

print thres $=0.05$ bonds

evaluate ( $\$ r m s \_b o n d s=\$ r e s u l t$ )

print thres $=5$. angles

evaluate ( $\left.\$ r m s \_a n g l e s=\$ r e s u l t\right)$

print thres $=5$. impropers

evaluate (\$rms_impropers $=\$$ result)

sani print threshold $=0.0 \mathrm{class} \mathrm{hnBw}$ end

evaluate ( $\left.\$ r m s \_s a n i \_h n B w=\$ r e s u l t\right)$

evaluate ( $\$$ R_sani_hnBw $=\$$ result $* 100 / 19.13$ )

evaluate (\$viol_sani_hnBw=\$violations)

sani print threshold $=0.0$ class $\mathrm{hnBf}$ end

evaluate (\$rms_sani_hnBf=\$result)

evaluate ( $\$ R_{-}$sani_hnBf=\$result*100/19.13)

evaluate（\$viol_sani_hnBf=\$violations)

sani print threshold $=0.0$ class hnAw end

evaluate ( $\left.\$ r m s \_s a n i \_h n A w=\$ r e s u l t\right)$

evaluate（\$R_sani_hnAw=\$result*100/19.13)

evaluate (\$viol_sani_hnAw=\$violations)

sani print threshold $=0.0$ class hnAf end

evaluate ( $\$ r m s \_s a n i \_h n A f=\$ r e s u l t$ )

evaluate ( $\$$ R_sani_hnAf $=\$$ result $* 100 / 19.13$ )

evaluate (\$viol_sani_hnAf=\$violations)

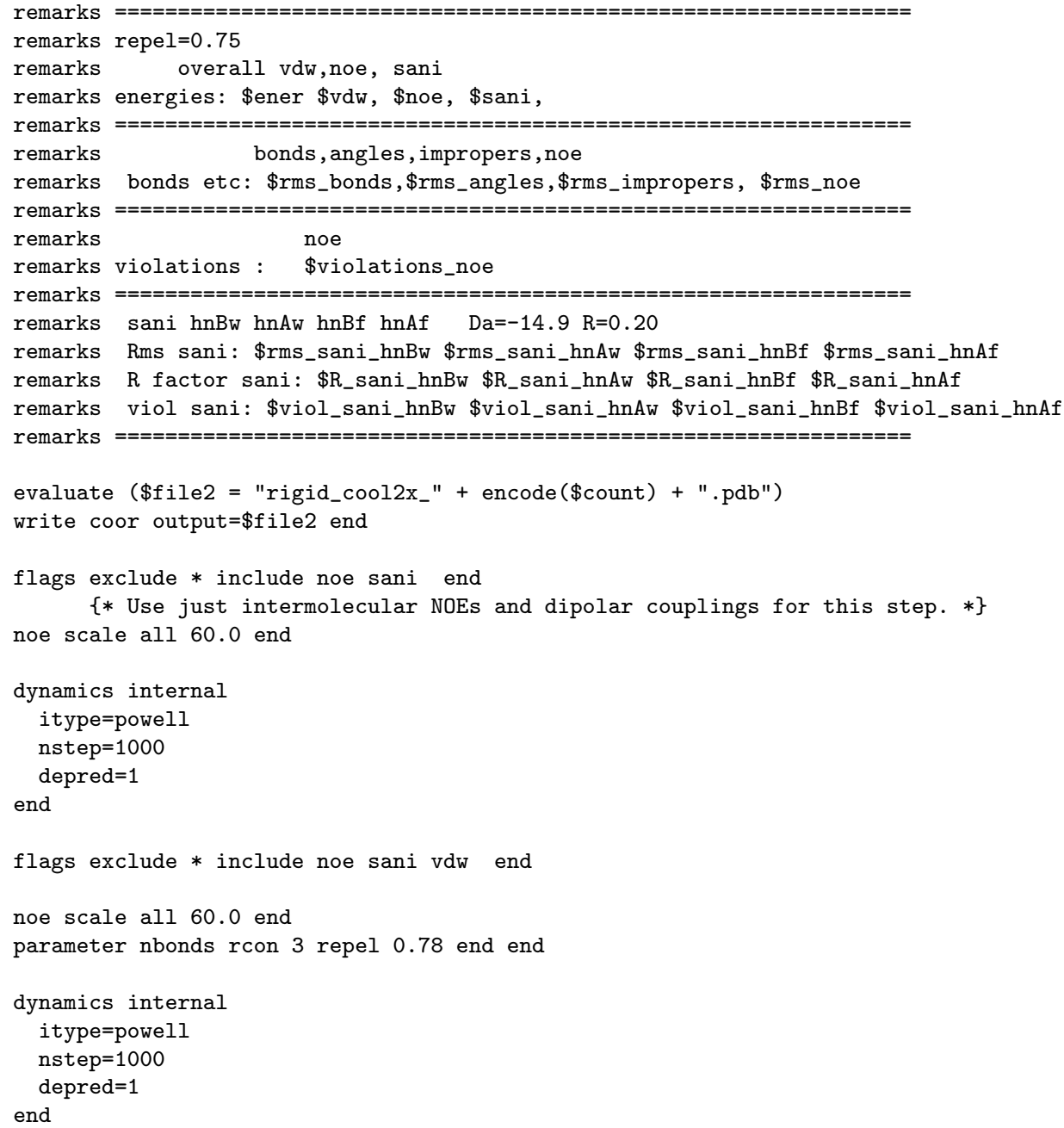




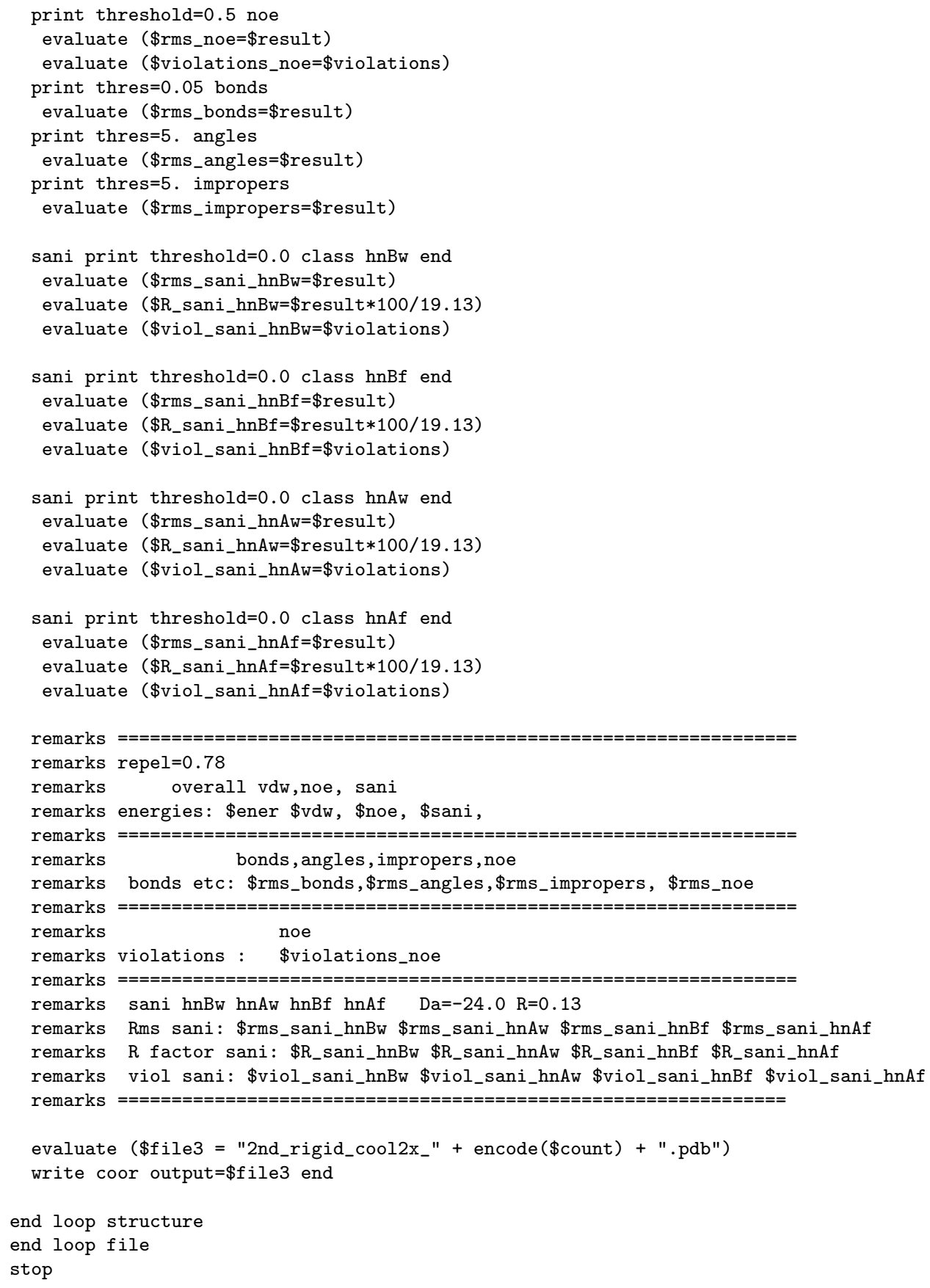




\section{D.3 Simulated annealing}

After the rigid-body docking protocol, a standard simulated annealing protocol based on a Powell minimization in cartesian coordinate space was applied and is given below.

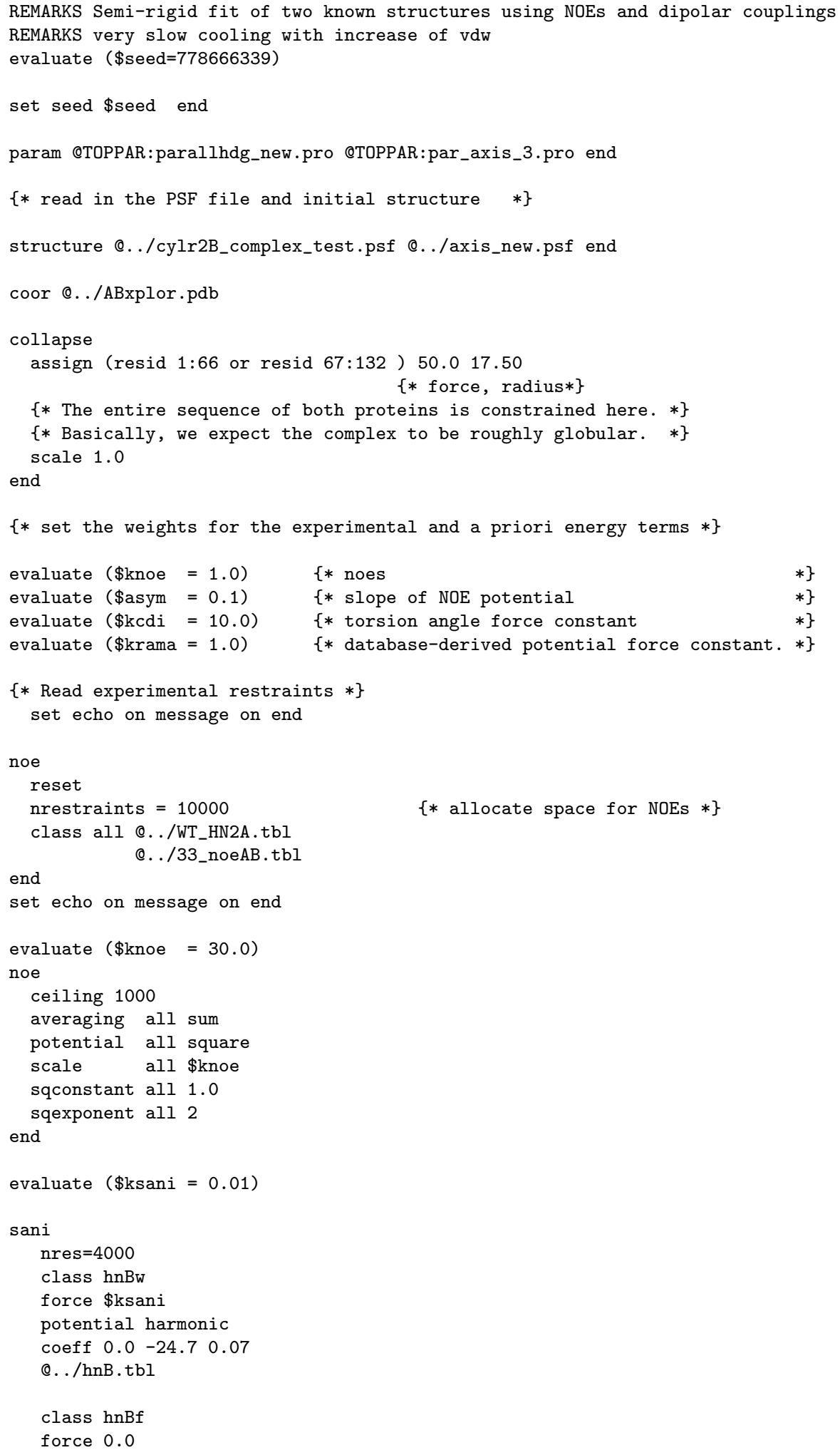




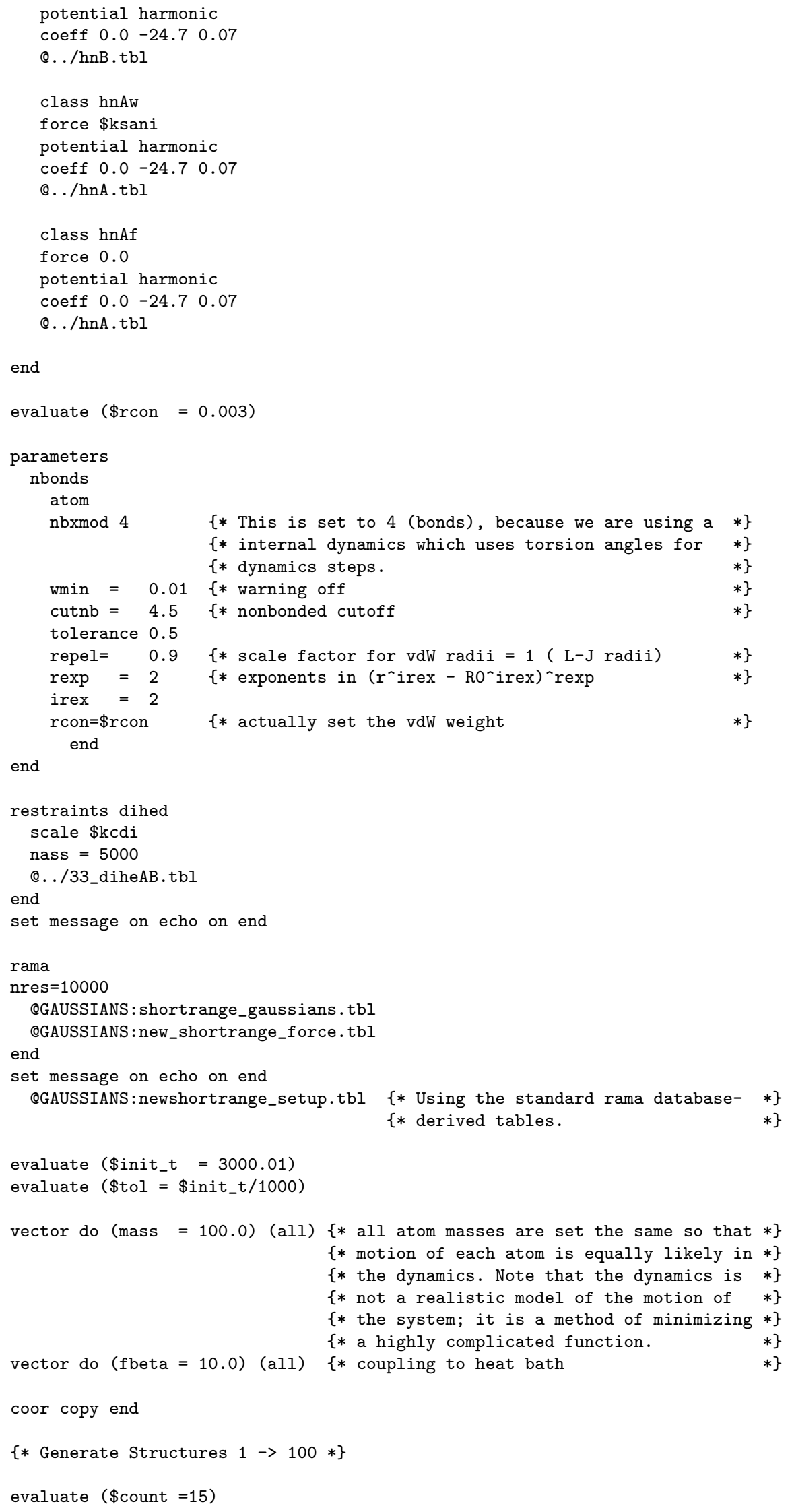

evaluate ( $\$$ init_t $=3000.01$ )

evaluate $(\$$ tol $=\$$ init_t $/ 1000)$

vector do (mass $=100.0$ ) (all) $\{*$ all atom masses are set the same so that $*$ \} $\{*$ motion of each atom is equally likely in $*$ \} $\{*$ the dynamics. Note that the dynamics is *\} 
while (\$count < 100) loop structur

evaluate $(\$$ count $=\$$ count +1$)$

$\{====>\}$

$\{*$ Filename $(s)$ for embedded coordinates. $*\}$

vector do $(\mathrm{x}=\mathrm{xcomp})(\mathrm{all})$

vector do ( $y=y c o m p)$ (all)

vector do $(z=z$ comp $)$ (all)

flags

exclude *

include bonds angles impr vdw noe cdih rama sani coll

$\{*$ turn on experimental data-based energy terms for dynamics and a priori $*$ \}

$\{*$ restraints, such as the rama torsion correlation term and the radius of $*$ \}

* gyration collapse term. end

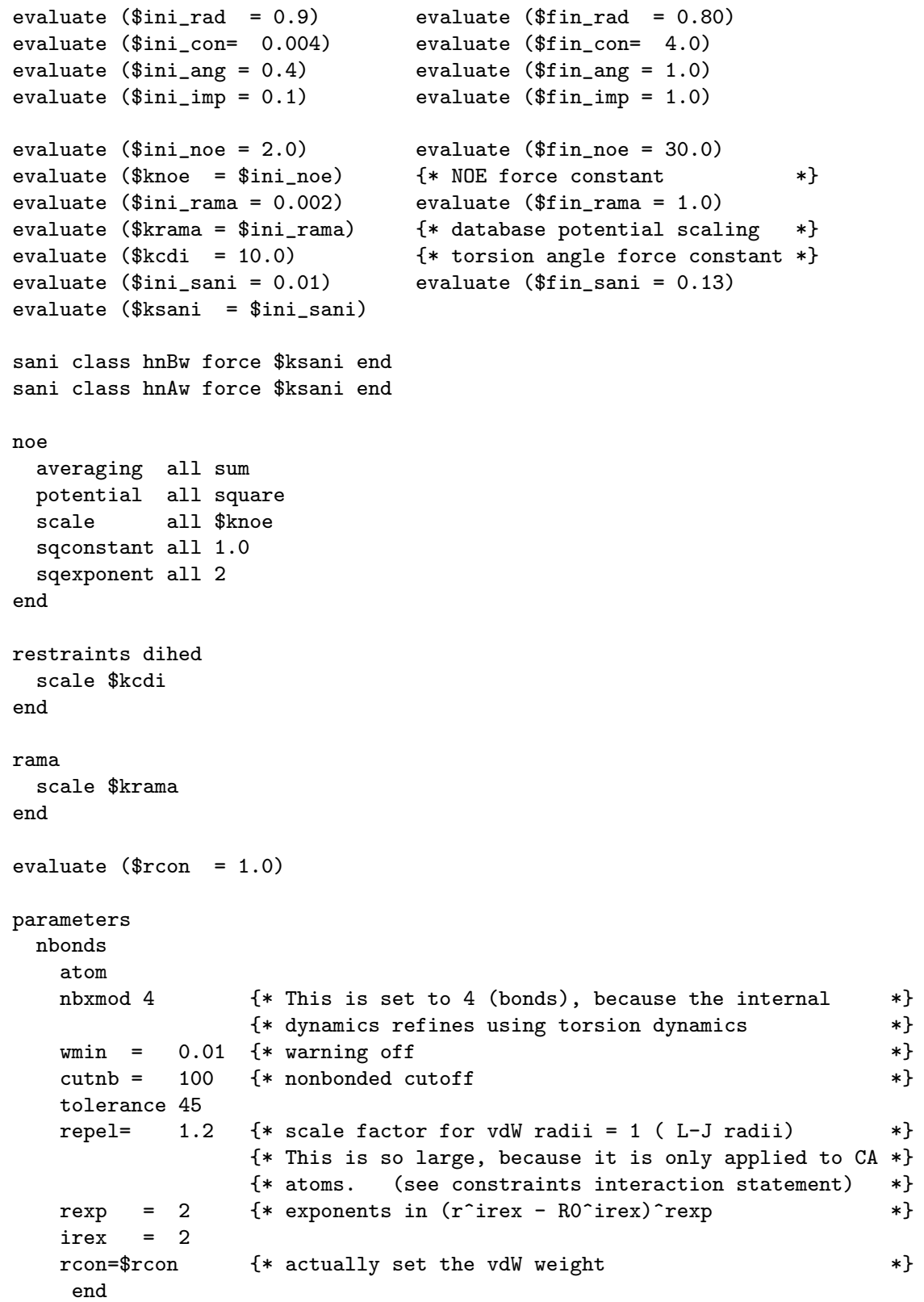




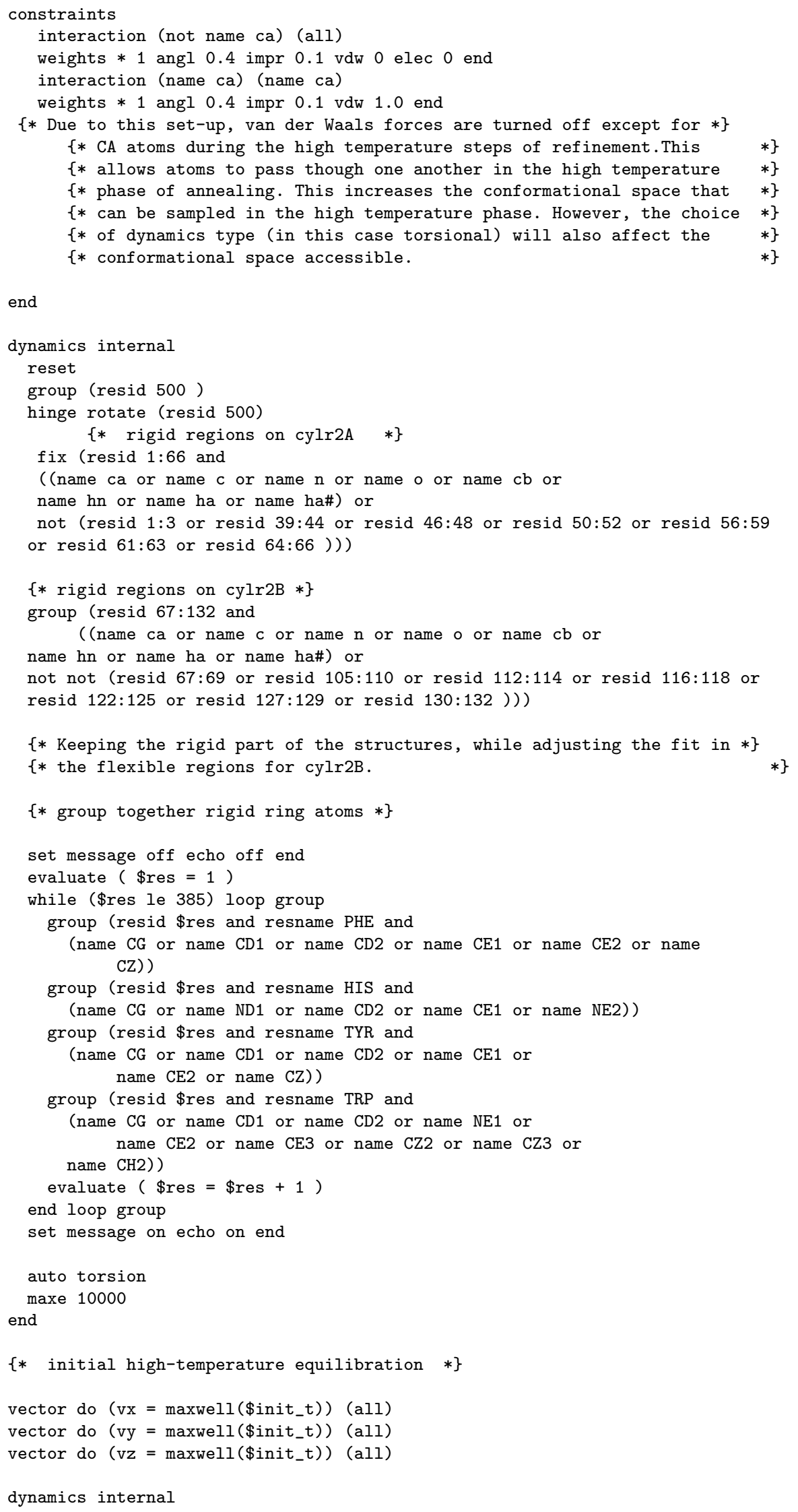

end

dynamics internal

reset

group (resid 500 )

hinge rotate (resid 500)

$\{*$ rigid regions on cylr $2 \mathrm{~A} \quad *\}$

fix (resid 1:66 and

( (name ca or name $c$ or name $\mathrm{n}$ or name or name $\mathrm{cb}$ or

name hn or name ha or name ha\#) or

not (resid 1:3 or resid $39: 44$ or resid $46: 48$ or resid 50:52 or resid 56:59

or resid $61: 63$ or resid $64: 66$ )))

$\{*$ rigid regions on cylr2B *\}

group (resid 67:132 and

( (name ca or name $c$ or name $\mathrm{n}$ or name or name $\mathrm{cb}$ or

name hn or name ha or name ha\#) or

not not (resid 67:69 or resid 105:110 or resid 112:114 or resid 116:118 or

resid $122: 125$ or resid $127: 129$ or resid 130:132)))

$\{*$ Keeping the rigid part of the structures, while adjusting the fit in $*$ \}

$\{*$ the flexible regions for cylr2B.

$\{*$ group together rigid ring atoms $*\}$

set message off echo off end

evaluate ( $\$$ res $=1$ )

while (\$res le 385) loop group

group (resid \$res and resname PHE and

(name CG or name CD1 or name CD2 or name CE1 or name CE2 or name CZ))

group (resid \$res and resname HIS and

(name CG or name ND1 or name CD2 or name CE1 or name NE2))

group (resid \$res and resname TYR and

(name CG or name CD1 or name CD2 or name CE1 or name CE2 or name CZ))

group (resid \$res and resname TRP and

(name CG or name CD1 or name CD2 or name NE1 or name CE2 or name CE3 or name CZ2 or name CZ3 or

name $\mathrm{CH} 2)$ )

evaluate $($ \$res $=$ \$res +1$)$

end loop group

set message on echo on end

auto torsion

maxe 10000

end

$\{*$ initial high-temperature equilibration $*\}$

vector do $(v x=\operatorname{maxwell}(\$$ init_t $))($ all $)$

vector do $\left(v y=\operatorname{maxwell}\left(\$ i n i t_{-} t\right)\right)($ all $)$

vector do $(v z=\operatorname{maxwell}(\$$ init_t $))($ all $)$

dynamics internal 


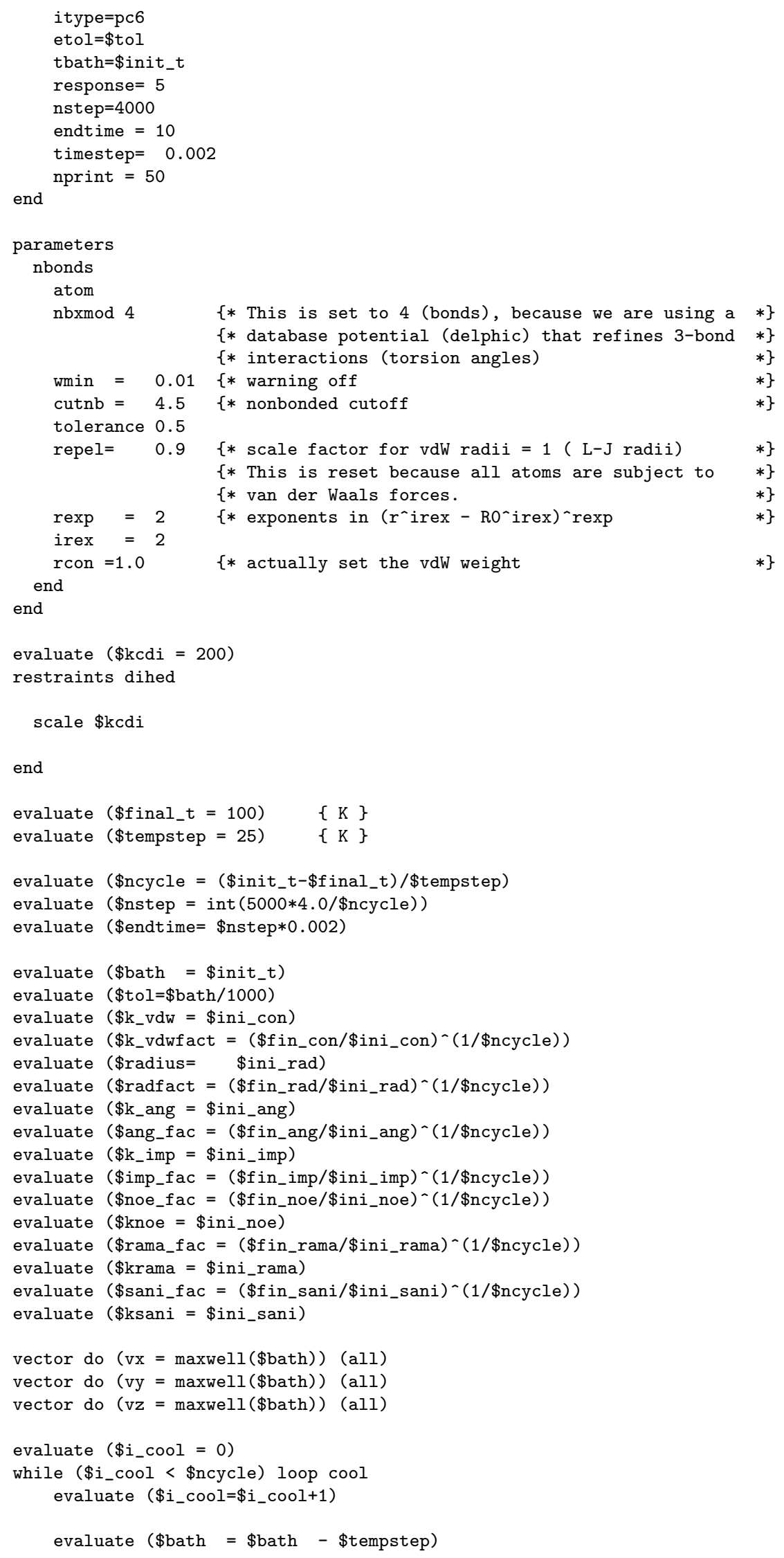




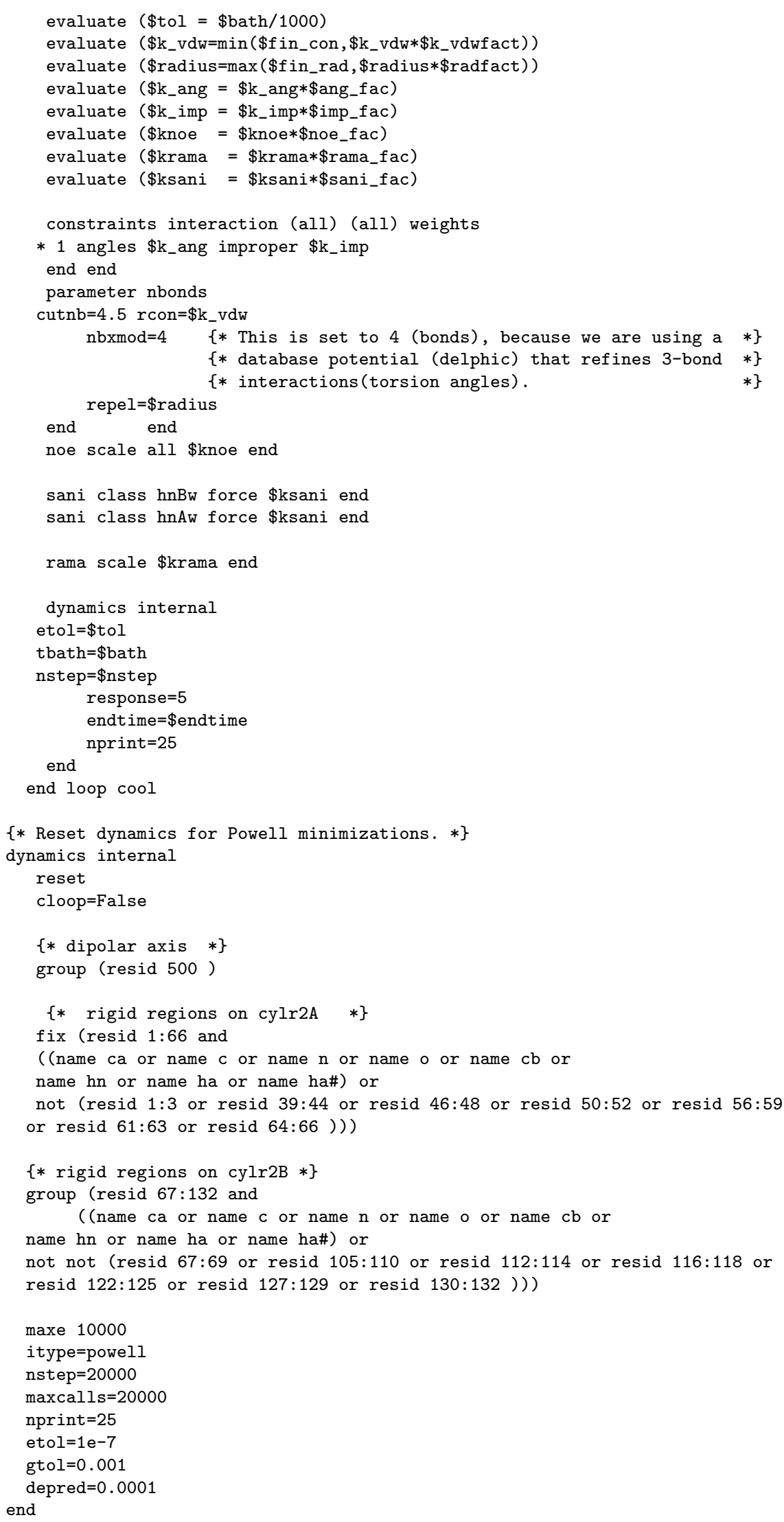




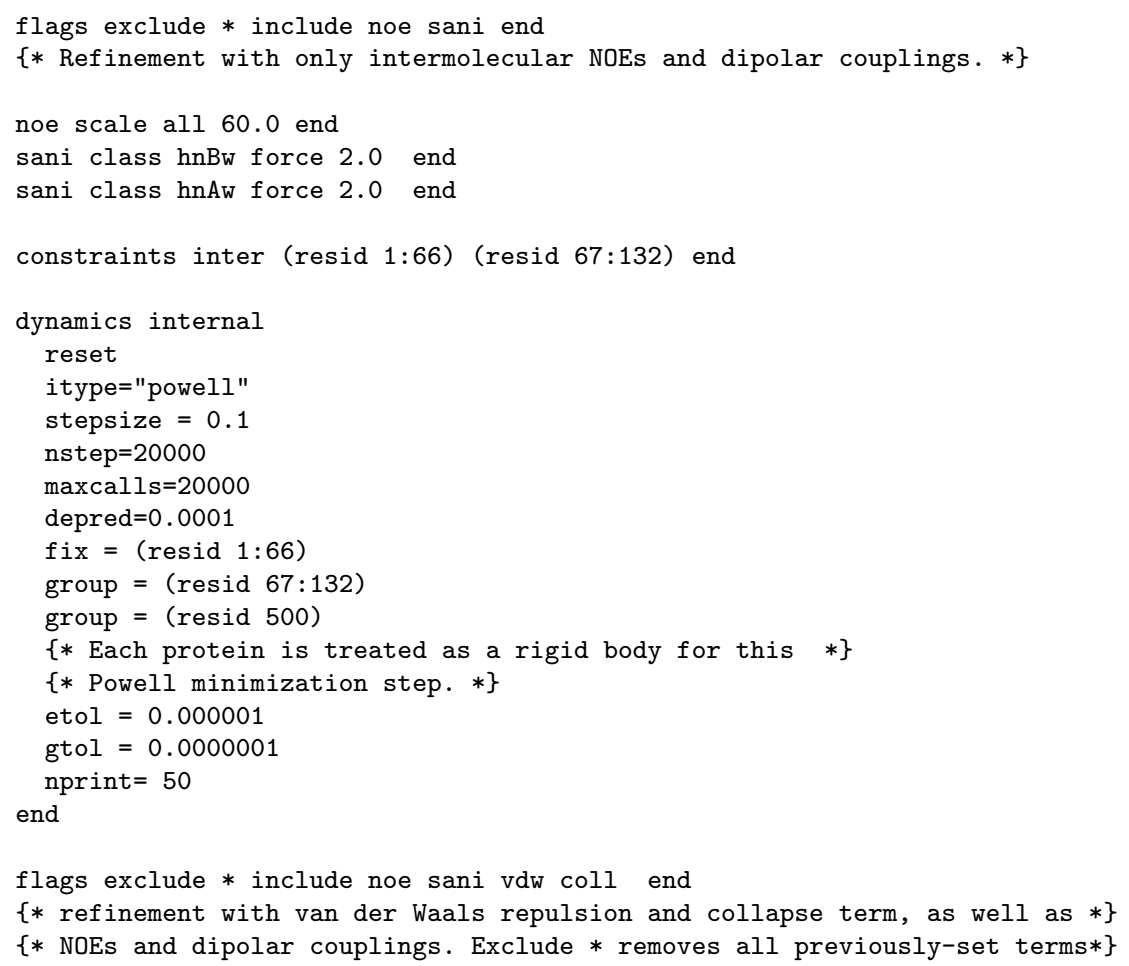




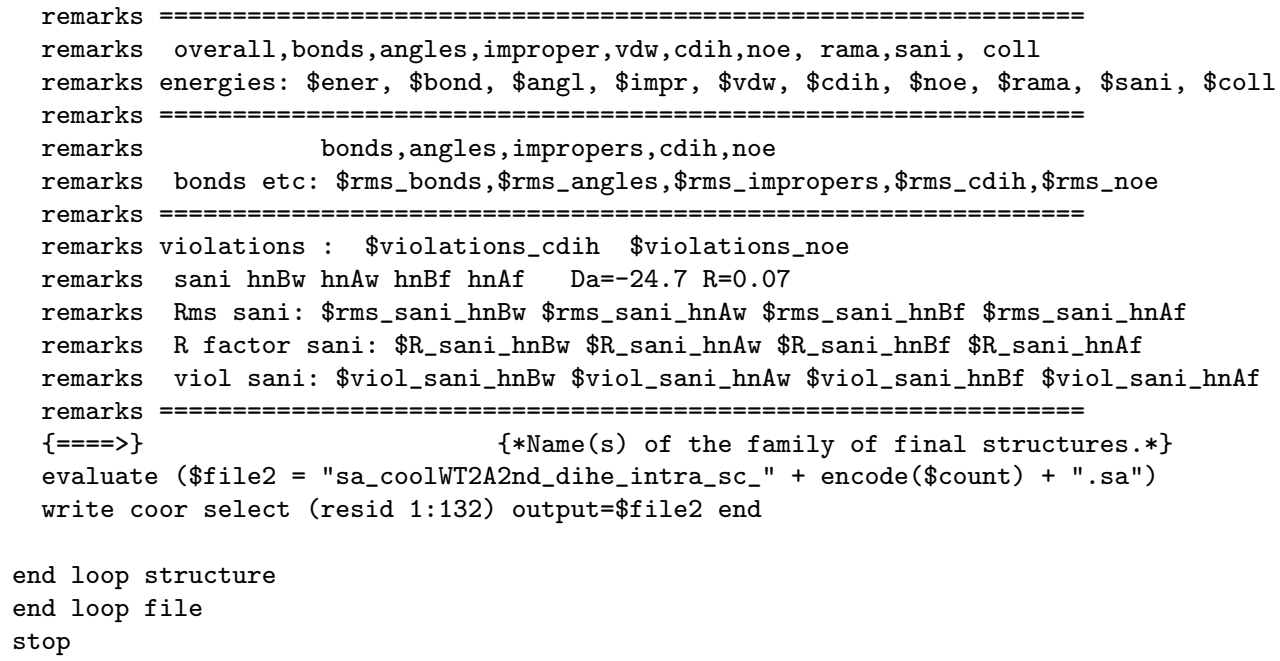




\title{
Curriculum Vitae
}

\author{
Personal data \\ Name \\ Sigrun Rumpel \\ Date of birth \\ November $23^{r d}, 1978$ \\ Place of birth \\ Göttingen, Germany \\ Citizenship \\ German
}

\begin{abstract}
Education and studies
1985-1991 Primary and grammar School, Göttingen, Germany

07/1998 General qualification for university entrance

11/1998 Beginning of biochemistry studies at the University of Bayreuth, Germany

10/2000 Intermediate diploma in biochemistry at the University of Bayreuth, Germany

2000-2002 Advanced studies of biochemistry at the University of Bayreuth, Germany

04/2002 Final examination in biochemistry at the University of Bayreuth, Germany

05/2002- Diploma thesis with the title "Expression and NMR-spectroscopic

11/2002 examination of a PTH(1-34)-analogon and the N-terminal domain of the PTH-receptor" at the chair of biopolymers from Prof. Dr. Paul Rösch

01/2003- PhD. thesis with the title "Protein NMR studies of two systems 09/2006 involved in bacterial pathogenicity " in the department of NMRbased structural biology of Prof. Dr. Christian Griesinger at the Max Planck Institute for Biophysical Chemistry in Göttingen, Germany
\end{abstract}

\section{Other experience}

1996-2002 Tennis trainer for children and adults with trainer certificate since 1997 


\title{
Lebenslauf
}

\author{
Persönliche Daten \\ Name \\ Sigrun Rumpel \\ Geburtstag \\ 23.11.1978 \\ Geburtsort \\ Göttingen \\ Staatsangehörigkeit deutsch
}

\section{Schulbildung}

1985-1989 Grundschule Herberhausen der Stadt Göttingen

1989-1991 Orientierungsstufe Lutherschule in Göttingen

1991-1998 Theodor-Heuss-Gymnasium in Göttingen

07/1998 Allgemeine Hochschulreife

\section{Studium}

11/1998 Beginn des Diplomstudienganges Biochemie an der Universität Bayreuth

10/2000 Vordiplom Biochemie an der Universität Bayreuth

2000-2002 Hauptstudium Biochemie an der Universität Bayreuth

04/2002 Diplomprüfung

05/2002- Anfertigung der Diplomarbeit mit dem Titel ,Expression und

11/2002 NMR-spektroskopische Untersuchung eines PTH(1-34)-Analogons und der N-terminalen Domäne des PTH-Rezeptors" am Lehrstuhl für Biopolymere von Prof. Dr. Paul Rösch

01/2003- Anfertigung der vorliegenden Arbeit mit dem Titel ,Untersuchun-

09/2006 gen mittels Protein NMR an zwei Systemen mit Einfluss auf bakterielle Pathogenität" in der Abteilung NMR-basierte Strukturbiologie von Prof. Dr. Christian Griesinger am Max Planck Institut für Biophysikalische Chemie in Göttingen

\section{Sonstige Tätigkeiten}

1996-2002 Tennistrainerin für Kinder und Erwachsene mit Trainerschein seit 1997 